\title{
Development and Integration of Light Water Reactor (LWR) Materials Corrosion Degradation Codes into Grizzly
}

Digby D. Macdonald, Jie Yang, Balazs Fekete, louri Balachov, Benjamin W Spencer

December 2019

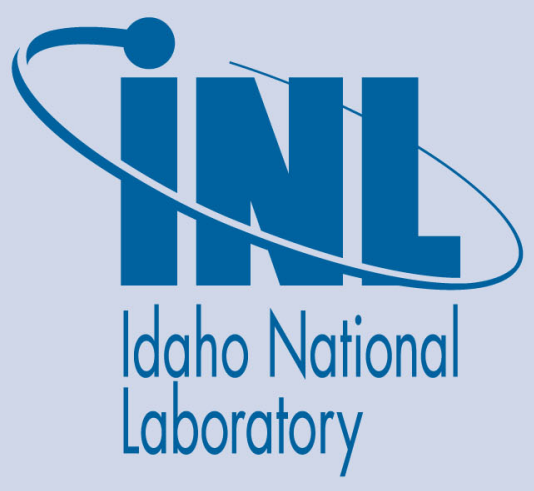

The INL is a U.S. Department of Energy National Laboratory operated by Battelle Energy Alliance 


\section{Development and Integration of Light Water Reactor (LWR) Materials Corrosion Degradation Codes into Grizzly}

Digby D. Macdonald, Jie Yang, Balazs Fekete, louri Balachov, Benjamin W Spencer

December 2019

Idaho National Laboratory Idaho Falls, Idaho 83415

http://www.inl.gov

Prepared for the U.S. Department of Energy Office of Nuclear Energy Under DOE Idaho Operations Office

Contract DE-AC07-05ID14517 


\title{
Nuclear Energy University Program Final Technical Report
}

\section{7-2019}

\section{Development and Integration of Light Water Reactor (LWR) Materials Corrosion Degradation Codes into Grizzly}

Digby D. Macdonald ${ }^{1}$, Jie Yang ${ }^{1}$, Balazs Fekete ${ }^{1}$, Iouri Balachov ${ }^{2}$, and Benjamin Spencer ${ }^{3}$

\author{
${ }^{1}$ Department of Nuclear Engineering, University of California at Berkeley \\ Berkeley, CA 94720, USA \\ 24-D Power, USA \\ ${ }^{3}$ Idaho National Engineering Laboratory, USA
}

Submitted to:

DOE/NEUP

Award DE-NE0008541

E-mail: macdonald@berkeley.edu

December 15, 2019 


\section{Abstract:}

The primary objectives of the proposed research was the development of deterministic, physico-electrochemical models for predicting the accumulation of localized corrosion damage (pitting corrosion, stress corrosion cracking and corrosion fatigue) in the primary coolant circuits of the currently operating fleet of Light Water Reactors (LWRs) and the embedment of the models into the Grizzly code currently currently being developed at the Idaho National Laboratory as part of their program on nuclear power plant component aging. Localized corrosion in LWR (BWR and PWR) primary coolant circuits (PCC) is primarily an electrochemical phenomenon, augmented by mechanics and microstructure, the rate of which is determined by certain electrochemical properties, such as the electrochemical corrosion potential (ECP), solution conductivity, temperature, $\mathrm{pH}$, flow rate, and the kinetics of the reduction of redox depolarizers (e.g. $\mathrm{O}_{2}, \mathrm{H}_{2} \mathrm{O}_{2}$, and $\mathrm{H}_{2}$ ) on the surfaces external to the crack, in addition to mechanical loading (stress intensity factor on the crack) and micro-structural/micro-chemical factors (grain size, precipitates, etc). Because the efficient control of environmentally-assisted cracking (EAC) damage accumulation requires the accurate control of these parameters, it is necessary to develop codes that can accurately predict ECP and crack growth rate (CGR) at any point in the primary coolant circuit (PCC) over wide ranges of temperature $\left(25{ }^{\circ} \mathrm{C}\right.$ to $\left.320{ }^{\circ} \mathrm{C}\right), \mathrm{pH}(6-8), \mathrm{ECP}(-0.9$ $\mathrm{V}_{\text {she }}$ to $\left.0.2 \mathrm{~V}_{\text {she }}\right)$, solution conductivity, flow rate $(1-6 \mathrm{~m} / \mathrm{s})$, and stress intensity factor $\left(5 \mathrm{MPa} . \mathrm{m}^{1 / 2}\right.$ - $50 \mathrm{MPa} \cdot \mathrm{m}^{1 / 2}$ ). Knowledge of these parameters, along with suitable damage prediction codes, would allow an operator to predict the accumulated damage in PCC as a function of the future operating history of the reactor (the "corrosion evolutionary path," CEP). In performing this study, we have further developed our previous prediction codes in the form of BWR_MASTER and PWR_MASTER by upgrading all sub-models for calculating radiolytic species concentration, $\mathrm{ECP}$, and crack growth rate (CGR) as a function of reactor operating variables (power, radiation density, temperature, location in the PCC, flow velocity, coolant $\mathrm{pH}$ and conductivity, and operating history). The codes have been used to predict the accumulation of IGSCC damage in Type 304 SS in the core shroud of a BWR over a fuel cycle and to estimate the damage at the same location during start-up, considering transients in reactor power, temperature, and conductivity (due to hide-out/hide-out return). The predicted damage is in good agreement with plant observation. Regarding PWRs, we have developed two new models for calculating CGR in millannealed, Alloy 600, by considerably upgrading the MPM (mixed potential model for estimating 
the ECP), the Coupled Environment Fracture Model (CEFM) that were originally developed to predict ECP and CGR in sensitized stainless steels, to predict CGR in nickel-base alloys, as well as developing a micro-void pressurization model for also estimating CGR in MA Alloy 600. Both CGR models yield CGRs that are in excellent agreement with the experiment. We have also successfully developed crack initiation models for both stainless steels and mill-annealed, Alloy 600. That for stainless steels is based on a pit being the initiation site, whereas in that for millannealed, Alloy 600 postulates that initiation occurs at emergent grain boundaries that have been wedged open by internal oxidation. Experiments show that the crack initiation time (CIT) is highly distributed and is a seemingly, random quantity that exhibits trends with various system properties, such as surface stress, hardness, yield strength, etc. Although not identified in the SoW, we have developed a theoretical framework for describing the distributions in the CIT by assuming a normal distribution in the number of initiation sites with respect to surface stress. It is well-known from experiment that CGRs are highly distributed quantities also, with almost all systems exhibiting log-normal distributions. In work outside of that proposed, we used the ANN and CEFM to confirm that a log-normal distribution in CGR is expected theoretically if the independent variables are normally distributed. This work essentially defines the accuracy that one might expect in the calculated CGR due to randomness in the independent variables. To provide fundamental, input data for the various models, we have made extensive measurement of the kinetic parameters (exchange current density and Tafel constants) for the oxygen electrode reaction (OER) and the hydrogen electrode reaction (OER) on stainless steels (Types 304 and 316) and nickel-base alloys (Alloys 600 and 690) in reactor coolant at temperatures to $300{ }^{\circ} \mathrm{C}$ and as a function of $\mathrm{pH},\left[\mathrm{O}_{2}\right]$, and $\left[\mathrm{H}_{2}\right]$. We have also optimized the Point Defect Model for passivity and passivity breakdown on measured electrochemical impedance spectroscopic (EIS) data for all alloys studied in this work to extract PDM parameter values that are then used to calculate the passive current density (general corrosion rate) and barrier layer thickness as a function of voltage, temperature, and $\mathrm{pH}$. Thus, we have, at last, a reasonably comprehensive database for model parameters. Also outside of the SoW, we have developed a new, innovative method for monitoring, in situ, the hydriding of zirconium alloy fuel cladding under reactor operating conditions by optimizing a modified PDM for hydride/oxide formation on experimental EIS data. We have demonstrated the technique on pure zirconium in PWR coolant at $250{ }^{\circ} \mathrm{C}$. Finally, also in addition to the SoW, we developed Fracture Impedance Spectroscopy, which draws an analogy 
with current flow in a passive electrical circuit in analyzing crack growth under fatigue loading conditions.

\section{The Accomplishments Achieved are Shown Below:}

1. We have found a slight decrease in CGR in Alloy 600 in PWR primary with increasing hydrogen concentration, especially in the low hydrogen concentration range and both the Artificial Neural Network and the CEFM models failed to confirm the existence of a peak in the CGR close to the potential of the $\mathrm{Ni} / \mathrm{NiO}$ phase boundary. We, therefore, consider this postulate to be unproven with resolution awaiting much more accurate CGR measurements.

2. Comparison of experimental and computed values showed that the Mixed Potential Model that includes the quantum mechanical correction for electronic charge carrier tunneling through the barrier layer, for the calculating ECP is viable and that the exchange current density calculated for the hypothetical, bare ("film-free") surface satisfactorily accounts for the ECP. This represents a major advance in the theory of electrochemical kinetics, because it is now only necessary to specify a single set of exchange current density and Tafel constants for each redox reaction, rather than a set for each value of the ECP (i.e., for each temperature, $\left[\mathrm{H}_{2}\right],\left[\mathrm{O}_{2}\right],\left[\mathrm{H}_{2} \mathrm{O}_{2}\right]$, flow velocity, etc.) in order to calculate the ECP and CGR under any given set of environmental conditions. This innovation greatly reduces the dimensionality of the problem of predicting the evolution of damage in nuclear power reactor primary coolant circuits.

3. We have updated the crack tip strain rate model using Hall's equation, which is based on the separation of the crack tip strain rate into quasi-stationary and crack advance components. A significant effect of yield strength on the CGR is predicted, resulting in the CGR increasing by up to $15 \%$ at a $\mathrm{K}_{\mathrm{I}}$ value of $40 \mathrm{MPa} \cdot \mathrm{m}^{1 / 2}$, when $\sigma_{\mathrm{y}}$ changes from 200 to $500 \mathrm{MPa}$. The theoretical model for the hydrogen-induced cracking based on grain boundary void pressurization by hydrogen was developed and incorporated into the CEFM. The calculated results indicate that the hydrogen evolution at the crack tip under PWR conditions has a significant effect on the CGR. The CGR increases with increasing ECP up to a maximum value in the range of $-1 \mathrm{~V}_{\text {she }}<\mathrm{ECP}<-0.5 \mathrm{~V}_{\text {she, }}$, and good agreement is found with experimental observations. The CGR is an increasing function of the temperature in the range of $200{ }^{\circ} \mathrm{C}$ 
and $350{ }^{\circ} \mathrm{C}$ and varies by three orders of magnitude due to thermally activated processes at the crack tip.

4. Experimental investigations have shown that cracks in stainless steels in simulated BWR primary coolant are often observed to nucleate within an emergent grain face (probably from a corrosion pit), grow transgranularly until it intersects a grain boundary and then grows intergranularly, after that. We have developed a model that predicts the time that it takes to nucleate and grow a critical pit, i.e., one whose stress intensity factor exceeds $\mathrm{K}_{\mathrm{ISCC}}$ for the prevailing stress (including residual and operational stresses) considering the properties of the external environment.

5. ECP is a strong function of $\left[\mathrm{O}_{2}\right],\left[\mathrm{H}_{2} \mathrm{O}_{2}\right]$, and $\left[\mathrm{H}_{2}\right]$, resulting in a sigmoid dependence of ECP on $\left[\mathrm{O}_{2}\right]$. Pit growth rate (PGR) increases exponentially with increasing ECP and is a strong function of conductivity as per the Coupled Environment Models. Increasing temperature results in a displacement of the ECP towards more negative values, hence decreasing the pit growth rate and increasing the crack initiation time. PGR increases with decreasing pit radius as for SCC, due to the IR potential drop down the pit cavity.

6. The literature survey revealed that the internal oxidation of Alloy 600 had received the most experimental support as a viable mechanism of stress corrosion cracking in PWRs. Based on the information collected from the literature, we have developed a model for calculating crack initiation time in PWRs. The model postulates that the water penetrates the emergent grain boundaries and reacts with the $\mathrm{Cr}$ of Alloy 600, forming a $\mathrm{Cr}_{2} \mathrm{O}_{3}$ oxide layer that wedge open the grain boundary. The model reproduces the effects of the following parameters: applied stress, temperature, cold work, grain boundary segregations, water chemistry, $\mathrm{pH}$, electrochemical potential.

7. The crack initiation time is a sensitive function of the temperature and ECP under PWR primary coolant conditions. Work was initiated on predicting the experimentally-observed, wide distribution in crack initiation time by assuming that the local crack initiation sites are normally distributed with respect to local, surface stress (PWR) and/or with respect to pit depth (BWR). We identify the next steps that should be taken to resolve the "greatest theoretical problem in corrosion science" and hence, in the prediction of localized corrosion damage in nuclear power reactor coolant circuits. 
8. We demonstrated, theoretically, using both the CEFM and ANN, that if the independent variables in the IGSCC in sensitized BWR primary coolant conditions are individually distributed normally, the resulting crack growth rate (CGR) is distributed log-normally, in agreement with experiment. These calculations define the ultimate accuracy with which the CGR can be determined under practical conditions.

9. The BWR_MASTER code has been made to be compatible with the macOS c compiler to facilitate its use with Grizzly, which is designed to run on UNIX-based operating systems such as macOS. A macOS-compatible version of the BWR_MASTER executable file has been created. Computations have been performed on macOS to confirm identity with results of BWR_MASTER computations performed under the Windows operating system. Calculation time for 60 years of BWR operation is under 5 minutes at iMac $2.7 \mathrm{GHz}$ Intel Core i5 processor, 8 GB $1333 \mathrm{MHz}$ DDR3 memory, 2 TB hard drive, and macOS Sierra operating system version 10.12.3. Interface files created for post-processing for data analysis and visualization with Excel and Gnuplot.

10. The sensitivity analysis of integral output parameters to variation of input parameters has been performed. The following integral parameters have been selected: concentration of hydrogen, oxygen and hydrogen peroxide, ECP, and crack growth rate. A total of 79 input parameters have been selected, including reactor modeling parameters, reaction rates, radiolytic yields of species, and input parameters for the Mixed Potential Model (MPM). Sensitivities have been estimated for each point along with the coolant flow under normal and hydrogen $\left(0.5 \mathrm{ppm} \mathrm{H}_{2}\right.$ in feedwater) water chemistry conditions.

11. Integral parameters are most sensitive to variations in radiolytic yields, Tafel constants and exchange current density for the redox reactions on stainless steels, and to uncertainty in the standard potentials for these reactions. Incorporation of the most recent data on radiolytic yields into BWR_MASTER resulted in much better agreement with the measured oxygen concentration and ECP from the Leibstadt BWR mini-test under normal water chemistry (NWC) and with the results from the $0.5 \mathrm{ppm}$ hydrogen water chemistry (HWC) in-plant tests. Re-evaluation of standard potentials for oxygen and hydrogen peroxide is recommended.

12. The generic chemical kinetics algorithm was implemented by replacing the "fixed" model radiolysis function Chemical_Kinetics to calculate gains and losses of species and by modifying the radiolysis functions for calculating radiolytic yields from neutron and gamma 
radiation, convection, and two-phase flow terms. A list of chemical species is defined in the input file YIELDS.DAT as string constants, which allows expansion or reduction of the number of species. Arbitrary lists of chemical reactions, reaction rates, and activation energies are taken from input file CHEMRAT.DAT, where chemical reactions are also defined as string variables.

13. Comparison of calculated distributions in ECP, CGR, and concentrations of $\mathrm{O}_{2}, \mathrm{H}_{2} \mathrm{O}_{2}$, and $\mathrm{H}_{2}$ along the BWR HTC under NWC and HWC conditions confirms that the previous "fixed" version of BWR_MASTER and modified BWR_MASTER with generic chemical kinetics delivered identical results. Trial simulation of species concentrations in BWR HTC has been performed with two "as is" alternative sets of chemical reactions: one by Christensen and another by Ishigure. Both sets have reaction rates for $288{ }^{\circ} \mathrm{C}$, which have been used as an input. No special treatment of reactions within the BWR_MASTER source code has been enacted. For the Christensen reaction set, calculated concentrations of $\mathrm{H}_{2}$ are in good agreement, except for the Core Bypass region under NWC, and the calculated concentrations of $\mathrm{H}_{2} \mathrm{O}_{2}$ deviate significantly from each other, both for NWC and HWC. This is attributed to under-prediction of the $\mathrm{H}_{2} \mathrm{O}_{2}$ decomposition rate, and calculated concentrations of $\mathrm{O}_{2}$ are in satisfactory agreement for NWC but deviate significantly under HWC for possibly the same reason.

14. The previously used CAM PSU data for chemical reactions are much more adequate and produce a better agreement with plant measure data than do the Christensen or Ishigure data sets.

15. An attempt to assess the feasibility and to estimate the cost/benefit ratio of reducing the number of chemical reactions was undertaken. Candidate reactions were removed from the input data set, as had been identified in the sensitivity analysis. Thus, if the calculated ECP was found to be insensitive to the kinetic parameters for a given reaction, the reaction was removed from the input file to yield a reduced reaction set. The impact of using the reduced reactions set on the calculated ECP under NWC was estimated. Maximal deviation of about $100 \mathrm{mV}$ was observed in the Upper Plenum and $25 \mathrm{mV}$ in the Recirculation Line and the Bottom of the Lower Plenum. While, in general, reduction of the reaction rates does not result in a more attractive cost/benefit ratio, in some practical cases, it may be justified, for example, in parametric scientific or engineering studies. 
16. An attempt to adjust reaction rates in the Christensen reaction set to match the calculated concentration of $\mathrm{H}_{2} \mathrm{O}_{2}$ with those calculated with the CAM_PSU reaction set has been made as an example. Agreement with CAM PSU based data has been improved significantly after increasing the rate of reaction \#33 (decomposition of $\mathrm{H}_{2} \mathrm{O}_{2}$ ) by two orders of magnitude. The Christensen data set must be adjusted in order to produce adequate simulation results for species concentrations, if desired. The use and adjustment of Ishigure's data set are not recommended for further consideration.

17. The generic kinetics algorithm was implemented into the UCB PWR_MASTER code. PWR simulations have been performed to check the results of the modified PWR_MASTER code. The BWR_MASTER code has been integrated with Grizzly by enabling it to use Grizzlygenerated stress intensity factors for predicting crack propagation. Simulation of crack propagation in BWR Core Shroud weld H6a has been performed for stress intensity factors from the original BWR_MASTER code, Grizzly (using a reduced order fracture model), and the J-Integral method.

18. The importance of incorporating electrochemical phenomena and transients during startups and shutdowns on crack propagation and predicted component lifetime has been demonstrated.

19. A comparison of deterministic and empirical predictions of IGSCC in sensitized austenitic stainless steel, in order to calculate the accumulated damage (crack depth versus time) in a BWR in-vessel component and estimate component's lifetime for given operating conditions is described.

20. We have set up a recirculating flow loop with a controlled hydrodynamic autoclave for performing experimental measurements with a volume flow velocity of $15 \mathrm{ml} / \mathrm{min}$ through the recirculation loop but with a linear flow velocity of up to several $\mathrm{m} / \mathrm{s}$ past the specimen to accurately reproduce the conditions that exist in a reactor HTC. We have developed a new working electrode with the PEEK washers for specimen sealing and successfully performed experiments up to $300^{\circ} \mathrm{C}$.

21. The effects of temperature and $\mathrm{pH}$ on the electrochemical behavior of Alloy 600, Alloy 690, Type 304L SS, and Type 316L SS were studied in oxygenated borate buffer solutions systematically by the measurements of open circuit potential (OCP), anodic potentiostatic polarization, Mott-Schottky analysis (MSA), and electrochemical impedance spectroscopy 
(EIS). We found that the deteriorative effect of the elevated temperature on the corrosion resistance of each alloy is much more significant than the improvement induced by increasing solution $\mathrm{pH}$.

22. We developed a Mixed Potential Model (MPM), containing the Point Defect Model (PDM) for describing the passive anodic dissolution of the alloy and the generalized Butler-Volmer equation for the cathodic oxygen reduction reaction. The revised model also includes a correction to the Generalized Butler-Volmer equation for quantum mechanical tunneling of electrons and electron holes through the barrier layer in response to a linearly-dependent thickness on voltage, as noted above. The MPM was optimized on the experimental EIS data, and several kinetic parameters were extracted. We found the passive films formed on Alloy 600, Alloy 690, Type 304L SS, and Type 316L SS have n-type semiconductor character, and the dominant defect is metal interstitial. We found that the defect density of passive film plays a more important role in the corrosion behavior of alloy than the film thickness.

23. We found that the corrosion resistance of nickel-base alloys is much higher than those of stainless-steels in the same corrosive environment. The defect densities of the barrier layers of the passive films formed on nickel-based alloys are much lower than those on the stainless steels, while the thickness of the barrier layers is very similar. This confirmed that the effect of defect density on the corrosion properties of the alloy is much more significant than that of film thickness.

24. We found that the activation energy for the formation of metal interstitials is much higher for nickel alloy compared with stainless steel. The concentration of Fe interstitials is much higher than $\mathrm{Ni}$ in the barrier layers on stainless steels; therefore, the passive films on stainless steels exhibit much higher defect densities of Fe interstitials and show much lower corrosion resistance than do the nickel alloys.

25. We found that the corrosion resistance of Alloy 690 is slightly higher than that of Alloy 600, while those of Type 304L SS and Type 316L SS show very similar resistance.

26. Also outside of the SoW, we have developed a new, innovative method for monitoring, in situ, the hydriding of zirconium alloy fuel cladding under reactor operating conditions by optimizing a modified PDM for hydride/oxide formation on experimental EIS data. We have demonstrated the technique on pure zirconium in PWR coolant at $250{ }^{\circ} \mathrm{C}$. 
27. Finally, we developed Fracture Impedance Spectroscopy, which draws an analogy with the current flow in a passive electrical circuit in analyzing crack growth under fatigue loading conditions.

\section{Publications, Conference Papers, and Presentations:}

1. J. Shi, B. Fekete, J. Wang, and D.D. Macdonald, Corros. Sci., 139, 58-67 (2018).

2. B. Fekete, J. Ai, J. Yang, J. Han, W. Maeng, and D.D. Macdonald, Theor. Appl. Fract. Mec., 95, 233-241 (2018).

3. D.D. Macdonald and B. Fekete, Eng. Fail. Anal., 102, 102-110 (2019).

4. J. Yang, Y.H. Li, A.N. Xu, B. Fekete, and D.D. Macdonald, J. Nucl. Mater., 518, 305-315 (2019).

5. J. Yang, Y.H. Li, and D.D. Macdonald, Effects of Temperature and $\mathrm{pH}$ on the Electrochemical Properties of Alloy 600 in Simulated Pressurized Water Reactor Primary Water, J. Nucl. Mater., $528,151850(2020)$.

6. I. Balachov, B. Fekete, and D.D. Macdonald, Parameter Sensitivity Analysis on Deterministic Models of Stress Corrosion Cracking in Light Water Nuclear Reactors, published in EPRI's 21st Water Chemistry Conference, San Francisco, Sept 2018.

7. B. Fekete, I. Balachov, and D.D. Macdonald, Deterministic Modeling of Stress Corrosion Cracking in Light Water Nuclear Reactors, presented in EPRI's 21st Water Chemistry Conference, San Francisco, Sept 2018.

8. J. Yang, Y.H. Li, and D.D. Macdonald, Experimental Measurement of Kinetic Parameters for Electrochemical Reactions on LWR Materials, presented in EPRI NPC Workshop, San Francisco, Sept 2018.

9. Y.H. Li, J. Yang, D.D. Macdonald, and S.Z. Wang, Study of Steady-state Electrochemical Reactions on Stainless Steels in Elevated Temperature Aqueous Systems, presented in EPRI's 21st Water Chemistry Conference, San Francisco, Sept 2018.

10. D.D. Macdonald presented invited seminars at Oak Ridge National Laboratory on the work on April 17 and 18, 2017. 
11. I. Balachov, B. Fekete, D.D. Macdonald, and B. Spencer, Lifetime Estimation of a BWR Core Shroud in Terms of IGSCC, submitted to Nuclear Science and Technology (2019).

12. J. Shi, R. Gupta, B. Fekete, J.H. Wang, and D.D. Macdonald, Theoretical Dependence of the Distribution in Crack Growth Rate in Type 304SS on Various Factors, submitted to Corrosion Science (2019).

13. J. Yang, Y.H. Li, and D.D. Macdonald, Effects of Temperature and $\mathrm{pH}$ on the Electrochemical Properties of Type 304L SS and Type 316L SS in Simulated Pressurized Water Reactor Primary Water, J. Nucl. Mater., in preparation (2019).

14. I. Balachov, B. Spencer, and D.D. Macdonald, Modeling the Accumulation of IGSCC Damage in BWR Core Shrouds During Reactor Start-up and Shut-down, in preparation (2019).

15. D.D. Macdonald, I.Balachov, J. Yang, and B. Fekete, Deterministic Prediction of Stress Corrosion Cracking Damage in Water-Cooled Nuclear Power Reactors, J. Nucl. Mater., in preparation (2019).

\section{Students and Researchers that Trained on the Project:}

- Two graduate students, two post-doctoral scholars, and three visiting scholars, carried out work on this project. 


\section{Table of Contents}

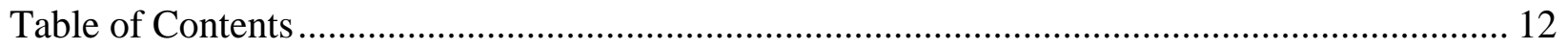

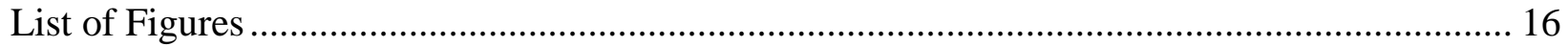

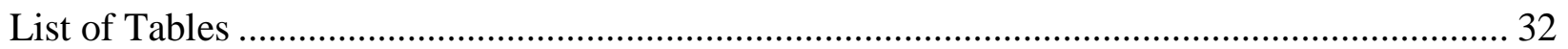

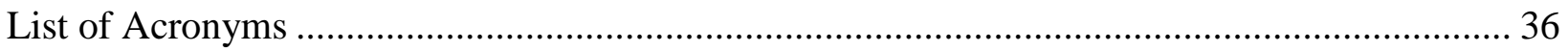

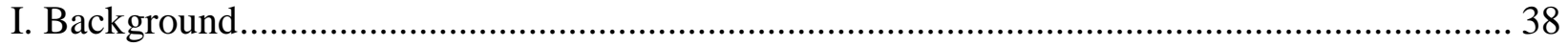

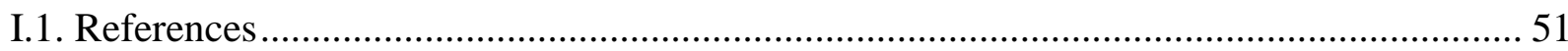

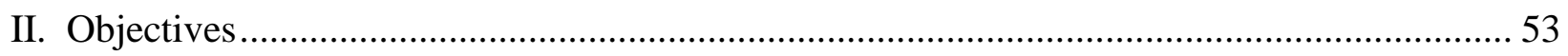

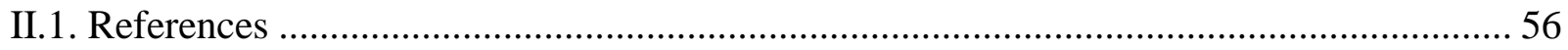

III. Upgrading existing LWR codes to currently operating LWRs............................................. 56

III.1. Quantum mechanical correction of ECP calculation ..................................................... 56

III.2. Upgrading the $\mathrm{pH}$ model in the PWR code................................................................ 59

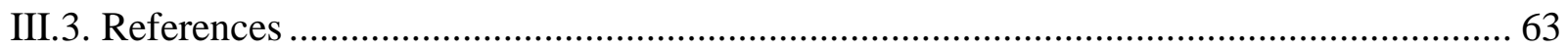

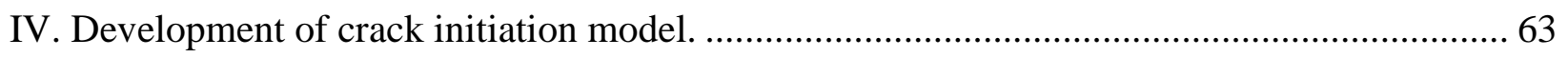

IV.1. Development of a crack initiation model for PWRs ....................................................... 63

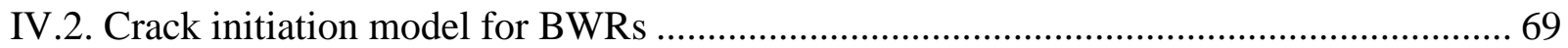

IV.3. Theory of the distribution in crack initiation time .......................................................... 74

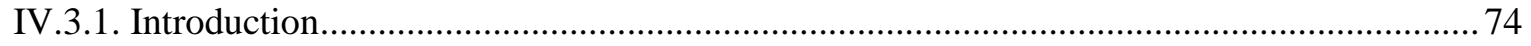

IV.3.2. Physico-electrochemistry of crack initiation …………………………………………....75

IV.3.3. Where should we go from here _................................................................................. 84

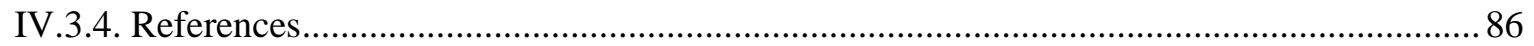

V. Further development of the coupled environment fracture model........................................... 87

V.1. Development of a hydrogen-induced cracking (HIC) model for Alloy 600 and incorporate

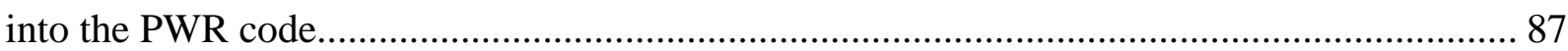

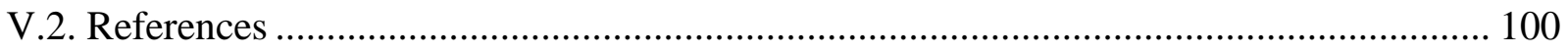

VI. An advanced coupled environment fracture model for hydrogen-induced cracking in Alloy 600

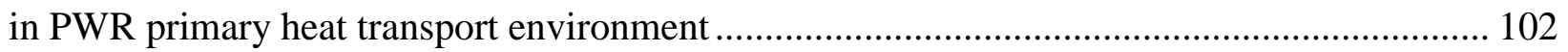

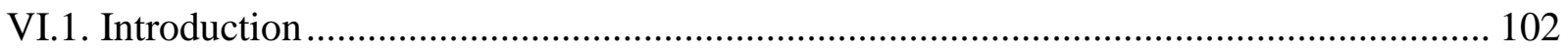

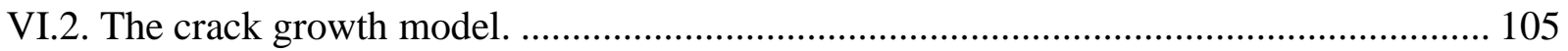

VI.3. Derivation of the equation for crack growth rate ......................................................... 107

VI.3.1. Basis of the coupled-environment fracture model (CEFM) .............................................. 107 


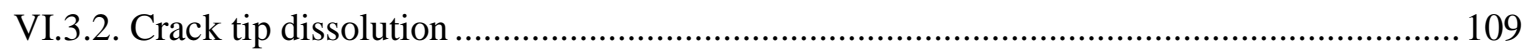

VI.3.3. Hydrogen ion concentration at the crack tip and hydrogen pressure in the voids ............ 111

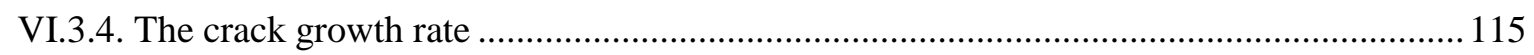

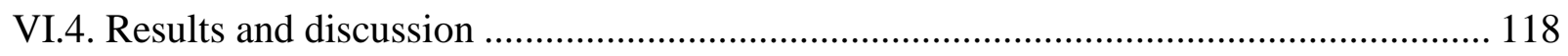

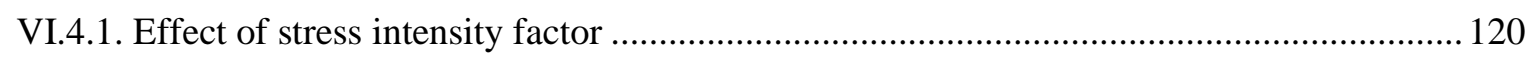

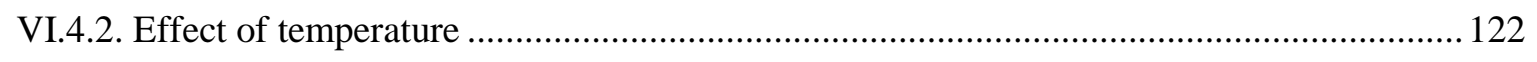

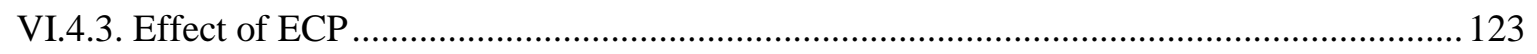

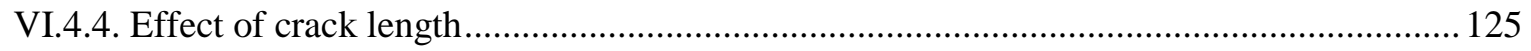

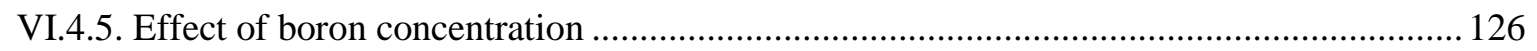

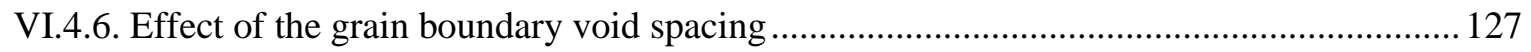

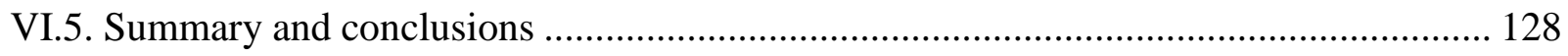

VI.6. Defining the mechanisms of hydriding failure of fuel cladding in pressurized water

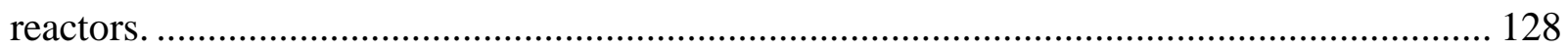

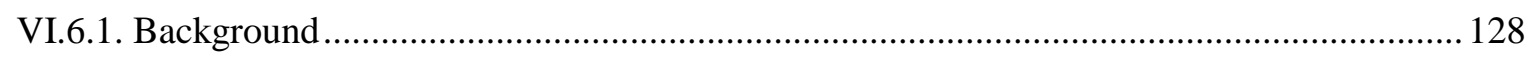

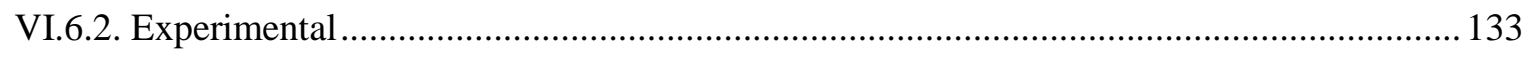

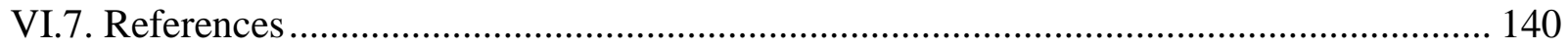

VII. The foundations of fracture impedance spectroscopy ............................................... 145

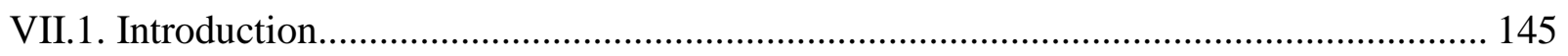

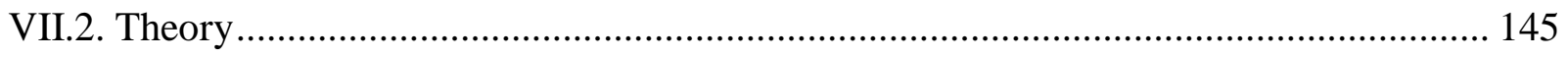

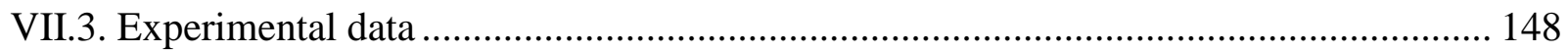

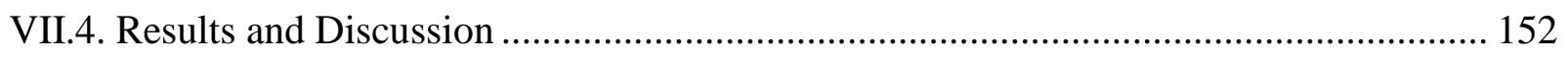

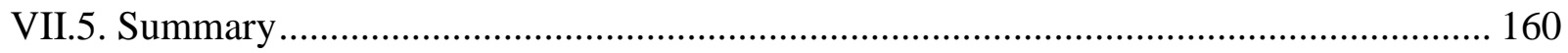

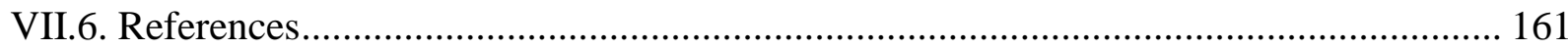

VIII. Characterization of the electrochemistry of LWR primary coolant circuits.................... 162

VIII.1. Characterization of the electrochemical conditions and CGR calculation in PWRs. . 162 IX. Theoretical dependence of the distribution in crack growth rate in Type 304SS on various

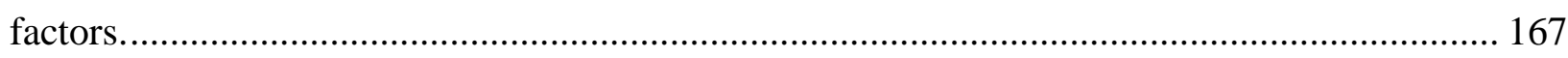

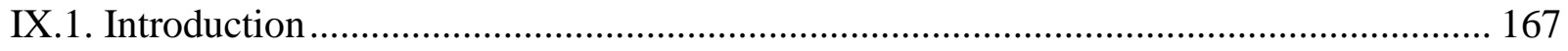

IX.2. Modeling of stress corrosion cracking ........................................................... 169

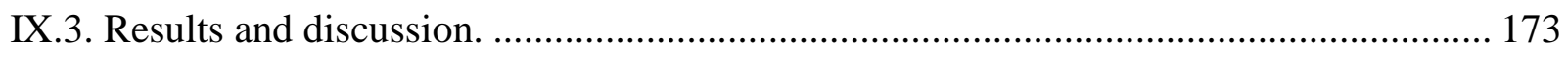

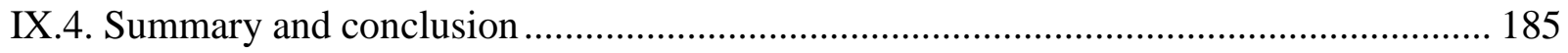

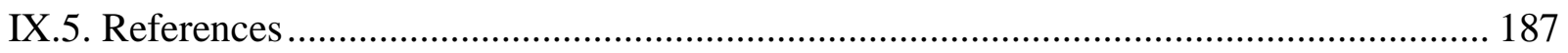


X. Experimental measurement of key model parameters ........................................................ 189

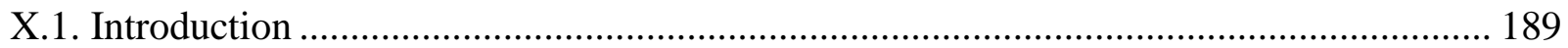

X.1.1. Background and literature survey ……………………………………………….... 189

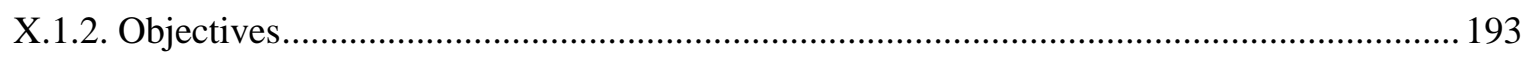

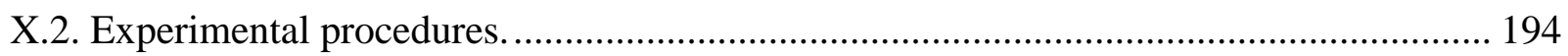

X.2.1. Apparatus setup and materials preparation. .................................................................... 194

X.2.2. Electrochemical measurements...................................................................................... 199

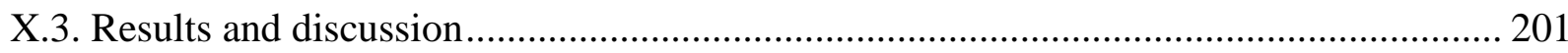

X.3.1. Effects of temperature and $\mathrm{pH}$ on the electrochemical properties of Alloys 600 and 690 in simulated pressurized water reactor primary water ..................................................................201

X.3.2. Effects of temperature and $\mathrm{pH}$ on the electrochemical properties of Type 304L SS and Type 316L SS in simulated pressurized water reactor primary water.................................................. 212

X.3.3. Effects of temperature and hydrogen concentration on the hydrogen electrode reactions for

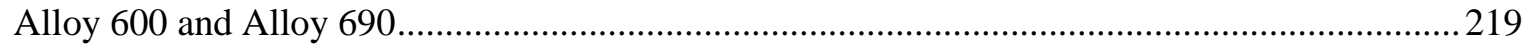

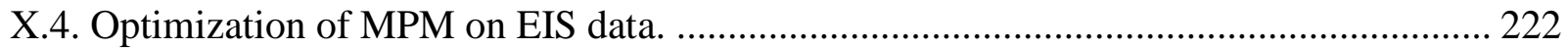

X.4.1. Point defect model (PDM) for Alloy 600, Alloy 690, Type 304L SS, and Type 316L SS.

X.4.2. Generalized Butler-Volmer equation for the cathodic reaction ........................................225

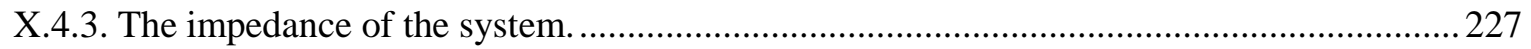

X.4.4. Optimize the MPM on the measured EIS data to extract model parameters.......................229

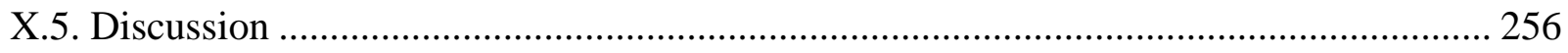

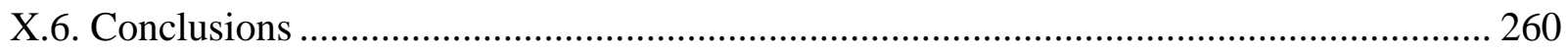

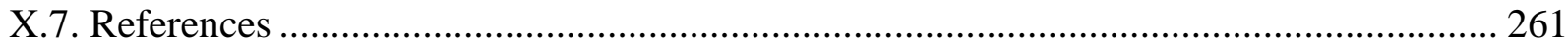

XI. Lifetime estimation of a BWR core shroud in terms of IGSCC ……………….................. 266

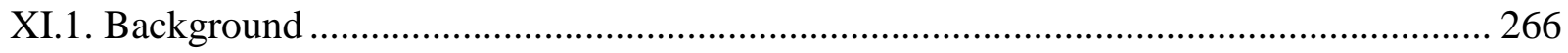

XI.2. Deterministic modeling of environment, crack propagation, and component's lifetime. 272

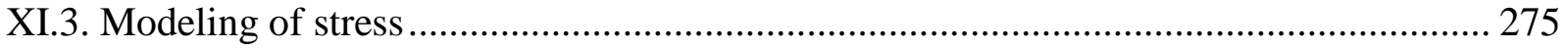

XI.4. Empirical modeling of crack propagation and component's lifetime............................. 276

XI.5. Comparison of stress intensity factors ………………………………………...... 277

XI.6. Comparison of crack growth rates ............................................................................ 278

XI.7. Comparison of predicted component lifetime................................................................ 281

XI.8. Summary and conclusions ………………………................................................. 283 


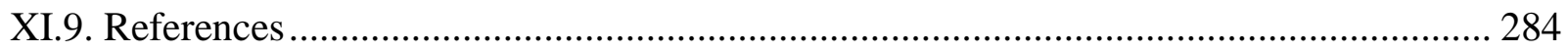

XII. Predicting transients in chemistry and crack growth rate in BWR start-ups....................... 288

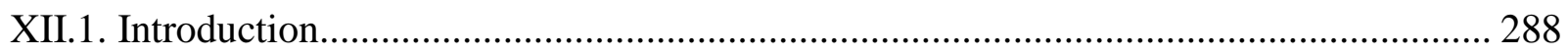

XII.2. Coolant chemistry and IGSCC damage transients during BWR start-up.................... 289

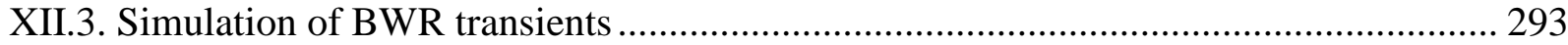

XII.4. Advantages of deterministic modeling of IGSCC damage in BWRs.......................... 297

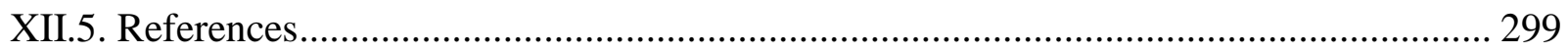

XIII. Modeling the electrochemistry and SCC damage accumulation in BWRs. ...................... 300

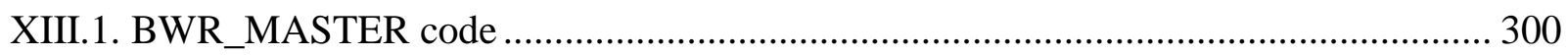

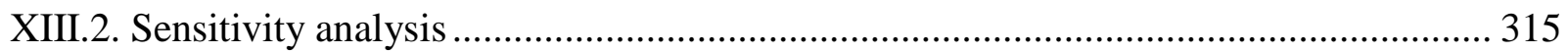

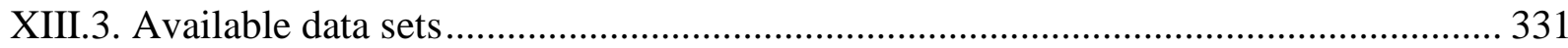

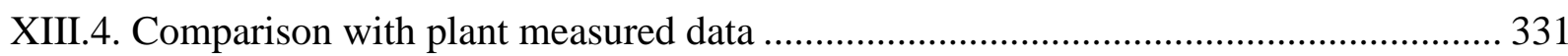

XIII.5. Implementation of generic chemical kinetics algorithm in BWR_MASTER radiolysis

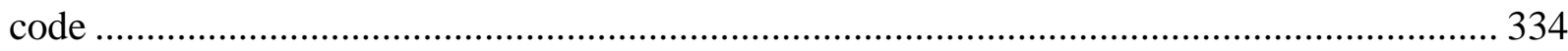

XIII.6. Simulation of BWR water chemistry, ECP and CGR with alternative chemical reaction

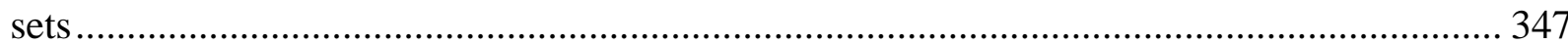

XIII.7. Comparison of calculated species concentrations and ECP with plant measured data353

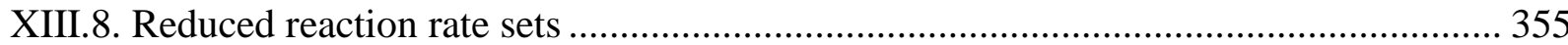

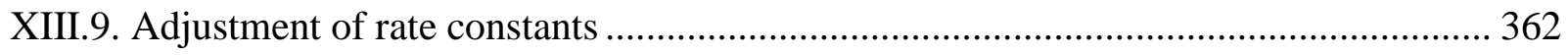

XIII.10. Implementation of the generic chemical kinetics algorithm in PWR_MASTER code 364

XIII.11. Integration BWR_MASTER with GRIZZLY .................................................... 373

XIII.12. Lifetime estimation of a BWR core shroud in terms of IGSCC .............................. 378

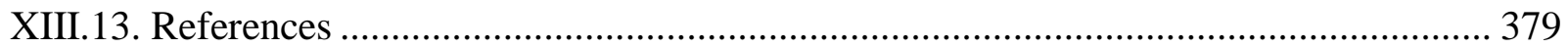

XIV. Integration of damage prediction codes into Grizzly and Raven...................................... 382

XIV.1. Grizzly analysis of stress intensity factors near the weld region of the core shroud. . 382

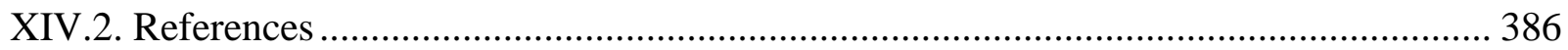

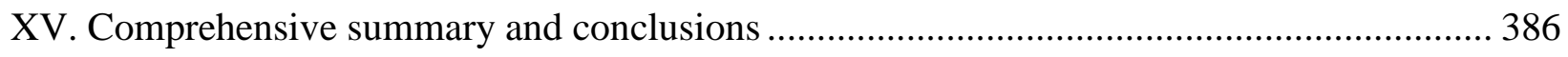

Appendix: Optimization of the mixed potential model on the experimental EIS data for passive films formed on Alloy 690, Type 304L SS, and Type 316L SS in oxygenated borate buffer solution 


\section{List of Figures}

Figure I.1. Structure of the algorithm. 41

Figure I.2. Predicted histories of growing cracks in the lower plenum of an operating, German BWR. Location No. 1 is at the bottom of the internal pump, and location No. 9 is at the bottom entrance to the core. Intermediate locations, Nos. 3, 5, and 7, represent locations near the bottom of the reactor vessel and at various elevations along the control rod drive structures. 44

Figure I.3. Predicted effect of HWC feedwater $\left[\mathrm{H}_{2}\right]=0.5 \mathrm{ppm}$ on the accumulated damage due to IGSCC in the core shroud of a BWR. The initial value of the crack length of $0.5 \mathrm{~cm}, 0.197$ in may be considered to be the depth of a detected crack, and the time in years as the remaining extended service time after introducing HWC.

Figure I.4. Predicted ECP around the PCC of a French PWR under normal, full power operating conditions. 46

Figure I.5. Percentage of IGSCC on the fracture surface as a function of applied potential on the standard hydrogen scale.

Figure I.6. Grizzly thermo-mechanical solution results for the RPV model showing (a) temperature contours and (b) von Mises stress contours at one point in time during a simulation of a PTS event.

Figure I.7. Global model of an RPV under PTS conditions (left) and sub-model of material in the vicinity of a postulated embedded circular crack (middle) used to calculate mechanical response and stress intensity factor (right) along the front of that crack.

Figure III.1. Comparison of measured and calculated ECP (measurements were conducted on Alloy 600 in water containing $1000 \mathrm{ppm}$ of boron, $2 \mathrm{ppm}$ of lithium, and $2 \mathrm{ppm}$ of dissolved hydrogen).

Figure III.2. $\mathrm{pH}$ in the PWR coolant containing boron and lithium: (a) Effect of lithium concentration and (b) Effect of boron concentration.

Figure III.3. Effect of temperature on $\mathrm{pH}$ in the PWR coolant containing boron and lithium. .. 61

Figure III.4. Comparison of pH values based on PHVALUE and PWR_MASTER. 62

Figure III.5. ECP and pH values in the PWR hot leg $\left(\mathrm{T}=326^{\circ} \mathrm{C}\right)$ over a fuel cycle for coordinated water chemistry. 62

Figure IV.1. Schematic of failure mechanism based on internal oxidation.............................. 65

Figure IV.2. Summary of governing equations of the model. 65 
Figure IV.3. Algorithm of the solution of internal oxidation-based crack initiation. 66

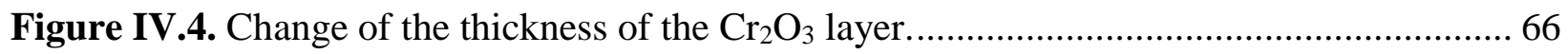

Figure IV.5. Variation of crack length and stress intensity factor vs. time. ........................... 67

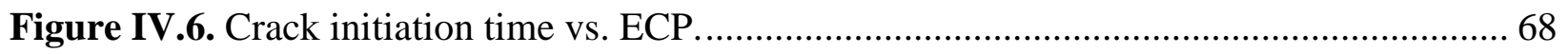

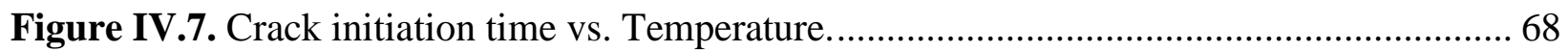

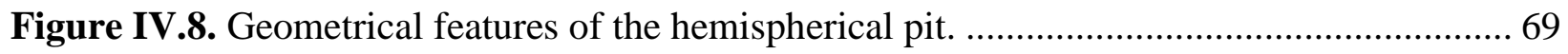

Figure IV.9. ECP as a function of $\left[\mathrm{O}_{2}\right]$ for various flow velocities as per the Coupled Environment

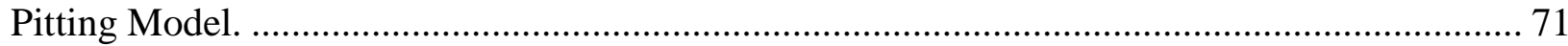

Figure IV.10. Variation of PGR in terms of conductivity................................................. 71

Figure IV.11. Variation of PGR in terms of ECP for different conductivities........................ 72

Figure IV.12. Effect of applied stress on critical crack length with $K_{I S C C}=8 M P a \sqrt{m} \ldots \ldots \ldots \ldots .73$

Figure IV.13. Effect of applied stress on crack initiation time with $\mathrm{R}_{\text {initial }}=1 x 10^{-5} \mathrm{~cm} \ldots \ldots \ldots .73$

Figure IV.14. SEM-SE images of the surface (upper) and BSE images showing the cross-section (lower) of a 60-grit finish plate specimen of MA Alloy 600 after exposure to simulated PWR

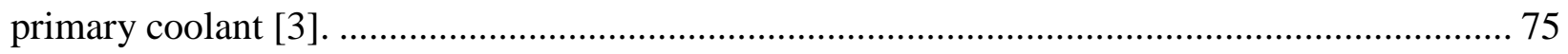

Figure IV.15. SEM-SE images of the surface showing surface cracks and high energy, emergent grain boundaries of multiple sizes after exposure of MA Alloy 600 to synthetic PWR primary coolant (See Ref. 3 for experimental details).............................................................. 76

Figure IV.16. Measured crack initiation time as a function of applied stress in PWR primary coolant at $360{ }^{\circ} \mathrm{C}$. The dashed lines define the bounds of the data. 77

Figure IV.17. The mean depth of the deepest pit in ALCAN aluminum alloy in Kingston tap water, as a function of time. Fitting to experimental data (black circles) has been performed by using the Hyperbolic dependencies [1]. 79

Figure IV.18. Schematic explanation of the origin of the distribution in critical crack length $(\Delta L)$ and hence in crack initiation time, $t_{\text {init }}$. Note that $\Delta K_{I}$ is the distribution in stress intensity factor for those sites on the surface for which $K_{I}>K_{I S C C}$. 79

Figure IV.19. Predicted ECP (a) and pit growth rate (PGR) as a function of ECP (b) and conductivity (c), as calculated from the Coupled Environment Pitting Model (CEPM). Note that the PGR is predicted to increase exponentially dependent upon the ECP and to increase with increasing solution conductivity [2]. 
Figure IV.20. Predicted critical crack length for crack initiation (a) and crack initiation time in Type 304 SS under BWR conditions $\left(288^{\circ} \mathrm{C}\right.$, pure water) as a function of the applied (residual + operational) stress [2] 82

Figure IV.21. Grain boundary oxidation/wedging mechanism for crack initiation in Alloy 600 in PWR primary coolant circuits. The model is based upon the proposal by Scott and Le Calvar [3].

Figure IV.22. Predicted $\mathrm{Cr}_{2} \mathrm{O}_{3}$ film growth within the emergent grain boundary (a) and calculated crack initiation time as a function of ECP (b) $\mathrm{pH}(\mathrm{c})$, and temperature (d). 83

Figure V.1. The schematic diagram of the interaction between the main crack and the voids located along the grain boundary.

Figure V.2. Variation of the CGR vs. the stress intensity factor for Alloy 600 in PWR primary heat transport circuits.

Figure V.3. Variation of the CGR vs. the ECP for Alloy 600 in PWR primary heat transport circuits for the Slip Dissolution-Repassivation mechanism (SDR) and together with the Grain Boundary Void Pressurization model (GBVP).

Figure V.4. Variation of the CGR vs. the grain boundary void spacing for Alloy 600 in PWR primary heat transport circuits. Experimental data point investigated by Transmission Electron Microscopy. 100

Figure VI.1. The schematic diagram of the interaction between the main crack and the voids located along the grain boundary. 105

Figure VI.2. The schematic diagram of the void growth, hydrogen diffusion, and stress distribution ahead of a crack tip. 114

Figure VI.3. Variation of the CGR vs. the stress intensity factor for Alloy 600 in PWR primary heat transport circuits.

Figure VI.4. Variation of the CGR vs. the temperature for Alloy 600 in PWR primary heat transport circuits. 123

Figure VI.5. Variation of the CGR vs. the ECP for Alloy 600 in PWR primary heat transport circuits for the Slip Dissolution-Repassivation mechanism (SDR) and together with the Grain Boundary Void Pressurization model (GBVP). 124

Figure VI.6. Variation of the CGR vs. the crack length for Alloy 600 in PWR primary heat transport circuits. 125 
Figure VI.7. Variation of the CGR vs. the boron concentration for Alloy 600 in PWR primary heat transport circuits.

Figure VI.8. Variation of the CGR vs. the grain boundary void spacing for Alloy 600 in PWR primary heat transport circuits. Experimental data point investigated by Transmission Electron Microscope (TEM) as per Shen and Shewmon.

Figure VI.9. Predicted variations of boron concentration, $\mathrm{pH}$ at the top and bottom of the core, and the hot-leg ECP of Alloys 600 and 690 as a function of time through a PWR fuel cycle. Coordinated water chemistry, $\left[\mathrm{H}_{2}\right]=5 \mathrm{ppm}\left[56 \mathrm{~cm}^{3}\left(\mathrm{STP} / \mathrm{kg} \mathrm{H}_{2} \mathrm{O}\right]\right.$ and $[\mathrm{Li}]=0-2 \mathrm{ppm}$. 130

Figure VI.10. Mechanism of cladding failure [1]. 131

Figure VI.11. Point defect model (PDM) for the oxidation/ hydriding of pure zirconium in PWR primary coolant. $\mathrm{Zr}_{\mathrm{Zr}}=$ zirconium cation in the hydride layer, $\mathrm{H}_{\mathrm{H}}=$ hydride anion of hydride sublattice, $\mathrm{V}_{\mathrm{H}}=$ hydrogen vacancy on hydride sublattice, $\mathrm{ZrH}_{2}=$ zirconium hydride [80]...... 132

Figure VI.12. Hydride redistribution induced by temperature gradients [64]. 133

Figure VI.13. Schematic of the recirculating flow loop with autoclave cell for high-temperature experimental measurements

Figure VI.14. The high temperature/high-pressure recirculating flow loop used for hightemperature experimental measurements.

Figure VI.15. Nyquist and Bode plots of the impedance of pure zirconium in simulated PWR primary environment at $250{ }^{\circ} \mathrm{C}$. Potential $=0.1 \mathrm{~V}_{\text {she, }}$, polarization time $=24$ hours, Solution: 0.1 $\mathrm{M} \mathrm{B}(\mathrm{OH})_{3}+0.001 \mathrm{M} \mathrm{LiOH}+22 \mathrm{~cm}^{3}(\mathrm{STP}) / \mathrm{kg} \mathrm{H} \mathrm{H}_{2} \mathrm{O}$. Figures show a comparison between the experimental data (points) and the impedance calculated from the optimized PDM, the parameters from which are given in Table VI.4 [80]. 136

Figure VI.16. Sensitivity of the calculated impedance of pure zirconium in simulated PWR primary coolant as a function of (b) the porosity and (f) thickness of the outer layer [80]....... 138 Figure VI.17. Comparison of the hydriding reaction rates of Zircaloy-2, Zircaloy-4, and ZIRLO [76]. 139

Figure VII.1. Experimental data of Al T2024-T3 taken from [6]. (a) Effect of loading amplitude. (b) Effect of $R$-ratio. 151

Figure VII.2. Experimental data of Alloy 718 taken from [7]. (a) Effect of loading amplitude. (b) Effect of R-ratio. 151 
Figure VII.3. Fracture impedance in terms of frequency of Al T2024-T3 in moist air at $\mathrm{T}=25{ }^{\circ} \mathrm{C}$ loading with a sinusoidal waveform.

Figure VII.4. Fracture impedance in terms of loading amplitude of Al T2024-T3 in moist air at $\mathrm{T}=25^{\circ} \mathrm{C}$ loading with sinusoidal waveform at $\mathrm{f}=30 \mathrm{~Hz}$.

Figure VII.5. Fracture impedance of Alloy 718 in moist air at $\mathrm{T}=650{ }^{\circ} \mathrm{C}$ loading with a triangular waveform: (a) Effect of loading amplitude and (b) Effect of R-ratio.... 156

Figure VII.6. Fracture impedance function in terms of the Laplace-frequency of AL 2024 T3 calculated for sinusoidal loading. 158

Figure VII.7. Crack Growth Rate in Al 2024-T3 obtained by the Fracture Impedance Method for sinusoidal loading and re-calculated for triangular loading using the same impedance function.

Figure VIII.1. Variation of ECP with hydrogen in steam generator U-tube hot leg over a 12-month fuel cycle.

Figure VIII.2. CGR in the steam generator U-tube as a function of $\left[\mathrm{H}_{2}\right]$ over a 12-month fuel cycle.

Figure VIII.3. Variation of crack length in the steam generator U-tube as a function of $\left[\mathrm{H}_{2}\right] .164$ Figure VIII.4. CGR in the steam generator U-tube at different stress intensity factors over a 12month fuel cycle. 165

Figure VIII.5. Variation of crack length in the steam generator U-tube at different stress intensity factors over a 12-month fuel cycle. 166

Figure VIII.6. CGR in the steam generator U-tube using different SCC models: (1) SDR mechanism, (2) SDR mechanism augmented by HIC. 166

Figure VIII.7. Crack length in the steam generator U-tube using different SCC models: (1) SDR mechanism, (2) SDR mechanism augmented by HIC. 167 Figure IX.1. Schematic of the differential aeration hypothesis for localized corrosion. $E^{M}{ }_{S}$ and $E^{L}{ }_{S}$ represent the electrostatic potentials in the solution vs. the metal on the standard hydrogen electrode scale at the crack mouth and the crack tip, respectively. 170 Figure IX.2. Histograms of the calculated CGRs under different ECP compared to the fitted lognormal distributions, (a) Results from the CEFM; (b) Results from the ANN. 176 Figure IX.3. Dependence of the mean value of CGR on ECP calculated using both the CEFM and ANN. 
Figure IX.4. Histograms of the calculated CGRs under different $K_{I}$ compared to the fitted lognormal distributions. (a) Results from the CEFM; (b) Results from the ANN. 179

Figure IX.5. Dependence of the mean value of CGR on $K_{I}$ calculated using both the CEFM and ANN. 180

Figure IX.6. Histograms of the calculated CGRs under different temperatures compared to the fitted log-normal distributions. (a) Results from the CEFM; (b) Results from the ANN..... 181

Figure IX.7. Dependence of the mean value of CGR on temperature calculated using both the CEFM and ANN. 182

Figure IX.8. Histograms of the calculated CGRs under different conductivity compared to the fitted log-normal distributions. (a) Results from the CEFM; (b) Results from the ANN. 183

Figure IX.9. Dependence of the mean value of CGR on conductivity calculated using both the CEFM and ANN. 184

Figure IX.10. Histograms of the calculated CGRs under different EPR compared to the fitted lognormal distributions. (a) Results from the CEFM; (b) Results from the ANN. 186 Figure IX.11. Dependence of the mean value of CGR on EPR calculated using both the CEFM and ANN. 187

Figure X.1. Schematic of the recirculating flow loop with autoclave cell for high temperature experimental studies. 195

Figure X.2. The assembly of the working electrode used for experimental measurements up to 300 ${ }^{\circ} \mathrm{C}$. 196

Figure X.3. Change of open circuit potential as a function of (a) temperature and (b) $\mathrm{pH}$ for Alloys 600 and 690 in borate buffer solutions containing 400 ppb dissolved oxygen. 201

Figure X.4. Change of passive current density as a function of film formation potential (potentiostatic test) with temperature for (a) Alloy 600 and (b) Alloy 690 in borate buffer solutions with $400 \mathrm{ppb}$ dissolved oxygen. 202

Figure X.5. Change of passive current density as a function of film formation potential (potentiostatic test) with $\mathrm{pH}$ for (a) Alloy 600 and (b) Alloy 690 in borate buffer solutions with 400 ppb dissolved oxygen. 203

Figure X.6. Mott-Schottky (M-S) plots for the passive film formed on (a) Alloy 600 and (b) Alloy 690 at $+0.3 \mathrm{~V}_{\text {OCP }}$ as a function of temperature in borate buffer solutions containing $400 \mathrm{ppb}$ dissolved oxygen. 204 
Figure X.7. Impedance plots of the measured EIS data in the Bode plane for two frequency step directions for passive films formed on Alloy 600 at the OCP in borate buffer solutions containing $400 \mathrm{ppb}$ dissolved oxygen at different temperatures. 205

Figure X.8. Impedance plots of the measured EIS data in the Nyquist plane for two frequency step directions for passive films formed on Alloy 600 at OCP in borate buffer solutions containing 400 ppb dissolved oxygen at different temperatures. 206

Figure X.9. Impedance plots of the measured EIS data in the Bode plane for two frequency step directions for passive films formed on Alloy 690 at the OCP in borate buffer solutions containing $400 \mathrm{ppb}$ dissolved oxygen at different temperatures. 207

Figure X.10. Impedance plots of the measured EIS data in the Nyquist plane for two frequency step directions for passive films formed on Alloy 690 at OCP in borate buffer solutions containing $400 \mathrm{ppb}$ dissolved oxygen at different temperatures. 208

Figure X.11. Impedance plots of the experimental data with the calculated values by KramersKronig transforms for Alloy 690 at OCP in a borate buffer solution containing 2000 ppm B + 2 ppm Li with $400 \mathrm{ppb}$ dissolved oxygen at different temperatures. 209

Figure X.12. Change of the absolute value of impedance with temperature for passive films formed on (a) Alloy 600 and (b) Alloy 690 at the OCP in borate buffer solutions containing 400 ppb dissolved oxygen.

Figure X.13. Change of the absolute value of impedance with $\mathrm{pH}$ for passive films formed on (a) Alloy 600 and (b) Alloy 690 at the OCP in borate buffer solutions containing 400 ppb dissolved oxygen. 210

Figure X.14. Impedance plots of the measured EIS data (a) at $+0.3 \mathrm{~V}_{\text {OCP }}$ in the Bode plane and (b) at $+0.3 \mathrm{~V}_{\mathrm{OCP}}$ in the Nyquist plane for two frequency step directions and (c) at the OCP and +0.3 $V_{\text {OCP }}$ in the Bode plane for passive films formed on Alloy 600 in borate buffer solutions containing $400 \mathrm{ppb}$ dissolved oxygen at different temperatures.

Figure X.15. Changes of the open circuit potential as a function of (a) temperature and (b) $\mathrm{pH}$ for Type 304L SS and Type 316L SS immersed in borate buffer solutions containing $400 \mathrm{ppb}$ dissolved oxygen.

Figure X.16. Change of passive current density as a function of film formation potential (potentiostatic test) with temperature for (a) Type 304L SS and (b) Type 316L SS in borate buffer solutions with $400 \mathrm{ppb}$ dissolved oxygen. 
Figure X.17. Change of passive current density as a function of film formation potential (potentiostatic test) with pH for (a) Type 304L SS and (b) Type 316L SS in borate buffer solutions with $400 \mathrm{ppb}$ dissolved oxygen.

Figure X.18. Mott-Schottky (M-S) plots for the passive film formed on (a) Type 304L SS and (b) Type $316 \mathrm{~L} \mathrm{SS}$ at $+0.3 \mathrm{~V}_{\text {OCP }}$ as a function of temperature in borate buffer solutions containing 400 ppb dissolved oxygen.

Figure X.19. Measured impedance plots in (a) Bode and (b) Nyquist planes at two frequency step directions for passive films formed on Type 304L SS at OCP in borate buffer solutions containing $400 \mathrm{ppb}$ dissolved oxygen at different temperatures. 216

Figure X.20. Measured impedance plots in (a) Bode and (b) Nyquist planes at two frequency step directions for passive films formed on Type 316L SS at OCP in borate buffer solutions containing $400 \mathrm{ppb}$ dissolved oxygen at different temperatures.

Figure X.21. Change of the absolute value of impedance with temperature for passive films formed on (a) Type 304L SS and (b) Type 316L SS at the OCP in borate buffer solutions containing 400 ppb dissolved oxygen. 218

Figure X.22. Change of the absolute value of the impedance with $\mathrm{pH}$ for passive films formed on (a) Type 304L SS and (b) Type 316L SS at the OCP in borate buffer solutions containing $400 \mathrm{ppb}$ dissolved oxygen.

Figure X.23. Measured equilibrium potentials of $W / W O 3$ electrode half-cell reaction (a) versus standard hydrogen electrode and (b) compares with the calculated value based on Nernst Equation.

Figure X.24. Effect of hydrogen concentration on the HER for Alloys 690 and 600............. 221

Figure X.25. Effect of temperature on the HER for Alloys 690 and 600.

Figure X.26. Schematic diagram of the interfacial reactions for defects generation and annihilation in the barrier layer of passive film formed on Alloy 600, Alloy 690, Type 304L SS, and Type 316L SS based on the Point Defect Model. $C r$ is chromium atom in metal, $V_{C r}^{\chi^{\prime}}$ is chromium vacancy in the barrier layer, $\mathrm{Cr}_{C r}$ is chromium cation in normal cation sublattice in the barrier layer, $V_{C r}$ is chromium atom vacancy in metal, $\mathrm{Cr}_{i}^{\chi^{+}}$is chromium cation interstitial in the barrier layer, $V_{\ddot{\mathrm{o}}}$ is an oxygen vacancy in the barrier layer, $\mathrm{Cr}^{\delta^{+}}(\mathrm{aq})$ is chromium cation in solution, $\mathrm{O}_{o}$ is oxide ion in anion sublattice in the barrier layer, $\mathrm{CrO}_{\frac{\chi}{2}}$ is stoichiometric barrier layer oxide, $\chi$ and $\delta$ are 
oxidation states of chromium in barrier layer and in solution, respectively, and all assumed to be

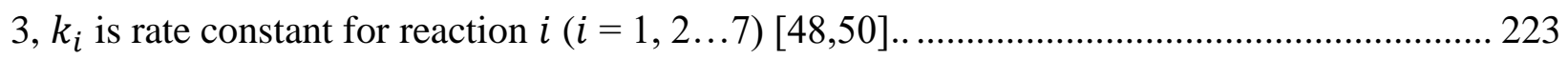

Figure X.27. Schematic of the electrical equivalent circuit for the entire electrochemical reaction system of Alloy 600, Alloy 690, Type 304L SS, and Type 316L SS.

Figure X.28. Comparison of the impedance plots between the experimental measured EIS data and the calculated EIS data from the Mixed Potential Model (MPM) in the Bode plane for passive films formed on Alloy 600 at OCP in borate buffer solutions containing $400 \mathrm{ppb}$ dissolved oxygen at different temperatures.

Figure X.29. Comparison of the impedance plots between the experimental measured EIS data and the calculated EIS data from the Mixed Potential Model (MPM) in the Nyquist plane for passive films formed on Alloy 600 at OCP in borate buffer solutions containing $400 \mathrm{ppb}$ dissolved oxygen at different temperatures. 231

Figure X.30. Comparison of the impedance plots between the experimental measured EIS data and the calculated EIS data from the Mixed Potential Model (MPM) in the (a) Bode and (b) Nyquist planes for passive films formed on Alloy 600 at $+0.3 \mathrm{~V}_{\text {OCP }}$ in borate buffer solutions containing $400 \mathrm{ppb}$ dissolved oxygen at different temperatures. 232

Figure X.31. Comparison of the impedance plots between the experimental measured EIS data and the calculated EIS data from the Mixed Potential Model (MPM) in the Bode plane for passive films formed on Alloy 690 at OCP in borate buffer solutions containing 400 ppb dissolved oxygen at different temperatures. 233

Figure X.32. Comparison of the impedance plots between the experimental measured EIS data and the calculated EIS data from the Mixed Potential Model (MPM) in the Nyquist plane for passive films formed on Alloy 690 at OCP in borate buffer solutions containing 400 ppb dissolved oxygen at different temperatures. 234

Figure X.33. Comparison of the impedance plots between the experimental measured EIS data and the calculated EIS data from the Mixed Potential Model (MPM) in the Bode plane for passive films formed on Type 304L SS at OCP in borate buffer solutions containing $400 \mathrm{ppb}$ dissolved oxygen at different temperatures. 235

Figure X.34. Comparison of the impedance plots between the experimental measured EIS data and the calculated EIS data from the Mixed Potential Model (MPM) in the Nyquist plane for 
passive films formed on Type 304L SS at OCP in borate buffer solutions containing $400 \mathrm{ppb}$ dissolved oxygen at different temperatures. 236

Figure X.35. Comparison of the impedance plots between the experimental measured EIS data and the calculated EIS data from the Mixed Potential Model (MPM) in the Bode plane for passive films formed on Type 316L SS at OCP in borate buffer solutions containing $400 \mathrm{ppb}$ dissolved oxygen at different temperatures. 237

Figure X.36. Comparison of the impedance plots between the experimental measured EIS data and the calculated EIS data from the Mixed Potential Model (MPM) in the Nyquist plane for passive films formed on Type 316L SS at OCP in borate buffer solutions containing $400 \mathrm{ppb}$ dissolved oxygen at different temperatures. 238

Figure X.37. Arrhenius linear plots of (a) the base rate constant $\left(k_{i}^{00}\right)$ of anodic metal passive dissolution and the hypothetical exchange current density $\left(\widehat{\iota_{0}}\right)$ of cathodic oxygen electrode reaction versus the reciprocal of temperature $1 / T$ and (b) the calculated activation energies of the anodic metal dissolution and the cathodic oxygen reduction reactions for Alloy 600 in borate buffer solutions containing $400 \mathrm{ppb}$ dissolved oxygen..

Figure X.38. Arrhenius linear plots of the base rate constant $\left(\left(k_{i}^{00}\right)\right.$ of anodic metal passive dissolution versus the reciprocal of temperature $1 / T$ and the calculated activation energies of the anodic metal dissolution reactions for Alloy 690 in borate buffer solutions containing $400 \mathrm{ppb}$ dissolved oxygen. 246

Figure X.39. Arrhenius linear plots of the base rate constant $\left(\left(k_{i}^{00}\right)\right.$ of anodic metal passive dissolution versus the reciprocal of temperature $1 / T$ and the calculated activation energies of the anodic metal dissolution reactions for Type 304L SS in borate buffer solutions containing $400 \mathrm{ppb}$ dissolved oxygen. 246

Figure X.40. Arrhenius linear plots of the base rate constant $\left(\left(k_{i}^{00}\right)\right.$ of anodic metal passive dissolution versus the reciprocal of temperature $1 / T$ and the calculated activation energies of the anodic metal dissolution reactions for Type 316L SS in borate buffer solutions containing $400 \mathrm{ppb}$ dissolved oxygen. 247

Figure X.41. Changes of transfer coefficient $\alpha i(i=2,3,5,6,7)$ for anodic metal passive dissolution of Alloy 600 with increasing temperature in borate buffer solutions containing $400 \mathrm{ppb}$ dissolved oxygen. 249 
Figure X.42. Effects of (a) temperature and (b) $\mathrm{pH}$ on the anodic passive current density for passive films formed on Alloy 600 under OCP in borate buffer solutions with 400 ppb dissolved oxygen 255

Figure X.43. Effects of (a) temperature and (b) $\mathrm{pH}$ on the thickness of passive films formed on Alloy 600 under OCP in borate buffer solutions with 400 ppb dissolved oxygen. 255

Figure X.44. Effects of (a) temperature and (b) $\mathrm{pH}$ on the defect density of passive films formed on Alloy 600 under OCP in borate buffer solutions with 400 ppb dissolved oxygen. 256

Figure X.45. Change of open circuit potential with temperature for Alloy 690, Alloy 600, Type 316L SS and Type 304L SS in a solution containing $\mathrm{H}_{3} \mathrm{BO}_{3}(2000$ ppm B, 200 ppm B, 0 ppm B) + $\mathrm{LiOH}(2 \mathrm{ppm} \mathrm{Li})$ with $400 \mathrm{ppb}$ dissolved oxygen. 256

Figure X.46. Comparison of the absolute value of impedance for passive films formed on different alloys at OCP in borate buffer solutions containing 400 ppb dissolved oxygen-containing (a) 2000 ppm B + 2 ppm Li, (b) 200 ppm B + 2 ppm Li, and (c) 0 ppm B + 2 ppm Li. 257

Figure X.47. Compare concentrations of (a) oxygen vacancy, (b) metal interstitial, and (c) total defect density in the barrier layers of passive films formed on Alloy 690, Alloy 600, Type 316L SS, and Type 304L SS. 258

Figure X.48. Calculated values of activation energy for the formation of metal interstitials for Alloy 690, Alloy 600, Type 316L SS, and Type 304L SS in three borate buffer solutions with 400 ppb dissolved oxygen. 259

Figure X.49. Calculated thickness of barrier layers for passive films formed on Alloy 690, Alloy 600, Type 316L SS, and Type 304L SS in borate buffer solutions with 400 ppb dissolved oxygen at different temperatures. 260

Figure XI.1. Synergy of driving forces for the stress corrosion cracking phenomenon........... 267

Figure XI.2. Locations and orientations of BWR shroud cracking [8]............................... 269

Figure XI.3. Location of BWR Shroud Welds and Off-axis Cracking. Nearly all off-axis cracking is between weld numbers $\mathrm{H} 3$ and H6a, as shown by the arrow [8]..................................... 271

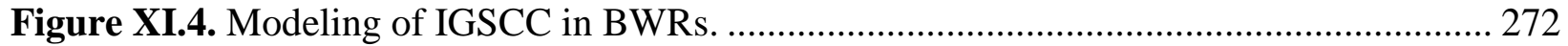

Figure XI.5. A Localized Model of the H6a Weld [43] .................................................. 276

Figure XI.6. Stress intensity factor at the crack tip during crack propagation. ..................... 278

Figure XI.7. Crack growth rate during crack propagation through the H6a weld.................... 279 
Figure XI.8. Predicted core shroud remaining lifetime based on crack propagation in H6a weld in the core shroud for BWR operating at full power throughout the calculation. 283

Figure XII.1. Typical transients in chloride concentration and conductivity in the start-up of a BWR [11]. 289

Figure XII.2. Comparison of transients in coolant sulfate concentration and reactor power for the start-up of a BWR [11]. 290

Figure XII.3. Transients in ECP, temperature, and $\left[\mathrm{H}_{2}\right]$ upon the start-up of a BWR on HWC. Also shown are ECP data for start-up under normal water chemistry (NWC). The ECP data were measured at the $\mathrm{R} / \mathrm{V}$ bottom drain line [11].

Figure XII.4. Transients in power and flow velocity (a), concentration of Hydrogen and Oxygen in Feedwater (b), and $[\mathrm{NaOH}],[\mathrm{HCl}]$ (c) during an idealized start of a BWR operating on HWC.

Figure XII.5. Time dependence of water temperature and water conductivity at H6a Core Shroud weld location during reactor startup under combined transient conditions.

Figure XII.6. Time dependence of ECP and CGR at H6a Core Shroud weld location during reactor startup under combined transient conditions. While ECP monotonically diminished during the transient, CGR exhibits a maximum at about 10 hours and a temperature of about $160{ }^{\circ} \mathrm{C} \ldots . . .296$ Figure XII.7. Time dependence of the crack length of a pre-existing, 0.1-cm deep crack at the H6a Core Shroud weld location and the incremental contribution of the crack advance at each time interval to the total crack advance over the 20-hr during reactor startup under combined transient conditions. 297

Figure XIII.1. Code development history. 301

Figure XIII.2. BWR recirculating coolant model: simplified reactor geometry (a) and HTC

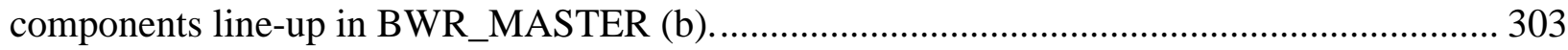

Figure XIII.3. A one-dimensional representation of BWR HTC in BWR_MASTER. ........... 304

Figure XIII.4. The BWR_MASTER input files............................................................. 304

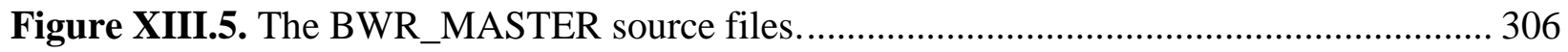

Figure XIII.6. The BWR_MASTER folder with input files in MAC OS Finder. ................... 306

Figure XIII.7. BWR_MASTER folder with input files in Terminal Window........................ 307

Figure XIII.8. BWR_MASTER folder with input files in the XQuarts window.................... 307

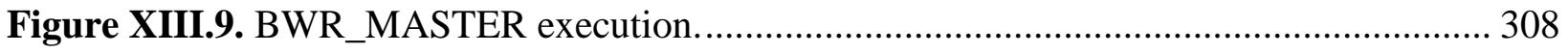


Figure XIII.10. The BWR_MASTER progress output. 308

Figure XIII.11. The BWR_MASTER progress output in a XQuartz window: beginning (a) and end (b). 309

Figure XIII.12. BWR_MASTER output files. .............................................................. 310

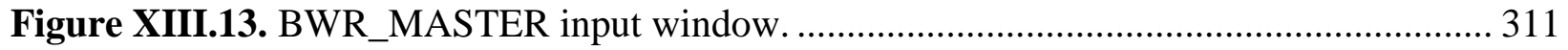

Figure XIII.14. BWR_MASTER progress window................................................... 311

Figure XIII.15. BWR_MASTER output files. ............................................................... 312

Figure XIII.16. BWR_MASTER interface file example: crack depth along HTC for each time point. 314

Figure XIII.17. Post-processing in Excel. ................................................................ 315

Figure XIII.18. Post-processing with Gnuplot. ............................................................... 316

Figure XIII.19. The sensitivity of $\mathrm{H}_{2}$ concentration to radiolytic yields.............................. 322

Figure XIII.20. The sensitivity of $\mathrm{O}_{2}$ concentration to radiolytic yields............................. 322

Figure XIII.21. The sensitivity of $\mathrm{H}_{2} \mathrm{O}_{2}$ concentration to radiolytic yields. ......................... 323

Figure XIII.22. The sensitivity of ECP SHE $_{\text {to }}$ to radiolytic yields. ........................................ 323

Figure XIII.23. The sensitivity of crack growth rate to radiolytic yields............................ 324

Figure XIII.24. The sensitivity of $\mathrm{H}_{2}$ concentration to the radiolytic yield of $\mathrm{e}^{-}$under gamma radiation.

Figure XIII.25. The sensitivity of $\mathrm{O}_{2}$ concentration to the radiolytic yield of $\mathrm{e}^{-}$under gamma radiation.

Figure XIII.26. The sensitivity of $\mathrm{H}_{2} \mathrm{O}_{2}$ concentration to the radiolytic yield of $\mathrm{e}^{-}$under gamma radiation.

Figure XIII.27. The sensitivity of ECPSHE to the radiolytic yield of $\mathrm{e}^{-}$under gamma radiation.

Figure XIII.28. The sensitivity of the crack growth rate to the radiolytic yield of $\mathrm{e}^{-}$under gamma radiation. 326

Figure XIII.29. Comparison of measured and calculated $\mathrm{H}_{2}$ concentration. 333

Figure XIII.30. Comparison of measured and calculated $\mathrm{O}_{2}$ concentration. 333

Figure XIII.31. Comparison of measured and calculated ECP $\left(\mathrm{V}_{\mathrm{SHE}}\right)$. 334

Figure XIII.32. Source code of generic chemical kinetics algorithm. 336 
Figure XIII.33. A fragment of the source code of the previously used "fixed" chemical kinetics algorithm.

Figure XIII.34. Comparison of ECP under Normal Water Chemistry conditions calculated with "Fixed" (old version of BWR_MASTER) and "Generic" kinetics algorithms (new version of BWR_MASTER).

Figure XIII.35. Comparison of ECP under Hydrogen Water Chemistry conditions calculated with "Fixed" (old version of BWR_MASTER) and "Generic" kinetics algorithms (new version of BWR_MASTER).

Figure XIII.36. Comparison of Crack Growth Rates under Normal Water Chemistry conditions calculated with "Fixed" (old version of BWR_MASTER) and "Generic" kinetics algorithms (new version of BWR_MASTER). 340

Figure XIII.37. Comparison of Crack Growth Rates under Hydrogen Water Chemistry conditions calculated with "Fixed" (old version of BWR_MASTER) and "Generic" kinetics algorithms (new version of BWR_MASTER). 340

Figure XIII.38. Comparison of oxygen concentration under Normal Water Chemistry conditions calculated with "Fixed" (old version of BWR_MASTER) and "Generic" kinetics algorithms (new version of BWR_MASTER).

Figure XIII.39. Comparison of oxygen concentration under Hydrogen Water Chemistry conditions calculated with "Fixed" (old version of BWR_MASTER) and "Generic" kinetics algorithms (new version of BWR_MASTER).

Figure XIII.40. Comparison of hydrogen peroxide concentration under Normal Water Chemistry conditions calculated with "Fixed" (old version of BWR_MASTER) and "Generic" kinetics algorithms (new version of BWR_MASTER). 342

Figure XIII.41. Comparison of hydrogen peroxide concentration under Hydrogen Water Chemistry conditions calculated with "Fixed" (old version of BWR_MASTER) and "Generic" kinetics algorithms (new version of BWR_MASTER).

Figure XIII.42. Comparison of hydrogen concentration under Normal Water Chemistry conditions calculated with "Fixed" (old version of BWR_MASTER) and "Generic" kinetics algorithms (new version of BWR_MASTER). 
Figure XIII.43. Comparison of hydrogen concentration under Hydrogen Water Chemistry conditions calculated with "Fixed" (old version of BWR_MASTER) and "Generic" kinetics algorithms (new version of BWR_MASTER). 343

Figure XIII.44. Comparison of ECP under Normal Water Chemistry (NWC) Conditions calculated using a generic kinetics algorithm with five reaction rates in CAM PSU set are set to zero (open circles) and the same reactions removed from CAM PSU set. 344

Figure XIII.45. Comparison of ECP under Hydrogen Water Chemistry (HWC) Conditions calculated using a generic kinetics algorithm with five reaction rates in CAM PSU set are set to zero (open circles) and the same reactions removed from CAM PSU set. 344

Figure XIII.46. YIELD.DAT file format. 345

Figure XIII.47. CHEMRATE.DAT file format. 346

Figure XIII.48. BWR_MASTER code printout file format. 347

Figure XIII.49. Comparison of hydrogen concentration under Normal Water Chemistry conditions calculated with Christensen set (open circles) and CAM PSU set (solid line)........ 350 Figure XIII.50. Comparison of hydrogen concentration under Hydrogen Water Chemistry conditions calculated with Christensen set (open circles) and CAM PSU set (solid line). 350

Figure XIII.51. Comparison of hydrogen peroxide concentration under Normal Water Chemistry conditions calculated with Christensen set (open circles) and CAM PSU set (solid line)..... 351

Figure XIII.52. Comparison of hydrogen peroxide concentration under Hydrogen Water Chemistry conditions calculated with Christensen set (open circles) and CAM PSU set (solid line).

Figure XIII.53. Comparison of oxygen concentration under Normal Water Chemistry conditions calculated with Christensen set (open circles) and CAM PSU set (solid line). 352

Figure XIII.54. Comparison of oxygen concentration under Hydrogen Water Chemistry conditions calculated with Christensen set (open circles) and CAM PSU set (solid line). 352 Figure XIII.55. Comparison of measured during Hydrogen Water Chemistry minitest and calculated ECP and species concentrations. 354

Figure XIII.56. The sensitivity of ECP in Core Bypass (a) and Recirculation Line (b) to 10x increase in reaction rates in CAM PSU reaction set under NWC. 357

Figure XIII.57. The sensitivity of species concentrations and ECP in core bypass to 10x increase in reaction rates in CAM PSU reaction set under NWC. 358 
Figure XIII.58. The sensitivity of species concentrations and ECP in recirculation line to 10x increase in reaction rates in CAM PSU reaction set under NWC.

Figure XIII.59. Calculated ECP under NWC with original CAM PSU data (solid line), with 5 reactions removed (open circles) and 10 reactions removed (closed circles)...... 360 Figure XIII.60. Calculated crack depth in the Bypass Region (a) and in the Recirculation Line (b) after 60 years of operation under NWC with original CAM PSU data (closed circles) and with 10 reactions removed (closed squares). 361

Figure XIII.61. Effect of the number of chemical reactions in the reaction rate set on simulation time. 361

Figure XIII.62. Comparison of hydrogen peroxide concentration under Normal Water Chemistry conditions calculated with Christensen's original set (open circles) and CAM PSU set (solid line).

Figure XIII.63. Comparison of hydrogen peroxide concentration under Normal Water Chemistry conditions calculated with adjusted Christensen original set (open triangles) and CAM PSU set (solid line). 363

Figure XIII.64. Source code of generic chemical kinetics algorithm. 366

Figure XIII.65. A fragment of the source code of the previously used "fixed" chemical kinetics algorithm. 367

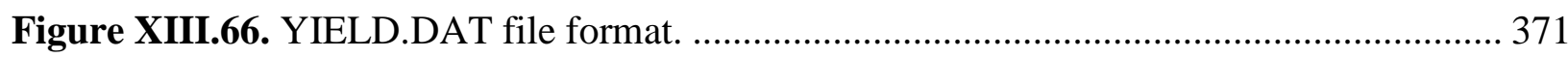

Figure XIII.67. CHEMRATE.DAT file format. ........................................................ 371

Figure XIII.68. PWR_MASTER code printout file format.............................................. 372

Figure XIII.69. A modified portion of the reactor description input file. ............................. 373

Figure XIII.70. The modified portion of printout file format. ....................................... 373

Figure XIII.71. Stress Intensity Factors input file format: number of input lines, crack depth in $\mathrm{cm}$, and stress intensity factor in $\mathrm{MPa} \mathrm{m}^{\wedge} 1 / 2$.

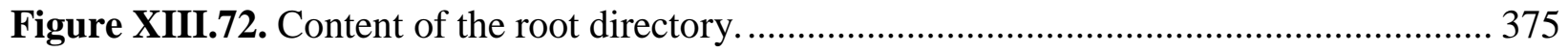

Figure XIII.73. Content of the "Run" sub-directory..................................................... 375

Figure XIII.74. Running BWR_MASTER simulation with external stress intensity factor data file. 376

Figure XIII.75. Running BWR_MASTER simulation with internally generated stress intensity factors. 376 
Figure XIII.76. Content of the "outputs" sub-directory. 377

Figure XIII.77. Comparison of crack propagation in H6a weld of BWR Core Shroud during 40 years of full power operation under normal water chemistry condition

Figure XIII.78. Predicted core shroud remaining lifetime based on crack propagation in H6a weld in the core shroud for BWR. 378

Figure XIV.1. Residual stress profile in the vicinity of a weld in a BWR core shroud from [7]. 384

Figure XIV.2. Through-wall residual axial stress showing the raw data extracted from the image in Figure XIV.1, as reported in [7], together with third and fourth-order polynomial fits of that data used in a weight function analysis in Grizzly. 385

Figure XIV.3. Stress intensity factor for a 360-degree circumferential crack computed using the weight function technique in Grizzly using the third and fourth-order polynomial expansions of the residual stress field shown in Figure XIV.2, along with the results computed in [7] 386

\section{List of Tables}

Table III.1. Default values for electrochemical parameters in the MPM and CEFM for Alloy 600.

Table III.2. Reaction set used for calculating the $\mathrm{pH}$ value in PWR coolant containing boric acid and lithium hydroxide.

Table VI.1. Parameter values for the slip-dissolution model............................................. 119

Table VI.2. Parameter values for the grain boundary crack growth model............................ 120

Table VI.3. Chemical composition of different zirconium alloys.

Table VI.4. PDM optimized parameters for the corrosion of pure zirconium in simulated PWR coolant at $250{ }^{\circ} \mathrm{C}[80]$.

Table VII.1. Laplace transform pairs for typical loading waveforms employed in fatigue experiments.

Table VII.2. Summary of the experimental data used in this study.................................... 150

Table IX.1. Summary of the random variables considered 174

Table IX.2. Numerical values of the distribution parameters of CGR by CEFM................... 175

Table IX.3. Numerical values of the distribution parameters of CGR by ANN. 175 
Table X.1. Chemical compositions of Alloy 600, Alloy 690, Type 304L SS and Type 316L SS (wt.\%)

Table X.2. The calculated $p H$ values of solution and the calculated standard $\left(E_{T}^{0}\right)$ and equilibrium $\left(E_{T}^{e}\right)$ potentials of $\mathrm{W} / \mathrm{WO}_{3}$ reference electrode as a function of temperature. 197

Table X.3. Dissolved hydrogen concentration in a solution containing 0 ppm B 2 ppm Li. 198

Table X.4. Calculated equilibrium potentials of hydrogen electrode reaction based-on Nernst equation. 200

Table X.5. Calculated defect density of passive films formed on Alloys 600 and 690 at +0.3 V OCP in borate buffer solutions containing $400 \mathrm{ppb}$ dissolved oxygen at different temperatures based on the slopes of Mott-Schottky plots. 204

Table X.6. Calculated defect density of passive films formed on Type 304L SS and Type 316L $\mathrm{SS}$ at $+0.3 \mathrm{~V}_{\text {OCP }}$ in borate buffer solutions containing $400 \mathrm{ppb}$ dissolved oxygen at different temperatures based on the slopes of Mott-Schottky plots. 215

Table X.7. Functional forms of the rate constants with applied potential for Reactions (1) - (7) and the exponential coefficients for these rate constants $[17,63]$ 225

Table X.8. Values of the parameters extracted from the optimization of the mixed potential model on the experimental EIS data for passive films formed on Alloy 600 at OCP in a solution containing 2000 ppm B + 2 ppm Li and 400 ppb dissolved oxygen at different temperatures. 240

Table X.9. Values of the parameters extracted from the optimization of the mixed potential model on the experimental EIS data for passive films formed on Alloy 600 at OCP in a solution containing 200 ppm B + 2 ppm Li and 400 ppb dissolved oxygen at different temperatures. 241

Table X.10. Values of the parameters extracted from the optimization of the mixed potential model on the experimental EIS data for passive films formed on Alloy 600 at $+0.3 \mathrm{~V}_{\text {ocP }}$ in a solution containing 200 ppm B + 2 ppm Li and 400 ppb dissolved oxygen at different temperatures... 242 Table X.11. Values of the parameters extracted from the optimization of the mixed potential model on the experimental EIS data for passive films formed on Alloy 600 in a solution containing 0 ppm B + 2 ppm Li and 400 ppb dissolved oxygen at different temperatures.

Table X.12. Calculated values of activation energies for anodic metal dissolution of Alloy 600, Alloy 690, Type 304L SS, and Type 316L SS in oxygenated borate buffer solution. 248 
Table X.13. Several properties that were calculated based on the extracted coefficients for passive films formed on Alloy 600 under OCP in a solution containing 2000 ppm B + 2 ppm Li, and 400 ppb dissolved oxygen at different temperatures.

Table X.14. Several properties that were calculated based on the extracted coefficients for passive films formed on Alloy 600 under OCP in a solution containing 200 ppm B + 2 ppm Li, and 400 ppb dissolved oxygen at different temperatures.

Table X.15. Several properties that were calculated based on the extracted coefficients for passive films formed on Alloy 600 at $+0.3 \mathrm{~V}_{\text {OCP }}$ in a solution containing $200 \mathrm{ppm} \mathrm{B}+2$ ppm Li, and 400 ppb dissolved oxygen at different temperatures. 253

Table X.16. Several properties that were calculated based on the extracted coefficients for passive films formed on Alloy 600 under OCP in a solution containing 0 ppm B +2 ppm Li, and 400 ppb dissolved oxygen at different temperatures. 254

Table X.17. OCP values for all alloys in borate buffer solutions with $400 \mathrm{ppb}$ dissolved oxygen. 257

Table XIII.1. Input files. 305

Table XIII.2. Output file names and contents. 313

Table XIII.3. List of input parameters for sensitivity analysis. 317

Table XIII.4. The highest sensitivity of $\mathrm{H}_{2}$ concentration to input parameters under normal and hydrogen water chemistry conditions. 318

Table XIII.5. The highest sensitivity of $\mathrm{O}_{2}$ concentration to input parameters under normal and hydrogen water chemistry conditions.

Table XIII.6. The highest sensitivity of $\mathrm{H}_{2} \mathrm{O}_{2}$ concentration to input parameters under normal and hydrogen water chemistry conditions 319

Table XIII.7. The highest sensitivity of ECPSHE to input parameters under normal and hydrogen water chemistry conditions. 320

Table XIII.8. The highest sensitivity of CGR to input parameters under normal and hydrogen water chemistry conditions. 320

Table XIII.9. Sensitivity to radiolytic yields. ............................................................. 321

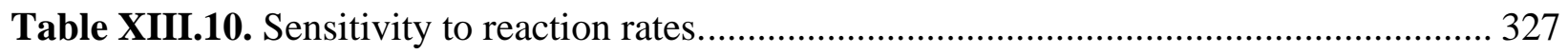

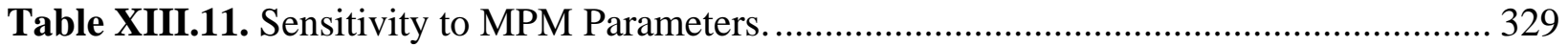

Table XIII.12. Sensitivity to Reactor Modeling Parameters. ............................................ 330 
Table XIII.13. Primary G-values for fast neutrons at high temperature............................... 332

Table XIII.14. Primary G-values for gamma radiation at high temperature ......................... 332

Table XIII.15. Christensen's (2002) reaction set. ............................................................. 348

Table XIII.16. Ishigure's (1987) reaction set................................................................... 349

Table XIII.17. Reduced reaction rate sets. .......................................................................... 360

Table XIII.18. Hydrogen peroxide decomposition reactions in Christensen data set.............. 362

Table XIII.19. List of additional chemical reactions......................................................... 364

Table XIII.20. PWR simulation results before and after replacement "abs" with "fabs." ........ 369

Table XIII.21. PWR simulation results after implementing a generic kinetics algorithm. ....... 369

Table XIII.22. PWR simulation results from UC Berkeley and from author........................ 370 


\section{List of Acronyms}

LWRs

ECP

EAC

CGR

PCC

CEP

CEFM

IGSCC

HWC

HIC

HER

OER

RPVs

PTS

PWR

BWR

MPM

ACGR

$\mathrm{CF}$

ANN

BL

OL

BL/OL
Light water reactors.

Electrochemical corrosion potential.

Environmentally-assisted cracking.

Crack growth rate.

Primary coolant circuit.

Corrosion evolutionary path.

Coupled environment fracture model.

Inter-granular cracking.

Hydrogen water chemistry.

Hydrogen-induced cracking.

Hydrogen electrode reaction.

Oxygen electrode reaction.

Reactor pressure vessels.

Pressurized thermal shock.

Pressurized water reactor.

Boiling water reactor.

Mixed potential model.

Advanced crack growth rate.

Corrosion fatigue.

Artificial neural network.

Barrier layer.

Outer layer.

Barrier/outer layer interface. 
TGSCC

HTC

SCC

SDR

GBVP

SIF

SG

PDM

OCP

EIS

MSA

HER

OER

XFEM

WF

SIFICs

OEM

IASCC

NWC

FEM
Transgranular stress corrosion cracking. Heat transport circuit. Stress corrosion cracking. Slip-dissulution repassivation. Grain boundary void pressurization. Stress intensity factor. Steam generator. Point defect model. Open circuit potential. Electrochemical impedance spectroscopy. Mott-Schottky analysis. Hydrogen electrode reaction. Oxygen electrode reaction. Extended finite element method. Weight function. Stress intensity factor influence coefficients. Original equipment manufacturer. Irradiation assisted stress corrosion cracking. Normal water chemistry. Finite element method. 


\section{Background}

The primary goal of the proposed research is the development of deterministic, physicoelectrochemical models for predicting the accumulation of localized corrosion damage (pitting corrosion, stress corrosion cracking and corrosion fatigue) in the primary coolant circuits of the currently operating fleet of Light Water Reactors (LWRs) and to embed the models into the Grizzly and code for modeling nuclear power plant component aging, which is based on Idaho National Laboratory's simulation environment (MOOSE). Localized corrosion in LWR (BWR and PWR) primary coolant circuits is primarily an electrochemical phenomenon [1], the rate of which is determined by certain electrochemical properties, such as the electrochemical corrosion potential (ECP), solution conductivity, temperature, $\mathrm{pH}$, flow rate, and the kinetics of the reduction of a cathodic depolarizer (e.g. $\mathrm{O}_{2}$ ) on the surfaces external to the crack, in addition to mechanical loading (stress intensity factor on the crack) and micro-structural/micro-chemical factors (grain size, precipitates, etc). Because the efficient control of environmentally-assisted cracking (EAC) damage accumulation requires the accurate control of these parameters, it is necessary to develop codes that can accurately predict their values and the crack growth rate (CGR) at any point in the primary coolant circuit (PCC) over wide ranges of temperature $\left(25^{\circ} \mathrm{C}\right.$ to $\left.320^{\circ} \mathrm{C}\right), \mathrm{pH}(6-8), \mathrm{ECP}$ $\left(-0.9 \mathrm{~V}_{\text {she }}\right.$ to $\left.-0.2 \mathrm{~V}_{\text {she }}\right)$, solution conductivity, flow rate $(1-6 \mathrm{~m} / \mathrm{s})$, and stress intensity factor $(5$ $\mathrm{MPa} . \mathrm{m}^{1 / 2}$ - $50 \mathrm{MPa} . \mathrm{m}^{1 / 2}$ ), reflecting the properties in reactor PCCs. Knowledge of these parameters, along with suitable codes, would allow one to predict the accumulated damage in PCC as a function of the future operating history of the reactor (the "corrosion evolutionary path," CEP). If this can be done successfully, tremendous economic and safety benefits would accrue to the operators because it would allow the reactors to be operated in a manner to reduce both the risk and cost of damage and to enhance safety. Of importance is that the codes would allow operators to explore "what if" options in plant operation, to define the resulting cost-benefit and safety-risk issues. We have previously developed codes of this Type, DAMAGE-PREDICTOR, ALERT, REMAIN, and FOCUS [1,2], for predicting EAC damage in the primary coolant circuits in BWRs and the codes have been used to predict damage in 14 BWRs world-wide. One of these codes (REMAIN) was installed in Siemens' reactors operating in Germany, and another was installed in Fermi II (ALERT) in Michigan. Generally, these codes have demonstrated a remarkable ability to predict EAC damage in operating BWRs, even though they lacked a viable model for crack initiation. A much less capable PWR code (PWR_ECP) was also developed, but it predicted only 
the ECP, because a viable coupled environment fracture model (CEFM) for estimating crack growth rate in non-stainless steels, such as Ally 600, does not currently exist.

The work developed advanced damage simulation codes for predicting EAC damage in the primary coolant circuits of currently operating (Generation II) LWRs; BWRs and PWRs of the generic General Electric and Westinghouse designs, respectively. In BWRs, the primary coolant is pure water while in PWRs it is a boric acid $\left[\mathrm{B}(\mathrm{OH})_{3}\right]+$ lithium hydroxide $[\mathrm{LiOH}]+\mathrm{H}_{2}$ solution, with the relative concentrations of the components being adjusted during operation, so that the $\mathrm{pH}$ follows a predetermined $\mathrm{pH}$ trajectory, in order to minimize corrosion and activity transport. Water radiolysis, and hence the formation of corrosive species, such as $\mathrm{O}_{2}$ and $\mathrm{H}_{2} \mathrm{O}_{2}$, is suppressed in the PCCs of PWRs by the added hydrogen and this protocol has also been adopted in BWRs in the form of Hydrogen Water Chemistry [3], in order to displace the electrochemical corrosion potential (ECP) below the critical potential of $-0.23 \mathrm{~V}_{\text {she }}$ for intergranular stress corrosion cracking (IGSCC) in sensitized Type 304 SS. About 20 years ago, the PI and his colleagues developed powerful codes to describe the impact of water radiolysis on the chemistry and electrochemistry of both BWRs and PWRs [1,2].

All objectives of this work were achieved, including the delivery of BWR_MASTER and PWR_MASTER to the Idaho National for embedment in Grizzly. In addition, several issues that were not included in the contracted deliverables were successfully addressed, including the theoretical assessment of the distribution in crack growth rate in Type 304 SS in BWR primary coolant in response to random (normal) distributions in the independent variables, incorporation of a correction to the theory of the electrochemical kinetics of redox reactions on passive metals due the quantum mechanical tunneling of electrons and electron holes across the barrier oxide layer, development of a new, innovative method for monitoring, in situ, the hydriding of zirconium alloy fuel cladding under reactor operating conditions by optimizing a modified PDM for hydride/oxide formation on experimental EIS data, which has been demonstrated on the hydriding of pure zirconium in PWR coolant at $250{ }^{\circ} \mathrm{C}$, and

\section{Boiling water reactors (BWRs)}

Over the past two decades, several water radiolysis codes that predict the concentrations

of radiolysis products (principally $\mathrm{H}_{2}, \mathrm{O}_{2}$, and $\mathrm{H}_{2} \mathrm{O}_{2}$ ) around the primary coolant circuits of 
BWRs and in other aqueous radiolytic systems have been developed by the PI and his colleagues [1-2, 4-8]. In this previous work [4-8], we combined a radiolysis code with a mixed potential model (MPM) [7] to predict ECP, and with the deterministic crack growth rate algorithm (coupled environment fracture model, CEFM) $[9,11]$ to form a general code, DAMAGEPREDICTOR [4-6], in order to predict damage due to SCC in BWR HTCs. These steadystate models were applied to fixed state points of operation to yield "snap-shots" of the developing damage in a differential sense (i.e., in terms of the CGR). However, the damage is cumulative, so that prediction of the extent of damage at any given time must integrate the crack growth rate over the history of the plant, which is an innovation that was subsequently added in the later codes ALERT, REMAIN, and FOCUS [12-14]. We emphasize that our approach is largely deterministic, in that the outputs of the component models are constrained by known physicochemical (natural) laws [12]. These algorithms have been successfully used in modeling fourteen BWRs operating in the US, Japan, and Europe. Two of these reactors (Duane Arnold and Dresden-2) were included in the original study of Ruiz et al. [15], who showed that the reactors exist at the opposite extremes in the spectrum of reactor response to hydrogen water chemistry (HWC). Indeed, DAMAGE-PREDICTOR was initially calibrated on data from only Dresden-2, by adjusting the rate constant for the thermal decomposition of $\mathrm{H}_{2} \mathrm{O}_{2}$ within the ranges of values that had been observed experimentally. The predicted values for $\left[\mathrm{O}_{2}\right]$ and $\left[\mathrm{H}_{2}\right]$ in the main steam line and in the liquid (recirculation system) phase, and the predicted ECP, as measured in autoclaves connected to the recirculation piping, were in good accord with plant data that had been reported by Ruiz et al. [15] and by Indig and Nelson [16].

In Additionally, the radiolysis/ECP/crack growth rate model (ALERT) has been used to model BWRs containing external coolant pumps in "double-blind" mini-tests (e.g., on the KKL "Leibstadt" BWR in Switzerland [12,13]), where the plant results (ECP, oxygen concentration) were not known to us in advance. Subsequent comparison of the predicted and measured data showed that the code provides for accurate simulation of reactor coolant electrochemistry. Importantly, no recalibration of DAMAGE-PREDICTOR has been necessary when simulating reactors across the entire HWC response spectrum. The code REMAIN was developed to model German BWRs that contain internal main coolant pumps. This code also contains deterministic models for calculating radiolytic species concentrations, ECP, and crack growth rate, but has been optimized for speed of execution, so that calculations may be performed in "real-time." Thus, by 
employing an optimized algorithm for solving simultaneous ordinary differential equations, and by writing the code in $\mathrm{C}++$, the time of execution on a $200 \mathrm{MHz} \mathrm{PC}$, compared with that of DAMAGE-PREDICTOR, has been reduced by a factor of 50 to 100 . This enhanced code permits a single state point to be simulated in 1-2 min, thereby making practical iterative calculations over a locus of the state points representing the CEP.

The main body of the algorithm (Figure I.1) is the water radiolysis model, which yields the concentrations of radiolysis products from the decomposition of water under neutron and gamma irradiation (and alpha irradiation in the case of PWRs), coupled with homogeneous and heterogeneous chemical reactions, liquid/steam transfer of volatile species $\left(\mathrm{H}_{2}\right.$ and $\left.\mathrm{O}_{2}\right)$, and fluid convection.

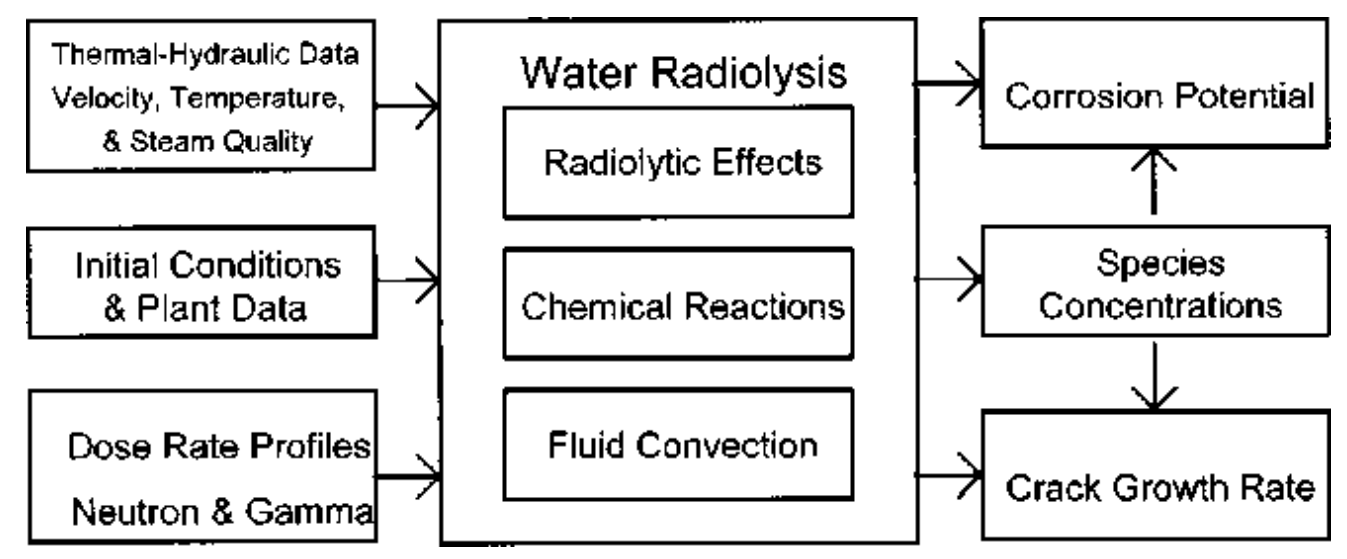

Figure I.1. Structure of the algorithm.

After the species concentrations have been determined around the entire PCC under steady-state conditions, the ECP is calculated using the MPM [7], and the crack growth rate is estimated using the coupled environment fracture model (CEFM) [9,17,18]. Auxiliary input parameters, such as flow velocity, coolant temperature, steam quality, and neutron and gamma dose rates in the coolant are obtained from other codes (e.g., thermohydraulic data from RETRAN). According to the CEFM, crack advance occurs via the slip- dissolutionrepassivation mechanism at the crack tip augmented by hydrogen-induced cracking (HIC) [18]. The dissolution of metal (crack growth) takes place when the oxide layer ruptures due to elastic and plastic deformation at the crack tip, and a bare metal surface is exposed to the crack environment. Provided that the crack aspect ratio $(x / a$, where $x$ is the crack length and $a$ is 
the crack mouth opening displacement) is greater than about 5, which is certainly the case for a long, tortuous intergranular crack in a sensitized stainless steel, particularly when oriented transverse to the fluid flow direction, the crack internal environment is hydrodynamically quiescent, and the CEFM can be used to calculate the crack growth rate $[1,2]$ accurately. The physico-electrochemical condition that must be satisfied by a growing crack is the conservation of charge, which requires that the net positive current exiting the crack mouth be consumed quantitatively on the external surfaces by various cathodic reactions (e.g., reduction of oxygen and hydrogen peroxide). Thus, the CEFM emphasizes that the crack internal and external environments are strongly coupled, a postulate that has been demonstrated unequivocally by experiment [18-20]. Indeed, the CEFM predicts that modifying the electrocatalytic properties of the external surface may mitigate or accelerate crack growth, depending on whether the reactions are inhibited or catalyzed, respectively [19]. Thus, we have shown theoretically that, if the exchange-current densities of the redox reactions (reduction of oxygen and hydrogen peroxide and the oxidation of hydrogen) are increased in the presence of a stoichiometric excess of hydrogen or are decreased with no restriction on the ratio of hydrogen to oxidizing species concentrations, the ECP is displaced in the negative direction and the crack growth rate is decreased. The first case is the basis of noble metal-enhanced hydrogen water chemistry (NMEHWC), as achieved by using noble metal coatings on the stainless-steel surfaces external to the crack [3]. Recent work [19] indicates that this strategy increases the exchange-current densities for the hydrogen electrode reaction (HER) and the oxygen electrode reaction (OER) at $288{ }^{\circ} \mathrm{C}$ by factors of about 20 and 3, respectively, as determined by fitting DAMAGEPREDICTOR to plant data. NMEHWC is now being applied in operating BWRs. The disadvantages of NMEHWC are the cost of the noble metal, the need to store hydrogen on-site, coolant circuit activation due to $\mathrm{Co}^{60}$ transport, the formation of $\mathrm{N}^{16}$, and the uncertainty (and unknown negative impact) associated with the introduction of new material into the PCC on future reactor operation. This latter factor may require that the reactor continue to operate on NMEHWC for the remainder of its life, or at least until a strategy is devised for removing unreactive noble metals from the surfaces [19]. On the other hand, inhibition of the redox reactions that occur on the stainless-steel surfaces external to the crack is predicted and found [20] to strongly decrease the crack growth rate, even in the absence of HWC. Inhibition may 
be achieved by a variety of means, including the deposition of a dielectric coating on the steel surface, as has been recently demonstrated in the laboratory [20].

The crack length, $x_{N}$, over the anticipated service time of a component, $T$, is obtained by an accumulation of the crack advances over $N$ periods $\Delta t_{1}, \ldots, \Delta t_{i}, \ldots, \Delta t_{N}$

$X_{i}=X_{i-1}+\mathrm{CGR}_{i} \bullet \Delta t_{i}, \quad i=1, \ldots, N$

The crack growth rate, CGR $i$, is presumed to be time-independent for each interval, $\Delta t_{i}$, but of course, it depends on the crack length (through $K_{I}$ and because of changes in the current and potential distributions in the crack internal and external environments) and hence changes from increment to increment. The initial crack length, $X_{0}$, corresponds to the depth of a preexisting crack (detected during an inspection or assumed for a safety analysis scenario). The algorithm outlined above for calculating the accumulated damage is essentially identical to that employed [21] to predict the lifetime of disks in low-pressure steam turbines under conditions where thin electrolyte films precipitate on the steel surface. Finally, we note, parenthetically, that the present calculations have been made possible by enhancing the mathematical and computational procedures to achieve reductions in the execution time of the code by a factor of about 100. Thus, a typical simulation involves a value of $N$ ranging from 40 to 100 . The nonenhanced DAMAGE-PREDICTOR typically requires $1-2.5 \mathrm{~h}$ to execute a single state point on a $200-\mathrm{MHz}$ computer to yield a total simulation time of up to $250 \mathrm{~h}$ (i.e., more than $11 \mathrm{~d}$ ). However, the enhanced code (in this case, REMAIN) performs the simulations in a few minutes, thereby rendering the calculations "practical" from a user's point of view. This is the most important issue for the acceptance of the codes.

The impact that hydrogen water chemistry (HWC) is predicted to have on the accumulated damage is illustrated for one case (Location, No. 1) in Figure I.2. Thus, the addition of $0.5 \mathrm{ppm}$ of $\mathrm{H}_{2}$ to the feedwater, under HWC operating conditions, is predicted to decrease the increment in accumulated damage at the bottom (exit) of an internal pump after $40 \mathrm{yr}$ of continuous operation (or approximately $0.75 \times 40 \times 365=10,950$ EFPDs, assuming an availability of 75 percent), by a factor of almost ten from $2.0-0.5=1.5 \mathrm{~cm}(0.591 \mathrm{in}$.) to $0.655-$ $0.50=0.155 \mathrm{~cm}(0.061 \mathrm{in}$.). 

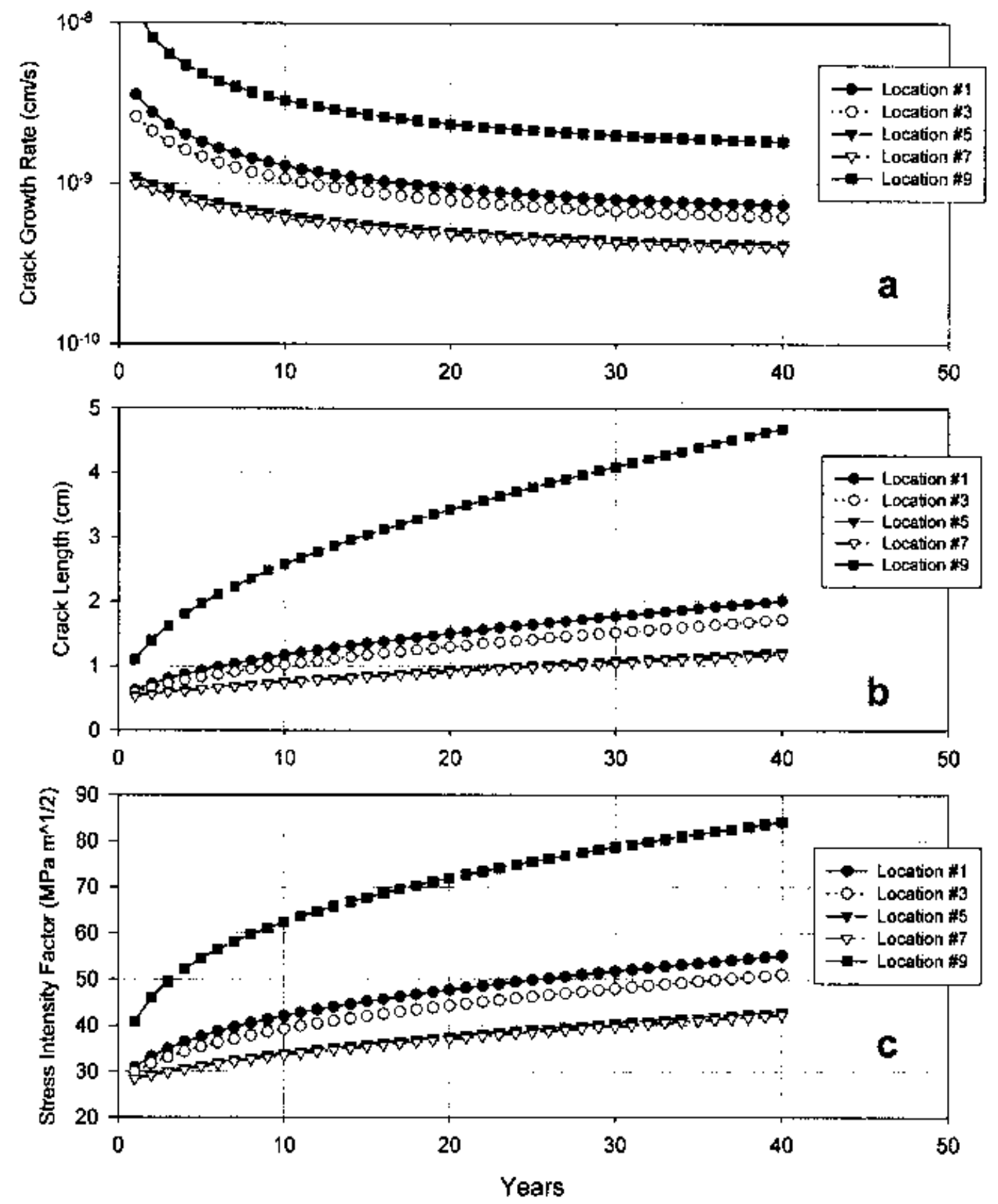

Figure I.2. Predicted histories of growing cracks in the lower plenum of an operating, German BWR. Location No. 1 is at the bottom of the internal pump, and location No. 9 is at the bottom entrance to the core. Intermediate locations, Nos. 3, 5, and 7, represent locations near the bottom of the reactor vessel and at various elevations along the control rod drive structures.

This is a substantial reduction in the extent of damage that could not have been estimated from the crack growth rates at a single state point alone (because of the different, nonlinear 
dependencies of crack growth rate on time for the two cases shown in Figure I.3) or by using a model that fails to recognize a dependence of crack growth rate on the electrochemical crack length.

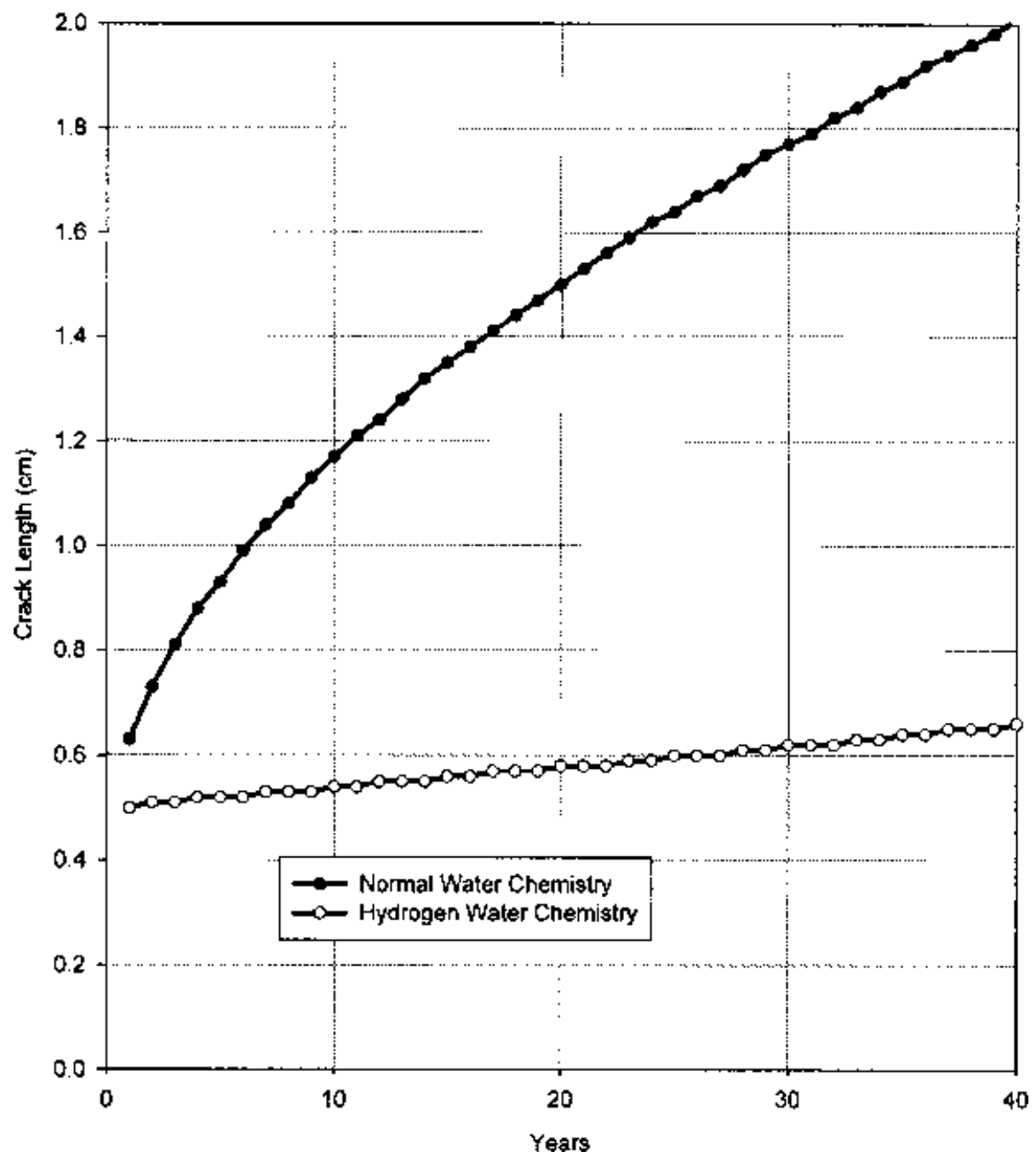

Figure I.3. Predicted effect of HWC feedwater $\left[\mathrm{H}_{2}\right]=0.5 \mathrm{ppm}$ on the accumulated damage due to IGSCC in the core shroud of a BWR. The initial value of the crack length of $0.5 \mathrm{~cm}, 0.197$ in may be considered to be the depth of a detected crack, and the time in years as the remaining extended service time after introducing HWC.

In this particular case, neither stress-controlled failure (i.e., $K_{I}>K_{I C}$ ) nor damagecontrolled failure $\left(X>X_{\text {lim }}\right)$ is predicted to occur, provided that $X_{\lim }>2 \mathrm{~cm}(0.787$ in.). These codes were not equipped with a crack initiation model so that damage could only be predicted, 
assuming the presence of a pre-existing crack. This important deficiency will be corrected in the proposed work.

\section{Pressurized water reactors (PWRs)}

About a decade ago, the PI developed a simulation code for PWRs (PWR_ECP) for the French vendor Framatome, but much less work has been reported on simulating pressurized water reactors than boiling water reactors. Its primary output was the ECP calculated at closely-spaced points around the primary coolant circuit. This code demonstrated that the PCC could not be regarded as being a "giant hydrogen electrode", with the ECP being clamped by the hydrogen electrode equilibrium reaction, as has been suggested by others, but rather it was found that the ECP displayed remarkably large variations around the circuit (Figure I.4).

\section{Normal Operation, Main Loop (Core tube 6) Cycle 3}

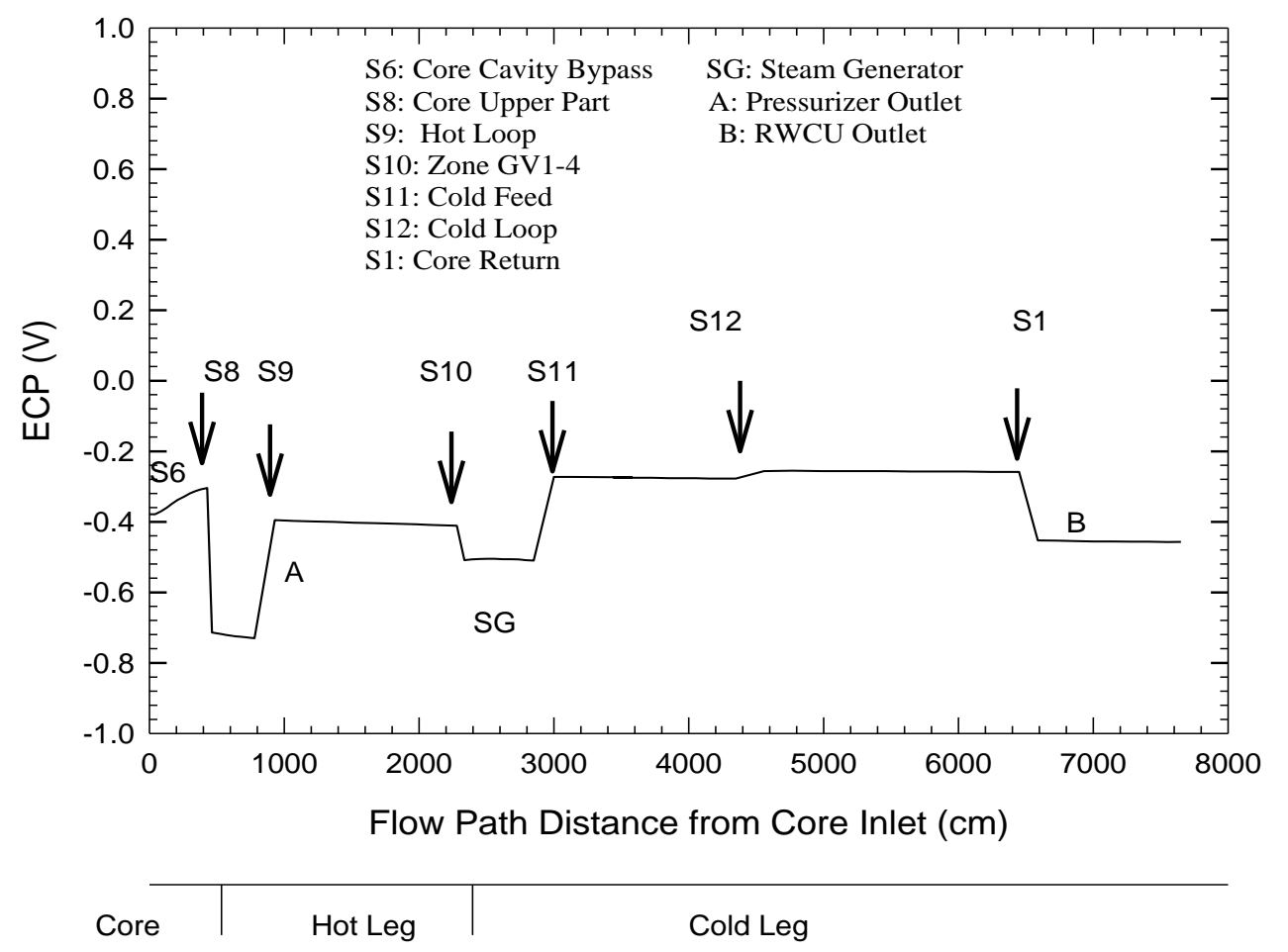

Figure I.4. Predicted ECP around the PCC of a French PWR under normal, full power operating conditions. $\left[\mathrm{H}_{2}\right]=25 \mathrm{cc}(\mathrm{STP}) / \mathrm{kg} \mathrm{H}_{2} \mathrm{O},[\mathrm{B}]=840 \mathrm{ppm},[\mathrm{Li}]=3.5 \mathrm{ppm}[1]$. 
Of particular importance is the prediction that the ECP, under current operating conditions of $30 \mathrm{cc}(\mathrm{STP}) / \mathrm{kg}$ of $\mathrm{H}_{2}$ results in a value of the ECP in the reactor vessel, the primary side of the steam generator, and in the pressurizer that is perilously close to, or even more negative than the critical potential for the occurrence of HIC of MA Alloy 600 (Figure I.5) [22]. As time has gone by, operators have progressively added more hydrogen to the PCCs of PWRs, thereby gradually displacing the ECP to more negative values. Thus, a central issue in this analysis will be the extent to which the ECP will be displaced in the negative direction by the adopted water chemistry protocol (primarily $\mathrm{pH}$ and $\left[\mathrm{H}_{2}\right]$ ), because of Totsuka and Smialowska's [22] important finding that MA Alloy 600 suffers intergranular, HIC when the potential is displaced below $-0.80 \mathrm{~V}_{\text {she }}$ (Figure I.5). This phenomenon represents a direct threat to the integrity of the PCC and is postulated by the PI to be responsible for a host of PWR operating problems, including the primary side stress corrosion cracking of steam generator tubes, cracking of stainless-steel baffle bolts, springs, and other internal components, cracking of CRD head penetrations, and cracking of pressurizer components. The developed PWR code will allow prediction of the most probable locations for HIC via calculation of the ECP and, when combined with crack initiation and crack growth rate models, will allow the prediction of the accumulated stress corrosion cracking damage over the corrosion evolutionary path of the reactor.

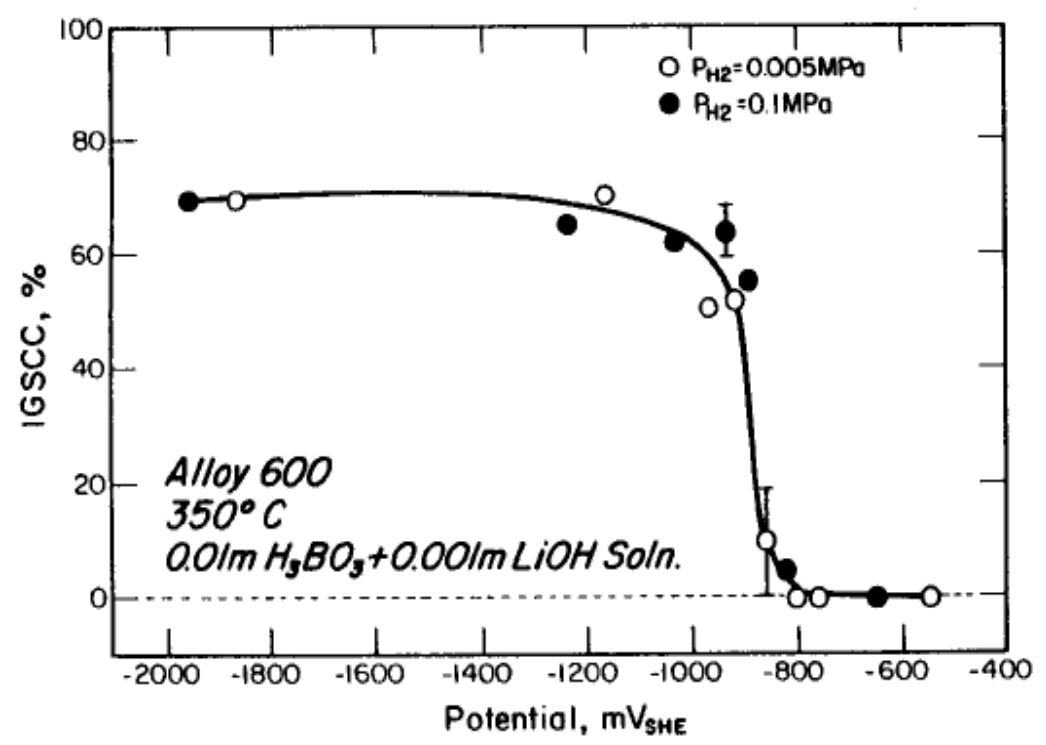

Figure I.5. Percentage of IGSCC on the fracture surface as a function of applied potential on the standard hydrogen scale. After Totsuka and Smialowska [22]. 


\section{Opportunities for code expansion and development}

The goal of this work is to augment the fracture mechanics capabilities in INL's Grizzly code, with models that account for environmental effects in the fracture of components in LWRs. The Grizzly simulation code is being developed at INL under the Risk-Informed Safety Margin Characterization Pathway of the Light Water Reactor Sustainability (LWRS) program to provide an improved scientific basis for decisions surrounding renewals of nuclear power plant licensed in the United States for operation beyond 60 years. Thus, Grizzly is intended to provide a multiphysics, multiscale computational platform to simulate both the aging processes and the effects of aging on the ability of these components to perform their intended tasks safely. The code is based on the finite element method and includes fracture mechanics capabilities for describing brittle fracture in irradiation-damaged Reactor Pressure Vessels (RPVs). Ultimately, Grizzly will address a wide range of aging issues in key components, including the reactor pressure vessel (RPV), core internals, containments, piping, concrete structures, and cables, but in order to do that it must incorporate an ability to predict environmental effects in elastic-plastic alloys, such as the stainless steels. However, as noted above, the code in its current form does not have any capabilities for predicting environmental effects in elastic-plastic alloys. Thus, by combining the LWR codes that will be developed in the proposed work with Grizzly, a powerful tool will be obtained that is greater than the sum of the individual parts. This is, because, if environmental effects are of interest (e.g., in IGSCC of the core shroud in a BWR), Grizzly can be used to compute the stress intensity factor as the crack propagates through a component of any geometry, particularly as the stresses (loading and residual) change (e.g., relax during shutdown) as the reactor progresses along the CEP. At the current time, the IGSCC code, ALERT, incorporates a stress intensity solution for an infinitely sharp crack existing in an infinite phase assuming LEFM. Because Grizzly also computes J-integrals, the mechanical basis of ALERT and the corresponding PWR code will be greatly enhanced to include almost any crack geometry, by incorporating small-scale yielding in elastic-plastic alloys, and by incorporating microstructural effects (see below). The advantage for INL's Grizzly code, is that by calling ALERT for IGSCC in BWRs or the equivalent code for PWRs, the INL codes will be applicable to predicting environmental fracture damage in a wide range of alloys in LWR primary coolant circuits and not be restricted to predicting brittle fracture only in irradiation-damaged RPVs, which is their primary target at present. Thus, the Grizzly and the environmentally-based LWR codes that will be developed in the proposed work for both 
current (Generation II) BWRs and PWRs will be rendered capable of predicting SCC damage in all components across the entire spectrum of metallurgy and mechanics that exists in the reactor primary coolant circuits. This capability is unprecedented in reactor damage simulation.

Grizzly is based on and relies heavily upon the open-source MOOSE framework developed at Idaho National Laboratory. MOOSE provides an environment for solving tightly coupled systems of partial differential equations on parallel computers using the finite element method. MOOSE provides the framework for rapid development of physics simulation codes as well as access to solvers appropriate for nonlinear multi-physics problems. In addition to providing a solution framework, MOOSE provides a set of modules for modeling physics that are shared across applications using this framework. This growing set of common modules currently includes capabilities for solid mechanics, heat conduction, contact, phase field methods, and partially saturated flow. Codes targeted at specific applications can be developed by combining and building upon the capabilities provided in the MOOSE modules.

The reactor pressure vessel (RPV) has been chosen as the first application for Grizzly because of its critical role in ensuring the safe operation of nuclear power plants, and because of the extreme difficulty involved in replacing or repairing the RPV. The susceptibility of an embrittled RPV to fracture during a pressurized thermal shock (PTS) loading event is a potential safety concern for license renewal. To model this problem, Grizzly currently provides a general 2D axisymmetric or 3D thermo-mechanics capability to model the global response of the vessel to PTS conditions. Figure I.6 shows representative simulation results for a thermomechanical simulation of an RPV under PTS loading.

It also provides a capability to transfer results to a 3D sub-model of the local region in the vicinity of a postulated flaw and provides fracture domain integral capabilities to evaluate mode- $I$ stress intensity factors along the front of a meshed crack, as shown in Figure I.7. The embrittling effects of neutron irradiation and thermal aging are accounted for by using the physically-based correlations in the EONY model [1] to calculate the transition temperature shift. Grizzly can perform fracture mechanics analysis either by using detailed 2D or 3D fracture mechanics models that directly represent the crack, or by using reduced order models based on those detailed models. 


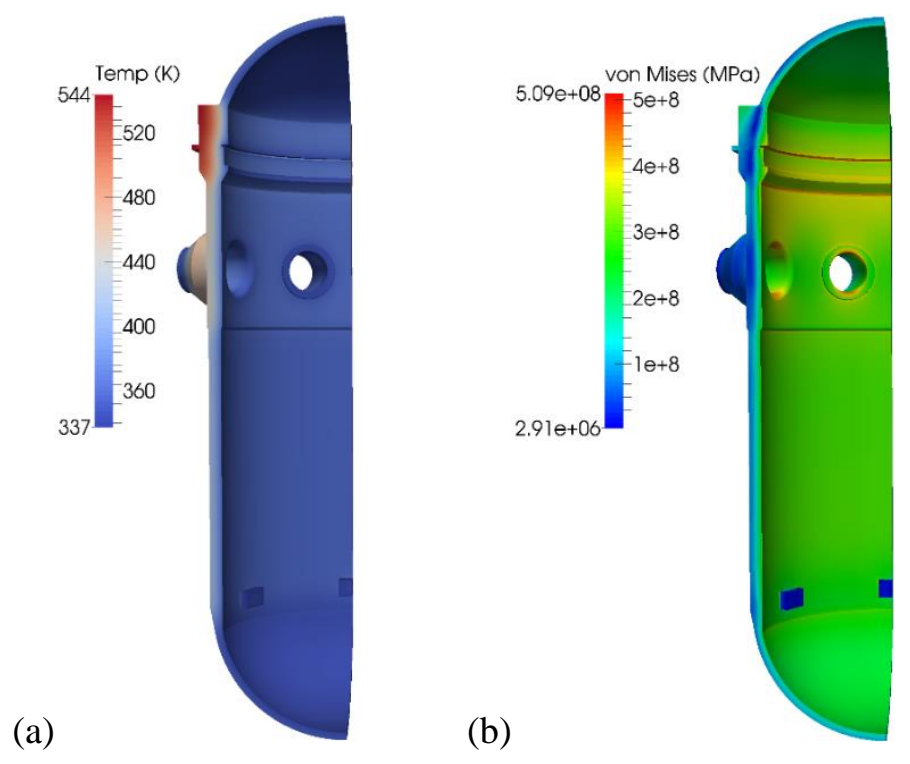

Figure I.6. Grizzly thermo-mechanical solution results for the RPV model showing (a) temperature contours and (b) von Mises stress contours at one point in time during a simulation of a PTS event.
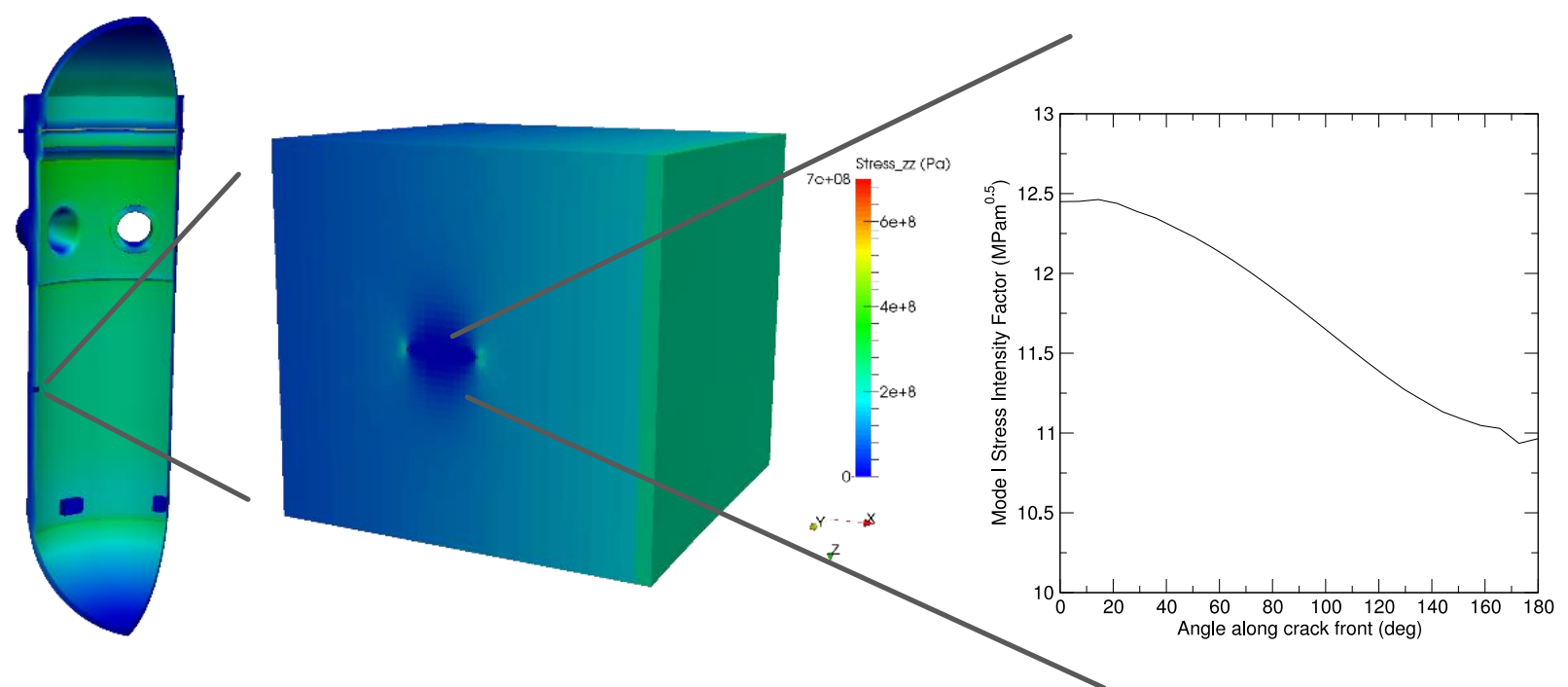

Figure I.7. Global model of an RPV under PTS conditions (left) and sub-model of material in the vicinity of a postulated embedded circular crack (middle) used to calculate mechanical response and stress intensity factor (right) along the front of that crack. 
Significant work is also underway to develop lower length scale models to model the microstructure evolution underlying the embrittlement process for RPV steel. Where applicable, models for this are being developed in Grizzly, including models for precipitate coarsening using cluster dynamics models, MOOSE's phase field capabilities, crystal plasticity, and cohesive zone models for modeling fracture test specimens. Atomistic scale models are run in other codes and provide input into these models.

Recent work has demonstrated Grizzly's ability to model arbitrary propagating cracks in 3D [23]. This is done using the extended finite element method (XFEM), which allows cracks to be represented at arbitrary locations relative to the finite element mesh. This capability is the topic of continuing development, and with additional work, is expected to allow for the propagation of arbitrary non-planar cracks in 3D driven by processes such as stress corrosion cracking. Accurately modeling this physical process requires using a combination of a sophisticated fracture mechanics code and environmental damage codes such as BWR_MASTER and PWR_MASTER.

\section{I.1. References}

[1] D.D. Macdonald and M. Urquidi-Macdonald, The Electrochemistry of Nuclear Reactor Coolant Circuits, Encyclopedia of Electrochemistry, A.J. Bard and M. Stratmann eds. Vol 5 Electrochemical Engineering, Edited by Digby D. Macdonald and Patrik Schmuki, WileyVCH Verlag GmbH \& Co. KGaA, Weinheim, pp. 665-720, (2007).

[2] D.D. Macdonald and I. Balachov, Modeling the Accumulation and Mitigation of SCC Damage in BWRs, Proc. 9-th. Int. Symp. Mats. Degrad. Nucl. Power Systs.-Water Reactors, (Ed. F. P. Ford, S. M. Bruemmer, and G. S. Was), TMS, Warrendale, PA., pp. 399-407 (1999).

[3] Y.J. Kim, L.W. Niedrach, and P.L. Andresen, Corrosion, 52, 738-743 (1996).

[4] T.K. Yeh, D.D. Macdonald, and A.T. Motta, Nucl. Sci. Eng., 121, 468-482 (1995).

[5] T.K. Yeh, D.D. Macdonald, and A.T. Motta, Nucl. Sci. Eng., 123, 295-304 (1996).

[6] T.K. Yeh, D.D. Macdonald, and A.T. Motta, Nucl. Sci. Eng., 123, 305-316 (1996).

[7] D.D. Macdonald, Corrosion, 48, 194-205 (1992). 
[8] X. Zhou, D.D. Macdonald, and I. Balachov, Enhancing the Operation of Boiling Water Reactors by Deterministic Simulation, Proc. Water Chemistry ‘98, 1998 JAIF Int. Conf. Water Chem. Nucl. Power Plants, Kashiwazaki, Japan, Oct. 13-16 (1998).

[9] D.D. Macdonald and M. Urquidi-Macdonald, Corros. Sci., 32, 51-81 (1991).

[10] D.D. Macdonald, Corrosion, 48, 194-205 (1992).

[11] D.D. Macdonald and M. Urquidi-Macdonald, Corros. Sci., 32, 51-81 (1991).

[12] D.D. Macdonald, PowerPlant Chemi., 6, 731 (2004).

[13] I. Balachov, G.R. Engelhardt, and D.D. Macdonald, The Electrochemistry of Stress Corrosion Cracking - from Theory to Damage Prediction in Practical Systems, Environment-Induced Cracking of Materials, Volume 2: Prediction, Industrial Development, and Evaluation, Elsevier, 55-92 (2008).

[14] D.D. Macdonald and G.R. Engelhardt, Predictive Modeling of Corrosion, In: Richardson J A et al. (eds.), Shrier's Corrosion, Amsterdam: Elsevier, 2, 1630-1679 (2010).

[15] C.P. Ruiz, Modeling Hydrogen Water Chemistry for BWR Applications, EPRI-NP-6386, Electric Power Research Institute, Palo Alto, CA, 1989.

[16] M. Indig and J.L. Nelson, Corrosion (Houston), 47, 202-209 (1991).

[17] D.D. Macdonald, P.C. Lu, M. Urquidi-Macdonald, and T.K. Yeh, Corrosion (Houston), 52, 768-785 (1996).

[18] M.P. Manahan, D.D. Macdonald, and A.J. Peterson, Corros. Sci., 37, 189-208 (1995).

[19] T.K. Yeh, M.S. Yu, and D.D. Macdonald, The Effect of Catalytic Coatings on IGSCC Mitigation for Boiling Water Reactors Operated Under Hydrogen Water Chemistry, Proceedings 8th International Symposium on the Environmental Degradation of Materials in Nuclear Power Systems - Water Reactors, NACE International, Houston, TX, 1, 551-558 (1997).

[20] X. Zhou, I. Balachov, and D.D. Macdonald, Corros. Sci., 40, 1349-1362 (1998).

[21] C. Liu and D.D. Macdonald, ASME J. Pressure Vessel Technol., 119, 393-400 (1997).

[22] N. Totsuka and Z. Szklarska-Smialowska, Corrosion, 43, 734 (1987). 
[23] B.W. Spencer, S.A. Pitts, L.Liu, M. Vyas, W. Jiang, A. Casagranda, and D.J. McDowell. Summary of Grizzly development for advanced reactor structural materials. INL/EXT-1955958, Idaho National Laboratory, September 2019.

\section{Objectives}

\section{Task 1: Upgrading existing LWR codes to currently operating LWRs.}

Our initial task will be to upgrade the codes that we developed up to two decades ago for modeling damage accumulation in the PCCs of LWRs, including the currently-operating Generation II BWRs and PWRs, to ensure that the codes are based on the latest mathematical techniques and technical information available. The upgrading will include insertion of an advanced Mixed Potential Model (MPM) for calculating the electrochemical corrosion potential (ECP); inclusion of advanced crack growth rate (ACGR) models; and insertion of models for crack initiation, in both the BWR and PWR codes, and inclusion of an algorithm for integrating the CGR in a PWR over the operating history of the reactor (such an algorithm already exists in the BWR codes). These upgrades will allow prediction of the accumulation of damage in stainless steels and nickel-based alloys, such as Type 304 SS and Alloy 600, respectively, in both types of reactors.

\section{Task 2: Development of crack initiation model.}

In this second task, we will develop a crack initiation model for IGSCC and corrosion fatigue $(\mathrm{CF})$, based upon the following mechanisms: decohesion of emergent grain boundaries, due to internal oxidation/hydrogen penetration (HIC-IGSCC); mechanical decohesion/internal oxidation followed by dissolution (AD-IGSCC); and pitting, resulting in crack nucleation via the Kondo criteria at the pit, which acts as a stress-raiser. The theoretical basis for the crack initiation model will be Damage Function Analysis [1], which will be adapted to describe the distribution of induction times in terms of the fundamental properties of the passive film (distribution in cation vacancy diffusivity) and to describe the growth nucleation feature (pit, separated, emergent grain boundary, etc) to the critical size at which a crack will nucleate. We have previously used DFA with considerable success to describe SCC and CF crack initiation from pits, subsequent crack growth, and eventual failure of steam turbine blades and disks. 


\section{Task 3: Further development of the coupled environment fracture model.}

Our recent Artificial Neural Network (ANN) analysis of an experimental database for IGSCC in Type 304 SS in BWR coolant environments [2] demonstrates that crack growth is primarily an electrochemical phenomenon, augmented by mechanics and microstructure. On the other hand, a similar analysis on fracture in Alloy 600 in PWR primary coolant shows that fracture in this alloy is primarily a mechanical phenomenon, augmented by electrochemistry and microstructure. These findings reflect a viable crack growth rate model must reproduce the "characters" of the phenomenon in the two alloys and these characters. The current CEFM approximately meets this condition, but the model needs to be adjusted to obtain a better fit between the experimental and theoretical characters. Thus, in this third task, we will further develop the CEFM, which has been used so successfully in simulation codes for stress corrosion cracking damage in BWR primary coolant circuits, to incorporate hydrogen-induced cracking (HIC) in the failure of Alloy 600, Alloy 690, and stainless steels in PWR PCCs. The CEFM will be modified to incorporate the injection of hydrogen into the metal at the crack tip, its transport via the stress field to creep-induced voids on the grain boundaries, recombination in the voids to augment the hydrostatic stress, and increased frequency of fracture of the ligament between the void and the crack tip to yield an increased crack growth rate. Thus, the resulting, modified CEFM will, therefore, permit prediction of crack growth rate (CGR) under both SCC (anodic dissolution) and HIC conditions in a single algorithm and hence will apply to both BWR and PWR primary coolant circuits.

\section{Task 4: Characterization of the electrochemistry of LWR primary coolant circuits.}

Using modified water radiolysis codes from Task 1, we will predict the variations in radiolytic species concentrations, ECP, redox potential (the ECP of an inert metal substrate, such as Pt), crack initiation time, and accumulated damage around PCCs of operating BWRs and PWRs along preconceived corrosion evolutionary paths (CEPs) through a fuel cycle. These predictions will be made, in the case of PWRs, by adopting appropriate [B] vs. [Li] trajectories and by varying the hydrogen level in the coolant, in order to ascertain those conditions that lead to the ECP being more negative than $-0.80 \mathrm{~V}_{\text {she }}$ (leading to hydrogen-induced cracking, HIC) or more positive than 
-0.65 $\mathrm{V}_{\text {she }}$ (corresponding to Intergranular Stress Corrosion Cracking, IGSCC) and hence representing a threat to the integrity of Alloy 600 and sensitized stainless steel components in PWRs. In the case of BWRs, we will explore the impact of catalyzing the decomposition of $\mathrm{H}_{2} \mathrm{O}_{2}$, catalysis/inhibition of the redox reactions that occur on the surfaces external to a crack, ultra-low conductivity operation, stress relief, and other strategies for controlling IGSCC over a wide range of CEPs in order to fully explore these options for mitigating damage in operating reactors.

\section{Task 5: Experimental measurement of key model parameters.}

The successful modeling of the accumulation of EAC in BWR and PWR primary coolant circuits will require the experimental measurement of important model parameters, most notably the kinetic parameters (exchange current densities and Tafel constants, reaction orders) for hydrogen evolution, the reduction of oxygen, and the electro-dissolution of the alloys. These data will be employed in the MPM and the CEFM to calculate the corrosion potential and the crack growth rate, respectively. Some data of this Type have been obtained by the PI and his students for Alloy 600 and 690, but only for temperatures up to $250{ }^{\circ} \mathrm{C}$ [2]. This work needs to be extended to higher temperatures and other alloys (e.g., the stainless steels). The data will be measured using the controlled hydrodynamic apparatus that we have previously employed in work of this type [2].

\section{Task 6: Integration of damage prediction codes into Grizzly.}

Following our extensive work on predicting environmentally-assisted cracking (EAC) damage in BWR primary coolant systems in the past, we will embed the developed codes into Grizzly and employ them to predict integrated cracking damage around the PCCs of BWRs and PWRs. Thus, PI \#2 will lead the incorporation of the codes (developed in Task 1 - 5), including the crack initiation algorithm (Task 2), an advanced CGR algorithm (Task 3), and experimentallymeasured parameter values (Task 5) into Grizzly, as appropriate, to yield a tool that will be capable of calculating the accumulated damage (crack length) in any component in the PCC along any preconceived CEP or operating history of the reactor. The codes will be supplied to PI \#2 by the PI, with assistance from PI \#1, in a mutually acceptable computer language. These codes, when combined with Grizzly will allow the operator to explore operating options and to schedule 
maintenance, so as to avoid safety issues and unscheduled downtime in both LWRs, in a much more accurate and effective manner than has been possible to do the past in BWRs, for example, using the codes REMAIN, ALERT, and FOCUS and will make these operating option available for the first time for PWRs.

\section{II.1. References}

[1] D.D. Macdonald and G.R. Engelhardt, Predictive Modeling of Corrosion. In: Richardson J A et al. (eds.), Shrier's Corrosion, Amsterdam: Elsevier, 2, 1630-1679 (2010).

[2] H. Kim and D.D. Macdonald. Corros. Sci., 52, 1139-1145 (2010).

\section{Upgrading existing LWR codes to currently operating LWRs.}

\section{III.1. Quantum mechanical correction of ECP calculation}

In its original form [1,2], the CEFM incorporates the generalized Butler-Volmer Equation to calculate the cathodic current based upon contributions from all three species (oxygen, hydrogen, and hydrogen peroxide) that are assumed to exist in the coolant, as is also described elsewhere in this paper. The CEFM, however, employs the MPM to estimate the electrochemical corrosion potential (ECP) on the external surface and in doing so tacitly recognizes that the cathodic reactions occur upon a passive surface [3]. Thus, in calculating ECP, the model should consider the presence of the barrier oxide layer (BOL) and the impact that this layer has on the exchange current density of the redox reaction.

The retardation of the redox reaction rate by the barrier layer (BL) can be accounted for in terms of the quantum mechanical tunneling of the charge carrier across the BL [4], with the current being given by:

$$
i=\hat{i_{0}} e^{-\hat{\beta} L_{s s}}
$$

where $\hat{\beta}$ is the tunneling constant and $\hat{i}_{0}$ is the "film-free" current density. Accordingly, the thickness of the oxide film $\left(L_{s s}\right)$ plays an important role in determining the reaction rate and hence in determining the exchange current density in Equation (III.1), which is defined as the current at 
the equilibrium potential $E_{O / R, j}^{e}$. Because the equilibrium potential for the redox reaction depends on $[\mathrm{O}]$ and $[\mathrm{R}]$ and since the thickness of the barrier layer depends upon the potential, the tunneling probability, and hence the exchange current density, varies with the composition of the system, such that as $[\mathrm{R}] /[\mathrm{O}]$ changes the thickness of the barrier layer varies and hence so does the exchange current density. In developing an electrochemical theory for charge transfer at oxidecovered metal surfaces, it is necessary to recognize that the independent variable that is available for experimental manipulation is generally the applied voltage, not the film thickness, as its experimental determination is exceedingly difficult in many cases [5]. Thus, in order to render a theory for charge transfer compatible with the experiment, it is necessary to include a theoretical relationship between the passive film thickness and the applied voltage into the MPM. The required relationship is readily provided by the PDM, which quantitatively predicts that the steadystate thickness of a barrier oxide layer on a metal varies linearly with potential [6] as

$$
L_{s s}=\frac{1-\alpha}{\hat{\varepsilon}} V+g
$$

where $\alpha$ is the polarizability of the BOL/solution interface, $\hat{\varepsilon}$ is the electric field strength in the BOL, $V$ is the applied voltage, and $g$ is a function that depends on $\mathrm{pH}$ and the rate constants for film formation at the metal/BOL interface, among other parameters. Substitution of Eq. (III.2) to Eq. (III.1) and by inserting the equilibrium potential for the redox reactions $\left(E^{e}\right)$ we obtain the exchange current density as

$$
i^{0}=\hat{i}_{0} e^{-\hat{\beta}\left(\frac{1-\hat{\beta}}{\varepsilon}\right) E^{e}} e^{-\hat{\beta} g}
$$

Eq. (III.3) allows calculation of the exchange current density of a redox couple on a passive metal from the exchange current density on a hypothetical film-free surface, the quantum mechanical tunneling probability, and the parameters of the PDM. It is important to recognize that in the case of many metals (e.g., chromium-containing alloys), the "film-free state" is hypothetical because no measurement can be made within the kinetic and thermodynamic stability ranges for water where this state can be practically realized. However, the value of defining this term is that it reduces the Butler-Volmer equation to a single independent variable $(E)$. 
The corrected exchange current density, as embodied in Eq. (III.3) is that on the passive surface at the barrier layer/outer layer (bl/ol) interface and not that on the bare metal and hence is impacted by quantum mechanical tunneling of charge carriers (electrons or electron holes) across the barrier layer. We used $i^{0}$, as given in Eq. (III.3), in the generalized Butler-Volmer Equations, to calculate cathodic current corresponding to hydrogen, oxygen and hydrogen peroxide. In the calculation that follows, we used the parameter values as tabulated in Table III.1.

Table III.1. Default values for electrochemical parameters in the MPM and CEFM for Alloy 600.

\begin{tabular}{llc}
\hline Parameter & Value & Unit \\
\hline Anodic Tafel constant for Alloy 600 dissolution, $b_{a}$ & 0.42 & $\mathrm{~V}$ \\
Cathodic Tafel constant $\left(\mathrm{H}_{2}\right), b_{c}$ & 0.083 & 1 \\
Anodic Tafel constant $\left(\mathrm{H}_{2}\right), b_{a}$ & 0.083 & 1 \\
Cathodic Tafel constant $\left(\mathrm{O}_{2}\right), b_{c}$ & 0.051 & 1 \\
Anodic Tafel constant $\left(\mathrm{O}_{2}\right), b_{a}$ & 0.051 & 1 \\
Cathodic Tafel constant $\left(\mathrm{H}_{2} \mathrm{O}_{2}\right), b_{c}$ & 0.051 & 1 \\
Anodic Tafel constant $\left(\mathrm{H}_{2} \mathrm{O}_{2}\right), b_{a}$ & 0.051 & $\mathrm{~A} / \mathrm{cm}^{2}$ \\
Bare surface current density & 0.02 & $\mathrm{~s}$ \\
Time at which repassivation begins, $t_{0}$ & 0.4 & $\mathrm{~A} / \mathrm{cm}^{2}$ \\
Passive current density at steady state & $1.79 \times 10^{-10}$ & $\mathrm{~V} / \mathrm{cm}^{2}$ \\
Electric field strength, $\varepsilon$ & $2.0 \times 10^{6}$ & $1 / \mathrm{cm}$ \\
Quantum Mechanical Tunneling constant, $\hat{\beta}$ & $3.5 \times 10^{8}$ & 1 \\
Polarizability of the interface, $\alpha$ & 0.7 & 0.2 \\
\hline Transfer coefficient, $\alpha_{2}$ & & $1 / \mathrm{V}$ \\
\hline
\end{tabular}

Park et al. [7] measured the ECP on Alloy 600 in boric acid solutions at a temperature between 150 and $316^{\circ} \mathrm{C}$. Figure III.1 shows the measured data along with the ECP calculated by 
taking into account quantum-mechanical tunneling of charge carriers across the BOL, as described above. The calculated values agree well with the experimentally measured data set, demonstrating that the model including the quantum mechanical theory for the calculating ECP is viable and that the exchange current density calculated for the passive surface satisfactorily accounts for the ECP.

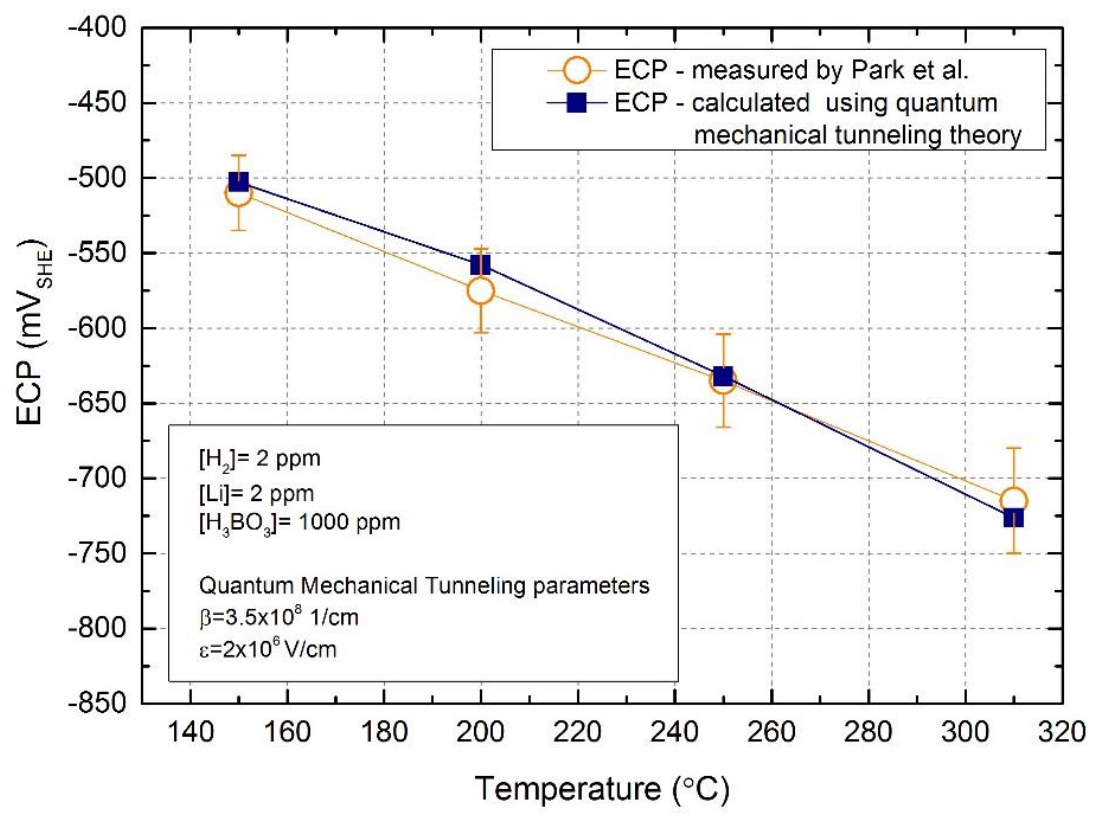

Figure III.1. Comparison of measured and calculated ECP (measurements were conducted on Alloy 600 in water containing 1000 ppm of boron, 2 ppm of lithium, and 2 ppm of dissolved hydrogen).

\section{III.2. Upgrading the pH model in the PWR code}

The electrochemical corrosion potential (ECP) depends on the material properties, and electrochemical corrosion characteristics (kinetic parameters such as the hydrogen electrode reaction and anodic metal dissolution current density and $\mathrm{pH}$ ) are necessary in order to calculate the ECP of the individual structural material in the PWR loops. The primary coolant in the PWR heat transport circuits comprises a boric acid/lithium hydroxide solution with the boron concentration ranging from $2000 \mathrm{ppm}$ at the beginning of a fuel cycle to about $10 \mathrm{ppm}$ at the end. Lithium is injected as $\mathrm{LiOH}$ in the primary coolant to control the $\mathrm{pH}$ during operation to minimize corrosion product transport and activation in the core region. Because oxide solubility and metal 
corrosion rate depend on $\mathrm{pH}$, it was important to include a model for the chemistry of the coolant from which the $\mathrm{pH}$ can be estimated for any given temperature, $[\mathrm{B}]$, and [Li]. The model in the present study was adopted from the thesis work of H.S. Kim [2] that makes use of the reaction set summarized in Table III.2. The species concentrations are calculated by the solution of the mass action equations together with the elemental and charge balance constraints. The calculation is carried out iteratively with the activity coefficients being estimated at each step from extended Debye-Huckel theory.

Table III.2. Reaction set used for calculating the $\mathrm{pH}$ value in PWR coolant containing boric acid and lithium hydroxide.

\begin{tabular}{ll}
\hline $\mathrm{B}(\mathrm{OH})_{3}+\mathrm{OH}^{-}=\mathrm{B}(\mathrm{OH})_{4}^{-}$ & $(\mathrm{R} 1)$ \\
$2 \mathrm{~B}(\mathrm{OH})_{3}+\mathrm{OH}^{-}=\mathrm{B}_{2}(\mathrm{OH})_{7}^{-}$ & $(\mathrm{R} 2)$ \\
$3 \mathrm{~B}(\mathrm{OH})_{3}+\mathrm{OH}^{-}=\mathrm{B}_{3}(\mathrm{OH})_{10}$ & $(\mathrm{R} 3)$ \\
$\mathrm{Li}^{+}+\mathrm{OH}^{-}=\mathrm{LiOH}$ & $(\mathrm{R} 4)$ \\
$\mathrm{Li}^{+}+\mathrm{B}(\mathrm{OH})_{4}^{-}=\mathrm{LiB}(\mathrm{OH})_{4}$ & $(\mathrm{R} 5)$ \\
$\mathrm{H}_{2} \mathrm{O}=\mathrm{H}^{+}+\mathrm{OH}^{-}$ & $(\mathrm{R} 6)$ \\
\hline
\end{tabular}

Figure III. 2 shows the theoretical dependence of $\mathrm{pH}$ at a constant temperature on boron and lithium concentrations calculated by the PHVALUE code. The effect of temperature on the $\mathrm{pH}$ value of the PWR primary water coolant was also calculated (Figure III.3).

The accuracy of this code has been verified by Framatome and can be used as a reference for $\mathrm{pH}$ calculations in the PWR coolant circuit. The seven species $\mathrm{pH}$ calculation model shown in Table III. 2 included in the PWR_MASTER model was originally based on Urquidi- Macdonald's nine species $\mathrm{pH}$ calculation model. The obtained $\mathrm{pH}$ value was provided as an input parameter in order to calculate the ECP values.

The $\mathrm{pH}$ algorithm in the predecessor code of PWR_MASTER has had several programing issues that have been eliminated in the new code. Now, it yields the same results as the validated FORTRAN based PHVALUE code, as shown in Figure III.4. 

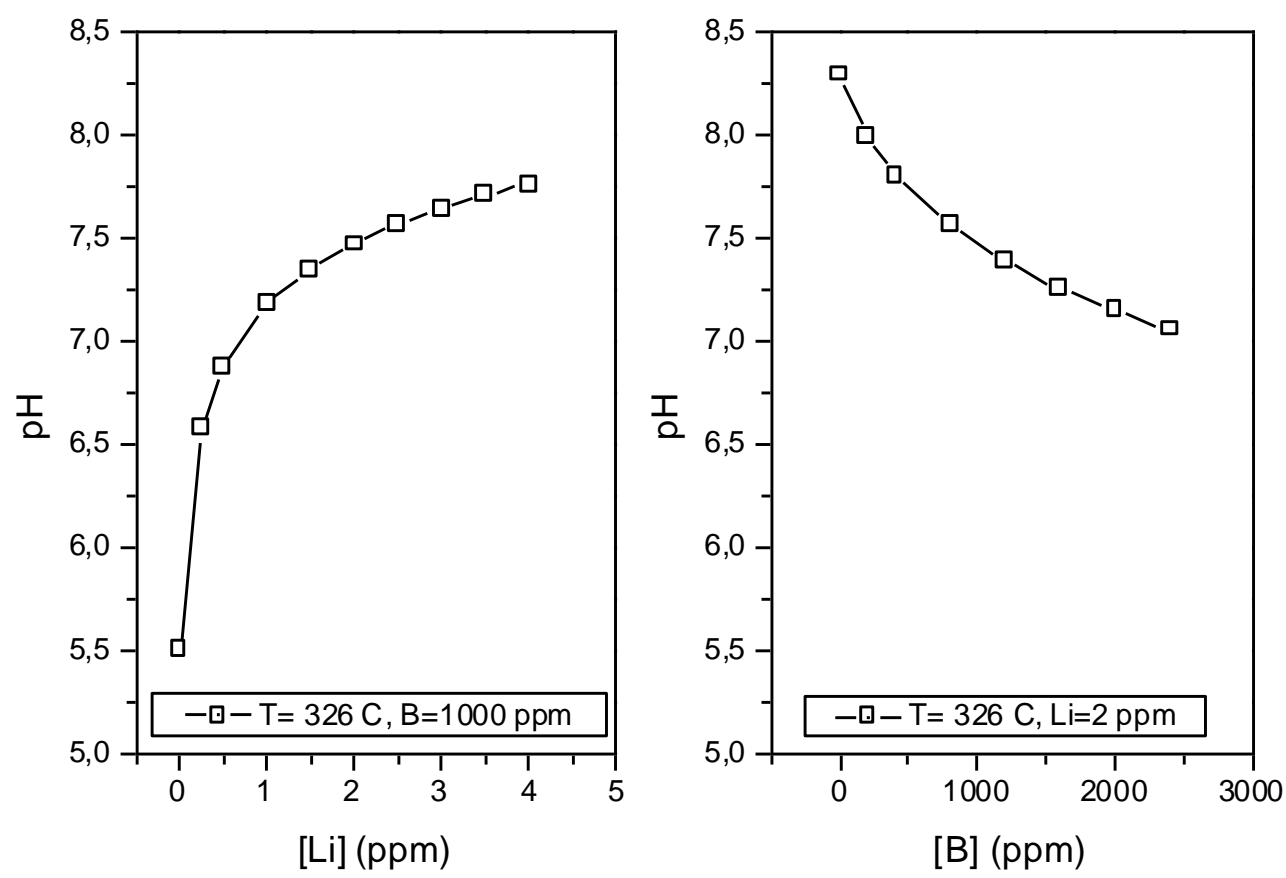

Figure III.2. $\mathrm{pH}$ in the PWR coolant containing boron and lithium: (a) Effect of lithium concentration and (b) Effect of boron concentration.

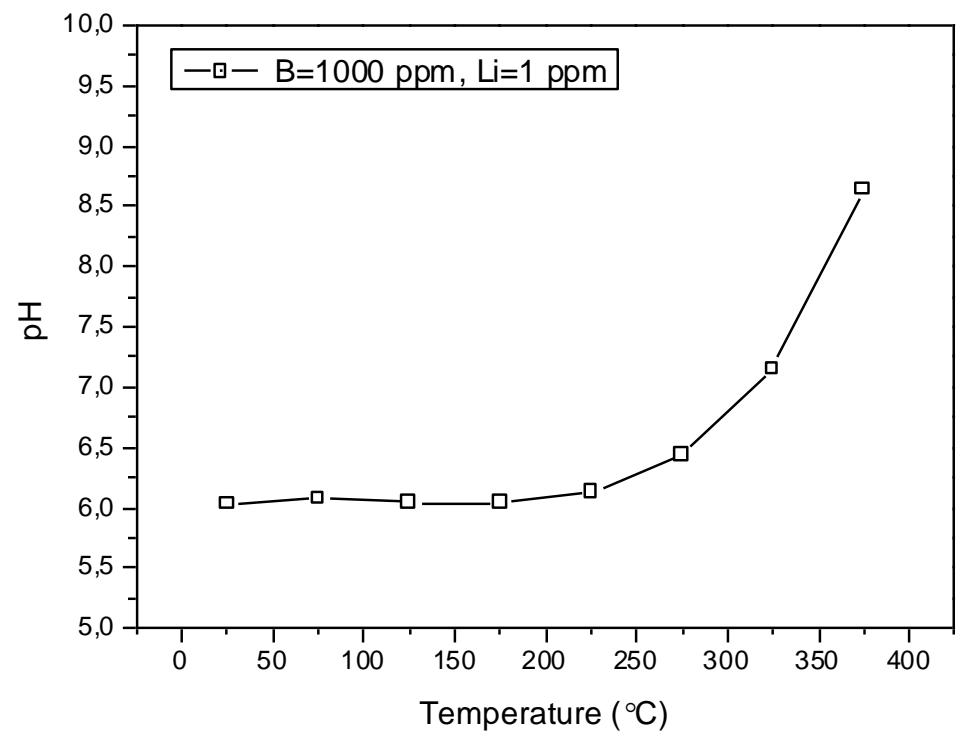

Figure III.3. Effect of temperature on $\mathrm{pH}$ in the PWR coolant containing boron and lithium. 


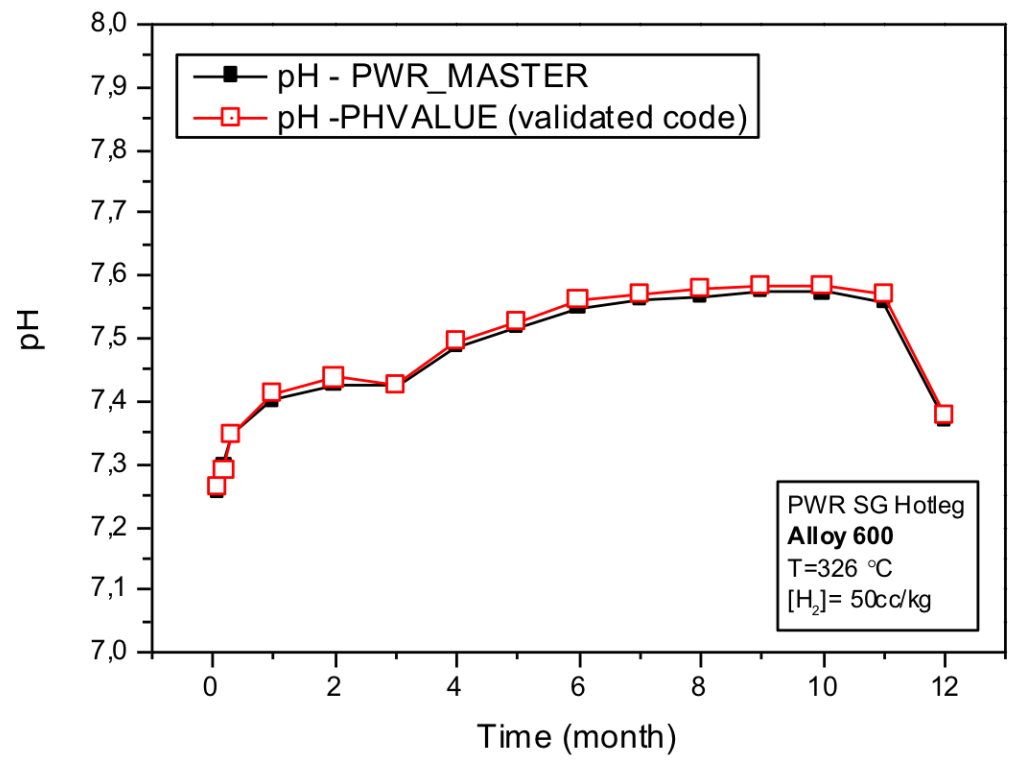

Figure III.4. Comparison of pH values based on PHVALUE and PWR_MASTER.

We have tested the code, and it yields the same $\mathrm{pH}$ values in terms of $\mathrm{T},[\mathrm{B}],[\mathrm{Li}]$ as PHVALUE. The electrochemical parameters calculated over a 12-month fuel cycle can be seen in Figure III.5 along with the boron and lithium concentrations corresponding to coordinated water chemistry protocol.

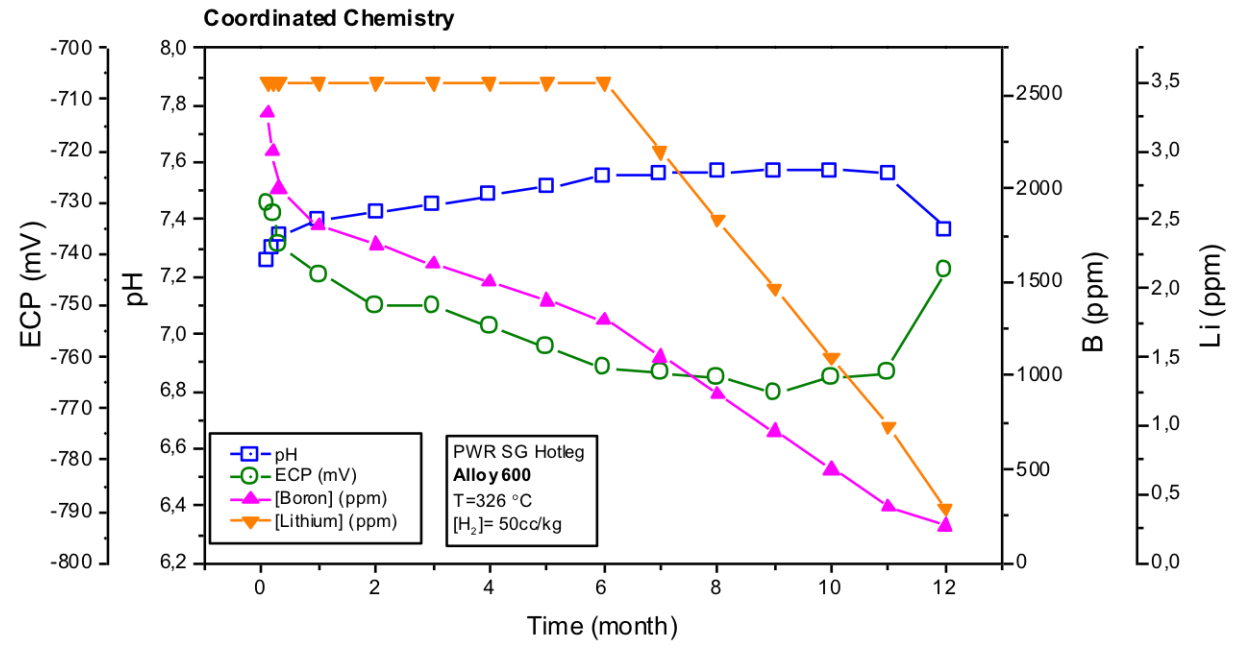

Figure III.5. ECP and $\mathrm{pH}$ values in the PWR hot leg $\left(\mathrm{T}=326^{\circ} \mathrm{C}\right)$ over a fuel cycle for coordinated water chemistry. 


\section{III.3. References}

[1] Program on Technology Innovation: Prediction and Evaluation of Environmentally Assisted Cracking in LWR Structural Materials, EPRI, Palo Alto, CA, EDF-SEPTEN, Hitachi Ltd., Japan Atomic Power Company, Kansai Electric Power Co., Inc., Mitsubishi Heavy Industries, Ltd., Swedish Radiation Safety Authority, Tohoku Electric Power Co., Inc., Tokyo Electric Power Company, Toshiba Corporation, Ishikawajima- Harima Heavy Industries Co., Ltd., and Tohoku University, 1019034 (2009).

[2] H.S. Kim, A Study for Modeling Electrochemistry in Light Water Reactors. Ph.D. Thesis. Pennsylvania State University, 2007.

[3] D.D. Macdonald, P.C. Lu, M. Urquidi-Macdonald, and T.K. Yeh, Corrosion, 52, 768-785 (1996).

[4] M. Kamrunnahar, J. Bao, and D.D. Macdonald, Corr. Sci., 47, 3111-3139 (2005).

[5] J. Bao and D.D. Macdonald, J. Electroanal. Chem., 600, 205-216 (2007).

[6] F. Sutanto and D.D. Macdonald, On the Role of Quantum Mechanics in Corrosion Processes, in Corrosion \& Prevention 2016, Auckland, NZ, 2016.

[7] J.H. Park, O.K. Chopra, K. Natesan, and W.J. Shack, Boric Acid Corrosion of Light Water Reactor Pressure Vessel Materials, ANL NUREG/CR-6875, 2005.

\section{Development of crack initiation model.}

\section{IV.1. Development of a crack initiation model for PWRs}

The literature survey revealed that the internal oxidation of Alloy 600 had received the most experimental support as a viable mechanism of stress corrosion cracking in PWRs. Based on the information collected from the literature, we have developed a model for calculating crack initiation time in PWRs. The model reproduces the water penetrates the emergent grain boundaries and reacts with the $\mathrm{Cr}$ of Alloy 600 , forming a $\mathrm{Cr}_{2} \mathrm{O}_{3}$ oxide layer that wedges the grain boundary. The model can reproduce the effects of the following parameters: applied stress, temperature, cold work, grain boundary segregations, water chemistry, $\mathrm{pH}$, and electrochemical potential. The 
model is implemented in $\mathrm{C}$ language resulting in the computer code PWR_INI. Features of the code are:

- PWR_INI is a computer code for modeling crack initiation time based on internal oxidation of grain boundaries in Pressurized Water Reactors.

- PWR_INI is written in C language and can be compiled on both Windows and macOS platforms.

- The program calculates the film thickness with the PDM and applies the Griffith criterion for modeling the delamination of grain boundaries.

- The Wilkinson and Vitek model calculate the mechanical creep rate.

- PWR_INI includes the effects of cold work and grain boundary carbide structure on initiation time.

The process of the failure mechanism illustrated in Figure IV.1. The process can be summarized as follows:

1. The high-temperature PWR coolant penetrates the emergent grain boundaries.

2. The water reacts with the $\mathrm{Cr}$ of Alloy 600 to form $\mathrm{Cr}_{2} \mathrm{O}_{3}$.

3. The growing oxide scale decreases the cohesive strength of the grain boundary.

4. The grain boundary fractures when the energy of the applied stress exceeds its cohesive strength, as described by the Griffith law.

5. The crack grows towards the next grain boundary inclusion, and the damage process starts over.

The model can be divided into two main parts: the electrochemical model and the mechanical model, as shown in Figure IV.2. The model employs the Point Defect Model to calculate the growth rate of the film within the grain boundaries in terms of various electrochemical parameters as corrosion potential, $\mathrm{pH}$ and temperature. The mechanical basis of the model is the Griffith fracture criterion, from which one can calculate the external energy necessary for the delamination of the grain boundary. In our model, we have modified the Griffith criterion to take into account the effect of the growing oxide layer on the energy balance. The algorithm of the solution can be seen in Figure IV.3. The model continuously checks the value of $K_{I}$ as the crack grows due to internal oxidation and mechanical creep. When $K_{I}>K_{I S C C}$ the model treats the failure 
in terms of macroscopic, linear elastic fracture mechanics, so this point considered as crack initiation time.
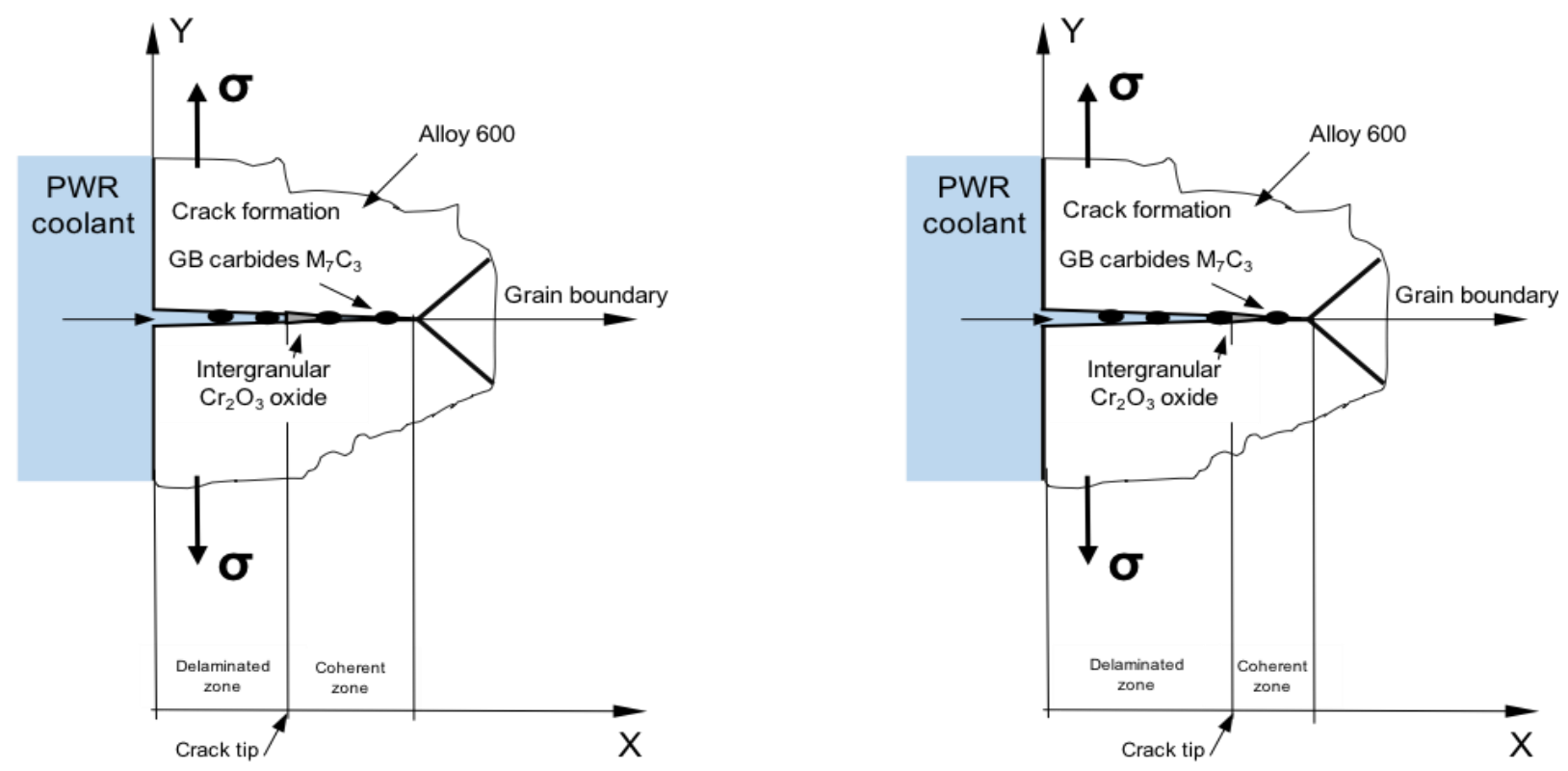

Figure IV.1. Schematic of failure mechanism based on internal oxidation.

\section{Electrochemistry}

Rate of change in the thickness of $\mathrm{Cr}_{2} \mathrm{O}_{3}$ layer:

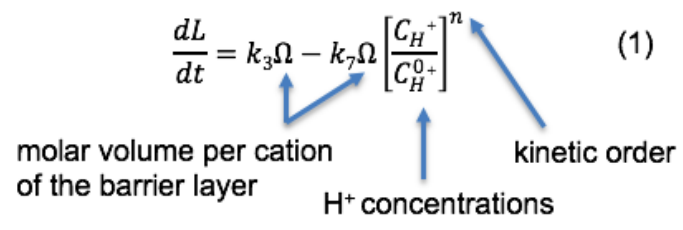

Reaction rate constants:

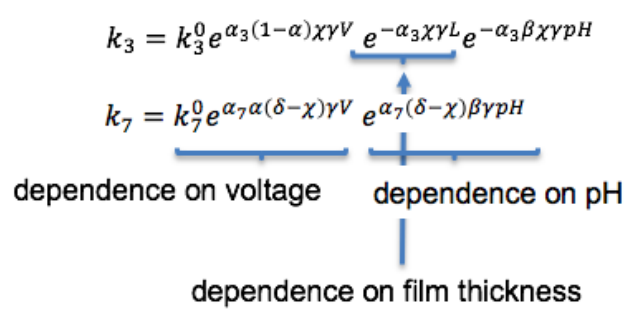

Griffith criterion of fracture of grain boundary:

$G \geq 2 \gamma_{s}-\gamma_{b}$

Mechanics

Modification the Griffith criterion:

$G \geq 2 \gamma_{s}-\gamma_{b}-\gamma_{C r_{2} O_{3}}$

$\gamma_{s}$ - Surface energy $\quad \gamma_{b}$ - "clean" grain boundary energy

$\gamma_{C r_{2} O_{3}}$ - grain boundary energy of the oxide

Elastic strain energy of external loads $(\sigma)$ :

$G=\frac{(1-v) \pi \sigma^{2} \mathbf{a}}{G_{\text {shear }}}$

Elastic strain energy of growing oxide:

$$
\gamma_{\mathrm{Cr}_{2} \mathrm{O}_{3}}=\frac{1}{2} \sigma_{e l} \varepsilon_{e l}=\frac{1}{2} E \varepsilon_{e l}^{2}
$$

$\varepsilon_{e l}=\frac{L-L_{0}}{L_{0}} \quad \begin{aligned} & - \text { Strain in the grain boundary due to oxide } \\ & \text { formation, where } L_{0} \text { is the grain boundary } \\ & \text { thickness }\end{aligned}$

+ Wilkinson and Vitek model for the purely mechanical creep

Figure IV.2. Summary of governing equations of the model. 


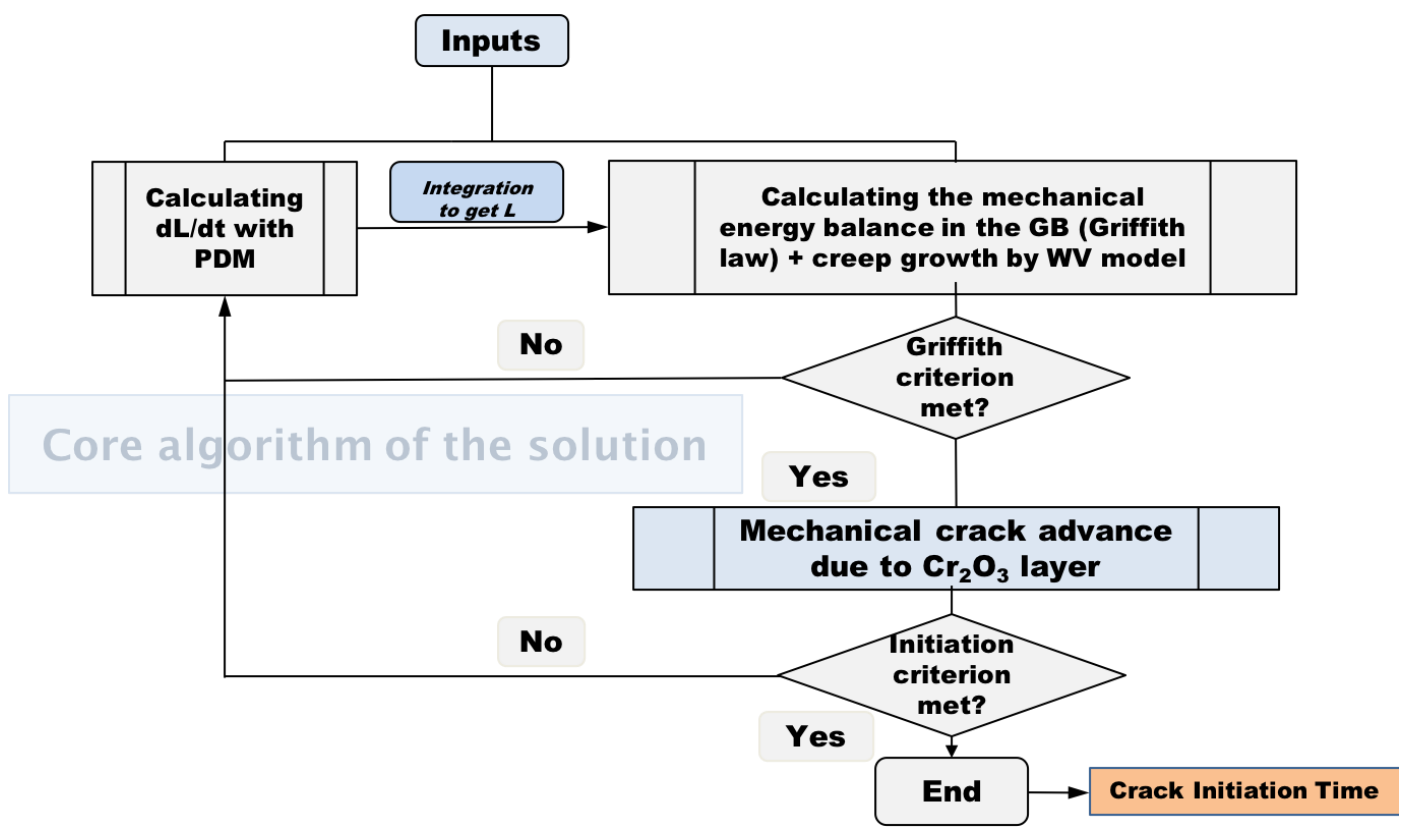

Figure IV.3. Algorithm of the solution of internal oxidation-based crack initiation.

The first step of the calculation is to determine the growth rate at a certain time point using the environmental variables as inputs (ECP, $\mathrm{pH}$, Temperature). Integrating the growth rate of the film results in the thickness as shown in Figure IV.4.

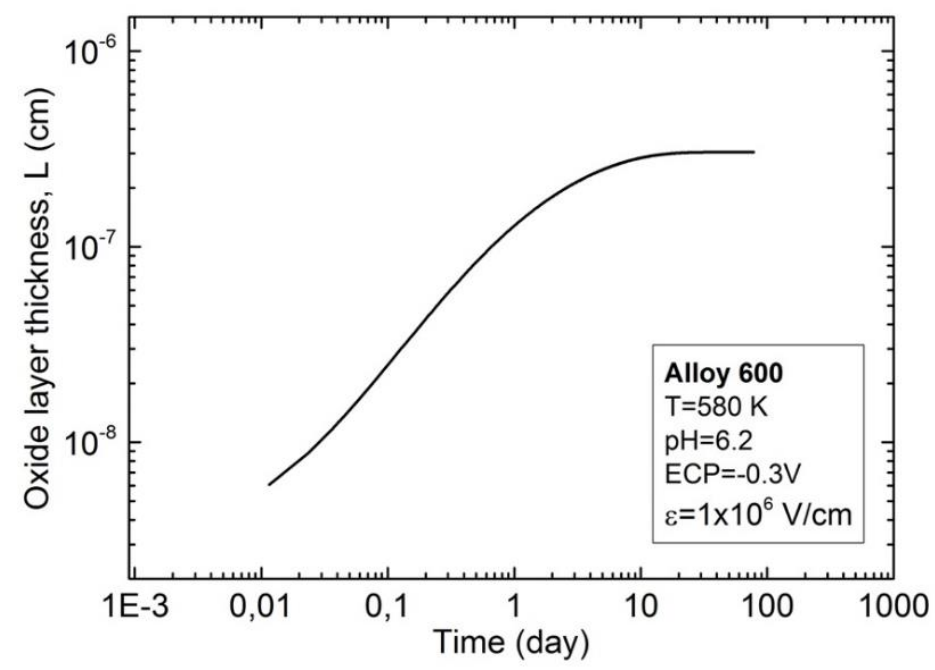

Figure IV.4. Change of the thickness of the $\mathrm{Cr}_{2} \mathrm{O}_{3}$ layer. 
It can be seen that the film thickness reaches the steady state value after about 10 days with the indicated parameters. A recent experimental study performed in the course of this NEUP project confirmed the value of the calculated film thickness. Figure IV.5 shows an example of the variation of the stress intensity factor along with the crack length. Assuming a certain crack shape and external mechanical load, the stress intensity factor increases with the increasing crack length. It is important to recognize that using this crack initiation criterion (initiation when $K_{I}>K_{I S C C}$ ), the initiation time is highly sensitive to the external load as sufficiently high external load can cause a higher stress intensity factor than the critical value. This emphasizes the importance of introducing a correct crack shape factor into the model.

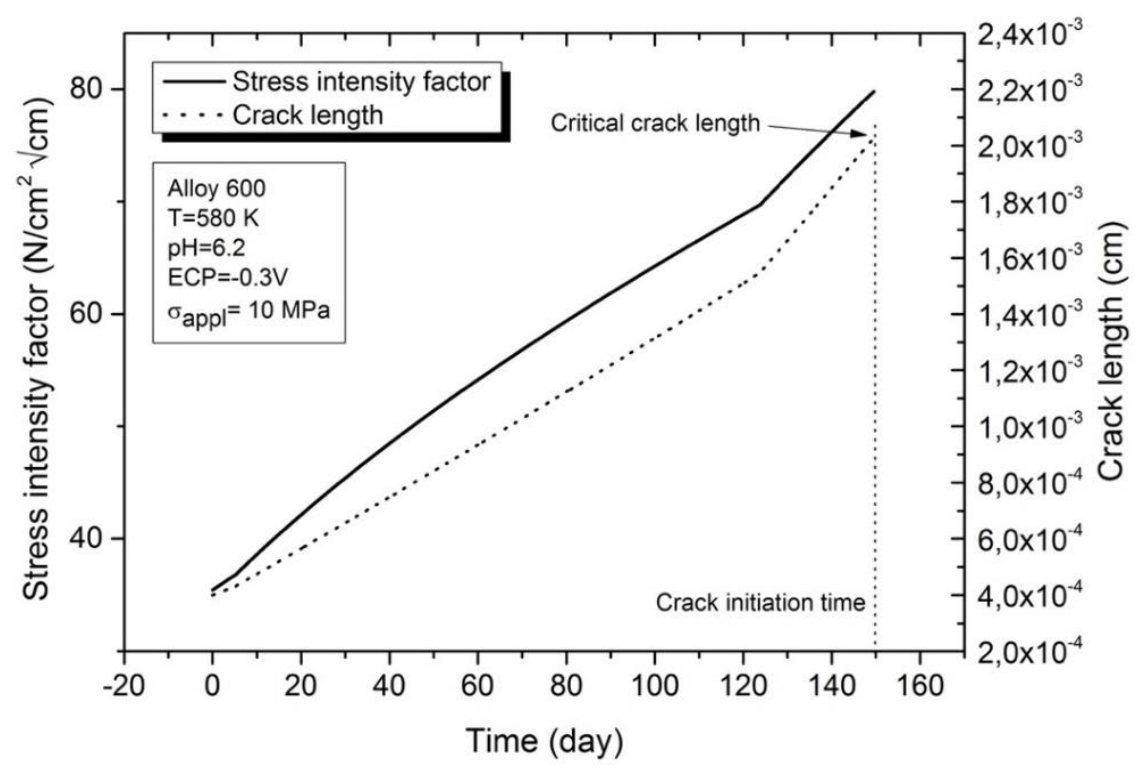

Figure IV.5. Variation of crack length and stress intensity factor vs. time.

Figure IV.6 shows the effect of ECP on crack initiation time for different temperatures. According to the PDM, increasing ECP increases the growth rate of the film. The internal oxidation model postulates that the oxide film wedges the grain boundaries developing macroscopic cracks so as increasing the film growth rate decreases the initiation time as depicted in Figure IV.6. The crack initiation time is a sensitive function of the temperature, as can be seen in Figure IV.7. This high sensitivity is understandable in the light of the fact that the temperature appears in various components of the model in PDM that results in the growth rate of the film in the mechanical creep rate model. Figure IV.7 also shows good agreement with the experimental 
data collected from the literature. The framework of the crack initiation model for PWRs has been completed, as described above. The computer code of the crack initiation model (PWR_INI) operates on both Windows and macOS platforms. Having been written, the code in $\mathrm{C}$ language enables straightforward insertion into the existing reactor codes or even in INL's Grizzly code.

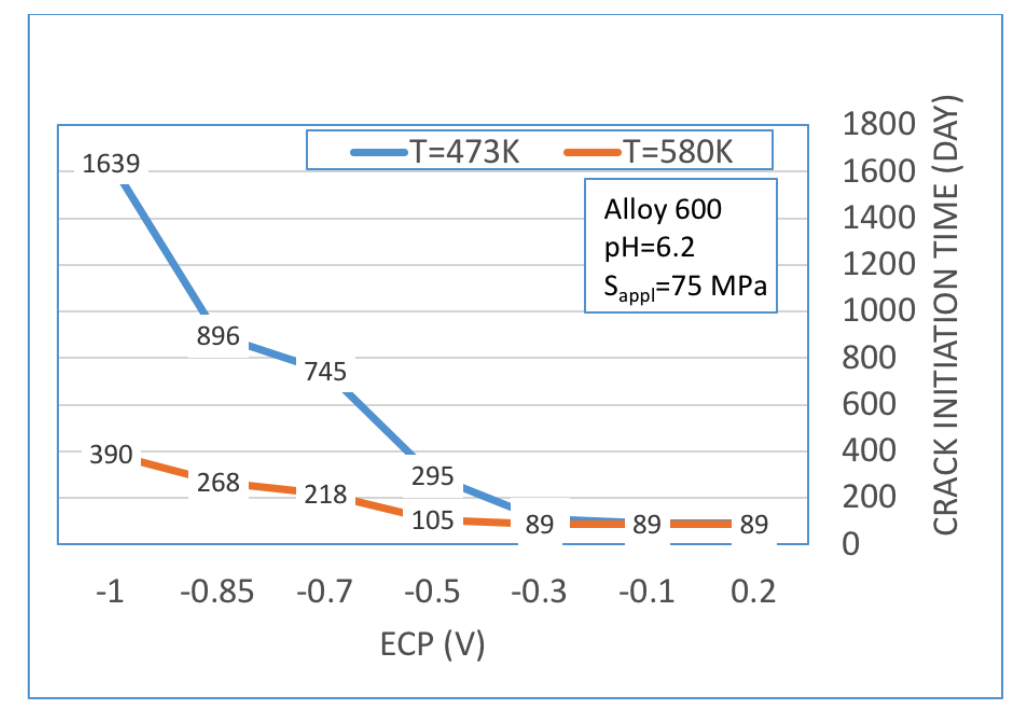

Figure IV.6. Crack initiation time vs. ECP.

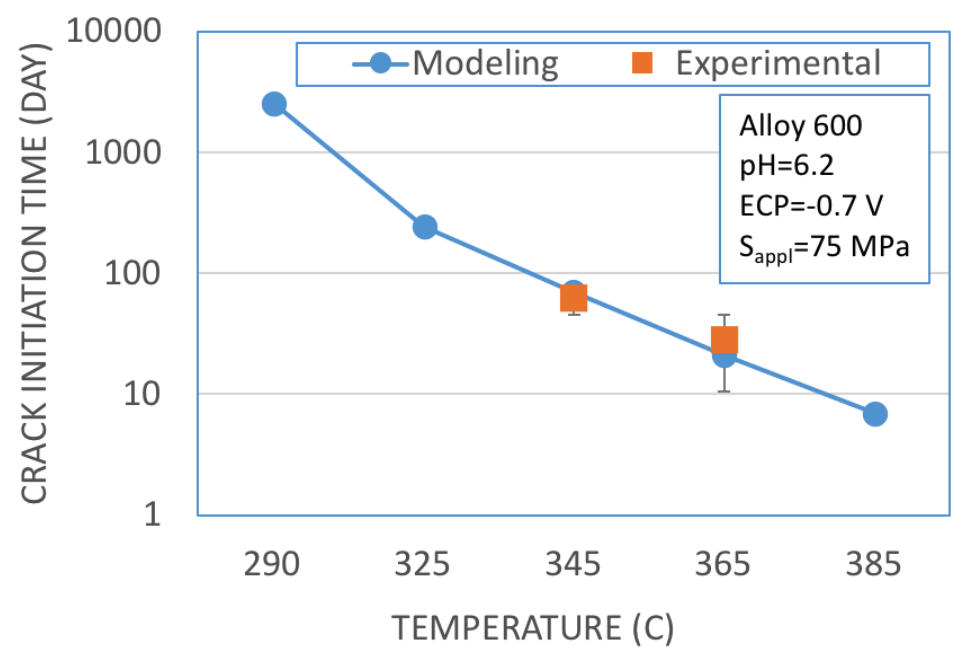

Figure IV.7. Crack initiation time vs. Temperature. 


\section{IV.2. Crack initiation model for BWRs}

A viable, mechanism-based model for crack initiation in BWRs has never been previously developed. Experimental investigations have shown that crack is often observed to nucleate within an emergent grain face (probably from a corrosion pit), grow transgranularly until it intersects a grain boundary, and then grow interganularly after that. Our goal has been to develop a model for calculating the crack initiation time in BWRs that considers the properties of the external environment. We have developed a model that predicts the time that it takes to nucleate and grow a critical pit; i.e., one whose stress intensity factor exceeds $K_{I S C C}$ for the prevailing stress includes residual and operational. The CEFM has been modified to calculate the growth rate of a hemispherical pit in terms of the properties of the pit external and internal environment. Figure IV.8 shows the postulated hemispherical pit in Type 304 SS exposed to the BWR environment.

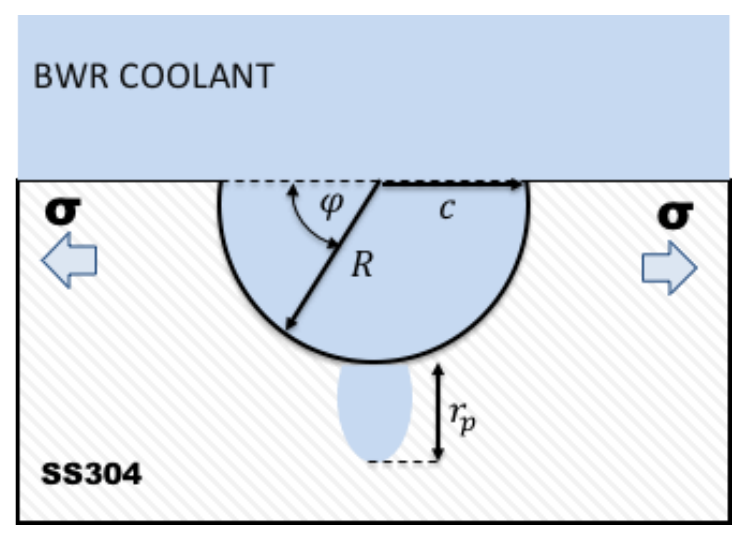

Figure IV.8. Geometrical features of the hemispherical pit.

According to Irwin's linear elastic analysis, the stress intensity factor along the crack front can be calculated by the following expression:

$K_{I}=\frac{\sigma \sqrt{\pi R}}{\theta}\left(\sin ^{2} \varphi+\cos ^{2} \varphi\right)^{\frac{1}{4}}$

where $\sigma$ is the applied stress, all other parameters are described in Figure IV.8. A more sophisticate fracture mechanical analysis requires to consider the effect of small-scale plasticity by taking into account the plastic zone size in the pit front:

$r_{p}=\frac{K_{I}^{2}}{4 \pi \sqrt{2} \sigma_{Y S}^{2}}$ 
where $\sigma_{Y S}$ is the yield stress. Recognizing that the plastic domain in the front of the pit has small resistance against the stress we can extend the pit depth by $r_{p}$ as:

$$
K_{I}=\frac{1.12 \sigma \sqrt{\pi R}}{\sqrt{\theta^{2}-0.212 \sigma^{2} / \sigma_{Y S^{2}}}}\left(\sin ^{2} \varphi+\cos ^{2} \varphi\right)^{\frac{1}{4}}
$$

We can express the critical pit depth by setting $K_{I}=K_{I S C C}$, and recognizing that $K_{I}$ reaches its maximal value at $\varphi=\frac{\pi}{2}$ :

$$
R_{\text {crit }}=\frac{\theta^{2}-0.212 \sigma^{2} / \sigma_{Y S}^{2}}{\pi} \frac{K_{I S C C}^{2}}{1.25 \sigma^{2}}
$$

We have developed a model that calculates the growth rate of a pit as well as the pit depth

as a function of time with the termination criteria (crack initiation) defined by $R_{p i t}=R_{\text {crit }}$. The electrochemical considerations of the model can be summarized as:

- Uniform current density distribution is assumed on the surface of the hemisphere.

- Passivity breakdown no longer considered being caused by mechanical loading (through fracture by crack tip strain) as in CEFM but by the low $\mathrm{pH}$ and presence of $\mathrm{Cl}^{-}$. Tafel's equation calculates the current density in the pit.

In the next few sections, the results presented corresponding to tracking the growth of a hemispherical pit with a certain initial radius (depth) in terms of various environmental parameters.

\section{Effect of electrochemical variables}

The PGR is a strong function of conductivity and to a lesser extent, the linear flow velocity of the coolant. The predicted dependence on the conductivity is due to the increased throwing power of the coupling current from the pit mouth, such that a larger area on the external surface is available for annihilating the coupling current due to the cathodic reactions of oxygen reduction and hydrogen evolution. The dependence on flow velocity is due to the increase in mass transport of oxygen to the external surface. The calculation does not consider "washing out" of the pit, which might be important for low aspect ratio, open pits.

The Pit Growth Rate (PGR) values in terms of ECP obtained for four different $\mathrm{NaCl}$ concentrations for Type 304 SS are compared in Figure IV.9. As the model confirms, $\mathrm{NaCl}$ is 
effective in increasing the solution conductivity and hence, the PGR. ECP is a strong function of $\left[\mathrm{O}_{2}\right],\left[\mathrm{H}_{2} \mathrm{O}_{2}\right]$, and $\left[\mathrm{H}_{2}\right]$, resulting in a sigmoid dependence of ECP on $\left[\mathrm{O}_{2}\right]$. Pit growth rate (PGR) increases exponentially with increasing ECP and is a strong function of conductivity as per Coupled Environment Models. The ECP decreases strongly (becomes more negative) as the temperature increases. The PGR decreases strongly with decreasing ECP. The decrease in the ECP more than offsets the increase in PGR due to activation, resulting in an apparent decrease in the PGR with increasing temperature.

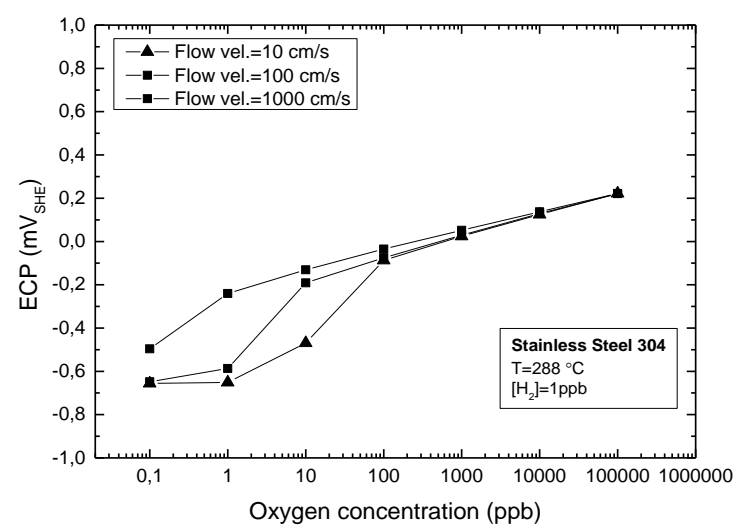

Figure IV.9. ECP as a function of $\left[\mathrm{O}_{2}\right]$ for various flow velocities as per the Coupled Environment Pitting Model.

Figure IV.10 shows the crack initiation time in terms of temperature for different ECP values.

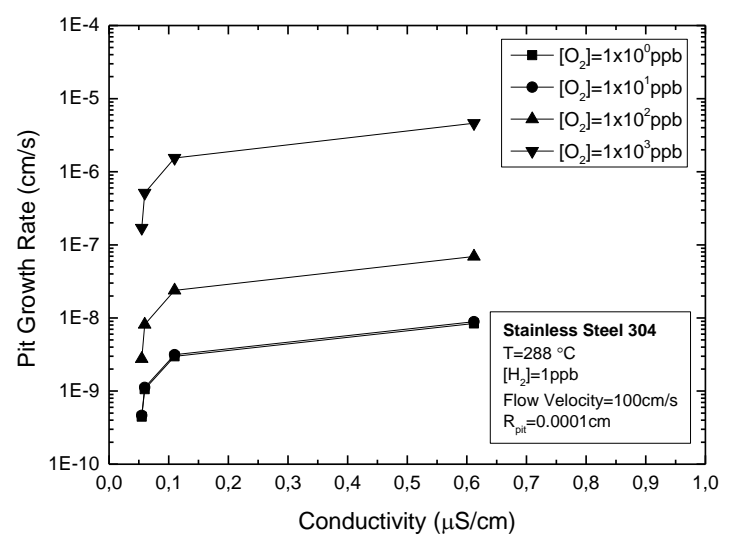

Figure IV.10. Variation of PGR in terms of conductivity. 
Increasing temperature results in the displacement of the ECP towards more negative values, hence decreasing the pit growth rate and increasing the crack initiation time. It is important to note that the ECP, as calculated by the Mixed Potential Model, is strongly dependent upon the temperature. Future work will include calculations with arbitrarily imposed ECP values to investigate the effect of ECP on PGR, independently of temperature. Figure IV.11 shows the effect of the flow velocity on the pit growth rate corresponding to different oxygen concentrations. The impact of the flow velocity at low oxygen concentrations is more significant as described by the generalized Butler-Volmer model.

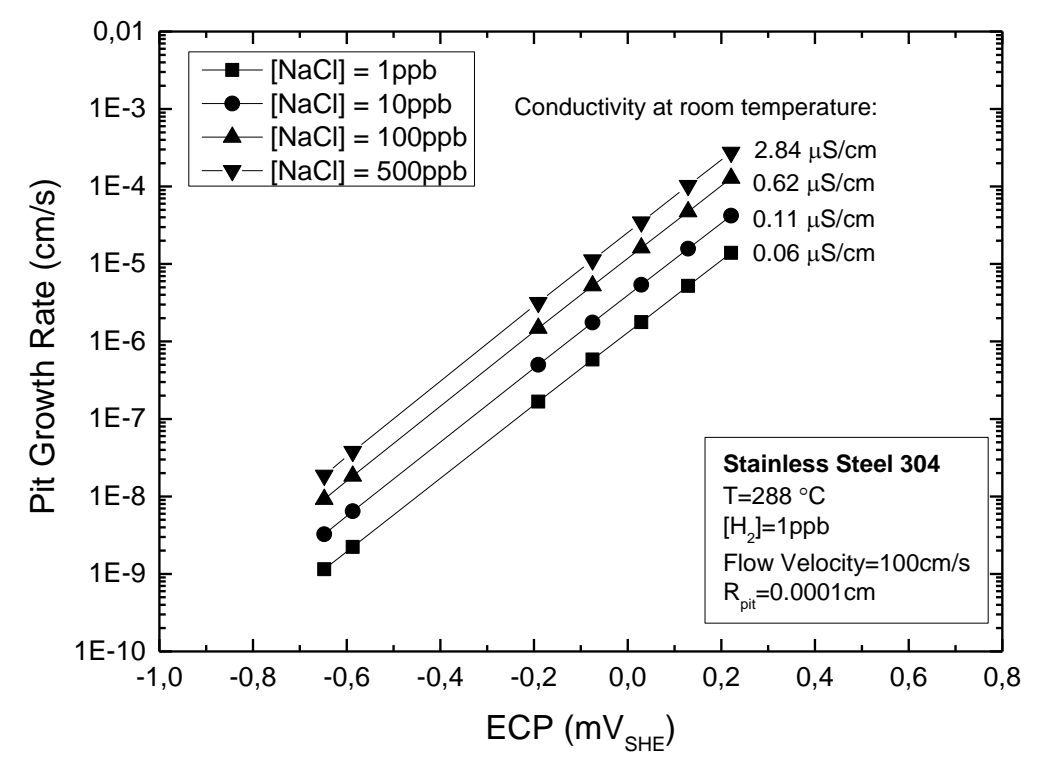

Figure IV.11. Variation of PGR in terms of ECP for different conductivities.

\section{Effect of mechanical variables}

The critical crack length obtained by Eq. (IV.4) in the variation of the applied stress is shown in Figure IV.12. The applied stress has a significant influence on the critical crack length at a low stress level that is gradually decreasing be moving towards higher stress levels. 


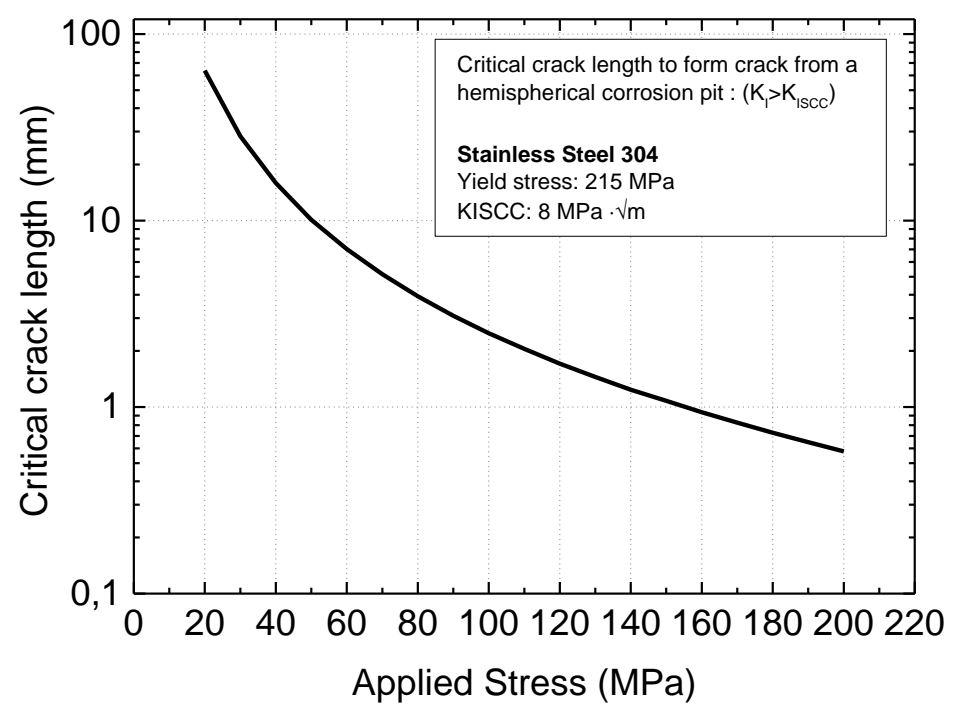

Figure IV.12. Effect of applied stress on critical crack length with $K_{I S C C}=8 M P a \sqrt{m}$.

The effect of applied stress on crack initiation time is shown in Figure IV.13. This figure also reflects the high sensitivity of critical crack length, and therefore, the crack initiation time in the lower range of the applied stresses.

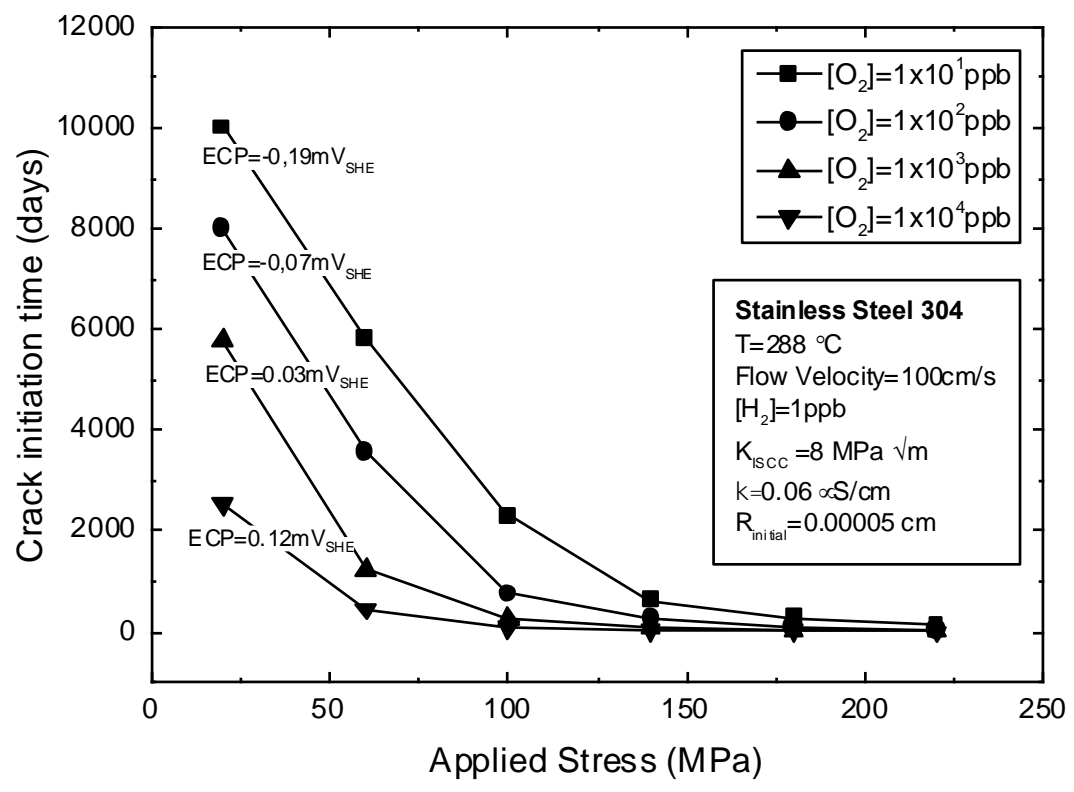

Figure IV.13. Effect of applied stress on crack initiation time with $\mathrm{R}_{\text {initial }}=1 \times 10^{-5} \mathrm{~cm}$. 
It is important to note that this effect is generally observed in the field of stress corrosion cracking, since the stress intensity factor has the same effect on crack growth rate close to $K_{I S C C}$, then it reaches a plateau as $K_{I}$ increasing toward higher values. Crack initiation time is predicted to decrease strongly with increasing applied stress but is also a sensitive function of ECP. This emphasizes the need for accurate estimates of the residual and operational stress and the ECP when predicting crack initiation times. The pit growth rate is predicted to increase exponentially with ECP, just like the CGR. PGR increases with decreasing pit radius as for SCC. This is due to the IR potential drop down the pit cavity.

Figure IV.13 also shows that the ECP has a significant impact on crack initiation time at low mechanical stress levels; however its impact became obsolete at higher stresses. This behavior corresponds to a general observation also in the field of stress corrosion cracking. At extreme high mechanical loading, the electrochemical contribution is almost negligible and vice-versa; with intensive electrochemical conditions, the mechanical effect loses its significance. $K_{I}$ for the pit is a strong function of the ECP, because of the strong, positive dependence of the PGR on ECP. Likewise, the pit radius is a strong, positive function of the ECP. Once the pit radius and $K_{I}$ exceed critical values, a crack nucleates.

\section{IV.3. Theory of the distribution in crack initiation time}

\section{IV.3.1. Introduction}

Attempts to measure crack initiation times (CITs) for materials in reactor coolant circuits, generally produce data that are highly scattered, suggesting that a fundamental limit may exist in the accuracy of measurement and the prediction of CITs. If so, attempts to measure increasingly more accurate CITs may prove to be futile and hence, a waste of precious resources of both time and money. In this Section, we outline why such a limitation may exist and discuss briefly what information may be gleaned from crack initiation time data, even if the sought-after accuracy is not attainable.

Damage Function Analysis (DFA) [1], which is a deterministic localized corrosion damage prediction protocol developed by Macdonald and his colleagues [1], successfully predicts the evolution of pit depth data for an ensemble of pits in an aluminum alloy in tap water, the evolution 
of pits on the inner surfaces of gas and oil pipelines, pitting in condensing heat exchangers, and in many other systems. A feature of DFA is that it is capable of accurately predicting damage by a prediction factor (PF) of 10 to 100 , where the PF is defined as the ratio of the prediction horizon to the calibration time. Empirical methods of extrapolation are seldom capable of achieving a PF of more than 2 to 3 .

\section{IV.3.2. Physico-electrochemistry of crack initiation}

One of the problems inherent in describing crack initiation is to specify the exact nature of the initiation site in terms of both geometry and chemistry. As shown in Figures IV.14 and IV.15, the surface of mill-annealed (MA), Alloy 600 is far from being atomically smooth; instead, the morphology is dominated by gouges and other machining features and corrosion-induced roughening.
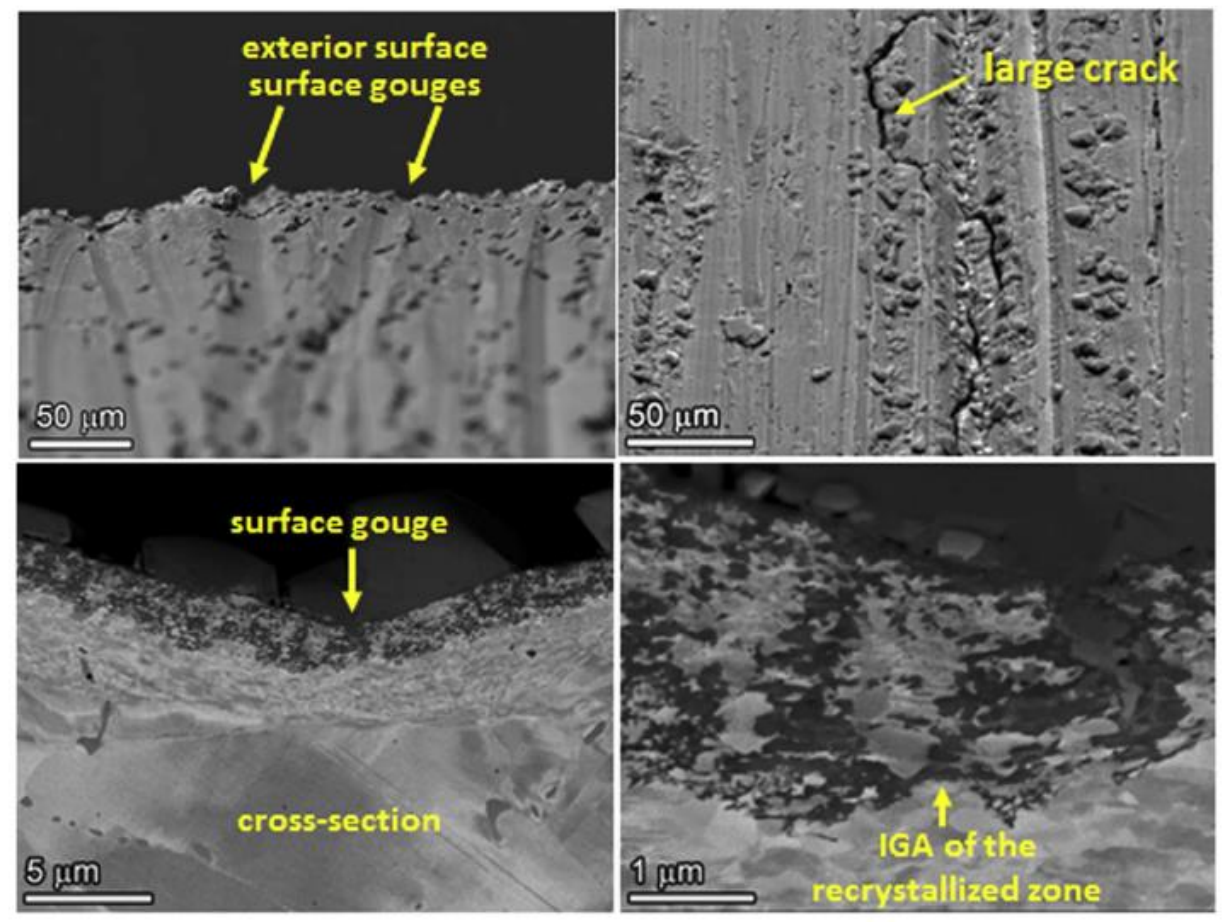

Figure IV.14. SEM-SE images of the surface (upper) and BSE images showing the cross-section (lower) of a 60-grit finish plate specimen of MA Alloy 600 after exposure to simulated PWR primary coolant [3]. 

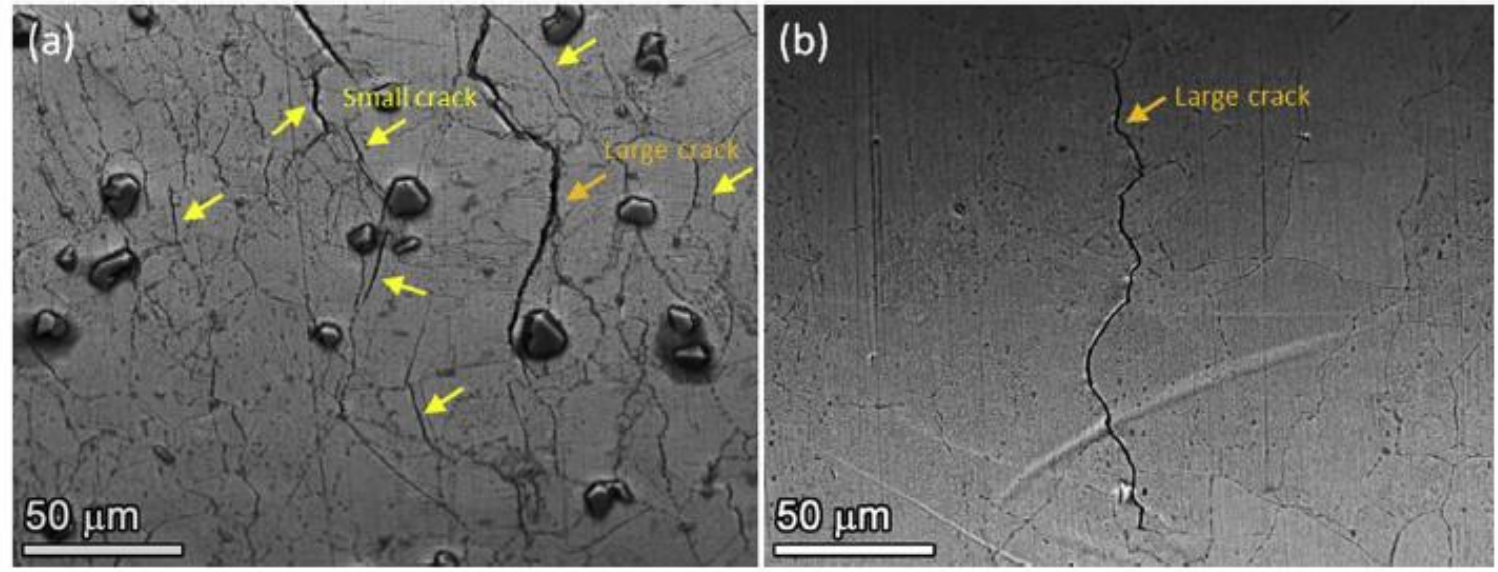

Figure IV.15. SEM-SE images of the surface showing surface cracks and high energy, emergent grain boundaries of multiple sizes after exposure of MA Alloy 600 to synthetic PWR primary coolant (See Ref. 3 for experimental details).

As shown in Figure IV.15, upper right, the surface also contains cracks, perhaps more accurately, intergranular penetrations that can form in the absence of applied stress (see also Figure IV.15, lower right), that can serve as the nucleation sites of IGSCC. In general, the surface of Alloy 600 exposed to PWR primary coolant containing $10-50 \mathrm{~cm}^{3}$ (STP)/kg $\mathrm{H}_{2} \mathrm{O}$ of hydrogen does not contain pits, because the ECP is far below the pitting potential, because the $\mathrm{pH}$ is relatively high $(\mathrm{pH} \approx 7)$, and because the chloride concentration is very low. Thus, generally, pits can be discounted as crack nucleation sites in MA Alloy 600 in PWR primary coolant. Given that a sharp surface crack has a much greater stress intensity factor than does a blunt, surface gouge, it is fair to assume that, for a given stress, IGSCC will tend to nucleate at the former rather than the latter. The essential question then is: What is the mechanism of surface crack formation? This question has been addressed by Scott and Le Calvar [2] by assuming that water penetrates emergent grain boundaries and forms oxides on the internal boundaries to wedge open the boundaries until $K_{I}>K_{I S C C}$. However, Scott and Le Calvar [2] did not express this model in analytical form, so that the initiation time could be calculated. This has been done in the present work, and we now have an analytical model for this crack initiation process.

Measured crack initiation time (CIT) data for stainless steels and Alloys 600/690/182 in high-temperature aqueous solutions simulating BWR primary and PWR primary coolants [pure water and $\mathrm{B}(\mathrm{OH})_{3} / \mathrm{LiOH}$, respectively], have been reported by a number of research groups; for 
example, by Bruemmer et.al. [3]. The data plotted in Figure IV.16 demonstrate that for applied stresses below $450 \mathrm{MPa}$, the initiation time is a very sensitive function of stress, CIT $=a / \sigma^{m}$, where the exponent $m$ appears to be 3-4. Since the yield stress shown in Figure IV.16 corresponds to the yield stress under the conditions of each experiment, the variation of the CIT presumably reflects the variation of some other parameter, such as temperature. A similar plot is given by Bruemmer et al. [3] for the measured CIT as a function of hardness. The important question then arises: Is the scatter in the measured CIT reflective of experimental uncertainty, or does it reflect a fundamental limitation imposed by the statistical nature of the phenomenon. Though the data are very scattered for the reasons captured in theory described below, a consensus has been achieved on the strong dependencies of the CIT on stress, cold work [3], and ECP, all of which, in principle, can be controlled in an operating reactor.

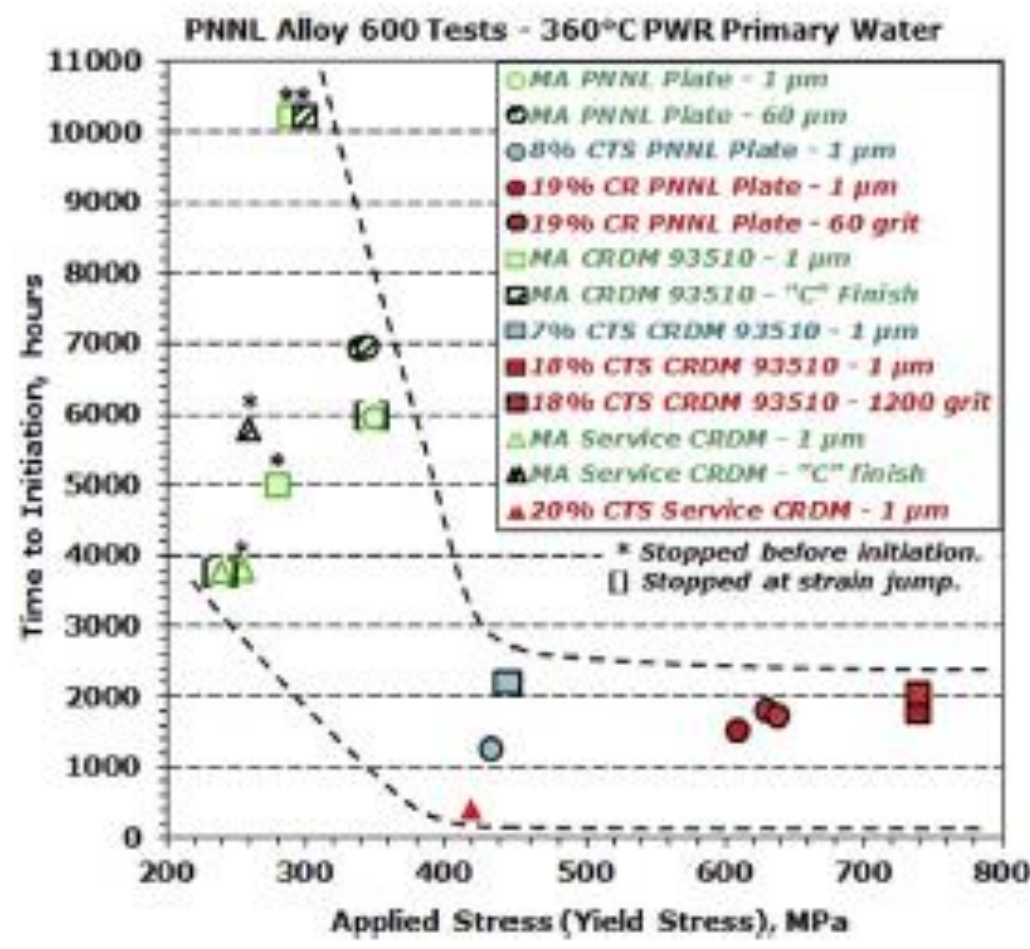

Figure IV.16. Measured crack initiation time as a function of applied stress in PWR primary coolant at $360{ }^{\circ} \mathrm{C}$. The dashed lines define the bounds of the data. After Bruemmer, et.al. [7].

It is evident from Figure IV.16 that the crack initiation time is a highly-distributed quantity, where the dashed lines are meant to define the range over which the data are distributed. Insufficient data are available to exactly define the nature of the distribution so that we must resort to theory, in the form of Damage Function Analysis [1], for guidance in this matter. It is evident that cracks nucleate at stress risers, in the form of corrosion pits in BWR primary coolant circuits and at emergent, open grain boundaries in the case of PWR primary coolant circuits. In both cases, 
the initiation site is a cavity of critical depth, $L_{c r i t}$, such that $K_{I}>K_{I S C C}$ for the prevailing stress. Accordingly, crack nucleation in both BWR and PWR primary coolant circuits can be described under a common theoretical framework that satisfies the Kondo Criteria (see Ref. [1]). Deterministic Extreme Value Statistics (DEVS), which is a component of the DFA protocol, predicts that the pit depth approaches a plateau asymptotically with time after extended exposure periods, as displayed in Figure IV.17. While these data are specifically for an aluminum alloy, the same trend is displayed for stainless steels, nickel-based alloys, and other material. The shape of this curve is due to pits repassivating (dying) as the population (ensemble) of pits evolves (cf, the evolution of an aging human population where the maximum age of individuals does not change significantly with time at the upper extreme; i.e., we all tend to die before reaching an age of 100). The data are plotted as the mean (average) \pm one standard deviation, $\sigma$, corresponding to a confidence level of $68 \%$. Stress corrosion and corrosion fatigue cracks often nucleate at corrosion pits, which act as stress risers, such that $K_{I}=Y \sigma \sqrt{L}>K_{I S C C}$. Thus, at the critical condition, $K_{I}>$ $K_{I S C C}$, the critical crack length becomes:

$$
L_{c r i t}=K_{I S C C}^{2} / Y^{2} \bar{\sigma}^{2}
$$

where $Y$ is the geometric factor that accounts for the pit shape and $\bar{\sigma}$ is the mean, local surface stress. On any real surface, the initiation sites are subject to a wide distribution of local stress state, due to machining operations, cold working, etc, such that there exists a distribution in $K_{I}$ that we identify by $\Delta K_{I}$. Because of the relationship between $K_{I}$ and $\sqrt{L}$, as expressed by Equation (IV.5), a corresponding distribution in critical crack length exists, $\Delta L_{\text {crit }}$. The slope of the curve drawn in Figure IV.18 is the global pit growth rate (i.e., the average for the ensemble). The slope tends to zero, not because the growth rate does not decrease (it does, as the pit depth increases, because of the increase in the IR potential drop down the cavity), but because there are no active pits left to grow due to repassivation. As time goes on for a given value of $\Delta K_{I}$, corresponding to the distribution in $\Delta \sigma$, the width of $\Delta L$ increases and in the limit of $\mathrm{t} \rightarrow \infty, \Delta L \rightarrow \infty$. This imposes a limit upon how accurately the crack initiation time may be determined. 


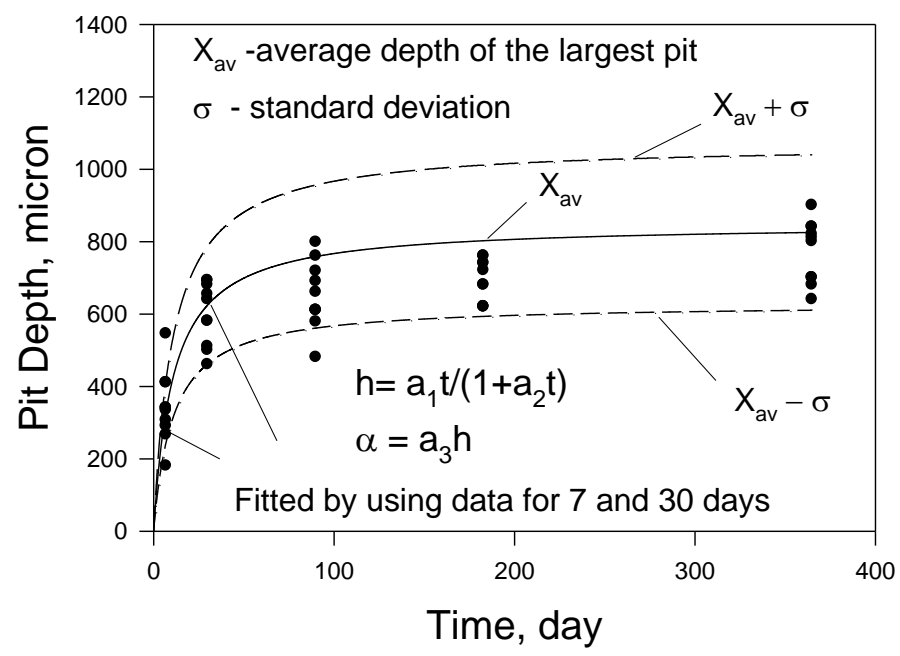

Figure IV.17. The mean depth of the deepest pit in ALCAN aluminum alloy in Kingston tap water, as a function of time. Fitting to experimental data (black circles) has been performed by using the Hyperbolic dependencies [1].

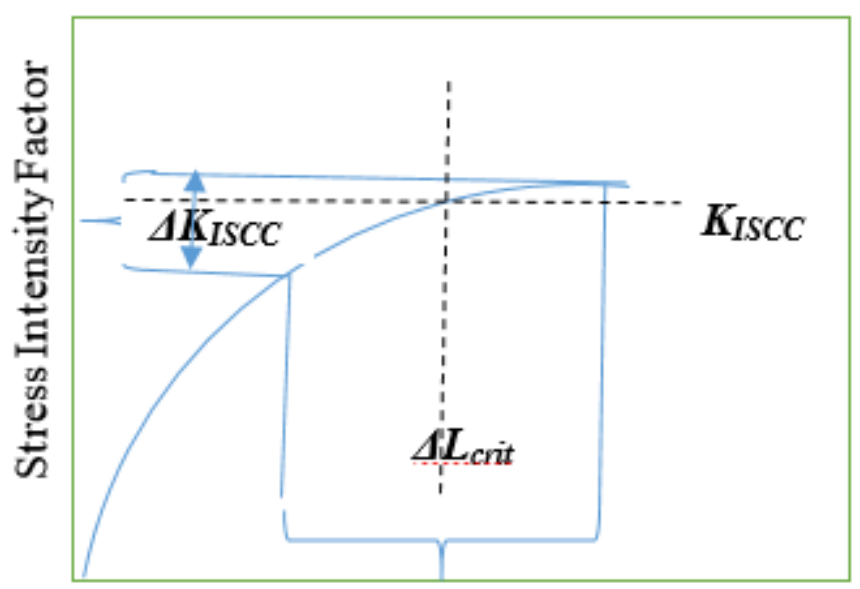

Figure IV.18. Schematic explanation of the origin of the distribution in critical crack length $(\Delta L)$ and hence in crack initiation time, $t_{\text {init. }}$. Note that $\Delta K_{I}$ is the distribution in stress intensity factor for those sites on the surface for which $K_{I}>K_{I S C C}$.

\section{Crack Length}

An outline of the theoretical approach being developed by the author is outlined below. Let us assume that the potential crack initiation sites (population, $N$ ) on a surface are distributed normally with respect to the local surface stress, $\sigma$, i.e.,

$$
\frac{d N}{d \sigma}=-\frac{1}{\sqrt{2 \pi \sigma^{2}}} e^{(\sigma-\bar{\sigma}) / 2 S D_{\sigma}^{2}}
$$


where $\bar{\sigma}$ is the mean stress for the population of the initiation sites and $S D_{\sigma}$ is the standard deviation in the normal distribution of the initiation sites with respect to the residual stress. The chain rule as: then gives the distribution in the initiation time

$$
\frac{d N}{d t_{\text {init }}}=\frac{d N}{d \sigma} / \frac{d t_{\text {init }}}{d \sigma}=\frac{d N}{d \sigma} / \frac{d L_{\text {crit }}}{d \sigma} x \frac{d L_{\text {crit }}}{d t_{\text {init }}}
$$

From Equation (IV.5), we have:

$$
\frac{d L_{c r i t}}{d \sigma}=-\frac{2 K_{I S C C}^{2}}{Y^{2} \sigma^{3}}
$$

Finally, the term $d L_{c r i t} / d t_{\text {init }}$ is readily calculated from the pit growth rate function for a pit of depth, $L_{\text {crit }}$, as:

$$
\frac{d L_{\text {crit }}}{d t_{\text {init }}}=R\left(L_{\text {crit }}\right)
$$

Substitution of Equations (IV.6), (IV.8), and (IV.9) into Equation (IV.7) then yields the distribution in the crack initiation time as:

$$
\frac{d N}{d t_{\text {init }}}=\frac{1}{\sqrt{2 \pi \sigma^{2}}} e^{(\sigma-\bar{\sigma}) / 2 S D_{\sigma}^{2}} \cdot \frac{Y^{2} \sigma^{3}}{2 K_{I S C C}^{2}} x R\left(L_{c r i t}\right)
$$

In this expression, the first factor introduces statistics into the problem, the second introduces mechanics, and the third $\left[R\left(L_{\text {crit }}\right)\right]$ introduces electrochemistry, all three of which must be contained within a successful theory for crack initiation. Examination of Equation (IV.10) indicates that the rate of crack initiation should increase strongly with the surface (residual and applied) stress (a cubic relationship) and with the PGR, $R\left(L_{\text {crit }}\right)$, to increase exponentially with ECP.

In the case of stainless steel in a BWR primary coolant circuit, the predicted ECP (a) as a function of oxygen concentration and flow velocity and calculated pit growth rate (PGR) as a function of ECP (b) and conductivity (c), as calculated from the Coupled Environment Pitting Model (CEPM) are presented in Figure IV.19. The ECP displays the well-known sigmoidal variation with $\left[\mathrm{O}_{2}\right]$ and displays the expected sensitivity to flow velocity at oxygen concentrations ranging from 1 to $100 \mathrm{ppb}$. Note that the PGR is predicted to increase exponentially with the ECP and to increase with increasing solution conductivity, illustrating the importance of accurately predicting the ECP and the solution chemistry. 

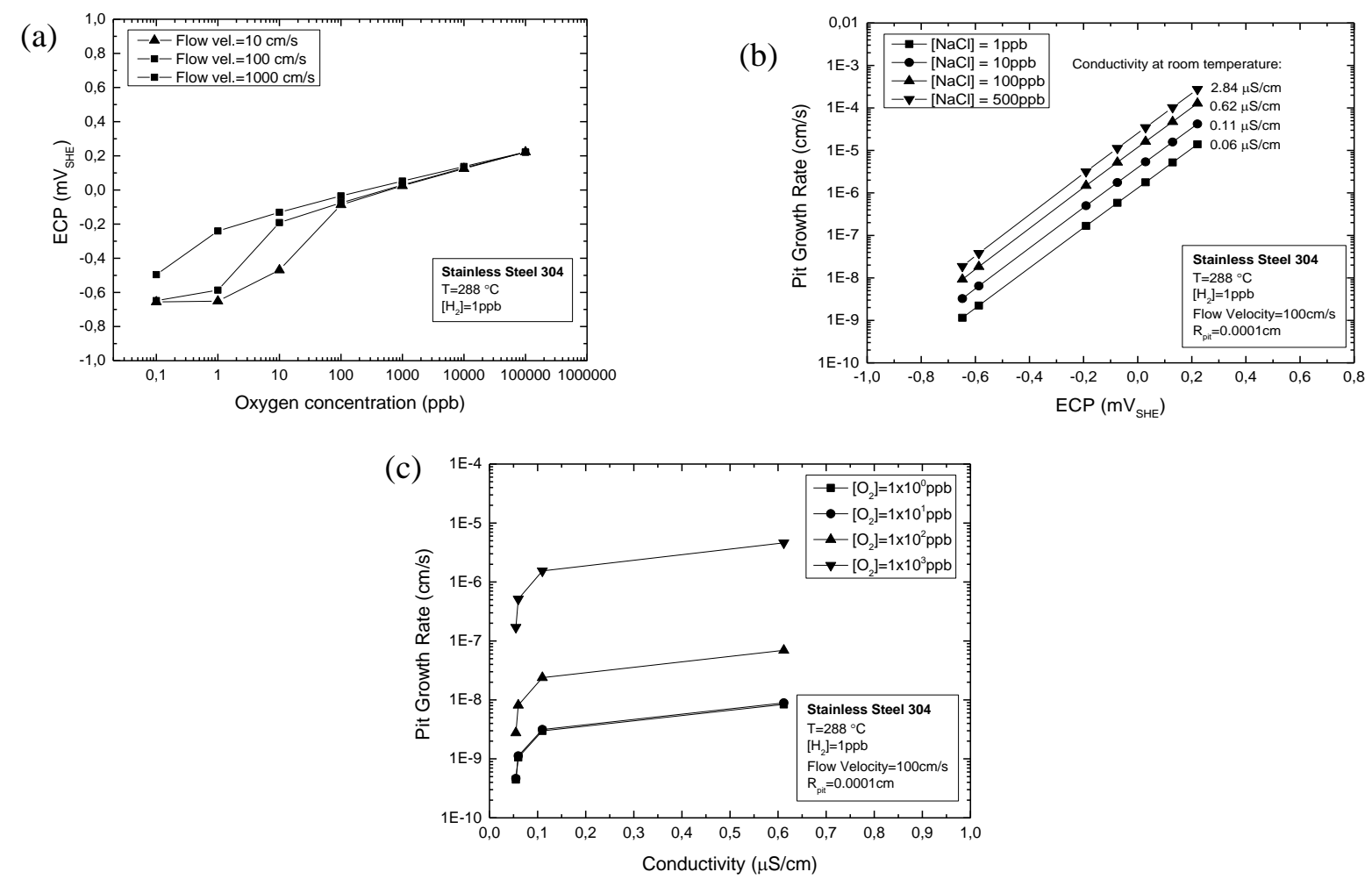

Figure IV.19. Predicted ECP (a) and pit growth rate (PGR) as a function of ECP (b) and conductivity (c), as calculated from the Coupled Environment Pitting Model (CEPM). Note that the PGR is predicted to increase exponentially dependent upon the ECP and to increase with increasing solution conductivity [2].

Over the past 3-years, under the sponsorship of DOE-NEUP, we have developed a sophisticated model for crack-initiation-at-a-single-pit, based on the concepts discussed above. Some of our predictions of crack initiation in Type 304 SS in BWR HTCs are shown in Figure IV.20. Thus, the model predicts that the critical pit depth (Figure IV.20a) and the initiation time (Figure IV.20b) decrease strongly with increasing stress and with increasing ECP. These are the expected dependencies. While the theory outlined above strictly applies to the nucleation of cracks at corrosion pits, the methodology applies to any type of nucleation site, such as emergent dislocation/slip systems, inclusions, oxidized grain boundaries, provided that the critical conditions and growth rate can be specified. For example, we have developed an emergent grain boundary wedging model for crack initiation in PWRs, as depicted in Figure IV.21, because the ECP in a PWR HTC is far too negative ( -0.85 to $-0.75 \mathrm{~V}_{\text {she }}$ ) for pitting to be a viable initiation 
mechanism. In this model, it is envisioned, after Scott and Le Calvar [3], that water penetrates down an emergent grain boundary and reacts with chromium to form $\mathrm{Cr}_{2} \mathrm{O}_{3}$, which occupies more than two times the volume of the metal from which it forms (i.e., the Pilling-Bedworth ratio). The growing oxide scale wedges open the boundary and decreases the cohesive strength of grain boundary, with a crack initiating when $L_{c r i t}=K_{I S C C}^{2} / Y^{2} \bar{\sigma}^{2}$.

(a)

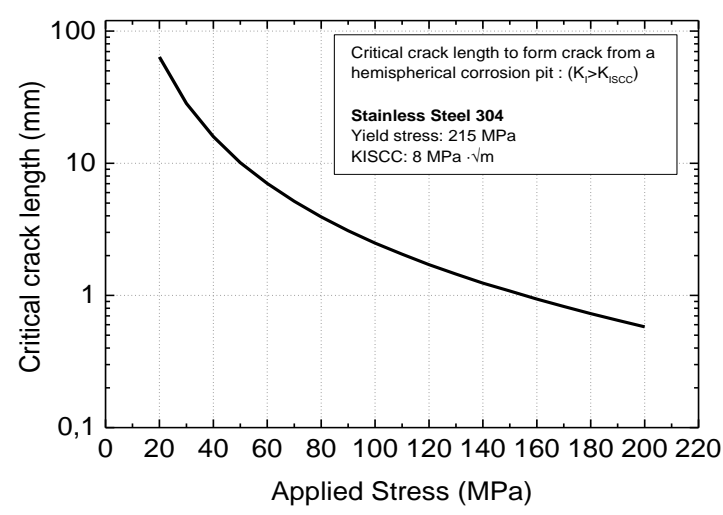

(b)

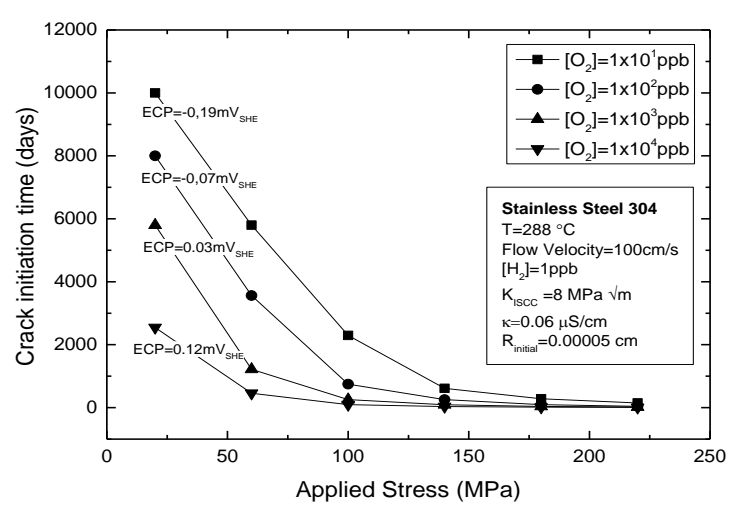

Figure IV.20. Predicted critical crack length for crack initiation (a) and crack initiation time in Type $304 \mathrm{SS}$ under BWR conditions $\left(288^{\circ} \mathrm{C}\right.$, pure water) as a function of the applied (residual + operational) stress [2].

The grain boundary fractures when the energy of the applied and residual stress exceeds its cohesive strength as described by the Griffith law. The crack grows towards the next grain boundary inclusion, and the damage process starts over, as depicted in Figure IV.21. The predicted growth of the $\mathrm{Cr}_{2} \mathrm{O}_{3}$ layer with the emergent grain boundary is displayed in Figure IV.22a. The variation of the thickness with time plateaus in about ten days of growth after reaching a steadystate where the rate of growth equals the rate of dissolution, and hence $d L / d t=0$, as expected from theory [see Equation (IV.11)] [4,5]. The predicted crack initiation time, such that Equation (IV.5) is satisfied, is shown as a function of ECP, pH, and temperature in Figures IV.22(b), (c), and (d), respectively. CITs of up to 10,000 days are predicted, although most are in the range of 100 to 1000 days. 


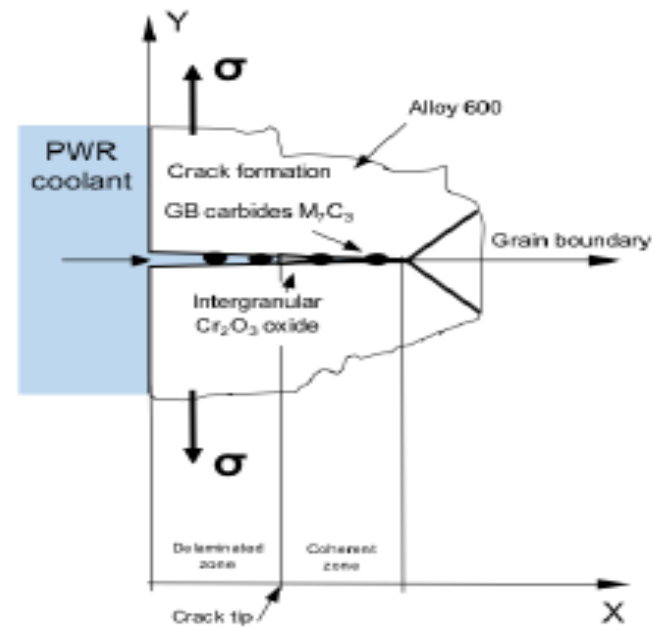

Figure IV.21. Grain boundary oxidation/wedging mechanism for crack initiation in Alloy 600 in PWR primary coolant circuits. The model is based upon the proposal by Scott and Le Calvar [3].
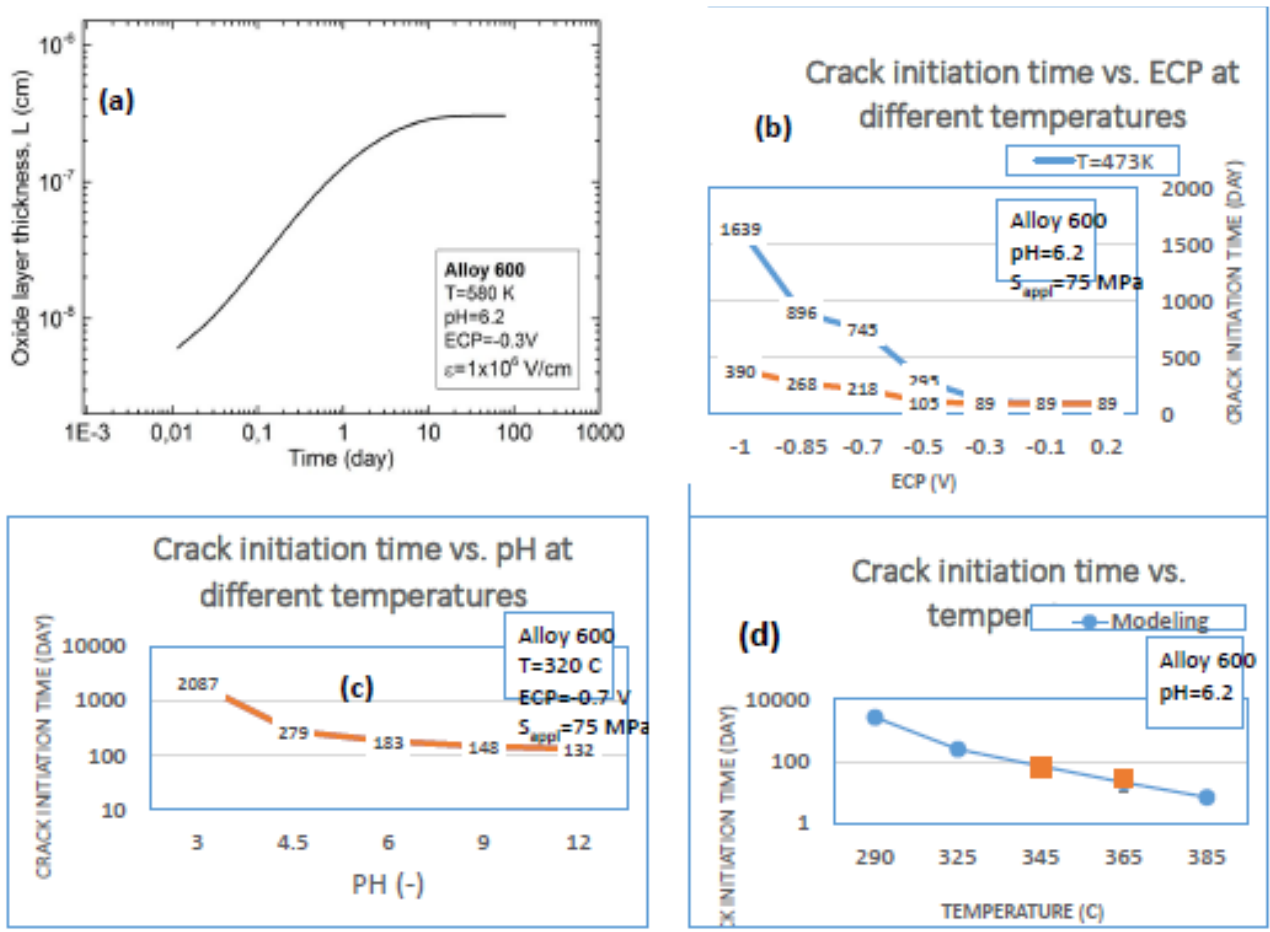

Figure IV.22. Predicted $\mathrm{Cr}_{2} \mathrm{O}_{3}$ film growth within the emergent grain boundary (a) and calculated crack initiation time as a function of ECP (b) $\mathrm{pH}(\mathrm{c})$, and temperature (d). 
The Point Defect Model (PDM) [5] describes the rate of growth of the chromic oxide within the separated grain boundary:

$$
\frac{d L}{d t}=\Omega k_{3}-\Omega k_{7}\left(\frac{C_{H^{+}}}{C_{H^{+}}^{0}}\right)^{n}
$$

where $L$ is the thickness of the $\mathrm{Cr}_{2} \mathrm{O}_{3}$ layer, $k_{3}$ and $k_{7}$ are the rate constants for oxide growth and dissolution, respectively, $\Omega$ is the mole volume of $\mathrm{Cr}_{2} \mathrm{O}_{3}$ per cation, and $n$ is the kinetic order of dissolution with respect to the concentration of $\mathrm{H}^{+}\left(C_{H^{+}}\right) . C_{H^{+}}^{0}$ is the standard state hydrogen ion concentration. The values of all of these parameters have been determined by optimizing the PDM on electrochemical impedance spectroscopic data for passive Alloy 600 in PWR primary coolant at temperatures to $300{ }^{\circ} \mathrm{C}[6]$.

From Figure IV.22a, it is predicted that the film grows sigmoidally over about 240 hours before reaching a steady-state, with the resulting wedging resulting in the initiation times shown in Figure IV.22b, IV.22c, and IV.22d as functions of ECP, pH, and temperature [7]. Increasing the ECP is predicted to decrease the CIT because the value of $k_{3}$ increases, and the oxide film grows faster. The decrease in the CIT with increasing $\mathrm{pH}$ is because the oxide within the wedged grain boundary dissolves less rapidly, and hence the oxide also grows more rapidly, resulting in more rapid wedging. Finally, the CIT is predicted to decrease with increasing temperature because the rate constants, $k 3$ and $k 7$, are temperature-dependent with the temperature dependence of $k 3$ being greater than that of $k_{7}$.

\section{IV.3.3. Where should we go from here}

To advance the field further, the following tasks in developing the technology for predicting crack initiation times (CITs) in BWR and PWR primary coolant heat transport circuits: Complete the development of the theory of crack initiation to include the distributions in key parameters, such as surface stress, and distributions due to passivity breakdown. In this regard, distribution functions for passivity breakdown and the nucleation of pits have been previously developed [8] and can be used directly in the proposed research. Likewise, the theory of pit growth and cracking in materials in BWR coolant circuits is also well-developed [9] and will be used directly in the proposed work. For PWRs, the model is based on the oxidative wedging of 
emergent grain boundaries, a model for which has already been developed for a single initiation site [4].

- Survey the literature and create a database of crack initiation time, as a function of relevant independent variables, including surface stress, degree of cold work, hardness, ECP, solution conductivity, temperature, substrate composition, inclusion density, grain size, and so forth. Noting that many of the important independent variables will not have been measured or reported, where possible, we can use various methods to predict the missing values, to "fill in the gaps." We have used this approach in the past with great effect in the development of Artificial Neural Networks (ANNs) for describing stress corrosion cracking in sensitized Type 304 SS in BWR coolant circuits [10] and mill-annealed Alloy 600 in PWR primary environments [11].

- Using Artificial Intelligence (AI) techniques, develop an Artificial Neural Network (ANN) to analyze the data contained within the database. The ANN should be trained via supervised learning on half of the database, while the remaining half will be used to evaluate the trained ANN. Development of an ANN has two important purposes:

1. To determine the mechanistic character of crack initiation, i.e., how much of the phenomenon is "mechanical" or "physical" (including "microstructural") versus how much is "electrochemical" in nature. We have previously used this approach with great success in determining the character of SCC and in formulating deterministic predictive models (i.e., the CEFM for SCC in both Type 304 SS [10] and Alloy 600 [11] in reactor coolant circuits.

2. If the underlying database is sufficiently extensive that robust training is achieved, the ANN can be used as a "stand-alone" tool for predicting crack initiation time (CIT), in the same manner that we have done with the crack growth rate. Because an ANN contains no underlying model, the predicted crack initiation times reflect hidden relationships between the dependent variable (CIT) and the various independent variables $\left(\mathrm{T}, \mathrm{ECP}\right.$, stress, hardness, $\left[\mathrm{Cl}^{-}\right], \mathrm{pH}$, flow velocity, inclusion density, grain size, etc) that are uncovered by training the net. Any viable physical model must account for these relationships.

3. Once the ANN is trained and the physical model has been verified, the models will be used to predict CITs over the operating histories of BWRs and PWRs as a function of 
the various independent variables. In particular, determine the sensitivity of the CIT to changes in each of the independent variables from the optimized weights of the trained ANN $[10,11]$ and in this manner we can ascertain the contribution from each variable to the CIT in a specific reactor coolant circuit under any given set of operating conditions.

- The final goal will be to answer the question: Does a fundamental limit exist in the prediction of crack initiation time, and, if so, what information can be obtained from experimental observation? This issue will be addressed by predicting the number of measurements that must be made in order to achieve the desired confidence level, given the statistical nature of the problem. The ultimate goal is to predict crack initiation time and the associated uncertainty in a deterministic manner such that the induction times can be used with engineering confidence.

\section{IV.3.4. References}

[1] D.D. Macdonald and G.R. Engelhardt, Shrier's Corrosion, 2, 1630-1679 (2010). Amsterdam: Elsevier.

[2] P.M. Scott and N. Le Calvar, The Minerals, Metals \& Materials Society, 1993.

[3] Z. Zhai, M.B. Toloczko, M.J. Olszta, and S. Bruemmer, Corros. Sci., 123, 76-87 (2017).

[4] B. Fekete, I. Balachov, and D.D. Macdonald, to be published (2019).

[5] D.D. Macdonald. J. Electrochem. Soc., 153(7), B213 (2006).

[6] B. Fekete, I. Balachov, and D.D. Macdonald, to be published (2019).

[7] J. Yang, Y. Li A. Xu, B. Fekete, and D.D. Macdonald, J. Nucl. Mat., 518, 305-315 (2019).

[8] D.D. Macdonald and M. Urquidi-Macdonald, Electrochim. Acta, 31, 1079 (1987).

[9] M.P. Manahan, K.E. Newman, D.D. Macdonald, and A.J. Peterson, Jr., Proc. EPRI Workshop on Secondary-Side Initiated IGA/SCC. p. 1-9. (October 14-15, 1993). Minneapolis, MN, Electric Power Research Institute, Palo Alto, CA.

[10] J.B. Shi and D.D. Macdonald, Corros. Sci., 89, 69-80 (2014).

[11] J.B. Shi and D.D. Macdonald, Corros. Sci., 92, 217-227 (2015). 


\section{Further development of the coupled environment fracture model}

\section{V.1. Development of a hydrogen-induced cracking (HIC) model for Alloy 600 and incorporate into the PWR code.}

The objective of this project task was to reconfigure the CEFM to incorporate hydrogen evolution at the crack tip as a theoretical model for predicting the HIC inter-granular stress corrosion cracking in aqueous environments by considering the effect of recombinant hydrogen pressure in the voids ahead of the crack tip together with the anodic dissolution. The calculation for the HIC inter-granular CGR was preliminarily carried out for Alloy 600, corresponding to PWR primary coolant circuit conditions containing boric acid and lithium hydroxide solution. The crack tip region of the crack growth model developed in this work is shown schematically in Figure V.1. The voids are assumed to nucleate ahead of the crack tip along a grain boundary and grow due to the effect of the crack stress field, which varies with distance from the crack tip. This model is an adaptation of the Wilkinson and Vitek creep model that was incorporated in the original CEFM.

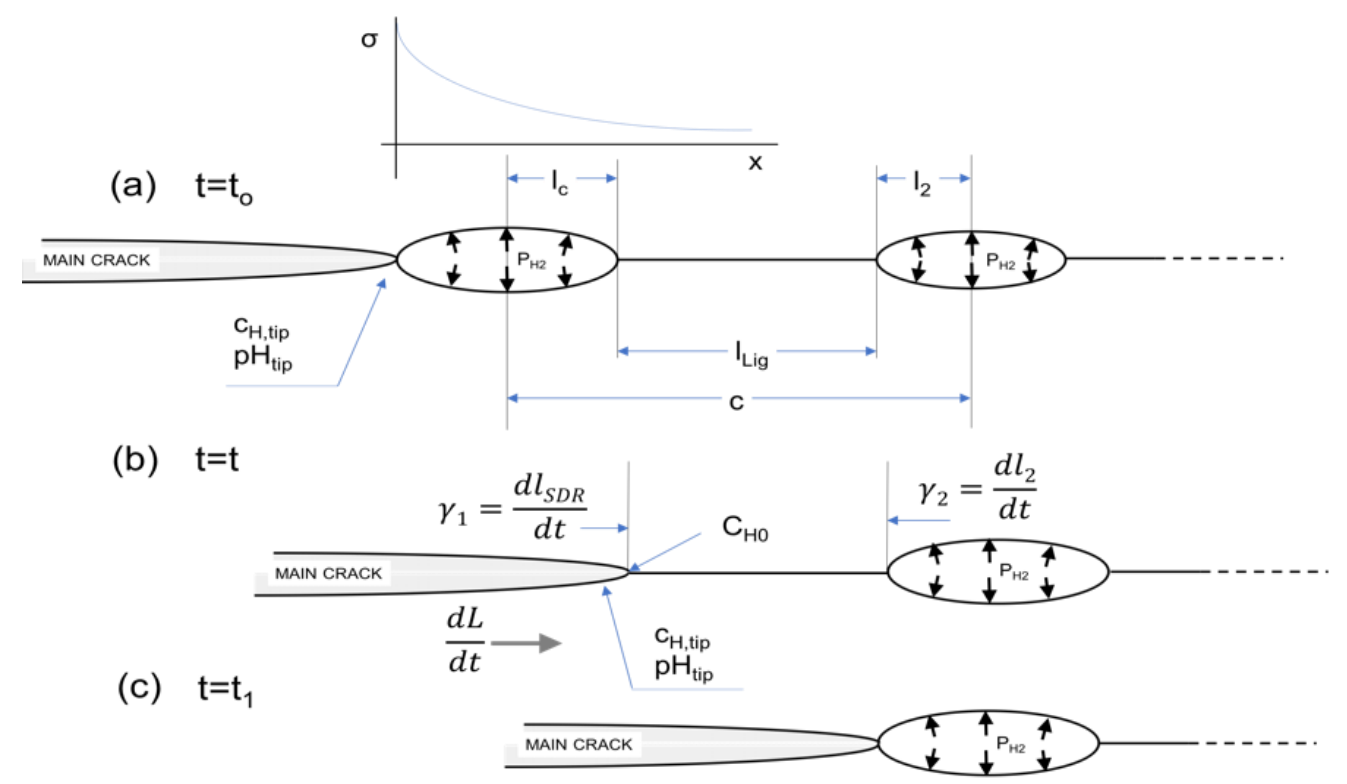

Figure V.1. The schematic diagram of the interaction between the main crack and the voids located along the grain boundary. 
In the original CEFM, increasing stress intensity factor, $K_{I}$ increases the frequency of rupture of the passive film at the crack tip and enhances the CGR. In the current model, the $K_{I}$ influences the void growth, too, as it increases the solubility of hydrogen in the metal and enhances the growth of grain boundary voids, thereby determining the hydrostatic stress field ahead of the crack tip.

\section{Derivation of the equation for crack growth rate}

Some assumptions are made to simplify the derivation below:

1. Cracking is assumed to be inter-granular;

2. The effect of hydrogen on void nucleation, microstructure, and mechanical property is neglected;

3. The crack is assumed to be parallel-sided;

4. The walls of the crack are assumed to be inert (passive), except those that result from microfracture events at the crack tip.

Assumptions (3) and (4) are essentially employed in the development of the original CEFM [1], and their validity has been discussed elsewhere [2-5]. Assumption (2) is adopted because we have no information indicating the contrary, and Assumption (1) is self-evident because IGSCC alone is being modeled. However, the same model, with minor modifications, may also apply to transgranular stress corrosion cracking (TGSCC).

\section{Basis of the coupled-environment fracture model (CEFM)}

The CEFM performs its calculations in two steps. In the first step, it calculates the electrochemical corrosion potential (ECP) of the external surface; in the second step, the CGR is estimated. The ECP sufficiently far from the crack is calculated using the Mixed Potential Model (MPM) [6] from the composition and properties of the system, such that the local electrochemical properties are unaffected by the presence of the crack, and, hence, that the potential in the solution with respect to the metal is equal to the negative of the free ECP (the metal is taken as the potential reference plane). 
In the CEFM, the CGR calculation relies on splitting the crack environment into the crackinternal and crack-external environments. A value of the electrochemical potential is firstly assumed at the crack mouth, i.e., the boundary between the crack-internal and crack-external environments, and then the potential is changed until the calculated crack-internal, and crackexternal currents match, i.e., the charge conservation [1], which can be stated as follows:

$$
i_{\text {crack }} A_{\text {crack } \cdot m o u t h}+\int_{S} i_{C}^{N} d s=0
$$

where $i_{C}^{N}$ is the net (cathodic) current density due to the charge transfer reactions on the external surface, and $d s$ is an increment in the external surface area. The subscript $S$ on the integral indicates that the integration is to be performed over the entire external surface; $A_{\text {crack mouth }}$ is the area of the crack mouth; $i_{\text {crack }}$ is the net (positive) current density exiting the crack mouth, which can be stated as follows:

$$
i_{\text {crack }}=F \sum_{i} z_{i} J_{i}=-F\left[\sum_{i} z_{i} D_{i} \frac{\partial C_{i}}{\partial l}+\sum_{i} \frac{F z_{i}^{2} D_{i}}{R T} C_{i} \frac{\partial \varphi}{\partial l}\right]
$$

where $z_{i}, J_{i}, D_{i}$, and $C_{i}$ are the charge number, flux, diffusion coefficient and concentration of species $i$, respectively; in the crack-internal environment, $F, l, R, T$, and $\varphi$ are Faraday's constant, the distance from the crack mouth, universal gas constant, Kelvin temperature, and the potential in the solution inside the crack, respectively. For hydrolyzed species $i$, where $z_{i}$ is the valence of the metal ion and is the hydrolysis state of the species $i$. For the calculation of the internal crack current, an electrochemical potential is assumed at the crack tip. This potential is changed until electro-neutrality is satisfied at the tip. For the calculation of the external crack current, a noniterative procedure is followed, including solving Laplace's equation:

$$
\nabla^{2} \varphi=0
$$

to yield $\varphi(x)$, such that electrical neutrality outside the crack is satisfied at all points (i.e., Poisson's equation with the charge density equal to zero). The electrochemical potential relatively far from the crack is assumed to be unchanged by the presence of the crack, and hence, is equal to the free corrosion potential (ECP). 
For highly resistive aqueous environments, the throwing power of the positive current from the crack mouth, which effectively determines the ability of reduction reactions occurring on the external surfaces to consume electrons released at the crack tip, has been predicted to play an essential role in determining the CGR $[8,9]$. The throwing power of the current from the crack mouth increases with increasing conductivity $\sigma_{c}$ of the external environment. According to dilute solution theory, the conductivity of the solution can be written as [10]:

$$
\sigma_{c}=\sum_{i}\left|z_{i}\right| \cdot C_{i} \cdot \lambda_{i}
$$

where $\lambda_{i}$ is the equivalent conductivity of the species $i$, which is a function of temperature [11].

\section{Crack tip dissolution}

In the CEFM, the micro-fracture events that occur at the crack tip were attributed to SDR. In the SDR mechanism, the crack growth rate is related to the electro-dissolution reactions that occur at the crack tip as the protective film is cyclically ruptured, followed by repassivation by increasing strain in the underlying matrix [8]. The electro dissolution reaction occurring at the crack tip from the oxidation of the steel is described in terms of Tafel's equation to yield the current averaged over a slip dissolution-repassivation cycle as:

$$
I_{0}=2 i_{0}^{0} A_{c t}\left(\frac{t_{0}}{t_{f}}\right) e^{\frac{E_{S}^{L}-E_{s}^{0}}{b_{a}}},
$$

where $i_{0}^{0}$ is the standard exchange current density for the dissolution reaction, $A_{c t}$ is the area at the crack tip over which dissolution occurs immediately upon rupture of the film, $E_{S}^{L}$ is the potential in the solution adjacent to the crack tip, $E_{S}^{0}$ is the (negative of the) standard potential ( $\left.E^{0}\right), b_{a}$ is Tafel's constant, $I_{0}$ is equal to the total current exiting the crack, $t_{f}$ is the time of cyclical fracture of the passive film at the crack tip, and $t_{0}$ is a constant determined from the repassivation transient [8].

The fracture frequency of the passive film at the crack tip is equal to the inverse of the period of cyclical fracture: 


$$
f_{r}=\frac{1}{t_{f}}=\frac{\dot{\varepsilon}_{c t}}{\varepsilon_{f}},
$$

where $\dot{\varepsilon}_{c t}$ is the strain rate and $\varepsilon_{f}$ is the strain of the passive film at the crack tip at fracture.

In this model, we use Hall's equation [12] for modeling the crack tip strain rate, which is based on the separation of the crack tip strain rate into quasi-stationary and crack advance components. This equation is given as:

$$
\dot{\varepsilon}_{c t}=\frac{n}{n+1} \frac{\sigma_{y}}{E}\left(\frac{\alpha_{c t} E J}{r \sigma_{y}^{2}}\right)^{\frac{n}{n+1}} \frac{\dot{J}}{J}+\frac{n}{n-1} \beta_{c t} \frac{\sigma_{y}}{E} \frac{\dot{a}}{r}\left\{\ln \left[\left(\frac{\lambda}{r}\right)\left(\frac{K}{\sigma_{y}}\right)^{2}\right]\right\}^{\frac{n+1}{n-1}},
$$

where $\alpha, \beta$ and $\lambda$ are dimensionless constants in the plastic strain calculation, $\sigma_{y}$ is the yield strength, $E$ is Young's modulus, $n$ is the strain-hardening exponent defined by Ramberg and Osgood, $r$ is the distance from the crack tip, $K$ is the stress intensity factor, $J$ is the J-integral, $\dot{J}$ is the change in $J$ with time, and $\dot{a}$ is crack growth rate. The model given by Eq. (V.7) is highly deterministic and can account for the cold work on the crack tip strain rate through the effects of strain hardening and yield strength. Given a typical value for $\varepsilon_{f}$ (e.g., 0.001), the fracture frequency can be calculated using Eq. (V.6).

Once $E_{S}^{0}$ and $E_{S}^{L}$ are known, the potential distribution down the crack (through distance $x$ ) may be estimated for the crack environment by solving Laplace's equation (Eq. (V.6)). However, since $E_{S}^{0}$ and $E_{S}^{L}$ depend upon $I_{0}$, the above calculation must be repeated iteratively (including the solution for $\left.E_{S}(x)\right)$ until convergence on the total current is obtained. The crack growth rate due to anodic dissolution is then calculated using Faraday's law:

$$
\frac{d l_{S D R}}{d t}=\frac{M I_{0}}{\rho_{m} z F A_{c t}},
$$

where $M$ is the atomic weight of the metal, $\rho_{m}$ is the metal density, $z$ is the oxidation number, and $F$ is Faraday's constant.

\section{Hydrogen ion concentration at the crack tip and hydrogen pressure in the voids}


In this work, hydrogen evolution at the crack tip is analyzed in terms of the electrochemical phenomena contained within the CEFM. In order to solve analytically for the hydrogen ion concentration at the main crack tip, we assume a linearly varying potential in the solution inside the crack (as indicated by the solution of the one-dimensional Laplace's equation). The potential of the solution from the crack tip to mouth decreases along a parabolic curve, as indicated by the more exact Poisson's equation. However, generally, such a linear assumption does not introduce an obvious discrepancy to the potential drop from the crack tip to mouth. Indeed, as we all recognize, modeling is always a compromise between tractability and reality.

$$
\partial \varphi / \partial l=\left(\varphi_{\text {tip }}-\varphi_{\text {mouth }}\right) / L=\varepsilon
$$

where $L, \varepsilon, \varphi_{\text {tip }}$, and $\varphi_{\text {mouth }}$ are the crack length (the distance from the crack mouth to tip), the potential gradient across the crack, the potential of the solution at the crack tip, and the potential of the solution at the crack mouth, respectively.

The current resulting from hydrogen ion transport is:

$$
I_{H^{+}}=F \cdot J_{H^{+}}=t_{H^{+}} I,
$$

where $I$, and $t_{H^{+}}$are the total current density through the crack mouth, and the fraction of that current carried by hydrogen ions (i.e., the transport number), respectively.

The transport equation can be written as follows with a boundary condition:

$$
\begin{aligned}
& \frac{\partial C_{H^{+}}}{\partial l}-\frac{F \varepsilon}{R T} C_{H^{+}}+\frac{f_{H^{+}} I}{F D_{H^{+}}}=0, \\
& \left.C_{H^{+}}\right|_{l=0}=C_{\text {bulk }, H^{+}},
\end{aligned}
$$

where $C_{b u l k, H^{+}}$is the hydrogen ion concentration in the bulk solution.

According to the CEFM, the solution to the above transport equation is:

$$
C_{H^{+}}(l)=\zeta+\left(C_{b u l k, H^{+}}-\zeta\right) \cdot \exp \left(\frac{F\left(\varphi_{\text {tip }}-\varphi_{\text {mouth }}\right)}{R T} \frac{l}{L}\right)
$$

where 


$$
\zeta=\frac{f_{H^{+}} I}{\left[D_{H^{+}} F^{2} \varepsilon / R T\right]}=\frac{\left[L \cdot I \cdot \sum_{i} n_{i} w_{i} \% / \sum_{i} z_{i} w_{i} \%\right]}{\left[D_{H^{+}} F^{2}\left(\varphi_{\text {tip }}-\varphi_{\text {mouth }}\right) / R T\right]}
$$

and $w_{i}$ is the weight percent of the electroactive species in the metal, respectively.

At the main crack tip, $l=L$, which yields:

$$
C_{t i p, H^{+}}=\zeta+\left(C_{b u l k, H^{+}}-\zeta\right) \cdot \exp \left(\frac{F\left(\varphi_{\text {tip }}-\varphi_{\text {mouth }}\right)}{R T}\right) \text {. }
$$

The hydrogen ion concentration at the main crack tip is assumed to be time-independent, corresponding to steady-state conditions. In the solution, the $\mathrm{H}^{+}$is assumed to be in approximate equilibrium with the reduced gaseous hydrogen.

$$
H^{+}+e^{-} \Leftrightarrow H_{2} / 2
$$

The Nernst equation gives the equilibrium potential of the hydrogen electrode reaction as

$$
E_{t i p}=E^{0}-\frac{2.303 R T}{2 F} \log _{10}\left(f_{H_{2}}\right)-\frac{2.303 R T}{F} p H
$$

where $E_{t i p}$ is the equilibrium potential at the crack tip, $E^{0}=0$ is the standard potential for the HER and $f_{H_{2}}$ is the dissolved hydrogen fugacity in the solution at the crack tip.

If the reduced gaseous hydrogen in the solution at the crack tip is assumed to be in approximate equilibrium with the dissolved hydrogen in the metal, the concentration of the dissolved hydrogen in the metal can be calculated according to Sievert's law:

$$
C_{H_{0}}=K_{s}\left(P_{H_{2}}^{R}+P_{H_{2}}^{A}\right)^{1 / 2}
$$

where $C_{H_{0}}$ is the equilibrium concentration of the dissolved hydrogen in the metal at the crack tip in the absence of stress, $P_{H_{2}}^{R}$ and $P_{H_{2}}^{A}$ are the reduced gaseous hydrogen pressure at the crack tip and the background gaseous hydrogen pressure (e.g., from a PWR primary coolant), respectively, and $K_{s}$ is Sievert's constant.

It is generally established that grain boundaries are short circuits for diffusion, and thus they can play an important role in the diffusion. The effect of the grain boundaries is particularly 
noticeable at relatively low temperatures, where bulk diffusion is less important [13,14]. Therefore, the grain boundary is assumed to be the preferred diffusion path.

From the theoretical considerations, it can be concluded that tensile stress often increases the solubility of hydrogen in the metal. For one-dimensional system, the concentration of hydrogen, $C_{H}(x, t)$, ahead of the main crack tip is determined by [15]:

$$
C_{H}(x, t)=C_{H_{0}}[1-\operatorname{erf}(z)] \exp \left[\bar{V}_{H} \cdot \sigma_{h(x)} /(R T)\right]
$$

where

$$
\begin{aligned}
& z=x /\left(2 \sqrt{D_{H} \cdot t}\right) \\
& D_{H}=D_{0} \exp [-Q /(R T)],
\end{aligned}
$$

and $D_{H}$ is the diffusivity; $t$ is the diffusion time; $x$ is the distance from the main crack tip into the metal; $D_{0}$ is the frequency factor; $Q$ is the activation energy of hydrogen diffusion along a grain boundary; $\bar{V}_{H}$ is the partial molar volume of the dissolved hydrogen; $C_{H_{0}}$ is the point source of hydrogen concentration at the main crack tip; and $\sigma_{h(x)}$ is the stress field ahead of the crack tip. As indicated by Eq. (V.19), the driving force for hydrogen transport in the stress field ahead of the crack is the gradient in the chemical potential for atomic hydrogen in the matrix by virtue of the partial molar volume of hydrogen being positive. Thus, the stress-related component of the chemical potential is $\sigma_{h(x)}$ and hydrogen diffused to the region of the matrix with the greatest free volume, which occurs at the point of maximum hydrostatic tensile stress ahead of the crack tip. This location coincides with the first void.

Due to local stress relaxation around a void ahead of the crack tip, resulting from the void formation and growth, part of the atomic hydrogen in the metal will recombine to form molecular hydrogen in the void. The gaseous hydrogen pressure in the $n$th void, $P_{H_{2}}\left(x_{n}^{\prime}, t\right)$, is assumed to be in approximate equilibrium with the local hydrogen concentration in the metal, and thus it can be written according to Sievert's law:

$$
P_{H_{2}}\left(x_{n}^{\prime}, t\right)=\left[C_{H}\left(x_{n}^{\prime}, t\right) / K_{s}\right]^{2}
$$


where $x_{n}^{\prime}$ is the distance from the main crack tip to the left side of the $n$th void.

In order to simplify the calculation, the gaseous hydrogen pressure, $P_{H_{2}}\left(x_{2}^{\prime}, t\right)$ inside the void nearest the main crack tip is assumed to be in approximate equilibrium with the dissolved hydrogen in the metal at point B (shown in Figure V.2), stated as:

$$
P_{H_{2}}\left(x_{2}^{\prime}, t\right)=\left[C_{H}\left(x_{2}^{\prime}, t\right) / K_{s}\right]^{2}
$$

where $x_{2}^{\prime}$ is the ligament width between the main crack tip and the void nearest the tip.

$K_{s}$ can be expressed as follows [16]:

$$
K_{s}=K_{s 0} \cdot \exp \left(-E_{s} / k_{b} T\right)
$$

where $K_{s 0}$ is a constant; $E_{s}$ is the activation energy, and $k_{b}$ is Boltzmann's constant.

\section{The crack growth rate}

At the moment, $t$, as shown in Figure V.1, the main crack continues to advance, and the void nearest the crack tip grows due to the effect of the main crack stress field. During the time interval $t_{1}-t_{0}$, the advance rate of the main crack tip is $\gamma_{1}$, and the growth rates of the void nearest the crack tip in the two directions shown in Figure V.2 are assumed to be $\gamma_{2}^{\prime}$ and $\gamma_{2}^{\prime \prime}$. The parameters $\gamma_{1}, \gamma_{2}^{\prime}$ and $\gamma_{2}^{\prime \prime}$ are functions of time and other parameters. Thus,

$$
c=l_{\mathrm{c}}+l_{2}^{0}+\int_{t_{0}}^{t_{1}} \gamma_{1} d t+\int_{t_{0}}^{t_{1}} \gamma_{2}^{\prime} d t
$$

where $l_{\mathrm{c}}$ and $l_{2}^{0}$ are the half lengths of the $1^{\text {st }}$ and $2^{\text {nd }}$ voids at the starting moment, $t_{0}$.

$$
\begin{array}{ll}
l_{\mathrm{c}}=y_{1} \cdot c & \left(0<y_{1} \leq 0.5\right), \\
l_{2}^{0}=y_{2} \cdot c & \left(0<y_{2}<0.5\right),
\end{array}
$$

where $y_{1}$ and $y_{2}$ are assumed to be constants.

The void growth rate depends on the applied stress, void size, and spacing [17]. 


$$
\gamma_{n}=d l_{n} / d t=\Phi\left(l_{n}, c\right) \cdot \sigma_{n}^{\beta} \quad(n=1,2, \cdots)
$$

where $l_{n}$ is the half-length of the $n$th void, and $\beta$ is a positive number, $\Phi$ is a function of the void size and void spacing as well as various material parameters, and its specific form depends on the mechanism considered. $\Phi$ can be written as follows if the void possesses an equilibrium shape, and $\beta=1$ [17].

$$
\Phi\left(l_{n}, c\right)=\frac{6 \lambda}{g(\theta) \cdot G} \frac{c}{l_{n}\left(c-2 l_{n}\right)^{2}},
$$

where

$$
\begin{aligned}
& g(\theta)=2(\theta-\sin \theta \cos \theta) / \sin ^{2} \theta, \\
& \lambda=\delta \cdot D \cdot \Omega \cdot G /\left(k_{b} T\right), \\
& D=D_{g b} \exp \left(-Q_{g b} / R T\right),
\end{aligned}
$$

and $\theta$ is the equilibrium dihedral angle at the void-grain boundary interface, and is assumed to be constant during the time interval, $t_{1}-t_{0} ; \delta$ is the diffusion width of a grain boundary; $D$ is the grain boundary diffusion coefficient; $D_{g b}$ is the frequency factor; $Q_{g b}$ is the activation energy of diffusion along a grain boundary; $\Omega$ is the atomic volume; $G$ is the shear modulus; and $k_{b}$ is Boltzmann's constant.

The growth rates, $\gamma_{2}^{\prime}$ and $\gamma_{2}^{\prime \prime}$, of the void nearest the main crack tip at the moment $t$ (shown in Figure V.2) can be written as:

$$
\begin{aligned}
& \gamma_{2}^{\prime}=\frac{d l_{2}^{\prime}}{d t}=\Phi\left(l_{2}^{\prime}, c\right) \cdot\left[\sigma_{h\left(x_{2}^{\prime}\right)}\right]^{\beta}=\frac{6 \lambda}{g(\theta) \cdot G} \frac{c}{l_{2}^{\prime}\left(c-2 l_{2}^{\prime}\right)^{2}} \cdot\left[\sigma_{h\left(x_{2}^{\prime}\right)}\right]^{\beta}, \\
& \gamma_{2}^{\prime \prime}=\frac{d l_{2}^{\prime \prime}}{d t}=\Phi\left(l_{2}^{\prime \prime}, c\right) \cdot\left[\sigma_{h\left(x_{2}^{\prime \prime}\right)}\right]^{\beta}=\frac{6 \lambda}{g(\theta) \cdot G} \frac{c}{l_{2}^{\prime \prime}\left(c-2 l_{2}^{\prime \prime}\right)^{2}} \cdot\left[\sigma_{h\left(x_{2}^{\prime \prime}\right)}\right]^{\beta},
\end{aligned}
$$

where $\sigma_{h\left(x_{2}^{\prime}\right)}$ and $\sigma_{h\left(x_{2}^{\prime \prime}\right)}$ are the stress at the two points B and C (shown in Figure V.2) and $x_{2}^{\prime}$ and $x_{2}^{\prime \prime}$ are the distances from the main crack tip to the two points B and C, respectively. 
In order to simplify the numerical analysis hereafter, $l_{2}^{\prime}$ and $x_{2}^{\prime}$ are taken to be an average value, as Wilkinson and Vitek [17] reported that in an elastic body, the average stress in the ligament between a crack and a void positioned ahead of the crack tip is essentially constant until the void has nearly linked up with the crack. Thus,

$$
\begin{aligned}
& \bar{l}_{2}=l_{2}^{0}+\left(c-l_{\mathrm{c}}-l_{2}^{0}\right) / y_{3}, \\
& \bar{x}_{2}^{\prime}=\left(c-l_{\mathrm{c}}-l_{2}^{0}\right) / y_{4},
\end{aligned}
$$

where $y_{3}$ and $y_{4}$ are assumed to be constants.

During the time interval from $t_{0}$ to $t_{1}$, the main crack continues to advance due to metal dissolution. However, in this work, only hydrogen-induced cracking is described, so the main crack tip is temporarily assumed to be static from $t_{0}$ to $t_{l}$. The advance of the crack tip during the time interval will involve a comprehensive theoretical CGR model that will be developed later. If the zone ahead of the main crack tip can be assumed to be approximately elastic, the stress field ahead of a Mode I crack tip in the absence of other local pressures is simply the elastic field as determined by the stress intensity factor, $K$, i.e.; [18]

$$
\sigma_{h(x)}=\frac{2}{3}(1+\gamma) \frac{K}{\left[2 \pi\left(x+x_{0}\right)\right]^{\alpha}}
$$

where $x$ is the distance from the main crack tip, $\gamma$ is the Poison's ratio of the metal, and

$$
x_{0}=\frac{1}{2 \pi}\left[\frac{2}{3}(1+\gamma) \frac{K}{\sigma_{\mathrm{t}}}\right]^{1 / \alpha} \text {. }
$$

The release of $\mathrm{H}_{2}$ into a void generates pressure within the void that increases with time. The hydrogen pressure adds to the hydrostatic stress on the void due to mechanical loading. The total stress at the left side of the $n$th void can be approximately written as follows while considering the gaseous hydrogen pressure inside the $n$th void:

$$
\sigma_{h\left(x_{n}^{\prime}\right)}=\frac{2}{3}(1+\gamma) \frac{K}{\left[2 \pi\left(x_{n}^{\prime}+x_{0}\right)\right]^{\alpha}}+P_{H_{2}}\left(x_{n}^{\prime}, t\right),
$$

where $P_{H}\left(x_{n}^{\prime}, t\right)$ is the hydrogen pressure inside the $n$th void.

At point B (shown in Figure V.1), the total stress can be approximately written: 


$$
\sigma_{h\left(x_{2}^{\prime}\right)}=\frac{2}{3}(1+\gamma) \frac{K}{\left[2 \pi\left(x_{2}^{\prime}+x_{0}\right)\right]^{\alpha}}+P_{H_{2}}\left(x_{2}^{\prime}, t\right) .
$$

The rate of change in the length of the transient ligament can be written as:

$$
\frac{d l_{2}}{d t}=\frac{6 \lambda \cdot c}{g(\theta) \cdot G} \frac{\left(\sigma_{h\left(\overline{x_{2}}\right)}\right)^{\beta}}{\overline{l_{2}}\left(c-2 \bar{l}_{2}\right)^{2}}
$$

Considering the anodic dissolution together with void growth by hydrogen pressurization, the average crack growth rate is given by:

$$
\frac{d L}{d t}=\frac{1}{1-\left(y_{1}+y_{2}+y_{5}\right)}\left[\frac{M I_{0}}{\rho_{m} z F A_{c t}}+\frac{6 \lambda \cdot c}{g(\theta) \cdot G} \frac{\left(\sigma_{h\left(\bar{x}_{2}^{\prime}\right.}\right)^{\beta}}{\bar{l}_{2}\left(c-2 \bar{l}_{2}\right)^{2}}\right] \text {. }
$$

where $y_{5} \cdot c$ is the ligament width at the moment when the transient ligament is considerably smaller than the void size, and the CGR begins to increase significantly, i.e., the time interval from this moment to the void linkage with the main crack is negligible, compared with the time interval $t_{1}-t_{0}$.

Figure V.2 shows the dependence of the CGR on the $\mathrm{K}_{\mathrm{I}}$, as predicted using our new model, along with some laboratory data. Good agreement between the CEFM-predicted results and experimentally measured data has been obtained during the whole range of stress intensity factors.

The ECP is calculated within CEFM from the concentrations of $\mathrm{H}_{2} \mathrm{O}_{2}, \mathrm{O}_{2}, \mathrm{H}_{2}, \mathrm{LiOH}$, and $\mathrm{H}_{3} \mathrm{BO}_{3}$ in the solution, from the kinetics of electrodissolution of the metal substrate, and the hydrodynamic conditions (flow rate and channel dimensions) using the mixed potential model. As can be seen from Figure V.3, the CGR increases with increasing ECP up to a maximum value in the range of $-1 \mathrm{~V}<\mathrm{ECP}<-0.5 \mathrm{~V}$, and good agreement is found with experimental observations. Generally, ECP has a positive impact on the CGR, and for the whole range of ECP we have studied, the CGR increases by almost an order of magnitude. 


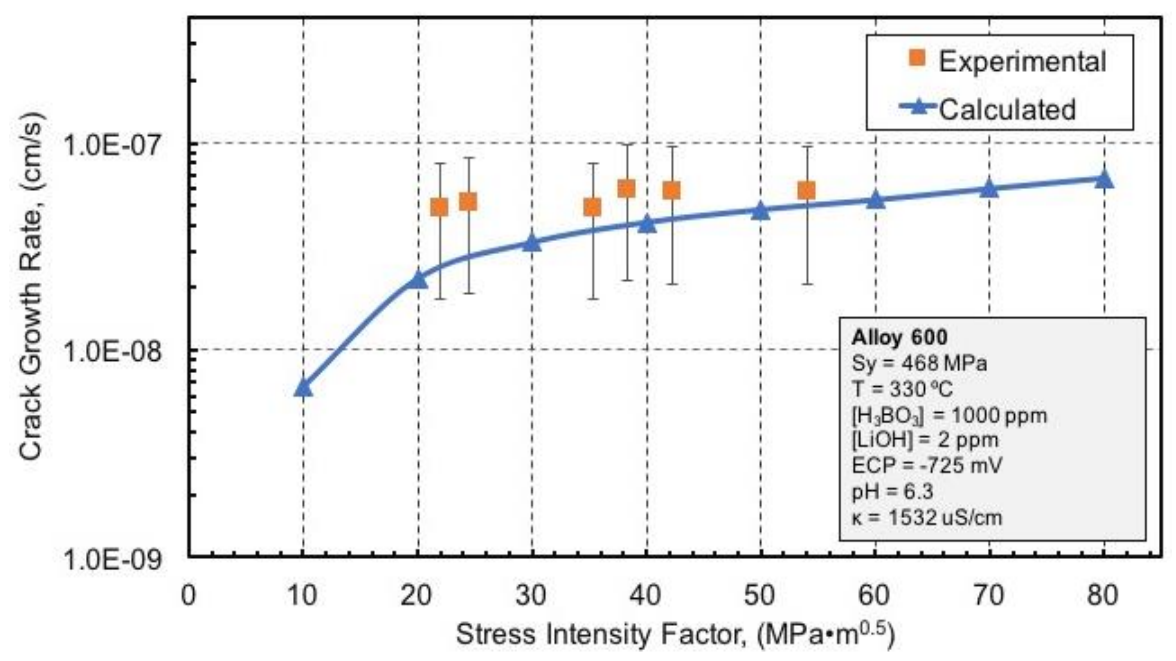

Figure V.2. Variation of the CGR vs. the stress intensity factor for Alloy 600 in PWR primary heat transport circuits.

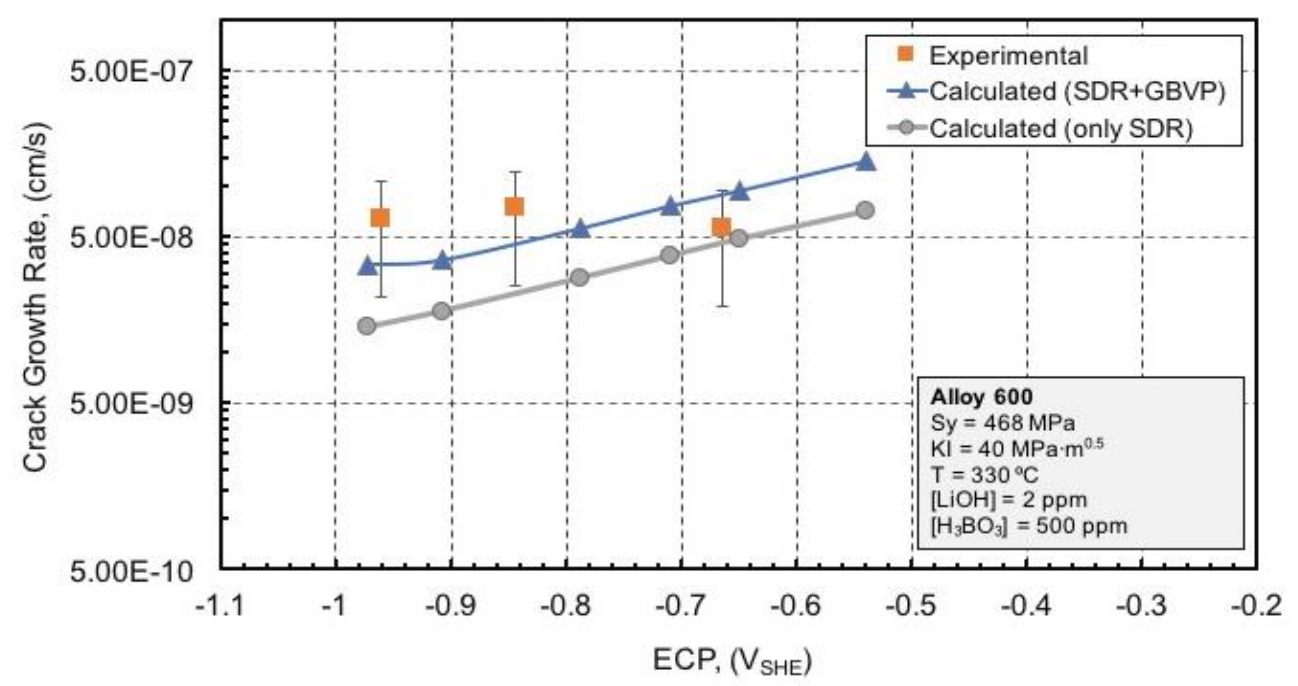

Figure V.3. Variation of the CGR vs. the ECP for Alloy 600 in PWR primary heat transport circuits for the Slip Dissolution-Repassivation mechanism (SDR) and together with the Grain Boundary Void Pressurization model (GBVP).

Figure V.3 also shows the dependence of CGR on ECP calculated considering only the SDR mechanism alone. It is important to recognize that at very low potentials, the CGR is moderately sensitive for the ECP compared to the SDR model, indicating that in this region, the void growth is the dominant factor over the anodic dissolution in determining the CGR. 
Figure V.4 shows another important effect that is the grain boundary void spacing $(c)$ on CGR along with the experimental result obtained by TEM investigations. Excellent agreement between the calculated results and the experiments is found. Figure V.4 indicates that increasing the void spacing in the range of $0.05 \mu \mathrm{m}-5.0 \mu \mathrm{m}$ the corresponding CGR decreases significantly, and at higher $c$ values the model yields the predictions of the original CEFM, demonstrating that the new model is an extension of the old CEFM, as intended.

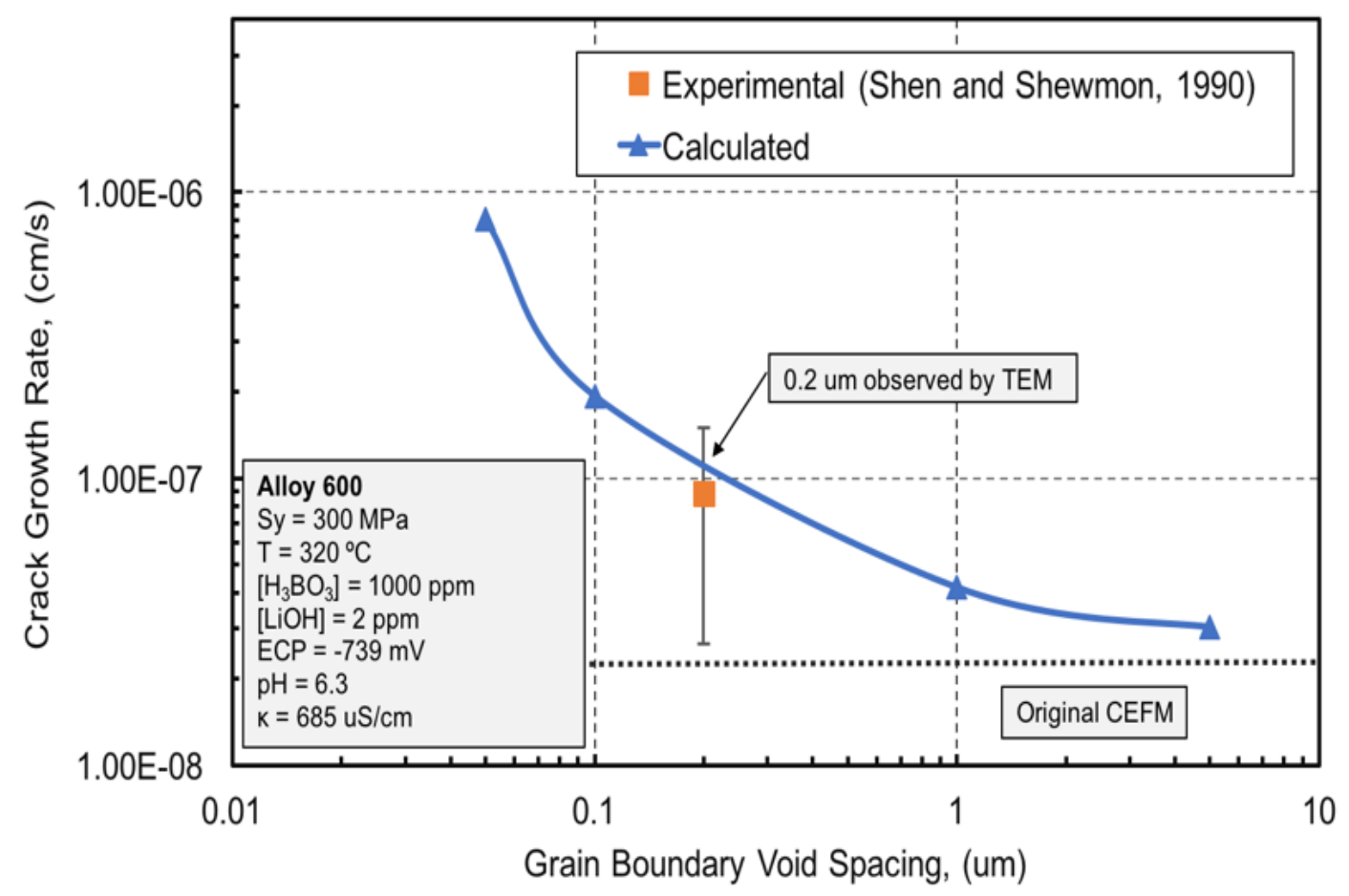

Figure V.4. Variation of the CGR vs. the grain boundary void spacing for Alloy 600 in PWR primary heat transport circuits. Experimental data point investigated by Transmission Electron Microscopy.

\section{V.2. References}

[1] D.D. Macdonald and M. Urquidi-Macdonald, Corros. Sci., 32, 51 (1991).

[2] D.D. Macdonald and M. Urquidi-Macdonald, Advanced Coupled Environment Fracture Model for Predicting Crack Growth Rates, in Proceedings of Parkins Symposium on Fundamental Aspects of Stress Corrosion Cracking, edited by S.M. Bruemmer, E.I. Meletis, R.H. Jones, Cincinnati, Ohio, Oct.21-24, 443 (1991). 
[3] D.D. Macdonald, P.C. Lu, M. Urquidi-Macdonald, and T.K. Yeh, Corrosion, 52, 768 (1996).

[4] G.R. Engelhardt, D.D. Macdonald, and M. Urquidi-Macdonald, Corros. Sci., 41, 2267 (1999).

[5] M. Vankeerberghen and D.D. Macdonald, Corros. Sci., 44, 1425 (2002).

[6] D.D. Macdonald, P.C. Lu, M. Urquidi-Macdonald, and T.K. Yeh, Corrosion 52, 768 (1996).

[7] N. Totsuka and Z. Szklarska-Smialowska, Corrosion, 44, 124 (1988).

[8] D.D. Macdonald and M. Urquidi-Macdonald, Corros. Sci., 32, 51 (1991).

[9] D.D. Macdonald and M. Urquidi-Macdonald, Advanced Coupled Environment Fracture Model for Predicting Crack Growth Rates, in Proceedings of Parkins Symposium on Fundamental Aspects of Stress Corrosion Cracking, edited by S.M. Bruemmer, E.I. Meletis, R.H. Jones, Cincinnati, Ohio, Oct.21-24, 443 (1991).

[10] A.S. Quist and W.L. Marshall, J. Phys. Chem., 69, 2984 (1965).

[11] D.S. Wilkinson, Models for Creep Crack Growth, in Deformation of Ceramics Materials II, edited by Tressler RE and Bradt RC, New York, Plenum Press, 507 (1984).

[12] M.M. Hall, Corros. Sci., 50, 2902 (2008).

[13] T. Karakasidis and M. Meyer, Phys. Rev. B, 55, 13853 (1997).

[14] Yongning Yu, Principles of Metallography, Beijing, Metallurgical Industry Press, 202, (2000).

[15] P. Doig and G.T. Jones, Metall. Trans. A, 8A, 1993 (1977).

[16] B. Zajec and V. Nemani, J. Vac. Sci. Technol., A 23, 322 (2005).

[17] D.S. Wilkinson and V. Vitek, Acta Metall., 30, 1723 (1982).

[18] P.C. Paris and G.C.M. Sih, Fracture Toughness Testing and its Applications, ASTM STP, 381,30 (1965). 


\section{An advanced coupled environment fracture model for hydrogen-induced cracking in Alloy 600 in PWR primary heat transport environment}

\section{VI.1. Introduction}

Stress corrosion cracking (SCC) is the most catastrophic form of corrosion. Extensive research has been carried out in this area, but the mechanism is still controversial in many cases. Since SCC is a corrosion-related process, its mechanism is inevitably correlated with the anodic reaction that occurs in the crack and cathodic reactions that occurs on the external surface as required by the Differential Aeration Hypothesis (DAH). The DAH provides the framework within which all localized corrosion processes can be interpreted. An important feature of the DAH is that the local anode and the local cathode are spatially separated. The local anode exists on the in-crack surfaces (including at the crack flanks and crack tip), and the cathode primarily exists on the bold, exposed external surfaces, which have the greatest access to the cathodic depolarizer. Because of the potential drop that exists between the crack tip and the external surface, a positive current flow from the crack-internal environment to the external surface through the crack mouth and an equivalent electronic "coupling current" flows through the metal in the same direction. The coupling current must be of sufficient magnitude to maintain the necessary separation between the local anode (in the crack) and the local cathode (on the external surface) and to maintain a suitably aggressive environment at the crack tip, in order that crack growth is sustained. Crack growth requires a cathodic reaction on the external surface that has an equilibrium potential that is more positive than the equilibrium potential of the anodic process occurring at the crack tip, which is the case here for a chromium-containing alloy such as Alloy 600. This ensures that $\Delta \mathrm{G}<0$ and hence that the corrosion reaction (crack propagation) is spontaneous thermodynamically because of the potential difference as discussed above.

With regards to the processes that are envisioned to occur at the crack tip, the two most important mechanisms, the anodic path dissolution (APD) and hydrogen-induced cracking (HIC), have frequently been proposed to explain crack growth. Extensive evidence for the HIC mechanism exists [1-3], including earlier work by one of the authors [4], which shows that crack advance occurs via microfracture events having a dimension of micrometers, which is consistent with HIC, but is inconsistent with APD and in particular with the slip-dissolution-repassivation (SDR) model [5-8]. In the HIC mechanism adopted in this work, hydrogen is envisaged to be 
injected into the matrix ahead of the crack tip via the hydrogen evolution reaction that occurs on the crack tip flanks, as the result of microfracture events occurring at the crack tip. The hydrogen is transported ahead of the crack tip via the stress field and recombines to form molecular hydrogen in voids that have nucleated ahead of the crack as the result of creep. The sites of void nucleation are postulated to be precipitates (e.g., chromium carbide, $\mathrm{Cr}_{23} \mathrm{C}_{7}$ ) on the grain boundaries. The microfracture events produce the anodic current that comprises the coupling current that flows out of the crack and is consumed on the external surfaces as demonstrated experimentally by Manahan, et.al. [4] and postulated theoretically in the Coupled Environment Fracture Model (CEFM) [8-12] as well as a small fraction of the current that is consumed on the crack tip flanks by hydrogen evolution. It is the "coupling current" that maintains the crack enclave in the aggressive conditions of high chloride concentration and high hydronium $\left(\mathrm{H}^{+}\right)$concentration simultaneously, which results from differential aeration. Thus, gaseous hydrogen artificially added in aqueous solutions is not the only source of the hydrogen present in metals and alloys, and the hydrogen evolved through cathodic reactions is also important or even dominant. Thermodynamically, a low pH at the crack tip and a potential drop inside a stress corrosion crack are usually necessary (but not sufficient) for the occurrence of hydrogen evolution at the crack tip. Pickering and co-workers $[13,14]$ and Vermilyea et al. [15] separately measured the potential drop inside a crevice or a crack, and they reported that the obvious potential drop is ascribed to the IR drop (ohmic potential drop). March [16], Gray [17] and Williams [18] separately experimentally confirmed that hydrogen gas evolves near the crack tip when the solution at the crack tip has a low $\mathrm{pH}$ value during stress corrosion cracking of austenitic stainless steels in boiling $\mathrm{MgCl}_{2}$ solution. Tien [19] investigated hydrogen transport by dislocations, and they found that large hydrogen pressures (up to $1000 \mathrm{MPa}$ for small voids of $0.01 \mu \mathrm{m}$ to $10 \mathrm{MPa}$ for larger voids of $1 \mu \mathrm{m}$ diameter) were developed at voids developed at micro-inclusions.

Considerable research has also been carried out in developing theoretical models of environment assisted cracking to explore the fracture mechanisms and estimate the chemistry and potential in a stress corrosion crack (SCC), and to predict the crack growth rate (CGR) in steels under aqueous solution conditions [5-12, 20-32]. Thus, Li et al. [20] explored the thermodynamic conditions for hydrogen generation inside a SCC, and they reported that the $\mathrm{H}^{+}$partial potential drop plays an important role in the hydrogen generation, that is the electrochemical potential difference of $\mathrm{H}^{+}$inside and outside the crack caused by the solution resistance expressed in the 
dimension of voltage. Turnbull [32] modeled the crack chemistry in sensitized stainless steel in boiling water reactor (BWR) coolant environments, where the medium is basically high temperature $\left(288^{\circ} \mathrm{C}\right)$ pure water of low conductivity, and he found that the crack tip potential is becoming more negative as the ECP became more positive for ECP $<-760 \mathrm{mV}_{\text {she. }}$ Gonzalez [2] et al. developed an HIC crack growth rate model in terms of the hydrogen pressure inside an internal crack in steel plates. Ford [5-7] et al. developed a model for environmental cracking, but the model, like many others, fails to invoke charge conservation explicitly. Accordingly, the model fails to meet the minimal conditions required for determinism, nor does it account for the influence of the external environment on crack growth in an analytical (as opposed to empirical) manner. This latter aspect is particularly important because the conductivity of the external environment may vary significantly if upset chemistry conditions are experienced. Macdonald and co-workers [8-11] developed a coupled-environment fracture model (CEFM) to estimate the CGR of Type 304 stainless steel under BWR coolant conditions. The CEFM takes into account mass transport by diffusion and ion migration in the crack, electrochemical reactions at the crack tip, hydrolysis of $\mathrm{Fe}^{2+}, \mathrm{Ni}^{2+}$, and $\mathrm{Cr}^{3+}$, and the potential drop from the crack tip to the crack mouth. Furthermore, charge conservation is invoked to match crack-internal and crack-external currents, and electro-neutrality is satisfied at the crack tip. Vankeerberghen and Macdonald [12] extended the CEFM to incorporate the effects of dilute sulphuric acid on the properties of the environment and hence, on the CGR and include the thermal activation of the crack tip strain rate. However, it has long been recognized that the CEFM needs to incorporate the role of hydrogen in affecting crack advance in order to provide a comprehensive theoretical model of inter-granular stress corrosion cracking that might be used to estimate CGR over the full spectrum of electrochemical conditions that exist water-cooled reactor coolant circuits, ranging from oxidizing as in BWRs under normal water chemistry operation to mildly reducing conditions under hydrogen water chemistry to the highly reducing conditions that exist in PWR primary circuits.

The objective of the present work is to reconfigure the CEFM to incorporate hydrogen evolution at the crack tip as a theoretical model for predicting the HIC inter-granular stress corrosion cracking in aqueous environments by considering the effect of recombinant hydrogen pressure in the voids ahead of the crack tip. The calculation for the HIC inter-granular CGR was preliminarily carried out for Alloy 600, corresponding to PWR primary coolant circuit conditions. 


\section{VI.2. The crack growth model.}

The crack tip region of the crack growth model developed in this work is shown schematically in Figure VI.1. The voids are assumed to nucleate ahead of the crack tip along a grain boundary and grow due to the effect of the crack stress field, which varies with distance from the crack tip [26,34,35]. This model is an adaptation of the Wilkinson and Vitek [34,35] creep model that was incorporated in the original CEFM. The centers of the voids are separated by a distance $c$, which may be identified with the average spacing of void nucleation sites, such as precipitates. We shall regard both the main crack and voids as infinite in the direction of the crack front so that the problem becomes one dimensional. At the starting moment $t_{0}$ [shown in Figure VI.1a], the void nearest the crack tip grows to a critical size, 2lc, (lc: half-length), and the first void is assumed to link up with the main crack immediately. Thus, the second void becomes the closest to the main crack tip. The main crack continues to advance due to the slip-dissolutionrepassivation mechanism (SDR) as modeled by the original CEFM, and the void nearest the tip grows [shown in VI.1b]. At the moment $\mathrm{t}_{1}$ [shown in VI.1c], the current first void links up with the main crack, and the main crack advances by a step distance c.

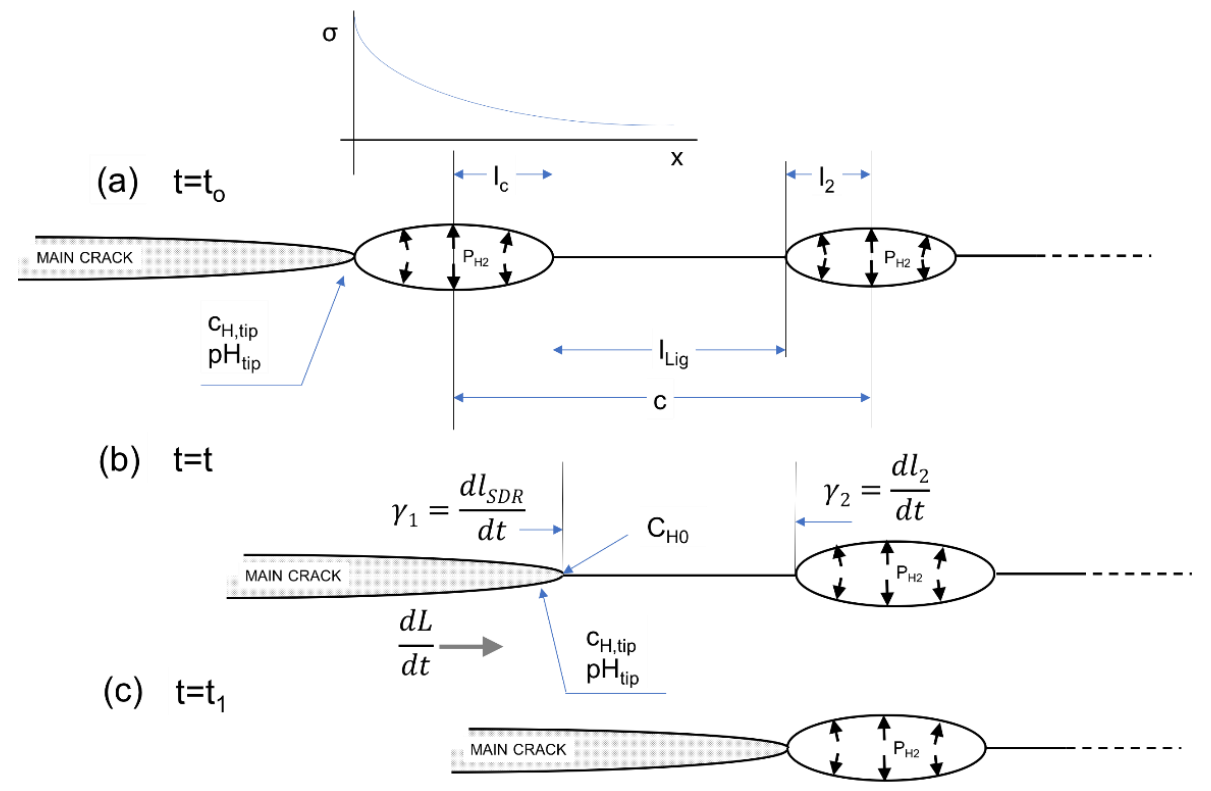

Figure VI.1. The schematic diagram of the interaction between the main crack and the voids located along the grain boundary. 
This process takes place cyclically, i.e., the main crack propagates step by step. Considering the void growth and linkage as the only mechanism of CGR [34]:

$$
\frac{d L}{d t}=\frac{c}{t_{1}-t_{0}}=f c
$$

where $f=1 /\left(t_{1}-t_{0}\right)$ is the fracture frequency. Note that in this model, the crack advance is via discrete events, which is consistent with the experimental findings of Manahan et al. [4]. The decrease of the length of the transient ligament $\left({ }^{l}\right.$ lig $)$ between the crack tip and the void including also the SDR mechanism can be written as:

$$
\frac{d l_{l i g}}{d t}=\gamma_{1}+\gamma_{2}=\frac{d l_{S D R}}{d t}+\frac{d l_{2}}{d t}
$$

where $\frac{d l_{S D R}}{d t}$ and $\frac{d l_{2}}{d t}$ are the rate of change in the transient ligament due to the anodic dissolution and the void growth, respectively. Assuming that the time required to link up the main crack with the closest void is negligible compared to the time interval $\left[t_{0}, t_{1}\right]$, the average crack growth rate can be written as:

$$
\frac{d L}{d t}=\frac{c}{l_{L i g}} \cdot \frac{d l_{L i g}}{d t}=\frac{c}{l_{L i g}} \cdot\left(\frac{d l_{S D R}}{d t}+\frac{d l_{2}}{d t}\right)
$$

Generally, the hydrostatic stress field (i.e., that due to mechanical loading) ahead of a crack tip is inevitably affected by the presence of voids, and thus the stress analysis and void growth are not strictly separable problems. However, Wilkinson and Vitek [34,35] reported that in an elastic body, the average stress in the ligament between a crack and a void positioned ahead of the crack tip is essentially constant until the void is nearly linked up with the crack. This means that the void is loaded by almost the same stress field as that of the crack alone until the ligament between the crack and the void is considerably smaller than the void size. Therefore, the stress distribution is assumed to depend only on the position ahead of the main crack tip without considering the presence of voids, so as to simplify the analysis. Meanwhile, the hydrostatic stress field ahead of 
the main crack tip is assumed to be time-independent, and the stress concentration beside the voids are neglected.

\section{VI.3. Derivation of the equation for crack growth rate}

Some assumptions are made to simplify the derivation below:

1. Cracking is assumed to be inter-granular;

2. The effect of hydrogen on void nucleation, microstructure, and mechanical property is neglected;

3. The crack is assumed to be parallel-sided;

4. The walls of the crack are assumed to be inert (passive), except those that result from microfracture events at the crack tip.

Assumptions (3) and (4) are essentially employed in the development of the original CEFM [8], and their validity has been discussed elsewhere [9-12]. Assumption (2) is adopted because we have no information indicating the contrary, and Assumption (1) is self-evident because IGSCC alone is being modeled. However, the same model, with minor modifications, may also be applicable to transgranular stress corrosion cracking (TGSCC).

\section{VI.3.1. Basis of the coupled-environment fracture model (CEFM)}

The CEFM performs its calculations in two steps. In the first step, it calculates the electrochemical corrosion potential (ECP) of the external surface; in the second step, the CGR is estimated. The ECP sufficiently far from the crack is calculated using the Mixed Potential Model (MPM) [10] from the composition and properties of the system, such that the local electrochemical properties are unaffected by the presence of the crack, and, hence, that the potential in the solution with respect to the metal is equal to the negative of the free ECP (the metal is taken as the potential reference plane).

In the CEFM, the CGR calculation relies on splitting the crack environment into the crackinternal and crack-external environments. An electrochemical potential is firstly assumed at the crack mouth, i.e., the boundary between the crack-internal and crack-external environments, and 
then the potential is changed until the calculated crack-internal, and crack-external currents match, i.e., the charge conservation [8], which can be stated as follows:

$$
i_{\text {crack }} A_{\text {crack } \cdot \text { mouth }}+\int_{S} i_{C}^{N} d s=0
$$

where $i_{C}^{N}$ is the net (cathodic) current density due to the charge transfer reactions on the external surface, and $d s$ is an increment in the external surface area. The subscript $S$ on the integral indicates that the integration is to be performed over the entire external surface; $A_{\text {crack mouth }}$ is the area of the crack mouth; $i_{\text {crack }}$ is the net (positive) current density exiting the crack mouth, which can be stated as follows:

$$
i_{\text {crack }}=F \sum_{i} z_{i} J_{i}=-F\left[\sum_{i} z_{i} D_{i} \frac{\partial C_{i}}{\partial l}+\sum_{i} \frac{F z_{i}^{2} D_{i}}{R T} C_{i} \frac{\partial \varphi}{\partial l}\right]
$$

where $z_{i}, J_{i}, D_{i}$, and $C_{i}$ are the charge number, flux, diffusion coefficient and concentration of species $i$, respectively; in the crack-internal environment, $F, l, R, T$, and $\varphi$ are Faraday's constant, the distance from the crack mouth, universal gas constant, Kelvin temperature, and the potential in the solution inside the crack, respectively. For hydrolyzed species $i, M(O H)_{n_{i}}^{z_{i}-n_{i}}$, where $n_{i}$ is the valence of the metal ion and $n_{i}$ is the hydrolysis state of the species $i$. For the calculation of the internal crack current, an electrochemical potential is assumed at the crack tip. This potential is changed until electro-neutrality is satisfied at the tip. For the calculation of the external crack current, a non-iterative procedure is followed, including solving Laplace's equation:

$$
\nabla^{2} \varphi=0
$$

to yield $\varphi(x)$, such that electrical neutrality outside the crack is satisfied at all points (i.e., Poisson's equation with the charge density equal to zero). The electrochemical potential relatively far from the crack is assumed to be unchanged by the presence of the crack, and hence, is equal to the free corrosion potential (ECP).

For highly resistive aqueous environments, the throwing power of the positive current from the crack mouth, which effectively determines the ability of reduction reactions occurring on the external surfaces to consume electrons released at the crack tip, has been predicted to play an 
essential role in determining the CGR [8,9]. The throwing power of the current from the crack mouth increases with increasing conductivity $\sigma_{c}$ of the external environment. According to dilute solution theory, the conductivity of the solution can be written as [36]:

$$
\sigma_{c}=\sum_{i}\left|z_{i}\right| \cdot C_{i} \cdot \lambda_{i}
$$

where $\lambda_{i}$ is the equivalent conductivity of the species $i$, which is a function of temperature [35]. For further details of the CEFM, the reader is referred to Refs. [8-12].

\section{VI.3.2. Crack tip dissolution}

In the CEFM, the micro-fracture events that occur at the crack tip were attributed to SDR. In the SDR mechanism, the crack growth rate is related to the electro-dissolution reactions that occur at the crack tip as the protective film is cyclically ruptured, followed by repassivation by increasing strain in the underlying matrix [8-10]. The electrodissolution reaction occurring at the crack tip from the oxidation of the steel is described in terms of Tafel's equation to yield the current averaged over a slip dissolution-repassivation cycle as:

$$
I_{0}=2 i_{0}^{0} A_{c t}\left(\frac{t_{0}}{t_{f}}\right) e^{\frac{E_{S}^{L}-E_{S}^{0}}{b_{a}}}
$$

where $i_{0}^{0}$ is the standard exchange current density for the dissolution reaction, $A_{c t}$ is the area at the crack tip over which dissolution occurs immediately upon rupture of the film, $E_{S}^{L}$ is the potential in the solution adjacent to the crack tip, $E_{S}^{0}$ is the (negative of the) standard potential ( $\left.E^{0}\right), b_{a}$ is Tafel's constant, $I_{0}$ is equal to the total current exiting the crack, $t_{f}$ is the time of cyclical fracture of the passive film at the crack tip, and $t_{0}$ is a constant determined from the repassivation transient [8].

The fracture frequency of the passive film at the crack tip is equal to the inverse of the time period of cyclical fracture: 


$$
f_{r}=\frac{1}{t_{f}}=\frac{\dot{\varepsilon}_{c t}}{\varepsilon_{f}},
$$

where $\dot{\varepsilon}_{c t}$ is the strain rate and $\varepsilon_{f}$ is the strain of the passive film at the crack tip at fracture.

In this model, we use Hall's equation [37] for modeling the crack tip strain rate, which is based on the separation of the crack tip strain rate into quasi-stationary and crack advance components. This equation is given as:

$$
\dot{\varepsilon}_{c t}=\frac{n}{n+1} \frac{\sigma_{y}}{E}\left(\frac{\alpha_{c t} E J}{r \sigma_{y}^{2}}\right)^{\frac{n}{n+1}} \frac{\dot{J}}{J}+\frac{n}{n-1} \beta_{c t} \frac{\sigma_{y}}{E} \frac{\dot{a}}{r}\left\{\ln \left[\left(\frac{\lambda}{r}\right)\left(\frac{K}{\sigma_{y}}\right)^{2}\right]\right\}^{\frac{n+1}{n-1}},
$$

where $\alpha, \beta$ and $\lambda$ are dimensionless constants in the plastic strain calculation, $\sigma_{y}$ is the yield strength, $E$ is Young's modulus, $n$ is the strain-hardening exponent defined by Ramberg and Osgood, $r$ is the distance from the crack tip, $K$ is the stress intensity factor, $J$ is the J-integral, $\dot{J}$ is the change in $J$ with time, and $\dot{a}$ is crack growth rate. The model is given by Eq. VI.10 is highly deterministic and has the capability to account for the cold work on the crack tip strain rate through the effects of strain hardening and yield strength. Given a typical value for $\varepsilon_{f}$ (e.g., 0.001), the fracture frequency can be calculated using Eq. VI.9.

Once $E_{S}^{0}$ and $E_{S}^{L}$ are known, the potential distribution down the crack (through distance $x$ ) may be estimated for the crack environment by solving Laplace's equation (Eq. VI.6). However, since $E_{S}^{0}$ and $E_{S}^{L}$ depend upon $I_{0}$, the above calculation must be repeated iteratively (including the solution for $\left.E_{S}(x)\right)$ until convergence on the total current is obtained. The crack growth rate due to anodic dissolution is then calculated using Faraday's law:

$$
\frac{d l_{S D R}}{d t}=\frac{M I_{0}}{\rho_{m} z F A_{c t}},
$$

where $M$ is the atomic weight of the metal, $\rho_{m}$ is the metal density, $z$ is the oxidation number, and $F$ is Faraday's constant. 


\section{VI.3.3. Hydrogen ion concentration at the crack tip and hydrogen pressure in the voids}

In this work, hydrogen evolution at the crack tip is analyzed in terms of the electrochemical phenomena contained within the CEFM. In order to solve analytically for the hydrogen ion concentration at the main crack tip, we assume a linearly varying potential in the solution inside the crack (as indicated by the solution of the one-dimensional Laplace's equation). Actually, the potential of the solution from the crack tip to mouth decreases along a parabolic curve, as indicated by the more exact Poisson's equation. However, generally, such a linear assumption does not introduce an obvious discrepancy to the potential drop from the crack tip to mouth. Indeed, as we all recognize, modeling is always a compromise between tractability and reality.

$$
\partial \varphi / \partial l=\left(\varphi_{\text {tip }}-\varphi_{\text {mouth }}\right) / L=\varepsilon
$$

where $L, \varepsilon, \varphi_{\text {tip }}$, and $\varphi_{\text {mouth }}$ are the crack length (the distance from the crack mouth to tip), the potential gradient across the crack, the potential of the solution at the crack tip, and the potential of the solution at the crack mouth, respectively.

The current resulting from hydrogen ion transport is:

$$
I_{H^{+}}=F \cdot J_{H^{+}}=t_{H^{+}} I
$$

where $I$, and $t_{H^{+}}$are the total current density through the crack mouth, and the fraction of that current carried by hydrogen ions (i.e., the transport number), respectively.

The transport equation can be written as follows with a boundary condition:

$$
\begin{aligned}
& \frac{\partial C_{H^{+}}}{\partial l}-\frac{F \varepsilon}{R T} C_{H^{+}}+\frac{f_{H^{+}} I}{F D_{H^{+}}}=0, \\
& C_{\left.H^{+}\right|_{l=0}}=C_{\text {bulk }, H^{+}},
\end{aligned}
$$

where $C_{b u l k, H^{+}}$is the hydrogen ion concentration in the bulk solution.

According to the CEFM, the solution to the above transport equation is: 


$$
C_{H^{+}}(l)=\zeta+\left(C_{b u l k, H^{+}}-\zeta\right) \cdot \exp \left(\frac{F\left(\varphi_{\text {tip }}-\varphi_{\text {mouth }}\right)}{R T} \frac{l}{L}\right)
$$

where

$$
\zeta=\frac{f_{H^{+}} I}{\left[D_{H^{+}} F^{2} \varepsilon / R T\right]}=\frac{\left[L \cdot I \cdot \sum_{i} n_{i} w_{i} \% / \sum_{i} z_{i} w_{i} \%\right]}{\left[D_{H^{+}} F^{2}\left(\varphi_{\text {tip }}-\varphi_{\text {mouth }}\right) / R T\right]},
$$

and $w_{i}$ is the weight percent of the electroactive species in the metal, respectively.

At the main crack tip, $l=L$, this yields:

$$
C_{t i p, H^{+}}=\zeta+\left(C_{b u l k, H^{+}}-\zeta\right) \cdot \exp \left(\frac{F\left(\varphi_{t i p}-\varphi_{\text {mouth }}\right)}{R T}\right)
$$

The hydrogen ion concentration at the main crack tip is assumed to be time-independent, corresponding to steady-state conditions. In the solution, the $\mathrm{H}^{+}$is assumed to be in approximate equilibrium with the reduced gaseous hydrogen.

$$
H^{+}+e^{-} \Leftrightarrow H_{2} / 2
$$

The equilibrium potential of the hydrogen electrode reaction is given by the Nernst equation:

$$
E_{t i p}=E^{0}-\frac{2.303 R T}{2 F} \log _{10}\left(f_{H_{2}}\right)-\frac{2.303 R T}{F} p H
$$

where $E_{t i p}$ is the equilibrium potential at the crack tip, $E^{0}=0$ is the standard potential for the HER and $f_{H_{2}}$ is the dissolved hydrogen fugacity in the solution at the crack tip.

If the reduced gaseous hydrogen in the solution at the crack tip is assumed to be in approximate equilibrium with the dissolved hydrogen in the metal, the concentration of the dissolved hydrogen in the metal can be calculated according to Sievert's law:

$$
C_{H_{0}}=K_{s}\left(P_{H_{2}}^{R}+P_{H_{2}}^{A}\right)^{1 / 2}
$$


where $C_{H_{0}}$ is the equilibrium concentration of the dissolved hydrogen in the metal at the crack tip in the absence of stress, ${ }^{H_{2}}$ and $P_{H_{2}}^{A}$ are the reduced gaseous hydrogen pressure at the crack tip and the background gaseous hydrogen pressure (e.g., from a PWR primary coolant), respectively, and Ks is Sievert's constant.

It is generally established that grain boundaries are short circuits for diffusion, and thus they can play an important role in the diffusion. The effect of the grain boundaries is particularly noticeable at relatively low temperatures, where bulk diffusion is less important $[38,39]$. Therefore, the grain boundary is assumed to be the preferred diffusion path.

From the theoretical considerations, it can be concluded that tensile stress often increases the solubility of hydrogen in the metal. For one-dimensional system, the concentration of hydrogen, $C_{H}(x, t)$, ahead of the main crack tip is determined by [22]:

$$
C_{H}(x, t)=C_{H_{0}}[1-\operatorname{erf}(z)] \exp \left[\bar{V}_{H} \cdot \sigma_{h(x)} /(R T)\right],
$$

where

$$
\begin{aligned}
& z=x /\left(2 \sqrt{D_{H} \cdot t}\right), \\
& D_{H}=D_{0} \exp [-Q /(R T)],
\end{aligned}
$$

$D_{H}$ is the diffusivity; $t$ is the diffusion time; $x$ is the distance from the main crack tip into the metal; $D_{0}$ is the frequency factor; $Q$ is the activation energy of hydrogen diffusion along a grain boundary; $\bar{V}_{H}$ is the partial molar volume of the dissolved hydrogen; $C_{H_{0}}$ is the point source of hydrogen concentration at the main crack tip; and $\sigma_{h(x)}$ is the stress field ahead of the crack tip. As indicated by Eq. VI.22, the driving force for hydrogen transport in the stress field ahead of the crack is the gradient in the chemical potential for atomic hydrogen in the matrix by virtue of the partial molar volume of hydrogen being positive. Thus, the stress-related component of the chemical potential is $\sigma_{h(x)} \cdot \bar{V}_{H}$, and hydrogen diffused to the region of the matrix with the greatest free volume, which occurs at the point of maximum hydrostatic tensile stress ahead of the crack tip. This location coincides with the first void. 
Due to local stress relaxation around a void ahead of the crack tip, resulting from the void formation and growth, part of the atomic hydrogen in the metal will recombine to form molecular hydrogen in the void. The gaseous hydrogen pressure in the $n$th void, $P_{H_{2}}\left(x_{n}^{\prime}, t\right)$, is assumed to be in approximate equilibrium with the local hydrogen concentration in the metal, and thus it can be written according to Sievert's law:

$$
P_{H_{2}}\left(x_{n}^{\prime}, t\right)=\left[C_{H}\left(x_{n}^{\prime}, t\right) / K_{s}\right]^{2},
$$

where $x_{n}^{\prime}$ is the distance from the main crack tip to the left side of the $n$th void.

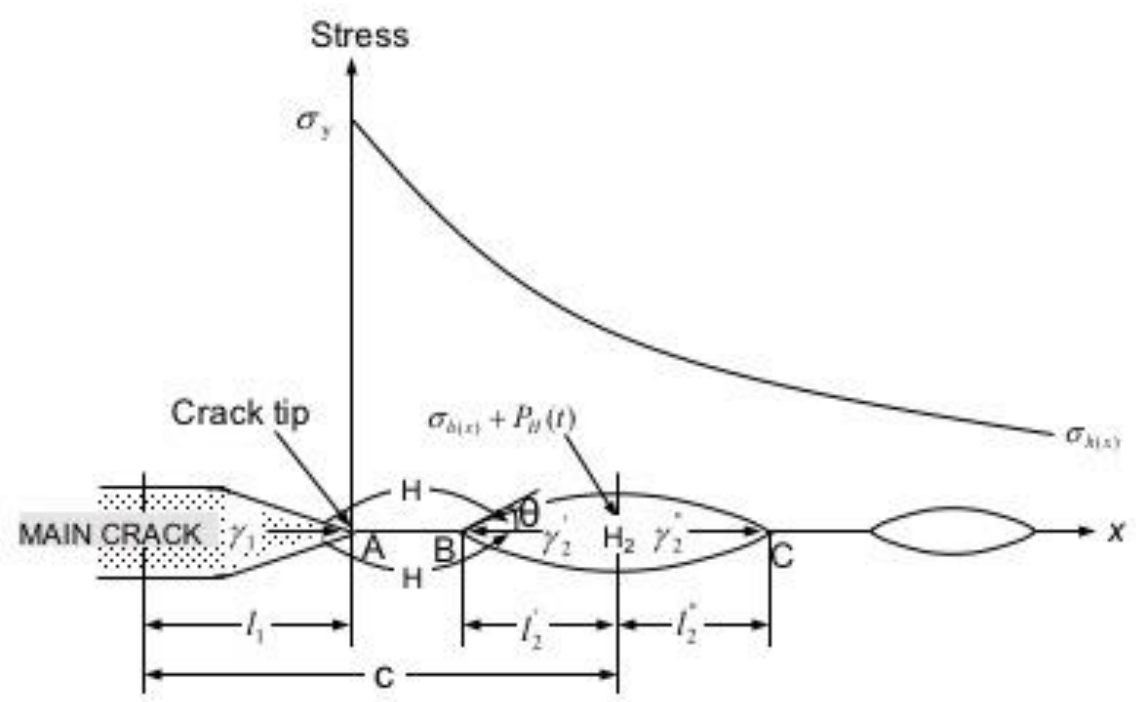

Figure VI.2. The schematic diagram of the void growth, hydrogen diffusion, and stress distribution ahead of a crack tip.

In order to simplify the calculation, the gaseous hydrogen pressure, $P_{H_{2}}\left(x_{2}^{\prime}, t\right)$ inside the void nearest the main crack tip is assumed to be in approximate equilibrium with the dissolved hydrogen in the metal at point B (shown in Figure VI.2), stated as:

$$
P_{H_{2}}\left(x_{2}^{\prime}, t\right)=\left[C_{H}\left(x_{2}^{\prime}, t\right) / K_{s}\right]^{2},
$$

where $x_{2}^{\prime}$ is the ligament width between the main crack tip and the void nearest the tip. Ks can be expressed as follows [40]: 


$$
K_{s}=K_{s 0} \cdot \exp \left(-E_{s} / k_{b} T\right)
$$

where $K_{s O}$ is a constant; $E_{s}$ is the activation energy, and $k_{b}$ is Boltzmann's constant.

\section{VI.3.4. The crack growth rate}

At the moment, $t$, as shown in Figure VI.1, the main crack continues to advance, and the void nearest the crack tip grows due to the effect of the main crack stress field. During the time interval $t_{1}-t_{0}$, the advance rate of the main crack tip is $\gamma_{1}$, and the growth rates of the void nearest the crack tip in the two directions shown in Figure VI.2 are assumed to be $\gamma_{2}^{\prime}$ and $\gamma_{2}^{\prime \prime}$. The parameters $\gamma_{1}, \gamma_{2}^{\prime}$ and $\gamma_{2}^{\prime \prime}$ are functions of time and other parameters. Thus,

$$
c=l_{\mathrm{c}}+l_{2}^{0}+\int_{t_{0}}^{t_{1}} \gamma_{1} d t+\int_{t_{0}}^{t_{1}} \gamma_{2}^{\prime} d t
$$

where $l_{\mathrm{c}}$ and $l_{2}^{0}$ are the half lengths of the $1^{\text {st }}$ and $2^{\text {nd }}$ voids at the starting moment, $t_{0 .}$

$$
\begin{array}{ll}
l_{\mathrm{c}}=y_{1} \cdot c & \left(0<y_{1} \leq 0.5\right), \\
l_{2}^{0}=y_{2} \cdot c & \left(0<y_{2}<0.5\right),
\end{array}
$$

where $y_{1}$ and $y_{2}$ are assumed to be constants.

The void growth rate depends on the applied stress, void size, and spacing [34].

$$
\gamma_{n}=d l_{n} / d t=\Phi\left(l_{n}, c\right) \cdot \sigma_{n}^{\beta} \quad(n=1,2, \cdots)
$$

where $l_{n}$ is the half-length of the $n$th void, and $\beta$ is a positive number, $\Phi$ is a function of the void size and void spacing as well as various material parameters, and its specific form depends on the mechanism considered. $\Phi$ can be written as follows if the void possesses an equilibrium shape, and $\beta=1[34]$.

$$
\Phi\left(l_{n}, c\right)=\frac{6 \lambda}{g(\theta) \cdot G} \frac{c}{l_{n}\left(c-2 l_{n}\right)^{2}}
$$

where 


$$
\begin{aligned}
& g(\theta)=2(\theta-\sin \theta \cos \theta) / \sin ^{2} \theta \\
& \lambda=\delta \cdot D \cdot \Omega \cdot G /\left(k_{b} T\right) \\
& D=D_{g b} \exp \left(-Q_{g b} / R T\right)
\end{aligned}
$$

and $\theta$ is the equilibrium dihedral angle at the void-grain boundary interface, and is assumed to be constant during the time interval, $t_{1}-t_{0} ; \delta$ is the diffusion width of a grain boundary; $D$ is the grain boundary diffusion coefficient; $D_{g b}$ is the frequency factor; $Q_{g b}$ is the activation energy of diffusion along a grain boundary; $\Omega$ is the atomic volume; $G$ is the shear modulus; and $k_{b}$ is Boltzmann's constant.

The growth rates, $\gamma_{2}^{\prime}$ and $\gamma_{2}^{\prime \prime}$, of the void nearest the main crack tip at the moment $t$ (shown in Figure VI.2) can be written as:

$$
\begin{aligned}
& \gamma_{2}^{\prime}=\frac{d l_{2}^{\prime}}{d t}=\Phi\left(l_{2}^{\prime}, c\right) \cdot\left[\sigma_{h\left(x_{2}^{\prime}\right)}\right]^{\beta}=\frac{6 \lambda}{g(\theta) \cdot G} \frac{c}{l_{2}^{\prime}\left(c-2 l_{2}^{\prime}\right)^{2}} \cdot\left[\sigma_{h\left(x_{2}^{\prime}\right)}\right]^{\beta}, \\
& \gamma_{2}^{\prime \prime}=\frac{d l_{2}^{\prime \prime}}{d t}=\Phi\left(l_{2}^{\prime \prime}, c\right) \cdot\left[\sigma_{h\left(x_{2}^{\prime \prime}\right)}\right]^{\beta}=\frac{6 \lambda}{g(\theta) \cdot G} \frac{c}{l_{2}^{\prime \prime}\left(c-2 l_{2}^{\prime \prime}\right)^{2}} \cdot\left[\sigma_{h\left(x_{2}^{\prime \prime}\right)}\right]^{\beta},
\end{aligned}
$$

where $\sigma_{h\left(x_{2}^{\prime}\right)}$ and $\sigma_{h\left(x_{2}^{\prime \prime}\right)}$ are the stress at the two points B and C (shown in Figure VI.2), and $x_{2}^{\prime}$ and $x_{2}^{\prime \prime}$ are the distances from the main crack tip to the two points $\mathrm{B}$ and $\mathrm{C}$, respectively.

In order to simplify the numerical analysis hereafter, $l_{2}^{\prime}$ and $x_{2}^{\prime}$ are taken to be an average value, as Wilkinson and Vitek [34,35] reported that in an elastic body, the average stress in the ligament between a crack and a void positioned ahead of the crack tip is essentially constant until the void has nearly linked up with the crack. Thus,

$$
\begin{aligned}
& \bar{l}_{2}=l_{2}^{0}+\left(c-l_{\mathrm{c}}-l_{2}^{0}\right) / y_{3}, \\
& \bar{x}_{2}^{\prime}=\left(c-l_{\mathrm{c}}-l_{2}^{0}\right) / y_{4},
\end{aligned}
$$

where $y_{3}$ and $y_{4}$ are assumed to be constants. 
During the time interval from $t_{0}$ to $t_{1}$, the main crack continues to advance due to metal dissolution and so on. But in this work, only hydrogen-induced cracking is described, so the main crack tip is temporarily assumed to be static from $t_{0}$ to $t_{1}$. The advance of the crack tip during the time interval will involve a comprehensive theoretical CGR model that will be developed later.

If the zone ahead of the main crack tip can be assumed to be approximately elastic, the stress field ahead of a Mode I crack tip in the absence of other local pressures is simply the elastic field as determined by the stress intensity factor, $K$, i.e.; [41]

$$
\sigma_{h(x)}=\frac{2}{3}(1+\gamma) \frac{K}{\left[2 \pi\left(x+x_{0}\right)\right]^{\alpha}}
$$

where $x$ is the distance from the main crack tip, $\gamma$ is the Poison's ratio of the metal, and

$$
x_{0}=\frac{1}{2 \pi}\left[\frac{2}{3}(1+\gamma) \frac{K}{\sigma_{\mathrm{t}}}\right]^{1 / \alpha}
$$

The release of $\mathrm{H}_{2}$ into a void generates pressure within the void that increases with time. The hydrogen pressure adds to the hydrostatic stress on the void due to mechanical loading. The total stress at the left side of the $n$th void can be approximately written as follows while considering the gaseous hydrogen pressure inside the $n$th void:

$$
\sigma_{h\left(x_{n}^{\prime}\right)}=\frac{2}{3}(1+\gamma) \frac{K}{\left[2 \pi\left(x_{n}^{\prime}+x_{0}\right)\right]^{\alpha}}+P_{H_{2}}\left(x_{n}^{\prime}, t\right),
$$

where $P_{H}\left(x_{n}^{\prime}, t\right)$ is the hydrogen pressure inside the $n$th void.

At point B (shown in Figure VI.2), the total stress can be approximately written:

$$
\sigma_{h\left(x_{2}^{\prime}\right)}=\frac{2}{3}(1+\gamma) \frac{K}{\left[2 \pi\left(x_{2}^{\prime}+x_{0}\right)\right]^{\alpha}}+P_{H_{2}}\left(x_{2}^{\prime}, t\right) \text {. }
$$

The rate of change in the length of the transient ligament can be written as:

$$
\frac{d l_{2}}{d t}=\frac{6 \lambda \cdot c}{g(\theta) \cdot G} \frac{\left(\sigma_{h\left(\bar{x}_{2}^{\prime}\right.}\right)^{\beta}}{\bar{l}_{2}\left(c-2 \bar{l}_{2}\right)^{2}} .
$$


Considering the anodic dissolution together with void growth by hydrogen pressurization, the average crack growth rate is given by combining Eq. VI.11, Eq. VI.29, Eq. VI.30 and Eq. VI.45 and then substituting the result into Eq. VI.3 to yield:

$$
\frac{d L}{d t}=\frac{1}{1-\left(y_{1}+y_{2}+y_{5}\right)}\left[\frac{M I_{0}}{\rho_{m} z F A_{c t}}+\frac{6 \lambda \cdot c}{g(\theta) \cdot G} \frac{\left(\sigma_{h\left(\bar{x}_{2}\right)}\right)^{\beta}}{\bar{l}_{2}\left(c-2 \bar{l}_{2}\right)^{2}}\right] \text {. }
$$

where $y_{5} \cdot c$ is the ligament width at the moment when the transient ligament is considerably smaller than the void size, and the CGR begins to increase significantly, i.e., the time interval from this moment to the void linkage with the main crack is negligible, compared with the time interval $t_{1}-t_{0}$.

\section{VI.4. Results and discussion}

The above model was used to preliminarily calculate IGCGR in Nickel-based Alloy 600 in pressurized water reactor (PWR) primary heat transport circuits, containing boric acid and lithium hydroxide solution. Parameter values used in the present calculations are summarized in Table VI.1 and Table VI.2, together with the sources from which they were taken.

The relevant electrochemical conditions are also tabulated on each graph, which was chosen to be identical to the conditions at which the selected experiments were carried out. Of course, in an actual environment, wide ranges exist in these parameters, because of different crack initiation times, prior CGR, residual and operating stresses, microstructure, and temperature/time heat input combinations. Furthermore, the present model does not consider neutron-catalyzed, inservice sensitization, which is possibly responsible for irradiation-assisted stress corrosion cracking (IASCC). Thus, the conditions chosen for the present analysis should be regarded as "standard conditions" that permit one to discern the effects of the environment alone on CGR, unencumbered by changes in the other parameters. Generally, the activation energy for diffusion along a grain boundary is lower than that for lattice diffusion. 
Table VI.1. Parameter values for the slip-dissolution model.

\begin{tabular}{lll}
\hline Parameter & Value & Comment/Source \\
\hline$\varepsilon_{f}$ & 0.001 & Reference [57] \\
$E$ & $197500 \mathrm{MPa}$ & Reference [58] \\
$\rho_{m}$ & $8.49 \mathrm{~g} / \mathrm{cm}^{3}$ & Reference [58] \\
$\sigma_{y}$ & $257 \mathrm{MPa}$ & Reference [57] \\
$\alpha_{c t}$ & 0.5 & Reference [37] \\
$\beta_{c t}$ & 5.46 & Reference [58] \\
$\lambda$ & 1.06 & Reference [58] \\
$L$ & $0.5 \mathrm{~cm}$ & - \\
$M$ & $56 \mathrm{~g} / \mathrm{mol}^{2}$ & Reference [59] \\
$z$ & 2 & Reference [58] \\
$A_{c t}$ & $0.001 \mathrm{~cm}{ }^{2}$ & Reference [10] \\
$Q_{c t}$ & $50000 \mathrm{~J} / \mathrm{mol}$ & Calibrated using a CGR vs temperature \\
& & experimental data \\
\hline
\end{tabular}

As for self-diffusion, the activation energy of diffusion along a grain boundary is about 0.4 $\sim 0.6$ times that of lattice diffusion [39]. In this study, the ratio is taken to be 0.45 for calculating the activation energy of self-diffusion along a grain boundary, $B_{i b}$, and 0.40 for calculating the activation energy of hydrogen diffusion along a grain boundary, $Q$, and the constants $y_{1}, y_{2}, y_{3}, y_{4}$, and $y_{5}$ mentioned above were set to be $0.45,0.25,5.0,2.0$ and 0.2 , respectively, based on the assumptions at derivation of the original model by Wilkinson and Vitek [34]. Stress inevitably concentrates on the main crack tip. However, it is hard, or it is even impossible to know the local stress at the crack tip exactly. In the present calculation, the local stress at the crack tip is assumed to be a little less than the yield stress of Alloy 600, or about $250 \mathrm{MPa}$. As for the partial molar 
volume of the dissolved atomic hydrogen in the metal $\bar{V}_{H}$, it has been reported that the value at room temperature is around $1.0 \times 10^{-6} \mathrm{~m}^{3} / \mathrm{mol}[33,48,49]$. An extensive review of the literature indicates that no data are available for $\bar{V}_{H}$ in high-temperature aqueous environments, so the value is assumed to be $2.0 \times 10^{-6} \mathrm{~m}^{3} / \mathrm{mol}$.

Table VI.2. Parameter values for the grain boundary crack growth model.

\begin{tabular}{lll}
\hline Parameter & Value & Comment/Source \\
\hline$\alpha$ & $1 / 2$ & $\begin{array}{l}\text { Assumed the effect of plastic creep near the } \\
\text { crack tip is negilible [34] }\end{array}$ \\
$\beta$ & 1 & For rounded, equilibrium shape [34] \\
$c$ & $0.2 \mu \mathrm{m}$ & Reference [51] \\
$K_{s 0}$ & $1.113 \mathrm{~mol} / \mathrm{m}^{3} \cdot \mathrm{Pa}^{1 / 2}$ & Reference [40] \\
$E_{s}$ & $2.563 \times 10^{-20} \mathrm{~J}$ & Reference [40] \\
$\theta$ & $75^{\circ}$ & Reference [42] \\
$\delta$ & $5 \times 10^{-10} \mathrm{~m}$ & Reference [43] \\
$\Omega$ & $1.177 \times 10^{-29} \mathrm{~m}^{3}$ & Reference [43] \\
$G$ & $7.309 \times 10^{10} \mathrm{~N} / \mathrm{m}^{2}$ & Reference [44] \\
$k_{b}$ & $1.38 \times 10^{-23} \mathrm{~J} /{ }^{\circ} \mathrm{K}$ & Boltzmann's constant \\
$\gamma$ & 0.3 & Poisson's ratio \\
$D_{g b}$ & $2.76 \times 10^{-4} \mathrm{~m}^{2} / \mathrm{s}$ & Reference [45,46] \\
$Q_{g b}$ & $250600 \mathrm{~J} / \mathrm{mol}^{-7}$ & Reference [45,46] \\
$D_{0}$ & $6.43 \times 10^{-7} \mathrm{~m}^{2} / \mathrm{s}$ & Reference [47] \\
$Q$ & $53450 \mathrm{~J} / \mathrm{mol}^{2}$ & Reference [47] \\
\hline
\end{tabular}

\section{VI.4.1. Effect of stress intensity factor}

In the original CEFM, increasing stress intensity factor (SIF) KI, increases the frequency of rupture of the passive film at the crack tip and enhances the CGR [8-10]. In the current model, 
the SIF has an effect on the void growth, too, as it increases the solubility of hydrogen in the metal and also enhances the growth of grain boundary voids, thereby determining the hydrostatic stress field ahead of the crack tip. Figure VI.3 shows the dependence of the CGR on the SIF, as predicted using the presented model, along with some laboratory data [50]. Good agreement between the CEFM-predicted results and experimentally measured data has been obtained during the whole range of stress intensity factors. In any event, for the conditions employed in calculating the CGR shown in Figure VI.3, the value of KISCC is estimated to be about $7 \mathrm{MPa} \cdot \sqrt{\mathrm{m}}$, although the exact value does appear to depend upon the conditions assumed, as expected. There are several studies supporting HIC as a key factor in the mechanism of crack advance in the SCC of Alloy 600 [51,5254]. As shown in our work on Type 304SS in high-temperature water [8], fracture-mechanics controls the microfracture frequency, but hydrogen embrittlement determines the microfracture size. As noted above, if the SDR model was correct, the microfracture dimension should be a few Burger's vectors or a few nanometers in magnitude, but experimentally it is founded to be $2-3 \mu \mathrm{m}$. Thus, if the SDR alone was viable, the event frequency would have to be in the kilohertz range rather than 0-2 Hz observed experimentally by analysis of the noise in the coupling current.

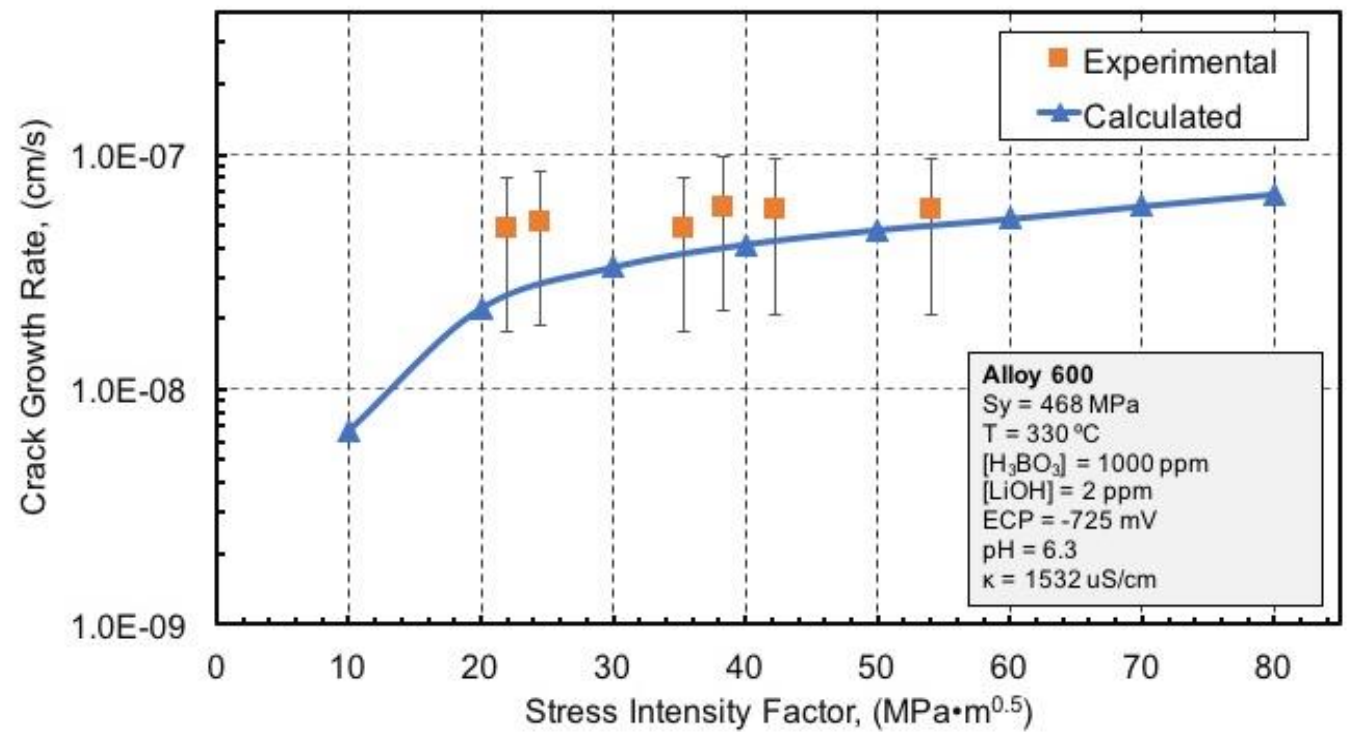

Figure VI.3. Variation of the CGR vs. the stress intensity factor for Alloy 600 in PWR primary heat transport circuits. 


\section{VI.4.2. Effect of temperature}

In terms of the anodic dissolution, the fracture of the passive film and repassivation processes in the crack tip are recognized to be thermally activated and hence, to be temperaturedependent. Temperature is a principal factor influencing the CGR in Alloy 600 in dilute aqueous solutions, and a value of $50 \mathrm{~kJ} / \mathrm{mol}$ has been obtained as the activation energy for the crack-tip strain rate by calibration of the model for selected CGR vs. experimental temperature data. Thus, the temperature dependence of the film rupture and repassivation process are included in the CEFM by using a temperature-dependent crack tip strain rate, since it is the crack tip strain rate that controls the film rupture frequency, which in turn controls (along with the kinetics of the reactions that occur on the external surface) the average crack tip current and hence the CGR [8]. The effect of temperature on the crack tip strain rate is expressed by an Arrhenius-Type expression around a reference temperature of $330^{\circ} \mathrm{C}$, i.e.

$$
\dot{\varepsilon}_{c t}(T)=\dot{\varepsilon}_{c t}\left(330{ }^{\circ} C\right) e^{\frac{Q_{c t}}{R}\left(\frac{1}{T}-\frac{1}{330+273.15}\right)},
$$

where $\dot{\varepsilon}_{c t}(T)$ and $\dot{\varepsilon}_{c t}\left(330{ }^{\circ} \mathrm{C}\right)$ are the crack tip strain rate at temperature $T(\mathrm{~K})$ and $330{ }^{\circ} \mathrm{C}$, respectively, $Q_{c t}$ is the thermal activation energy for the crack tip strain rate in $\mathrm{J} / \mathrm{mol}$, and $R$ the universal gas constant in $\mathrm{J} / \mathrm{mol} / \mathrm{K}$. The grain boundary diffusion in the void pressurization model is postulated to be thermally activated, which is described by Eqs. VI.32-VI.35, as well as the hydrogen evolution at the crack tip and diffusion within the metal, are temperature-dependent, too. It is important to recognize, however, that the temperature-dependence of the crack growth rate is also determined by the temperature-dependencies of the solution properties (e.g., conductivity) and of the various electrochemical processes, such as the kinetics of the charge transfer reactions that occur on the external surfaces, the kinetics of metal dissolution at the crack tip and so forth. Figure 4 shows the effect of temperature on CGR along with other test conditions. As can be seen from this figure, good agreement is obtained between the calculated and the experimental values [55], which demonstrate that the thermal activation energy for crack tip strain rate was set correctly, and the GBVP model accurately accounts for the effect of temperature on CGR. The CGR is an increasing function of the temperature in the range of $200{ }^{\circ} \mathrm{C}$ and $350{ }^{\circ} \mathrm{C}$ and varies strongly (by 3 order of magnitude), as expected. 


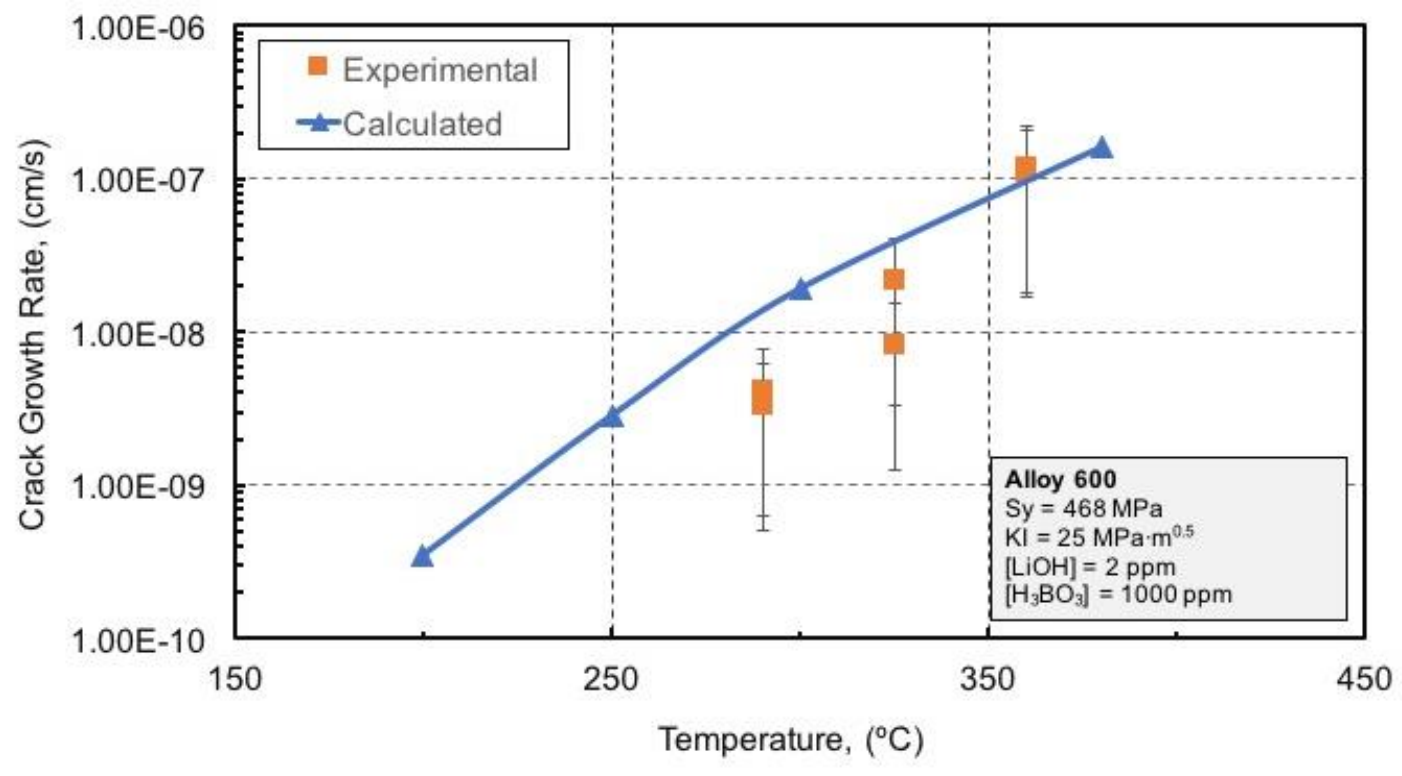

Figure VI.4. Variation of the CGR vs. the temperature for Alloy 600 in PWR primary heat transport circuits.

\section{VI.4.3. Effect of ECP}

ECP is calculated within CEFM from the concentrations of $\mathrm{H}_{2} \mathrm{O}_{2}, \mathrm{O}_{2}, \mathrm{H}_{2}, \mathrm{LiOH}$, and $\mathrm{H}_{3} \mathrm{BO}_{3}$ in the solution, from the kinetics of electrodissolution of the metal substrate, and from the hydrodynamic conditions (flow rate and channel dimensions) using the MPM. However, the CGR is a sensitive function of the concentrations of electroactive species in solution (e.g., $\mathrm{O}_{2}, \mathrm{H}_{2}$, and $\mathrm{H}_{2} \mathrm{O}_{2}$ ), so that a close relationship between CGR and ECP is expected theoretically. Figure VI.4 shows the predicted dependence of CGR on ECP; all the other input parameters are as indicated in the figure. As can be seen from Figure VI.5, the CGR increases with increasing ECP up to a maximum value in the range of $-1 \mathrm{~V}<\mathrm{ECP}<-0.5 \mathrm{~V}$, and good agreement is found with experimental observations [55]. Generally, ECP has a positive impact on the CGR, and for the whole range of ECP we have studied, the CGR increases by almost an order of magnitude. These results agree well with the Artificial Neural Network-based sensitivity analysis result for Alloy 600 [56], in which the ECP comes out as the third most important parameter in determining the CGR after temperature and $\mathrm{K}_{\mathrm{I}}$. Figure VI.5 also shows the dependence of CGR on ECP calculated considering only the SDR mechanism alone. It is important to recognize that at very low 
potentials, the CGR is moderately sensitive for the ECP compared to the SDR model, indicating that in this region, the void growth is the dominant factor over the anodic dissolution in determining the CGR.

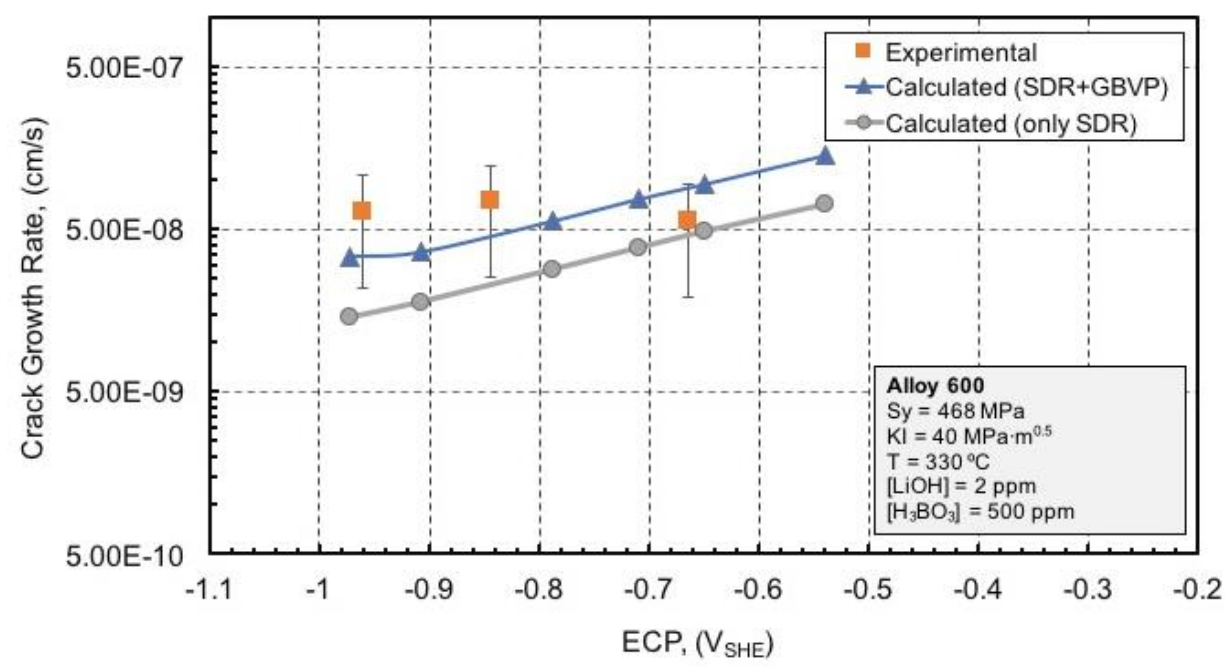

Figure VI.5. Variation of the CGR vs. the ECP for Alloy 600 in PWR primary heat transport circuits for the Slip Dissolution-Repassivation mechanism (SDR) and together with the Grain Boundary Void Pressurization model (GBVP).

It is also important to note that the ECP is imposed on the external surface, but it is the potential at the crack tip that is important with regards to local hydrogen evolution and hydrogen injection into the crack tip alloy matrix. The potential at the crack tip is calculated in the CEFM, and it becomes more negative at the crack tip as the externally-imposed potential (ECP) is made more positive. For the assumed conditions (PWR primary coolant), the potential at the crack tip $\left(E_{\text {tip }}\right)$ is calculated to be $-0.9 \mathrm{~V}_{\text {she }}$ while the ECP on the external surface is $-0.73 \mathrm{~V}_{\text {she. }}$. These values satisfy the condition of $E_{t i p}<\mathrm{ECP}$ for spontaneous crack growth. Furthermore, the $\mathrm{pH}$ at the crack tip is calculated in the CEFM to be 2.6, so that under those conditions, the thickness of the passive film is small; however, the crack tip still undergoes cyclic fracture. Also, the thin film allows results in the injection of hydrogen into the metal matrix that recombines in the voids and increases the pressure, as calculated by Sievert's law (Eq. VI.25). The rate of the hydrogen evolution reaction on the residual passive film at the crack tip is a sensitive function of the film thickness. Quantum mechanics shows that the exchange current density changes as $e^{(-\beta L)}$ where $\beta$ is the 
tunneling constant, and $L$ is the barrier layer thickness. Thus, any decrease in $L$ at the crack tip, which occurs because of the low $\mathrm{pH}(2.6)$ and the low potential (-0.9 Vshe) means that the crack tip is a preferred location for hydrogen evolution and the injection of atomic hydrogen into the matrix, even in the absence of film rupture because of creep. Rupture of the film means that over some fraction of the microfracture cycle, the average film thickness will be even lower, and hence, hydrogen injection will be even higher than in the scenario that film fracture did not occur.

\section{VI.4.4. Effect of crack length}

Due to the strong coupling between the internal and external crack environments, a dependence of CGR on crack length is expected under constant $\mathrm{K}_{\mathrm{I}}$ conditions. This dependence is shown in Figure VI.6, where the predicted CGR is plotted as a function of the crack length as the crack length is increased from $0.01 \mathrm{~cm}$ to $5.00 \mathrm{~cm}$. The CGR is predicted to moderately decrease, as expected from the CEFM. Calculations were performed for $K_{I}=80 \mathrm{MPa} \sqrt{\mathrm{m}}$ and for other parameter values tabulated in the figure. These parameters were set to be identical in [51], where the measured crack growth rate, along with the corresponding crack length, is given.

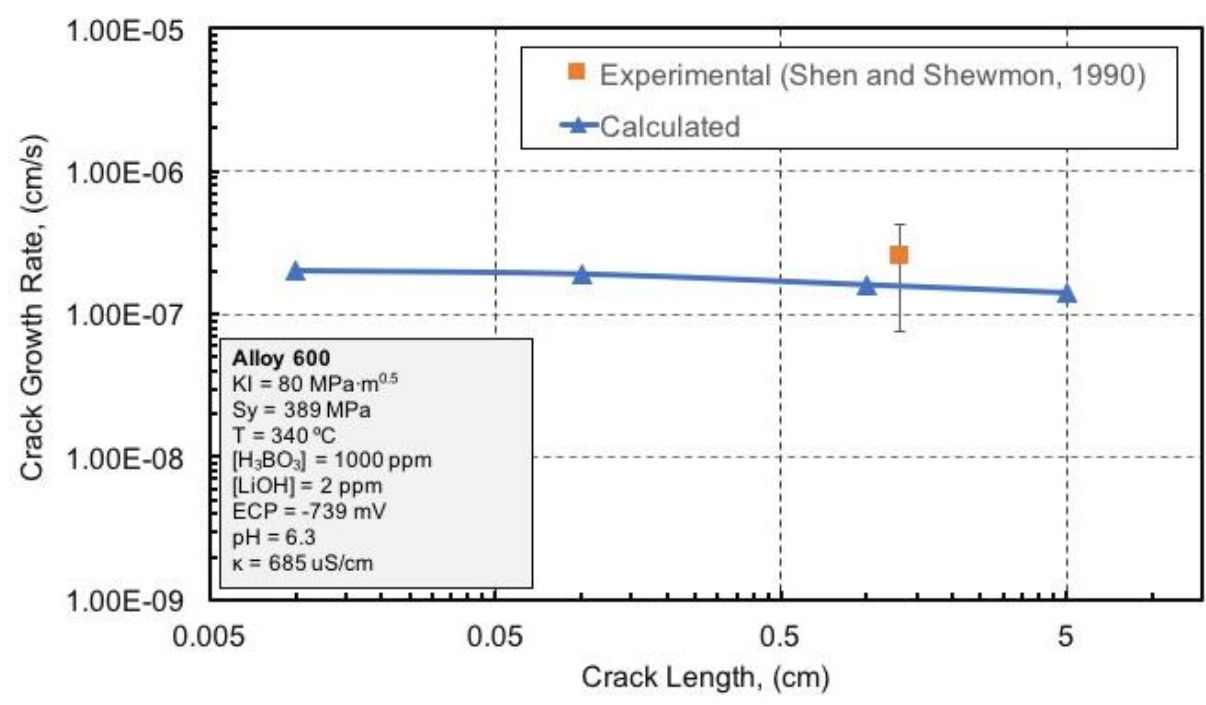

Figure VI.6. Variation of the CGR vs. the crack length for Alloy 600 in PWR primary heat transport circuits. 
As can be seen in Figure VI.6, the results calculated by the present model are in good agreement with the experimentally measured data. However, it is important to note that the dependence of CGR on crack length is based upon the electrochemical effect and is due to the decreasing coupling current with increasing crack length (increasing potential drop down the crack) recognizing that the CGR is a linear function of the coupling current at least for Type 304 $\mathrm{SS}$ in high-temperature water [60]. Due to the high value of stress intensity factor, creep became dominant and partially obscured the electrochemical influence on CGR.

\section{VI.4.5. Effect of boron concentration}

Figure VI.7 shows the effect boron (as boric acid $\left[\mathrm{H}_{3} \mathrm{BO}_{3}\right]$ ) on CGR in Alloy 600, which indicates that there is only a moderate effect of the boron content on the CGR in the region from 250 to $1200 \mathrm{ppm}$. Rebak et al. [50], also found that the CGR was not dependent on boric acid concentration, because boric acid is a weak acid and it becomes weaker as temperature increases. The present model predicts that the CGR is a positive function of the boron content, which corresponds to experimental observations [56].

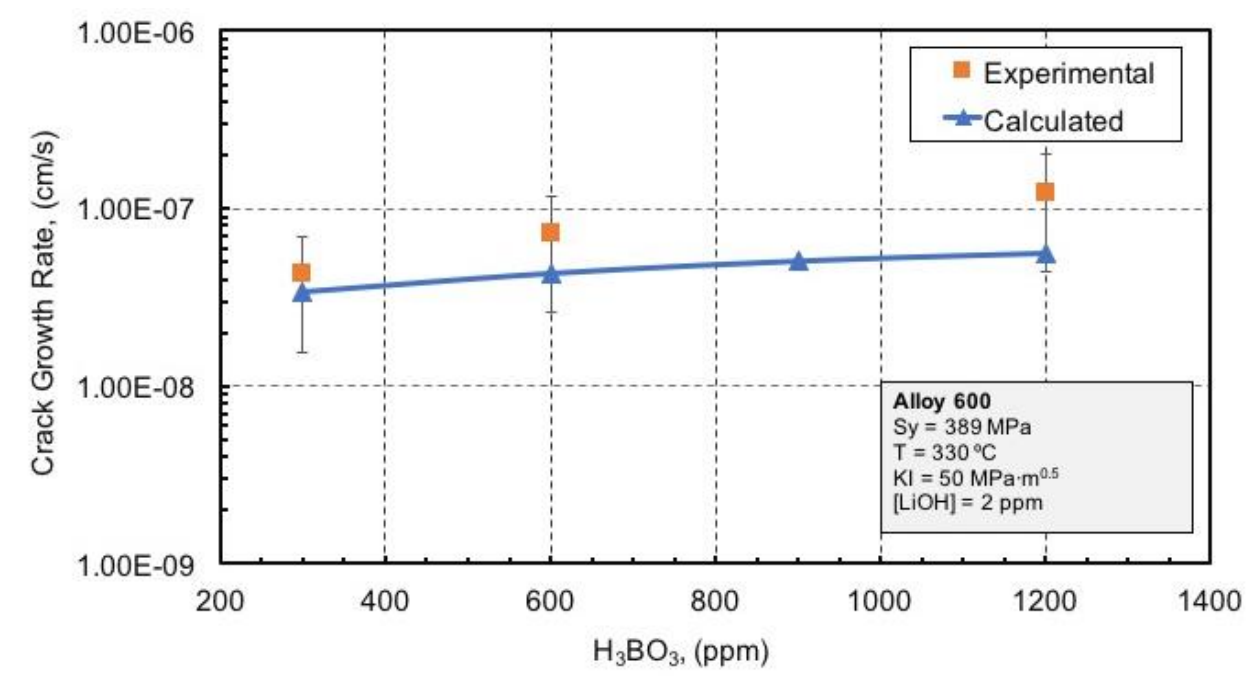

Figure VI.7. Variation of the CGR vs. the boron concentration for Alloy 600 in PWR primary heat transport circuits. 


\section{VI.4.6. Effect of the grain boundary void spacing}

Shen and Shewmon [51] showed for Alloy 600 that in high-temperature deaerated water, cracks are initiated by the nucleation of a high density of voids on the grain boundary under the combined action of the applied stress with hydrogen injected from corrosion. The voids then grow together by grain boundary diffusion to give local failure. They found the void spacing in the area of the crack tip to be $0.2 \mu \mathrm{m}$ by transmission electron microscopy. Figure VI.8 shows the effect of the grain boundary void spacing $(c)$ on CGR, along with the experimental result given in [51]. Excellent agreement between the calculated results and the experiments is found. Figure VI.8 indicates that increasing the void spacing in the range of $0.05 \mu \mathrm{m}-5.0 \mu \mathrm{m}$ the corresponding CGR decreases significantly, and at higher $c$ values the model yields the predictions of the original CEFM, demonstrating that the new model is an extension of the old CEFM, as intended.

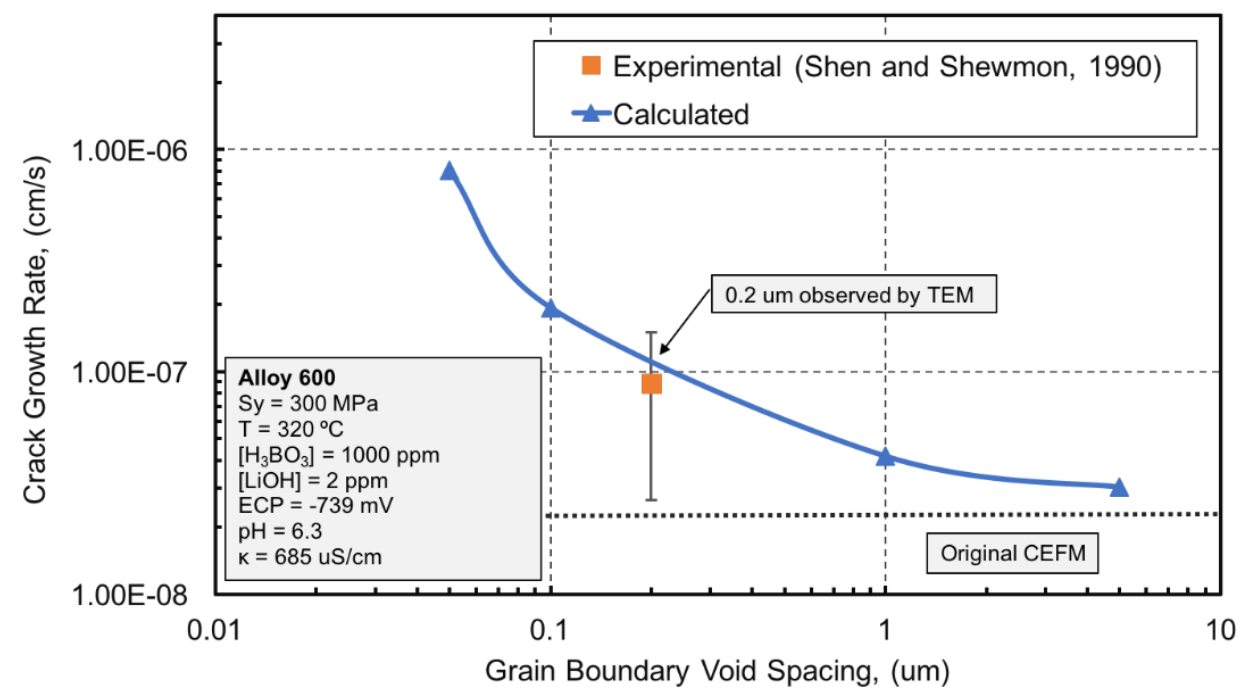

Figure VI.8. Variation of the CGR vs. the grain boundary void spacing for Alloy 600 in PWR primary heat transport circuits. Experimental data point investigated by Transmission Electron Microscope (TEM) as per Shen and Shewmon.

It is important to note that the present model does not include effects from the microstructure of the material and residual stresses [61-63]. Although microstructural effects were not considered in a recent Artificial Neural Network (ANN) analysis of SCC in Alloy 600 in PWR Primary Coolant [56], the ANN successfully reproduced the CGR vs independent variables [K Yield Strength, temperature, solution conductivity, Electrochemical Corrosion Potential, $\mathrm{pH}$ (B 
and $\mathrm{Li}$ concentrations)] when tested against an evaluation data set suggesting that either all specimens had similar microstructures or the microstructural features do not strongly influence the CGR. This issue has yet to be resolved and was considered to be well beyond the scope of the present paper.

\section{VI.5. Summary and conclusions}

In the present work, we have developed a theoretical model for the hydrogen-induced cracking to estimate the inter-granular crack growth rate in Alloy 600 in aqueous environments. In this model, a crack growth is assumed to occur by the combined effect of the anodic dissolution and the nucleation and growth of voids ahead of the crack tip along a grain boundary, and then linkage with the main crack due to stress-induced fracture of the remaining ligaments to yield the micro-fracture events that have been previously reported for IGSCC in Alloy 600 in high temperature water. Under proper conditions, hydrogen evolution at the crack tip injects atomic hydrogen into the crack tip matrix. The hydrogen atoms diffuse ahead of the crack tip and recombine to form $\mathrm{H}_{2}$ in the voids. The hydrogen generates pressure within a void that increases with time. The hydrogen pressure adds to the hydrostatic stress on the void due to mechanical loading, and once the effective stress exceeds the fracture stress of the ligament, the void links with the crack marking a micro-fracture event. The theoretical model for the hydrogen-induced cracking based on grain boundary void pressurization was incorporated into the CEFM. The CGR was calculated for Alloy 600 in PWR heat transport circuits for different electrochemical and mechanical conditions. The calculated results indicate that the hydrogen evolution at the crack tip under PWR conditions has a significant effect on the CGR.

\section{VI.6. Defining the mechanisms of hydriding failure of fuel cladding in pressurized water reactors.}

\section{VI.6.1. Background}

Hydriding is recognized as a principal failure mechanism of zirconium alloy fuel cladding in Pressurized Water Reactors [64]. While the formation of hydrides has been well described phenomenologically [64-77], the mechanistic details are still lacking. The need for precise 
mechanistic detail stems from the possibility of designing new zirconium alloys that are significantly more resistant to hydriding failure than are the current alloys (Zircaloy 4, ZIRLO, MS, etc.). If such a cladding can be designed and its enhanced resistance demonstrated, its use as fuel cladding in PWRs would allow the fuel to be taken to considerably higher "burn-up" [60-70 gigawatt-days per metric ton of uranium (GWd/MTU)] than is now practiced [<50 GWd/MTU]. This, in turn, would have a significant impact on the economics of power production, would conserve resources of reactor fuel by the increased utilization of ${ }^{235} \mathrm{U}_{92}$, and would enhance safety and operation (the latter arising from fewer failed fuel incidences).

The compositions of some commercial fuel cladding used in nuclear power reactors are shown in Table VI.3 [64]. Of particular interest in this work are Zircaloy-4, ZIRLO, and MS, as all three are used in PWRs World-wide. The most common of these alloys in Zircaloy-4, which has the composition Zr-Sn (1.2-1.7 wt.\%)-Fe (0.18-0.24 wt.\%)-Cr (0.07-0.13-1.7 wt.\%). The compositions of the other alloys are given in Table VI.3.

Table VI.3. Chemical composition of different zirconium alloys.

\begin{tabular}{|c|c|c|c|c|c|c|}
\hline \multirow[t]{2}{*}{ Name of alloy } & \multicolumn{5}{|c|}{ Alloying elements (weight \%) } & \multirow[t]{2}{*}{ Utilization } \\
\hline & Tin & Iron & Chromium & Nickel & Niobium & \\
\hline Zircaloy-1 & 2.50 & - & - & - & - & Not suitable for reactor operation \\
\hline Zirealoy-2 & 1.50 & 0.12 & 0.10 & 0.05 & - & Boiling water reactor, CANDU \\
\hline Zircaloy 4 & 1.50 & 0.20 & 0.10 & - & - & Pressurized water reactor \\
\hline ZRLLOTre & 1.02 & 0.10 & - & - & 1.01 & Pressurized water reactor \\
\hline MSru & - & 0.05 & 0.015 & - & 1.00 & Pressurized water reactor \\
\hline
\end{tabular}

More recently, metal-clad zirconium alloys (e.g., Westinghouse's chromium-clad, EnCore, and coated AXIOM advanced nuclear fuels) have been introduced to inhibit hydriding and hence to extend the fuel to higher burn-up. However, we do not consider these advanced cladding materials in the present work, because the emphasis was on devising a technique for monitoring hydriding in situ under typical reactor operating conditions. We considered that the development of such a technique to be particularly important because it would permit hydrogen pick-up in realtime as the reactor transitioned through a fuel cycle. This is so because hydriding is a strong function of the electrochemical corrosion potential (ECP) of the cladding, and the ECP is predicted to trend to more negative values as burn-up proceeds, as shown in Figure VI.9. 


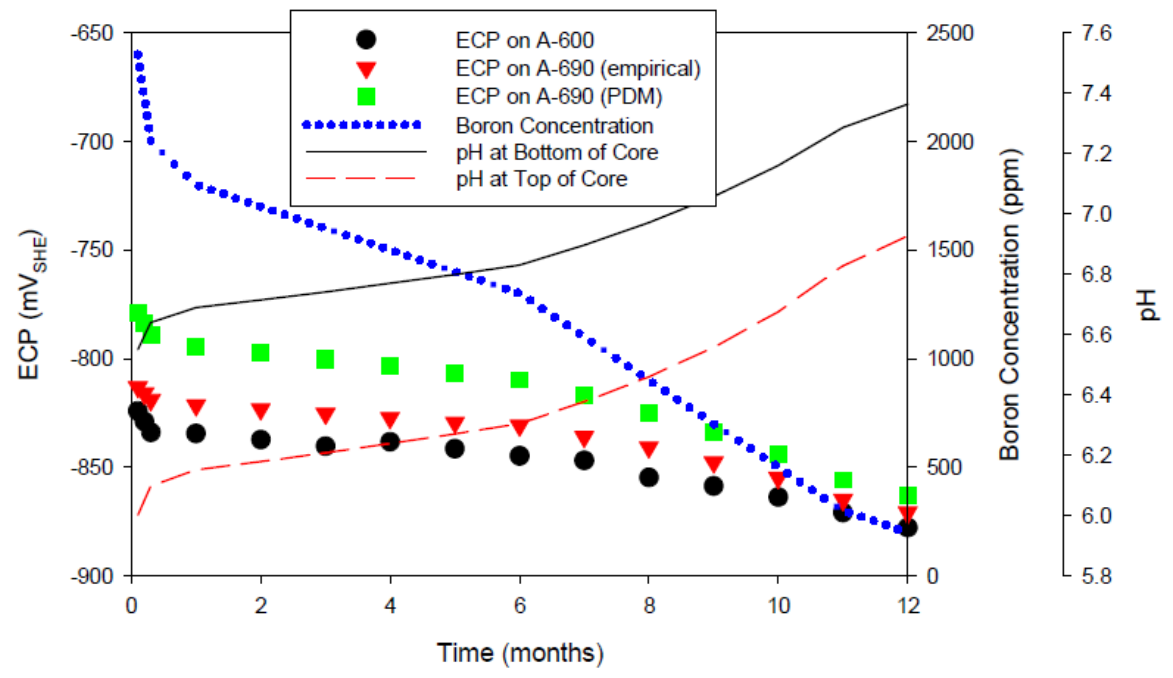

Figure VI.9. Predicted variations of boron concentration, $\mathrm{pH}$ at the top and bottom of the core, and the hot-leg ECP of Alloys 600 and 690 as a function of time through a PWR fuel cycle. Coordinated water chemistry, $\left[\mathrm{H}_{2}\right]=5 \mathrm{ppm}\left[56 \mathrm{~cm}^{3}\left(\mathrm{STP} / \mathrm{kg} \mathrm{H} \mathrm{H}_{2} \mathrm{O}\right]\right.$ and $[\mathrm{Li}]=0-2 \mathrm{ppm}$.

In this figure, the calculated ECP values correspond to those of Alloys 600 and 690 in the hot-leg of the steam generator. However, these values are posited to also correspond to those of various $\mathrm{Zr}$ alloys, because the chemistry of the environment $\left(\left[\mathrm{H}_{2}\right]\right.$ and $\left.\mathrm{pH}\right)$ dominates the ECP and by the kinetics of the passive dissolution of the alloys. In two cases [Alloy 690 (empirical) and Alloy 690 (PDM)] the passive current density is calculated using an empirical correlation between the steady-state passive current density and by using the Point Defect Model, respectively, where the PDM parameters were obtained by optimization of the model onto experimental electrochemical impedance (EIS) data. PDM-derived values are considered to be more reliable. The reader will note that the ECP changes from about $-780 \mathrm{mV}_{\text {she }}$ at the beginning of the fuel cycle to about $-870 \mathrm{mV}_{\text {she }}$ at the end, a change of almost $100 \mathrm{mV}$.

As emphasized by Suman, et al. [64], the failure of fuel cladding is a complex phenomenon, involving chemical and corrosion, mechanical (stress from fuel swelling), thermal, and operational factors, as summarized in Figure VI.10. In this proposal, we focus on corrosion and hydriding, because they are the root cause of cladding failures in PWRs. 


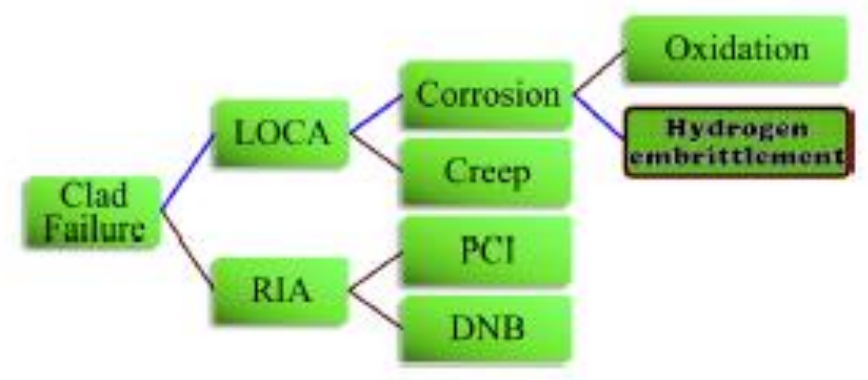

Figure VI.10. Mechanism of cladding failure [1].

The mechanism of the oxidation of zirconium and zirconium alloys has been extensively studied [64-73]. While most researchers have interpreted the oxidation kinetics of zirconium in terms of a simplistic diffusion model, leading to the rate law, $\Delta w^{n}=k t$ with $n=2-5$ [68], comprehensive electrochemical impedance spectroscopic (EIS) studies of the oxidation of pure $\mathrm{Zr}$ in high temperature, oxygenated water shows that the PDM provides a better mechanistic description of the film growth process [78-80].

EIS is an electrochemical technique in which a small amplitude, sinusoidal voltage $(\tilde{V})$ is applied between the metal and a reference electrode in the solution (and, therefore, across the passive film) and the resulting sinusoidal current response $(\tilde{I})$ is used to define the impedance $(Z=$ $\left.\frac{\widetilde{V}}{\tilde{I}}=Z^{\prime}-j Z^{\prime \prime}\right)$, where $Z^{\prime}$ and $Z^{\prime \prime}$ are the real and imaginary components that are plotted in the Nyquist. Theoretically, the impedance, when measured over an infinite bandwidth of frequency, contains all of the information that can be gleaned from a system via electrochemical excitation. Typically, the maximum bandwidth extends from $10^{6} \mathrm{~Hz}$ to $10^{-4} \mathrm{~Hz}$; at higher frequencies, stray capacitance and inductance distort the response, and at lower frequencies, stability imposes a practical limit (note a $10^{-4} \mathrm{~Hz}$ sinusoid takes $10,000 \mathrm{~s}$ or $2.8 \mathrm{hrs}$ to be applied). Because of the small voltage amplitude (typically $10 \mathrm{mV}$ peak-to-peak), the impedance can be interpreted in terms of linearized electrochemical models (electrochemical kinetics is inherently non-linear). However, the real power of EIS lies in the fact that values for parameters in parameter-rich models, such as the Point Defect Model (PDM), which typically contain 15-20 unknowns, may be determined by measuring the impedance at the requisite number of frequencies, with each frequency providing an independent observation. 
The work of Ai, et.al. [78-80] shows that in non-hydrogenated environments (e.g., BWR coolant) the oxide typically comprises a bilayer structure, with a inner barrier layer of oxygendeficient zirconium oxide $\left(\mathrm{ZrO}_{2-\mathrm{y}}\right)$ that grows into the metal via the generation of oxygen vacancies at the metal/barrier layer $(\mathrm{m} / \mathrm{bl})$ interface and an outer layer that forms via dissolution/reprecipitation of the oxide at the barrier layer/solution (bl/s) interface and/or the hydrolysis of $\mathrm{Zr}^{4+}$ interstitials transmitted through the barrier layer [79]. In the case of the corrosion of pure zirconium in hydrogenated PWR primary coolant, the barrier layer is found to comprise hydrogendeficient zirconium hydride $\left(\mathrm{ZrH}_{2-\mathrm{x}}\right)$, while the outer layer comprises porous, stoichiometric $\mathrm{ZrO}_{2}$ that forms via the hydrolysis of $\mathrm{ZrH}_{2-\mathrm{x}}$ at the bl/s (ol) interface (Figure VI.11) [80].

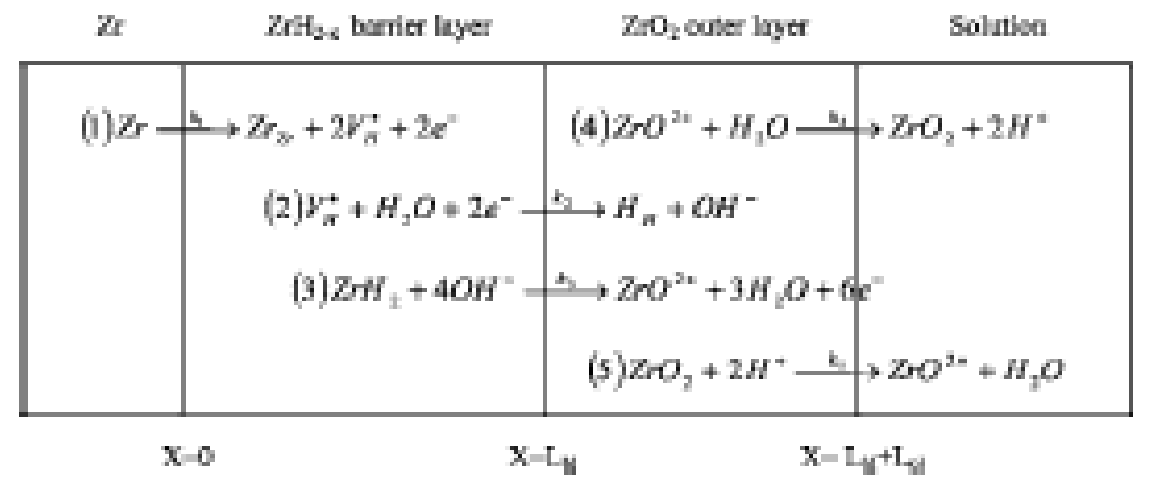

Figure VI.11. Point defect model (PDM) for the oxidation/ hydriding of pure zirconium in PWR primary coolant. $\mathrm{Zr}_{\mathrm{Zr}}=$ zirconium cation in the hydride layer, $\mathrm{H}_{\mathrm{H}}=$ hydride anion of hydride sublattice, $\mathrm{V}_{\mathrm{H}}=$ hydrogen vacancy on hydride sublattice, $\mathrm{ZrH}_{2}=$ zirconium hydride [80] .

In both cases, the corrosion resistance is determined by the porosity of the outer layer. Furthermore, hydride platelets are found to precipitate below the $\mathrm{m} / \mathrm{bl}$ interface with their number density decreasing with distance from the $\mathrm{m} / \mathrm{bl}$ interface, demonstrating that they form from atomic hydrogen that diffuses through the metal from the interface, where it is generated [73].

The $\mathrm{ZrH}_{2-\mathrm{x}}$ phase is brittle, as is the $\mathrm{ZrO}_{2}$ oxide phase, and both appear to contribute to the hydrogen "embrittlement" of zirconium and its alloys in PWR service. However, the hydride is found to migrate in the presence of a temperature gradient, with the movement toward the cold end of the gradient (outside of the tube), as illustrated in Figure VI.12 [64]. Parenthetically, this is an irreversible thermodynamic phenomenon that is readily interpreted in terms of thermal diffusion, although to the author's knowledge, this has never been done. 


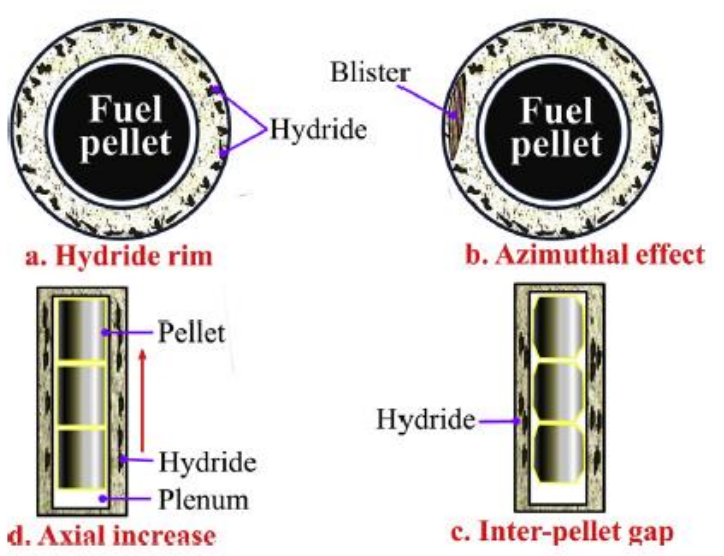

Figure VI.12. Hydride redistribution induced by temperature gradients [64].

Without question, electrochemical impedance spectroscopy (EIS) is the most powerful method for investigating corrosion phenomena. Its power lies in its ability to interrogate systems over a very wide range in frequency (practically from $10^{-2}$ to $10^{5} \mathrm{~Hz}$ ), permitting the detection and definition of relaxation processes having widely differing relaxation times in a single experiment.

\section{VI.6.2. Experimental}

The experimental data used to develop this in situ method for monitoring the hydriding of zirconium were originally obtained using the apparatus shown schematically in Figure VI.13. The apparatus is the forerunner and is essentially identical to that described in Chapter X. The experimental work was carried out in a unique high temperature/high-pressure recirculating flow loop depicted in Figure VI.13.

The flow loop comprises a reservoir [McMaster-Carr, 4.5 L capacity], a high pressure positive displacement pump (MRP051-8KCBM4NMGFSE1122, Milton Roy), a hydraulic accumulator (22155A-5100, Giant Pump Accumulator Pulse Dampener), a regenerative heat exchanger, an autoclave cell with a magnetically activated stirrer, an electric heater (Parr 4562 stirred reactor), a cooling heat exchanger, and a back pressure regulator (KPB1P0A412P20000, Swagelok). The actual experimental temperature in the autoclave cell (indicated in the yellowdashed box in Figure VI.14) and the rotating speed of the stirrer (300 rpm, indicated in the red dashed box in Figure VI.14, are controlled by a PARR 4843 controller. 


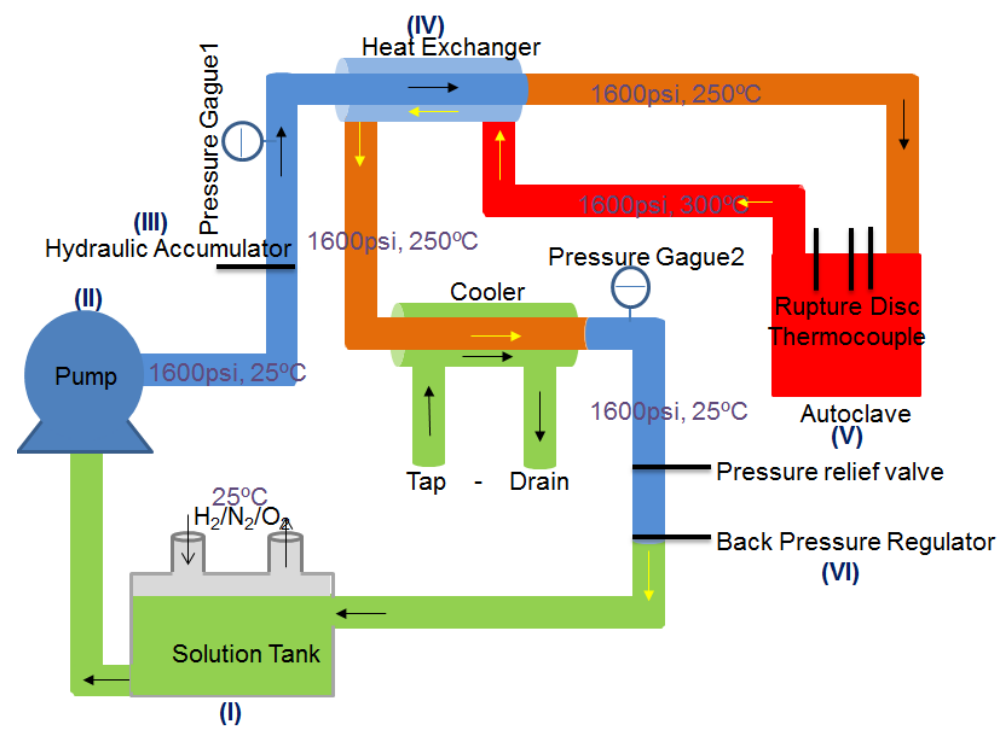

Figure VI.13. Schematic of the recirculating flow loop with autoclave cell for high-temperature experimental measurements.

A cell of similar design [81] achieved linear flow velocities through the annular flow channel of 1.58 meters per second, thereby successfully simulating the flow characteristics in a PWR core. The working, reference and counter electrodes are the specimen, a platinized platinum reference electrode, and the Alloy 600 inner wall of the annulus, and the flow channel, respectively.

Experimental work on zirconium alloy hydriding was carried out in borate buffer solution containing $\mathrm{H}_{3} \mathrm{BO}_{3}(2000,200,0 \mathrm{ppm} \mathrm{B})+\mathrm{LiOH}(2 \mathrm{ppm} \mathrm{Li})$. The solution in the reservoir was initially purged by sparging the solution at ambient temperature with pure $N_{2}$ gas $(99.998 \%$, Praxair) at atmospheric pressure for over $24 \mathrm{~h}$ before the solution was continuously pumped into the autoclave. The dissolved $H_{2}$ concentration [25 $\left.\mathrm{cm}^{3}(\mathrm{STP}) / \mathrm{kg} \mathrm{H} \mathrm{H}_{2} \mathrm{O}\right]$ ) in the solution was established by sparging the solution in the reservoir with a $\mathrm{H}_{2}$ mixture at the appropriate pressure, as calculated from Henry's law. All experiments were carried out at $250{ }^{\circ} \mathrm{C}$, with this upper limit being determined by the use of PTFE (polytetrafluoroethylene). Optimization of the PDM (Figure VI.11) on the experimental EIS data (Figure VI.15) yields the parameter values listed in Table VI.4. 
As shown in the Table VI.4, the EIS data were measured at three voltages $(-0.1,0$, and 0.1 $\mathrm{V}$ vs. Pt, i.e., $-0.85 \mathrm{~V}_{\text {she, }}-0.75 \mathrm{~V}_{\text {she, }}$ and $-0.65 \mathrm{~V}_{\text {she, }}$, respectively, see Figure VI.15), which effectively span the expected range for ECP of zirconium alloys in the core of an operating PWR. The last column gives the mean of each parameter and the standard deviation as calculated from the data for all three voltages. These mean values were then used to calculate the "simulated" impedance shown in Figure VI.15.

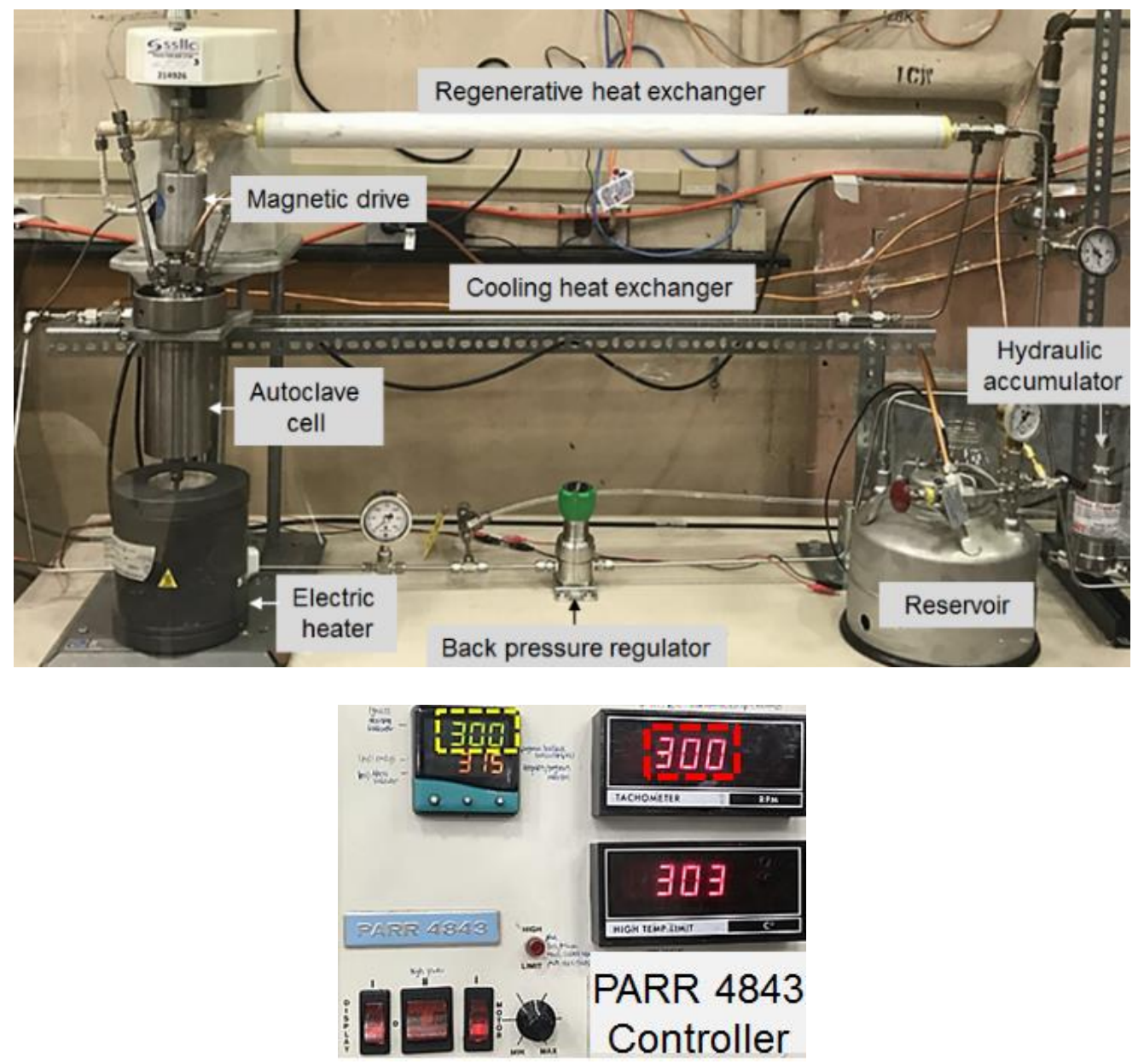

Figure VI.14. The high temperature/high-pressure recirculating flow loop used for hightemperature experimental measurements. 

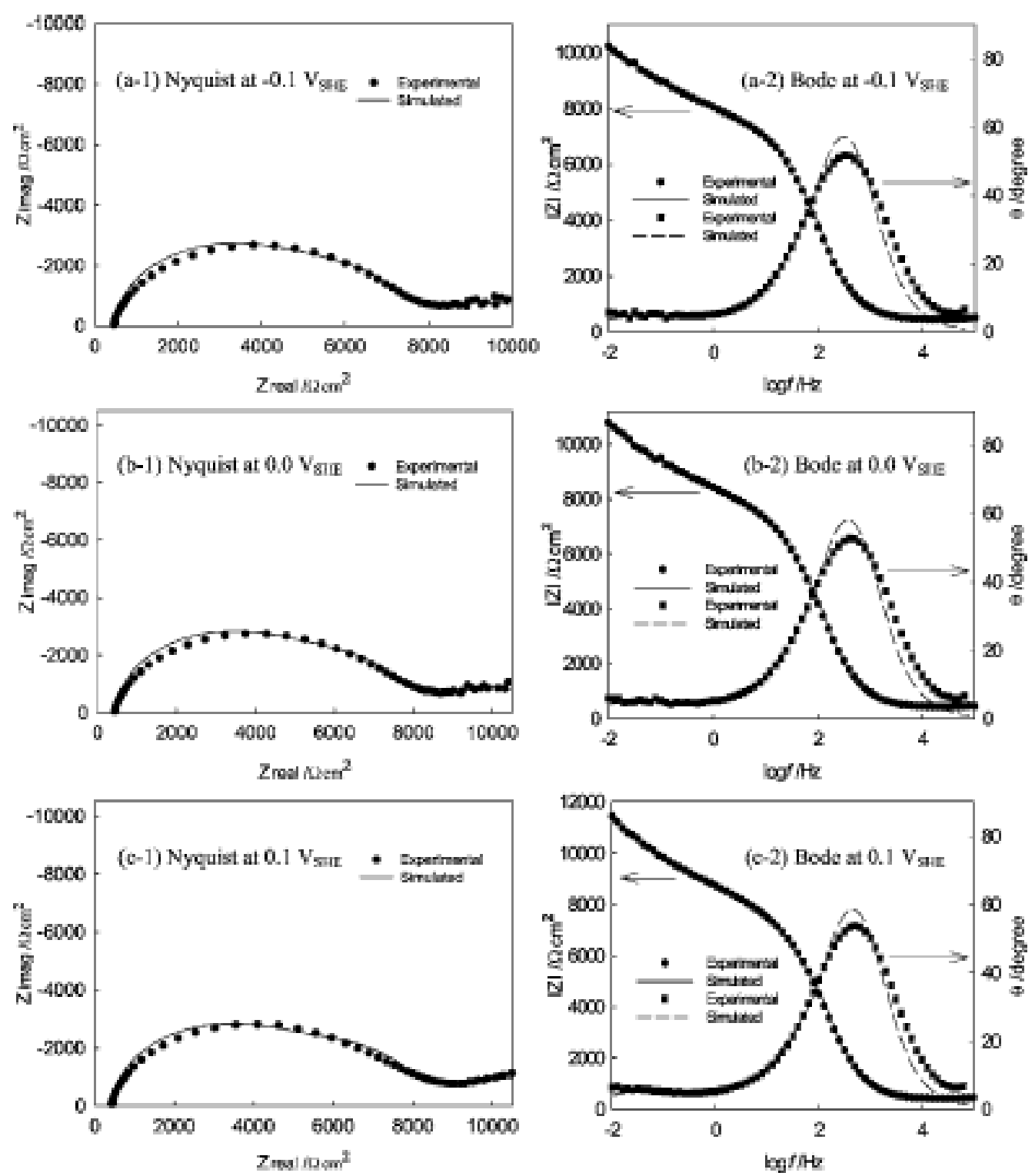

Figure VI.15. Nyquist and Bode plots of the impedance of pure zirconium in simulated PWR primary environment at $250{ }^{\circ} \mathrm{C}$. Potential $=0.1 \mathrm{~V}_{\text {she }}$, polarization time $=24$ hours, Solution: 0.1 $\mathrm{M} \mathrm{B}(\mathrm{OH})_{3}+0.001 \mathrm{M} \mathrm{LiOH}+22 \mathrm{~cm}^{3}(\mathrm{STP}) / \mathrm{kg} \mathrm{H} \mathrm{H}_{2} \mathrm{O}$. Figures show a comparison between the experimental data (points) and the impedance calculated from the optimized PDM, the parameters from which are given in Table VI.4 [80]. 
Table VI.4. PDM optimized parameters for the corrosion of pure zirconium in simulated PWR coolant at $250{ }^{\circ} \mathrm{C}[80]$.

$\begin{array}{lllll}E_{a p p}, \mathrm{~V} \text { vs } \mathrm{NHE}^{\#} & -0.1 & 0 & 0.1 & \text { Average Values }\end{array}$

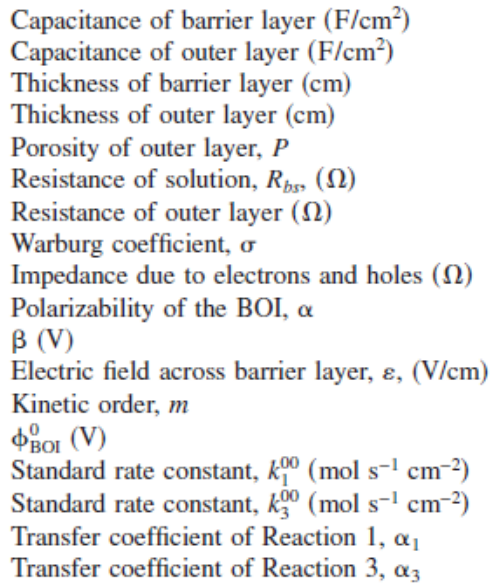

$3.20 \times 10^{-6}$
$3.57 \times 10^{-7}$
$1.89 \times 10^{-7}$
$1.44 \times 10^{-5}$
0.141
510
4430
3941
6282
0.484
-0.005
-648743
0.452
-0.041
$1.67 \times 10^{-9}$
$5.53 \times 10^{-4}$
0.363
0.114

$2.91 \times 10^{-6}$
$2.90 \times 10^{-7}$
$1.58 \times 10^{-7}$
$1.51 \times 10^{-5}$
0.139
493
4680
3543
6926
0.413
-0.005
-560714
0.473
-0.048
$1.47 \times 10^{-9}$
$4.61 \times 10^{-4}$
0.317
0.128

$2.41 \times 10^{-6}$
$2.47 \times 10^{-7}$
$1.39 \times 10^{-7}$
$1.56 \times 10^{-5}$
0.140
474
4640
4600
7500
0.406
-0.005
-595318
0.468
-0.048
$1.71 \times 10^{-9}$
$4.75 \times 10^{-4}$
0.335
0.110

$492 \pm 18$ $(4.03 \pm 0.57) \times 10^{3}$

$0.434 \pm 0.050$ -0.005 , Assumed $-(6.02 \pm 0.46) \times 10^{5}$ $0.464 \pm 0.012$ $-0.046 \pm 0.005$ $(1.62 \pm 0.15) \times 10^{-9}$ $(4.96 \pm 0.57) \times 10^{-4}$ $0.338 \pm 0.025$ $0.117 \pm 0.011$

"NHE is defined as the equilibrium potential for the hydrogen electrode reaction (HER) in the test solution, $0.1 \mathrm{~m} \mathrm{~B}(\mathrm{OH})_{3}+0.001 \mathrm{~m} \mathrm{LiOH}+22 \mathrm{~cm}^{3}(\mathrm{STP}) / \mathrm{kg} \mathrm{H}_{2} \mathrm{O} \mathrm{H}_{2}$ at $250^{\circ} \mathrm{C}$. The equilibrium potential for the HER in the test solution is calculated to be $-0.75 \mathrm{~V}_{\text {she }}$.

As seen, the experimental and calculated data almost perfectly match, demonstrating that the PDM with the parameter values shown in Table VI.4 accurately describes the mechanism of corrosion. Additional power of this Type of analysis lies in sensitivity analysis, in which the impact of each parameter, in turn on the impedance is readily determined. An example of sensitivity analysis is shown in Figure VI.16, in which the porosity and thickness of the outer (porous $\mathrm{ZrO}_{2}$ ) layer were systematically varied.

The reader will note that the variation of these parameters has a huge impact on the impedance and, therefore, on the corrosion resistance. On the other hand, the variation of the parameters for the $\mathrm{ZrH}_{2-\mathrm{x}}$ barrier layer had little impact on the impedance [80]. Accordingly, the corrosion resistance, $\mathrm{Z}(\omega \rightarrow 0)$, where $\omega$ is the angular frequency, and the rate of hydriding is determined entirely by the porous, outer layer and not by the barrier layer. This is a common observation for "valve" metals, such as $\mathrm{Zr}, \mathrm{Ti}, \mathrm{Al}, \mathrm{Nb}, \mathrm{Ta}$, etc. Thus, if one wishes to control the rate of hydriding, then one must control the resistance of the outer layer, either by controlling the 
porosity or thickness, or both or by the use of an impervious coating. We know of no other method that could have provided this vital insight on an in-situ basis.
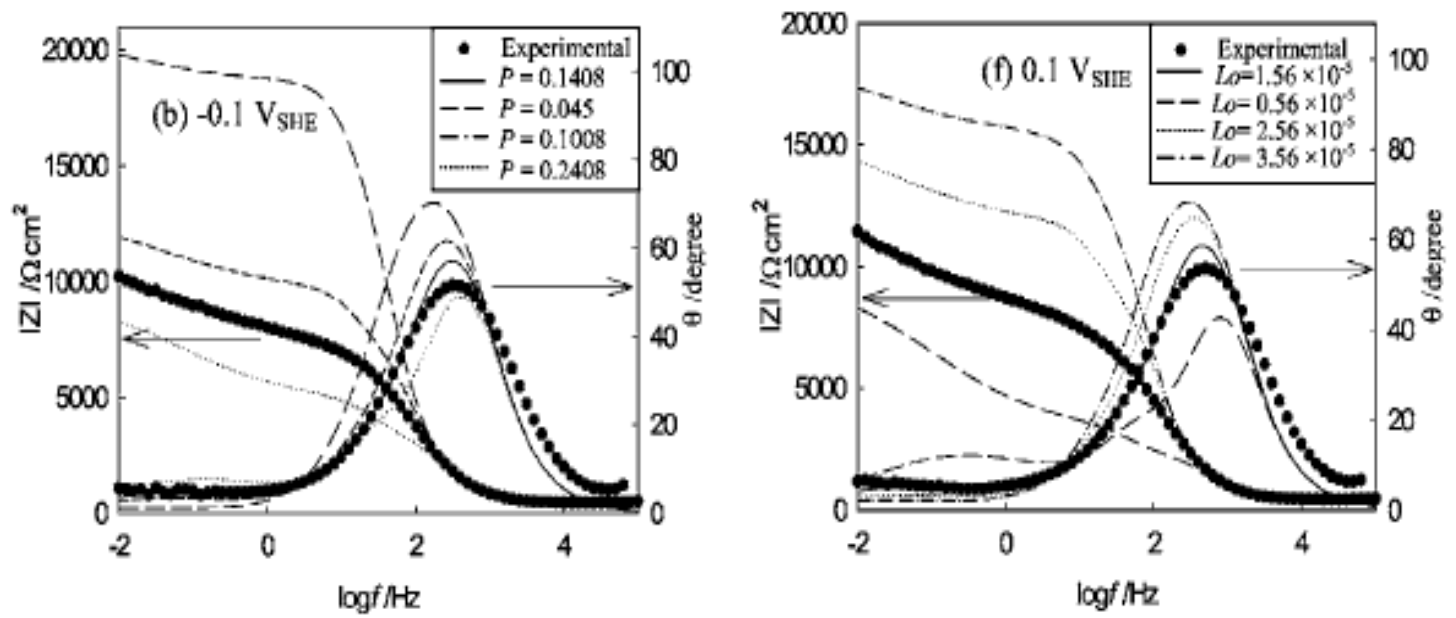

Figure VI.16. Sensitivity of the calculated impedance of pure zirconium in simulated PWR primary coolant as a function of (b) the porosity and (f) thickness of the outer layer [80].

Because the three alloys listed in Table VI.3 differ only in the concentrations of minor alloying elements ( $\mathrm{Sn}, \mathrm{Fe}, \mathrm{Cr}$ ), it is not expected that the rate of hydriding will differ remarkably from one alloy to another. This is because of the rate of the cathodic reaction, $\mathrm{H}_{2} \mathrm{O}+\mathrm{e}^{-} \rightarrow \mathrm{H}+$ $\mathrm{OH}^{-}$, which occurs at the bl/s interface, is controlled by the transport of water through the porous outer layer. However, it appears that the ol, comprising porous, pure $\mathrm{ZrO}_{2}$, is insensitive to the identity and concentration of the alloying elements ( $\mathrm{Sn}, \mathrm{Fe}, \mathrm{Cr}$ ). Accordingly, the properties of the outer layer should also be independent of the concentrations of $\mathrm{Sn}, \mathrm{Fe}$, and $\mathrm{Cr}$, so that little difference is expected in the hydriding rates. This expectation is borne out by the experimental findings of Kim and Kim [76], shown in Figure 17. The rate constants referred to by Kim and Kim [76] are essentially that of Reaction 1 of the PDM (Figure VI.11). The base rate constant for this reaction $\left(k_{1}^{00}\right)$ is listed in Table VI.4 and is found to be essentially independent of potential, as required by electrochemical kinetic theory. However, the rate constant, $k_{1}=$ $k_{1}^{00} e^{a_{1 V}} e^{b_{1} L_{b l}} e^{c_{1 p H}} A_{1}$, where $a_{1}, b_{1}, c_{1}$, and $A_{1}$ are constants that are defined in terms of various fundament parameters [82]. Theory shows that $a_{1 V}+b_{1} L_{b l}=$ constant, so that $k_{1}$, too, is voltageindependent, but theory also shows that $L_{b l}=[(1-\alpha) / \varepsilon] V+G$, where $G$ is constant. Because hydriding is a cathodic reaction, $\mathrm{Zr}+2 \mathrm{H}_{2} \mathrm{O}+2 \mathrm{e}^{-} \rightarrow \mathrm{ZrH}_{2}+2 \mathrm{OH}^{-}$(the addition of Reactions 1 and 
two times Reaction 2, Figure VI.11, such that the hydrogen vacancy, $V_{H}^{*}$, cancels) the barrier layer thickness $\left(L_{b l}\right)$ increases as the potential is made more negative, this being a consequence of the electric field strength $(\varepsilon)$ being negative (see Table VI.4). Parenthetically, in the case of oxide formation $\left(\mathrm{Zr}+2 \mathrm{H}_{2} \mathrm{O} \rightarrow \mathrm{ZrO}_{2}+4 \mathrm{H}^{+}+4 \mathrm{e}^{-}\right), L_{b l}$ increases as the voltage is made more positive because $\varepsilon$ is positive $[75,82]$. In light of the above, we conclude that the barrier layer thickness is a more appropriate measure of hydriding susceptibility.

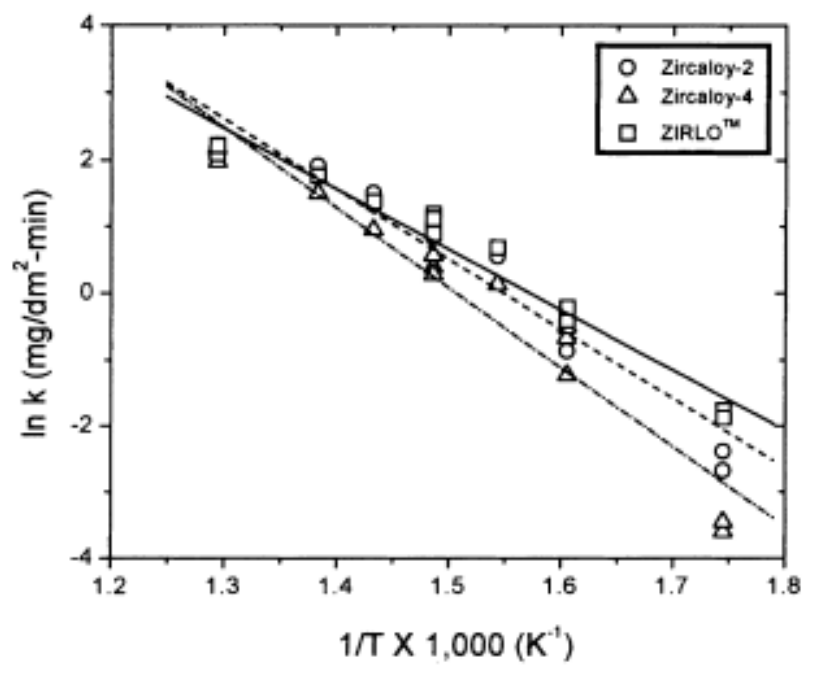

Figure VI.17. Comparison of the hydriding reaction rates of Zircaloy-2, Zircaloy-4, and ZIRLO [76].

To date, the work described above has only been performed on pure zirconium. So that these findings are relevant to PWR operation, the same studies need to be carried out on Zircaloy4, ZIRLO, and MS under conditions that exist in those reactors $\left(330^{\circ} \mathrm{C}, \mathrm{ECP}=-0.75\right.$ to $\left.-0.85 \mathrm{~V}_{\text {she }}\right)$, where the ECP is the electrochemical corrosion potential. Of particular importance is the correlation of the tendencies of these alloys to hydride with the properties of the inner, $\mathrm{ZrH}_{2-\mathrm{x}}$ barrier layer, and the outer $\mathrm{ZrO}_{2}$ layer. Because the bl forms directly from the alloy substrate, any impact that the alloying elements might have on the properties of the passive film are likely to be seen in the rate constants of the bl reactions contained within the PDM (Figure VI.11). Importantly, optimization of the PDM on the experimental EIS data allows us complete access to the rate constants and all other parameters of both the barrier layer and the outer layer, as demonstrated above. We know of no other technique that can provide that information as efficiently. 
The future implementation of the methods described above is the subject of a preapplication to the 2020 NEUP solicitation.

\section{VI.7. References}

[1] N. Totsuka and Z. Szklarska-Smialowska, Corrosion, 44, 124 (1988).

[2] J.L. Gonzalez, R. Ramirez, J.M. Hallen, and R.A. Guzman, Corrosion, 53, 935 (1997).

[3] L. Qiao, X. Mao, and W. Chu, Metall. Mater. Trans., 26A, 1777 (1995).

[4] M.P. Manahan Sr, D.D. Macdonald, and A.J. Peterson Jr, Corros. Sci., 37, 189 (1995).

[5] F.P. Ford and M.J. Silverman, Corrosion, 36, 597 (1980).

[6] F.P. Ford and P.L. Andresen, The Theoretical Prediction of the Effect of the System Variables on the Cracking of Stainless Steels and Its Use in Design, Paper No.83, Corrosion/87, NACE, Houston, TX (1987).

[7] F.P. Ford, D.F. Taylor, P.L. Andresen, and R.G. Ballinger, Corrosion-Assisted Cracking of Stainless and Low-alloy Steels in LWR Environments, NP-5064M, Final Report, Electric Power Research Institute, Palo Alto, CA (1987).

[8] D.D. Macdonald and M. Urquidi-Macdonald, Corros. Sci., 32, 51 (1991).

[9] D.D. Macdonald and M. Urquidi-Macdonald, Advanced Coupled Environment Fracture Model for Predicting Crack Growth Rates in Proceedings of Parkins Symposium on Fundamental Aspects of Stress Corrosion Cracking, edited by S.M. Bruemmer, E.I. Meletis, R.H. Jones, Cincinnati, Ohio, Oct.21-24, 443 (1991).

[10] D.D. Macdonald, P.C. Lu, M. Urquidi-Macdonald, and T.K. Yeh, Corrosion, 52, 768 (1996).

[11] G.R. Engelhardt, D.D. Macdonald, and M. Urquidi-Macdonald, Corros. Sci., 41, 2267 (1999).

[12] M. Vankeerberghen and D.D. Macdonald, Corros. Sci., 44, 1425 (2002).

[13] R.C. Rolfe, K.G. Weil, B.A. Shaw, and H.W. Pickering, J. Electrochem. Soc., 152, B82 (2005).

[14] H.W. Pickering, Corros. Sci., 29, 325 (1989). 
[15] D.A. Vermilyea and C.S. Tedmon, J. Electrochem. Soc., 117, 437 (1970).

[16] M. March and R.F. Hockman, Corrosion, 26, 5 (1970).

[17] H. Gray, Corrosion, 28, 47 (1972).

[18] P. Williams, C.A. Evens Jr., M.L. Grossbeck, and H.K. Birnbaum, Anal. Chem., 48, 964 (1976).

[19] J.K Tien, A.W. Thompson, I.M. Bernstein, and R.J. Richards, Metall. Tran. A, 7A, 821 (1976).

[20] R. Li and M.G.S. Ferreira, Corros. Sci., 38, 317 (1996).

[21] A. Turnbull, Corros. Sci., 39, 789 (1997).

[22] P. Doig and G.T. Jones, Metall. Trans. A, 8A, 1993 (1977).

[23] T. Boellinghaus and H.Hoffmeister, Corrosion, 56, 611 (2000).

[24] J.P. Chateau, D. Delafosse, and T. Magnin, Acta Mater., 50, 1523 (2002).

[25] J.P. Chateau, D. Delafosse, and T. Magnin, Acta Mater., 50, 1507 (2002).

[26] P. Shewmon and P. Anderson, Acta Mater., 46, 4861 (1998).

[27] A.Turnbull, D.H.Ferriss, and H. Anzai, Mater. Sci. Eng. A, 206, 1 (1996).

[28] B.D. McLaughlin, Modell. Simul. Mater. Sci. Eng., 5, 129 (1997).

[29] J. Kameda and M.L. Jokl, Scripta Metall., 16, 325 (1982).

[30] M. Gao and R.P. Wei, Metall. Trans. A, 16A, 2039 (1985).

[31] R.E. Stoltz, N.R. Moody, and M.W. Perra, Metall. Trans. A, 14A, 1528 (1983).

[32] A. Turnbull and J.G.N. Thomas, J. Electrochem. Soc., 129, 1412 (1982).

[33] P. Doig, Mater. Sci. Eng., 48, 181 (1981).

[34] D.S. Wilkinson and V. Vitek, Acta Metall., 30, 1723 (1982).

[35] D.S. Wilkinson, Models for Creep Crack Growth, in Deformation of Ceramics Materials II, edited by Tressler RE, and Bradt RC, New York, Plenum Press, 507 (1984).

[36] A.S. Quist and W.L. Marshall, J. Phys. Chem., 69, 2984 (1965).

[37] M.M. Hall, Corros. Sci., 50, 2902 (2008). 
[38] T. Karakasidis and M. Meyer, Phys. Rev. B, 55, 13853 (1997).

[39] Y.N. Yu, Principles of Metallography, Beijing, Metallurgical Industry Press, 202 (2000).

[40] B. Zajec and V. Nemani, J. Vac. Sci. Technol., A23, 322 (2005).

[41] P.C. Paris and G.C.M. Sih, ASTM STP, 381, 30 (1965).

[42] T. Takasugi and V. Vitek, Metall. Trans. A, 12A, 659 (1981).

[43] T.J. Chuang, K.I. Kagawa, J.R. Rice, and L.B. Sills, Acta Metall., 27, 265 (1979).

[44] C.T. Lynch, CRC Handbook of Materials Science, Cleveland, OH: CRC Press, 69 (1975).

[45] Y. Iijima, K. Kimura, and K. Hirano, Acta Metall., 36, 2811 (1988).

[46] G. Heffich, H. Mehrer, and K. Maier, Scripta Metall., 11, 795 (1977).

[47] X. Sun, J. Xu, and Y. Li, Acta Metall., 37, 2171 (1989).

[48] L.C. Hwang and T.P. Pering, Mater.Chem. \& Phys., 36, 231 (1994).

[49] J. Toribio, J. Strain Anal. Eng. Design, 35, 189 (2000).

[50] R. Rebak, A. McIlree, and Z. Szklarska-Smialowska, Proc. Fifth Int. Symp. Environmental Degradation of Materials in Nuclear Power Systems-Water Reactors, 511 (1991).

[51] C.H. Shen and P.G. Shewmon, Metall. Trans. A, 21, 1261 (1990).

[52] N. Totsuka and Z. Szklarskasmialowska, Corrosion, 43, 734 (1987).

[53] N. Totsuka, E. Lunarska, G. Cragnolino, and Z. Szklarskasmialowska, Corrosion, 43, 505 (1987).

[54] Y. Shen and P.G. Shewmon, Metall. Trans. A, 22, 1857 (1991).

[55] Materials Reliability Program (MRP) Crack Growth Rates for Evaluating Primary Water Stress Corrosion Cracking (PWSCC) of Thick-Wall Alloy 600 Materials (MRP-55) Revision 1, EPRI, Palo Alto, CA, 1006695, (2002).

[56] J. Shi, J. Wang, and D. D. Macdonald, Corros. Sci. 92, 217 (2015).

[57] Y. Yi and G. S. Was, Metall. Mater. Trans A, 32A, 553 (2001). 
[58] J. Kwon, Y.S. Yi, and J.S. Kim, Proceedings of the 12th International Conference on Environmental Degradation of Materials in Nuclear Power System, 661 (2005).

[59] S.A. Attanasio and J.S. Fish, Kapl Atomic Power Laboratory, (1999).

[60] H.S. Kwon, A. Wuensche, and D.D. Macdonald, Corrosion, 56, 482 (2000).

[61] S. Yamazaki, Z. Lu, Y.Ito, Y. Takeda, and T. Shoji, Corros. Sci., 50, 835 (2008).

[62] J. Hou, Q.J. Peng, T. Shoji, J.Q. Wang, EH. Han, and W. Ke, Corros. Sci., 53, 2956 (2011).

[63] S.M. Bruemmer, L.A. Charlot, and C.H. Henager, Corrosion, 44 (1998) 782.

[64] S. Suman, Int. J. Hyd. Ener., 40, 5976-5994 (2015).

[65] F.J. Erbacher and S. Leistikow, Zircaloy Fuel Cladding Behavior in a Loss-of-Coolant Accident: A Review, Zirconium in the Nuclear Industry: Seventh International Symposium, ASTM STP 939, R.B. Adamson and L.F.P. Van Swam, Eds., American Society for Testing and Materials, Philadelphia, 451-488 (1987).

[66] D.G. Franklin and P.M. Lang, Zirconium-Alloy Corrosion: A Review Based on an International Atomic Energy Agency (IAEA) Meeting, Zirconium in the Nuclear Industry: Ninth International Symposium, ASTM STP 1132, C.M. Eucken and A.M. Garde, Eds., American Society for Testing and Materials, Philadelphia, 3-32 (1991).

[67] Journal of ASTM International Selected Technical Papers STP1529, Zirconium in the Nuclear Industry: 16th International Symposium, ASTM International 100 Barr Harbor Drive PO Box C700 West Conshohocken, PA 19428-2959 (2019).

[68] K.R.F. Silva1, D.S. dos Santos1, A.F. Ribeiro1, and L.H. Almeidal, Hydrogen Diffusivity and Hydride Formation in Rich-Zirconium Alloys used in Nuclear Reactors, Defect and Diffusion Forum, ISSN: 1662-9507, Trans Tech Publications Ltd, Switzerland, 297-301, 722-727 (2010).

[69] E. Eidelpesa, L.F. Ibarraa, and R.A. Medina, Nucl. Eng. Des., 350, 116-130 (2019).

[70] S. Rajasekhara, P.G. Kotula, D.G. Enos, B.L. Doyle, and B.G. Clark, J. Nucl. Mat., 489, $222-$ 228 (2017). 
[71] J. Wei, P. Frankel, M. Blat, A. Ambard, R.J. Comstock, L. Hallstadius, S. Lyon, R.A. Cottis, and M. Preuss, Corros. Eng. Sci. Tech., 47, 516-528 (2012).

[72] O.F. Courty, A.T. Motta, C.J. Piotrowski, and J.D. Almer, J. Nucl. Mat., 461, 180-185 (2015).

[73] O.N. Pierron, D.A. Ross, A.T. Motta, and K.S. Chan, J. Nucl. Mat., 322, 21 (2003).

[74] C.E. Coleman and J.F.R. Ambler, Susceptibly of Zirconium Alloys to Delayed Hydrogen Cracking, Zirconium in the Nuclear Industry, ASTM STP 633, A.L. Lowe and G.W. Parry, Eds., American Society for Testing and Materials, 589-607 (1977).

[75] B.A. Cheadle, C.E. Coleman, and J.F.R. Ambler, Prevention of Delayed Hydride Cracking in Zirconium Alloys, Zirconium in the Nuclear Industry: Seventh International Symposium. ASTM STP 939, R.B. Adamson and L.F.P. Van Swam, Eds., American Society for Testing and Materials, Philadelphia,. 224-240 (1987).

[76] Y.S. Kim and S.K. Kim, J. Nucl. Mat., 270, 147-153 (1999).

[77] E. Eidelpesa, L.F. Ibarraa, and R.A. Medina, Nucl. Eng. Des., 351, 116-130 (2019).

[78] J. Ai, Y. Chen, D.D. Macdonald, and M. Urquidi-Macdonald, J. Nucl. Mat., 379, 162-168 (2008).

[79] J. Ai, Y. Chen, D.D. Macdonald, and M. Urquidi-Macdonald, J. Electrochem. Soc., 154, C43C51 (2006).

[80] J. Ai, Y. Chen, D.D. Macdonald, and M. Urquidi-Macdonald, J. Electrochem. Soc., 154, C52C59 (2006).

[81] M. Karaminezhaad-Ranjbar, J. Mankowski, and D.D. Macdonald, Pitting Corrosion of Inconel 600 in High-Temperature Chloride Solutions Under Controlled Hydrodynamic Conditions, Corrosion/84, Paper 168, 15p (1984). (NACE, Houston, TX).

[82] D.D. Macdonald, J. Electrochem. Soc., 139, 3434-3449 (1992). 


\section{The foundations of fracture impedance spectroscopy}

\section{VII.1. Introduction}

A pressing problem in the prediction of fatigue damage in structures is how to translate information developed from laboratory studies under well-defined loading conditions (sinusoidal, saw-tooth, trapezoidal, etc.) to real engineering structures that are subject to complex loading waveforms in order to predict the accumulation of fatigue damage (crack length). Typical examples are aircraft that operate under turbulent flight conditions and are subject to chaotic, cyclic loading with Fourier components ranging over wide ranges of frequency and amplitude and having random phase (i.e., "white noise" loading) [1]. Historically, fatigue studies of materials of aerospace structures have been carried out in the laboratory under pristine loading conditions, such as sinusoidal, square wave, positive saw-tooth, negative saw-tooth, and trapezoidal loading as a function of frequency and stress intensity factor range. Furthermore, fatigue studies are rarely performed at loading frequencies that cover the full spectrum of loads experienced in service, particularly at low frequencies, because of the excessive experimental times. Thus, the very lowest loading frequency is arguably the reciprocal of the flight time, which for a 5-hour flight is $5.6 \cdot 10^{-}$ ${ }^{5} \mathrm{~Hz}$. However, few experimental studies have been performed at frequencies lower than about $0.01 \mathrm{~Hz}$. Accordingly, a pressing need exists to bridge the gap between the lowest operating frequency and that which is feasible in the laboratory.

The method proposed here attempts to provide this bridge making use of Linear Systems Theory and the concept of a transfer function and builds upon work carried out more than twentyfive years ago by the first author and one of his students - Fracture Impedance Spectroscopy (FIS) $[2,3]$. In this paper, the framework of the theory and its application for two different aerospace alloys are presented.

\section{VII.2. Theory}

The hypothesis upon which the fracture impedance concept is based is that an analogy may be drawn between the flow of current in an electrical circuit and the propagation of a crack in a material in response to an imposed driving force (voltage in the case of an electrical circuit and stress intensity factor, or some function thereof, in the case of fracture). Briefly, this method 
defines an "impedance" for crack growth in a metal sample [e.g., a C(T) specimen] when subjected to transient loading (e.g. a step in the load or periodic loading of some specified waveform), by transforming the perturbation and response (time-dependent crack growth rate) from the time domain into the Laplace frequency domain to define the fracture impedance, $Z_{F}(s)$, as

$$
Z_{F}(s)=\mathcal{L}[P(t)] / \mathcal{L}\left[R_{F}(t)\right]
$$

where $\mathcal{L}$ signifies the Laplace transform

$$
\bar{X}(s)=\int_{0}^{\infty} X(t) e^{-s t} d t
$$

in which $\bar{X}(s)$ is the Laplace transform of $X(t), P(t)$ is the imposed perturbation in the stress intensity function, $R_{F}(t)$ is the response (crack growth rate), and $s$ is the Laplace frequency. Provided that the system is linear [which can be assured through an appropriate choice of the perturbation function, $P(t)$ ], $Z_{F}(s)$ is independent of $P$ and $R_{F}$ and is a pure material's property, so that for any arbitrary loading waveform, $\bar{P}^{\prime}(t)$, inverting Equation VII.1 yields:

$$
\bar{R}_{F}(s)=\bar{P}^{\prime}(s) / Z_{F}(s) .
$$

The value of the fracture impedance concept is that the inverse transform of Equation VII.1 will allow the prediction of fatigue damage in structures under arbitrary loading conditions (e.g., under chaotic loading as for an aircraft structure in turbulent air). Thus, noting that a properly defined $Z_{F}(s)$ is a material's property that is independent of the loading waveform, we can write:

$$
R_{F}(t)=\mathcal{L}^{-1}\left[\bar{P}^{\prime}(s) / Z_{F}(s)\right]
$$

A possible form of the arbitrary loading waveform:

$$
\bar{P}^{\prime}(t)=K_{e f f}(t)=K_{I}(t)-K_{t h}
$$

with chaotic loading defined by $K_{I}(t)$ as recorded by accelerometers or strain gauges and a threshold value, $K_{t h}$, which corresponds to a certain material exposed to a specific environment.

Integration in Laplace space is achieved by dividing the right side of Equation VII.4 by the Laplace frequency, $s$, to give the crack length at any time, $t$, as 


$$
a(t)=\mathcal{L}^{-1}\left[\bar{P}^{\prime}(s) / s Z_{F}(s)\right]
$$

Thus, by using Equations VII.1 and VII.6, the crack growth rate in the field may be predicted from the measured load and from the fracture impedance that was determined from laboratory experiments under pristine loading conditions.

It is important to note that FIS is perfectly general concept and that the Laplace transform of the arbitrary (complex) load on the structure (e.g., an aircraft wing) is readily derived numerically from Equation VII.4 using the frequency range indicated by the sampling theorem:

$$
\frac{1}{2 \Delta t}>s>\frac{2}{T}
$$

where $\Delta t$ is the time between neighboring data samples (inverse of the sampling frequency) and $T$ is the total time record. For example, if the sampling frequency is $1000 \mathrm{~Hz}(\Delta t=1 \mathrm{~ms})$ and $\mathrm{T}=$ $1000 \mathrm{~s}$, the frequency bandwidth is from $500 \mathrm{~Hz}$ to $0.002 \mathrm{~Hz}$.

The attainment of linear systems behavior may be achieved in two ways. Firstly, the perturbation driving the crack could be made sufficiently small that non-linear terms (e.g., exponential functions) may be "linearized" by expansion. Secondly, a functional form of the driving force may be chosen, such that the transfer function (fracture impedance) is independent of the driving force. The second method is preferred, because little restriction exists on the size of the perturbation, whereas, in the first case, the perturbation may be so small that it becomes difficult to determine the transient in the crack growth rate. Thus, a major challenge in further developing this technique is to identify the functional form of the driving force that yields behavior in conformity with linear systems theory, and it may be that the preferred functional form will differ from system-to-system. In the example given below, it is evident that linear behavior was not achieved with the driving force assumed, because the fracture impedance is a function of the step in the stress intensity factor. However, the fracture process may be represented by an equivalent electrical circuit, as discussed below, but it is not known whether this is generally true. In the case of the work of Chung and Macdonald [2-5], linear behavior was not evident because the fracture impedance function was found to be a function of the driving force (a step in $K_{I}$ using a tapered DCB specimen such that $K_{I}$ is independent of crack length), so that the inversion represented by Equation VII.4 would not be expected to be valid. 
Fatigue experiments are performed by imposing a periodic load of period $T$ on a precracked specimen and then reporting the increase in the length of the crack per cycle $(d L / d N)$. Multiplication by the frequency (inverse of the period, i.e., $f=1 / T$ ) yields the crack growth rate $(d L / d t)$. In making this transition, it is important to recognize that the increase in crack length per cycle may be equated with the average of the crack extension over the cycle; that is;

$$
\overline{\overline{C G R}}=\frac{\overline{d a}}{d t}=\frac{f}{T} \int_{0}^{T} C G R(t) d t=\frac{1}{T^{2}} \int_{0}^{T} C G R(t) d t
$$

where CGR(t) is the rate of crack growth over the loading cycle, the Laplace transform of which is given in Equation VII.8. Noting that the integral in Equation VII.8 in the steady-state for a given loading frequency is a constant, the Laplace transform of Equation VII.8 is then written as:

$$
L\left(\frac{\overline{d a}}{d t}\right)=\frac{1}{s T^{2}} \int_{0}^{T} C G R(t) d t
$$

However, it is important to note that the unloading of a crack over part of the loading cycle may reduce the crack growth rate to zero due to crack closure. In this case, the period, $T$, in Equation VII.8, corresponds to the time of tensile loading (i.e., the time over which the crack is open) and that this time might be significantly different (shorter) than the loading period. The impact that unloading may have on the predicted accumulated damage must be carefully analyzed.

\section{VII.3. Experimental data}

It is important to emphasize that the application of this method requires high-quality experimental data that have been acquired by loading specimens exposed to the same environment, on a wide range of loading frequency $(0.001-100 \mathrm{~Hz})$, applying different loading waveforms and loading amplitudes (Table VII.1). Few data exist that meet all of the above requirements. Experimental data of two different aerospace alloys taken from the literature were used to test the fracture impedance concept. Fatigue data of Al T2024-T3 alloy [6] and Alloy 718 [7] were used in this study tested by different environmental and mechanical conditions, as summarized in Table VII.2. 
Table VII.1. Laplace transform pairs for typical loading waveforms employed in fatigue experiments.

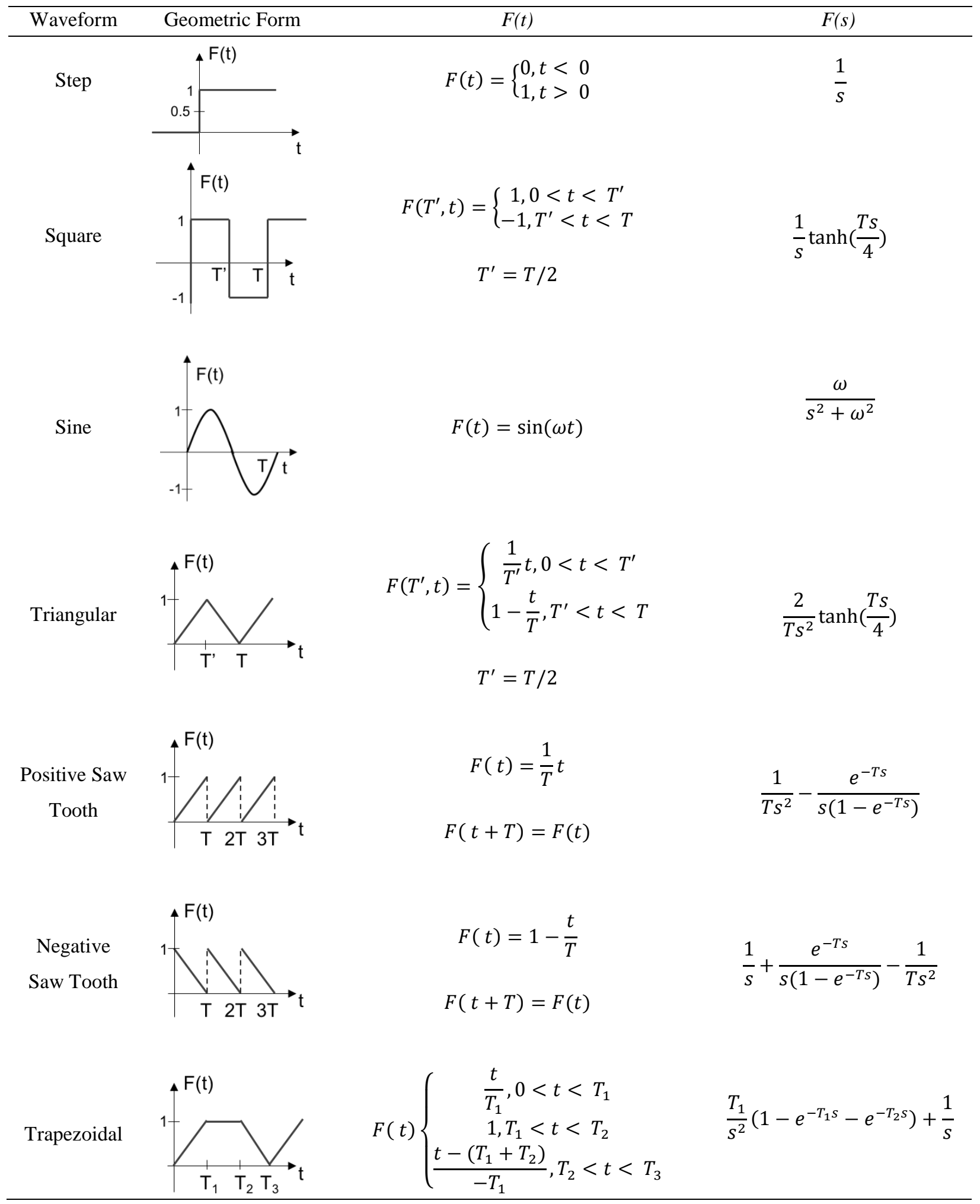


Table VII.2. Summary of the experimental data used in this study.

\begin{tabular}{rcc}
\hline & Al T2024-T3 & Alloy 718 \\
Young modulus & $73.1 \mathrm{GPa}^{*}$ & $172 \mathrm{GPa}^{* *}$ \\
Yield strength & $345 \mathrm{MPa}^{*}$ & $1027 \mathrm{MPa}^{* *}$ \\
Poisson's ratio & $0.33^{*}$ & $0.29 * *$ \\
Environment & moist air, $25^{\circ} \mathrm{C}$ & moist air, $650{ }^{\circ} \mathrm{C}$ \\
Stress intensity factor range & $6.0-42.0 \mathrm{MPa} \mathrm{m}^{0.5}$ & $27.8-40.0 \mathrm{MPa} \mathrm{m}^{0.5}$ \\
Frequency range & $0.22-30 \mathrm{~Hz}$ & $0.001-100 \mathrm{~Hz}$ \\
Loading waveform & sinusoidal & triangular \\
R-ratio range & $-1.0-0.0$ & $0.1-0.8$ \\
\end{tabular}

Figure VII.1 and Figure VII.2 show the crack growth rate data in terms of the frequency along with the stress intensity factor and $R$-ratio dependency. 

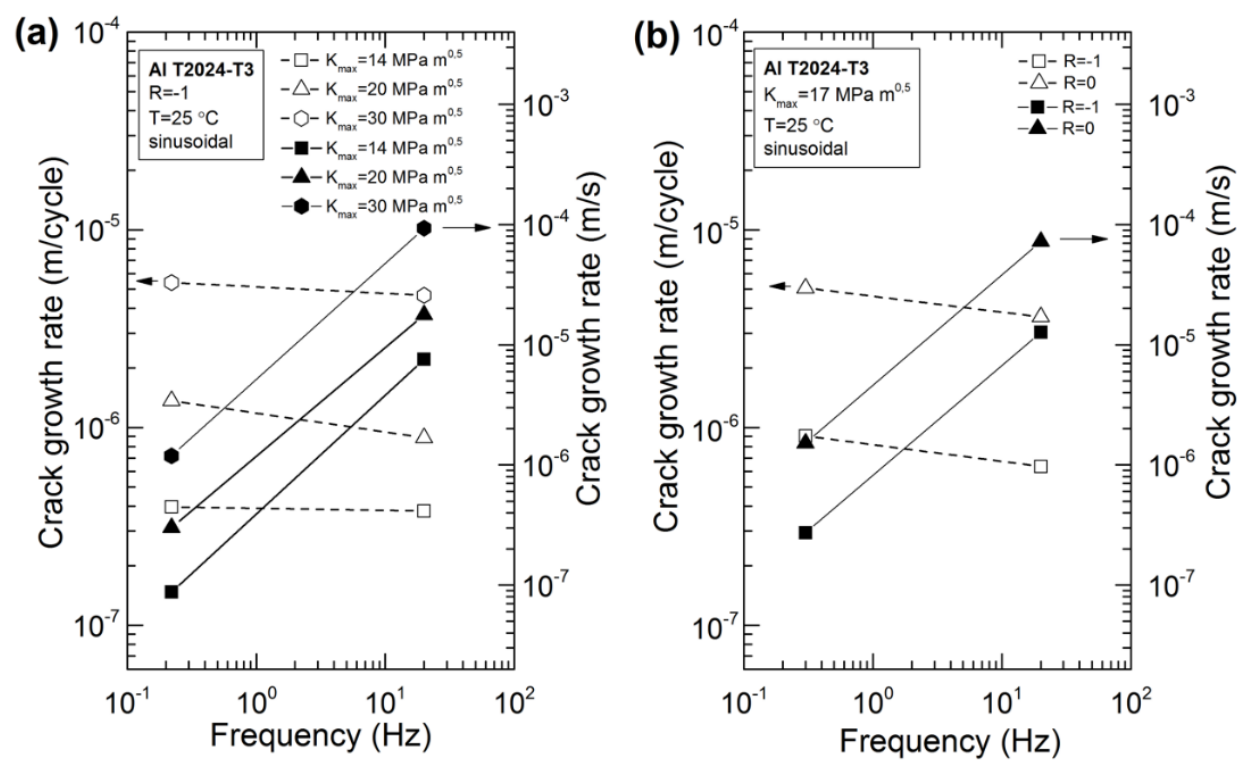

Figure VII.1. Experimental data of Al T2024-T3 taken from [6]. (a) Effect of loading amplitude. (b) Effect of $R$-ratio.
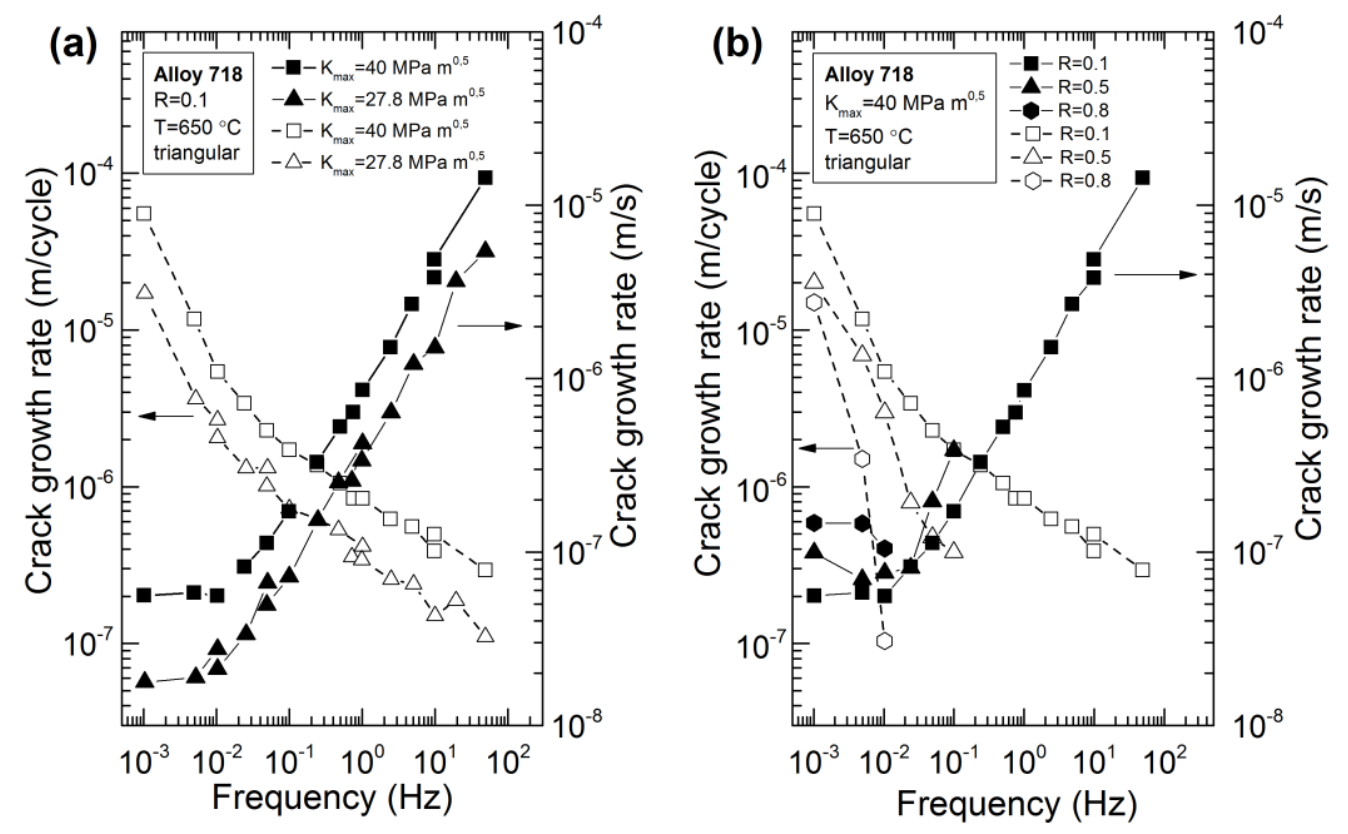

Figure VII.2. Experimental data of Alloy 718 taken from [7]. (a) Effect of loading amplitude. (b) Effect of R-ratio. 


\section{VII.4. Results and Discussion}

\section{Al T2024-T3}

Assuming a sinusoidal mechanical loading waveform, the stress intensity function in terms of time can be written as:

$$
P(t)=K_{\max } \sin (\omega t)+K_{\text {mean }},
$$

where $\omega=2 \pi f$ is the angular frequency with the frequency $f$. Given the value of the stress intensity factor range $\Delta K$, and the load ratio, $R$, the mean and maximum value of $K$ can be calculated as:

$$
\begin{gathered}
K_{\text {mean }}=\frac{K_{\max }+R K_{\max }}{2} \\
K_{\max }=\frac{\Delta K}{1-R}
\end{gathered}
$$

Replacing the stress intensity factor in this equation by the $J$ integral, that is $J_{\max }=\left(1-v^{2}\right) \frac{K_{\max }{ }^{2}}{E}$ and $J_{\text {mean }}=\left(1-v^{2}\right) \frac{K_{\text {mean }}{ }^{2}}{E}$ by considering linear elastic material:

$$
P(t)=J_{\max } \sin (\omega t)+J_{\text {mean }},
$$

The Laplace transform of this function:

$$
\mathcal{L}[P(t)]=\frac{J_{\text {mean }}}{s}+\frac{J_{\max } \omega}{s^{2}+\omega^{2}}
$$

Using Equation VII.1 and combining with Equations VII.9 and VII.13 the fracture impedance for pristine sinusoidal loading waveform is given as:

$$
Z_{F}(s)=\mathcal{L}[P(t)] / \mathcal{L}\left[R_{F}(t)\right]=\left(\frac{J_{\text {mean }}}{s}+\frac{J_{\text {max }} \omega}{s^{2}+\omega^{2}}\right) / \overline{C G R} .
$$

Using experimental data of Figure VII.3, the transfer function was calculated for Al T2024T3, as shown in Figure VII.5 for different frequencies and loading amplitudes. As can be seen, with increasing frequency the fracture impedance decreases; however, it also depends upon the value of the load ratio, $R$. Note that $R=-1$ means fully reversed loading with zero mean stress on the crack tip, whereas $R=0$ means the load has only tensile effect on the crack with a zero- 
minimum stress. It is seen from Figure VII.5 that the material has lower fracture impedance at $R$ $=0$, as the range of the applied load comprises only a tensile component, which is more effective in causing crack advance than is the fully reversed loading including a half period of crack "closing." The effect of the range of $J$-integral in the fracture impedance is shown in Figure VII.6. As can be seen, the fracture impedance is strongly dependent on the applied $J$-integral range. It is important to note that the $J$-integral was directly calculated from the stress intensity factor, which is a linear elastic quantity; thus, it cannot account for the effect of plasticity around the crack tip. Generally, the damage parameters in the field of fatigue and fracture of materials are more reliable if they comprise both the stress and strain, to become energy-Type parameters, rather than based on the stress or strain alone. It is widely accepted that the damage of the material is proportional to the mechanical strain energy deposited in their microstructure. Thus, we can conclude that the restriction of the stress intensity factor, that is the material is assumed to behave as linear elastic, may cause an apparent decrease in the fracture impedance, as it is not proportional to the damage (CGR) because the load increased beyond the value that the material response can be modeled with the tools of linear elastic fracture mechanics. The dimension of the plastically deformed region, $r_{p}$, ahead of the crack tip increase exponentially with the stress intensity factor [8]. In an attempt to eliminate the effect of the loading amplitude from the fracture impedance, the stress intensity factor, $K_{I}$ was corrected using the $r_{p}$, as:

$$
K_{I}^{C}=K_{I} \frac{\sqrt{\pi\left(a+\frac{K_{I}^{2}}{2 \pi \sigma_{Y}^{2}}\right)}}{\sqrt{\pi a}}
$$

where $a$ is the crack length and $\sigma_{Y}$ is the yield strength. 


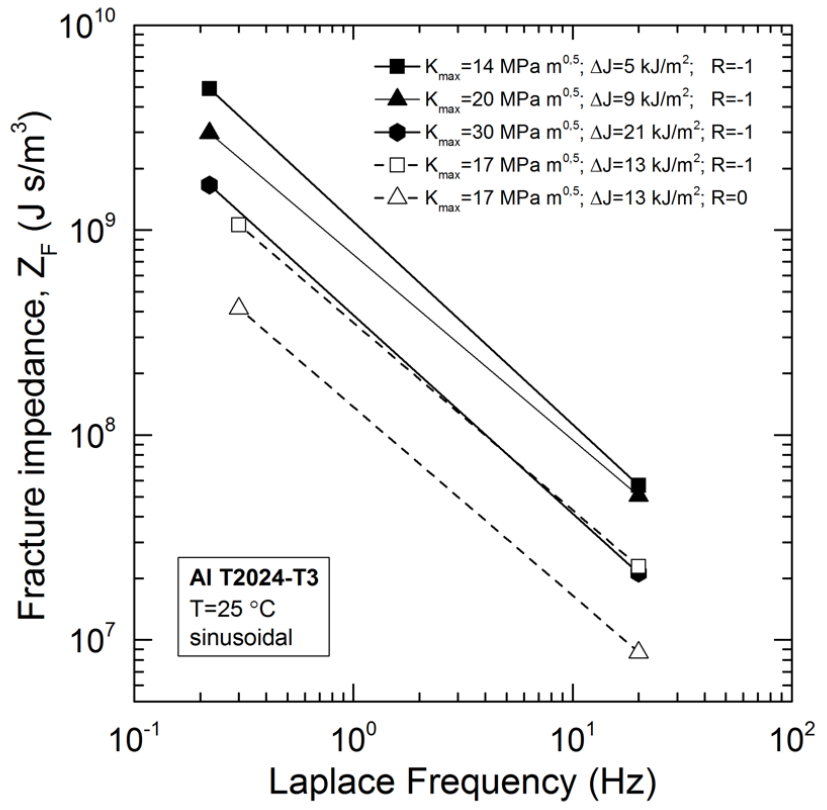

Figure VII.3. Fracture impedance in terms of frequency of $\mathrm{Al} T 2024-\mathrm{T} 3$ in moist air at $\mathrm{T}=25^{\circ} \mathrm{C}$ loading with a sinusoidal waveform.

Using $K_{I}^{C}$, the corresponding $J$-integral range was determined to obtain the fracture impedance, $Z_{F}(s)$, as seen in Figure VII.4. It is seen that the variation of the $Z_{F}(s)$ is lower, considering small scale yielding using this simple model than that of assuming linear elastic fracture mechanics. Note that this model cannot account for the plasticity at higher loading amplitudes, so further research should consider experiments that are conducted with controlled $J$ integral that may result in the elimination of the loading range dependency from fracture impedance function. 


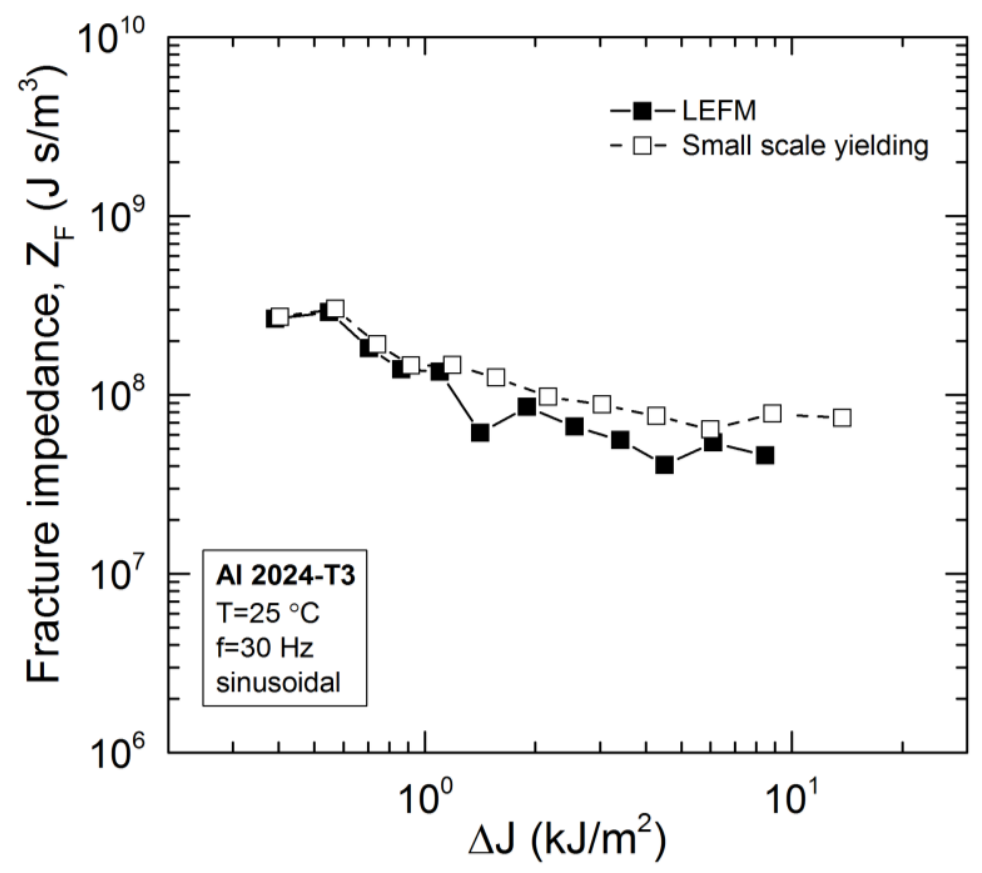

Figure VII.4. Fracture impedance in terms of loading amplitude of Al T2024-T3 in moist air at $\mathrm{T}=25^{\circ} \mathrm{C}$ loading with sinusoidal waveform at $\mathrm{f}=30 \mathrm{~Hz}$.

\section{Alloy 718}

Assuming a triangular mechanical loading waveform, the loading function in terms of time can be written:

$$
F\left(T^{\prime}, t\right)=\left\{\begin{array}{c}
\frac{J_{\text {max }}}{T^{\prime}} t+J_{\text {mean }}, 0<t<T^{\prime} \\
J_{\text {max }}-\frac{t}{T}+J_{\text {mean }}, T^{\prime}<t<T
\end{array}\right.
$$

where $T^{\wedge}=T / 2$. The Laplace transform of this function:

$$
\mathcal{L}[P(t)]=\frac{2 J_{\text {max }}}{T s^{2}} \tanh \left(\frac{T s}{4}\right)+\frac{J_{\text {mean }}}{s}
$$

Using Equation VII.1 and combining with Equations VII.9 and VII.18 the fracture impedance for pristine triangular loading waveform is given as: 


$$
Z_{F}(s)=\mathcal{L}[P(t)] / \mathcal{L}\left[R_{F}(t)\right]=\left[\frac{2 J_{\text {max }}}{T s^{2}} \tanh \left(\frac{T s}{4}\right)+\frac{J_{\text {mean }}}{s}\right] / \overline{C G R} .
$$

Using experimental data of Alloy 718 plotted in Figure VII.2, the transfer function was calculated, as shown in Figure VII.5, for different frequencies and loading amplitudes. As can be seen, with increasing frequency, the fracture impedance decreases; however, it also depends upon the value of the load ratio, $R$. Figure VII.5a shows a similar trend in terms of the loading intensity as observed in Al T2024-T3. Increasing the $R$-ratio decreases the fracture impedance, as can be seen in Figure VII.5b, which is, again, similar to the behavior of Al T2024-T3. This trend reflects the increasing mean stress over time around the crack tip. It is also important to note that the trends reported in Figures VII.3 and VII.5 might be significantly different in a more aggressive environment and/or at high temperature due to the influence of stress corrosion cracking and enhanced creep.
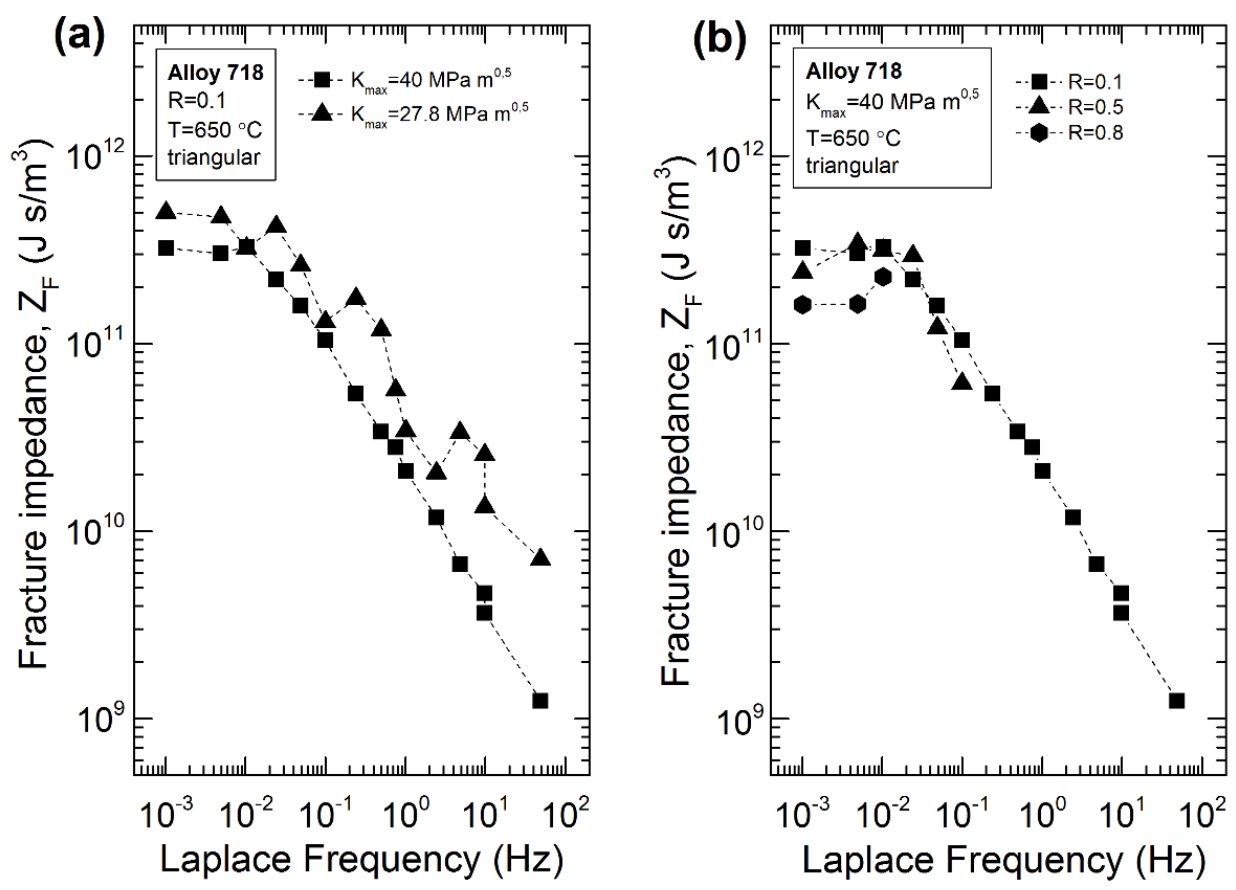

Figure VII.5. Fracture impedance of Alloy 718 in moist air at $\mathrm{T}=650{ }^{\circ} \mathrm{C}$ loading with a triangular waveform: (a) Effect of loading amplitude and (b) Effect of R-ratio. 
It is seen that the impedance $Z_{F}(s)$ decreases with the increasing Laplace frequency $s$ for both of the materials involved in this study. Accordingly, the electrical analog for crack propagation in this material is simply a resistance $\left(R_{A C}\right)$ and capacitance $(C)$ in series. However, the data that are shown in Figures VII.3 and VII.5 also reveal that the resistance, but not the capacitance, is a function of the applied stress intensity; that is

$$
Z_{F}(s)=R_{A C}\left(K_{I}\right)+\frac{1}{s C}
$$

It is clear, therefore, that although the system is nonlinear, and $Z_{F}(s)$ is not expected to obey the Kramers-Kronig transforms [9-11], the process is readily described by an electrical equivalent circuit, which is physically realistic, as indicated below. Nevertheless, the observed non-linearity (dependence of $R_{A C}$ on $K_{I}$ ) needs to be explored and eliminated, in order that the method might be used to estimate the crack growth rate under any other loading waveform, as noted above. Finally, it is evident that $R_{A C}$ (resistance to crack growth, c.f., resistance to current flow in an electrical circuit characterizes the crack growth rate whereas $C$ corresponds to energy storage, presumably due to strain of the matrix ahead of the crack tip. In the work of Chung and Macdonald [2-5] on fracture in AISI 4340 in $\mathrm{NaCl}$ solution under cathodic potential control, the electrical equivalent

circuit for fracture was found to be a resistance and inductance in series resulting in the fracture impedance increasing linearly with the Laplace frequency. The inductance also corresponds to energy storage, and in the electrical sense, energy is stored in a fluctuating magnetic field. Thus, we have two different energy storage modes that will need to be explored in future work. In the case of AISI 4340 [2-5], $R_{A C}$ was found to be independent of $K_{I}$, implying that the crack growth rate is also independent of $K_{I}$. This corresponds to fracture occurring in the Stage II region of CGR vs. $K_{I}$. Then, by representing fracture by an equivalent electrical circuit, we have achieved separation of crack growth and energy storage phenomena in fracture.

\section{Prediction of damage}

In this final section, we wish to demonstrate the process of predicting fatigue damage from the fracture impedance that was derived from some other loading waveform. The crack growth rate was calculated using the fracture impedance for Al T2024-T3 as determined using sinusoidal 
loading, and then the crack growth rate for triangular loading was determined. In Figure VII.6, the fracture impedance function in terms of the Laplace-frequency derived assuming the analog circuit of the growing crack was determined to be a resistance and capacitance in series. The average of the fracture impedance at each frequency value was taken, and curve fitting was performed using those data to obtain $R_{A C}$ and $C$ values in $Z_{F}(s)$. The values of $R_{A C}$ and $C$ obtained by curve-fitting are also depicted in Figure VII.6.

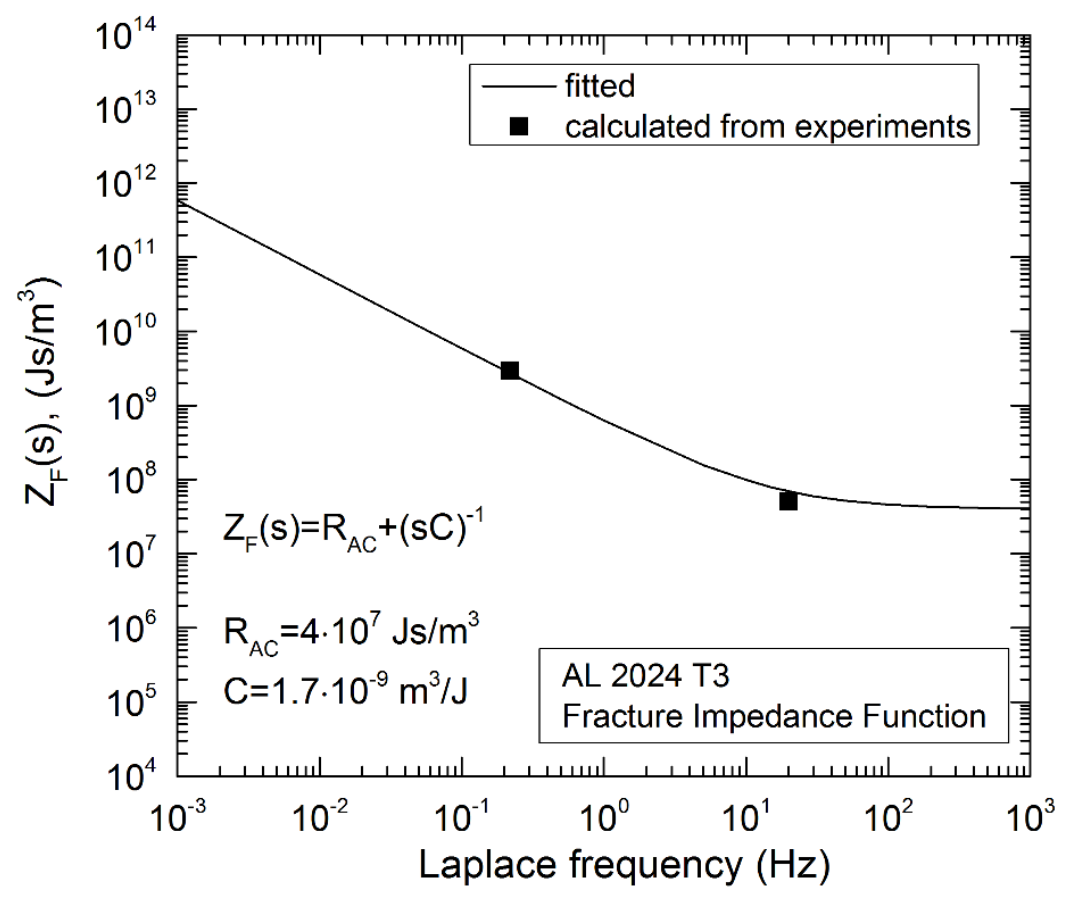

Figure VII.6. Fracture impedance function in terms of the Laplace-frequency of AL 2024 T3 calculated for sinusoidal loading.

Substituting Eq. VII.14 and Eq. VII.20 into Eq. VII.4 and taking into account that for $R=-$ 1 the $J_{\text {mean }}=0$, the crack growth rate for sinusoidal loading can be written as follows:

$$
R_{F}(t)=\mathcal{L}^{-1}\left[\bar{P}^{\prime}(s) / Z_{F}(s)\right]=\mathcal{L}^{-1}\left[\begin{array}{c}
\frac{I \max \omega}{s^{2}+\omega^{2}} \\
R_{A C} \\
R_{A C}+\frac{1}{s C}
\end{array}\right],
$$

that is after taking the inverse Laplace-transform: 


$$
R_{F}(t)=\frac{J_{\max }}{R_{A C}} \frac{1}{1+\frac{1}{\left(\omega R_{A C} C\right)^{2}}}\left[\frac{1}{\omega R_{A C} C} \cos (\omega t)+\sin (\omega t)\right]
$$

For triangular loading, the above equations are written as follows:

$$
R_{F}(t)=\mathcal{L}^{-1}\left[\bar{P}^{\prime}(s) / Z_{F}(s)\right]=\mathcal{L}^{-1}\left[\frac{\frac{2 J \max }{T s^{2}} \tanh \left(\frac{T s}{4}\right)}{R_{A C}+\frac{1}{s C}}\right]
$$

and

$$
R_{F}(t)=\frac{C J_{\max }}{2 T}-\frac{C J_{\max } e^{\frac{-t}{C R_{A C}}}}{2 T}
$$

After integration Eq. VII.22 and Eq. VII.24 over the loading cycle, the average crack growth rate per cycle can be obtained. Figure VII.7 shows the crack growth rate values expressed in $\mathrm{m} / \mathrm{s}$ in terms of the Laplace frequency for different loading amplitudes.

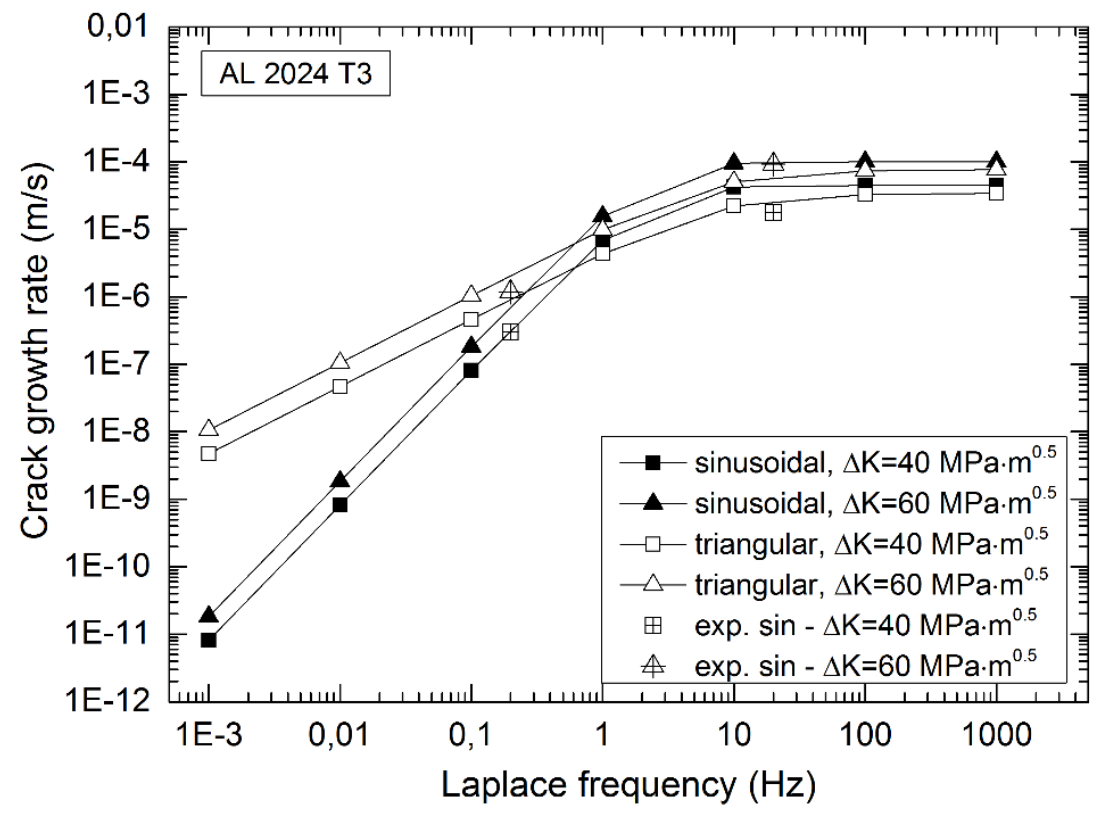

Figure VII.7. Crack Growth Rate in Al 2024-T3 obtained by the Fracture Impedance Method for sinusoidal loading and re-calculated for triangular loading using the same impedance function.

As can be seen for lower frequencies, the crack growth rate is higher with triangular loading, whereas at higher frequencies, the crack growth rate estimated to be higher with sinusoidal loading. We could not locate data against which the results directly could be compared to the 
entire range of frequency. However, experimental observations [12,13] show that for higher frequencies, the sinusoidal loading waveform causes a higher crack growth rate in comparison with the triangular loading waveform. The trend of the results is in good agreement with the experimental observations that support the viability of the Fracture Impedance Spectroscopy concept.

\section{VII.5. Summary}

The method proposed here attempts to bridge the gap between laboratory fatigue experiments and fatigue of arbitrarily loaded engineering structures by making use of Linear Systems Theory and the concept of fracture impedance. This study can be summarized as follows:

- "Fracture impedance" for crack growth in a metal sample subjected to transient loading (e.g., a step in the load or periodic loading of some specified waveform) has been defined by transforming the perturbation and response (time-dependent crack growth rate) from the time domain into the Laplace frequency domain.

- It is seen that fracture impedance of crack growth of AL T2024-T3 and Alloy 718 fatigued in laboratory air calculated from the range of $J$-integral decreases with increasing the Laplace frequency, under the specified range.

- The fracture impedance depends upon the applied loading amplitude. One possible explanation is that the dependence is due to the restriction of the stress intensity factor that is the material assumed to behave linearly elastically. That may cause an apparent decrease in the fracture impedance, as the stress intensity factor is not proportional to the damage (CGR) after the load increased beyond the value that the material response can be modeled with the tools of linear elastic fracture mechanics.

- The elimination of the effect of the loading amplitude on the fracture impedance may be attempted by using data generated from $J$-integral controlled experiments.

- The process of predicting fatigue crack growth rate from the fracture impedance that was derived from some other loading waveform was successfully demonstrated, and the trends of the results are in agreement with experimental observations. 
Based upon our conclusions above, testing the FIS theory more accurately will require high-quality experimental data generated on the wide ranges of frequency and $J$-integral, with appropriate resolution along with both parameters (e.g., $f=0.001-100 \mathrm{~Hz} ; \Delta J_{I}=1-25 \mathrm{~kJ} / \mathrm{m}^{2}$ ). Future work will explore the efficacy of the fracture impedance concept as a means of transferring laboratory fatigue data to the field with the specific purpose of predicting the accumulation of damage under any arbitrary loading waveform, such as those experienced by airframes under normal flight conditions or ships hulls also under normal operating conditions. Future experimental work will involve measurement of crack growth rate transients for alloy/environment couples of interests in industrial applications under pristine loading conditions so that the fracture impedance can be determined. In future studies, we will also measure accumulated fatigue damage (crack length) under "pure" loading waveforms (sinusoidal, saw-tooth, trapezoidal), and the observed damage will be compared with that calculated from the fracture impedance. Finally, fatigue damage should be measured on selected systems under arbitrary, complex waveforms to demonstrate the entire prediction process, including the numerical Laplace and inverse Laplace transformations. The successful development of this technology could have an enormous impact on the prioritization of inspection and in maintaining safety-related engineering structures.

\section{VII.6. References}

[1] D.R. Donaldson and W.E. Anderson, Crack propagation behavior of some airframe materials, in Proc. Crack Propag. Symp., 375-441 (1961).

[2] H. Chung and D.D. Macdonald, Study of Transient Crack Growth in AISI 4340 Steel in NaCl Solution, Paper No. 21, Corrosion/83, NACE, Houston, TX (1983).

[3] H. Chung and D.D. Macdonald, Corrosion, 41, 151-159 (1985).

[4] H. Chung, A Study of Transient Crack Growth in AISI 4340 Steel in NaCl Solution, Ph.D. dissertation, Ohio State University, Columbus, OH, (1983).

[5] D.D. Macdonald and M.C.H. McKubre, Corrosion of Materials, Chapter in Impedance Spectroscopy. Edited by J. R. Macdonald. Wiley Interscience, NY (1987).

[6] A. Oldersma and R.J.H. Wanhill: Variability of Fatigue Crack Growth Properties for 2024-T3 Aluminium Alloy, National Aerospace Laboratory Nlr., The Netherlands, (1996). 
[7] T. Weerasooriya, Effect of Frequency on Fatigue Crack Growth Rate of Inconel 718 at High Temperature, Fracture Mechanics: Nineteenth Symposium, ASTM STP 969, T.A. Cruse, Ed., American Society for Testing and Materials, Philadelphia, 907-923 (1988).

[8] D. Broek, Elementary Engineering Fracture Mechanics, Martinus Nijhoff Publishers (1982)

[9] Andrzej Lasia, Electrochemical Impedance Spectroscopy, and its Applications, Springer New York (2004).

[10] E. Barsukov and J. Ross Macdonald, Impedance Spectroscopy: Theory, Experiment, and Applications, Wiley (2005).

[11] D.D. Macdonald, Electrochim. Acta, 51, 1376-1388 (2006).

[12] J.H. Bulloch and L.W. Buchanan, Corros. Sci., 24, 661-674 (1984).

[13] H. Dietmann, T. Bhonghibhat, and A. Schmid, Multiaxial Fatigue Behavior of Steels under In-Phase and Out-of-Phase Loading Including different waveforms and frequencies, Third International Conference on Biaxial/Multiaxial Fatigue, Stuttgart, (1989).

\section{Characterization of the electrochemistry of LWR primary coolant circuits.}

VIII.1. Characterization of the electrochemical conditions and CGR calculation in PWRs.

The ECP is one of the most important parameters in terms of Stress Corrosion Cracking (SCC) of the Heat Transport Circuit (HTC) loop as it provides the driving force of the dissolution of metal and also of the hydrogen-induced cracking. It is also the most important parameter of the accumulation of activated corrosion products into the oxide films. The mechanical load is settled in basic design, and it is not a controllable parameter; therefore, the only parameter which the utilities of Nuclear Power Plants can control in order to mitigate the SCC is the properties of the electrochemical corrosion environment. Our PWR code is equipped with a radiolysis model and a chemical reaction model, in order to calculate the individual species concentrations, $\mathrm{pH}$ calculation model, and the Mixed Potential Model (MPM). In the following paragraphs, selected results are shown focusing on CGR in terms of various electrochemical and mechanical conditions. 
ECP values obtained from the calculation using four different hydrogen-concentration for Alloy 600, at the steam generator tube were compared in Figure VIII.1 for the hot side of the Utubes over a fuel cycle. As the model confirms, the hydrogen is effective in the reduction of dissolved coolant oxygen and decreases the ECP toward more negative values. Generally, it is beneficial for the anodic dissolution mechanism; however, it increases the risk of hydrogeninduced cracking. In the following figures, we calculate the CGR in terms of various parameters.

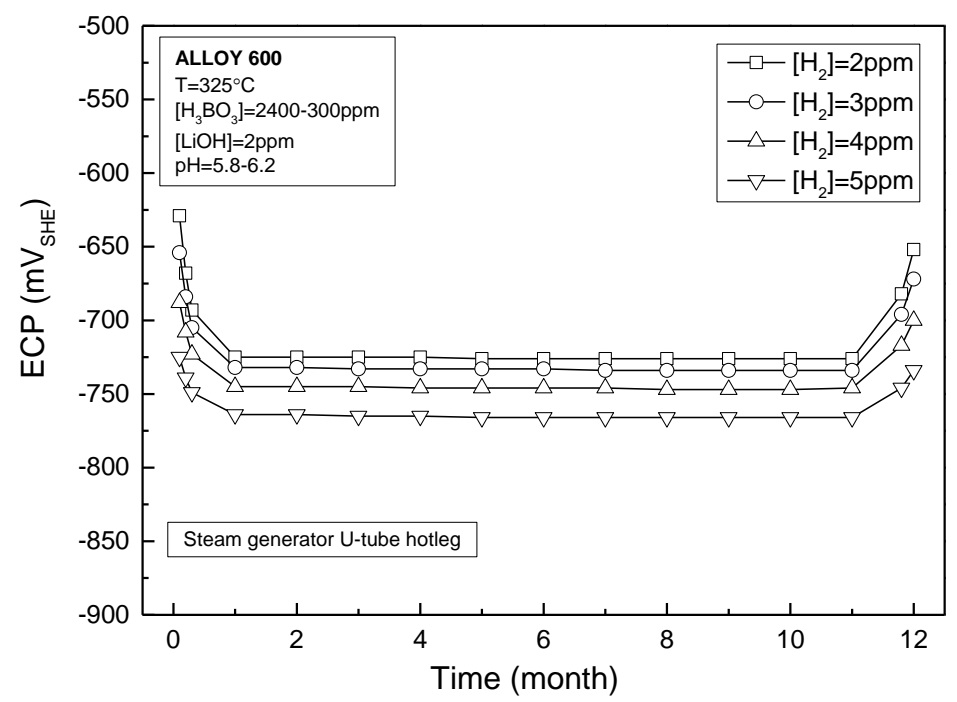

Figure VIII.1. Variation of ECP with hydrogen in steam generator U-tube hot leg over a 12-month fuel cycle.

Figure VIII.2 and VIII.3 show the CGR and crack length, respectively, as a function of time through a fuel cycle. These data were calculated for various hydrogen concentrations in the coolant. As can be seen from the figures, the change in the CGR with increasing hydrogen concentration is not significant. It is important to note that in our model incorporates the slipdissolution/repassivation (SDR) mechanism as well as the grain boundary void pressurization (GBVP) based hydrogen embrittlement model. This means the two phenomena are competing, as the ECP has a positive impact on the SDR, whereas it has a negative impact on GBVP.

In Figure VIII.3, the position of the wall thickness is also indicated, showing that having a $0.01 \mathrm{~cm}$ crack at the beginning of the cycle, the crack crosses the wall of the steam generator tube after about 6 months. 
Figure VIII.4 shows the CGR in terms of the stress intensity factor (SIF) in the steam generator U-tube hot leg. As can be seen from the figure, the CGR is a heavily sensitive function of the SIF.

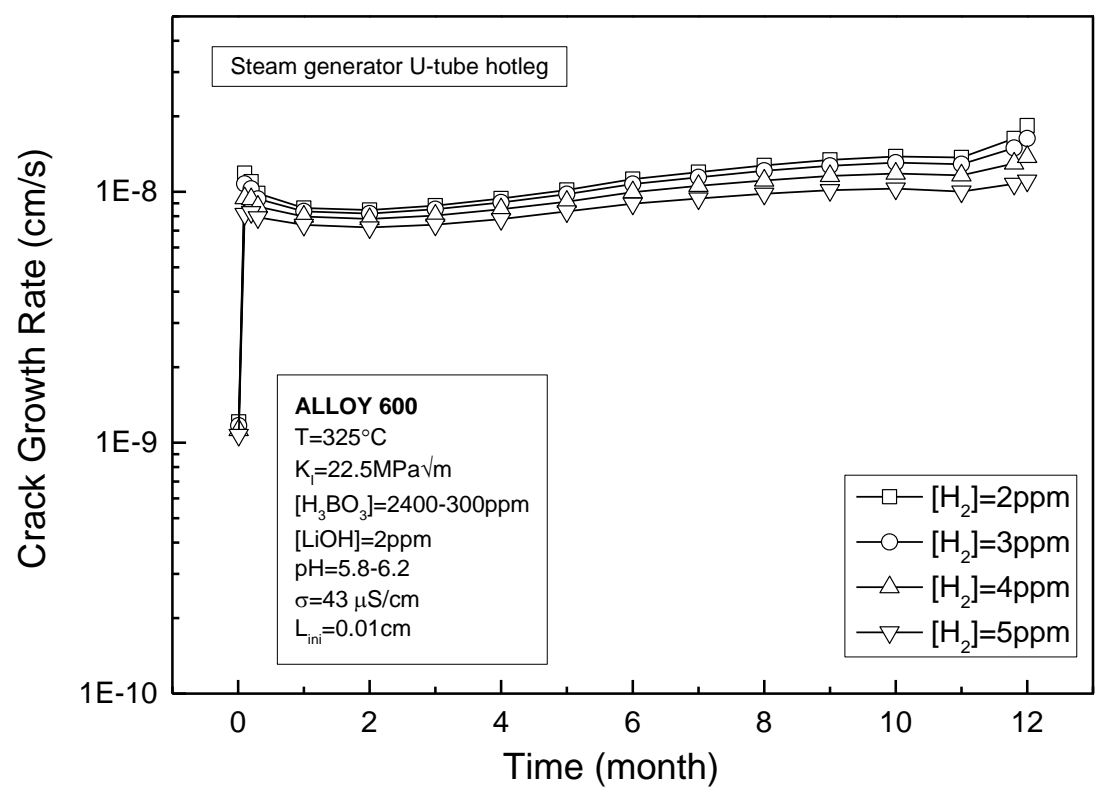

Figure VIII.2. CGR in the steam generator U-tube as a function of $\left[\mathrm{H}_{2}\right]$ over a 12-month fuel cycle.

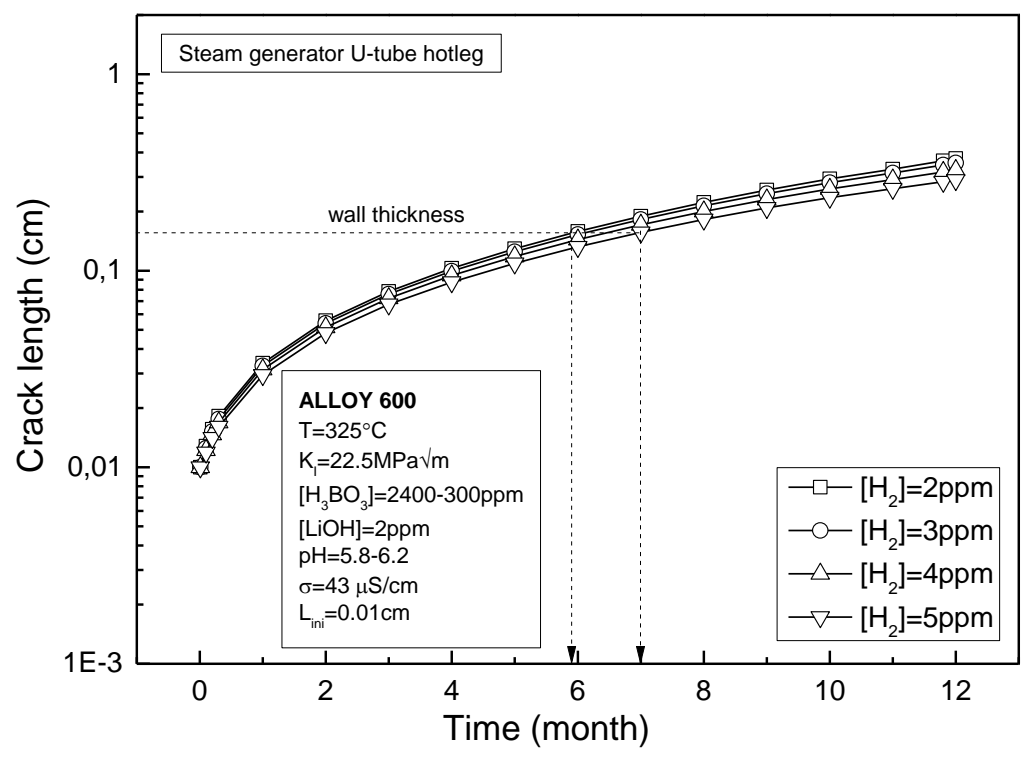

Figure VIII.3. Variation of crack length in the steam generator U-tube as a function of $\left[\mathrm{H}_{2}\right]$. 
It is important to recognize that the stress intensity factor is being used in various mechanical components throughout our model:

- The GBVP model that is based on Wilkinson and Vitek creep growth rate model.

- The SDR model as described by the CEFM.

- Previous work on Artificial Neural Network modeling of CGR in Alloy 600 in PWR primary coolant environments showed that the cracking is mainly a mechanical phenomenon augmented by electrochemistry that is in good agreement with our results displayed Figure VIII.1 through Figure VIII.5.

Figures VIII.6 and VIII.7 show the CGR and crack length, respectively, as a function of time over a 12-month fuel cycle, calculated using the two different models. One model includes only the SDR as the crack advance mechanism; the other includes the SDR augmented by HIC. It can be seen that the hydrogen re-combination in the voids has a significant impact on CGR by pressurizing the voids, therefore, increasing the fracture frequency of the grain boundary.

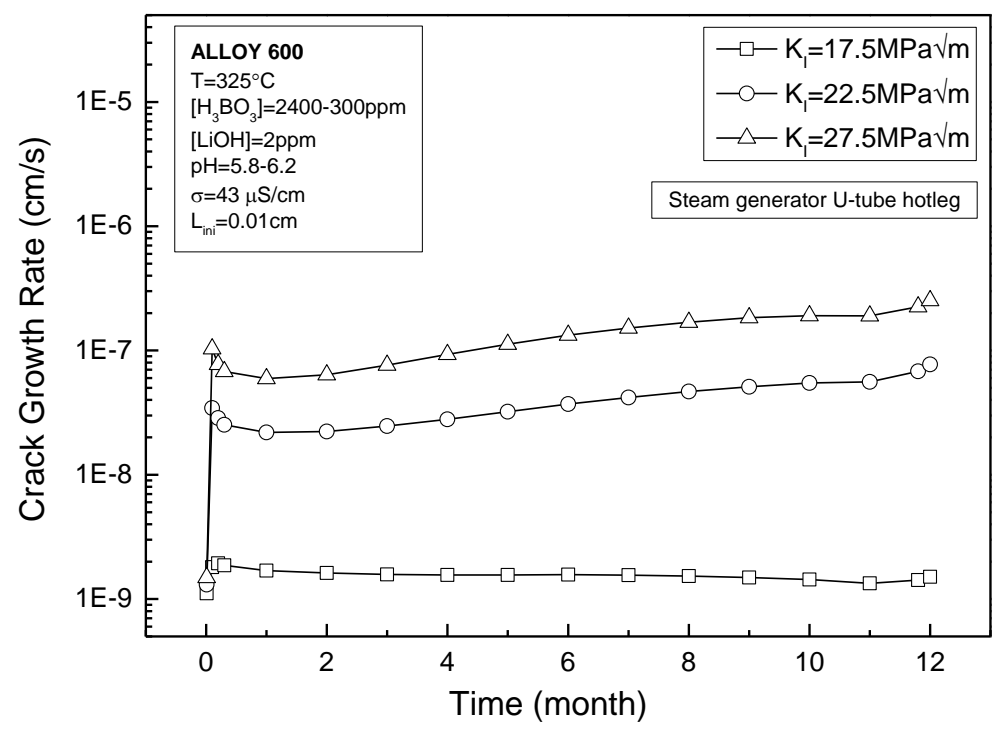

Figure VIII.4. CGR in the steam generator U-tube at different stress intensity factors over a 12month fuel cycle. 


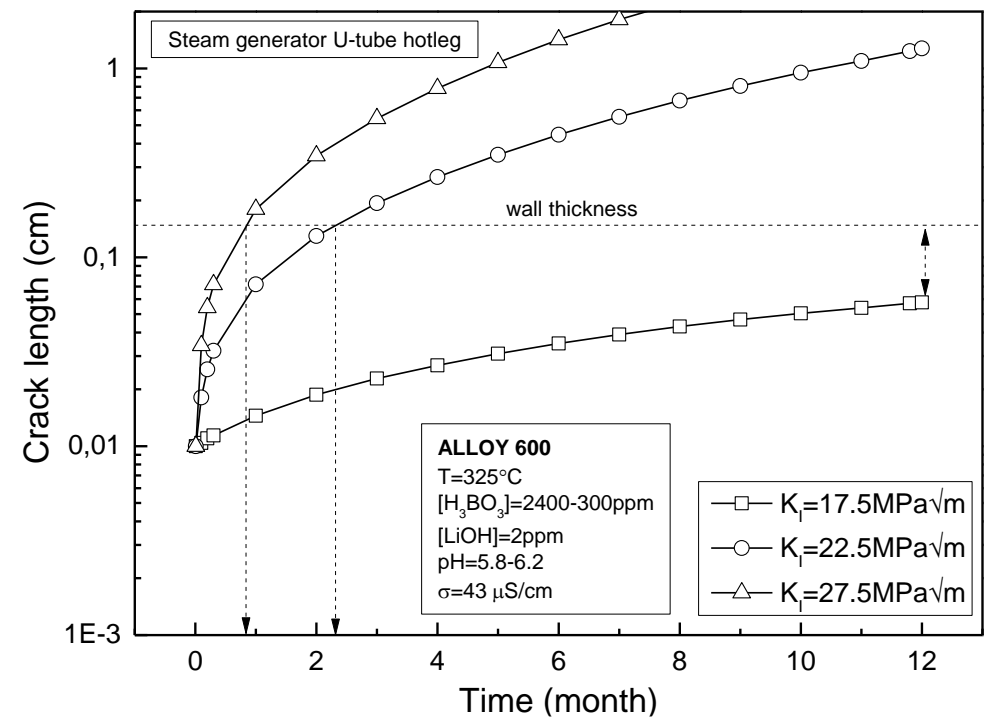

Figure VIII.5. Variation of crack length in the steam generator U-tube at different stress intensity factors over a 12-month fuel cycle.

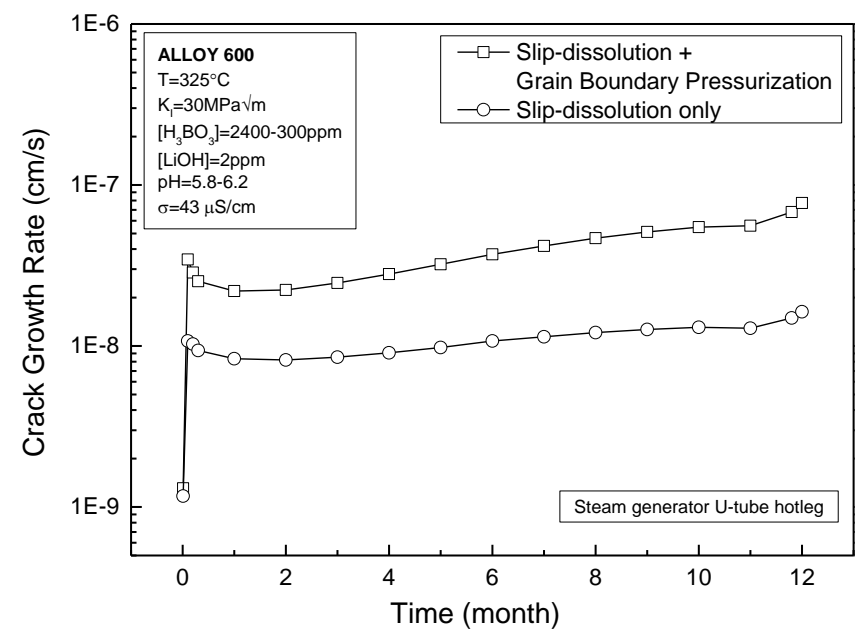

Figure VIII.6. CGR in the steam generator U-tube using different SCC models: (1) SDR mechanism, (2) SDR mechanism augmented by HIC. 


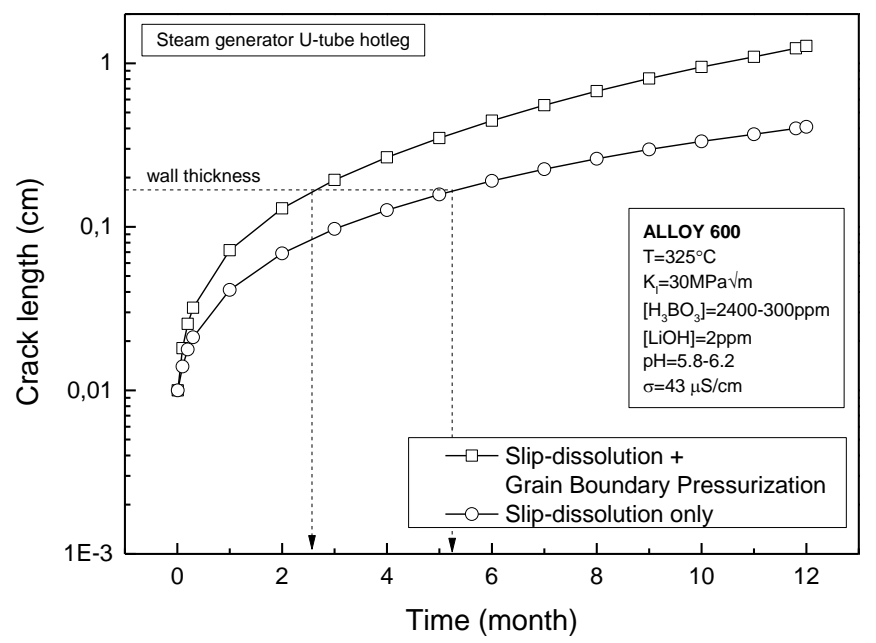

Figure VIII.7. Crack length in the steam generator U-tube using different SCC models: (1) SDR mechanism, (2) SDR mechanism augmented by HIC.

\section{Theoretical dependence of the distribution in crack growth rate in Type 304SS on various factors.}

\section{IX.1. Introduction}

In the early 1970s, numerous cases of intergranular stress corrosion cracking occurred in boiling water reactors (BWRs) in AISI 304 austenitic stainless steel [1]. The root cause of this cracking is a combination of tensile stress, an oxidizing environment, and a sensitized material. Stress corrosion cracking (SCC) continues to be a safety concern in light water reactors (LWRs), although methods, such as Hydrogen Water Chemistry (HWC), have been developed to at least partially mitigate the problem. As noted above, SCC involves complex interactions between metallurgy, stress, and the external environment. For these reasons, assessing failures of in-vessel stainless steel (SS) components in BWR from intergranular SCC has proven to be difficult, primarily because of any given system (e.g., BWR piping) is rarely sufficiently well characterized in terms of all three factors. Furthermore, the uncertainty in the empirical database makes the prediction of SCC challenging, although this has been overcome, to a significant extent, by the development of deterministic models [1].

Structural components in a nuclear power plant are designed to resist different types of loading, such as static loads, including operating pressure and stress-induced thermal loads, as well 
as transient loading from power cycling, rotating machinery, etc [2]. Because the operating and environmental conditions vary over both the short term (e.g., due to variations in reactor power) and over the long term (over multiple refueling cycles) during the lifetime of the power plant, the life of the structural components induced by SCC should be assessed by incorporating these changes in a probabilistic sense. The approach developed here is to calculate the distribution in the crack growth rate (CGR) deterministically using the Coupled Environment Fracture Model (CEFM) [1,3-6] or an empirically-trained, Artificial Neural Network (ANN) [7] by assuming normal distributions in the independent variables $\left(T, \mathrm{ECP}, \mathrm{pH}\right.$, conductivity, DoS, $K_{I}$, etc) at a given state point (as defined by the mean values for the independent variables). This process may then repeat over the corrosion evolutionary path (CEP, operating history of the reactor comprising a continuous series of state points) of the reactor as defined by the variations in the mean values of the independent variables to ultimately predict the probability of failure. This latter aspect will be addressed in a subsequent paper.

Several studies have been reported on assessing probabilities of SCC in different components of power plants and other energy-related systems. Thus, Jain et al. [8,9] used Bayesian networks to predict the probability of high-pH SCC failures in pipelines and discussed the effects of stress and other factors that affect high pH SCC. It was demonstrated that his model could be used to assess the probability of failure due to SCC as a function of time for different pipeline segments. Priya et al. [10] computed the failure probabilities of a piping component from SCC as a function of time using the Monte Carlo simulation technique, they took the degree of sensitization, applied stress, time to initiation of SCC, initial crack length, and initiation crack growth velocity into account as random variables. The simulation results were found to be in satisfactory agreement with the relevant experimental observations from the literature. By characterizing Intergranular Stress Corrosion Cracking (IGSCC) using a single damage parameter and using the general methodology recommended in the modified computer program, "Piping Reliability Analysis Including Seismic Events," Guedri [11] assessed the IGSCC of stainless steel piping in a probabilistic manner. Anoop [12] computed the fuzzy failure probabilities of a piping component from SCC with time using an approach combining the vertex method with the Monte Carlo simulation technique. Based on the source and type of uncertainty, the relevant variables were treated as random or fuzzy, to take into account the effect of uncertainty. From the results obtained, it is noted that the fuzzy-probabilistic approach presented shows promise for safety 
assessment of nuclear power plant pipelines. Andresen and Ford [13] proposed the slip dissolution/film rupture model to predict the observed crack growth rates for the stainless steels, nickel alloys, and low-alloy steels in high temperature water systems. It was demonstrated that distributions in the independent variables are easily input into the model, and a variety of approaches can be used for statistical and probabilistic treatment.

In this analysis, a piping component made of Type 304 stainless steel is considered for the analysis, because this type of steel is highly susceptible to SCC in BWR primary coolant environments. Based on the models we have proposed previously to predict the crack growth rate in sensitized Type 304SS and other alloys in aqueous environments, including high-temperature water systems [3-6,12-22], the degree of sensitization, conductivity, $\mathrm{pH}$, electrochemical potential, stress intensity factor, and temperature are considered as random, independent variables which obey normal distributions. Then we compute the effect of the distribution in each variable on the distribution in the dependent variable, the crack growth rate of Type 304SS under different environmental conditions.

The objectives of the present work are to define the probabilistic natures of both the theoretical model for crack advance (the CEFM) and the Artificial Neural Network (ANN), the latter of which contains no theoretical basis (i.e., contains no physico-electrochemical model or mechanism but is purely empirical in nature) and to determine if the two approaches yield similar results. This is done by distributing each independent variable ( $T$, ECP, $K_{I}$, yield strength, etc.) normally about a mean with an assigned standard deviation and inquiring into the nature of the distribution in the dependent variable (crack growth rate, CGR).

\section{IX.2. Modeling of stress corrosion cracking}

\section{Coupled environment fracture model (CEFM)}

As a deterministic, physico-electrochemical model for predicting crack growth rate, the coupled environment-fracture model (CEFM) is used in this paper to account for the effect of variations in the independent variables on the crack growth rate. In this section, a brief overview of the CEFM is presented. Since its inception in 1985 [3,4], the CEFM has been used extensively and successfully to model crack growth rate in sensitized Type 304SS in boiling water reactor 
(BWR) primary coolant environments and in dilute sulfate solutions over the temperature range of 50-300 ${ }^{\circ} \mathrm{C}[5,6]$. The CEFM code has been modified and calibrated to predict crack growth rate in marine aluminum alloys (e.g., AA 5083) in $\mathrm{NaCl}$ brine [14], in ASTM A470/471 turbine disk steels in simulated steam condensate [15], in Alloy 22 in simulated Yucca Mountain surface deliquescent environments [16], in Alloy 600 in prototypical PWR primary coolant [17], and has been recently used to account for the evolution of semi-elliptical surface cracks [18]. Of particular importance is the ANN analysis of the empirical CGR data for Type 304 SS [7] and for Alloy 600 [19], because those defined the "character" of IGSCC in these alloys by establishing relationships between the dependent variable (CGR) and the various independent variables ( $T, K_{I}$, yield strength, ECP, etc). The CEFM was found to provide an accurate description of the character in both cases $[7,19]$.

The physic-electrochemical basis of the CEFM is shown in Figure IX.1.

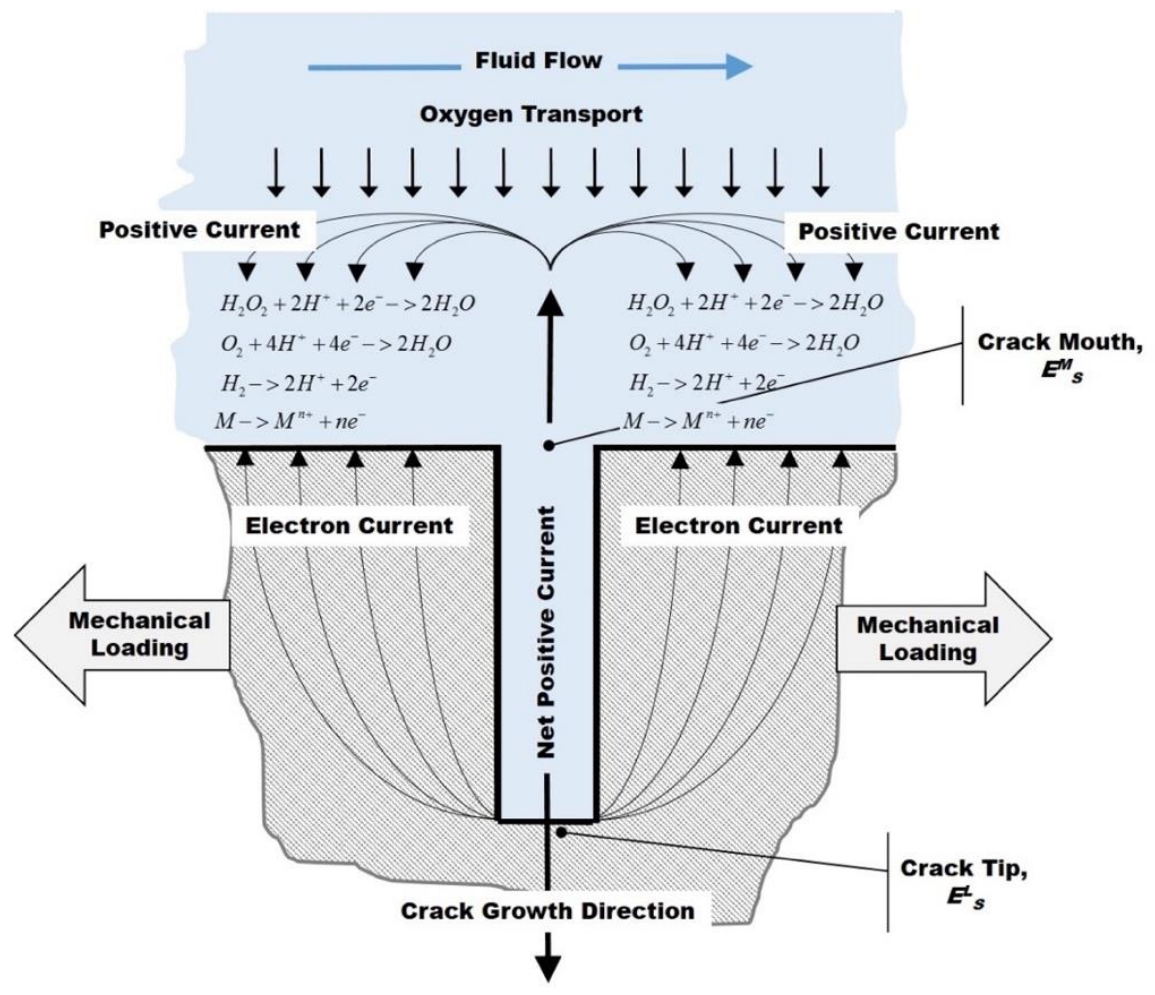

Figure IX.1. Schematic of the differential aeration hypothesis for localized corrosion. $E^{M} S$ and $E^{L} S$ represent the electrostatic potentials in the solution vs. the metal on the standard hydrogen electrode scale at the crack mouth and the crack tip, respectively. 
In this model, which is based upon the differential aeration hypothesis (DAH), the crack advance is assumed to occur via the slip/dissolution/passivation mechanism augmented with hydrogen-induced cracking (HIC), with the governing equation being a statement of charge conservation that is expressed as follows:

$$
i_{\text {crack }} A_{\text {crack }\lfloor\text { mouth }}+\int_{s} i_{C}^{N} \mathrm{~d} s=0
$$

or equivalently

$$
\int_{s} i^{N} \mathrm{~d} s^{\prime}=0
$$

(both have been used in various versions of the model), where $i_{\text {crack }}$ is the net (positive) current density flowing from the crack mouth, $A_{\text {crack } \text { mouth }}$ is the crack mouth area, $i_{C}{ }^{N}$ is the net (cathodic) current density due to charge transfer reactions on the external surface, $i^{N}$ is the net current density, and $\mathrm{d} s$ and $\mathrm{d} s^{\prime}$ are areal increments on the external surface and on the entire area (including the area inside the crack), respectively. The subscript $s$ on the integral indicates that the integration is to be performed over the areas just defined.

The CEFM model performs its calculation in two steps [3,4]. Firstly, it calculates the electrochemical potential of the external surface $\left(E_{c o r r}\right)$, and secondly, the crack growth rate is estimated. The electrochemical potential sufficiently far from the crack is calculated using the mixed potential model from the composition and properties of the system [20] such that the local electrochemical properties are unaffected by the presence of the crack, and, hence, that the potential in the solution with respect to the metal is equal to the negative of the free corrosion potential (the ECP). This condition holds for distances of a few $(<10)$ crack mouth opening displacements (CMODs) on either side of the crack center line, as determined by numerical simulation [6]. The crack growth rate calculation depends on splitting the crack environment into the crack-internal and the crack-external environment and by noting that the transport (NernstPlanck) and potential distribution (Poisson's or Laplace's) equations for both environments are coupled by common boundary conditions (concentrations and electrostatic potential) at the crack mouth. In order to solve for the crack growth rate, an electrochemical potential at the crack mouth (the boundary between the crack-internal and -external environments) is assumed, and this value is then changed iteratively, until the current in crack-internal and crack-external match and hence Equation IX.1 is satisfied. Thus, the crack-internal and external currents are calculated for a 
particular electrochemical potential at the crack mouth and for the prevailing ECP, and this calculation is repeated iteratively until the crack mouth potential at which charge is conserved is determined. For the calculation of the internal crack current, an electrochemical potential is assumed at the crack tip, and this value is changed until the electro-neutrality condition is satisfied at the crack tip within each iteration of the crack mouth potential. The calculation is repeated iteratively on the crack mouth potential until the charge is conserved. The crack internal current is then obtained by solving the Nernst-Planck equations for each of the ten species in the system $\left[\mathrm{H}^{+}, \mathrm{Fe}^{2+}, \mathrm{Fe}(\mathrm{OH})^{+}, \mathrm{Ni}^{2+}, \mathrm{Ni}(\mathrm{OH})^{+}, \mathrm{Cr}^{3+}, \mathrm{Cr}(\mathrm{OH})_{2}{ }^{+}, \mathrm{Na}^{+}, \mathrm{Cl}^{-}, \mathrm{OH}^{-}\right]$, with the appropriate boundary conditions of $C_{i}^{\text {mouth }}=C_{i}^{b}, i=1,10$, where $C_{i}^{\text {mouth }}$ and $C_{i}^{b}$ are the species concentrations at the crack mouth and in the bulk solution, respectively, recognizing that the source of the current is alloy dissolution at the crack tip as described by the slip/dissolution/repassivation mechanism. For $\mathrm{Fe}^{2+}, \mathrm{Fe}(\mathrm{OH})^{+}, \mathrm{Ni}^{2+}, \mathrm{Ni}(\mathrm{OH})^{+}, \mathrm{Cr}^{3+}, \mathrm{Cr}(\mathrm{OH})_{2}{ }^{+}, C_{i}^{b}=0$, but for $\mathrm{H}^{+}, \mathrm{OH}^{-}, \mathrm{Na}^{+}$, and $\mathrm{Cl}^{-}$the bulk concentrations are finite, depending upon the composition of the solution, with $C_{H^{+}}^{b} C_{O H^{-}}^{b}=K_{W}$. The boundary conditions for each specie at the crack tip is expressed in terms of the kinetics of dissolution of each component of the alloy recognizing that electroneutrality must hold; i.e., $\sum_{i=1}^{10} z_{i} C_{i}^{t i p}=0$, where $C_{i}^{t i p}$ is the concentration of species $i$ at the crack tip. Note, that the potentials are defined as electrostatic potential in the solution with respect to the metal, which is assumed to be an equi-potential phase. Accordingly, the potential at the surface remote from the crack mouth is simple $-E_{\text {corr }}$ and that at the crack tip is $-E^{L}$. For the calculation of the external current, a 40-term Fourier series solution to Laplace's equation is employed, as described elsewhere [3]. Importantly, measurement of the coupling current and the noise contained therein [21] demonstrates that the micro-fracture frequency is much too low, and the micro-fracture dimension is much too high to be attributed to classical slip-dissolution-repassivation and that the crack growth rate is determined by hydrogen-induced cracking (HIC), probably via the formation of strain-induced martensite on the grain boundaries ahead of the crack tip. The larger microfracture dimensions have been confirmed by analyzing the noise in the coupling current due to IGSCC in sensitized Type 304 SS in thiosulfate-containing solutions [22,23] and fracture in AISI 4340 steel in $\mathrm{NaOH}$ solutions [24] both being recognized as classical HIC systems. Finally, we note that an analytical form of the CEFM has been developed with the important advantage of computational speed [25]. 


\section{Artificial neural network model}

As a non-deterministic, empirical model, artificial neural networks (ANNs) have nonlinear mapping structures that can be applied for predictive modeling and classification. They are inspired by the structure of the cerebral cortex portion of the brain, where billions of neurons (in humans and higher-order animals) are interconnected to process a variety of complex information. A neural network consists of artificial neurons joined together in layers. Typically, there is an input layer through which the data are presented to the network and an output layer where the network response is obtained. Between these two layers, there exist one or more layers of neurons called "hidden layers." The strength of the interconnections between a neuron in any given layer with all of the neurons in neighboring layers is determined by the weight and bias associated with them. Establishment of the weights essentially imbues the net with "memory" and enables the relationships between the output (in our case the CGR) and each of the independent input variables ( $T$, ECP, Conductivity, $K_{I}, \mathrm{pH}$, and DoS) to be defined. For more details about the training and prediction of crack growth rate in Type 304SS and Alloy 600 in high-temperature reactor coolant using ANN, please see [7,19].

\section{IX.3. Results and discussion.}

In this study, the stochastic distribution crack growth rate due to SCC in sensitized Type 304SS is simulated using both the CEFM model and the ANN. The details regarding the random variables considered in this study are given in Table IX.1. When we study the variation effect of one variable on the CGR, all the other variables except the one under study are assigned their undistributed default values (Table IX.1) while values for the variable under investigation are randomly chosen from a database that contains 1000 data points.

A principal finding of this study is that while an independent variable ( $T, \mathrm{ECP}, K_{I}, \mathrm{DoS}$, conductivity, etc) may be distributed normally, reflecting a stochastic input, the dependent variable (CGR) is invariable log-normally distributed as indicated by analysis of data obtained from the theoretical model (CEFM) and the experimental data in the literature via the ANN. A log-normal (or lognormal) distribution is a continuous probability distribution of a random variable whose logarithm is normally distributed. 
Table IX.1. Summary of the random variables considered.

\begin{tabular}{llll}
\hline Variable & Distribution & Mean value & Standard deviation \\
\hline ECP $\left(\mathrm{V}_{\mathrm{SHE}}\right)$ & Normal & 0.1 & 0.05 \\
Conductivity $(\mu \mathrm{s} / \mathrm{cm})$ & Normal & 2.6 & 0.13 \\
Temperature $\left({ }^{\circ} \mathrm{C}\right)$ & Normal & 288 & 5 \\
Stress intensity factor $\left(\mathrm{MPa} \cdot V_{\mathrm{m}}\right)$ & Normal & 30 & 5 \\
Degree of sensitization $\left(\mathrm{C} / \mathrm{cm}^{2}\right)$ & Normal & 15 & 5 \\
\hline
\end{tabular}

Thus, if the random variable, $\mathrm{X}$, is log-normally distributed, then $\mathrm{Y}$ is normally-distributed. The probability density function for a log-normal distribution is given mathematically as:

$P(Y)=\frac{1}{X \sigma \sqrt{2 \pi}} \exp \left[-\frac{(Y-\mu)^{2}}{2 \sigma^{2}}\right]$

where the mean is $\exp \left(\mu+\sigma^{2} / 2\right)$, the median is $\exp (\mu)$, Mode is $\exp \left(\mu-\sigma^{2}\right)$, and the variance is $\left[\exp \left(\sigma^{2}-1\right)\right] \exp \left(2 \mu+\sigma^{2}\right)$. Numerical values of these parameters for the CGR distribution in terms of the various input parameters are tabulated in Table IX.2 and Table IX.3.

\section{Distribution of CGR with respect to ECP}

Histograms of the calculated CGR as a function of ECP are shown in Figure IX.2 as calculated from the deterministic CEFM and the empirical ANN. 
Table IX.2. Numerical values of the distribution parameters of CGR by CEFM.

\begin{tabular}{c|c|c|c|c}
\hline Input Variable & Mean & Median & Mode & Variance \\
\hline ECP $\left(V_{\text {SHE }}\right)$ & $9.80 \mathrm{E}-09$ & $5.77 \mathrm{E}-09$ & $1.99 \mathrm{E}-09$ & $1.02 \mathrm{E}-16$ \\
\hline Conductivity $(\mu \mathrm{s} / \mathrm{cm})$ & $1.06 \mathrm{E}-08$ & $6.23 \mathrm{E}-09$ & $2.11 \mathrm{E}-09$ & $1.24 \mathrm{E}-16$ \\
\hline \begin{tabular}{c} 
Temperature $\left({ }^{\circ} \mathrm{C}\right)$ \\
\hline $\begin{array}{c}\text { Stress intensity factor } \\
\left(\mathrm{MPa} \cdot \mathrm{m}^{1 / 2}\right)\end{array}$
\end{tabular} & $1.36 \mathrm{E}-08$ & $7.47 \mathrm{E}-09$ & $2.22 \mathrm{E}-09$ & $2.30 \mathrm{E}-16$ \\
\hline $\begin{array}{c}\text { Degree of } \\
\text { sensitization }\left(\mathrm{C} / \mathrm{cm}^{2}\right)\end{array}$ & $1.21 \mathrm{E}-08$ & $6.63 \mathrm{E}-09$ & $1.97 \mathrm{E}-09$ & $1.81 \mathrm{E}-16$ \\
\hline
\end{tabular}

Table IX.3. Numerical values of the distribution parameters of CGR by ANN.

\begin{tabular}{c|c|c|c|c}
\hline Input Variable & Mean & Median & Mode & Variance \\
\hline ECP $\left(V_{\text {SHE }}\right)$ & $1.12 \mathrm{E}-08$ & $9.35 \mathrm{E}-09$ & $6.33 \mathrm{E}-09$ & $6.86 \mathrm{E}-17$ \\
\hline Conductivity $(\mu \mathrm{s} / \mathrm{cm})$ & $3.25 \mathrm{E}-08$ & $2.31 \mathrm{E}-08$ & $1.15 \mathrm{E}-08$ & $7.78 \mathrm{E}-16$ \\
\hline Temperature $\left({ }^{\circ} \mathrm{C}\right)$ & $7.30 \mathrm{E}-09$ & $5.43 \mathrm{E}-09$ & $3.00 \mathrm{E}-09$ & $3.55 \mathrm{E}-17$ \\
\hline $\begin{array}{c}\text { Stress intensity factor } \\
\left(\mathrm{MPa} \cdot \mathrm{m}^{1 / 2}\right)\end{array}$ & $1.86 \mathrm{E}-08$ & $1.57 \mathrm{E}-08$ & $1.10 \mathrm{E}-08$ & $1.81 \mathrm{E}-16$ \\
\hline $\begin{array}{c}\text { Degree of } \\
\text { sensitization }\left(\mathrm{C} / \mathrm{cm}^{2}\right)\end{array}$ & $5.42 \mathrm{E}-09$ & $4.48 \mathrm{E}-09$ & $3.05 \mathrm{E}-09$ & $1.59 \mathrm{E}-17$ \\
\hline
\end{tabular}




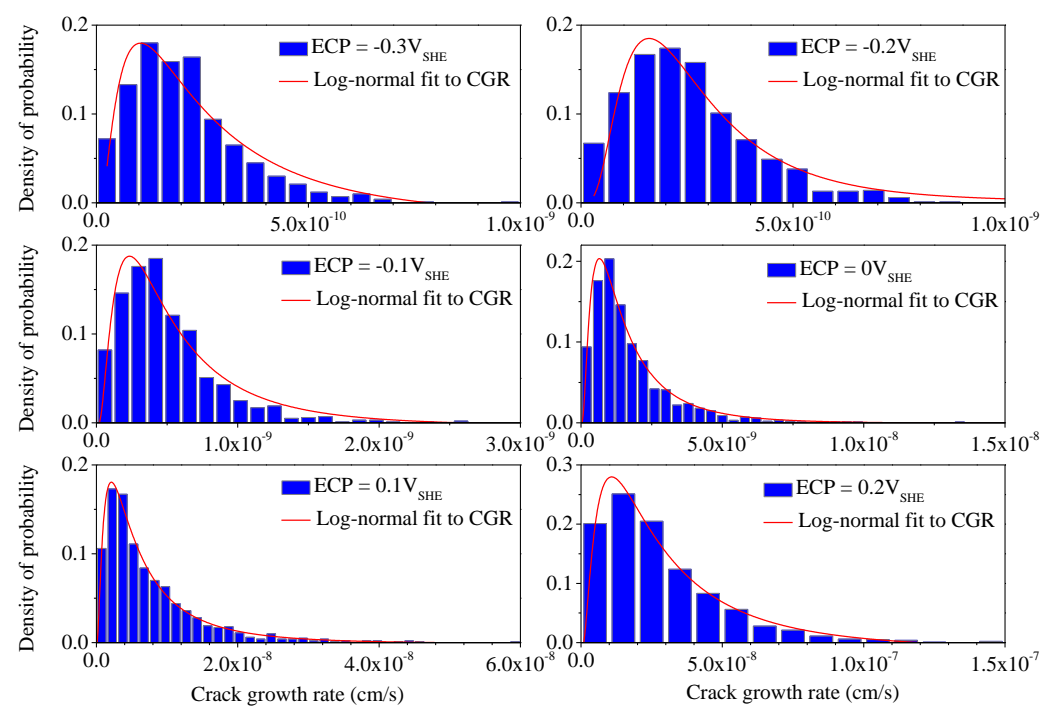

(a)

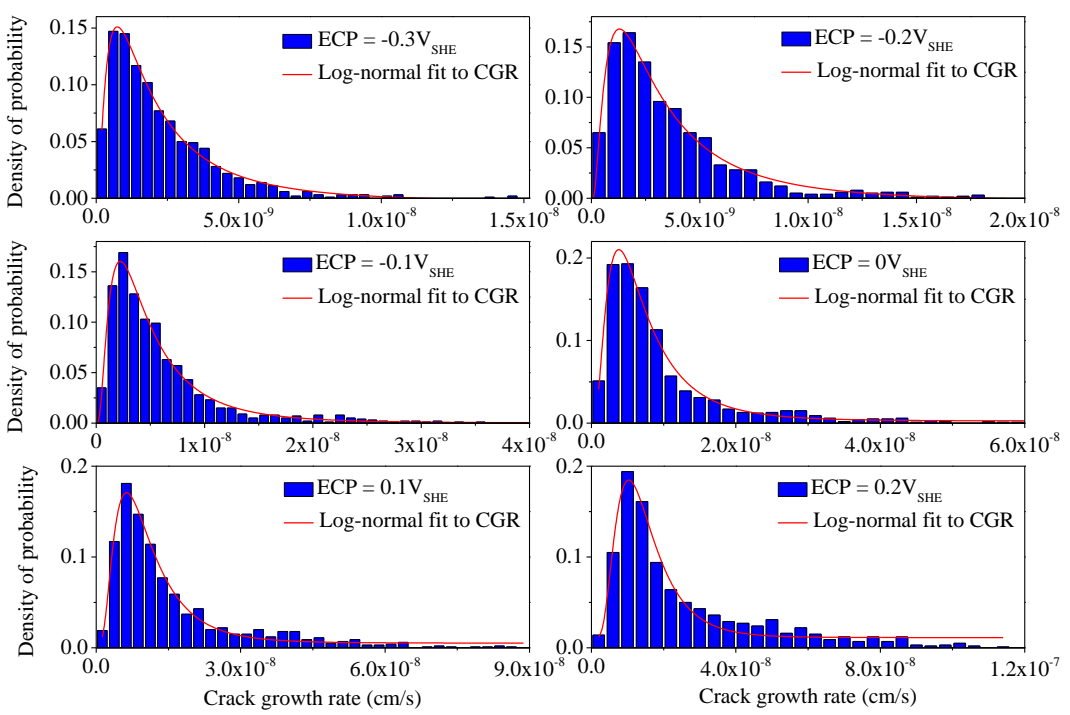

(b)

Figure IX.2. Histograms of the calculated CGRs under different ECP compared to the fitted lognormal distributions, (a) Results from the CEFM; (b) Results from the ANN.

All of the other input parameters (default values) are as shown in Table IX.1. The lognormally fitted curves are also shown in the figure. As can be seen from this figure, with an increase of ECP, the CGR increases accordingly. Note that the log-normal distribution "moves" 
to the right with increasing ECP, in the direction of increasing CGR, while the standard deviation increases, making the distribution wider. This indicates that if the input parameters are randomly distributed around a certain value, the calculated CGR will also have stochastic character, which approximates to a log-normal distribution.

Figure IX.3 shows the dependence of the mean value of CGR on ECP.

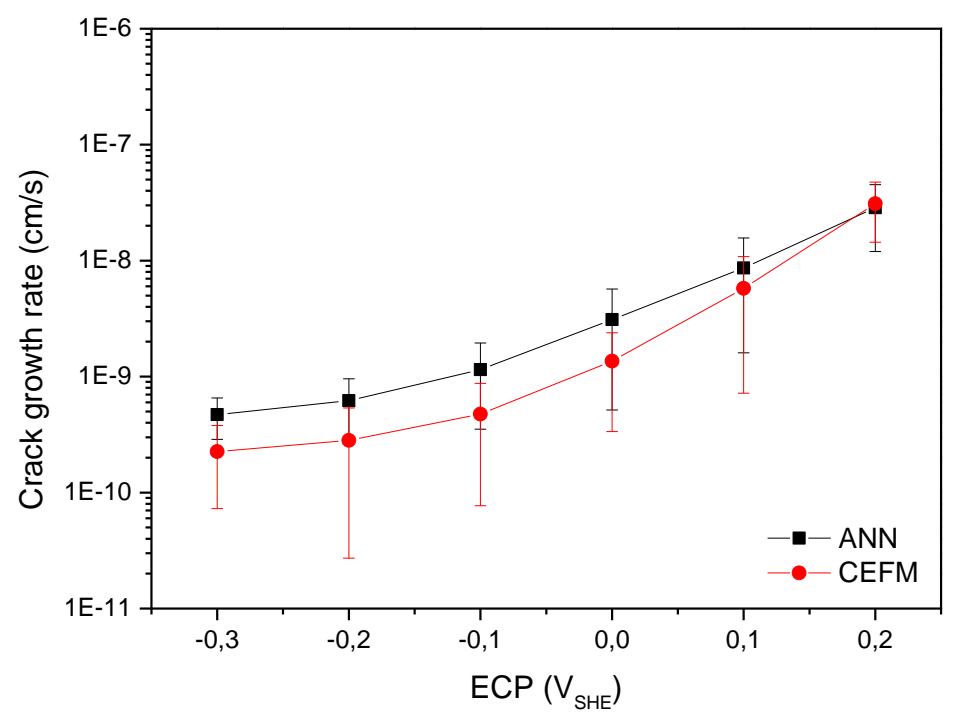

Figure IX.3. Dependence of the mean value of CGR on ECP calculated using both the CEFM and ANN.

Both the CEFM and the ANN predict that the CGR increases with increasing ECP. Furthermore, the CEFM predicts the well-known sigmoid relationship between the CGR and the ECP. Note that the CEFM predicts the creep rate for Type 304 SS when the ECP is lower than $0.23 \mathrm{~V}_{\mathrm{SHE}}$; however, there is no such trend for ANN. This is because the CGR is so small at lower ECP $\left(-0.23 \mathrm{~V}_{\mathrm{SHE}}\right)$ that it was never included in the experimental database upon which the ANN is trained. Accordingly, the predicted CGR from the ANN for lower ECP values are slightly higher than those calculated from the CEFM, but this is an artifact of the CGR database not including data for the creep CGR limit. However, as the ECP increases, the predicted results from both the CEFM and the ANN come into better agreement as electrochemical effects become increasingly dominant. 


\section{Distribution of CGR with respect to $K_{I}$}

Histograms of the calculated CGR as a function of $K_{I}$ are shown in Figure IX.4 as calculated from both the CEFM and the ANN. All of the other input parameters have their default values as shown in Table IX.1. The log-normal distribution provides an adequate description of the CGR distributions, as can be seen from the curves in Figure IX.4. For the predicted results from the CEFM, as shown in Figure IX.4a, the log-normal distribution "moves" a little to the right as $K_{I}$ increase from $20 \mathrm{MPa} \cdot \mathrm{m}^{1 / 2}$ to $30 \mathrm{MPa} \cdot \mathrm{m}^{1 / 2}$, meanwhile, when $K_{I}$ is above $30 \mathrm{MPa} \cdot \mathrm{m}^{1 / 2}$, there is almost no effect of $K_{I}$ on the CGR. However, for the results obtained from the ANN, with the increasing $K_{I}$, the CGR increases gradually. As indicated in Figure IX.4b, the peak value of the log-normal fitted curve moves to the right, combined with an increase of the standard deviation, making the distribution wider.

The mean value and the standard deviation of the CGR calculated from both the CEFM and the ANN are shown in Figure IX.5. As depicted above, the ANN predicts that the CGR increases with increasing $K_{I}$; however, the results from the CEFM show little effect of $K_{I}$ on the CGR, as expected from Stage II behavior. Note that the CEFM predicted results and those simulated by the ANN are in good agreement (within the combined standard deviations), in general.

\section{Distribution of CGR with respect to temperature.}

Figure IX.6a and IX.6b show the histograms for the effect of temperature on the distribution in the CGR. All the other input parameters are as shown in Table IX.1. As can be seen from Figure IX.6a, there is little effect of temperature on the CGR, as the temperature increases, except that the tail of the distribution in the CGR moves a little to the left. Figure IX.6b shows the same effect of temperature on CGR as determined from the ANN. As reported elsewhere [26,27], there should be a peak in CGR versus temperature at $150{ }^{\circ} \mathrm{C}$ to $200^{\circ} \mathrm{C}$, because of two competing effects: the increase in CGR due to increasing temperature upon the kinetics of the cathodic depolarizer reaction on the surfaces external and due to an increase in the crack tip strain rate and the observed decrease in corrosion potential for constant $\left[\mathrm{O}_{2}\right]$ and flow rate $[1,20,28]$. 


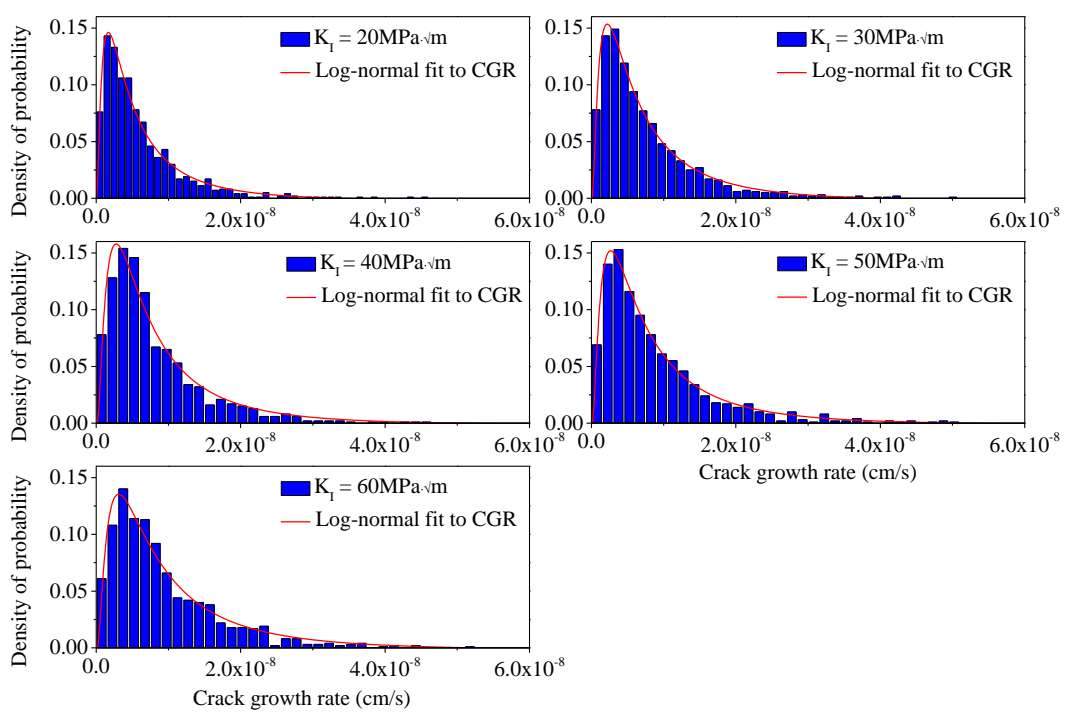

(a)

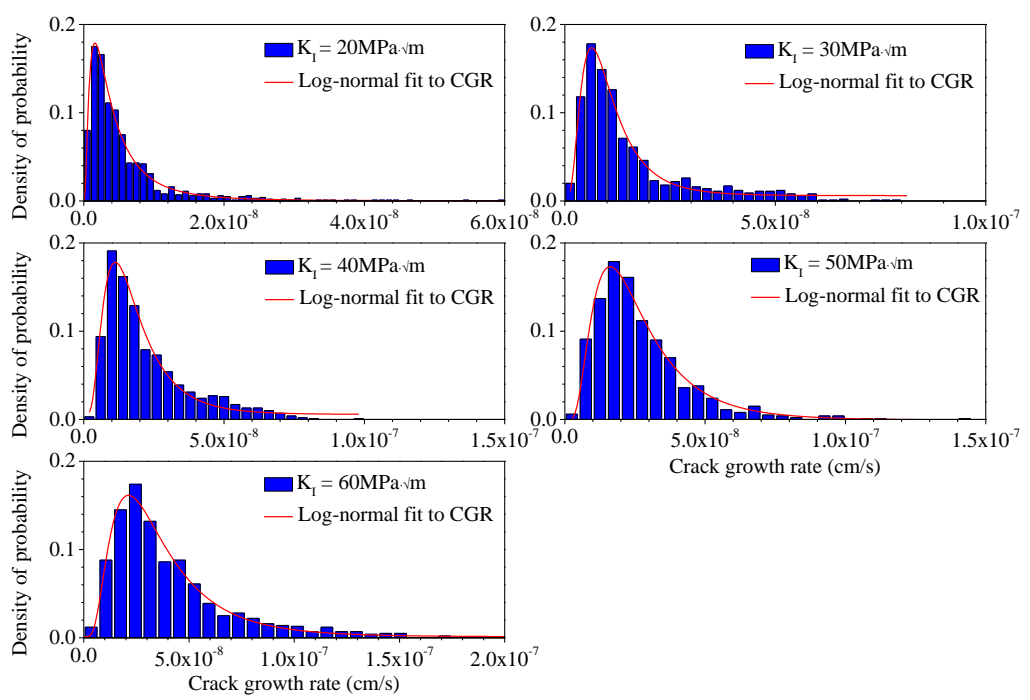

(b)

Figure IX.4. Histograms of the calculated CGRs under different $K_{I}$ compared to the fitted lognormal distributions. (a) Results from the CEFM; (b) Results from the ANN. 


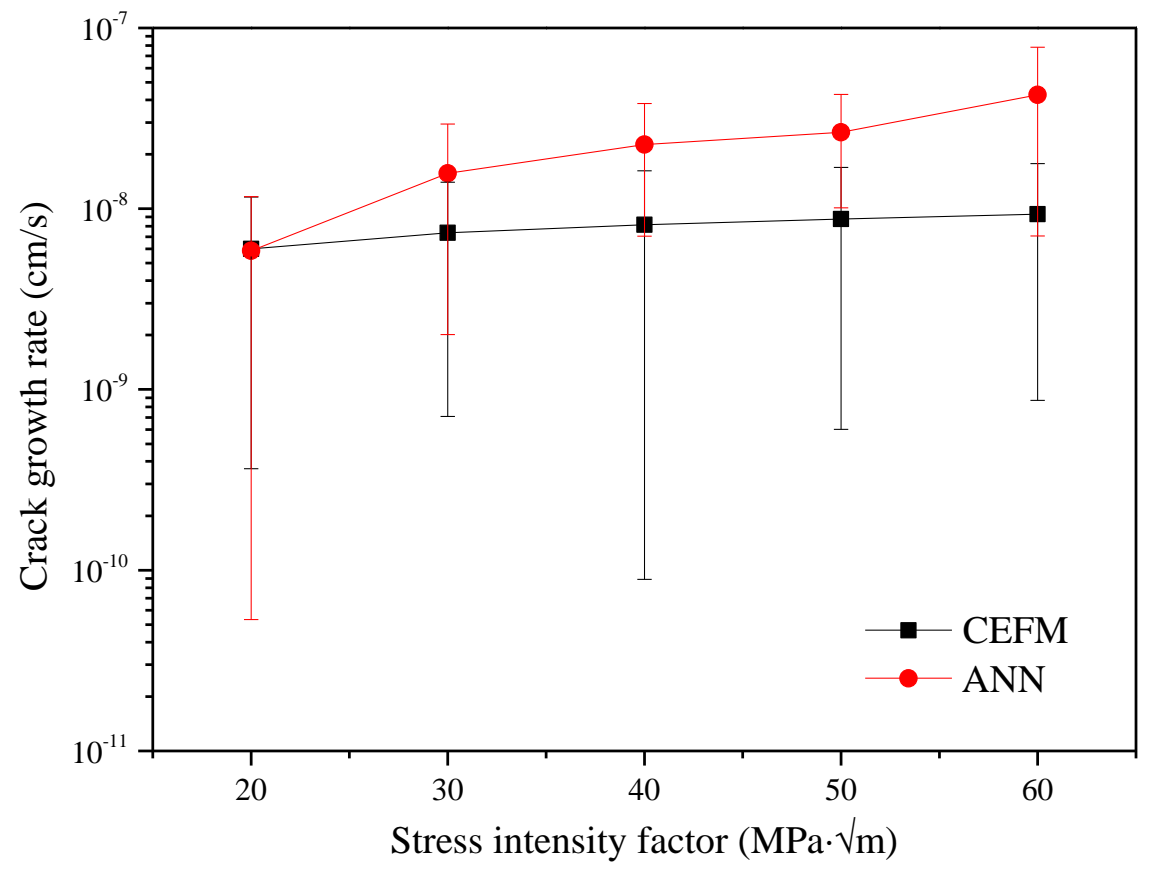

Figure IX.5. Dependence of the mean value of CGR on $K_{I}$ calculated using both the CEFM and ANN.

However, in the present case, there is little effect of temperature on the CGR, because the results obtained in our paper were obtained under the assumption that all the other parameters have constant values (e.g., ECP, conductivity, pH). The CEFM and the ANN allow us to study the effect of each variable on the CGR independently. The fitted log-normal distribution curves are also shown in Figure IX.6, which shows that the log-normal distribution function provides a good description of the CGR. 

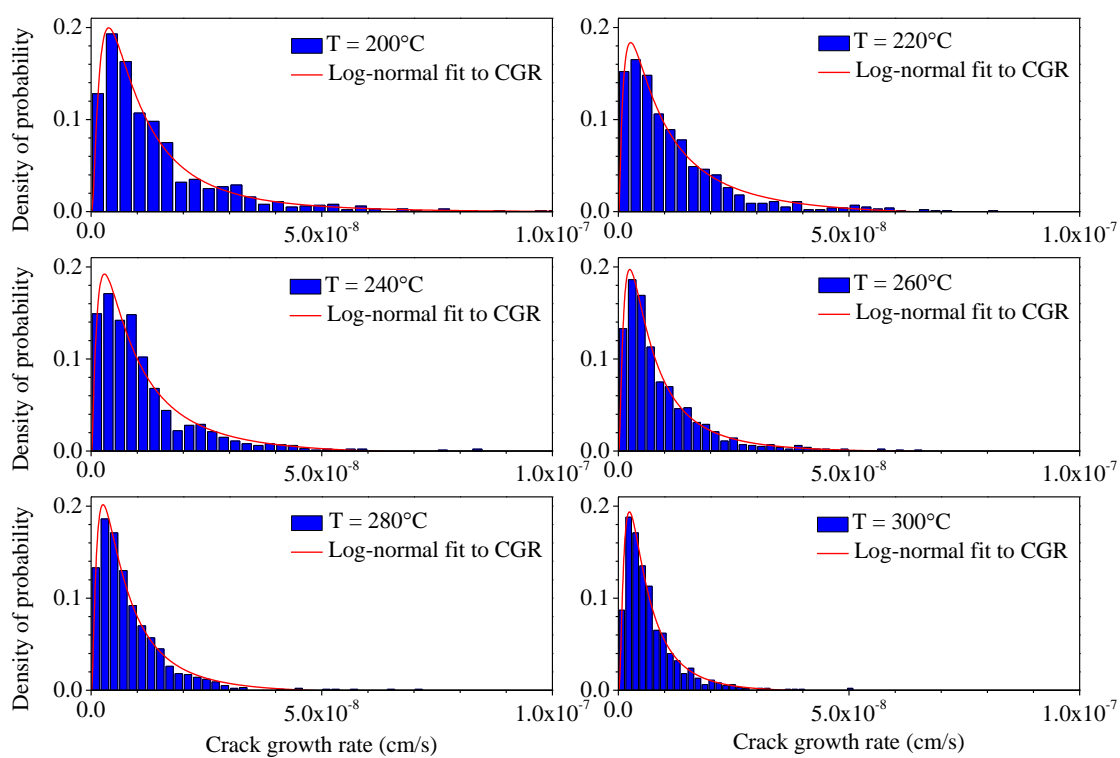

(a)
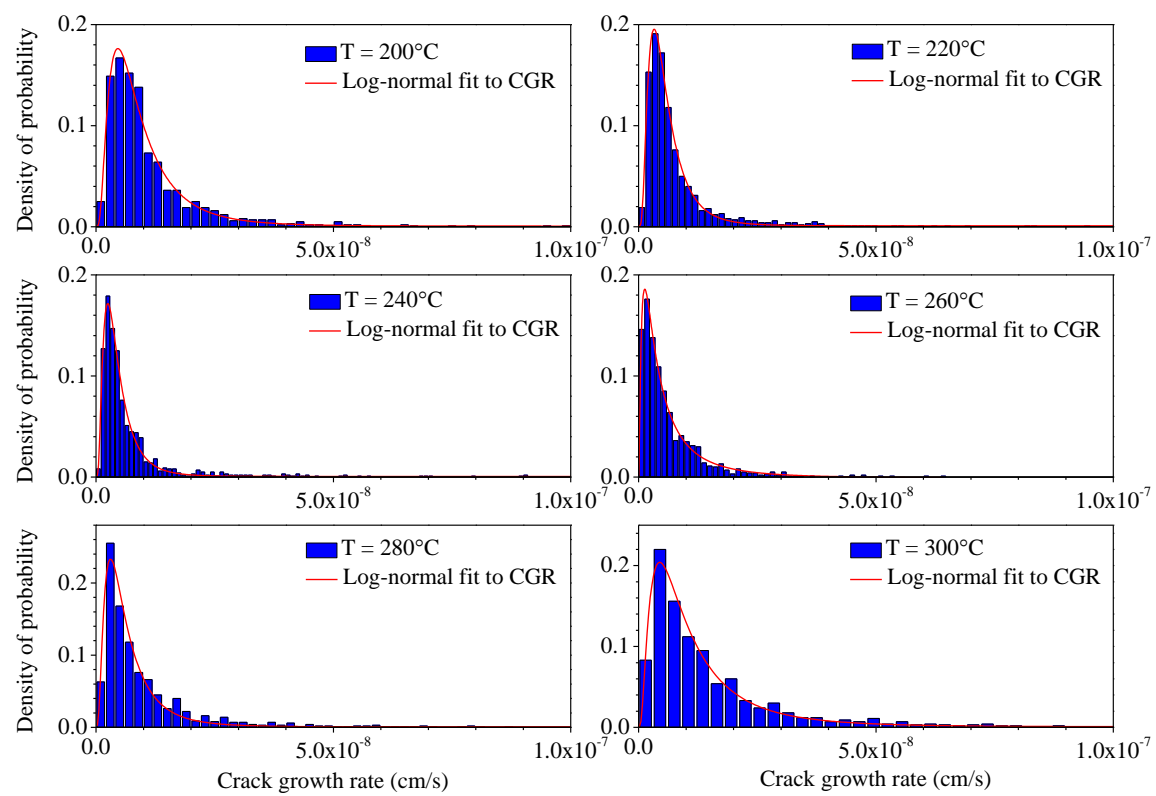

(b)

Figure IX.6. Histograms of the calculated CGRs under different temperatures compared to the fitted log-normal distributions. (a) Results from the CEFM; (b) Results from the ANN. 
Figure IX.7 shows the dependence of the mean CGR on temperature. The results from both the CEFM and the ANN show little effect of temperature on CGR when the other variables are kept constant, as discussed above. Good agreement is obtained between the CGR predicted from both the CEFM and the ANN.

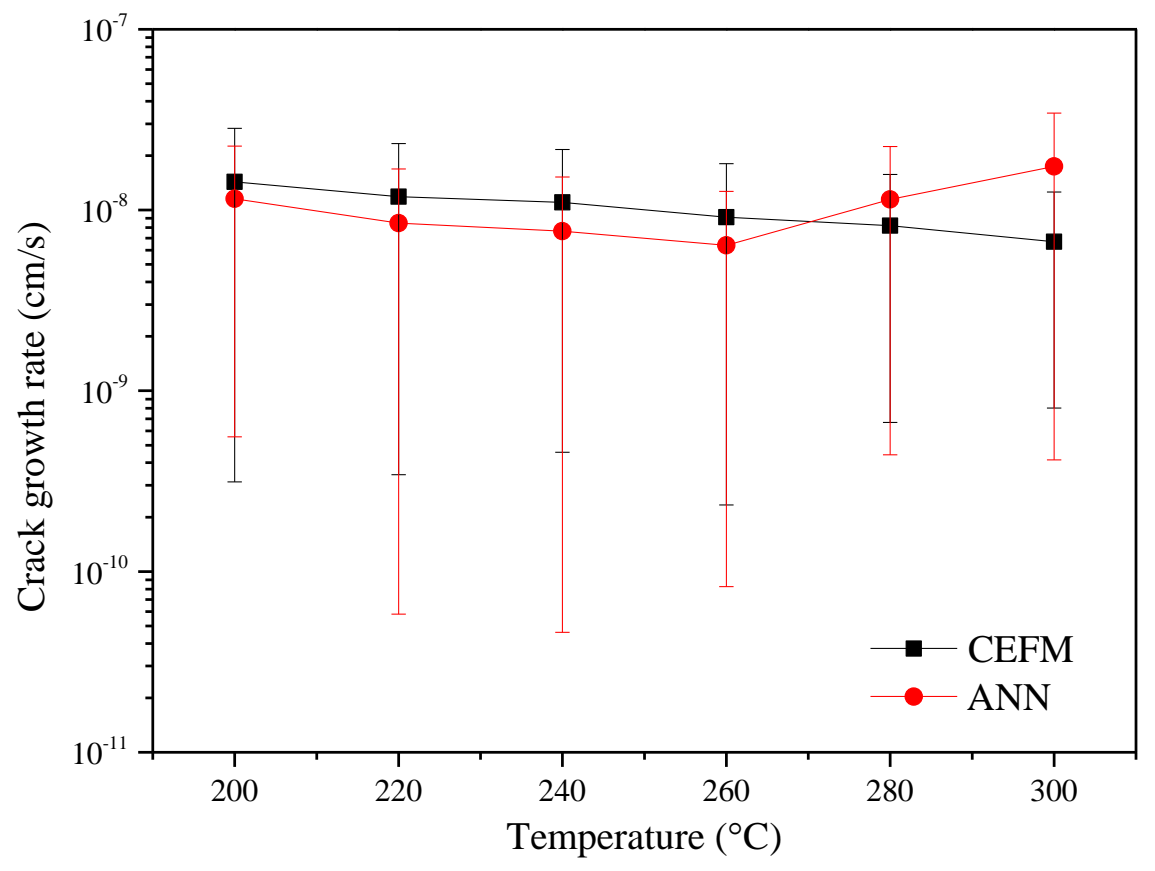

Figure IX.7. Dependence of the mean value of CGR on temperature calculated using both the CEFM and ANN.

\section{Distribution of CGR with respect to conductivity}

According to the coupled environment fracture model [5], the principal effect of increasing conductivity is to increase the throwing power of the current from the crack mouth, such that a larger area on the external surface may contribute to the annihilation of the coupling current via the redox reactions Figure IX.8a-b show the histogram of CGR as a function of conductivity. As can be seen from Figure IX.8a and IX.8b, as the conductivity increases from $2.6 \mu \mathrm{s} / \mathrm{cm}$ to $3.4 \mu \mathrm{s} / \mathrm{cm}$, the CGR distribution moves to the right, this phenomenon is more obvious for the prediction 
results from the ANN. The log-normal distribution fitted line is also shown in Figure IX.8, which gives an adequate description of the CGR distributions.

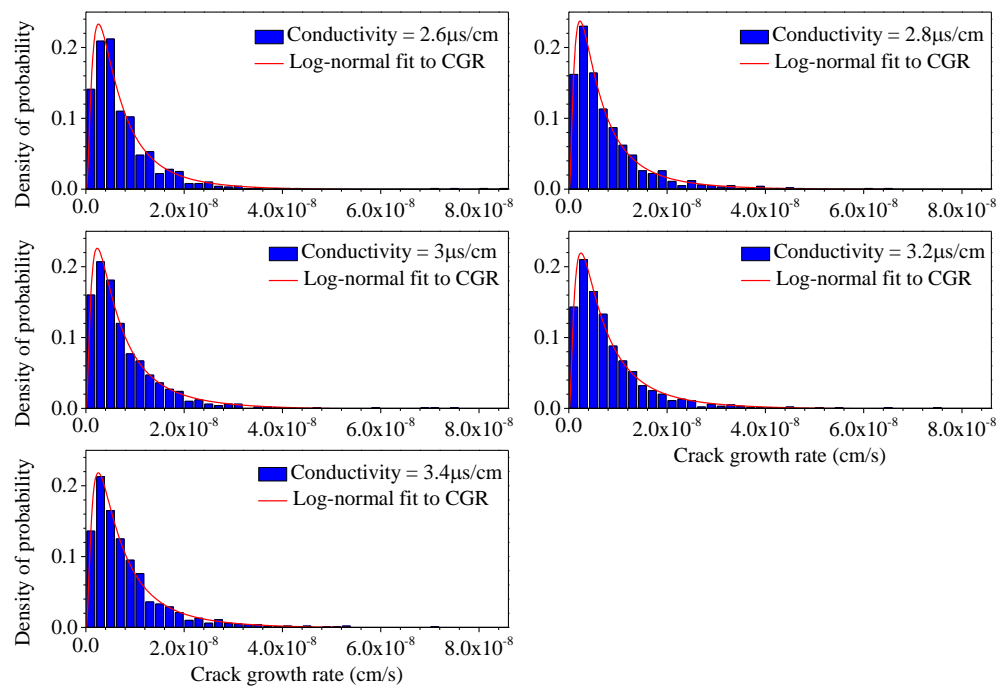

(a)

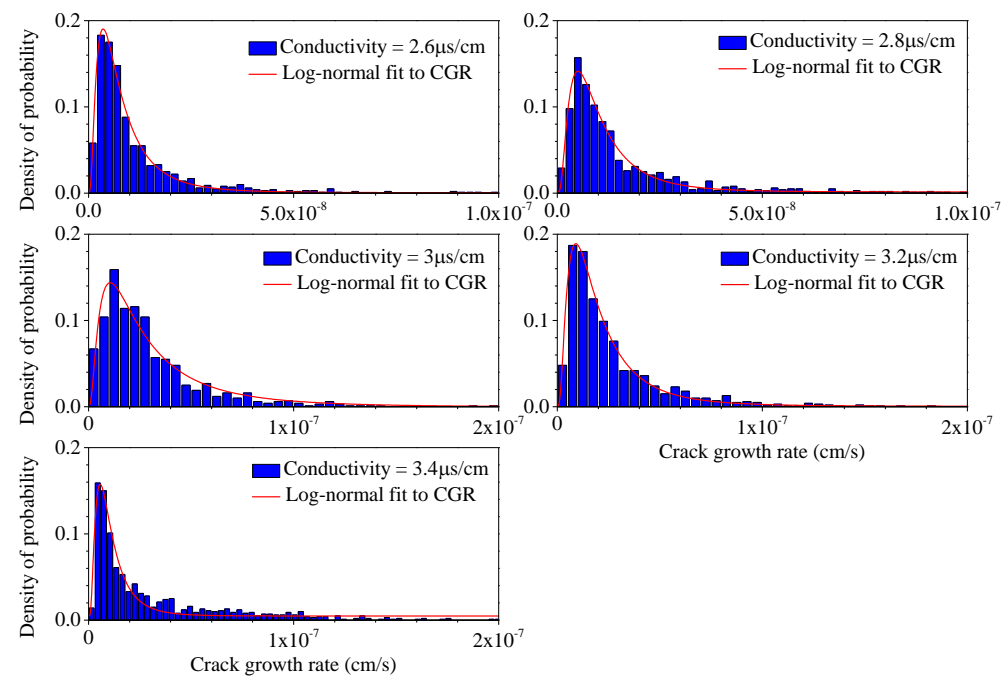

(b)

Figure IX.8. Histograms of the calculated CGRs under different conductivity compared to the fitted log-normal distributions. (a) Results from the CEFM; (b) Results from the ANN. 
Figure IX.9 shows the dependence of mean CGR on conductivity. The CEFM model predicts there is almost no effect of conductivity (between $2.6 \mu \mathrm{s} / \mathrm{cm}$ to $3.4 \mu \mathrm{s} / \mathrm{cm}$ ) on CGR, while the ANN predict a small increase in CGR with increasing conductivity. In general, the results calculated from both the CEFM model and the ANN agree well.

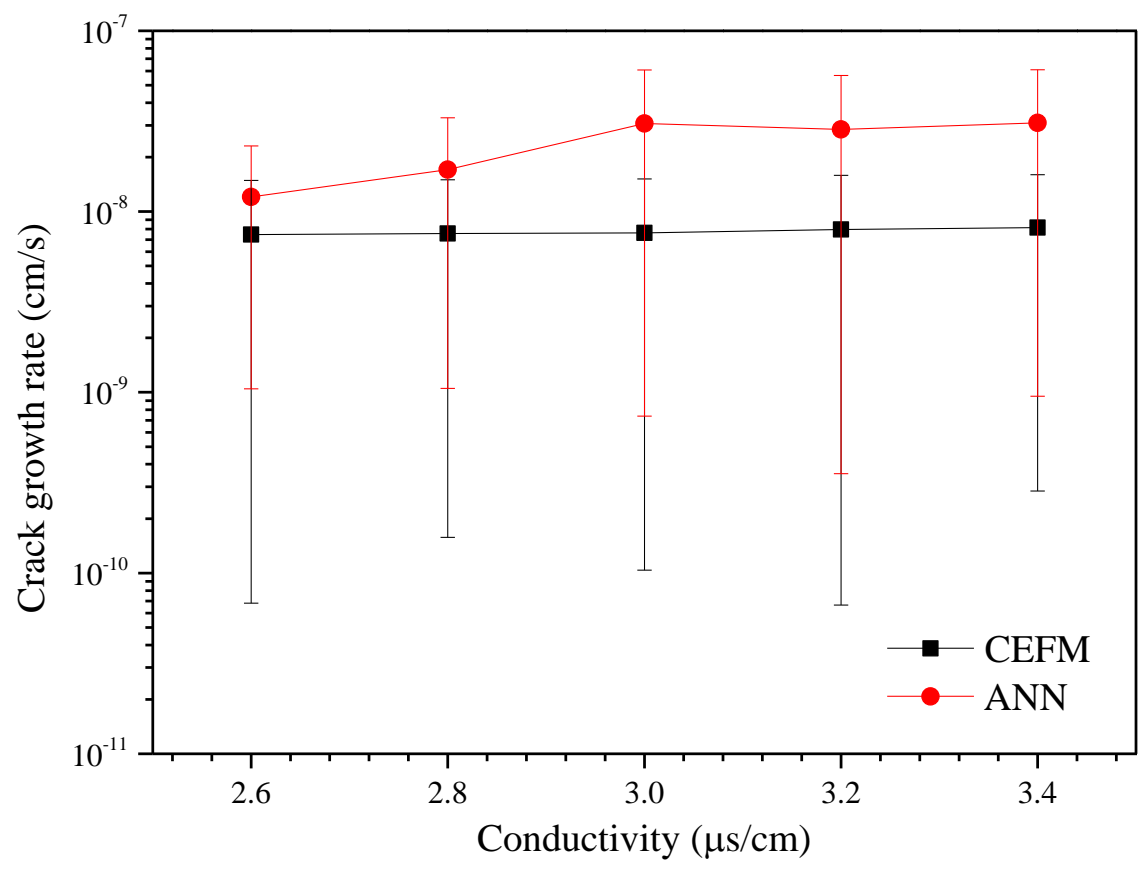

Figure IX.9. Dependence of the mean value of CGR on conductivity calculated using both the CEFM and ANN.

\section{Distribution of CGR with respect to the degree of sensitization (DOS)}

The IGSCC susceptibility of Type 304 SS in high-temperature water is known empirically to be a function of the degree of grain boundary sensitization. (i.e., the extent of chromium depletion in the matrix adjacent to grain boundary due to $\mathrm{Cr}_{23} \mathrm{C}_{6}$ precipitation on the boundary). The degree of sensitization, DoS, is commonly determined using the double loop, electrochemical potentio-kinetic reaction method, DL-EPR, and is expressed as an "EPR" or "DoS" number with units of $\mathrm{C} / \mathrm{cm}^{2}$. In a previous paper [7], based on the predictions of the ANN, we proposed a calibration equation to account for the effect of sensitization on CGR in the CEFM. Here, we use 
both the ANN and the CEFM to study distribution characteristics of the CGR when the EPR (DoS) is normally distributed, as shown in Figure IX.10a and IX.10b, respectively. As can be seen from Figure IX.10a and IX.10b, the distribution of CGR mainly concentrate on the left side of the graph when the EPR is $10 \mathrm{C} / \mathrm{cm}^{2}$, however, as the EPR increase to $20 \mathrm{C} / \mathrm{cm}^{2}$ the distribution of CGR moves to the right and becomes wider, which means the standard deviation increases with the increase in the CGR. The fitted log-normal distribution lines are also shown in Figures IX.10a and IX.10b, which give an adequate description of the distribution of CGR.

The dependence of the mean CGR on EPR is shown in Figure IX.11. An increase of CGR with increasing EPR can be seen from Figure IX.11; this agrees well with the findings of other studies [29].

\section{IX.4. Summary and conclusion}

The distributions in the CGR in Type 304 SS in high-temperature water systems have been modeled using the CEFM and an ANN. Six independent variables $\left(K_{I}, T\right.$, Conductivity, EPR, $\mathrm{ECP}$, and $\mathrm{pH}$ ) were chosen as input parameters to these two models. All of the input parameters were assigned normal distributions, and the distributions in the CGR under different conditions were calculated. Both the CEFM and the ANN account for the dependence of the variables of the CGR, and good agreement between the results from these two models have been obtained. Given that the input parameters obey normal distribution, the CGR distribution closely approximates that of a log-normal distribution. 


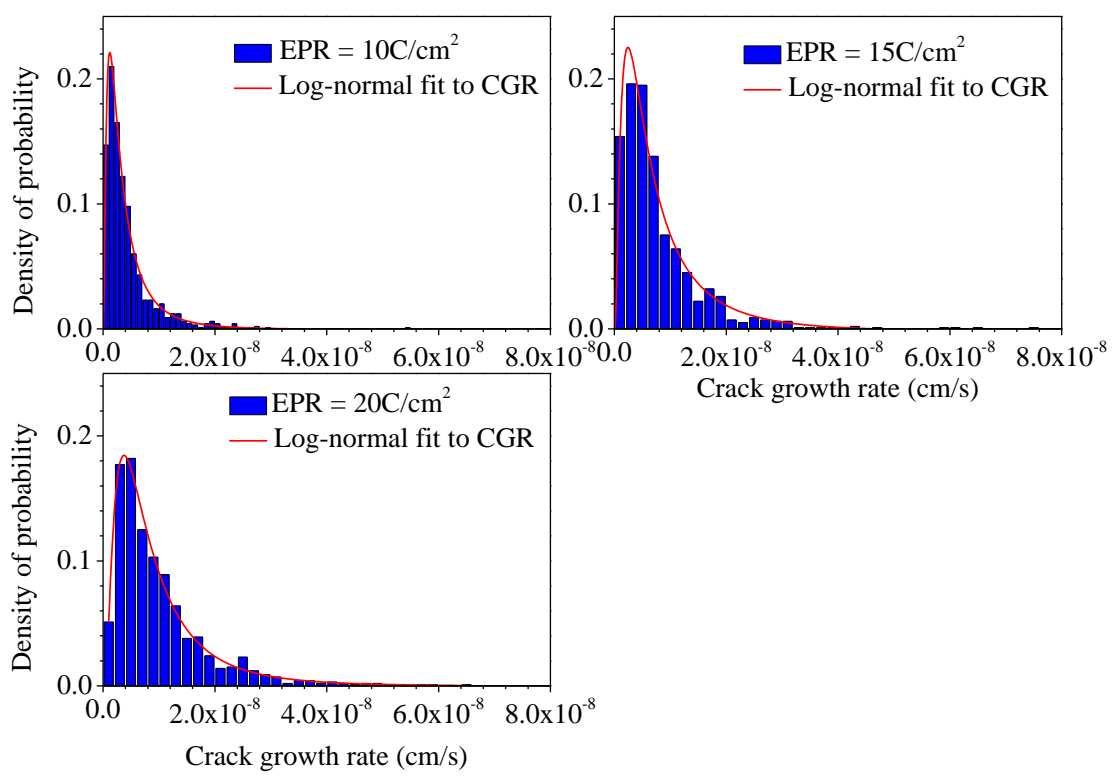

(a)

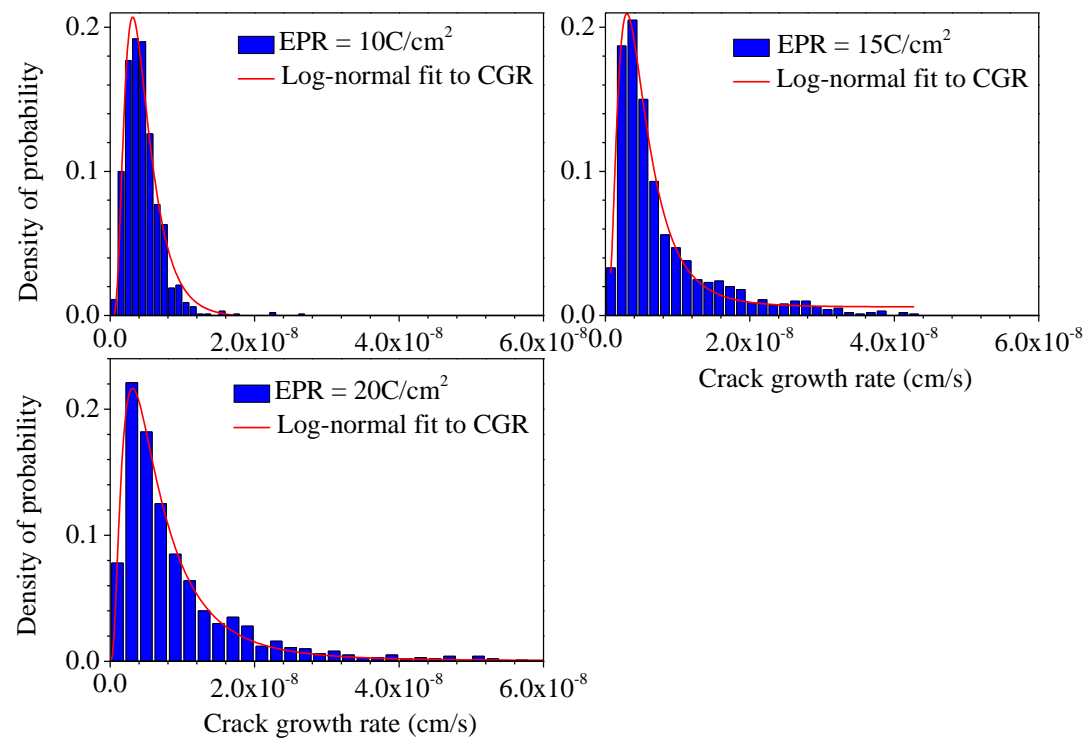

(b)

Figure IX.10. Histograms of the calculated CGRs under different EPR compared to the fitted lognormal distributions. (a) Results from the CEFM; (b) Results from the ANN. 


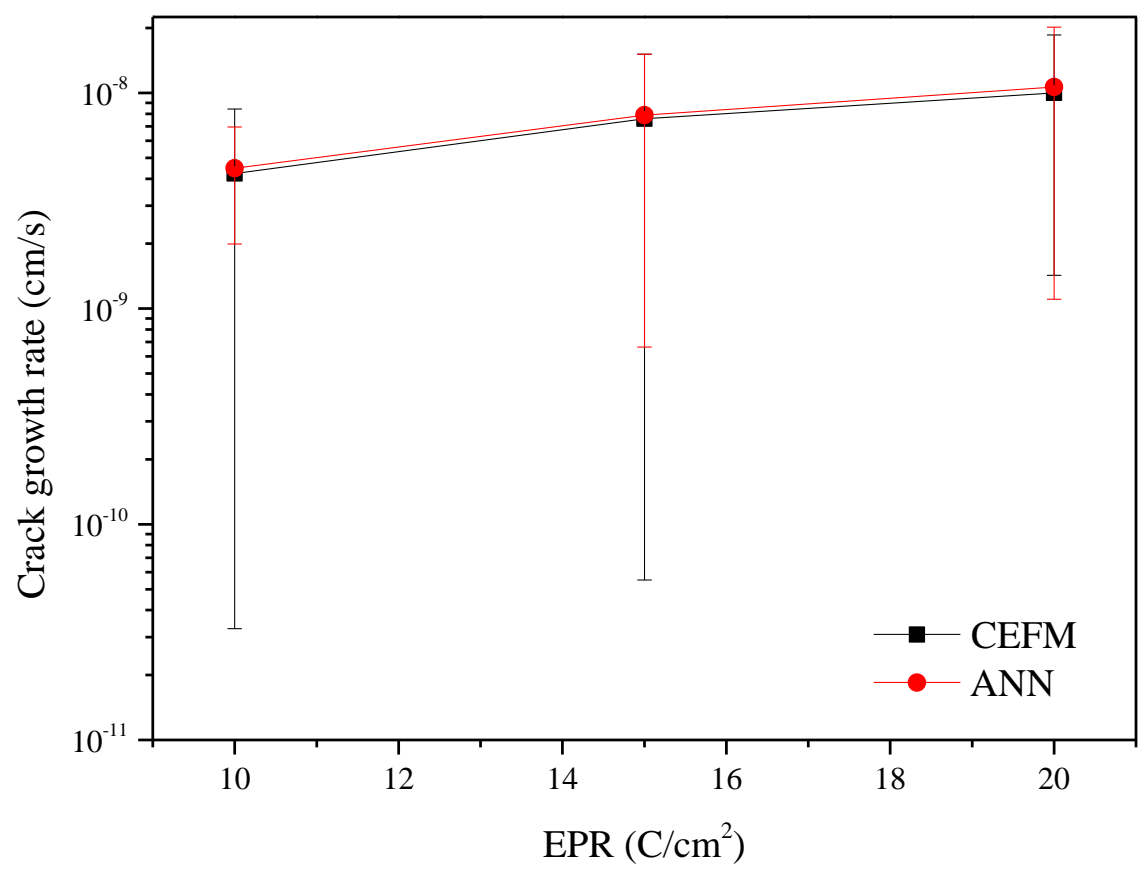

Figure IX.11. Dependence of the mean value of CGR on EPR calculated using both the CEFM and ANN.

\section{IX.5. References}

[1] D.D. Macdonald and M. Urquidi-Macdonald, Electrochemistry of nuclear reactor coolant circuits, Wiley Online Library (2007).

[2] V. Nevdacha and Y. Yagozinskyy, Dynamic strain aging of deformed nitrogen-alloyed AISI 316 stainless steels, Corrosion Issues in Light Water Reactors: Stress Corrosion Cracking, 103, (2007).

[3] D.D. Macdonald and M. Urquidi-Macdonald, Corros. Sci., 32, 51-81 (1991).

[4] D.D. Macdonald and M. Urquidi-Macdonald, An advanced coupled environment fracture model for predicting crack growth rates in Parkins Symposium on Fundamental Aspects of Stress Corrosion Cracking, 443-455 (1991).

[5] D.D. Macdonald, P.C. Lu, M. UrquidiMacdonald and T.K. Yeh, Corrosion, 52, 768-785 (1996). 
[6] M. Vankeerberghen and D.D. Macdonald, Corros. Sci., 44, 1425-1441 (2002).

[7] J. Shi, J. Wang, and D.D. Macdonald, Corros. Sci., 89, 69-80 (2014).

[8] S. Jain, F. Ayello, J.A. Beavers, and N. Sridhar, Development of a probabilistic model for stress corrosion cracking of underground pipelines using Bayesian networks: a concept, in: 2012 9th International Pipeline Conference, American Society of Mechanical Engineers, Calgary, Alberta, Canada, 615-625 (2012).

[9] S. Jain, J.A. Beavers, F. Ayello, and N. Sridhar, Corrosion, 2013, (2013).

[10] C. Priya, K.B. Rao, M.B. Anoop, N. Lakshmanan, V. Gopika, H.S. Kushwaha, and R.K. Saraf, P. I. Mech. Eng. C. J. Mec., 219, 607-624 (2005).

[11] A. Guedri, Int. J. Pres. Ves. Pip., 111, 1-11 (2013).

[12] M.B. Anoop, K. Balaji Rao, and N. Lakshmanan, Int. J. Pres. Ves. Pip., 85, 238-247 (2008).

[13] P.L. Andresen and F.P. Ford, Int. J. Pres. Ves. Pip., 59, 61-70 (1994).

[14] S.K. Lee, P. Lv, and D.D. Macdonald, J. Solid State Electr., 17, 2319-2332 (2013).

[15] W.Y. Maeng, and D.D. Macdonald, Corros. Sci., 50, 2239-2250 (2008).

[16] S.K. Lee and D.D. Macdonald, Stress Corrosion Cracking of Alloy 22, in 224th ECS Meeting, The Electrochemical Society, San Francisco, CA, 1781-1781 (2013).

[17] J. Shi, B. Fekete, J. Wang, and D.D. Macdonald, Corros. Sci., 193, 58-67 (2018).

[18] S. Lee and D.D. Macdonald, Development of semi-elliptical surface cracks in lightly sensitized Al-Mg alloys, in 224th ECS Meeting, The Electrochemical Society, San Francisco, CA, 1780-1780 (2013).

[19] J. Shi, J. Wang, and D.D. Macdonald, Corros. Sci., 89, 69-80 (2014).

[20] D.D. Macdonald, Corrosion, 48, 194-205 (1992).

[21] M.P. Manahan, D.D. Macdonald, and A.J. Peterson, Corros. Sci., 37, 189-208 (1995).

[22] M. Gomez-Duran and D.D. Macdonald, Corros. Sci., 45, 1455-1471 (2003).

[23] M. Gomez-Duran and D.D. Macdonald, Corros. Sci., 48, 1608-1622 (2006).

[24] S. Liu and D.D. Macdonald, Corrosion, 58, 835-845 (2002). 
[25] G.R. Engelhardt, D.D. Macdonald and M. Urquidi-Macdonald, Corros. Sci., 41, 2267-2302 (1999).

[26] P. Andresen, Corrosion, 49, 714-725 (1993).

[27] BWRVIP-226: BWRVIP Vessel and Internals Project, Feasibility Evaluation of BWR Hydrogen Injection for ECP Reduction During Startup, EPRI,Palo Alto, CA:1019074, (2009).

[28] H.S. Kwon, A. Wuensche, and D.D. Macdonald, Corrosion, 56, 482-491 (2000).

[29] S. Roychowdhury, V. Kain, S. Neogy, D. Srivastava, G.K. Dey, and R.C. Prasad, Acta. Mater., 60, 610-621 (2012).

\section{Experimental measurement of key model parameters}

\section{X.1. Introduction}

\section{X.1.1. Background and literature survey}

Alloy $600(\mathrm{Ni}-16 \mathrm{Cr}-9 \mathrm{Fe})$ as the standard material of steam generator tubing has been widely applied in the primary coolant circuit of pressurized water reactor (PWR) primary coolant circuit owing to its good resistance to general corrosion and pitting, which is attributed to the protective nature of the substituted, defective chromium oxide that constitutes the barrier layer of the passive film. However, the susceptibility of milled-annealed Alloy 600 to stress corrosion cracking (SCC) in primary coolant circuits of nuclear reactors is a still ongoing issue [1-7]. The SCC susceptibility, to a certain degree, depends upon the electrochemical properties of passive film formed on alloys, including not only the electronic character, but also the growth/destruction rates driven by the environmental potential, etc., and the electrochemical corrosion potential (ECP) [2,8-12]. To avoid and mitigate SCC damage, it is very important to understand the correlations between the protectiveness and the properties of the passive film, especially the kinetics of some atomic-scale processes occurring at the substrate/film interface contributing to the growth of the passive film (reflecting the ability for maintaining the film protectiveness). The composition, microstructure, and electronic properties of the passive film formed on Alloy 600 in high temperature have been investigated by several researchers [2,5,7,9,13-23]. These reports generally conclude that the passive film of Alloy 600 has a duplex structure, comprising a 
chromium-rich inner (barrier) layer and a nickel-ferrite rich outer layer. The very thin and compact barrier layer, which grows into the substrate alloy, acts like physico-electrochemical barrier against the species transfer between the alloy substrate and the solution, thus strengthening the corrosion resistance of the alloy. On the other hand, the precipitated, thick, and non-uniform outer layer greatly dominates the total film thickness under normal operating conditions. Hamdani [7] reported that the Cr-enriched inner layer formed on Alloy 600 in steam exhibited a p-Type semiconductivity and that the cation vacancy was a dominant point defect, while Montemor et al. [19] suggested that the outer layer of the passive film had a control on the semi-conductivity of Alloy 600. Contrariwise, Loucif et al. [18] found n-Type properties of the passive film on Alloy 600. Some researchers also suggested that for $\mathrm{Cr}$-rich oxides, the $\mathrm{Cr}$ interstitial was a dominant defect at a low oxygen concentration, while the Cr vacancy was a dominant defect at a high oxygen concentration [20-23]. The semi-conductivity of the passive film formed on the metal or alloy depends on which point defect within the film is predominant among the cation interstitials, cation vacancies, and/or oxygen vacancies. Nevertheless, the defect structure of the passive film forms on Alloy 600 has not been definitively established, but it may be represented as $(\mathrm{Cr}, \mathrm{Fe}, \mathrm{Ni})_{2 \pm \mathrm{x}} \mathrm{O}_{3-\mathrm{y}}$ where $x$ and $y$ define the non-stoichiometry of the oxide. The Fe and Ni content is considered to be due to interstitials moving through the barrier layer from the metal to the solution. Thus, if the metal stoichiometry is $2+x$, the barrier layer is invariably $n$-Type because both metal interstitials and oxygen vacancies are electron donors, but if it is 2-x, the barrier layer may be n-Type if 2-y > $2-x$ or p-Type if 2-x > 2-y. It is recommended that the ECP value of Alloy 600 in PWR should be maintained between $-0.835 \mathrm{~V}_{\mathrm{SHE}}$ and $-0.5 \mathrm{~V}_{\mathrm{SHE}}$ to prevent hydrogen-induced inter-granular cracking (IGSCC) [24] and SCC [25]. The protective character of the passive film that forms on Alloy 600 and the value of ECP can be adjusted directly by controlling the electrochemical corrosion environment [26,27].

Many failures have occurred of steam generator (SG) tubes fabricated from Alloy 600 in PWRs because of SCC [3,28]. Comparatively, the higher chromium content of Alloy 690 $(\mathrm{Ni}-30 \mathrm{Cr}-10 \mathrm{Fe})$ is now being frequently used as the SG tube material as an alternative to Alloy 600 , owing to the better SCC resistance [1,29]. No material failures of Alloy 690 in operating, commercial PWRs have been reported to date, although there are reports of Alloy 690 being vulnerable to SCC in laboratory studies [30,31]. The composition and structure of the passive film formed on Alloy 690 in the PWR primary environment have been systematically studied by many 
researchers $[13,19,32-35]$. This previous work generally reports that the film has a bilayer structure with a nickel-ferrite rich outer layer and a chromium-rich inner layer, which may be described as a highly point-defective but substituted chromic oxide, $\mathrm{Fe}_{u} \mathrm{Ni}_{v} \mathrm{Cr}_{2+x} \mathrm{O}_{3-y}$, containing significant amounts of $\mathrm{Fe}, \mathrm{Cr}$, and $\mathrm{Ni}$, probably as interstitials with $\mathrm{Cr}$ also being the lattice cationic species. These studies conclude that the chromium oxide barrier layer dominates the semi-conductivity of the passive film on Alloy 690, while the porous outer layer plays an insignificant role in determining the total film thickness.

Austenitic stainless steels are widely applied as structural materials in PWR nuclear power plants owing to good mechanical and corrosion properties [36-37]. However, localized corrosion including SCC is the problem for the application. The nucleation and propagation of SCC are influenced by the properties of the passive film significantly. Several researchers [38-43] found that the passive films formed on stainless steels have a bi-layer structure with a $\mathrm{Cr}$-rich inner layer and a Fe-rich outer layer. Sun et al. [36] found the passive film formed on Type 304 SS shows different semiconductor behavior in different potential ranges. They also suggested that the film formed on Type $304 \mathrm{SS}$ at $25^{\circ} \mathrm{C}$ is a single layer structure, while film formed between $100{ }^{\circ} \mathrm{C}$ and $300{ }^{\circ} \mathrm{C}$ presents a bi-layer structure. However, Nicic et al. [44] reported that the passive film formed on Type 316L SS in borate buffer solution is n-Type in electronic character and no p-Type semiconductor behavior was found.

As an electrochemical process, SCC is always significantly affected by the properties of the passive film on the alloy, although only a few SCC mechanisms have been proposed that explicitly involve the passive film on surfaces external to the crack in the crack propagation process [2,8-9,45-46]. One model, the Coupled Environment Fracture Model (CEFM) [10-11,47] that explicitly attributes the impedance of the external surface in controlling the coupling current that, in turn, determines the crack growth rate (CGR), such that a lower impedance results in a higher coupling current and hence in a higher CGR. Therefore, it is important to fully characterize the properties of the passive film to understand and ultimately to prevent the evolution of SCC damage. The Point Defect Model (PDM), which was developed by Macdonald and co-workers [17,48-55] for describing the growth and breakdown of passive films at the atomic scale, has successfully accounted for the experimental data from studies on many metals and alloys. In the present work, the Mixed Potential Model (MPM), including quantum mechanical tunneling of 
charge carriers across the barrier layer to the oxygen reduction reaction center [10,56-57], which incorporates the PDM, is used to interpret the experimental electrochemical data. Thus, the MPM comprises the PDM to describe the partial anodic process of passive dissolution of Alloy 600, Alloy 690, Type 304L SS and Type 316L SS, and the Butler-Volmer equation is employed to account for the partial cathodic process of oxygen reduction, with the net current being given by the sum of the two partial currents. As noted above, the passive film on each alloy has a bilayer structure, including the thin inner barrier and porous, thicker outer layer. The inner layer contains high concentrations of point defects (cation and anion vacancies, and cation interstitials), which impart semiconductor properties to the barrier layer (bl). The bl grows into metal substrate directly via the generation of oxygen vacancies at the metal/barrier layer $(\mathrm{m} / \mathrm{bl})$ interface while the outer layer (ol) grows outward from the barrier/outer layer (bl/ol) interface via the hydrolysis and precipitation of oxides, oxyhydroxides, and hydroxides of cations (principally $\mathrm{Fe}$ and $\mathrm{Ni}$ ) that are transmitted through the bl. As shown elsewhere, the bl generally dominates the passivity of metals or alloys, except for the valve metals (e,g., $A l, T i, T a, Z r, N b, W$ ) where a highly-resistive, outer layer dominates the impedance of the interface [48-54]. The point defects are generated or annihilated at the boundaries of the barrier layer, that is, at the $\mathrm{bl} / \mathrm{ol}$ interface or at the $\mathrm{m} / \mathrm{bl}$ interface. The outer layer, which is generally non-defective and hence of poor electronic and ionic conductivity, grows by the precipitation of cations that are transmitted through the barrier layer and react with anions or the solvent $\left(\mathrm{H}_{2} \mathrm{O}\right)$ in the solution. Because the outer layer is generally porous, and hence is electrolytically-conductive, its contribution to the impedance of the passivity of the passive film is frequently ignored for alloys [35,48], but recent work [51,53,58-59] shows that a significant potential drop may exist across the ol that can have a significant impact on the kinetics of the electrochemical reactions occurring at the bl interfaces, depending upon the substrate.

Stress corrosion cracking in reactor materials has been demonstrated both theoretically and experimentally to be a sensitive function of the ECP [12]. Under open-circuit conditions, the open circuit potential (OCP) depends on the water chemistry parameters, such as the temperature, $\mathrm{pH}$, and dissolved oxygen, hydrogen peroxide, hydrogen concentration [27,60-62]. Since control of the mechanical stress is generally ineffective as a mitigation strategy, except in a few specific instances, such as the annealing of residual stress, it is critical to identify the optimal conditions of water chemistry to control the OCP and hence to minimize the SCC damage to Alloy 600, Alloy 
690, Type 304L SS, Type 316L SS components during plant operation. The OCP is the mixed potential arising from charge conservation of the combined partial cathodic (reduction of $\mathrm{H}_{2} \mathrm{O}, \mathrm{O}_{2}$, and $\mathrm{H}_{2} \mathrm{O}_{2}$ ) and anodic (metal passive dissolution) reactions. The OCP can be calculated using the MPM, which requires knowledge of the correlations of the anodic and cathodic current densities with temperature, reactant $\left(\mathrm{H}_{2}, \mathrm{O}_{2}, \mathrm{H}_{2} \mathrm{O}_{2}\right)$ concentrations, mass-transfer rates, potential, and $\mathrm{pH}$ [61,63]. At the present time, the electrochemical kinetic parameters for the derivation of these correlations can only be acquired experimentally.

Kim et al. [35] reported anodic potentiostatic polarization and electrochemical impedance spectroscopy (EIS) measurements on Alloy 600 and Alloy 690 in simulated primary water at different temperatures. Their experimental temperature was limited to $250{ }^{\circ} \mathrm{C}$, because of the restriction of Teflon (PTFE) for sample sealing (exposing only one surface) and insulation. Li et al. [64] recorded the OCP of Alloy 690 up to $300{ }^{\circ} \mathrm{C}$ with the exposure of the whole surface to the solution. They found that the OCP decreased continuously from $25^{\circ} \mathrm{C}$ to $250^{\circ} \mathrm{C}$ and then showed insignificant change to $300{ }^{\circ} \mathrm{C}$. Until now, the effects of temperature and $\mathrm{pH}$ on the electrochemical behavior of Alloy 600, Alloy 690, Type 304L SS, and Type 316L SS have not been thoroughly explored, and no comprehensive EIS or polarization measurements have been carried out at temperatures exceeding $250{ }^{\circ} \mathrm{C}$ with successful sample sealing (one uniform surface of exposure).

Although PWR primary circuits are pressurized with hydrogen, the present work on the investigation of the passive film was carried out in oxygenated environments as part of a campaign to explore the electrochemical and corrosion behaviors of reactor materials under wide ranges in the redox properties of the environment. The cathodic hydrogen electrode reactions were studied in hydrogenated solutions.

\section{X.1.2. Objectives}

In this research, an apparatus was developed from a previous design [35,65-67] with the PTFE washer for specimen sealing being replaced by PEEK (Drake Plastics Ltd) for performing experiments up to $300{ }^{\circ} \mathrm{C}$.

In order to investigate the effects of temperature and $\mathrm{pH}$ on the passive dissolution of Alloy 600 (Deutsche Nickel America), Alloy 690 (American Special Metals Corporation), Type 304L 
SS and Type 316L SS (TW Metals), the OCP, anodic potentiostatic polarization at selected anodic polarization potentials, Mott-Schottky Analysis (MSA), and EIS measurements at the OCP and $+0.3 \mathrm{~V}_{\mathrm{OCP}}(\mathrm{OCP}+0.3 \mathrm{~V})$ were carried out in three different buffer solutions containing boric acid $\left(\mathrm{H}_{3} \mathrm{BO}_{3}\right.$, Alfa Aesar) and lithium hydroxide ( $\mathrm{LiOH}$, Alfa Aesar). Solutions containing $2000 \mathrm{ppm}$ $B+2 \mathrm{ppm} L i$ and $200 \mathrm{ppm} B+2 \mathrm{ppm} L i$ were used to simulate the electrochemical corrosion environment in primary circuits of PWRs at the beginning and end of a fuel cycle, respectively [35]. A solution containing $0 \mathrm{ppm} B+2 \mathrm{ppm} L i$ was also used for investigating the effect of $\mathrm{pH}$. All solutions were oxygenated by sparging with a mixture of nitrogen and oxygen $\left(1.0 \% \mathrm{O}_{2}\right)$, the concentration of dissolved oxygen was maintained at $400 \mathrm{ppb}$. A computer code based on the MPM, combining both the PDM for anodic metal passive dissolution and the Butler-Volmer equation for the cathodic reaction, was developed to interpret the experimental EIS data. The MPM was optimized on the measured EIS data and various kinetic parameters were derived to provide an electrochemical basis for understanding the effect(s) of temperature and $\mathrm{pH}$ on corrosion behavior of the alloy, and derive the correlations of the anodic current density with the water chemistry parameters (temperature, $\mathrm{pH}$, reactant concentration, potential), which will then be used to calculate the ECP using MPM [26,63].

In order to investigate the effects of hydrogen concentration, solution temperature and $\mathrm{pH}$ on the hydrogen electrode reaction (HER) and derive the correlations of the cathodic current density with the water chemistry parameters, the cathodic and anodic potentiostatic polarization measurements are being performed on alloys in hydrogenated solutions.

\section{X.2. Experimental procedures.}

\section{X.2.1. Apparatus setup and materials preparation.}

Figures X.1 and X.2 show the schematic and real recirculating flow loop with hydrodynamic autoclave vessel (SS 316) for performing electrochemical measurements up to 300 ${ }^{\circ} \mathrm{C}$ with a volume flow velocity of $15 \mathrm{ml} / \mathrm{min}$ through the recirculation loop, respectively. 


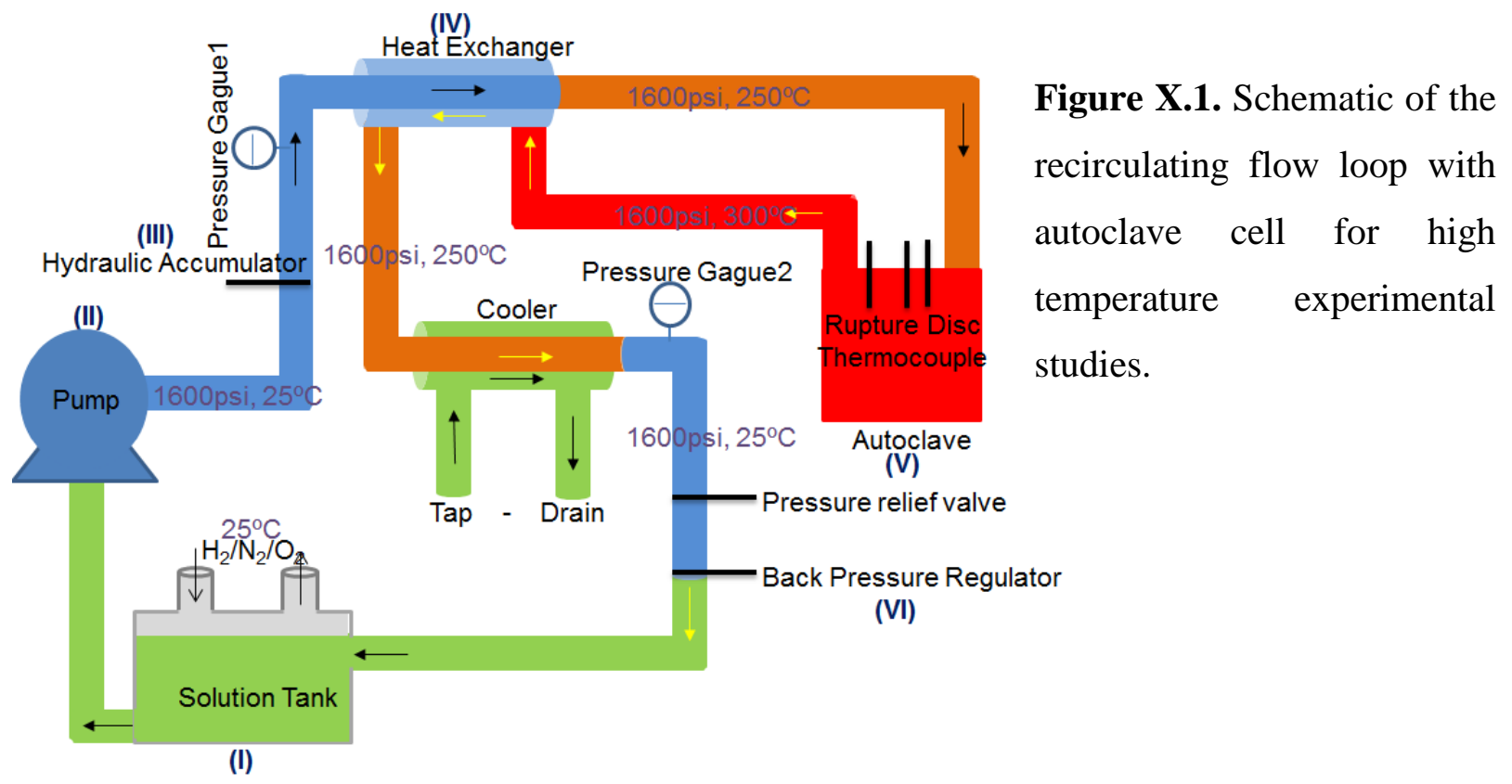

The components of the flow loop have been described systematically in Figure VI.14. The working, reference and counter electrodes were the specimen, a $W / W O_{3}$ electrode (research on the anodic metal dissolution reaction) or a Pt electrode (research on the cathodic hydrogen electrode reaction), and the flow channel, respectively. Figure X.2 shows the assembly of the working electrode. PEEK washer was used for specimen sealing in order to perform experiments up to $300{ }^{\circ} \mathrm{C}$. The Alloy 600, Alloy 690, Type 304L SS, and Type 316L SS specimens were machined into tubes (19.1 mm OD x $12.7 \mathrm{~mm}$ ID x $6.4 \mathrm{~mm}$ length). The chemical compositions of alloys are given in Table X.1. The specimens were ground using silicon carbide $(\mathrm{SiC})$ papers up to 2500 grit and installed into a holder with an exposed surface area of $3.8 \mathrm{~cm}^{2}$ (Figure X.2). Two specimens were electrically isolated from each other using PEEK washers and compressed axially by a CONAX metal ferrule, compression sealing assembly to seal all the interfaces tightly. The compressing force between the specimen and PEEK washer can be increased by tightening the 316 SS cone cap to obtain a good seal. The conductive wires for specimens were insulated by shrinkable PTFE tubing, which, because the tubing is not under high mechanical loading, does not flow at even $300{ }^{\circ} \mathrm{C}$ but still provides effective insulation. 


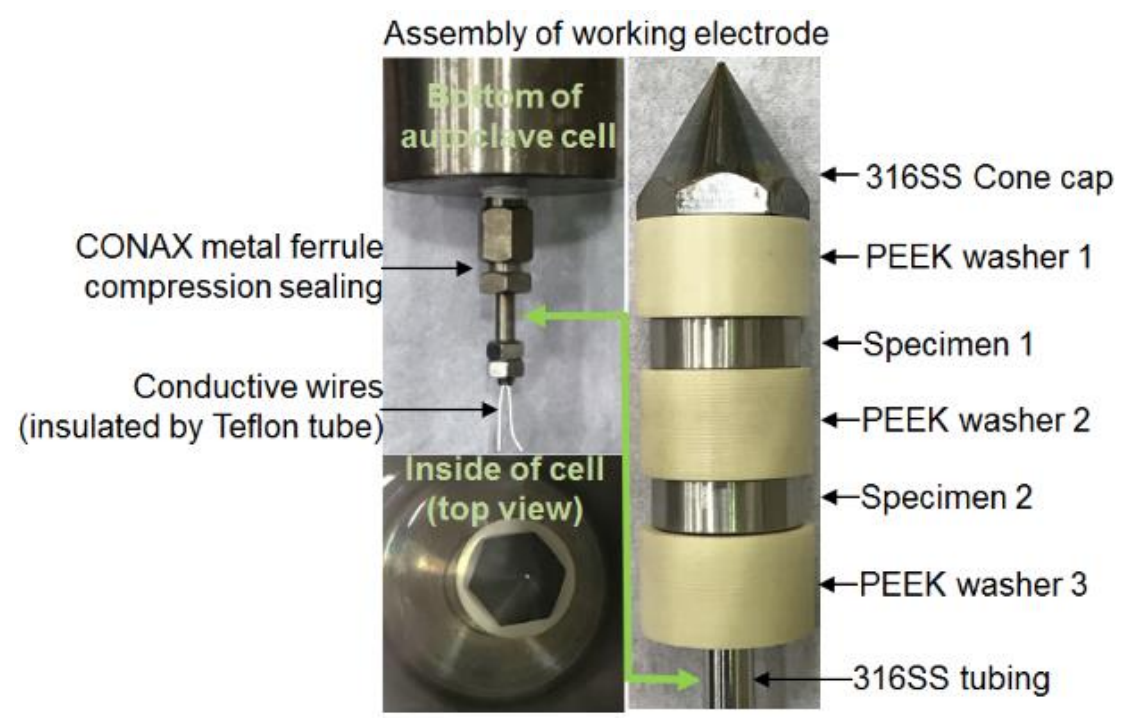

Figure X.2. The assembly of the working electrode used for experimental measurements up to $300{ }^{\circ} \mathrm{C}$.

Table X.1. Chemical compositions of Alloy 600, Alloy 690, Type 304L SS and Type 316L SS (wt.\%).

\begin{tabular}{l|l|l|l|l|l|l|l|l}
\hline Alloy & $\mathrm{Ni}$ & $\mathrm{Cr}$ & $\mathrm{Fe}$ & $\mathrm{Mn}$ & $\mathrm{Si}$ & $\mathrm{C}$ & $\mathrm{Cu}$ & $\mathrm{S}$ \\
\hline 600 & Balance & 16.04 & 9.02 & 0.20 & 0.31 & 0.071 & 0.045 & 0.001 \\
\hline 690 & Balance & 29.15 & 10.0 & 0.11 & 0.22 & 0.03 & 0.02 & 0.001 \\
\hline TYPE & 8.13 & 18.67 & Balance & 0.67 & 0.34 & 0.02 & - & 0.002 \\
\hline TYPE & 10.06 & 16.18 & Balance & 0.90 & 0.38 & 0.021 & - & 0.001 \\
\hline
\end{tabular}

Experimental measurements for the anodic metal dissolution investigation were carried out in oxygenated borate buffer solution containing $\mathrm{H}_{3} \mathrm{BO}_{3}(2000,200,0 \mathrm{ppm} \mathrm{B})+\mathrm{LiOH}(2 \mathrm{ppm} \mathrm{Li})$ at $150{ }^{\circ} \mathrm{C} 200{ }^{\circ} \mathrm{C}, 250{ }^{\circ} \mathrm{C}$, and $300{ }^{\circ} \mathrm{C}$. The solution in the reservoir was initially purged by sparging with pure $N_{2}$ gas $(99.998 \%$, Praxair) through the solution for over $24 \mathrm{~h}$ at room temperature and 1 atmosphere before the solution was continuously pumped into the autoclave. The dissolved $\mathrm{O}_{2}$ concentration $(400 \mathrm{ppb})$ in the solution that was established by sparging with a $\mathrm{O}_{2}+\mathrm{N}_{2}$ mixture $\left(1.0 \% \mathrm{O}_{2}\right)$ and $\left[\mathrm{O}_{2}\right]$ was continuously measured in return to the reservoir using an AMTAST Portable Dissolved Oxygen and Temperature Meter. The equilibrium potentials of $W / W O_{3}$ reference electrode half-cell reaction $\left(\mathrm{WO}_{3}+6 \mathrm{H}^{+}+6 e^{-}=W+3 \mathrm{H}_{2} \mathrm{O}\right)$ at different temperatures were calculated according to the Nernst Equation: 
$E_{T}^{e}=E_{T}^{0}+2.303 \cdot \frac{R T}{n F} \cdot \lg \frac{\left\{H^{+}\right\}^{6}}{\left\{H_{2} O\right\}^{3}}$

where $E_{T}^{e}$ is the equilibrium potential of the electrode at a temperature $T$ (Kelvin), $E_{T}^{0}$ is the standard potential at temperature $T, E_{T}^{0}=-\Delta G^{0} / 6 F, \Delta G^{0}$ is the change of standard Gibbs energy of the full-cell reaction $\left(\mathrm{WO}_{3}+3 \mathrm{H}_{2}=W+3 \mathrm{H}_{2} \mathrm{O}\right)$, which was calculated using HSC-5 Chemistry software, $F$ is Faraday's constant (the charge on a mole of electrons, $95484.56 \mathrm{C} \cdot \mathrm{mol}^{-}$ $\left.{ }^{1}\right), R$ is the ideal gas constant $\left(8.314 \mathrm{~J} \cdot \mathrm{mol}^{-1} \cdot \mathrm{K}^{-1}\right), n$ is the number of moles of electrons transferred in the half-cell reaction, $\left\{\mathrm{H}^{+}\right\}$is the activity of concentration of hydrogen ions in solution. $\left\{\mathrm{H}_{2} \mathrm{O}\right\}$ is the activity of water, which is defined as approximately as being 1.0 because the solution is dilute.

Equation X.1 is simplified as:

$E_{T}^{e}=E_{T}^{0}-2 \cdot 10^{-4} \cdot T \cdot p H$

where the $p H$ values of the solutions at high temperatures were calculated using the $p H$ code pH_Calc that was developed by Macdonald and co-workers. Table X.2 summarizes the calculated $p H$ values and equilibrium potentials of the $W / W O_{3}$ reference electrode at different temperatures.

Table X.2. The calculated $p H$ values of solution and the calculated standard $\left(E_{T}^{0}\right)$ and equilibrium $\left(E_{T}^{e}\right)$ potentials of $\mathrm{W} / \mathrm{WO}_{3}$ reference electrode as a function of temperature.

\begin{tabular}{l|l|l|l|l}
\hline$T\left({ }^{0} \mathrm{C}\right)$ & $p H$ & $\Delta G^{0}(\mathrm{~J})$ & $E_{T}^{0}\left(\mathrm{~V}_{\mathrm{SHE}}\right)$ & $E_{T}^{e}\left(\mathrm{~V}_{\mathrm{SHE}}\right)$ \\
\hline 150 & 5.91 & 78801 & -0.1361 & -0.638 \\
200 & 5.98 & 88300 & -0.1525 & -0.720 \\
250 & 6.20 & 97292 & -0.1681 & -0.819 \\
300 & 6.71 & 105740 & -0.1827 & -0.954 \\
\hline
\end{tabular}

For the research on hydrogen electrode reactions, a platinum plate was used for the reference electrode. The solution was deoxygenated by the mixed gas of $95 \% \mathrm{Ar}+5 \% \mathrm{H}_{2}$ for more than $12 \mathrm{~h}$ before the solution was continuously fed into the autoclave cell. The mixed gas $\left(95 \% \mathrm{Ar}+5 \% \mathrm{H}_{2}\right)$ pressure was adjusted to $0.068 \mathrm{~atm}, 0.68 \mathrm{~atm}$, and $6.8 \mathrm{~atm}$, respectively, to investigate the effect of $H_{2}$ concentration on the HER. The dissolved hydrogen concentration $m_{H_{2}}^{T}$ (Table X.3) was calculated according to Henry's law: 
$m_{H_{2}}^{T}=K_{H_{2}}^{T} \cdot f_{H_{2}}^{T}=K_{H_{2}}^{T_{0}} \cdot f_{H_{2}}^{T_{0}}$

where $T$ is temperature (K), $K_{H_{2}}^{T}$ is Henry's law constant $(\mathrm{mol} / \mathrm{kg} / \mathrm{atm}), f_{\mathrm{H}_{2}}^{T}$ is fugacity of $\mathrm{H}_{2}$ at temperature $T$ (atm), $T_{0}$ is $298.15 \mathrm{~K}, K_{H_{2}}^{T_{0}}$ is Henry's law constant of $H_{2}$ in the water at $298.15 \mathrm{~K}$, which is $7.8 \times 10^{-4} \mathrm{~mol} / \mathrm{l} / \mathrm{atm}[68], f_{H_{2}}^{T_{0}}$ is fugacity of $H_{2}$ at $298.15 \mathrm{~K}(\mathrm{~atm})$.

Table X.3. Dissolved hydrogen concentration in a solution containing $0 \mathrm{ppm} \mathrm{B}+2 \mathrm{ppm} \mathrm{Li}$.

\begin{tabular}{lll}
\hline Mixed gas $\left(95 \% \mathrm{Ar}+5 \% \mathrm{H}_{2}\right)(\mathrm{atm})$ & $\mathrm{H}_{2}$ gas pressure $(\mathrm{atm})$ & Dissolved $\mathrm{H}_{2}(\mathrm{~mol} / \mathrm{l})$ \\
0.068 & 0.0034 & $2.65 \times 10^{-6}$ \\
0.68 & 0.034 & $2.65 \times 10^{-5}$ \\
6.8 & 0.34 & $2.65 \times 10^{-4}$ \\
\hline
\end{tabular}

The equilibrium potential of the hydrogen electrode reaction $\left(2 H^{+}+2 e^{-} \rightleftharpoons H_{2}\right)$ was calculated based on the Nernst equation:

$E_{T}^{e}=E_{T}^{0}-\frac{2.303 R T}{2 F} \cdot \lg f_{H_{2}}^{T}-\frac{2.303 R T}{F} \cdot p H$

where $E_{T}^{e}$ is the equilibrium potential of HER in the given environment $(\mathrm{V}), E_{T}^{0}$ is the standard quilibrium potential of HER at temperature T (zero), $R$ is the ideal gas constant $\left(8.314 \mathrm{~J} \cdot \mathrm{mol}^{-1} \mathrm{~K}^{-}\right.$ ${ }^{1}$ ), $F$ is Faraday's constant (the charge on a mole of electrons, $96485 \mathrm{C} / \mathrm{mol}$ ), $p H$ is the solution $p H$.

$f_{H_{2}}^{T}=\frac{m_{H_{2}}^{T}}{K_{H_{2}}^{T}}$

$K_{H_{2}}^{T}=K_{H_{2}}^{T_{0}} \cdot \exp \left[\frac{-\Delta_{\text {sol }} H}{R}\left(\frac{1}{T}-\frac{1}{T_{0}}\right)\right]$

where the value of $\frac{-\Delta_{\text {sol }} H}{R}($ in $\mathrm{K})$ is 500 [68].

Table X.4 shows the calculated equilibrium potentials of HER at different temperatures $\left(150,200,250\right.$ and $\left.300{ }^{\circ} \mathrm{C}\right)$ in solutions of $2000 \mathrm{ppm} \mathrm{B}+2 \mathrm{ppm} \mathrm{Li,} 200 \mathrm{ppm} \mathrm{B}+2 \mathrm{ppm} \mathrm{Li}$, 0 ppm $B+2$ ppm $L i$ with different hydrogen concentrations of $2.65 \times 10^{-6}, 2.65 \times 10^{-5}, 2.65 \times$ $10^{-4}, 2.65 \times 10^{-3} \mathrm{~mol} / \mathrm{l}$. 


\section{X.2.2. Electrochemical measurements.}

The electrochemical measurements of the OCP, anodic potentiostatic polarization, MSA, and EIS were performed in oxygenated solutions containing $2000 \mathrm{ppm} \mathrm{B}+2 \mathrm{ppm} L i, 200 \mathrm{ppm} B$ $+2 \mathrm{ppm} L i$, and $0 \mathrm{ppm} B+2 \mathrm{ppm} L i$ at $150{ }^{\circ} \mathrm{C}, 200{ }^{\circ} \mathrm{C}, 250{ }^{\circ} \mathrm{C}$, and $300{ }^{\circ} \mathrm{C}$ using a Gamry instrument (PCI4), to study the influence of temperature and $\mathrm{pH}$ on the passivity of Alloy 600, Alloy 690, Type 304L SS and Type 316L SS. The logarithm of the steady-state passive current density was recorded as a function of the anodic polarization potentials $(+0.1,+0.2,+0.3 \mathrm{vs}$. OCP $)$. Each potential was held for 20 to $60 \mathrm{~min}$ for steady-state to as indicated by a constant current.

MSA was performed to study the electronic characteristics of the passive films formed on alloys at $+0.3 \mathrm{~V}_{\text {OCP }}(+0.3$ vs. OCP) since the capacitance behavior of a passive film/solution interface is similar to that of a semiconductor/solution interface. It is assumed that the capacitance of Helmholtz double layer $\left(C_{d l}\right)$ is much larger than the capacitance of the "space-charge region" of the film $\left(C_{s c}\right)$, hence the measured capacitance $(C)$ of film/solution interface layer is equal to $C_{s c}$. A sinusoidal excitation (amplitude of $10 \mathrm{mV}$ ) at a constant frequency of $0.5 \mathrm{kHz}$ and a potential step rate of $3.6 \mathrm{mV} / \mathrm{s}$ in the cathodic direction from the film formation potential $(+0.3$ $\mathrm{V}_{\mathrm{OCP}}$ ) was applied. This procedure is adopted to render the MSA into better compliance with the Mott-Schottky theory (MST).

Thus, by making capacitance measurements "on-the-fly" as the potential is stepped in the negative direction the thickness of the barrier layer can be held constant and the crystallographic defect structure can be "frozen-in", so that the change in capacitance with voltage is due only to the electronic defect structure, in compliance with MST that assumes that the dopant level is fixed and is not a function of voltage. According to MST, the effect of the applied potential on the measured capacitance $(C)$ can be expressed as [69-71]:

$C^{-2}=\frac{2}{q e e_{0} N}\left(V-V_{f b}-\frac{K T}{q}\right)$

where $q$ is the elementary charge $\left(1.6 \times 10^{-19} C\right), e$ is the permittivity of the passive film (30), $e_{0}$ is the permittivity of vacuum $\left(8.85 \times 10^{-14} \mathrm{~F} / \mathrm{cm}\right), N$ is the charge carrier density, $V$ is the applied voltage, $V_{f b}$ is the flat-band potential, $K$ is the Boltzmann constant $\left(1.38 \times 10^{23} \mathrm{~J} / K\right)$, and $T$ is the Kelvin temperature. EIS plots in the Bode and Nyquist planes were recorded at the OCP and +0.3 $\mathrm{V}_{\mathrm{OCP}}$ with an excitation voltage of $10 \mathrm{mV}$. The stability of the measured EIS data was confirmed 
experimentally by recording data from a high frequency of $10 \mathrm{KHz}$ to a low frequency of $0.02 \mathrm{~Hz}$ and then immediately recording the impedance in the reverse direction. The validity of the impedance data and their conformity with the stability, causality, and linearity constraints were also evaluated using the Kramers-Kronig transforms. The successful transformations demonstrate that the system conforms with these constraints of Linear Systems Theory.

Table X.4. Calculated equilibrium potentials of hydrogen electrode reaction based-on Nernst equation.

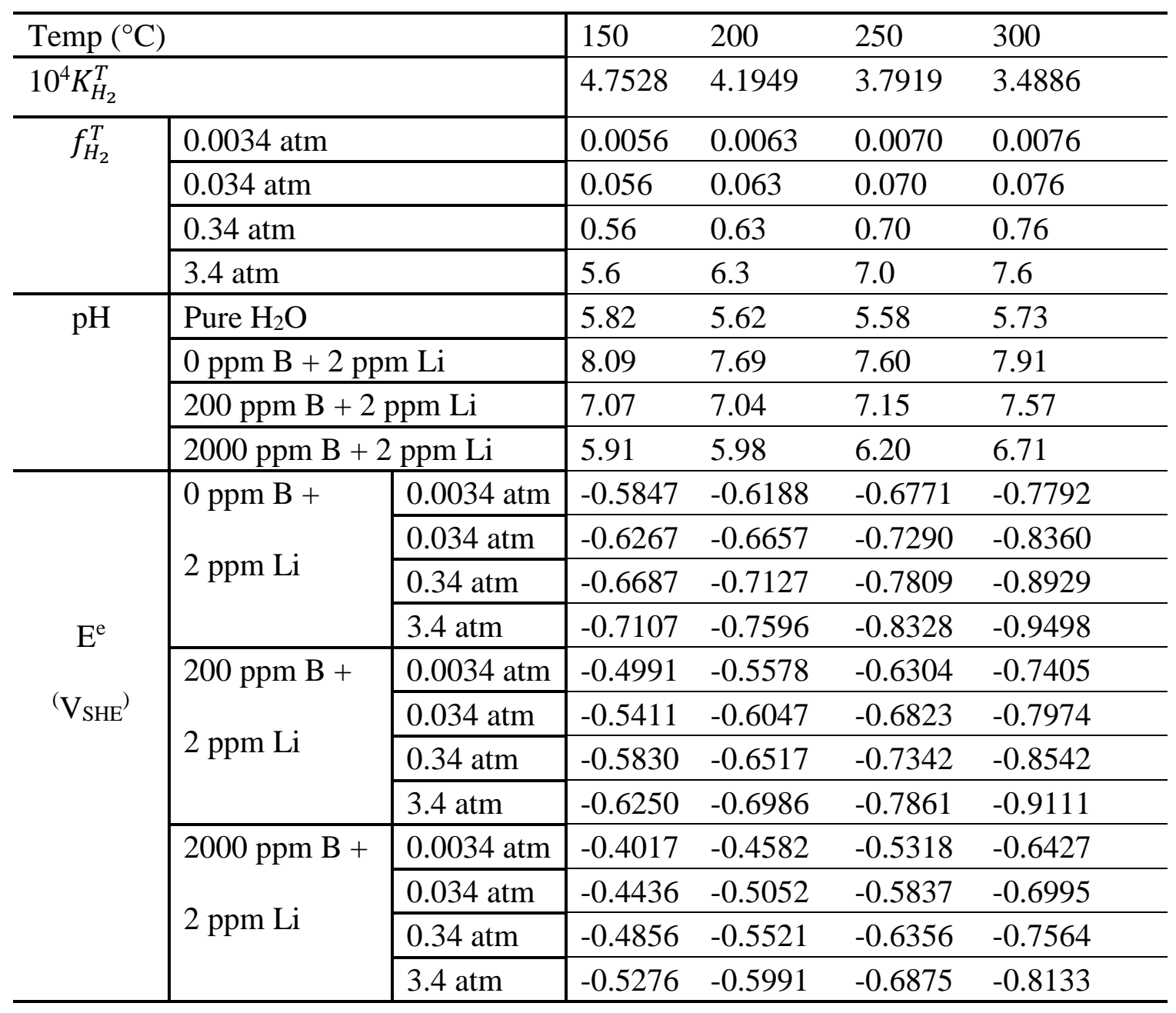

Cathodic and anodic potentiostatic polarization measurements are being performed on Alloys 600 and 690 simultaneously using two Gamry instruments (PCI4) in solutions containing 0 ppm $B+2$ ppm $L i, 200$ ppm $B+2$ ppm $L i, 2000$ ppm $B+2$ ppm $L i$ with hydrogen concentrations of $2.65 \times 10^{-6} \mathrm{M}, 2.65 \times 10^{-5} \mathrm{M}, 2.65 \times 10^{-4} \mathrm{M}$, and $2.65 \times 10^{-3} \mathrm{M}$ at $150^{\circ} \mathrm{C}, 200^{\circ} \mathrm{C}$, 
$250{ }^{\circ} \mathrm{C}$ and $300{ }^{\circ} \mathrm{C}$ to investigate the kinetic parameters of HER. Each polarization was held for 5-120 min to reach a steady-state.

\section{X.3. Results and discussion}

\section{X.3.1. Effects of temperature and $\mathrm{pH}$ on the electrochemical properties of Alloys 600 and 690 in simulated pressurized water reactor primary water}

As shown in Figure X.3, the open circuit potential of Alloys 600 and 690 decreases significantly with increasing temperature in each solution (Figure X.3a) while it only slightly decreases with increasing $\mathrm{pH}$ at the same temperature (Figure X.3b).

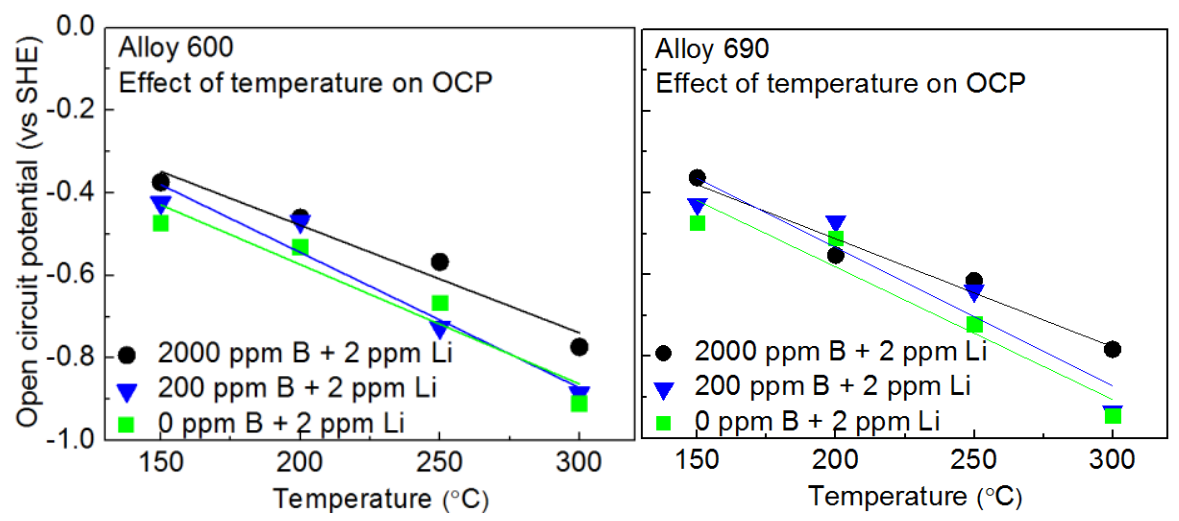

(a)

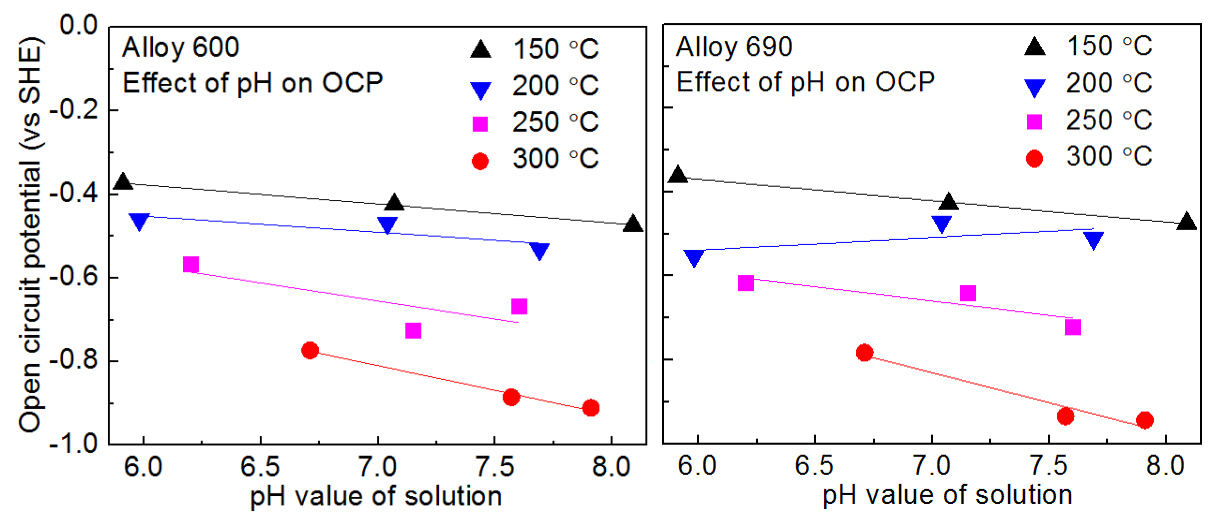

(b)

Figure X.3. Change of open circuit potential as a function of (a) temperature and (b) $\mathrm{pH}$ for Alloys 600 and 690 in borate buffer solutions containing 400 ppb dissolved oxygen. 
At the same temperature, within experimental uncertainty, the current density is independent of the passive film formation potential for Alloy 600 and Alloy 690, indicated in Figure X.4a and X.4b, respectively.

(a)
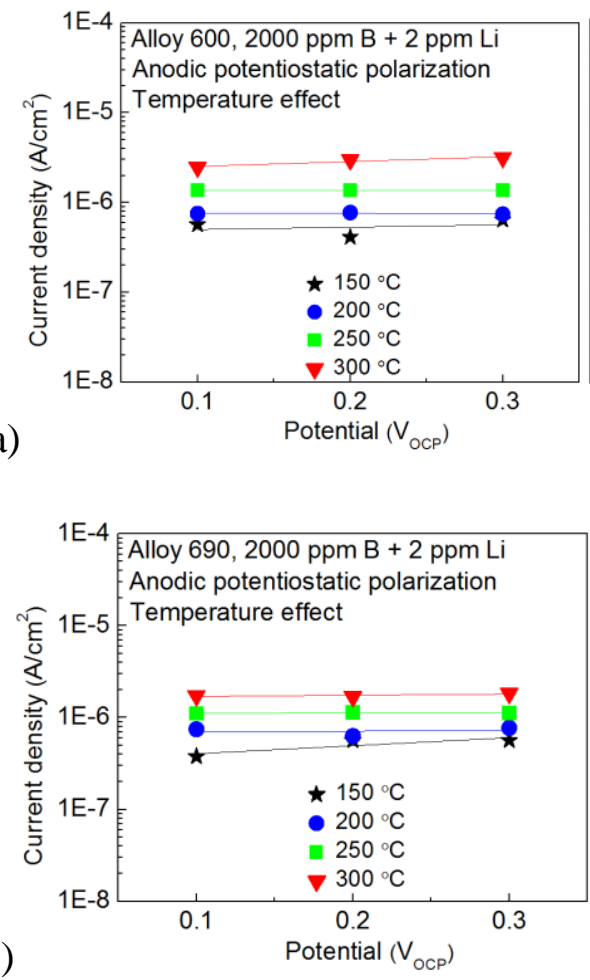

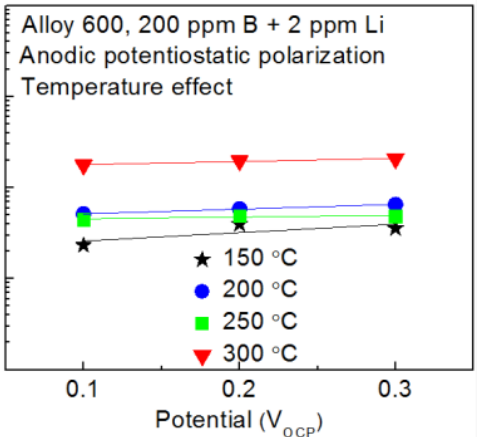

Alloy 600,0 ppm B + 2 ppm Li Anodic potentiostatic polarization Temperature effect

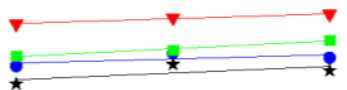

$\star 150^{\circ} \mathrm{C}$

- $200^{\circ} \mathrm{C}$

$=250^{\circ} \mathrm{C}$

v $300^{\circ} \mathrm{C}$

Potential $\left(\mathrm{V}_{\mathrm{OCP}}\right)$

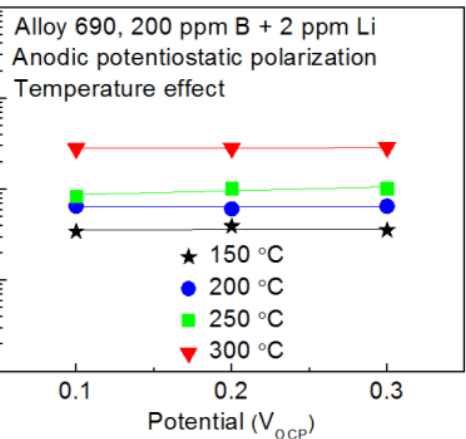

Alloy 690, 0 ppm B + 2 ppm Li Anodic potentiostatic polarization Temperature effect
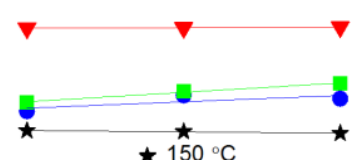

- $200^{\circ} \mathrm{C}$

- $250^{\circ} \mathrm{C}$

0.2

Potential $\left(\mathrm{V}_{\mathrm{OCP}}\right)$

Figure X.4. Change of passive current density as a function of film formation potential (potentiostatic test) with temperature for (a) Alloy 600 and (b) Alloy 690 in borate buffer solutions with 400 ppb dissolved oxygen.

This indicates that the dominant point defect within the barrier layer is the cation interstitial or oxygen vacancy, either of which yields a passive current density that is independent of the applied voltage, provided that no change occurs in the oxidation state of the cation upon ejection from the barrier layer. This corresponds to the barrier layer being of n-Type electronic character, as predicted by the PDM, and which agrees with other reports that the passive films formed on Alloy 600 and Alloy 690 are n-Type semiconductors, due to the chromium oxide rich barrier layer $[19,35,48-50]$ containing a high concentration of cation interstitials. At the same polarization potential, the passive current density of each alloy increases obviously with increasing temperature (Figure X.4) while decreases slightly with increasing pH (Figure X.5). This indicates that the 
general corrosion resistance decreases significantly as the temperature is elevated while increasing with increasing slightly solution $\mathrm{pH}$. These are the expected trends.
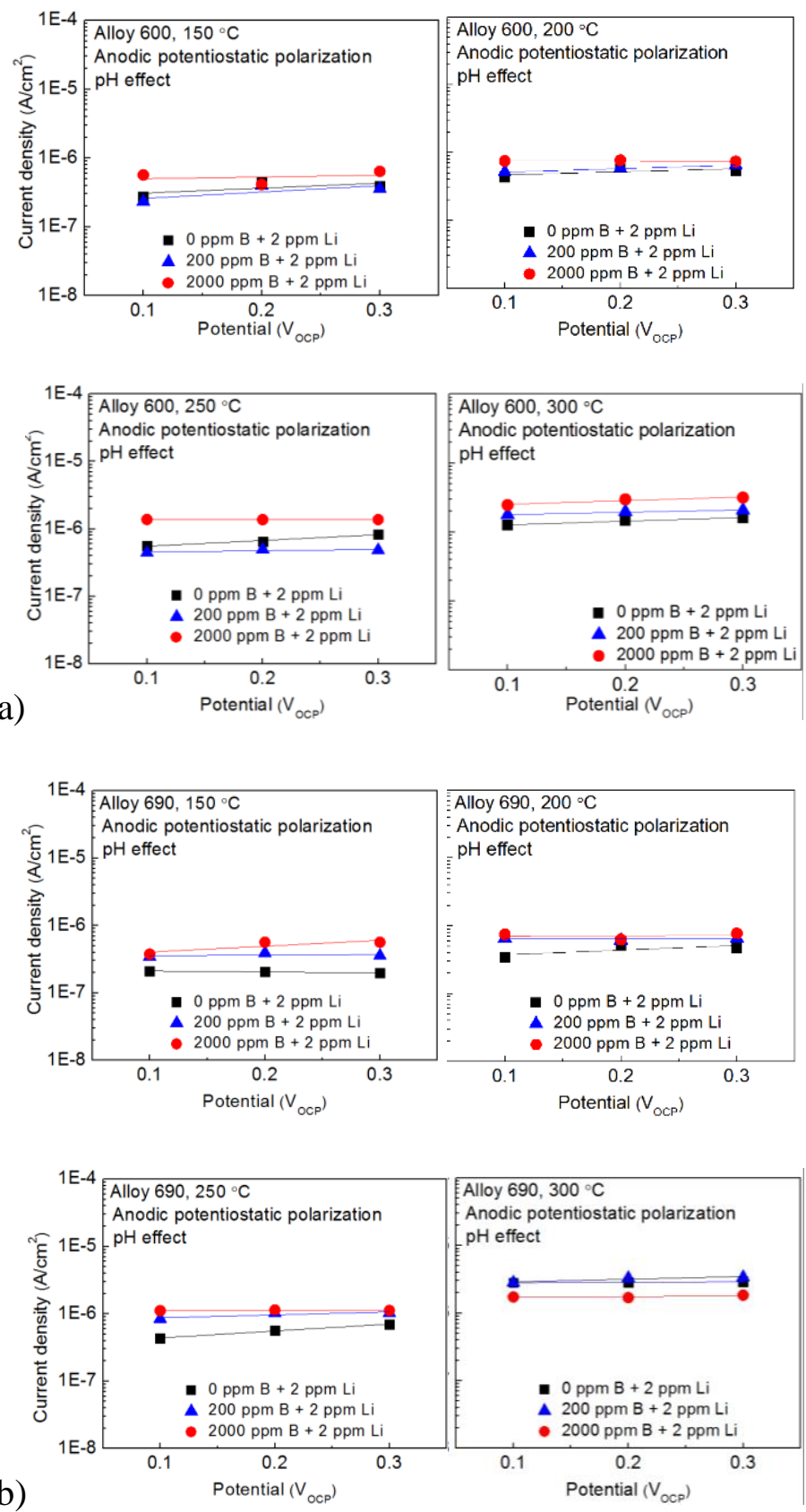

Figure X.5. Change of passive current density as a function of film formation potential (potentiostatic test) with $\mathrm{pH}$ for (a) Alloy 600 and (b) Alloy 690 in borate buffer solutions with 400 ppb dissolved oxygen. 
As shown in Figure X.6, the positive slope indicates the n-Type electronic characteristics of the barrier layers of the passive films formed on Alloy 600 (Figure X.6a) and Alloy 690 (Figure X.6b) in oxygenated borate buffer solutions, corresponding to the findings in Figures X.4 and X.5.

(a)
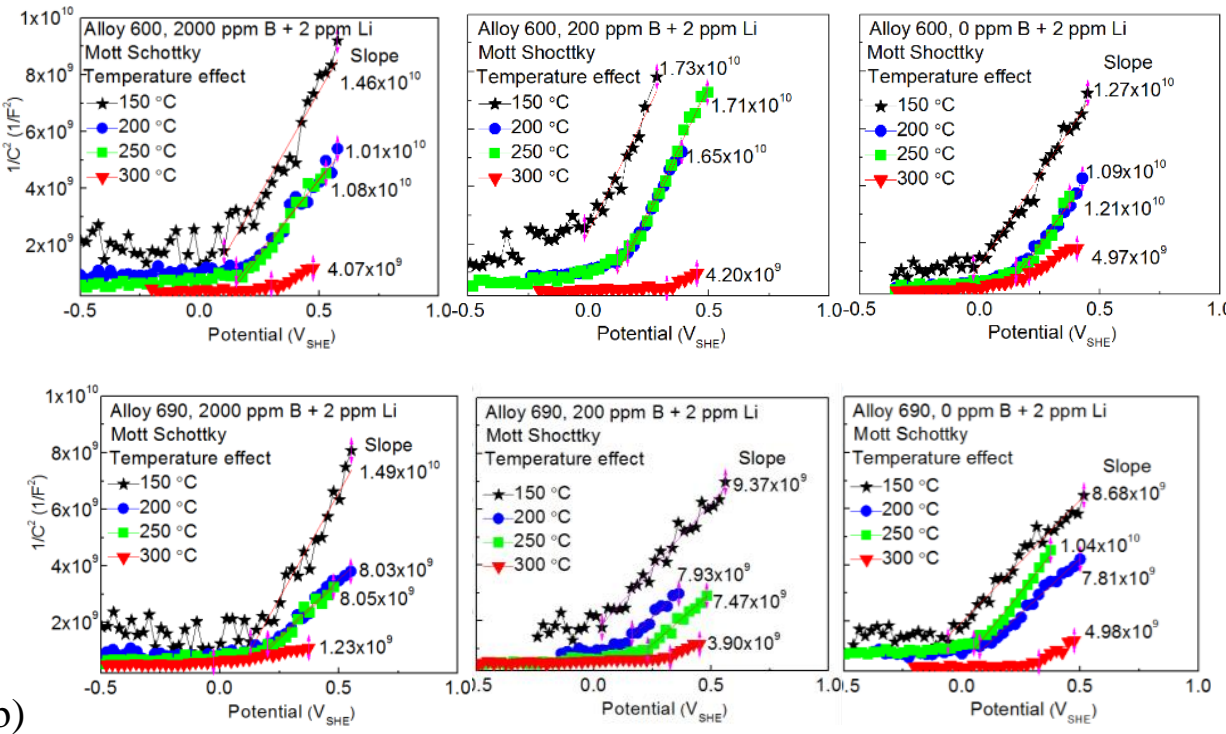

Figure X.6. Mott-Schottky (M-S) plots for the passive film formed on (a) Alloy 600 and (b) Alloy 690 at +0.3 V dissolved oxygen.

The charge carrier density (defect density) was calculated based on the slopes of the M-S plots, as shown in Table X.5.

Table X.5. Calculated defect density of passive films formed on Alloys 600 and 690 at +0.3 VocP in borate buffer solutions containing $400 \mathrm{ppb}$ dissolved oxygen at different temperatures based on the slopes of Mott-Schottky plots.

\begin{tabular}{l|l|l|l|l|l|l}
\hline \multicolumn{7}{c}{ Defect density $\left(\mathrm{mol} \cdot \mathrm{cm}^{-3}\right)$} \\
\hline \multirow{2}{*}{$T\left({ }^{\circ} \mathrm{C}\right)$} & \multicolumn{7}{l}{$2000 \mathrm{ppm} \mathrm{B}+2 \mathrm{ppm} \mathrm{Li}$} & \multicolumn{2}{l}{$200 \mathrm{ppm} \mathrm{B}+2 \mathrm{ppm} \mathrm{Li}$} & \multicolumn{2}{l}{$0 \mathrm{ppm} \mathrm{B}+2 \mathrm{ppm} \mathrm{Li}$} \\
\cline { 2 - 7 } & Alloy 600 & Alloy 690 & Alloy 600 & Alloy 690 & Alloy 600 & Alloy 690 \\
\hline 150 & $5.4 \times 10^{-4}$ & $5.3 \times 10^{-4}$ & $4.5 \times 10^{-4}$ & $8.4 \times 10^{-4}$ & $6.2 \times 10^{-4}$ & $9.0 \times 10^{-4}$ \\
200 & $7.7 \times 10^{-4}$ & $9.7 \times 10^{-4}$ & $1.2 \times 10^{-3}$ & $9.9 \times 10^{-4}$ & $7.2 \times 10^{-3}$ & $1.0 \times 10^{-3}$ \\
250 & $7.2 \times 10^{-4}$ & $9.7 \times 10^{-4}$ & $4.6 \times 10^{-4}$ & $1.1 \times 10^{-3}$ & $6.5 \times 10^{-4}$ & $7.5 \times 10^{-4}$ \\
300 & $1.9 \times 10^{-3}$ & $6.4 \times 10^{-3}$ & $1.9 \times 10^{-3}$ & $2.0 \times 10^{-3}$ & $1.6 \times 10^{-3}$ & $1.6 \times 10^{-3}$ \\
\hline
\end{tabular}


In the same solution, the defect density increases significantly with the increase in temperature under the experimental uncertainty, indicating a decreased corrosion resistance. While, at the same temperature, its change with $\mathrm{pH}$ is not that significant. This agrees with the results in Figures X.4 and X.5. Figures X.7 to X.10 present the measured EIS plots for the passive films that formed on Alloy 600 and Alloy 690 in the borate buffer solutions at the OCP.
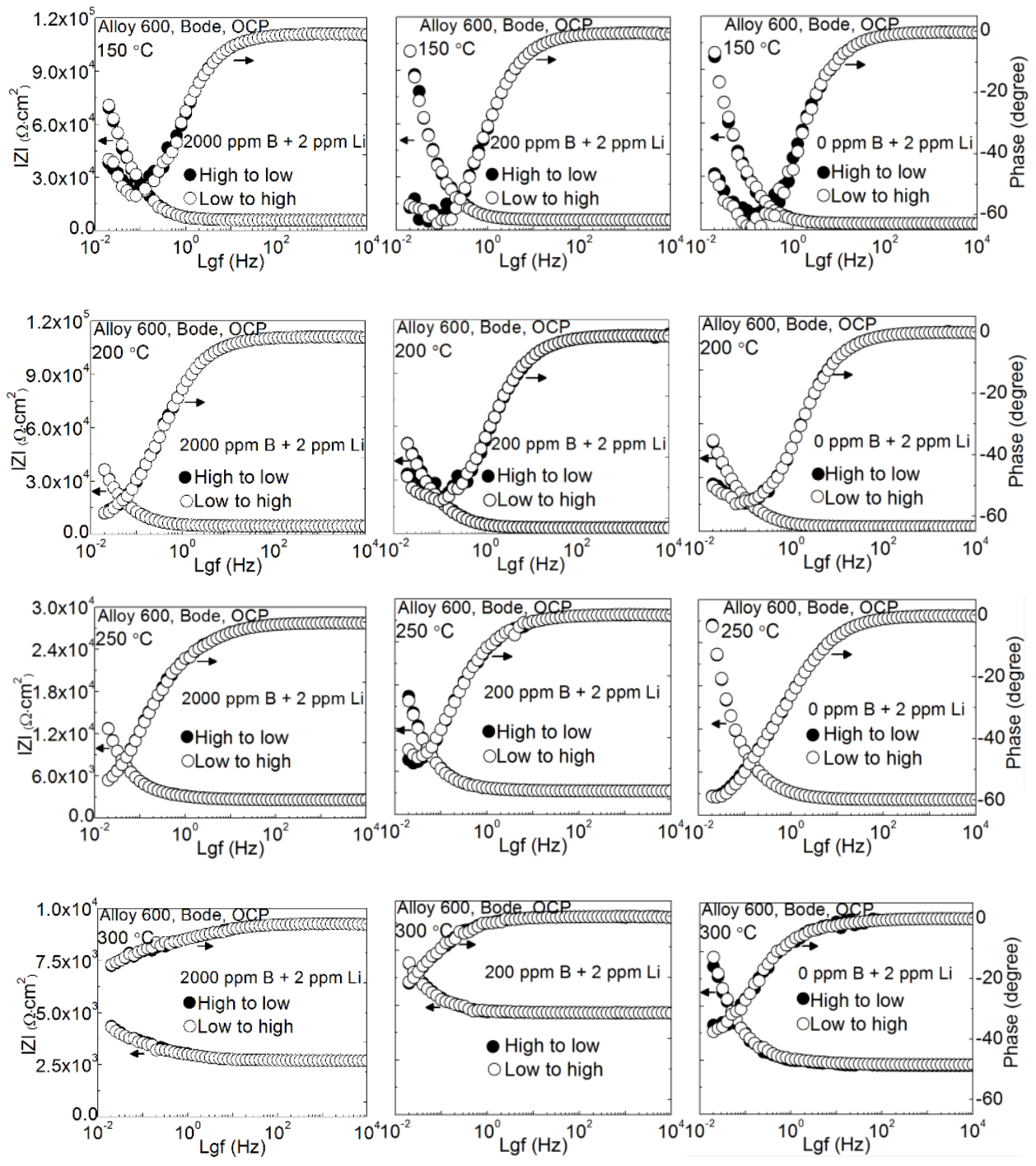

Figure X.7. Impedance plots of the measured EIS data in the Bode plane for two frequency step directions for passive films formed on Alloy 600 at the OCP in borate buffer solutions containing $400 \mathrm{ppb}$ dissolved oxygen at different temperatures. 
The measured impedance data in the two frequency stepping directions (high-to-low and low-to-high) coincide at the same frequency in both the Bode (Figures X.7 and X.9) and Nyquist (Figures X.8 and X.10) planes.
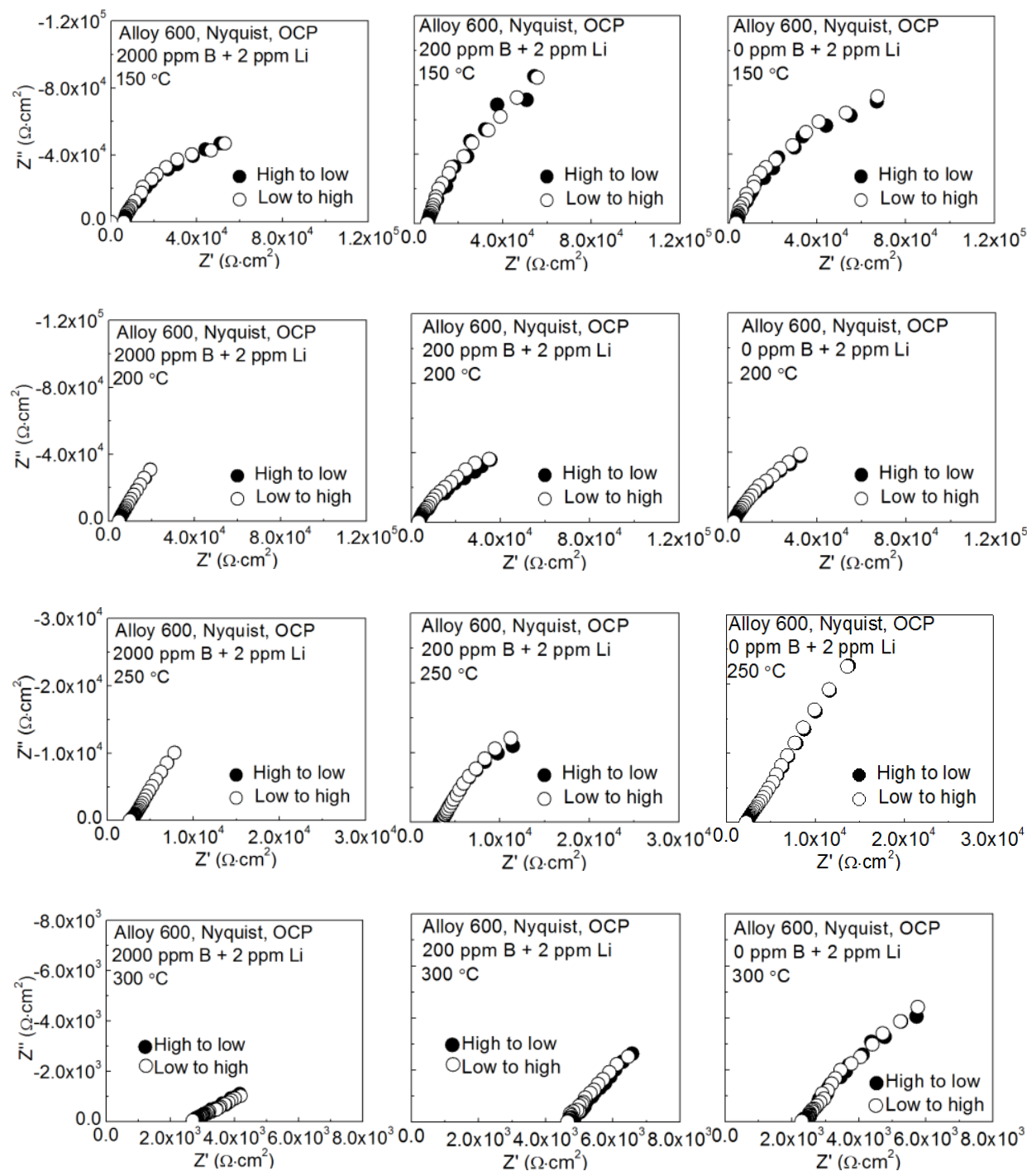

Figure X.8. Impedance plots of the measured EIS data in the Nyquist plane for two frequency step directions for passive films formed on Alloy 600 at OCP in borate buffer solutions containing 400 ppb dissolved oxygen at different temperatures.

This confirms the stability of the system during the measurement. As shown in Figure $\mathrm{X} .11$, the real and imaginary components of the impedance as calculated by Kramers-Kronig transformation from the real to the imaginary axes completely match with the experimental data 
for Alloy 690. This demonstrates that the system satisfies the linearity, stability, and causality constraints of Linear Systems Theory, and hence that the electrochemical response of the system to a small-amplitude potential perturbation can be described by linear response theory including linearized electrochemical models [72-74]. Thus, the quality of the measured EIS data is assured.
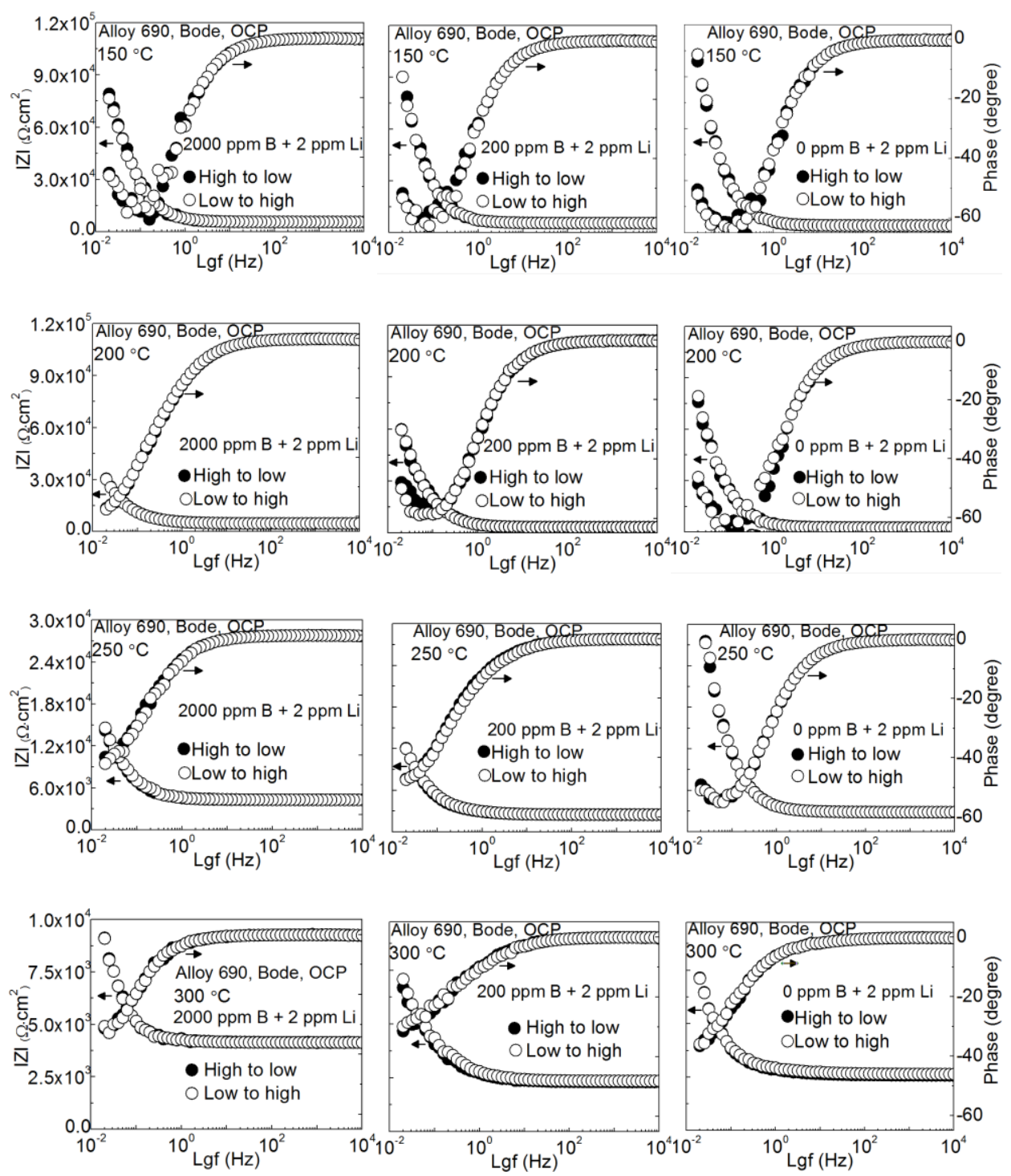

Figure X.9. Impedance plots of the measured EIS data in the Bode plane for two frequency step directions for passive films formed on Alloy 690 at the OCP in borate buffer solutions containing $400 \mathrm{ppb}$ dissolved oxygen at different temperatures. 

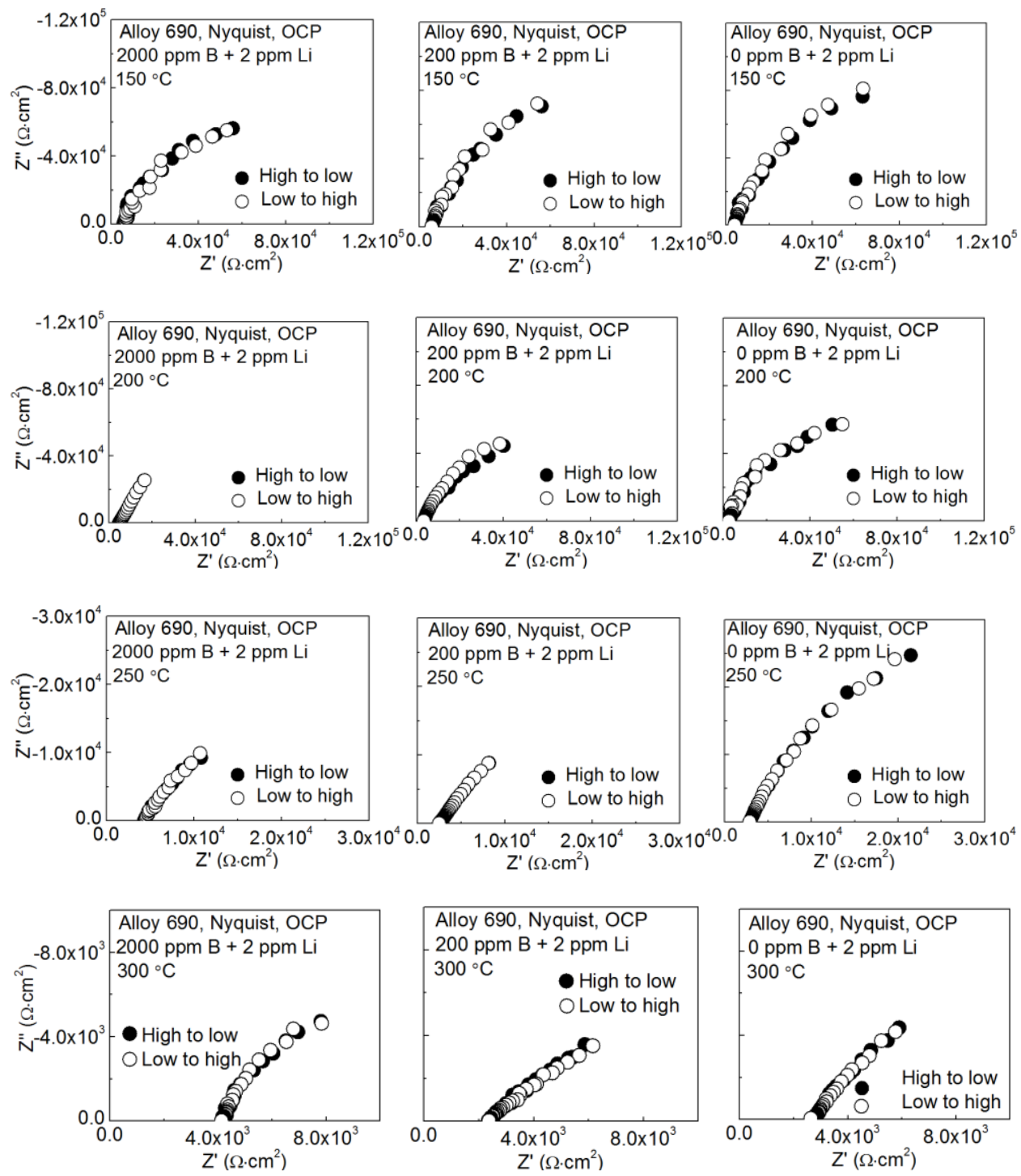

Figure X.10. Impedance plots of the measured EIS data in the Nyquist plane for two frequency step directions for passive films formed on Alloy 690 at OCP in borate buffer solutions containing $400 \mathrm{ppb}$ dissolved oxygen at different temperatures. 


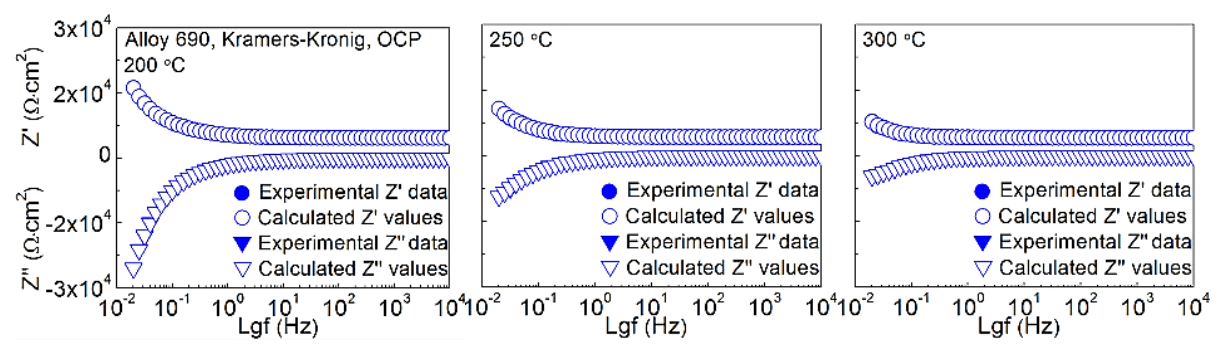

Figure X.11. Impedance plots of the experimental data with the calculated values by KramersKronig transforms for Alloy 690 at OCP in a borate buffer solution containing 2000 ppm B + 2 ppm Li with 400 ppb dissolved oxygen at different temperatures.

The effects of temperature and $\mathrm{pH}$ on the absolute value of impedance for passive films that formed on Alloys 600 and 690 under OCP were investigated, as presented in Figures X.12 and X.13, respectively.

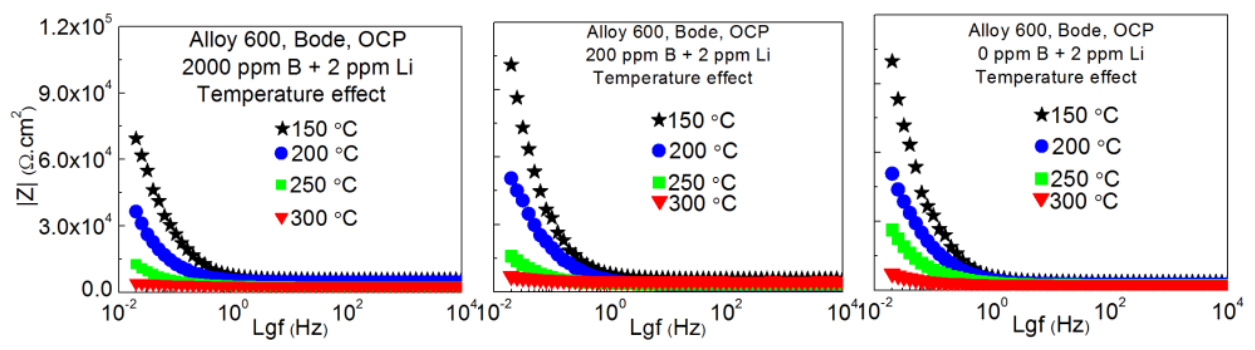

(a)

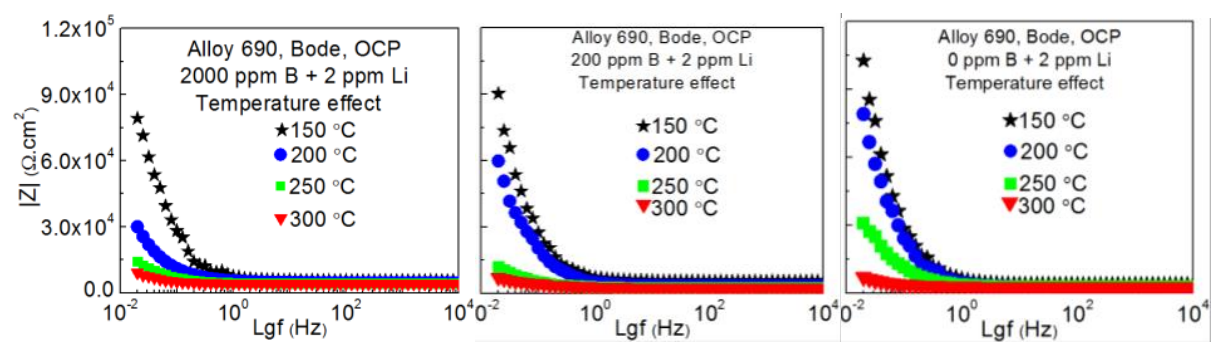

(b)

Figure X.12. Change of the absolute value of impedance with temperature for passive films formed on (a) Alloy 600 and (b) Alloy 690 at the OCP in borate buffer solutions containing 400 ppb dissolved oxygen.

The impedance decreases sharply with the increase in temperature for each alloy (Figure X.12), while it increases mildly with the increasing $\mathrm{pH}$ (Figure X.13). This indicates a greatly 
decreased general corrosion resistance with the elevated temperature and the slight improved corrosion resistance with the increased $\mathrm{pH}$ of the solution, which agree with the findings of passive current (Figures X.4 and X.5) and defect density (Table X.5).
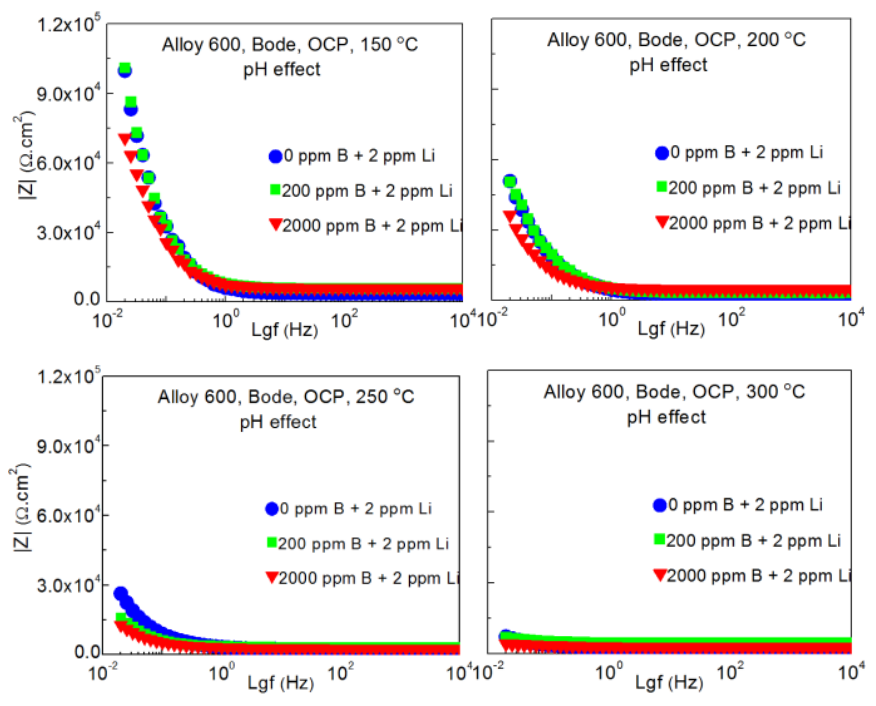

(a)
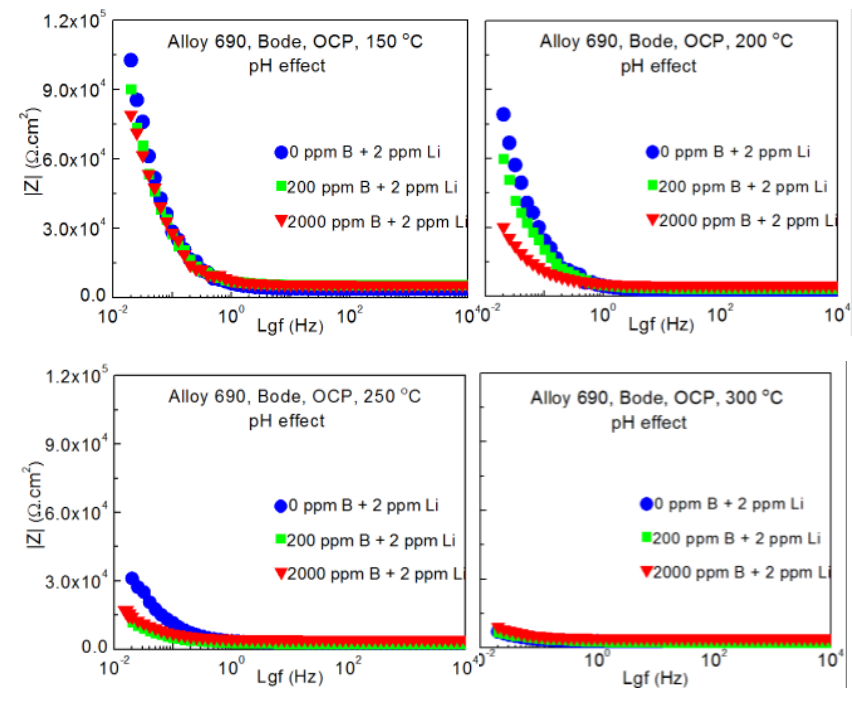

(b)

Figure X.13. Change of the absolute value of impedance with $\mathrm{pH}$ for passive films formed on (a) Alloy 600 and (b) Alloy 690 at the OCP in borate buffer solutions containing 400 ppb dissolved oxygen.

Figure X.14 presents the experimental EIS results for the passive films formed on Alloy 600 at $+0.3 \mathrm{~V}_{\text {OCP }}\left(-0.124 \mathrm{~V}_{\text {SHE }}\right.$ at $150{ }^{\circ} \mathrm{C},-0.169 \mathrm{~V}_{\text {SHE }}$ at $200{ }^{\circ} \mathrm{C},-0.426 \mathrm{~V}_{\text {SHE }}$ at $250{ }^{\circ} \mathrm{C},-0.585$ 
$\mathrm{V}_{\text {SHE }}$ at $300{ }^{\circ} \mathrm{C}$ ). The film stability was confirmed by the consistency of the plots obtained by the two frequency stepping directions in both the Bode (Figure X.14a) and Nyquist planes (Figure X.14b). At each temperature, the passive film formed at $+0.3 \mathrm{~V}_{\text {OCP }}$ has a higher impedance modulus than that formed at the OCP, indicating an improved corrosion resistance (Figure X.14c).

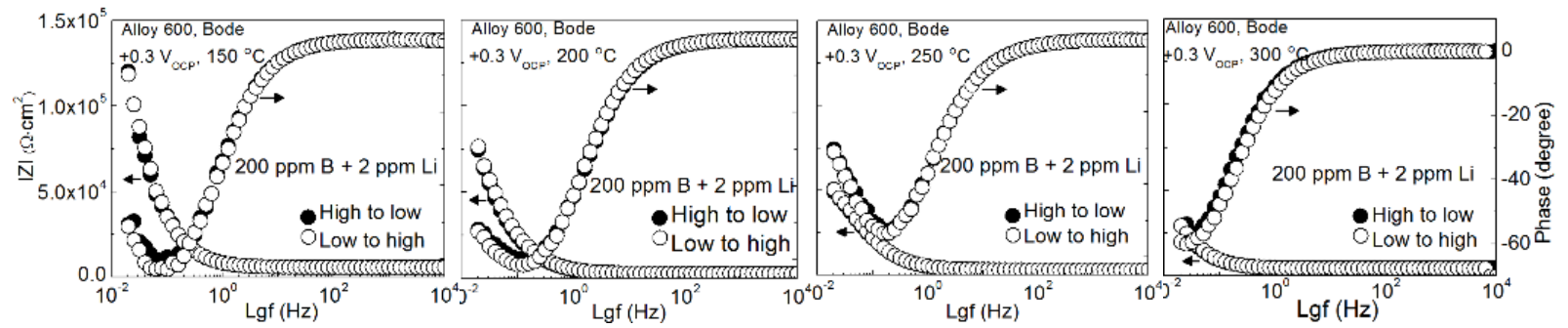

(a)
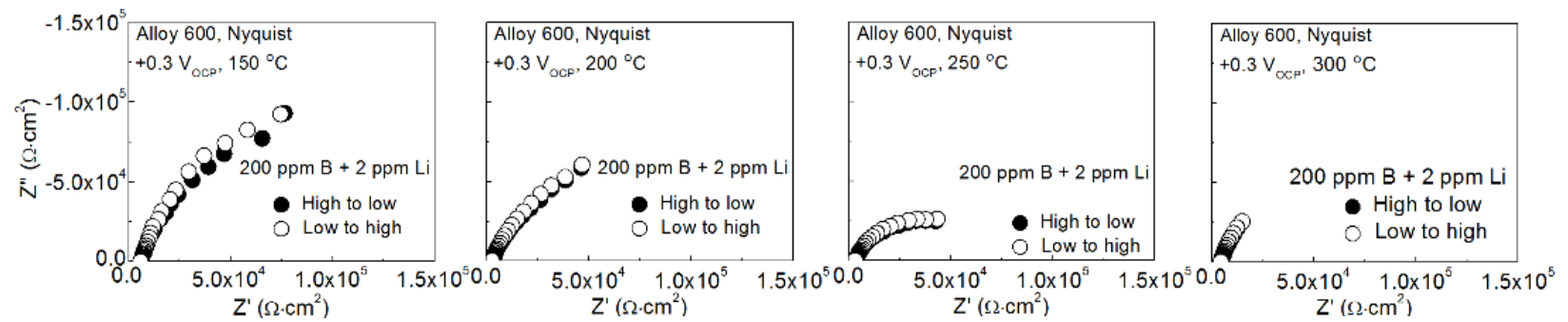

(b)

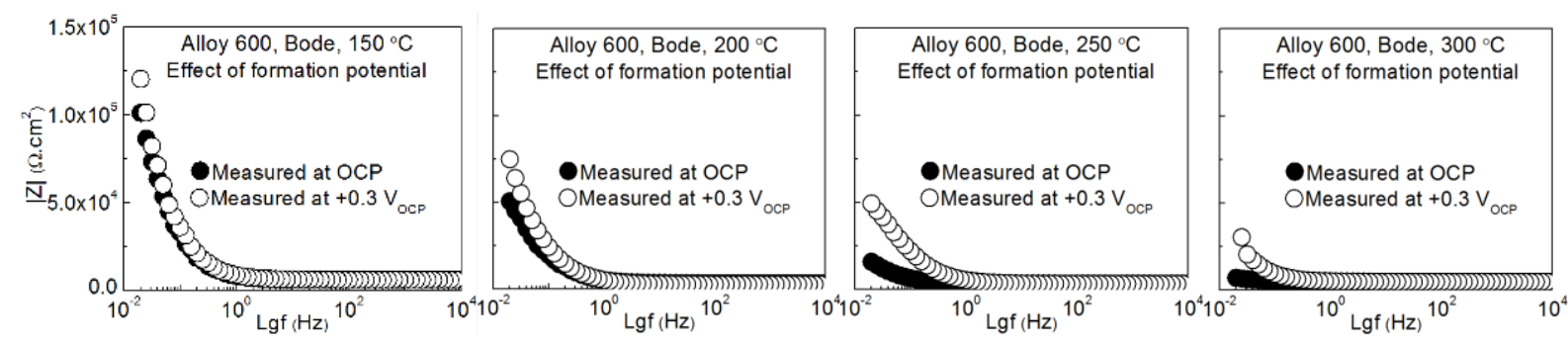

(c)

Figure X.14. Impedance plots of the measured EIS data (a) at $+0.3 \mathrm{~V}_{\mathrm{OCP}}$ in the Bode plane and (b) at $+0.3 \mathrm{~V}$ OCP in the Nyquist plane for two frequency step directions and (c) at the OCP and +0.3 $\mathrm{V}_{\text {OCP }}$ in the Bode plane for passive films formed on Alloy 600 in borate buffer solutions containing $400 \mathrm{ppb}$ dissolved oxygen at different temperatures. 


\section{X.3.2. Effects of temperature and $\mathrm{pH}$ on the electrochemical properties of Type 304L SS and}

Type 316L SS in simulated pressurized water reactor primary water

As shown in Figure X.15, the OCP of Type 304L SS and Type 316L SS in the same solution decreases significantly with increasing temperature (Figure X.15a) while it only slightly decreases with increasing $\mathrm{pH}$ at the same temperature (Figure X.15b).
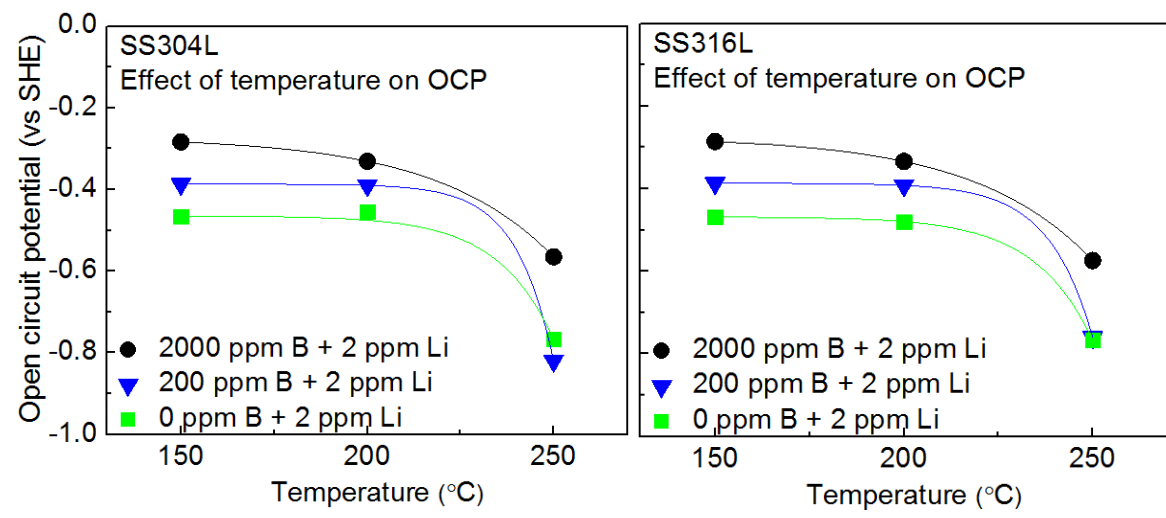

(a)

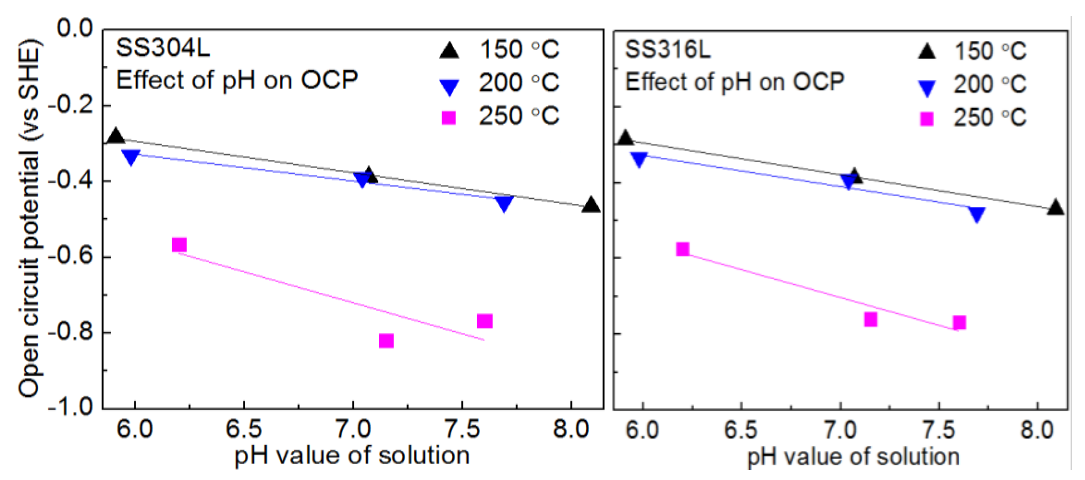

(b)

Figure X.15. Changes of the open circuit potential as a function of (a) temperature and (b) $\mathrm{pH}$ for Type 304L SS and Type 316L SS immersed in borate buffer solutions containing $400 \mathrm{ppb}$ dissolved oxygen.

The anodic potentiostatic polarization plots for Type 304L SS and Type 316L SS are presented in Figures X.16 and X.17. The passive current density is generally independent of the applied potential, indicating the dominant point defect in the barrier layer of the passive film is the 
cation interstitial or oxygen vacancy. This consistent with the n-type electronic properties of the barrier layer according to the PDM. The high interstitial and oxygen vacancy concentrations render the barrier layer n-Type in electronic character because both defects are electron donors. The passive current density of each alloy significantly increases with the elevated temperature (Figure X.16), while it decreases slightly with the increased pH (Figure X.17). This result agrees with the above findings in Figures X.4 and X.5.
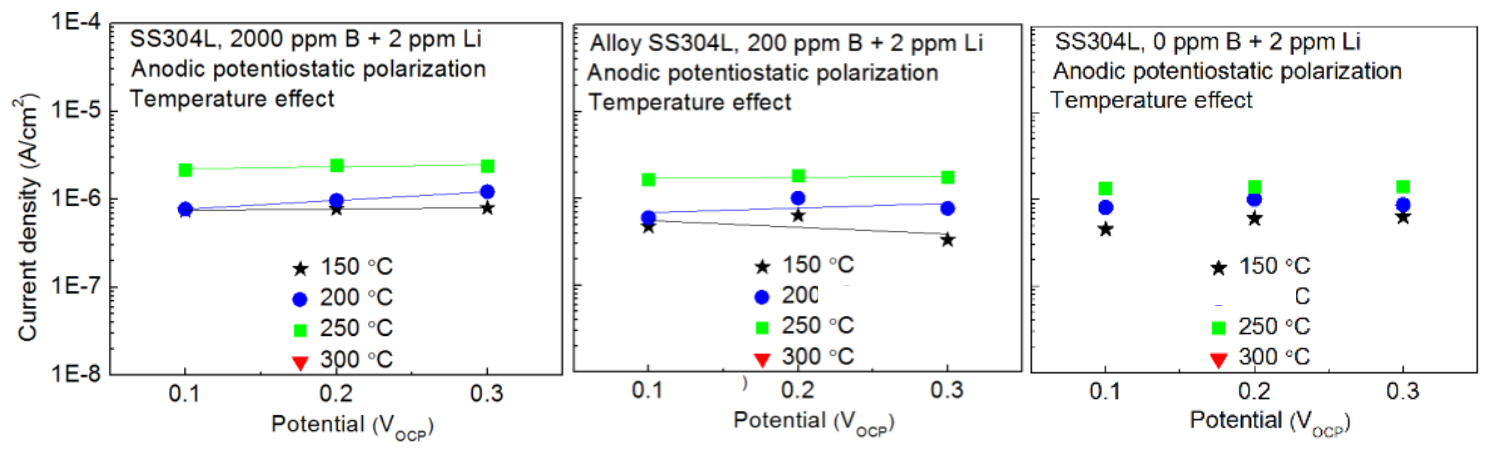

(a)
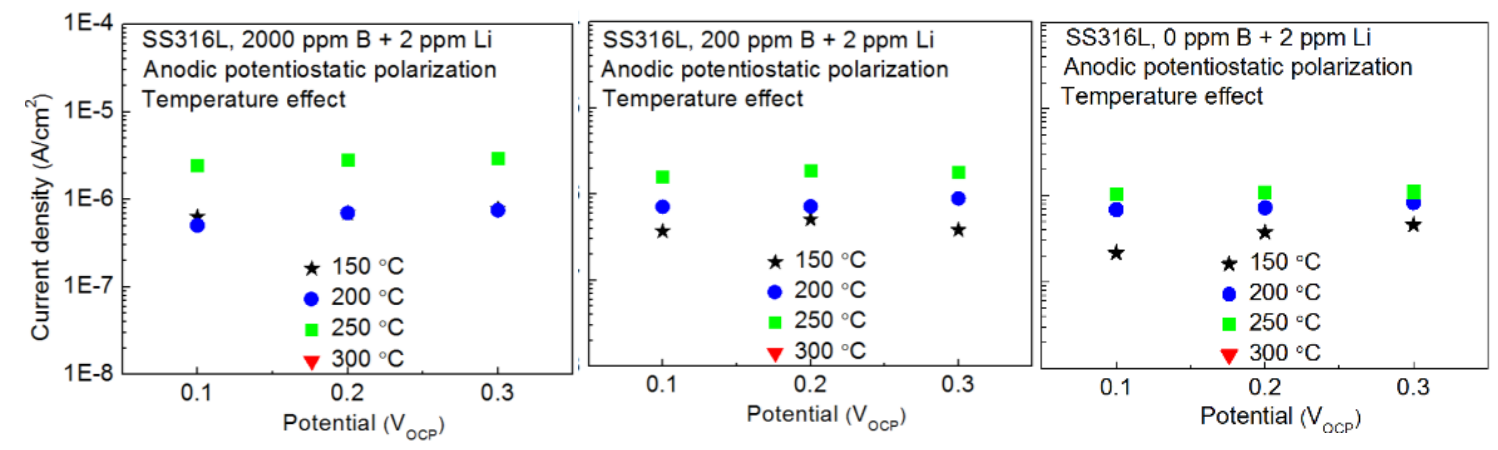

(b)

Figure X.16. Change of passive current density as a function of film formation potential (potentiostatic test) with temperature for (a) Type 304L SS and (b) Type 316L SS in borate buffer solutions with $400 \mathrm{ppb}$ dissolved oxygen. 

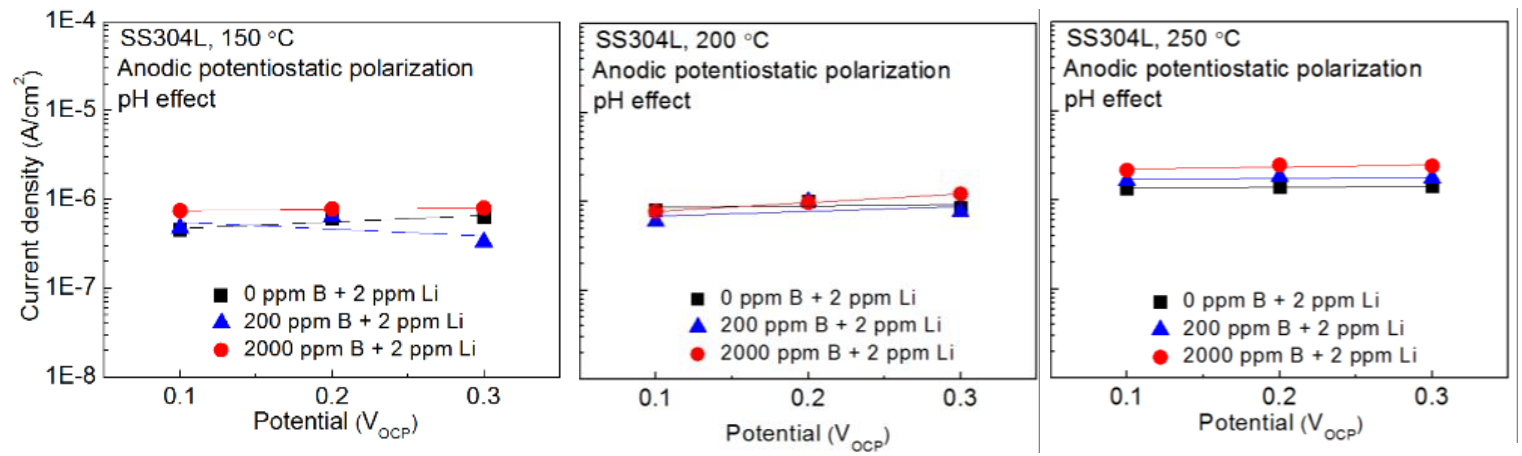

(a)
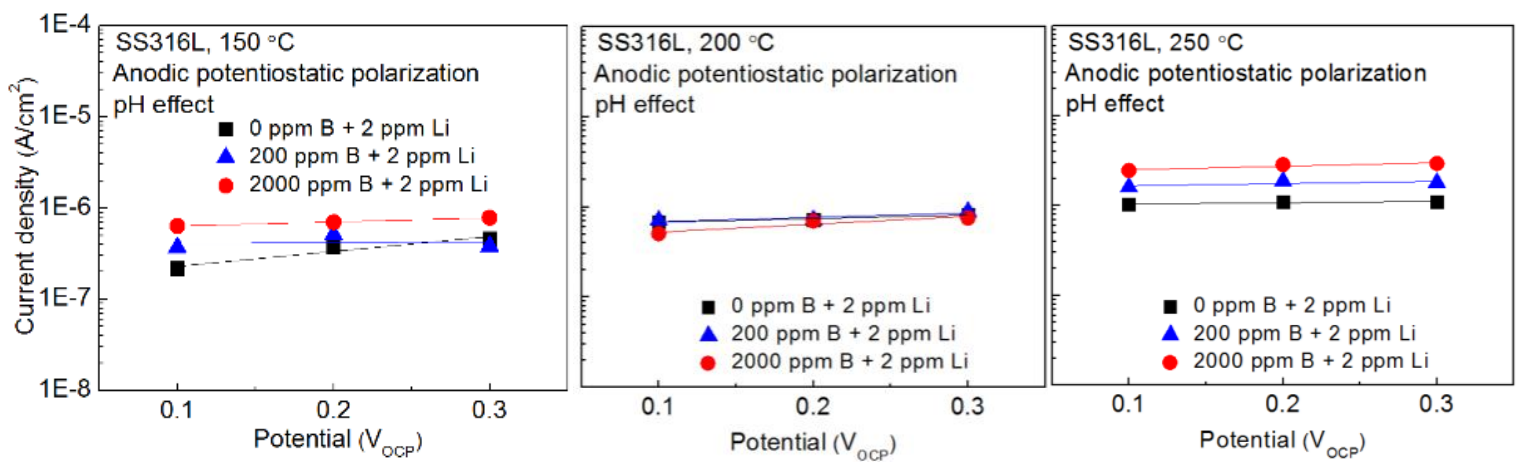

(b)

Figure X.17. Change of passive current density as a function of film formation potential (potentiostatic test) with pH for (a) Type 304L SS and (b) Type 316L SS in borate buffer solutions with $400 \mathrm{ppb}$ dissolved oxygen.

As shown in Figure X.18, the positive slope of M-S plots indicates the n-Type electronic characteristics of the barrier layers of the passive films formed on Type 304L SS and Type 316L SS in oxygenated borate buffer solutions, corresponding to the findings in Figures X.16 and X.17. The charge carrier density (defect density) was calculated based on the slopes of the M-S plots (Table X.6). The defect density increases obviously with increasing temperature, while it changes insignificantly with $\mathrm{pH}$. 

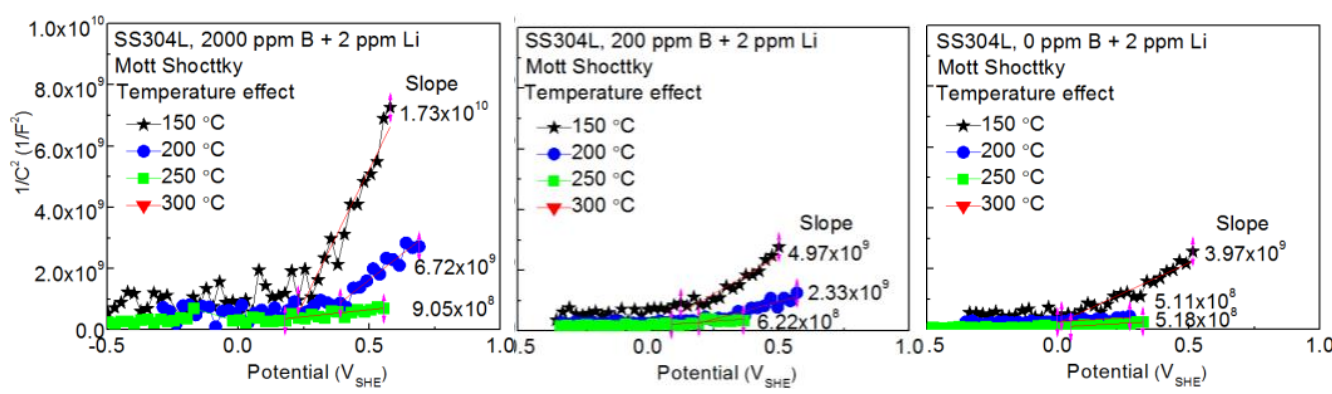

(a)
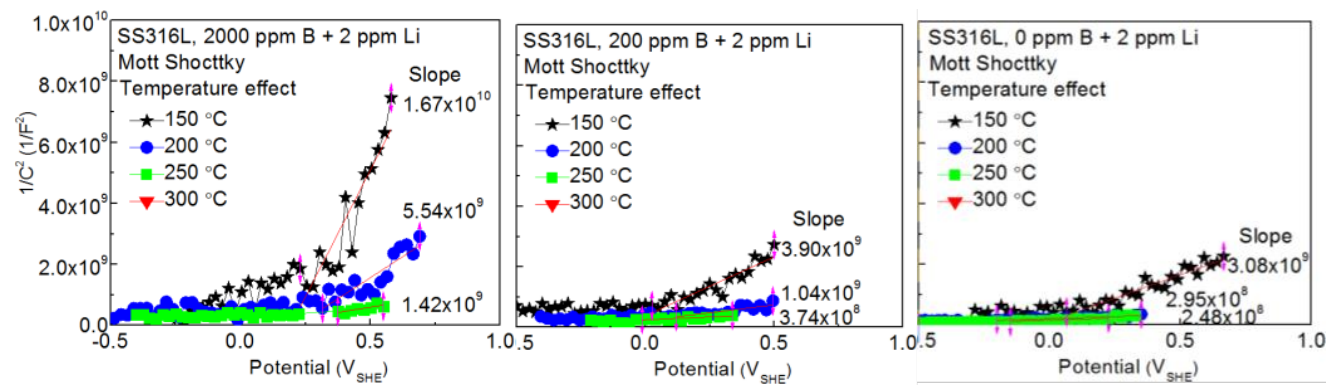

(b)

Figure X.18. Mott-Schottky (M-S) plots for the passive film formed on (a) Type 304L SS and (b) Type $316 \mathrm{~L} \mathrm{SS}$ at $+0.3 \mathrm{~V}_{\text {OCP }}$ as a function of temperature in borate buffer solutions containing 400 ppb dissolved oxygen.

Table X.6. Calculated defect density of passive films formed on Type 304L SS and Type 316L $\mathrm{SS}$ at $+0.3 \mathrm{~V}_{\text {OCP }}$ in borate buffer solutions containing $400 \mathrm{ppb}$ dissolved oxygen at different temperatures based on the slopes of Mott-Schottky plots.

\begin{tabular}{c|l|l|c|c|c|c}
\hline \multicolumn{7}{c}{ Defect density $\left(\mathrm{mol} \cdot \mathrm{cm}^{-3}\right)$} \\
\hline \multirow{3}{*}{$T\left({ }^{0} \mathrm{C}\right)$} & $2000 \mathrm{ppm} \mathrm{B}+2 \mathrm{ppm} \mathrm{Li}$ & $200 \mathrm{ppm} \mathrm{B}+2 \mathrm{ppm}$ & \multicolumn{2}{c}{$0 \mathrm{ppm} \mathrm{B}+2 \mathrm{ppm} \mathrm{Li}$} \\
\cline { 2 - 7 } & Type & Type & Type & Type & Type & Type \\
& $304 \mathrm{~L} \mathrm{SS}$ & $316 \mathrm{~L} \mathrm{SS}$ & $304 \mathrm{~L} \mathrm{SS}$ & $316 \mathrm{~L} \mathrm{SS}$ & $304 \mathrm{~L} \mathrm{SS}$ & $316 \mathrm{~L} \mathrm{SS}$ \\
\hline 150 & $4.5 \times 10^{-4}$ & $4.7 \times 10^{-4}$ & $1.6 \times 10^{-3}$ & $2.0 \times 10^{-3}$ & $2.0 \times 10^{-3}$ & $2.5 \times 10^{-3}$ \\
200 & $1.2 \times 10^{-3}$ & $1.4 \times 10^{-3}$ & $3.4 \times 10^{-3}$ & $7.5 \times 10^{-3}$ & $1.5 \times 10^{-2}$ & $2.7 \times 10^{-2}$ \\
250 & $8.6 \times 10^{-3}$ & $5.5 \times 10^{-3}$ & $1.3 \times 10^{-2}$ & $2.1 \times 10^{-2}$ & $1.5 \times 10^{-2}$ & $3.2 \times 10^{-2}$ \\
\hline
\end{tabular}

Figures X.19 and X.20 show the measured EIS plots in the high-to-low and low-to-high frequency stepping directions for Type 304L SS and Type 316L SS at the OCP in the Bode plane and the Nyquist plane, respectively. 
(a)
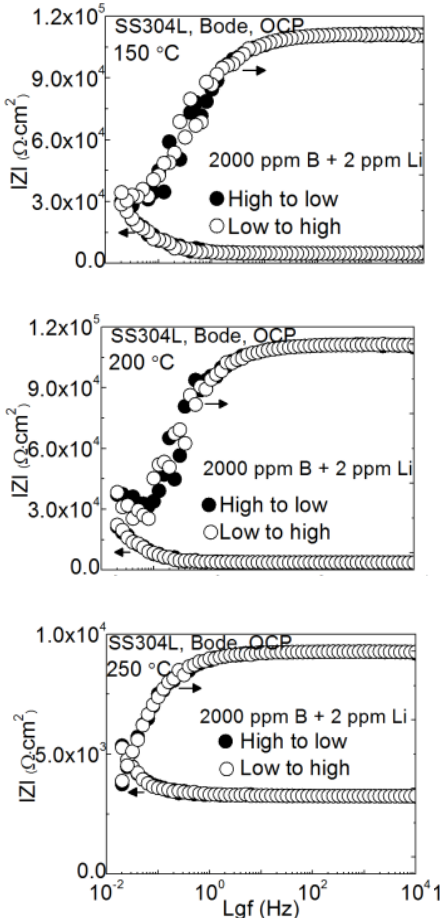

(b)
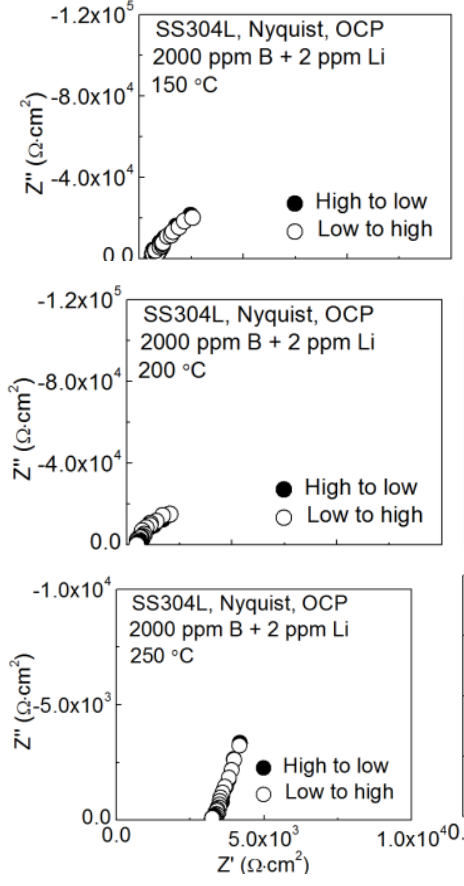
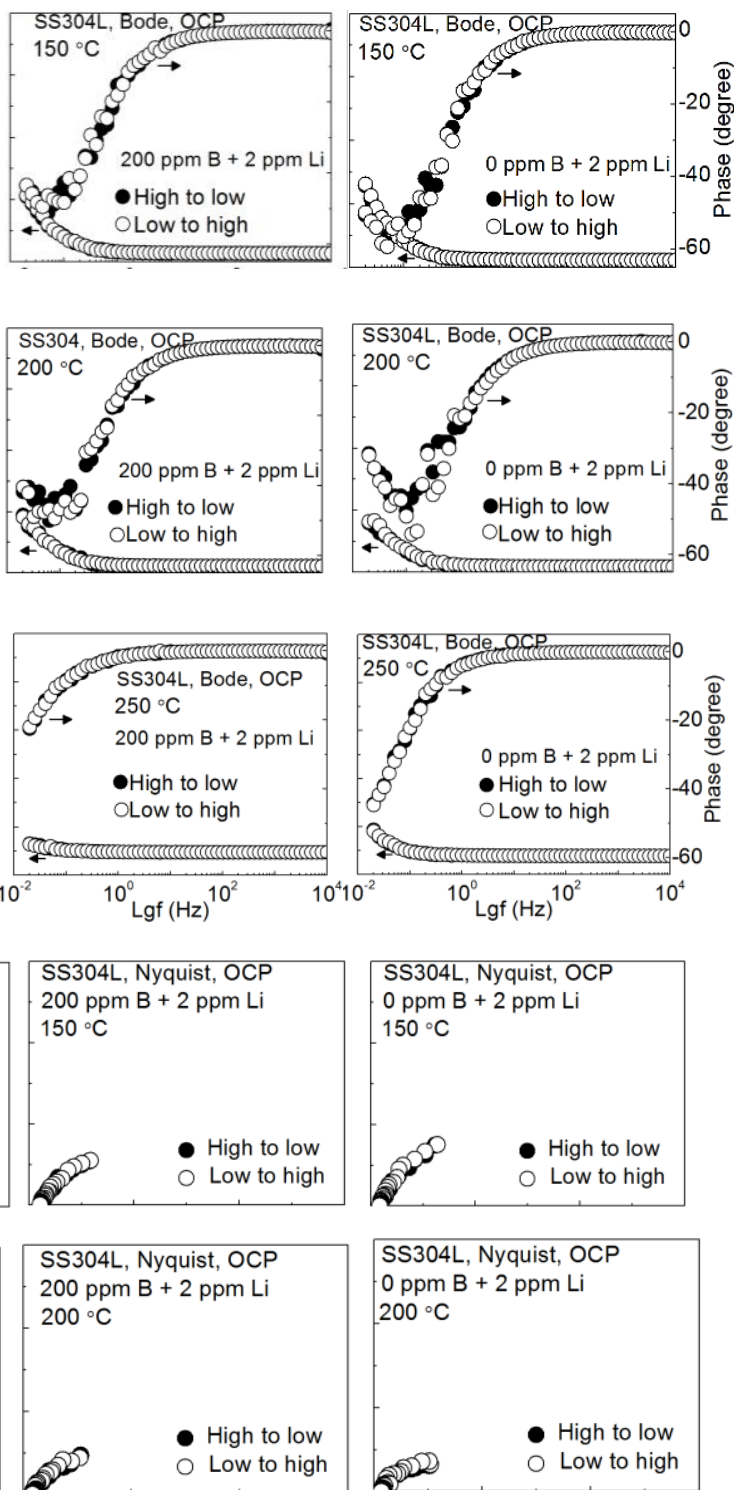

\section{SS304L, Nyquist, OCP}

0 ppm B +2 ppm Li

$200{ }^{\circ} \mathrm{C}$

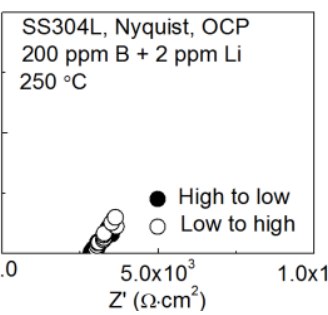

(200

High to low Low to high

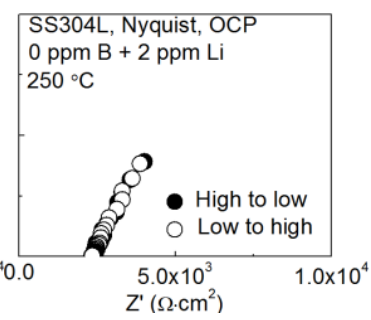

Figure X.19. Measured impedance plots in (a) Bode and (b) Nyquist planes at two frequency step directions for passive films formed on Type 304L SS at OCP in borate buffer solutions containing $400 \mathrm{ppb}$ dissolved oxygen at different temperatures.

The recorded impedance data in the two different frequency stepping directions are coincident, indicating the high stability of the passive film. 
(a)
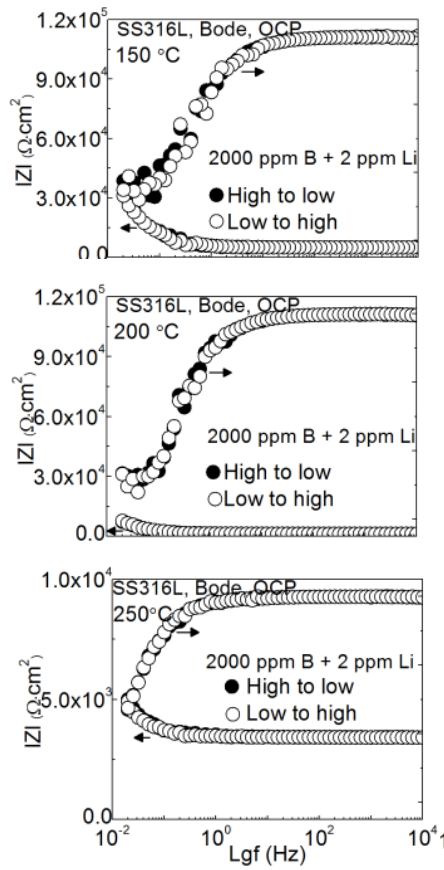

(b)
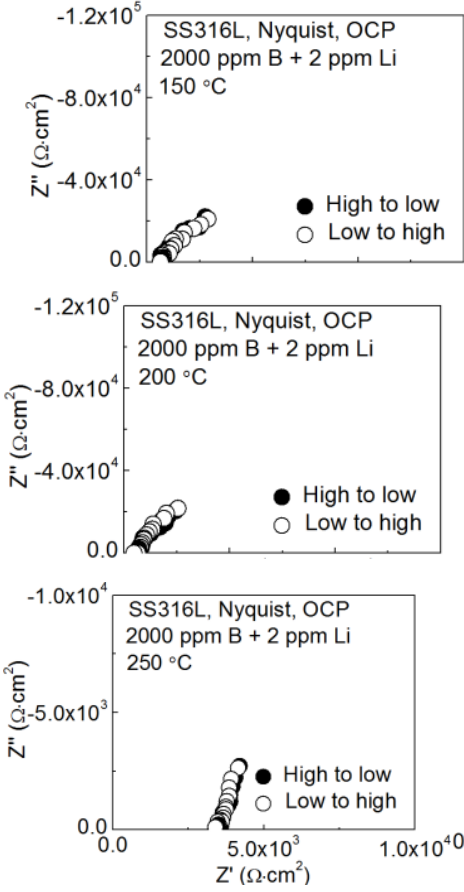
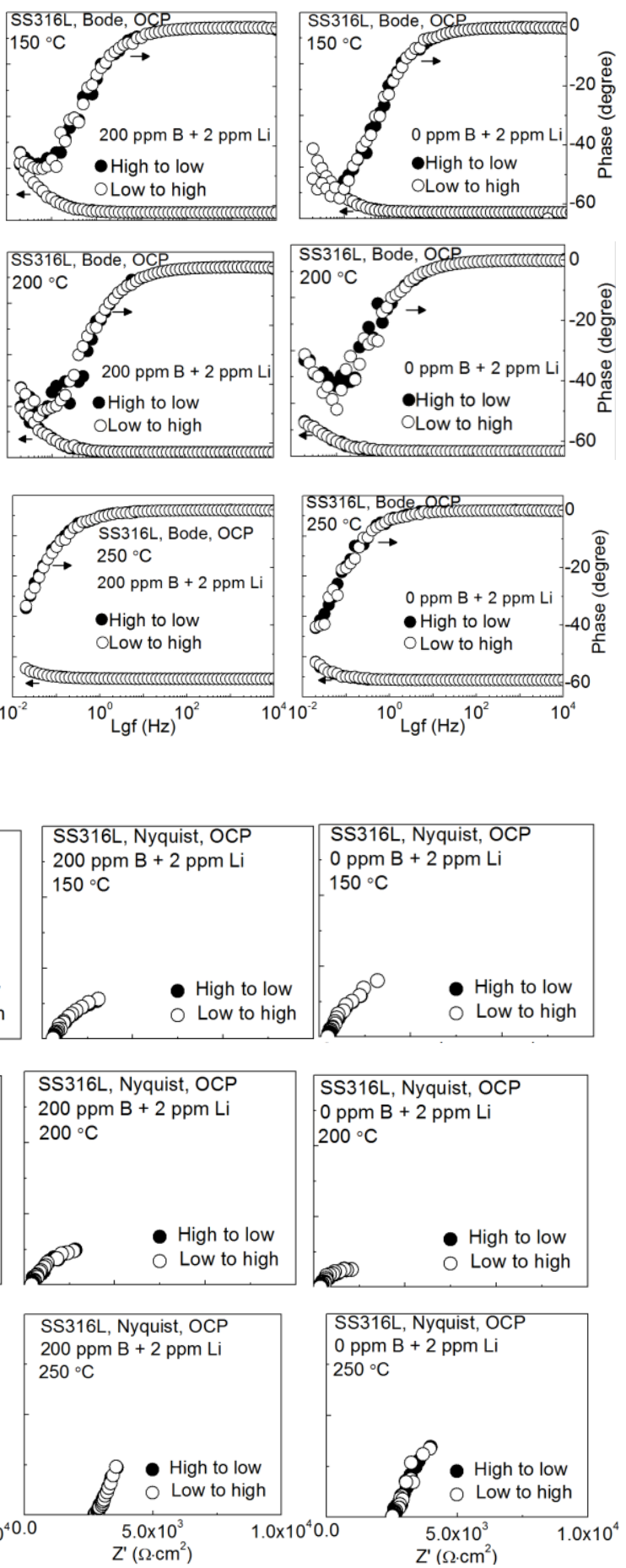

Figure X.20. Measured impedance plots in (a) Bode and (b) Nyquist planes at two frequency step directions for passive films formed on Type 316L SS at OCP in borate buffer solutions containing $400 \mathrm{ppb}$ dissolved oxygen at different temperatures. 
The effects of temperature and $\mathrm{pH}$ on the absolute value of impedance for passive films that formed on Type 304L SS and Type 316L SS at OCP are indicated in Figures X.21 and X.22. The impedance decreases strongly with increasing temperature (Figure X.21), while it increases mildly with increasing $\mathrm{pH}$. This indicates a greatly decreased corrosion resistance by elevating the temperature and the slight improvement of corrosion resistance by increasing the solution $\mathrm{pH}$, corresponding with the passive current (Figures X.16 and X.17) and defect density (Table X.6) findings.

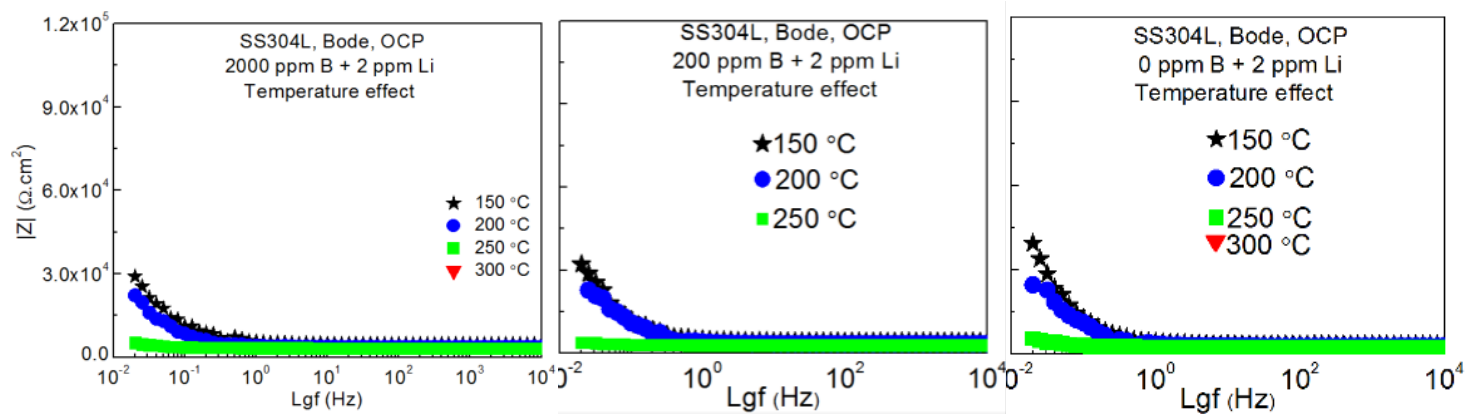

(a)

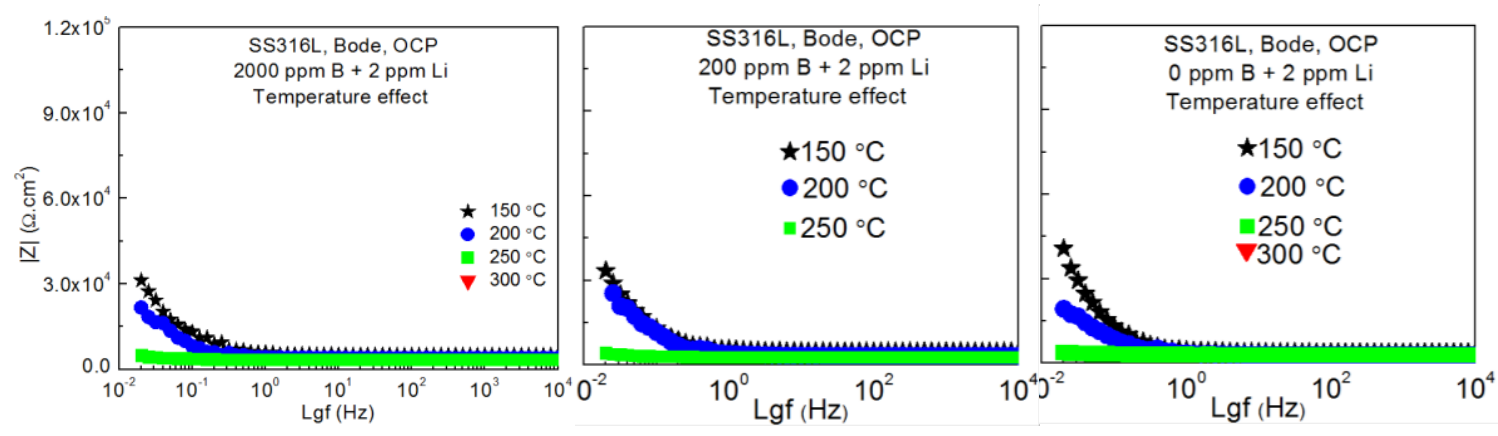

(b)

Figure X.21. Change of the absolute value of impedance with temperature for passive films formed on (a) Type 304L SS and (b) Type 316L SS at the OCP in borate buffer solutions containing 400 ppb dissolved oxygen. 

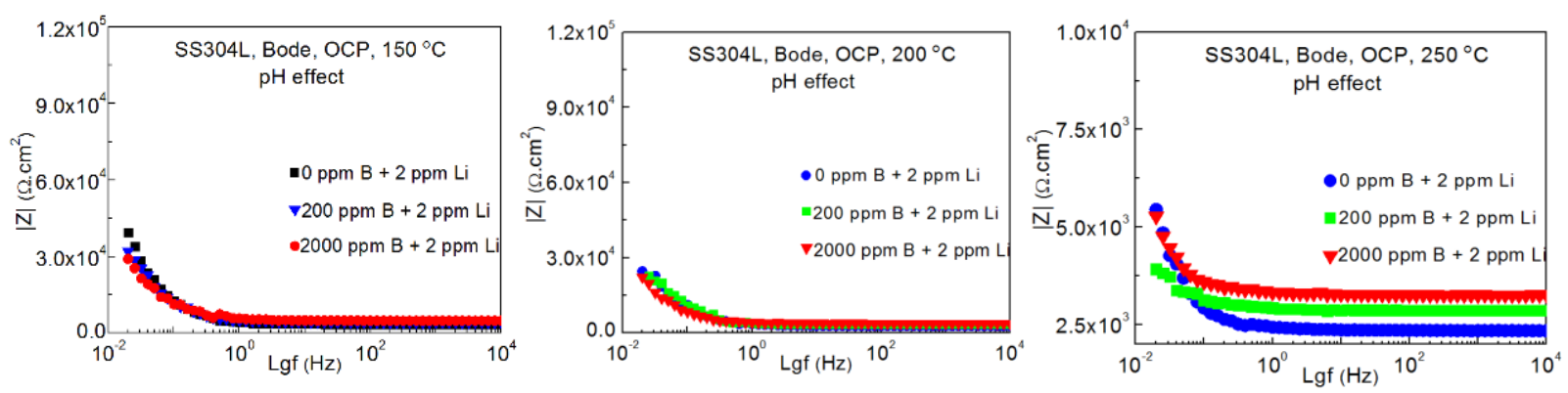

(a)
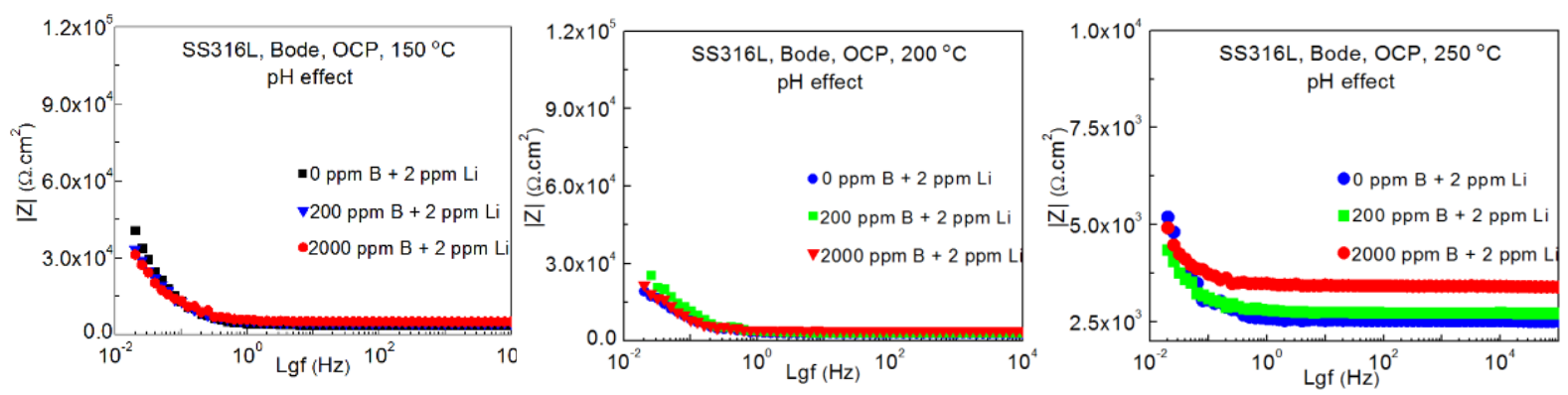

(b)

Figure X.22. Change of the absolute value of the impedance with $\mathrm{pH}$ for passive films formed on (a) Type 304L SS and (b) Type 316L SS at the OCP in borate buffer solutions containing $400 \mathrm{ppb}$ dissolved oxygen.

\section{X.3.3. Effects of temperature and hydrogen concentration on the hydrogen electrode reactions for Alloy 600 and Alloy 690}

For the above experimental measurements (investigation of the effects of temperature and $\mathrm{pH}$ on the anodic passive dissolution of each alloy), the $W / W O_{3}$ electrode was used as reference electrode. Here the equilibrium potentials of the $W / W O_{3}$ electrode half-cell reaction at different temperatures were measured against the hydrogen electrode in borate buffer solution with different hydrogen concentrations. The measured equilibrium potential of the $W / W O_{3}$ electrode is plotted versus the standard hydrogen electrode, indicated in Figure X.23. 

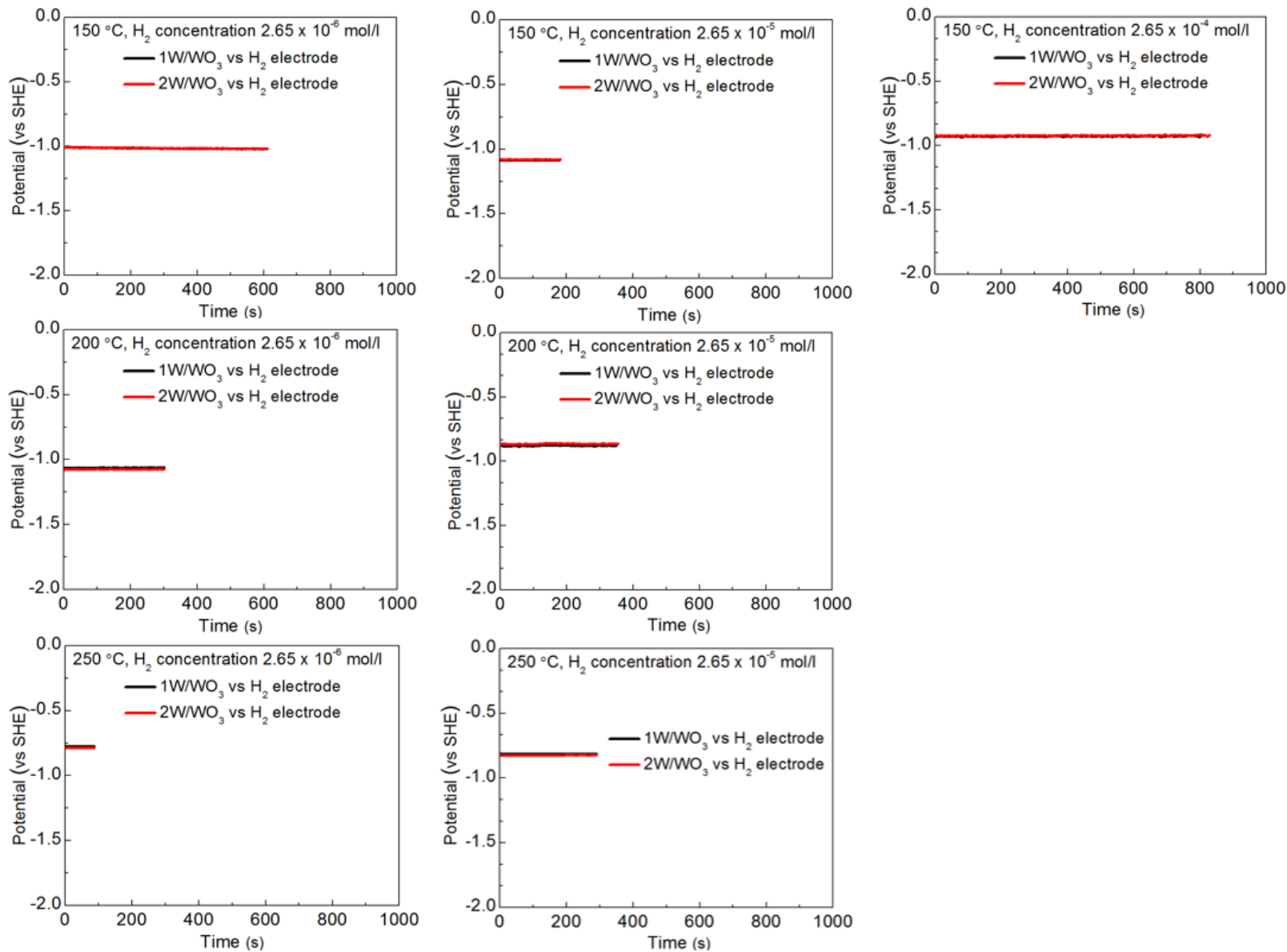

(a)

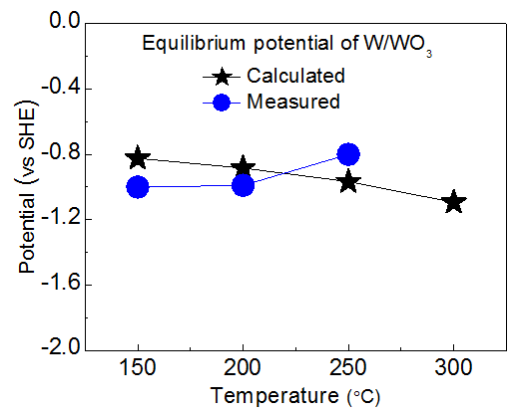

(b)

Figure X.23. Measured equilibrium potentials of $W / W O_{3}$ electrode half-cell reaction (a) versus standard hydrogen electrode and (b) compares with the calculated value based on Nernst Equation.

At each condition, the measured potential difference between two identical $\mathrm{W} / \mathrm{WO}$ electrodes in the autoclave cell is insignificant, indicating the repeatability. At the same 
temperature, the potential does not change obviously with the change of hydrogen concentration, indicating that the equilibrium potential of the $W / W O_{3}$ electrode is independent of the redox condition. The measured and calculated equilibrium potentials of $W / W O_{3}$ half-cell reaction is compared in Figure X.23b.

The logarithm of the steady-state current density was recorded as a function of the polarization potentials, as shown in Figures X.24 and X.25. The effects of hydrogen concentration (Figure X.24) and temperature (Figure X.25) on the HER were investigated. It seems the effect of temperature is more significant than the hydrogen concentration on the HER. With increasing temperature, the cathodic current density at the same polarization potential and the anodic limiting current density increased.
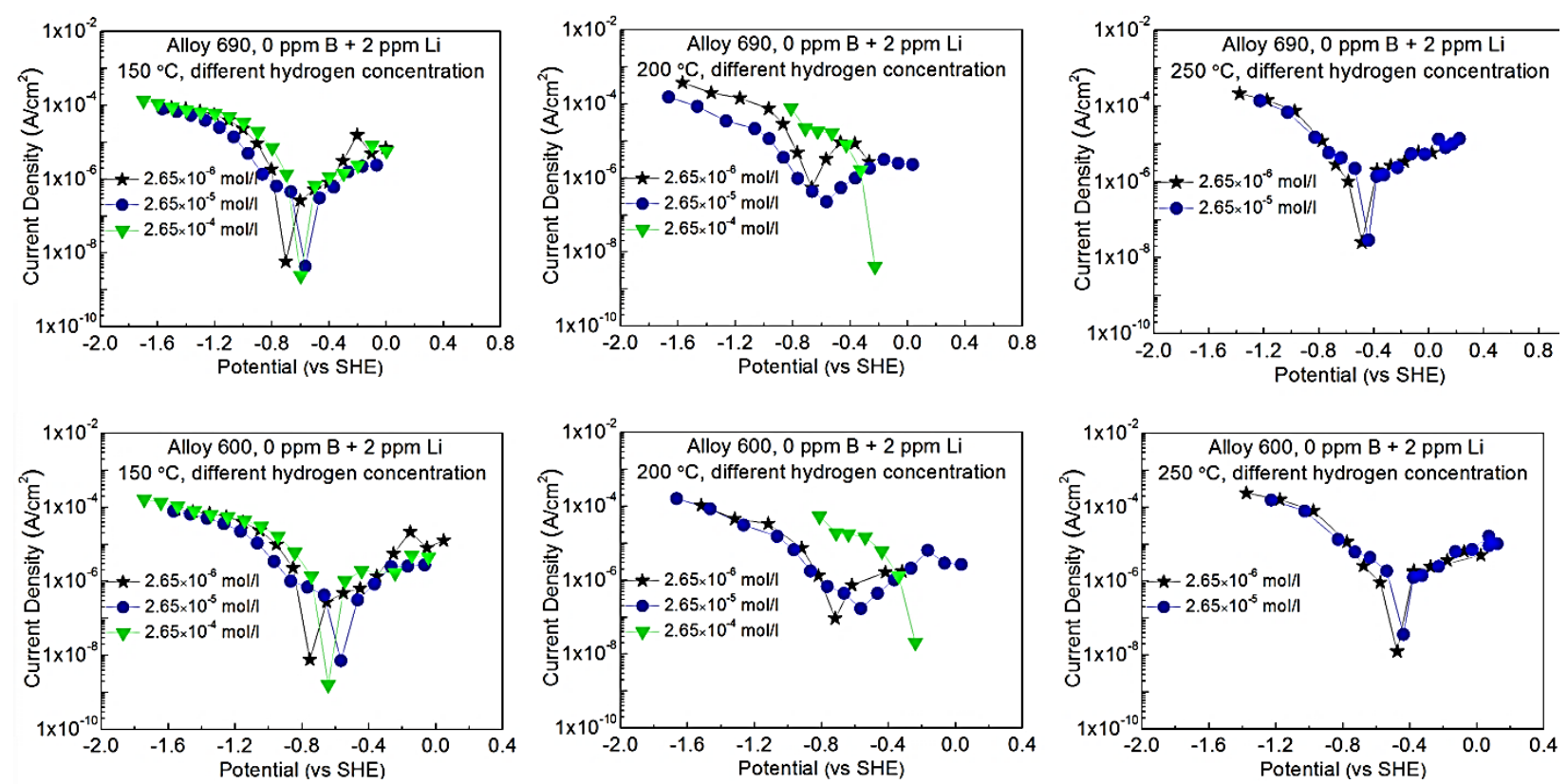

Figure X.24. Effect of hydrogen concentration on the HER for Alloys 690 and 600. 

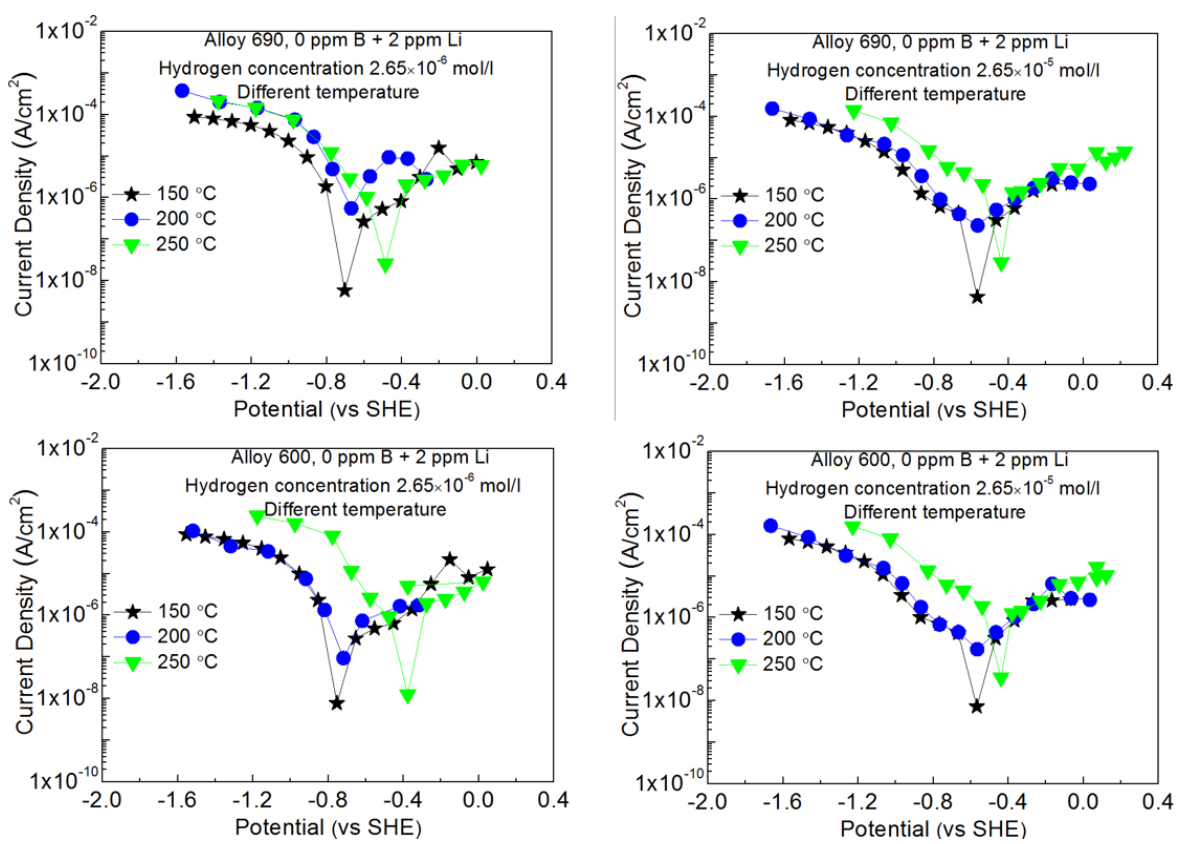

Figure X.25. Effect of temperature on the HER for Alloys 690 and 600.

\section{X.4. Optimization of MPM on EIS data.}

The MPM was optimized on the above experimental EIS data to extract values for the kinetic and other parameters in the model and to understand the effects of temperature and $\mathrm{pH}$ on the passive films formed on Alloy 600, Alloy 690, Type 304L SS, and Type 316L SS. As noted above, the MPM contains the PDM for the anodic passive metal dissolution and the generalized Butler-Volmer equation for the cathodic reaction (oxygen reduction).

\section{X.4.1. Point defect model (PDM) for Alloy 600, Alloy 690, Type 304L SS, and Type 316L SS.}

For the passive films formed on Alloy 600, Alloy 690, Type 304L SS, and Type 316L SS, the barrier layer comprises defective iron- and nickel-containing chromic oxide $(\mathrm{Fe}, \mathrm{Ni}, \mathrm{Cr})_{2+x} \mathrm{O}_{3-y}$, which primarily controls the corrosion resistance. Figure X.26 shows the electrochemical reactions that are envisioned in the PDM to occur at the $\mathrm{m} / \mathrm{bl}(x=L)$ and bl/ol $(x=0)$ interfaces and give rise to the generation, annihilation, and the final concentrations of the point defects in the barrier layer of each alloy [19,35,48-50]. For simplicity, only the related-Cr reactions are shown in the model. 


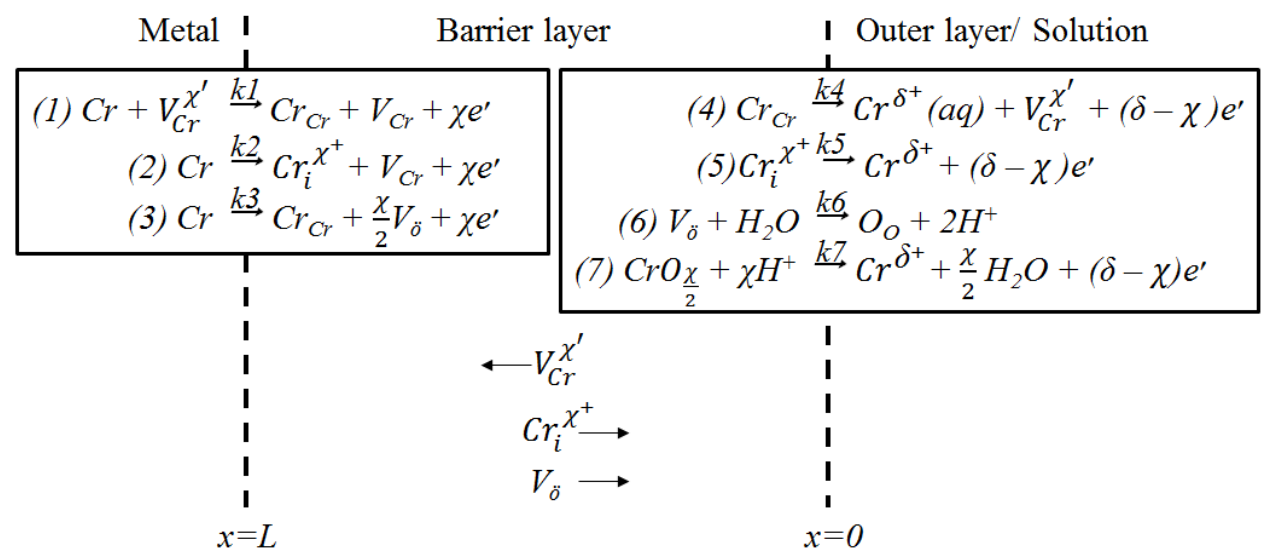

Figure X.26. Schematic diagram of the interfacial reactions for defects generation and annihilation in the barrier layer of passive film formed on Alloy 600, Alloy 690, Type 304L SS, and Type 316L SS based on the Point Defect Model. $\mathrm{Cr}$ is chromium atom in metal, $V_{C r}^{\chi^{\prime}}$ is chromium vacancy in the barrier layer, $C r_{C r}$ is chromium cation in normal cation sublattice in the barrier layer, $V_{C r}$ is chromium atom vacancy in metal, $C r_{i}^{\chi^{+}}$is chromium cation interstitial in the barrier layer, $V_{\ddot{0}}$ is an oxygen vacancy in the barrier layer, $\mathrm{Cr}^{\delta^{+}}$(aq) is chromium cation in solution, $O_{o}$ is oxide ion in anion sublattice in the barrier layer, $\mathrm{CrO}_{\frac{\chi}{2}}$ is stoichiometric barrier layer oxide, $\chi$ and $\delta$ are oxidation states of chromium in barrier layer and in solution, respectively, and all assumed to be $3, k_{i}$ is rate constant for reaction $i(i=1,2 \ldots 7)[48,50]$.

Reactions (1) and (4) indicate the annihilation and generation, respectively, of chromium cation vacancies $V_{C r}^{\chi^{\prime}}$ (electron acceptor) in the barrier layer, which dominates the semiconductor properties of a p-Type film. Reactions (2) and (5) show the formation of $\mathrm{Cr}_{i}^{\chi^{+}}, \mathrm{Fe}_{i}^{\chi^{+}}$, or $\mathrm{Ni}_{i}^{\chi^{+}}$ interstitials, which act as electron donors in the barrier layer, and the ejection of the same species at the outer layer/solution, respectively. Reaction (3) is responsible for the growth of the defective chromic oxide barrier layer through the generation of oxygen vacancies (electron donors) at the $\mathrm{m} / \mathrm{bl}$ interface. Reaction (6) indicates the annihilation of oxygen vacancies at the bl/ol interface. Reactions (2), (3), (5), (6) determine the electronic character for an n-Type film. Reaction (7) leads to the dissolution of the barrier layer. Reactions (1), (2), (4), (5), and (6) are lattice conservative, in that their occurrence does not result in the movement of the corresponding 
interface with respect to the laboratory frame of reference, while the Reactions (3) and (7) are lattice non-conservative and these two reactions determine the thickness of the barrier layer.

The rates of the above Reactions (1)-(7) depend on the rate constants, $k_{i}$, which are functions of the applied external potential $(V)$, the potential drop across the outer layer $\left(I_{\text {total }}\right.$. $\left.R_{o l}\right)$, which determines the potential at the bl/ol interface, the barrier layer thickness $(L)$, and the solution $p H$, are expressed as [17,48-54,7-76]:

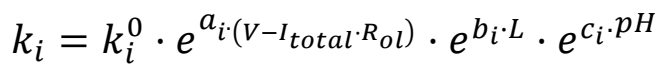

where $k_{i}^{0}$ is the standard rate constant for Reaction $i, I_{\text {total }}$ is the total current density (measured by potentiostatic polarization) for the system at steady state, which is the sum of the anodic $\left(I_{a}\right)$ and cathodic $\left(I_{c}\right)$ partial current densities assuming that neutral-charged $O_{2}$ is transported through the ol to the bl/ol interface where charge transfer (oxygen reduction) occurs, $R_{o l}\left(\Omega . \mathrm{cm}^{2}\right)$ is the specific resistance of the outer layer of the passive film. $a_{i}, b_{i}$, and $c_{i}$ are the exponential coefficients of the rate constants and are shown in Table X.7 as defined in terms of fundamental quantities. The change of film thickness with time due to the Reactions (3) and (7) is expressed as $[17,48-54,75-76]$ :

$d L / d t=\Omega k_{3}-\Omega k_{7}\left(C_{H^{+}} / C_{H^{+}}^{0}\right)^{n}$

where $\Omega$ is the mole volume of the bl per cation $\left(\mathrm{Cr}^{3+}\right)$ in the barrier layer and is calculated to be $14.59 \mathrm{~cm}^{3} / \mathrm{mol}, C_{H^{+}}$is the concentration of hydrogen ion at bl/ol interface, $C_{H^{+}}^{0}$ is the standard state concentration of hydronium and defined as $1 \mathrm{~mol} / \mathrm{cm}^{3}$ to ensure the units of $k_{7}$ are independent of the kinetic order $n$ of the dissolution reaction with respect to $C_{H^{+}}(0<n<1)$. The rate constants of $k_{3}$ and $k_{7}$ as defined by Equation X.7 are substituted into Equation X.8 noting that $b_{7}=0$. The thickness of the barrier layer in the steady-state is given by $d L / d t=0$. The simplified form for barrier layer thickness for no change in the metal ion oxidation state upon ejection from the bl/ol interface into the solution in the steady-state is expressed as $[17,48-54,75-$ 76]:

$L_{S S}=\left(\frac{1-\alpha}{\varepsilon}\right)\left(V-I_{\text {total }} R_{o l}\right)+\frac{1}{\varepsilon}\left(\frac{2.303 n}{\alpha_{3} \chi \gamma}-\beta\right) p H+\frac{1}{\alpha_{3} \varepsilon \chi \gamma} \ln \left[\frac{k_{3}^{0}}{k_{7}^{0}}\right]$

Of course, at the OCP, $I_{\text {total }}=0$, so that $I_{\text {total }} R_{o l}$ is also zero, regardless of the specific resistance of the outer layer. However, at any other potential, $I_{\text {total }} \neq 0$, and hence, the potential 
drop across the ol must be included in the analysis. The anodic passive current density $I_{a}$ arising from Reactions (1) - (7) is expressed as [17,48-54,75-76]:

$I_{a}=F \cdot\left[\chi k_{1} C_{V}^{L}+\chi k_{2}+\chi k_{3}+(\delta-\chi) k_{4}+(\delta-\chi) k_{5} C_{i}^{0}+(\delta-\chi) k_{7}\left(\frac{C_{H^{+}}}{C_{H^{+}}^{0}}\right)^{n}\right]$

where $C_{V}^{L}$ and $C_{i}^{0}$ are the concentrations of chromium cation vacancies at the $\mathrm{m} / \mathrm{bl}$ interface and metal $(\mathrm{Cr}, \mathrm{Ni}, \mathrm{Fe})$ interstitials at the bl/ol interface, respectively. Since the passive films formed on Alloy 600, Alloy 690, Type 304L SS and Type 316L SS show n-Type semiconductivity and assuming that the oxidation state of the chromium ions is same in film and solution $(\delta=\chi)$, Equation X.10 is simplified to:

$I_{a}=F \cdot\left(\chi k_{2}+\chi k_{3}\right)$

Table X.7. Functional forms of the rate constants with applied potential for Reactions (1) - (7) and the exponential coefficients for these rate constants $[17,63]$.

\begin{tabular}{|c|c|}
\hline Reactions (1) - (3) & 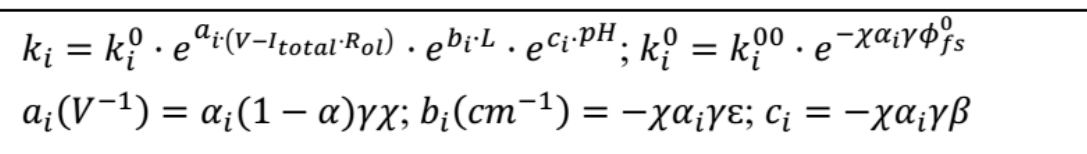 \\
\hline Reactions (4) - (6) & 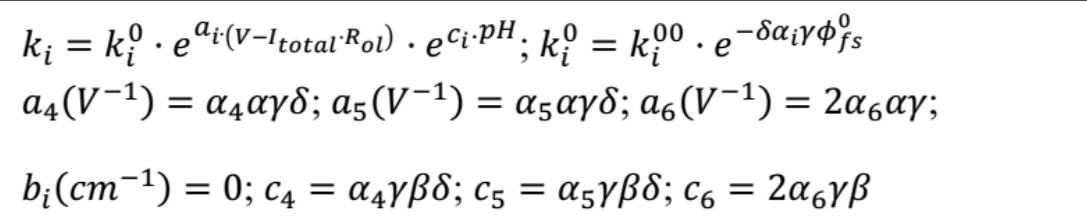 \\
\hline Reaction (7) & $\begin{array}{l}k_{7}=k_{7}^{0} \cdot e^{\left.a_{7 \cdot\left(V-I_{\text {total }} R_{o l}\right)}\right)} e^{c_{7} \cdot p H} ; k_{7}^{0}=k_{7}^{00} \cdot e^{(\delta-\chi) \alpha_{7} \gamma \phi_{f s}^{0}} \\
a_{7}\left(V^{-1}\right)=\alpha_{7} \alpha \gamma(\delta-\chi) ; b_{7}\left(\mathrm{~cm}^{-1}\right)=0 ; c_{7}=\alpha_{7} \beta \gamma(\delta-\chi)\end{array}$ \\
\hline \multicolumn{2}{|c|}{$\begin{array}{l}k_{i} \text {-Rate constants for Reaction } i ; \quad k_{i}^{0} \text {-Standard rate constant; } k_{i}^{00} \text {-Base rate constant; } a_{i}, b_{i} \text {, } \\
c_{i} \text {-Exponential coefficients; } V \text {-Applied external potential; } I_{\text {total }} \text {-Total current density; } R_{o l} \text {-Resistance } \\
\text { of film outer layer; L-Barrier layer thickness; } \phi_{f s}^{0} \text {-Standard potential of film/solution interface; } \alpha- \\
\text { Polarizability of the bl/ol interface; } \alpha_{i} \text {-Transfer coefficients; } \gamma=F / R T, F \text { is Faraday's constant, } R \\
\text { represents molar gas constant, and } T \text { is temperature in Kelvin; } \beta \text {-Effect of } p H \text { on the potential drop } \\
\text { across the bl/ol interface. }\end{array}$} \\
\hline
\end{tabular}

\section{X.4.2. Generalized Butler-Volmer equation for the cathodic reaction}

The dominant cathodic reaction for all alloys in this study is oxygen reduction which is expressed as the following Reaction (8): 
$\mathrm{O}_{2}+2 \mathrm{H}_{2} \mathrm{O}+4 e^{-} \rightarrow 4 \mathrm{OH}^{-}$

Based on the generalized Butler-Volmer equation, the cathodic current density $I_{c}$ is expressed [61] as:

$I_{C}=\frac{-e^{-\alpha_{c} \cdot \frac{F}{R T} \cdot\left(V-V_{e q}\right)}}{\frac{1}{i_{0}}-\frac{e^{-\alpha_{c} \cdot \frac{F}{R T} \cdot\left(V-V_{e q}\right)}}{i_{l, c}}}$

in which we assume that because of the large overpotential $\left(O C P-V_{e q}\right)$ the reaction is irreversible in the forward (reduction) direction to give the cathodic partial reaction current density as represented by Equation X.12. In this expression, $\alpha_{c}$ is the transfer coefficient of the oxygen reduction, $V_{e q}$ is the equilibrium potential of the oxygen electrode reaction under the specific conditions, $i_{0}$ is the exchange current density, and $i_{l, c}$ is the mass-transfer limited current density. Because Equation X.12 applies strictly to charge transfer at a bare (oxide-free) surface for which $i_{0}$ is a potential-independent constant, it is necessary to include a correction for the presence of the passive film. Thus, in the case of a passive surface, such as that which exists on Alloy 600, Alloy 690, Type 304L SS and Type 316L SS, the thickness of the bl increases linearly with potential [Equation (X.9)] and because charge carriers (electrons and electron holes) must quantummechanically tunnel across the barrier layer and the exchange current density is potentialdependent. It is important to recognize that the exchange current density corresponds to the redox current for the reaction; in this case, the oxygen electrode reaction (OER), at its equilibrium potential, which itself is a function of $\left[\mathrm{O}_{2}\right], \mathrm{pH}$, and temperature that also control the thickness of the barrier layer. Following our previous work [56-57,77-79], we write the exchange current density as:

$i_{0}=\widehat{\iota_{0}} \exp \left(-\hat{\beta} L_{s S}\right)$

where $\widehat{\iota_{0}}$ is the hypothetical exchange current density on a bare (oxide-free) metal surface and $\hat{\beta}$ is the tunneling constant $[10,77,80]$. Simplifying Equation (X.9), we write

$L_{S S}=\frac{1-\alpha}{\varepsilon} V+g$

and substitution into Equation (X.13) yields:

$i_{0}=\widehat{\iota_{0}} \exp \left[-\hat{\beta}\left(\frac{1-\alpha}{\varepsilon}\right) V\right] \exp (-\hat{\beta} g)$ 
Equation (X.15) is then combined with Equation (X.12) to describe the kinetics of the OER on the passive alloy surface.

\section{X.4.3. The impedance of the system.}

The above Reactions (1)-(7) (Figure X.26) and Reaction (8) contribute to the impedance of the entire system, which is indicated by the electrical equivalent circuit in Figure X.27 and which is measured by EIS.

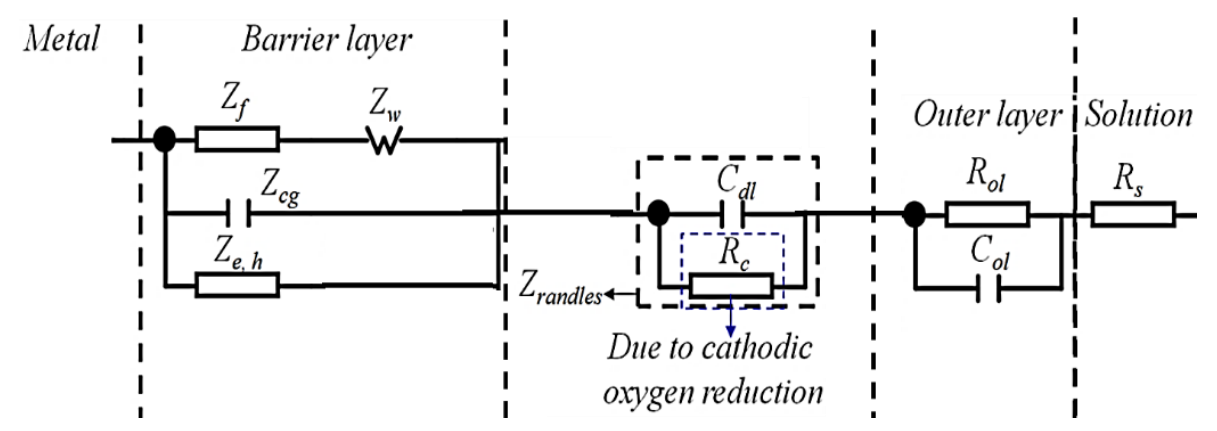

Figure X.27. Schematic of the electrical equivalent circuit for the entire electrochemical reaction system of Alloy 600, Alloy 690, Type 304L SS, and Type 316L SS.

The total impedance $Z_{\text {total }}$ includes the impedance of the barrier layer $\left(Z_{b l}\right.$, containing the $\mathrm{m} / \mathrm{bl}$ and $\mathrm{bl} / \mathrm{ol}$ interfaces and barrier layer itself), the impedance of the outer layer $\left(Z_{o l}\right)$, a Warburg impedance $\left(Z_{w}\right)$ for the transport of the dominant point defect within the bl, capacitances for the bl $\left(C_{g}\right)$ and ol $\left(C_{o l}\right)$, and the resistance of the solution $\left(R_{S}\right)$ between the outer layer and the tip of the reference electrode is expressed as [48-54]:

$Z_{\text {total }}=Z_{b l}+Z_{\text {Zrandles }}+Z_{o l}+R_{s}=\frac{1}{\left(Z_{f}+Z_{w}\right)^{-1}+Z_{c g}^{-1}+Z_{e, h}{ }^{-1}}+Z_{\text {zrandles }}+Z_{o l}+R_{s}$

where $Z_{f}$ is the Faradaic impedance due to the electrochemical reactions occurring at the $\mathrm{m} / \mathrm{bl}$ and $\mathrm{bl} / \mathrm{ol}$ interfaces, which is given by:

$Z_{f}=Y_{f}^{-1}$

$Y_{f}=F \cdot\left(\chi k_{2} a_{2}+\chi k_{3} a_{3}\right)+F \Omega\left(\chi k_{2} b_{2}+\chi k_{3} b_{3}\right)\left[k_{3} a_{3}-k_{7} a_{7} \cdot\left(C_{H^{+}} / C_{H^{+}}^{0}\right)^{n}\right]\left(j \omega-\Omega k_{3} b_{3}\right)^{-1}$ 
$Z_{w}$ is the Warburg impedance arising from the migration of dominant point defect in the barrier layer [75] and is expressed as:

$Z_{w}=\frac{\sigma}{\sqrt{\omega}}-j \frac{\sigma}{\sqrt{\omega}}$

where $\omega$ is the angular frequency, and $\sigma$ is the Warburg coefficient. $Z_{c g}$ is the impedance of the geometric capacitance of the dielectric barrier layer, $Z_{e, h}$ is the electronic impedance due to the movement of the electrons and/or electron holes. The Warburg impedance is added because of the assumption that the interfacial reactions are irreversible, as indicated in Figure X.27, resulting in the concentrations of neither the metal interstitial nor the oxygen vacancy appearing in the right sides of the expressions for the rates of the reactions. Accordingly, the transport of these defects across the barrier layer is decoupled from the interfacial kinetics. The impedance, $Z_{\text {randles }}$, is the impedance attributed to the redox reaction (OER) at bl/ol interface (oxygen reduction coupled with $C_{d l}$ ), which is indicated by:

$Z_{\text {randles }}=\frac{1}{Z_{C_{d l}}^{-1}+Z_{R_{C}}^{-1}}$

$Z_{C_{d l}}$ is the impedance of the double layer at the bl/ol interface, $Z_{R_{C}}$ is the charge transfer impedance due to the cathodic oxygen reduction reaction, which is derived from Equation (X.21) as:

$Z_{R_{C}}=\left(\frac{d I_{c}}{d V}\right)^{-1}$

$\frac{d I_{C}}{d V}=\frac{-\alpha_{c} F}{R T} \cdot I_{C}+\left\{\frac{\alpha_{c} F}{R T i_{l, c}}+\frac{\widehat{\beta}(1-\alpha)}{\varepsilon i_{0} \cdot \exp \left[-\frac{\alpha_{c} F}{R T}\left(V-V_{e q}\right)\right]}\right\} \cdot I_{C}^{2}$

The impedance of the outer layer $Z_{o l}$ is expressed as:

$Z_{o l}=\left[\frac{1}{R_{o l}}+Z_{C_{o l}}^{-1}\right]^{-1}$

where $Z_{C_{o l}}$ is the impedance arising from the geometric capacitance of the outer layer and $R_{o l}$ is due to the resistance of the solution in the pores of the outer layer. 


\section{X.4.4. Optimize the MPM on the measured EIS data to extract model parameters.}

The anodic and cathodic electrochemical Reactions (1)-(8) contribute to the total impedance of the system, which is measured by EIS and calculated by the MPM from the optimized parameter values. In performing the following analysis, the real and imaginary parts of the impedance are plotted in the Nyquist plane for specific frequencies and the absolute value of impedance and the phase angle $\left[\tan ^{-1}\left(-Z^{\prime \prime} / Z^{\prime}\right)\right]$ are plotted as a function of $\log (f)$ in the Bode plane with a comparison being made between the measured EIS data and that calculated from the MPM using the optimized parameter values. Optimization of the MPM on the experimental EIS data was performed using the Igor Pro, genetically-inspired (version 6.37, WaveMetrics Inc.) algorithm. The optimization was performed on all the impedance data sets collected periodically according to the following procedures: Firstly, the values of $\beta$ (influence of $p H$ on the potential drop across the bl/ol interface), $\hat{\beta}\left(\mathrm{cm}^{-1}\right), \delta$ (oxidation state of the cation in solution), and $\chi$ (oxidation state of the cation in barrier layer) are assumed to be $-0.01,0.21 \times 10^{8}$, 3 , and 3 , respectively [55,79]; secondly, optimize and lock the parameters like $\alpha$ (polarizability of the bl/ol interface), $\alpha_{i}$ (transfer coefficient for Reaction $i$ ), and $n$ (kinetic order of film dissolution with respect to the concentration of hydrogen ions); thirdly, optimize and lock the parameters of $k_{i}^{00}$ (base rate constant for Reaction $i$ )and $\varepsilon$ (electrical field strength); finally, optimize and obtain the equivalent circuit parameters,such as $\sigma$ (Warburg coefficient, determining the Warburg impedance, $Z_{w}$ ), $R_{S}$ (solution resistance), $R_{e, h}$ (electronic resistance), $C_{g}$ (geometric capacitance), $C_{d l}$ (capacitance of double layer), $R_{o l}$ (resistance of the outer layer of the passive film), $C_{o l}$ (capacitance of the outer layer of the passive film), $\alpha_{c}$ (transfer coefficient of cathodic oxygen electrode reaction), $\widehat{\iota_{0}}$ (hypothetical exchange current density of oxygen reaction), and $i_{l, c}$ (limiting current density of oxygen reduction).

Figures X.28 to X.36 show a comparison of the impedance calculated from the optimized parameters and the measured EIS data for Alloy 600, Alloy 690, Type 304L SS, and Type 316L $\mathrm{SS}$ in borate buffer solution under open circuit and $+0.3 \mathrm{~V}_{\mathrm{OCP}}$ conditions as a function of temperature. For each alloy, the impedance data calculated from the MPM equations correlate very well with the experimental data in both the Nyquist and Bode planes. Thus, the impedance of each alloy is accurately represented by the MPM, and the impedance model can be used with confidence to interpret the experimental data. 

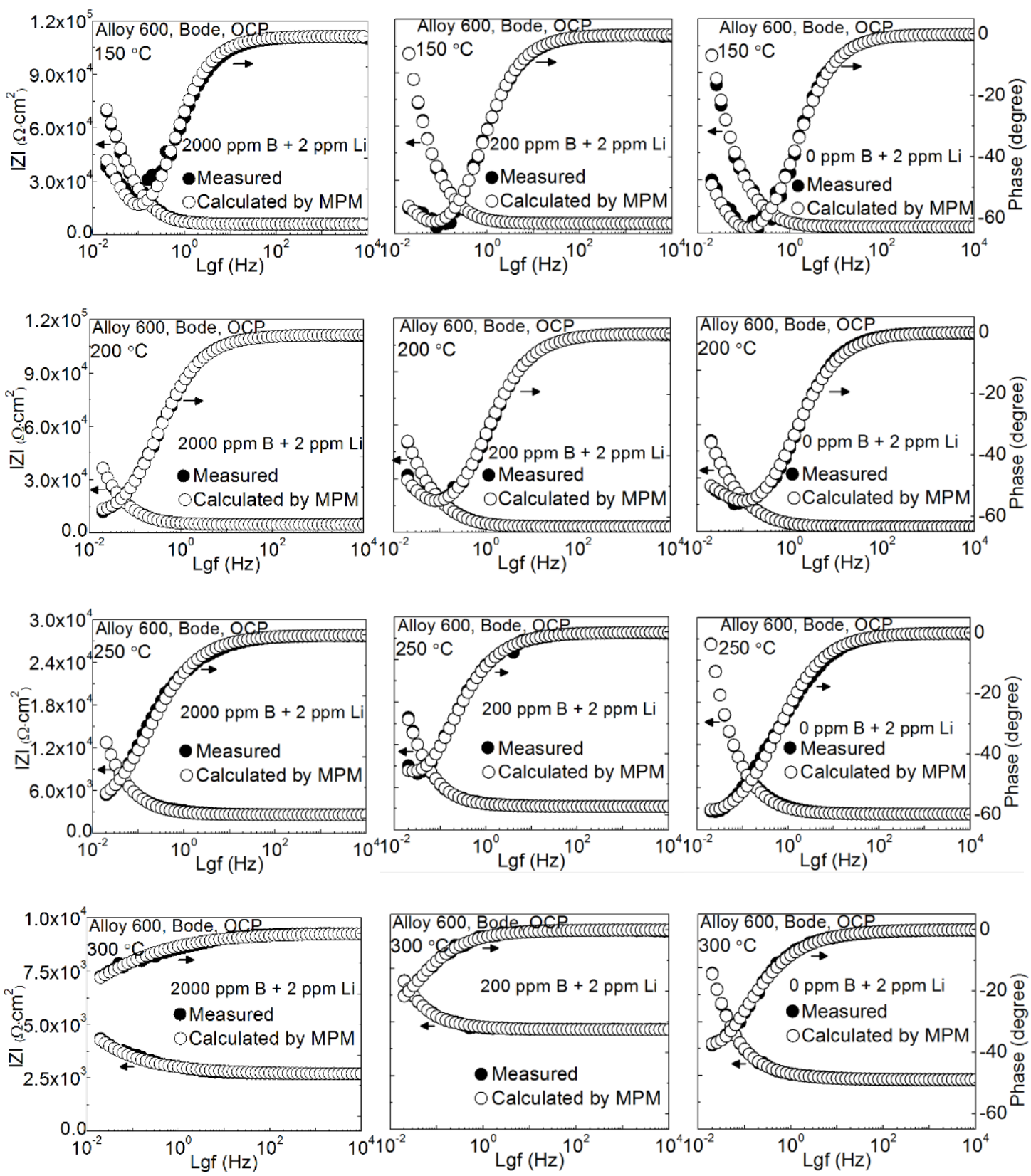

Figure X.28. Comparison of the impedance plots between the experimental measured EIS data and the calculated EIS data from the Mixed Potential Model (MPM) in the Bode plane for passive films formed on Alloy 600 at OCP in borate buffer solutions containing $400 \mathrm{ppb}$ dissolved oxygen at different temperatures. 

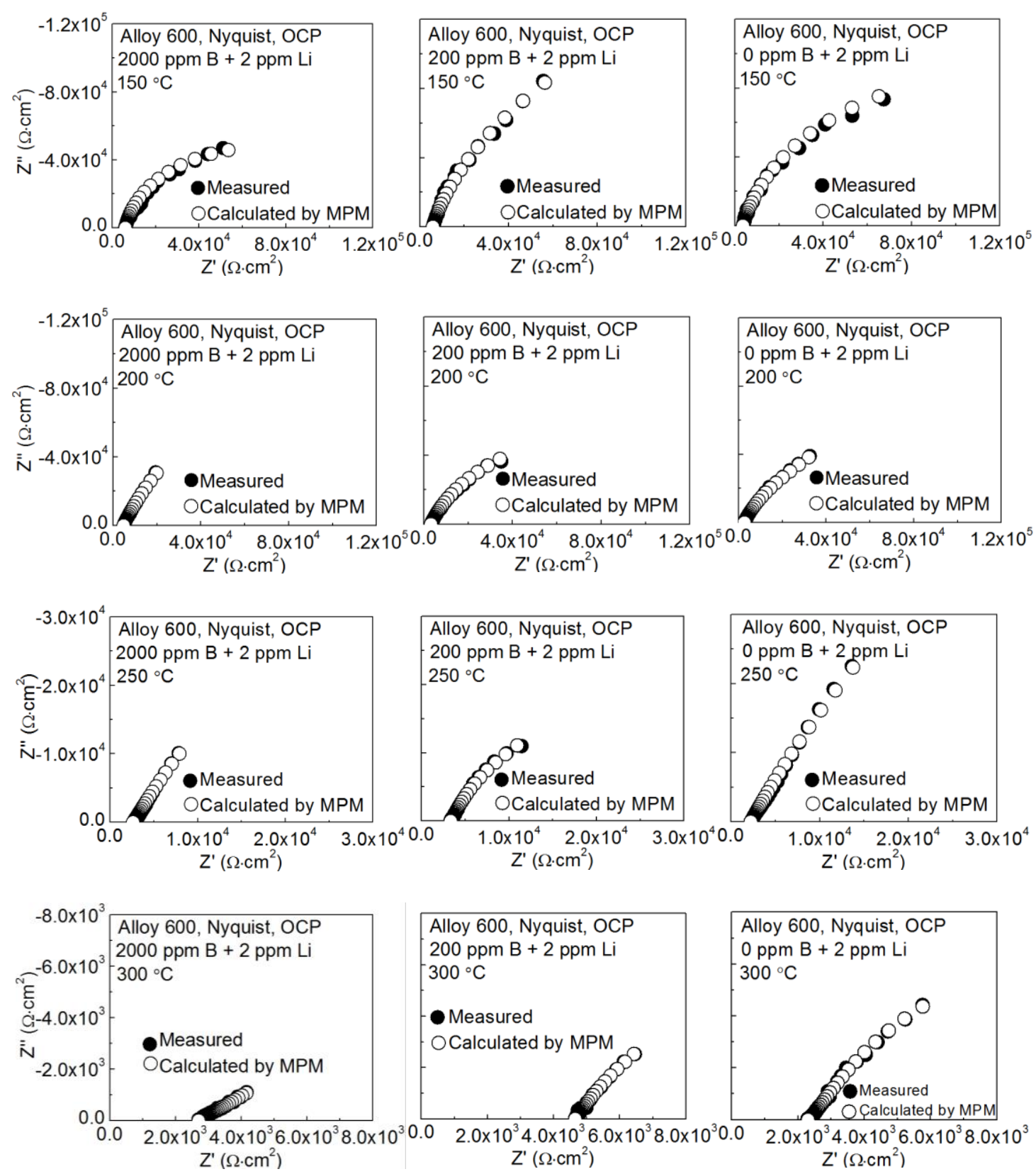

Figure X.29. Comparison of the impedance plots between the experimental measured EIS data and the calculated EIS data from the Mixed Potential Model (MPM) in the Nyquist plane for passive films formed on Alloy 600 at OCP in borate buffer solutions containing $400 \mathrm{ppb}$ dissolved oxygen at different temperatures. 

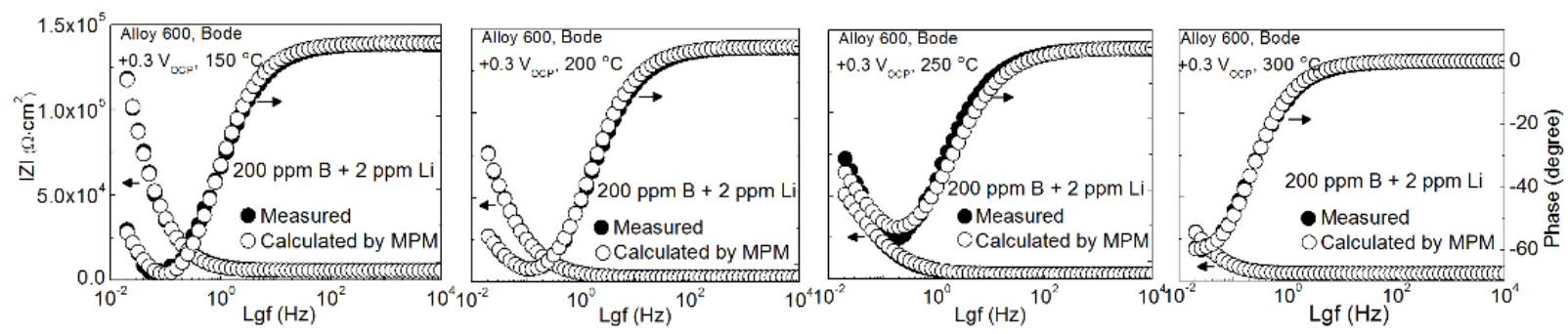

(a)
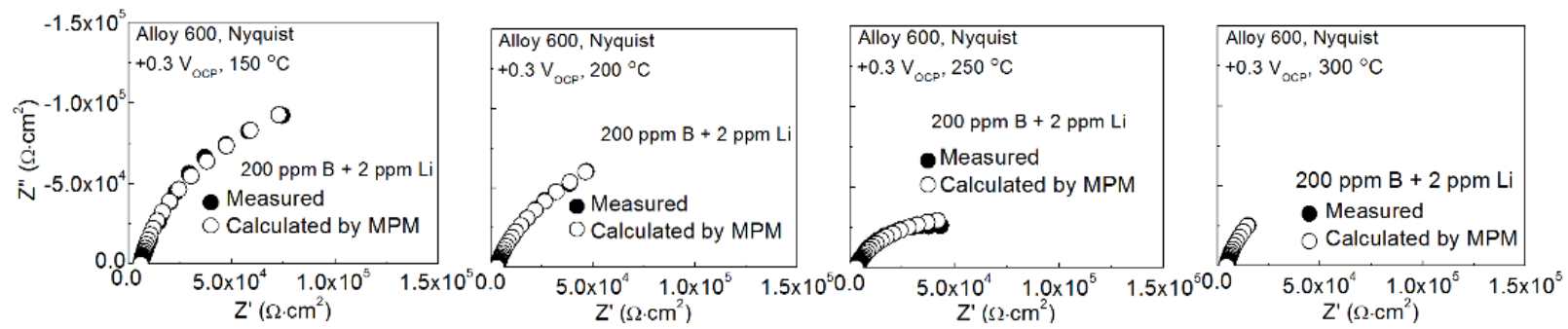

(b)

Figure X.30. Comparison of the impedance plots between the experimental measured EIS data and the calculated EIS data from the Mixed Potential Model (MPM) in the (a) Bode and (b) Nyquist planes for passive films formed on Alloy 600 at $+0.3 \mathrm{~V}_{\text {OCP }}$ in borate buffer solutions containing $400 \mathrm{ppb}$ dissolved oxygen at different temperatures. 

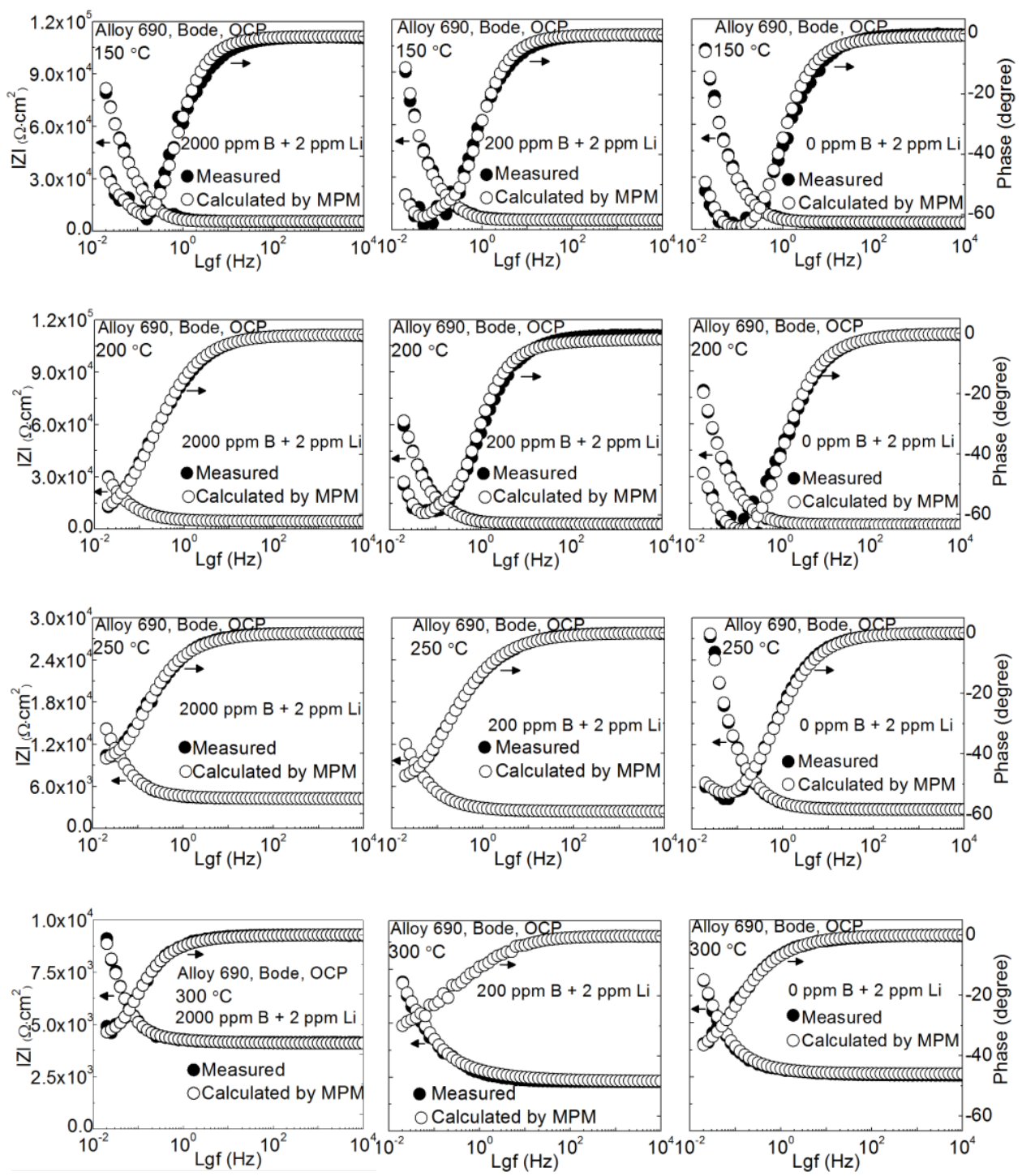

Figure X.31. Comparison of the impedance plots between the experimental measured EIS data and the calculated EIS data from the Mixed Potential Model (MPM) in the Bode plane for passive films formed on Alloy 690 at OCP in borate buffer solutions containing 400 ppb dissolved oxygen at different temperatures. 

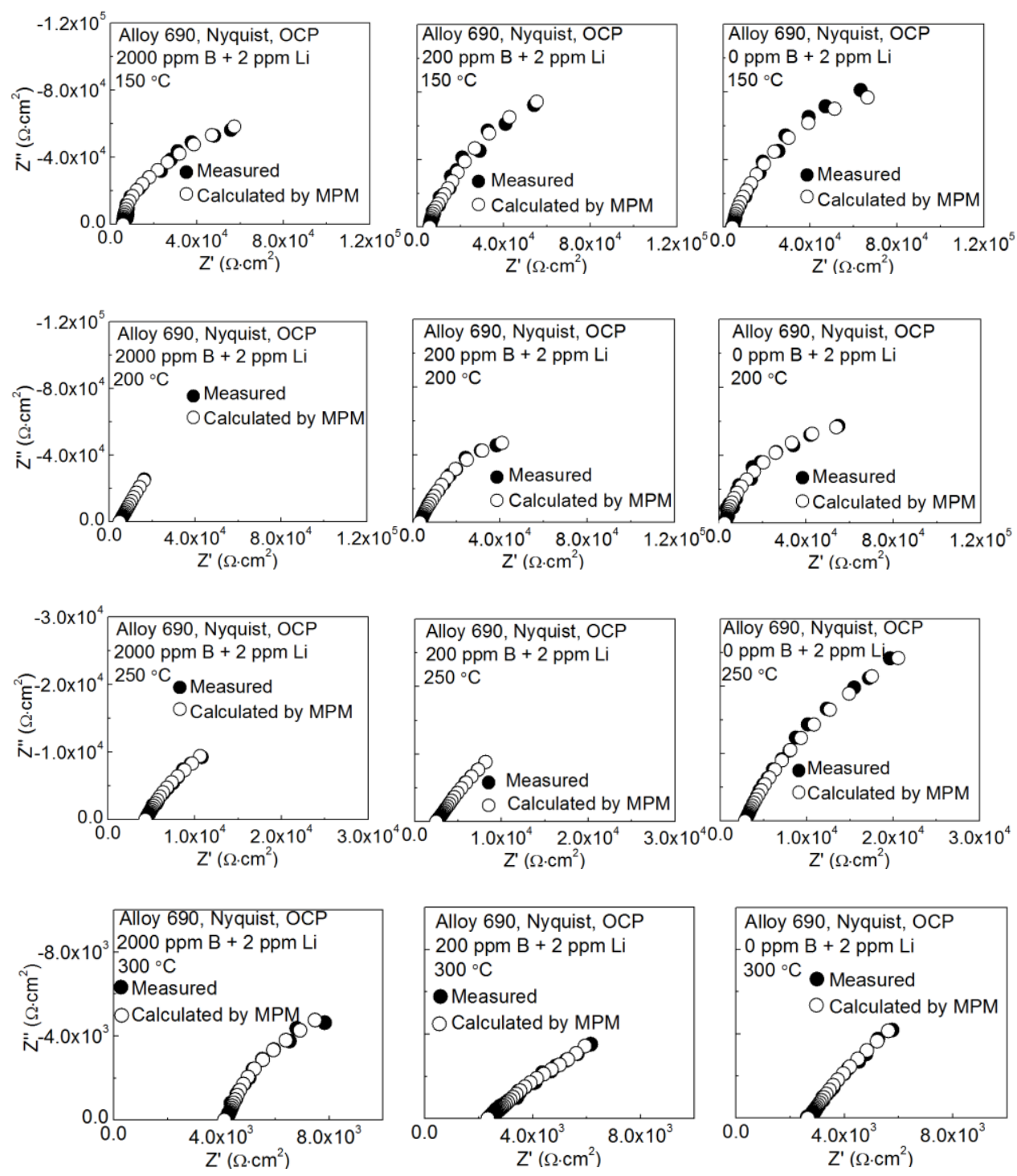

Figure X.32. Comparison of the impedance plots between the experimental measured EIS data and the calculated EIS data from the Mixed Potential Model (MPM) in the Nyquist plane for passive films formed on Alloy 690 at OCP in borate buffer solutions containing $400 \mathrm{ppb}$ dissolved oxygen at different temperatures. 

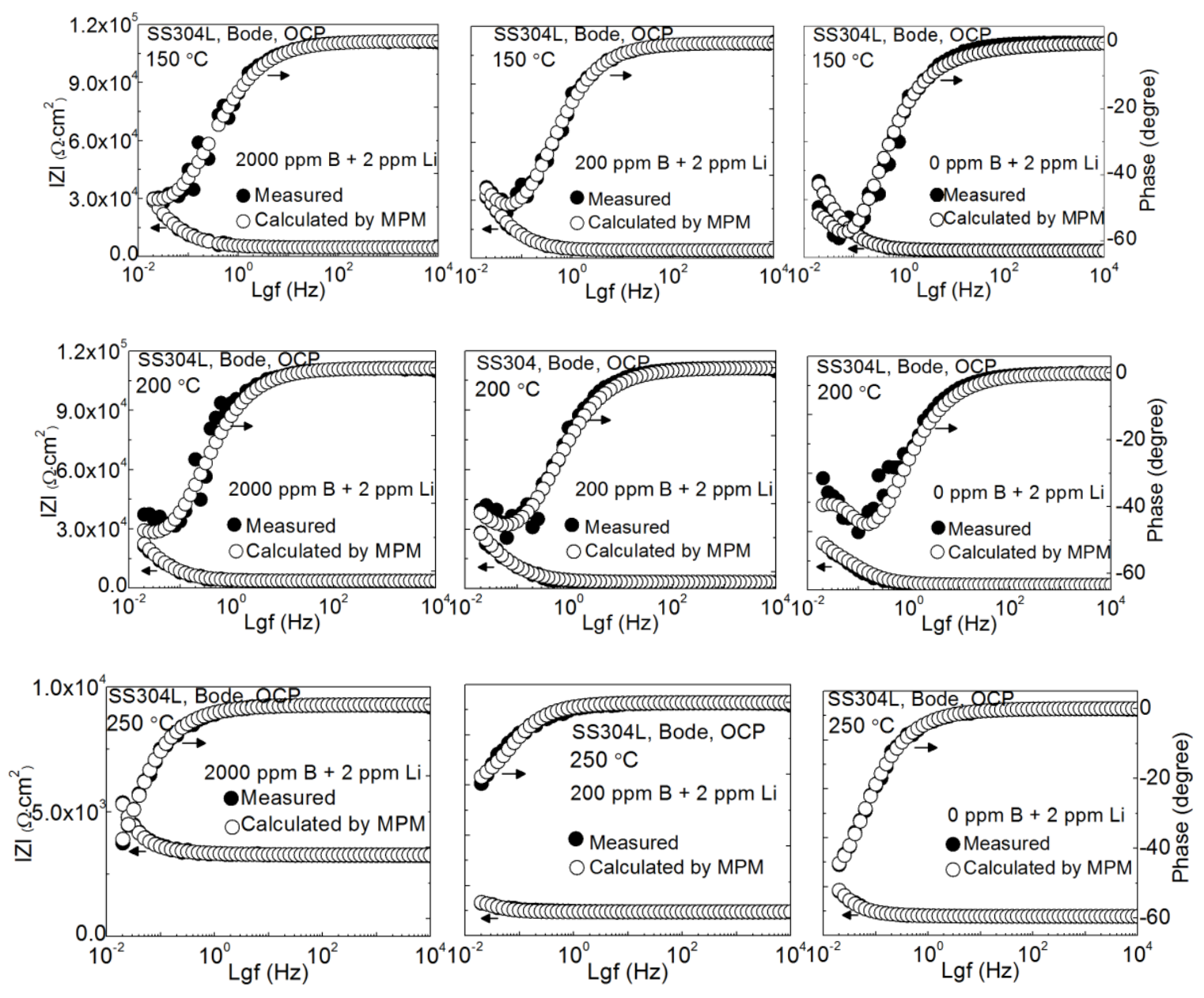

Figure X.33. Comparison of the impedance plots between the experimental measured EIS data and the calculated EIS data from the Mixed Potential Model (MPM) in the Bode plane for passive films formed on Type 304L SS at OCP in borate buffer solutions containing $400 \mathrm{ppb}$ dissolved oxygen at different temperatures. 

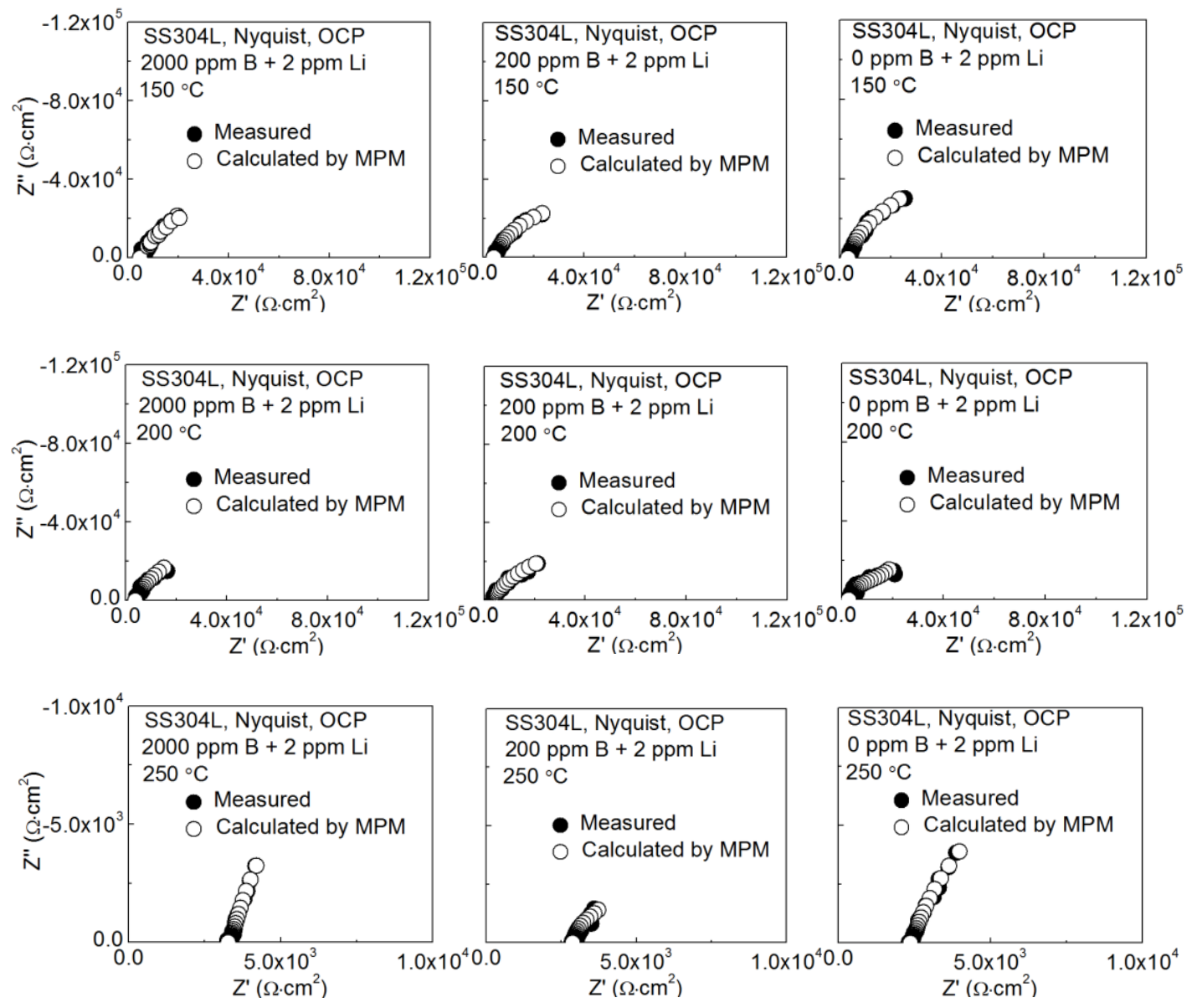

Figure X.34. Comparison of the impedance plots between the experimental measured EIS data and the calculated EIS data from the Mixed Potential Model (MPM) in the Nyquist plane for passive films formed on Type 304L SS at OCP in borate buffer solutions containing $400 \mathrm{ppb}$ dissolved oxygen at different temperatures. 

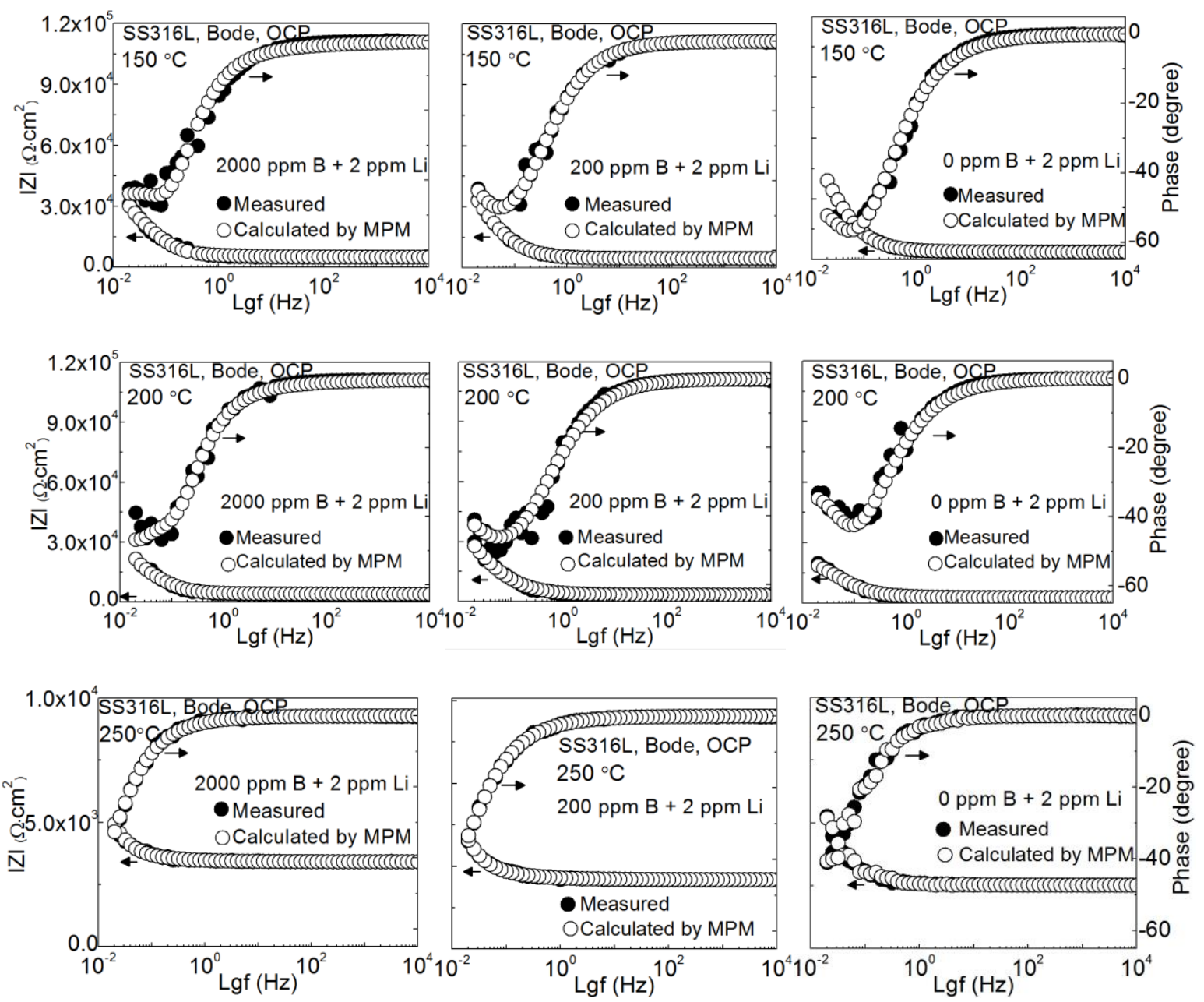

Figure X.35. Comparison of the impedance plots between the experimental measured EIS data and the calculated EIS data from the Mixed Potential Model (MPM) in the Bode plane for passive films formed on Type 316L SS at OCP in borate buffer solutions containing $400 \mathrm{ppb}$ dissolved oxygen at different temperatures. 

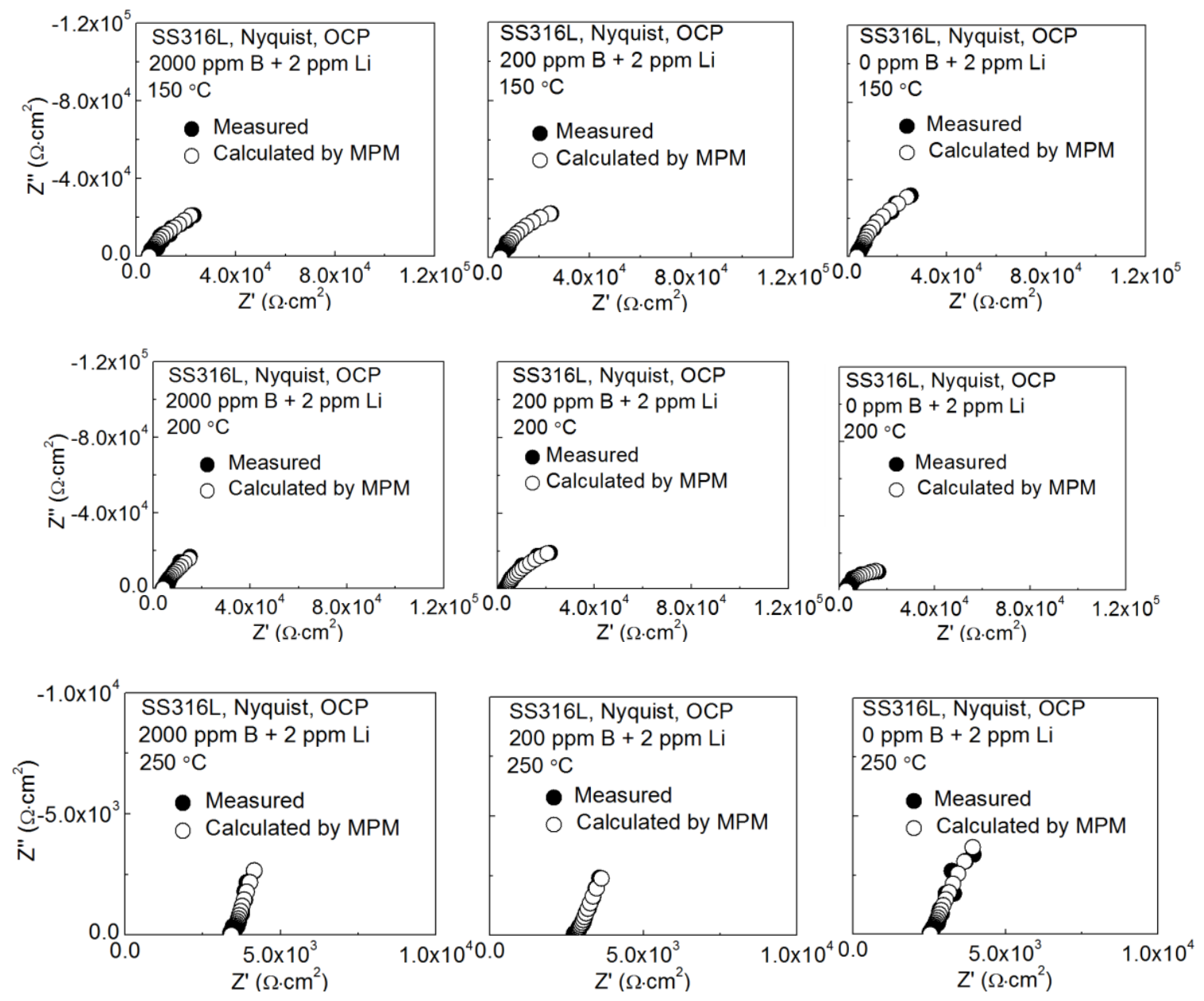

Figure X.36. Comparison of the impedance plots between the experimental measured EIS data and the calculated EIS data from the Mixed Potential Model (MPM) in the Nyquist plane for passive films formed on Type 316L SS at OCP in borate buffer solutions containing $400 \mathrm{ppb}$ dissolved oxygen at different temperatures.

As noted above, values for the various model parameters in the MPM were extracted with minimized error (optimization) between the experimental EIS data and simulated values for passive films formed on Alloy 600, Alloy 690, Type 304L SS, and Type 316L SS in solutions with $400 \mathrm{ppb}$ dissolved oxygen containing 2000 ppm $B+2$ ppm $L i, 200$ ppm $B+2$ ppm $L i$, and 0 ppm $B+2 \mathrm{ppm} L i$ at different temperatures. The extracted and calculated model parameters for Alloy 600 are show from Table X.8 to X.16. All these parameters for Alloy 690, Type 304L SS, and Type 316L SS are included in the appendix (Tables A.1 to A.18). The effect of $\mathrm{pH}$ (at the same temperature) on each parameter is not as significant as the effect of temperature (at the same $\mathrm{pH}$ ). 
With increasing temperature (in each solution): the electrical field strength $(\varepsilon)$, solution resistance $\left(R_{S}\right)$, the resistance of the passive film outer layer $\left(R_{o l}\right)$ and electronic resistance $\left(R_{e, h}\right)$ decreased; the Warburg coefficient $(\sigma)$, geometric capacitance $\left(C_{g}\right)$, cathodic oxygen reduction transfer coefficient $\left(\alpha_{c}\right)$, hypothetical exchange current density $\left(\widehat{\iota}_{0}\right)$ and limiting current density $\left(i_{l, c}\right)$ increased; and the capacitance of double layer $\left(C_{d l}\right)$ and the outer layer $\left(C_{o l}\right)$ show no systematic change. The reduction of $\varepsilon$ with increasing temperature is induced by thermal diode effect [81] in which electrons are thermally excited into the conduction band and move to quench the electric field. The resistance of the solution $\left(R_{S}\right)$ decreased with increasing temperature because of the increased conductivity of the borate buffer solution. The electronic resistance $R_{e, h}$ is always in orders of magnitude higher than that the total impedance at the lowest frequency at each temperature; such that it has little influence on the total impedance of the passive film because $R_{e, h}$ is in parallel with $Z_{c g}$ and the sum of $Z_{f}$ and $Z_{w}$, and hence the total impedance of the barrier layer is determined by the lowest value among them. As expected, the hypothetical exchange current density $\left(\widehat{l_{0}}\right)$ increased with increasing temperature. However, there is no obvious change in the mass-transfer limited current density $\left(i_{l, c}\right)$ for oxygen reduction which is mainly controlled by the oxygen concentration rather than by the temperature. As shown in Tables X.9 and X.10, for the passive films formed on Alloy 600 at the same temperature but different potentials (OCP and $\left.+0.3 \mathrm{~V}_{\mathrm{OCP}}\right)$ in a solution containing $200 \mathrm{ppm} B+2 \mathrm{ppm} L i$, the parameters such as the polarizability $\alpha$, electric field strength across barrier layer $\varepsilon$, the transfer coefficients $\alpha_{i}$, the base rate constants $k_{2}^{00}$, which are independent of the applied potential; this satisfies the criteria for acceptable impedance analysis via MPM optimization [17]. 
Table X.8. Values of the parameters extracted from the optimization of the mixed potential model on the experimental EIS data for passive films formed on Alloy 600 at OCP in a solution containing $2000 \mathrm{ppm} \mathrm{B}+2 \mathrm{ppm} \mathrm{Li}$ and $400 \mathrm{ppb}$ dissolved oxygen at different temperatures.

\begin{tabular}{|c|c|c|c|c|c|}
\hline Parameters & $150^{\circ} \mathrm{C}$ & $200^{\circ} \mathrm{C}$ & $250^{\circ} \mathrm{C}$ & $300^{\circ} \mathrm{C}$ & Method \\
\hline$O C P\left(V_{S H E}\right)$ & -0.374 & -0.460 & -0.567 & -0.773 & Measured \\
\hline$p H$ & 5.91 & 5.98 & 6.20 & 6.71 & $\begin{array}{l}\text { Calculated by } \\
\text { pH Calc }\end{array}$ \\
\hline$\varepsilon\left(\mathrm{V} \cdot \mathrm{cm}^{-1}\right)$ & $1.1 \times 10^{6}$ & $9.1 \times 10^{5}$ & $8.7 \times 10^{5}$ & $8.4 \times 10^{5}$ & $3^{\text {rd }}$ stage \\
\hline$\sigma$ & $1.0 \times 10^{4}$ & $1.0 \times 10^{5}$ & $3.0 \times 10^{5}$ & $4.4 \times 10^{5}$ & $3^{\text {rd }}$ stage \\
\hline$R_{s}(\Omega)$ & 6049 & 4759 & 2601 & 2691 & $3^{\text {rd }}$ stage \\
\hline$R_{e, h}\left(\Omega \cdot \mathrm{cm}^{2}\right)$ & $3.1 \times 10^{5}$ & $3.0 \times 10^{5}$ & $2.0 \times 10^{5}$ & $5.0 \times 10^{4}$ & $3^{\text {rd }}$ stage \\
\hline$C_{g}\left(\mathrm{~F} \cdot \mathrm{cm}^{-2}\right)$ & $6.3 \times 10^{-5}$ & $1.4 \times 10^{-4}$ & $4.0 \times 10^{-4}$ & $1.2 \times 10^{-3}$ & $3^{\text {rd }}$ stage \\
\hline$C_{d l}\left(\mathrm{~F} \cdot \mathrm{cm}^{-2}\right)$ & $7.1 \times 10^{-3}$ & $1.1 \times 10^{-4}$ & $1.7 \times 10^{-5}$ & $9.2 \times 10^{-3}$ & $3^{\text {rd }}$ stage \\
\hline$R_{o l}\left(\Omega \cdot \mathrm{cm}^{2}\right)$ & 4907 & 3712 & 3710 & 503 & $3^{\text {rd }}$ stage \\
\hline$C_{o l}\left(\mathrm{~F} \cdot \mathrm{cm}^{-2}\right)$ & $2.0 \times 10^{-3}$ & $5.5 \times 10^{-2}$ & $9.7 \times 10^{-3}$ & $9.0 \times 10^{-2}$ & $3^{\text {rd }}$ stage \\
\hline$\alpha_{c}$ & 0.30 & 0.45 & 0.50 & 0.66 & $3^{\text {rd }}$ stage \\
\hline$\widehat{\tau_{0}}\left(\mathrm{~A} \cdot \mathrm{cm}^{-2}\right)$ & $4.5 \times 10^{-9}$ & $6.6 \times 10^{-9}$ & $6.4 \times 10^{-8}$ & $6.2 \times 10^{-7}$ & $3^{\text {rd }}$ stage \\
\hline$i_{l, c}\left(\mathrm{~A} \cdot \mathrm{cm}^{-2}\right)$ & $1.0 \times 10^{-5}$ & $2.5 \times 10^{-5}$ & $2.5 \times 10^{-5}$ & $7.2 \times 10^{-5}$ & $3^{\text {rd }}$ stage \\
\hline$k_{2}^{00}\left(\mathrm{~mol} \cdot \mathrm{cm}^{-2} \cdot \mathrm{s}^{-1}\right)$ & $2.0 \times 10^{-9}$ & $1.2 \times 10^{-7}$ & $2.0 \times 10^{-6}$ & $1.5 \times 10^{-4}$ & $2^{\text {nd }}$ stage \\
\hline$k_{3}^{00}\left(\mathrm{~mol} \cdot \mathrm{cm}^{-2} \cdot \mathrm{s}^{-1}\right)$ & $1.1 \times 10^{-8}$ & $1.0 \times 10^{-7}$ & $1.0 \times 10^{-7}$ & $1.0 \times 10^{-6}$ & $2^{\text {nd }}$ stage \\
\hline$k_{5}^{00}\left(\mathrm{~cm} \cdot \mathrm{s}^{-1}\right)$ & $4.0 \times 10^{-6}$ & $4.0 \times 10^{-6}$ & $8.9 \times 10^{-6}$ & $1.6 \times 10^{-5}$ & $2^{\text {nd }}$ stage \\
\hline$k_{6}^{00}\left(\mathrm{~cm} \cdot \mathrm{s}^{-1}\right)$ & $6.4 \times 10^{-6}$ & $7.4 \times 10^{-6}$ & $6.1 \times 10^{-5}$ & $8.8 \times 10^{-5}$ & $2^{\text {nd }}$ stage \\
\hline$k_{7}^{00}\left(\mathrm{~mol} \cdot \mathrm{cm}^{-2} \cdot \mathrm{s}^{-1}\right)$ & $4.5 \times 10^{-9}$ & $5.7 \times 10^{-9}$ & $6.5 \times 10^{-9}$ & $3.0 \times 10^{-8}$ & $2^{\text {nd }}$ stage \\
\hline$n$ & 0.65 & 0.65 & 0.56 & 0.58 & $1^{\text {st }}$ stage \\
\hline $\begin{array}{l}\alpha ; \alpha_{2} ; \\
\alpha_{3} ; \alpha_{5} ; \\
\alpha_{6} ; \alpha_{7}\end{array}$ & $\begin{array}{l}0.60 ; 0.44 ; \\
0.58 ; 0.40 \\
0.53 ; 0.88\end{array}$ & $\begin{array}{l}0.55 ; 0.46 ; \\
0.48 ; 0.40 \\
0.52 ; 0.76\end{array}$ & $\begin{array}{l}0.60 ; 0.51 \\
0.42 ; 0.35 \\
0.44 ; 0.70\end{array}$ & $\begin{array}{l}0.70 ; 0.52 \\
0.37 ; 0.30 \\
0.30 ; 0.67\end{array}$ & $1^{\text {st }}$ stage \\
\hline
\end{tabular}


Table X.9. Values of the parameters extracted from the optimization of the mixed potential model on the experimental EIS data for passive films formed on Alloy 600 at OCP in a solution containing $200 \mathrm{ppm} \mathrm{B}+2 \mathrm{ppm} \mathrm{Li}$ and $400 \mathrm{ppb}$ dissolved oxygen at different temperatures.

\begin{tabular}{|c|c|c|c|c|c|}
\hline Parameters & $150^{\circ} \mathrm{C}$ & $200^{\circ} \mathrm{C}$ & $250^{\circ} \mathrm{C}$ & $300^{\circ} \mathrm{C}$ & Method \\
\hline$O C P\left(V_{S H E}\right)$ & -0.424 & -0.469 & -0.726 & -0.885 & Measured \\
\hline$p H$ & 7.07 & 7.04 & 7.15 & 7.57 & $\begin{array}{l}\text { Calculated by } \\
\text { pH Calc }\end{array}$ \\
\hline$\varepsilon\left(\mathrm{V} \cdot \mathrm{cm}^{-1}\right)$ & $1.3 \times 10^{6}$ & $1.1 \times 10^{6}$ & $8.7 \times 10^{5}$ & $8.1 \times 10^{5}$ & $3^{\text {rd }}$ stage \\
\hline$\sigma$ & $4.8 \times 10^{4}$ & $7.8 \times 10^{4}$ & $8.1 \times 10^{4}$ & $3.0 \times 10^{5}$ & $3^{\text {rd }}$ stage \\
\hline$R_{s}(\Omega)$ & 5880 & 3203 & 3318 & 4650 & $3^{\text {rd }}$ stage \\
\hline$R_{e, h}\left(\Omega \cdot \mathrm{cm}^{2}\right)$ & $8.5 \times 10^{5}$ & $1.6 \times 10^{5}$ & $1.0 \times 10^{5}$ & $5.0 \times 10^{4}$ & $3^{\text {rd }}$ stage \\
\hline$C_{g}\left(\mathrm{~F} \cdot \mathrm{cm}^{-2}\right)$ & $4.8 \times 10^{-5}$ & $8.0 \times 10^{-5}$ & $3.7 \times 10^{-4}$ & $1.4 \times 10^{-3}$ & $3^{\text {rd }}$ stage \\
\hline$C_{d l}\left(\mathrm{~F} \cdot \mathrm{cm}^{-2}\right)$ & $1.3 \times 10^{-3}$ & $2.9 \times 10^{-3}$ & $9.1 \times 10^{-3}$ & $8.9 \times 10^{-4}$ & $3^{\text {rd }}$ stage \\
\hline$R_{o l}\left(\Omega \cdot \mathrm{cm}^{2}\right)$ & 12050 & 3200 & 3195 & 501 & $3^{\text {rd }}$ stage \\
\hline$C_{o l}\left(\mathrm{~F} \cdot \mathrm{cm}^{-2}\right)$ & $8.8 \times 10^{-3}$ & $7.4 \times 10^{-2}$ & $2.2 \times 10^{-3}$ & $1.5 \times 10^{-2}$ & $3^{\text {rd }}$ stage \\
\hline$\alpha_{c}$ & 0.29 & 0.47 & 0.48 & 0.66 & $3^{\text {rd }}$ stage \\
\hline$\tau_{0}\left(\mathrm{~A} \cdot \mathrm{cm}^{-2}\right)$ & $4.4 \times 10^{-9}$ & $6.3 \times 10^{-9}$ & $6.2 \times 10^{-8}$ & $6.1 \times 10^{-7}$ & $3^{\text {rd }}$ stage \\
\hline$i_{l, c}\left(\mathrm{~A} \cdot \mathrm{cm}^{-2}\right)$ & $9.7 \times 10^{-6}$ & $2.5 \times 10^{-5}$ & $2.5 \times 10^{-5}$ & $7.1 \times 10^{-5}$ & $3^{\text {rd }}$ stage \\
\hline$k_{2}^{00}\left(\mathrm{~mol} \cdot \mathrm{cm}^{-2} \cdot \mathrm{s}^{-1}\right)$ & $1.1 \times 10^{-9}$ & $1.2 \times 10^{-7}$ & $1.5 \times 10^{-5}$ & $2.0 \times 10^{-4}$ & $2^{\text {nd }}$ stage \\
\hline$k_{3}^{00}\left(\mathrm{~mol} \cdot \mathrm{cm}^{-2} \cdot \mathrm{s}^{-1}\right)$ & $1.3 \times 10^{-8}$ & $1.0 \times 10^{-7}$ & $5.0 \times 10^{-7}$ & $1.0 \times 10^{-6}$ & $2^{\text {nd }}$ stage \\
\hline$k_{5}^{00}\left(\mathrm{~cm} \cdot \mathrm{s}^{-1}\right)$ & $4.7 \times 10^{-6}$ & $5.1 \times 10^{-6}$ & $4.3 \times 10^{-5}$ & $5.5 \times 10^{-5}$ & $2^{\text {nd }}$ stage \\
\hline$k_{6}^{00}\left(\mathrm{~cm} \cdot \mathrm{s}^{-1}\right)$ & $2.4 \times 10^{-5}$ & $3.2 \times 10^{-5}$ & $1.5 \times 10^{-4}$ & $1.8 \times 10^{-4}$ & $2^{\text {nd }}$ stage \\
\hline$k_{7}^{00}\left(\mathrm{~mol} \cdot \mathrm{cm}^{-2} \cdot \mathrm{s}^{-1}\right)$ & $4.0 \times 10^{-9}$ & $5.4 \times 10^{-9}$ & $1.3 \times 10^{-8}$ & $2.2 \times 10^{-8}$ & $2^{\text {nd }}$ stage \\
\hline$n$ & 0.54 & 0.55 & 0.57 & 0.51 & $1^{\text {st }}$ stage \\
\hline $\begin{array}{l}\alpha ; \alpha_{2} ; \\
\alpha_{3} ; \alpha_{5} ; \\
\alpha_{6} ; \alpha_{7}\end{array}$ & $\begin{array}{l}0.60 ; 0.44 \\
0.58 ; 0.40 \\
0.53 ; 0.88\end{array}$ & $\begin{array}{l}0.55 ; 0.46 ; \\
0.48 ; 0.40 \\
0.52 ; 0.76\end{array}$ & $\begin{array}{l}0.60 ; 0.51 \\
0.42 ; 0.35 \\
0.44 ; 0.70\end{array}$ & $\begin{array}{l}0.70 ; 0.52 \\
0.37 ; 0.29 \\
0.30 ; 0.67\end{array}$ & $1^{\text {st }}$ stage \\
\hline
\end{tabular}


Table X.10. Values of the parameters extracted from the optimization of the mixed potential model on the experimental EIS data for passive films formed on Alloy 600 at $+0.3 \mathrm{~V}_{\text {OCP }}$ in a solution containing 200 ppm B +2 ppm Li and 400 ppb dissolved oxygen at different temperatures.

\begin{tabular}{|c|c|c|c|c|c|}
\hline Parameters & $150^{\circ} \mathrm{C}$ & $200^{\circ} \mathrm{C}$ & $250^{\circ} \mathrm{C}$ & $300^{\circ} \mathrm{C}$ & Method \\
\hline$+0.3 V_{O C P}\left(V_{S H E}\right)$ & -0.124 & -0.169 & -0.426 & -0.585 & Measured \\
\hline$p H$ & 7.07 & 7.04 & 7.15 & 7.57 & $\begin{array}{l}\text { Calculated by } \\
\mathrm{pH} \mathrm{Calc}\end{array}$ \\
\hline$\varepsilon\left(\mathrm{V} \cdot \mathrm{cm}^{-1}\right)$ & $1.3 \times 10^{6}$ & $1.1 \times 10^{6}$ & $8.7 \times 10^{5}$ & $8.1 \times 10^{5}$ & $3^{\text {rd }}$ stage \\
\hline$\sigma$ & $2.1 \times 10^{4}$ & $7.5 \times 10^{4}$ & $6.0 \times 10^{4}$ & $1.8 \times 10^{5}$ & $3^{\text {rd }}$ stage \\
\hline$R_{s}(\Omega)$ & 5972 & 3010 & 2920 & 4531 & $3^{\text {rd }}$ stage \\
\hline$R_{e, h}\left(\Omega \cdot \mathrm{cm}^{2}\right)$ & $8.5 \times 10^{5}$ & $2.6 \times 10^{5}$ & $1.0 \times 10^{5}$ & $9.9 \times 10^{4}$ & $3^{\text {rd }}$ stage \\
\hline$C_{g}\left(\mathrm{~F} \cdot \mathrm{cm}^{-2}\right)$ & $4.3 \times 10^{-5}$ & $5.5 \times 10^{-5}$ & $6.2 \times 10^{-5}$ & $2.1 \times 10^{-4}$ & $3^{\text {rd }}$ stage \\
\hline$C_{d l}\left(\mathrm{~F} \cdot \mathrm{cm}^{-2}\right)$ & $8.1 \times 10^{-4}$ & $2.2 \times 10^{-4}$ & $8.3 \times 10^{-2}$ & $1.9 \times 10^{-4}$ & $3^{\text {rd }}$ stage \\
\hline$R_{o l}\left(\Omega \cdot \mathrm{cm}^{2}\right)$ & 15179 & 3459 & 3463 & 3177 & $3^{\text {rd }}$ stage \\
\hline$C_{o l}\left(\mathrm{~F} \cdot \mathrm{cm}^{-2}\right)$ & $4.1 \times 10^{-3}$ & $7.1 \times 10^{-2}$ & $7.0 \times 10^{-4}$ & $5.7 \times 10^{-3}$ & $3^{\text {rd }}$ stage \\
\hline$\alpha_{c}$ & 0.29 & 0.47 & 0.48 & 0.66 & $3^{\text {rd }}$ stage \\
\hline$\widehat{\tau}_{0}\left(\mathrm{~A} \cdot \mathrm{cm}^{-2}\right)$ & $4.4 \times 10^{-9}$ & $6.3 \times 10^{-9}$ & $6.2 \times 10^{-8}$ & $6.1 \times 10^{-7}$ & $3^{\text {rd }}$ stage \\
\hline$i_{l, c}\left(\mathrm{~A} \cdot \mathrm{cm}^{-2}\right)$ & $9.7 \times 10^{-6}$ & $2.5 \times 10^{-5}$ & $2.5 \times 10^{-5}$ & $7.1 \times 10^{-5}$ & $3^{\text {rd }}$ stage \\
\hline$k_{2}^{00}\left(\mathrm{~mol} \cdot \mathrm{cm}^{-2} \cdot \mathrm{s}^{-1}\right)$ & $1.1 \times 10^{-9}$ & $1.2 \times 10^{-7}$ & $1.5 \times 10^{-5}$ & $2.0 \times 10^{-4}$ & $2^{\text {nd }}$ stage \\
\hline$k_{3}^{00}\left(\mathrm{~mol} \cdot \mathrm{cm}^{-2} \cdot \mathrm{s}^{-1}\right)$ & $1.3 \times 10^{-8}$ & $1.0 \times 10^{-7}$ & $5.0 \times 10^{-7}$ & $1.0 \times 10^{-6}$ & $2^{\text {nd }}$ stage \\
\hline$k_{5}^{00}\left(\mathrm{~cm} \cdot \mathrm{s}^{-1}\right)$ & $4.7 \times 10^{-6}$ & $5.1 \times 10^{-6}$ & $4.3 \times 10^{-5}$ & $5.5 \times 10^{-5}$ & $2^{\text {nd }}$ stage \\
\hline$k_{6}^{00}\left(\mathrm{~cm} \cdot \mathrm{s}^{-1}\right)$ & $2.4 \times 10^{-5}$ & $3.2 \times 10^{-5}$ & $1.5 \times 10^{-4}$ & $1.8 \times 10^{-4}$ & $2^{\text {nd }}$ stage \\
\hline$k_{7}^{00}\left(\mathrm{~mol} \cdot \mathrm{cm}^{-2} \cdot \mathrm{s}^{-1}\right)$ & $4.0 \times 10^{-9}$ & $5.4 \times 10^{-9}$ & $1.3 \times 10^{-8}$ & $2.2 \times 10^{-8}$ & $2^{\text {nd }}$ stage \\
\hline$n$ & 0.56 & 0.55 & 0.57 & 0.50 & $1^{\text {st }}$ stage \\
\hline $\begin{array}{l}\alpha ; \alpha_{2} ; \\
\alpha_{3} ; \alpha_{5} ; \\
\alpha_{6} ; \alpha_{7}\end{array}$ & $\begin{array}{l}0.60 ; 0.44 ; \\
0.58 ; 0.40 ; \\
0.53 ; 0.88\end{array}$ & $\begin{array}{l}0.55 ; 0.46 ; \\
0.48 ; 0.40 \\
0.52 ; 0.76\end{array}$ & $\begin{array}{l}0.61 ; 0.51 \\
0.42 ; 0.35 \\
0.44 ; 0.70\end{array}$ & $\begin{array}{l}0.70 ; 0.52 \\
0.37 ; 0.29 \\
0.30 ; 0.67\end{array}$ & $1^{\text {st }}$ stage \\
\hline
\end{tabular}


Table X.11. Values of the parameters extracted from the optimization of the mixed potential model on the experimental EIS data for passive films formed on Alloy 600 in a solution containing 0 ppm $\mathrm{B}+2 \mathrm{ppm} \mathrm{Li}$ and $400 \mathrm{ppb}$ dissolved oxygen at different temperatures.

\begin{tabular}{|c|c|c|c|c|c|}
\hline Parameters & $150^{\circ} \mathrm{C}$ & $200^{\circ} \mathrm{C}$ & $250^{\circ} \mathrm{C}$ & $300^{\circ} \mathrm{C}$ & Method \\
\hline$O C P\left(V_{S H E}\right)$ & -0.474 & -0.531 & -0.667 & -0.911 & Measured \\
\hline$p H$ & 8.09 & 7.69 & 7.60 & 7.91 & $\begin{array}{l}\text { Calculated by } \\
\text { pH Calc }\end{array}$ \\
\hline$\varepsilon\left(\mathrm{V} \cdot \mathrm{cm}^{-1}\right)$ & $1.3 \times 10^{6}$ & $1.0 \times 10^{6}$ & $8.4 \times 10^{5}$ & $8.3 \times 10^{5}$ & $3^{\text {rd }}$ stage \\
\hline$\sigma$ & $1.0 \times 10^{4}$ & $6.3 \times 10^{4}$ & $3.0 \times 10^{5}$ & $3.1 \times 10^{5}$ & $3^{\text {rd }}$ stage \\
\hline$R_{s}(\Omega)$ & 3610 & 2679 & 2214 & 2301 & $3^{\text {rd }}$ stage \\
\hline$R_{e, h}\left(\Omega \cdot \mathrm{cm}^{2}\right)$ & $6.82 \times 10^{5}$ & $1.94 \times 10^{5}$ & $1.94 \times 10^{5}$ & $1.89 \times 10^{5}$ & $3^{\text {rd }}$ stage \\
\hline$C_{g}\left(\mathrm{~F} \cdot \mathrm{cm}^{-2}\right)$ & $4.7 \times 10^{-5}$ & $7.8 \times 10^{-5}$ & $1.8 \times 10^{-4}$ & $8.0 \times 10^{-4}$ & $3^{\text {rd }}$ stage \\
\hline$C_{d l}\left(\mathrm{~F} \cdot \mathrm{cm}^{-2}\right)$ & $1.3 \times 10^{-3}$ & $4.3 \times 10^{-4}$ & $4.6 \times 10^{-5}$ & $4.2 \times 10^{-3}$ & $3^{\text {rd }}$ stage \\
\hline$R_{o l}\left(\Omega \cdot \mathrm{cm}^{2}\right)$ & 7507 & 3497 & 3483 & 1954 & $3^{\text {rd }}$ stage \\
\hline$C_{o l}\left(\mathrm{~F} \cdot \mathrm{cm}^{-2}\right)$ & $7.7 \times 10^{-2}$ & $5.7 \times 10^{-2}$ & $9.9 \times 10^{-3}$ & $4.5 \times 10^{-3}$ & $3^{\text {rd }}$ stage \\
\hline$\alpha_{c}$ & 0.30 & 0.47 & 0.51 & 0.76 & $3^{\text {rd }}$ stage \\
\hline$\widehat{\tau_{0}}\left(\mathrm{~A} \cdot \mathrm{cm}^{-2}\right)$ & $4.2 \times 10^{-9}$ & $6.2 \times 10^{-9}$ & $6.2 \times 10^{-8}$ & $6.0 \times 10^{-7}$ & $3^{\text {rd }}$ stage \\
\hline$i_{l, c}\left(\mathrm{~A} \cdot \mathrm{cm}^{-2}\right)$ & $1.0 \times 10^{-5}$ & $2.4 \times 10^{-5}$ & $2.5 \times 10^{-5}$ & $7.1 \times 10^{-5}$ & $3^{\text {rd }}$ stage \\
\hline$k_{2}^{00}\left(\mathrm{~mol} \cdot \mathrm{cm}^{-2} \cdot \mathrm{s}^{-1}\right)$ & $2.0 \times 10^{-9}$ & $1.3 \times 10^{-7}$ & $1.2 \times 10^{-6}$ & $4.8 \times 10^{-3}$ & $2^{\text {nd }}$ stage \\
\hline$k_{3}^{00}\left(\mathrm{~mol} \cdot \mathrm{cm}^{-2} \cdot \mathrm{s}^{-1}\right)$ & $2.0 \times 10^{-8}$ & $1.1 \times 10^{-7}$ & $5.0 \times 10^{-7}$ & $2.5 \times 10^{-6}$ & $2^{\text {nd }}$ stage \\
\hline$k_{5}^{00}\left(\mathrm{~cm} \cdot \mathrm{s}^{-1}\right)$ & $3.7 \times 10^{-5}$ & $3.3 \times 10^{-5}$ & $5.9 \times 10^{-5}$ & $8.9 \times 10^{-4}$ & $2^{\text {nd }}$ stage \\
\hline$k_{6}^{00}\left(\mathrm{~cm} \cdot \mathrm{s}^{-1}\right)$ & $1.9 \times 10^{-5}$ & $2.3 \times 10^{-5}$ & $6.7 \times 10^{-5}$ & $9.1 \times 10^{-5}$ & $2^{\text {nd }}$ stage \\
\hline$k_{7}^{00}\left(\mathrm{~mol} \cdot \mathrm{cm}^{-2} \cdot \mathrm{s}^{-1}\right)$ & $3.5 \times 10^{-9}$ & $4.6 \times 10^{-9}$ & $1.2 \times 10^{-8}$ & $2.3 \times 10^{-8}$ & $2^{\text {nd }}$ stage \\
\hline$n$ & 0.50 & 0.51 & 0.55 & 0.50 & $1^{\text {st }}$ stage \\
\hline $\begin{array}{l}\alpha ; \alpha_{2} ; \\
\alpha_{3} ; \alpha_{5} ; \\
\alpha_{6} ; \alpha_{7}\end{array}$ & $\begin{array}{l}0.59 ; 0.44 ; \\
0.58 ; 0.42 \\
0.56 ; 0.88\end{array}$ & $\begin{array}{l}0.55 ; 0.46 \\
0.48 ; 0.42 \\
0.55 ; 0.76\end{array}$ & $\begin{array}{l}0.60 ; 0.50 \\
0.48 ; 0.40 \\
0.44 ; 0.70\end{array}$ & $\begin{array}{l}0.70 ; 0.53 ; \\
0.35 ; 0.35 \\
0.26 ; 0.67\end{array}$ & $1^{\text {st }}$ stage \\
\hline
\end{tabular}

The activation energy $E_{a i}$ for Reactions (2), (3), (5), (6), and (7) were calculated using the Arrhenius equation in linear plot form:

$\ln \left(k_{i}^{00}\right)=\frac{-E_{a}}{R} \cdot \frac{1}{T}+\ln \left(A^{\prime}\right)$

where $k_{i}^{00}$ is the base rate constant for metal dissolution reaction, and $A^{\prime}$ is the pre-exponential factor (constant). 
The activation energy for the reaction of oxygen reduction $\left(E_{c}\right)$ was calculated using the Arrhenius equation:

$\widehat{\iota_{0}}=A e^{\frac{-E_{C}}{R T}}$

where $\mathrm{A}$ is the pre-exponential factor (constant). The linear relation between $\ln \left(\widehat{\iota}_{0}\right)$ and $\frac{1}{T}$ was acquired by taking the natural logarithm of Equation (X.25):

$\ln \left(\widehat{l_{0}}\right)=\frac{-E_{c}}{R} \cdot \frac{1}{T}+\ln (A)$

Figures X.37 to X.40 present the Arrhenius linear plots of the base rate constant $\left(k_{i}^{00}\right)$ of passive anodic dissolution versus the reciprocal of temperature $1 / T$ and the calculated activation energies of the anodic metal dissolution reactions for Alloy 600, Alloy 690, Type 304L SS, and Type 316L SS in three borate buffer solutions containing $400 \mathrm{ppb}$ dissolved oxygen. Table X.12 indicates the calculated values of activation energies for anodic reactions of Alloy 600, Alloy 690, Type 304L SS and Type 316L SS. For each alloy, the activation energy for Reaction (2) is the highest, the changes in the base rate constant $\left(k_{i}^{00}\right)$ and hypothetical exchange current density $\left(\widehat{\iota_{0}}\right)$ are insignificant with the change of solution $\mathrm{pH}$ (Figures X.37 to X.40). With increasing solution $\mathrm{pH}$, the activation energy $E_{a i}(i=2,3,5,7)$ increases and $E_{a 6}$ decreases for the anodic metal dissolution reactions, whereas the activation energy for cathodic oxygen reduction $E_{c}$ did not change significantly (Figure X.37b). 

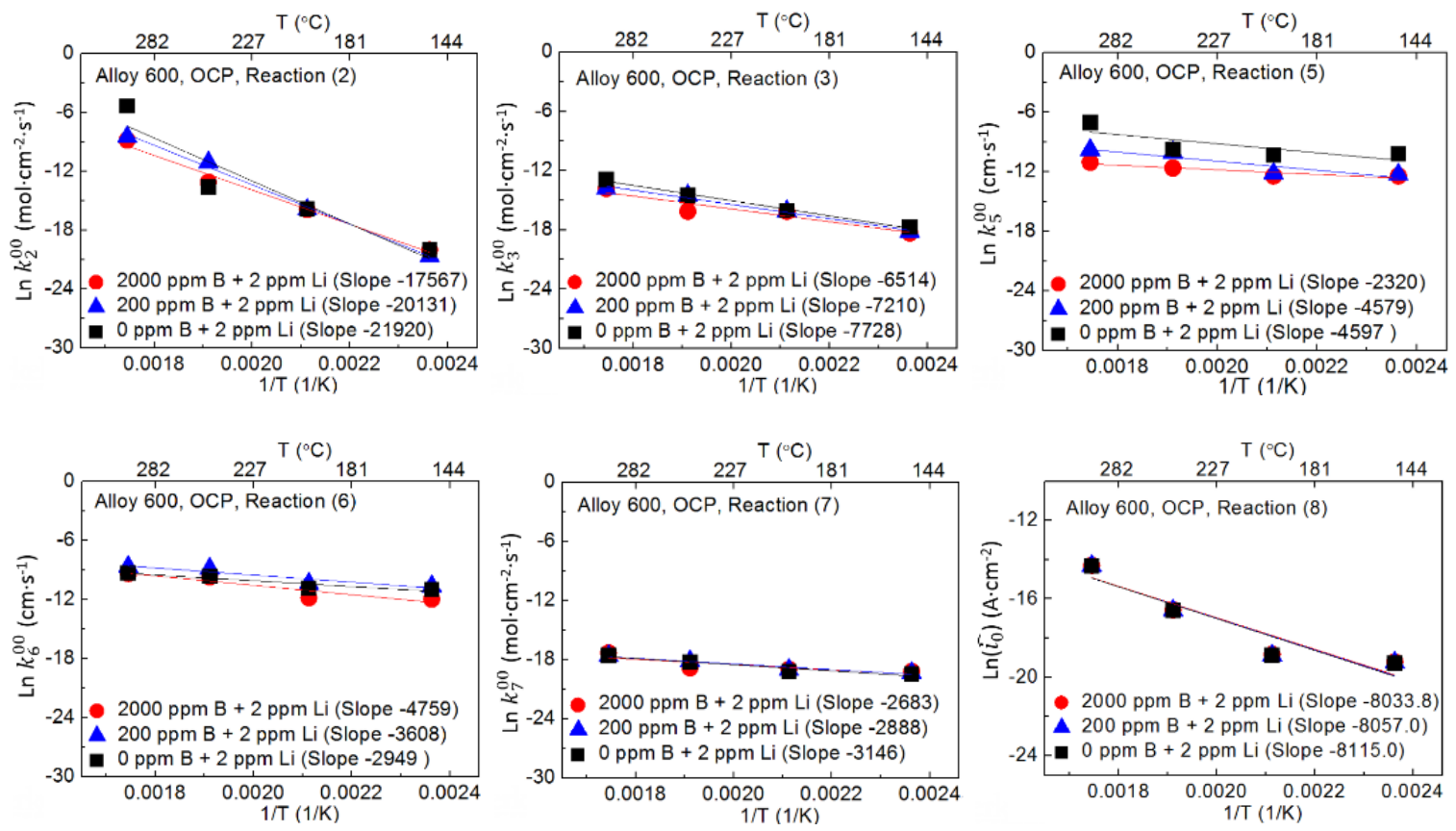

(a)
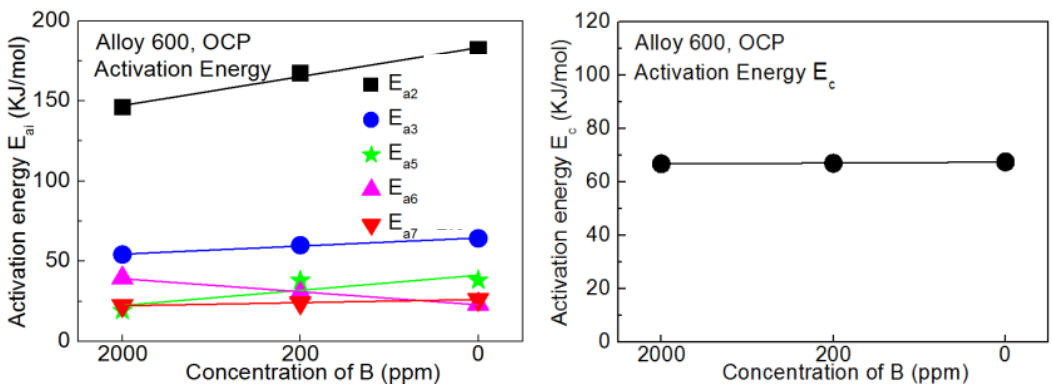

(b)

Figure X.37. Arrhenius linear plots of (a) the base rate constant $\left(k_{i}^{00}\right)$ of anodic metal passive dissolution and the hypothetical exchange current density $\left(\widehat{\iota_{0}}\right)$ of cathodic oxygen electrode reaction versus the reciprocal of temperature $1 / T$ and (b) the calculated activation energies of the anodic metal dissolution and the cathodic oxygen reduction reactions for Alloy 600 in borate buffer solutions containing $400 \mathrm{ppb}$ dissolved oxygen. 

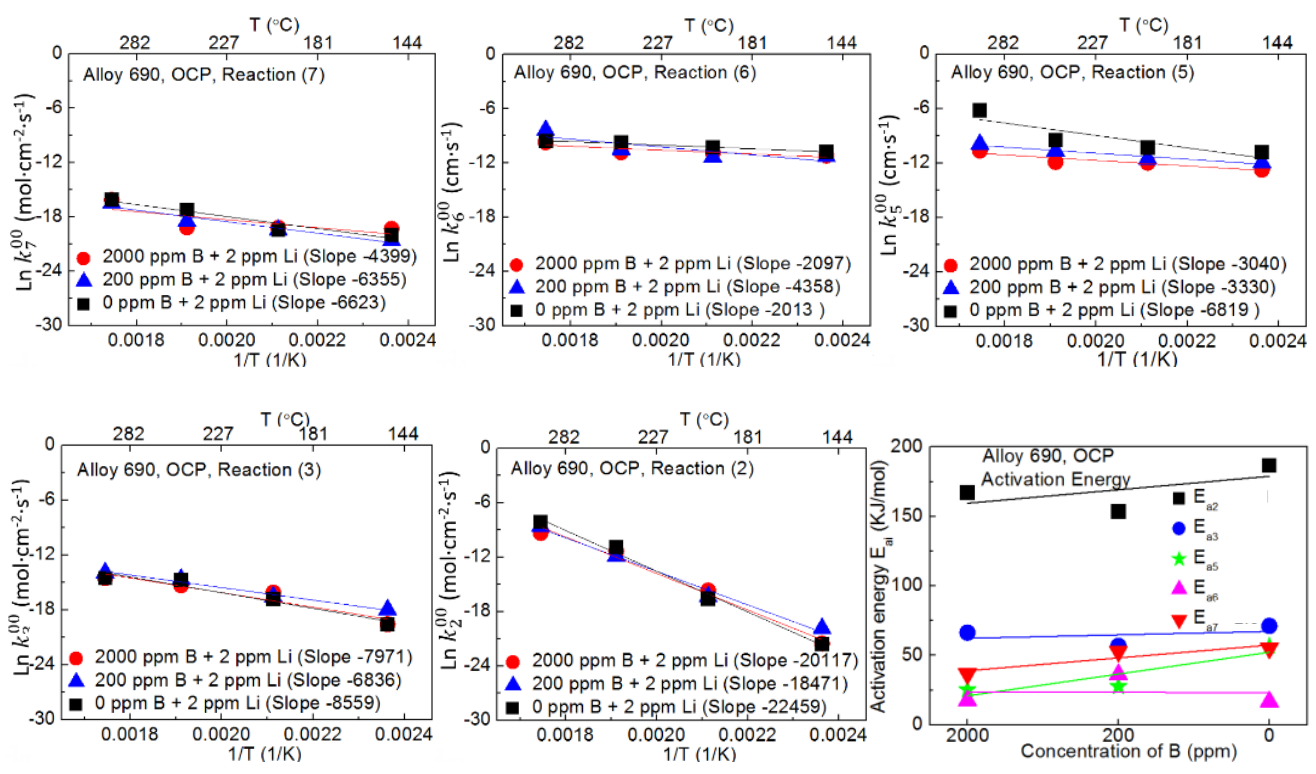

Figure X.38. Arrhenius linear plots of the base rate constant $\left(k_{i}^{00}\right)$ of anodic metal passive dissolution versus the reciprocal of temperature $1 / T$ and the calculated activation energies of the anodic metal dissolution reactions for Alloy 690 in borate buffer solutions containing 400 ppb dissolved oxygen.
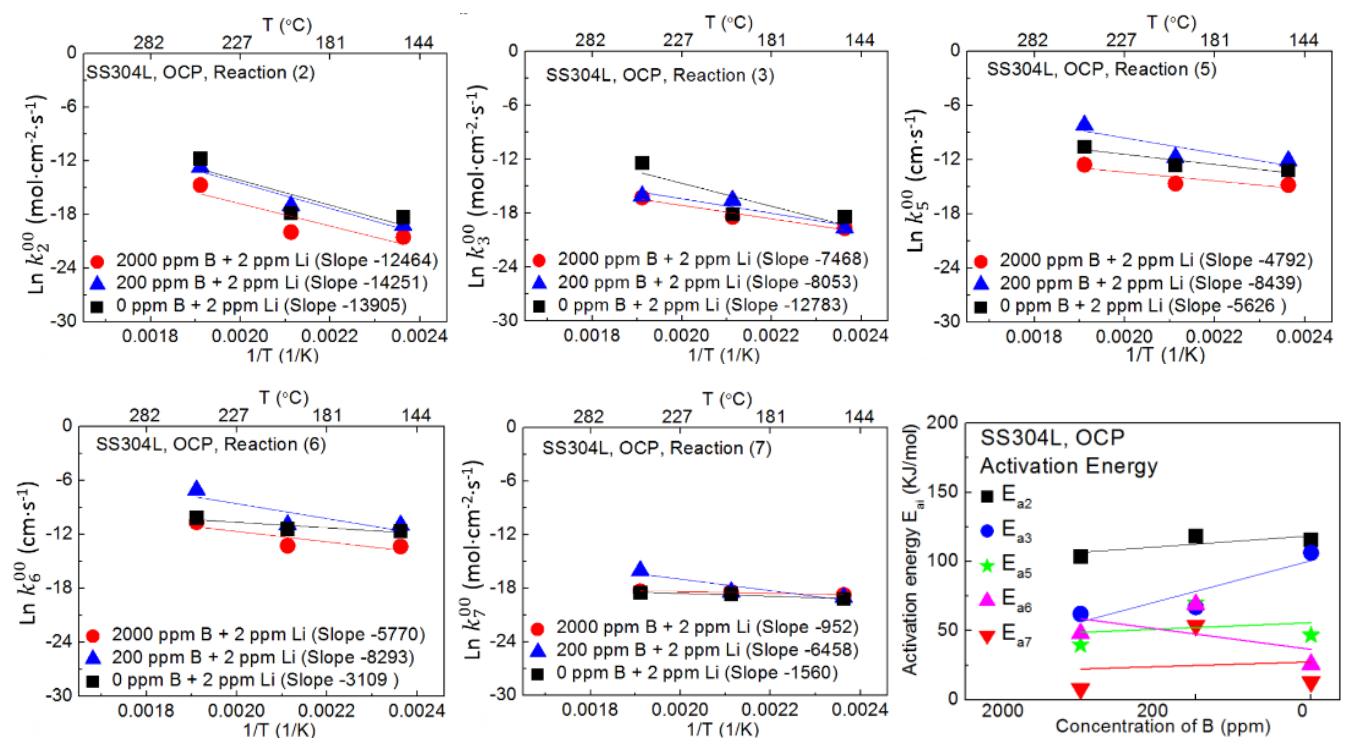

Figure X.39. Arrhenius linear plots of the base rate constant $\left(k_{i}^{00}\right)$ of anodic metal passive dissolution versus the reciprocal of temperature $1 / T$ and the calculated activation energies of the anodic metal dissolution reactions for Type 304L SS in borate buffer solutions containing $400 \mathrm{ppb}$ dissolved oxygen. 

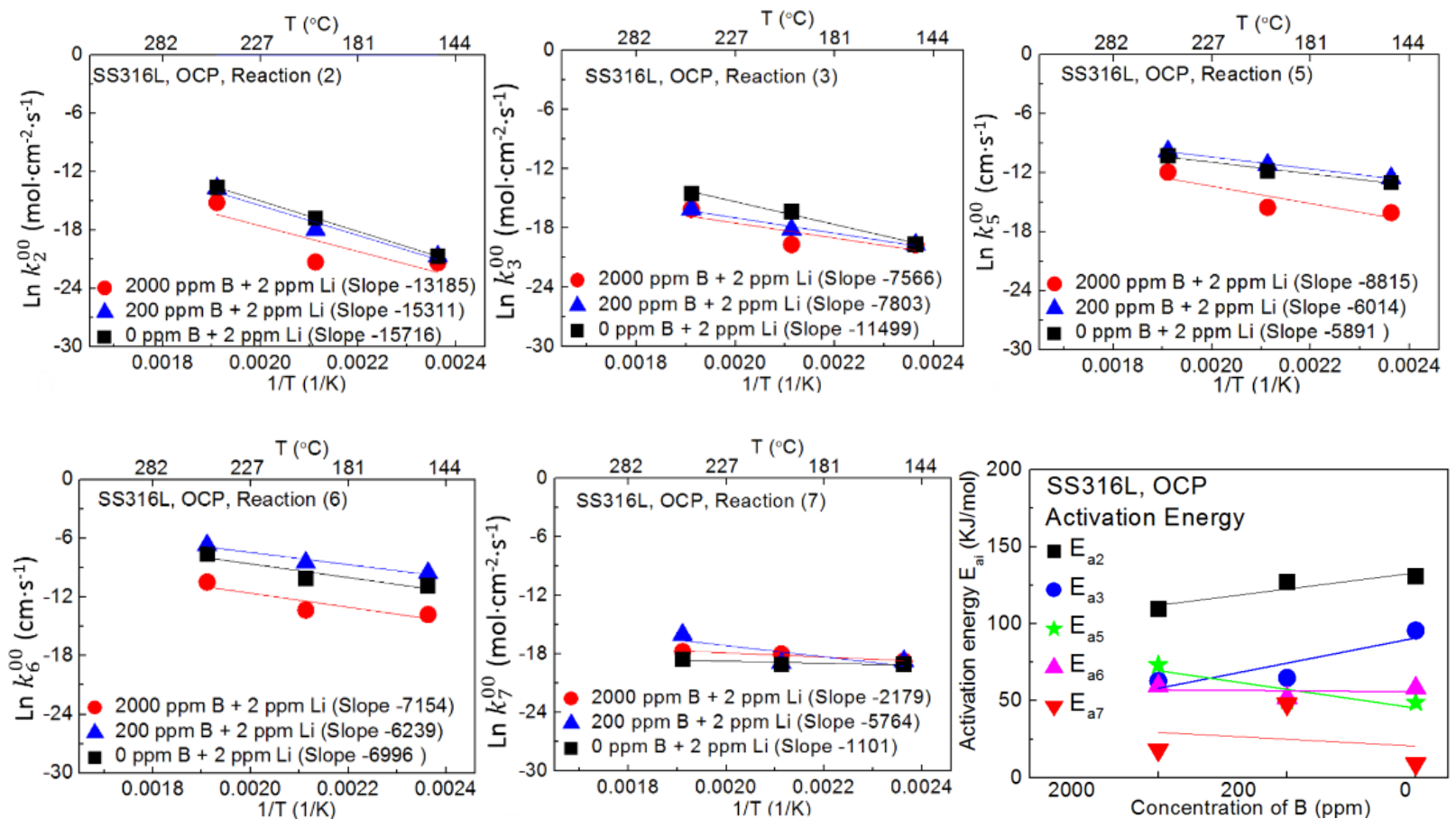

Figure X.40. Arrhenius linear plots of the base rate constant $\left(k_{i}^{00}\right)$ of anodic metal passive dissolution versus the reciprocal of temperature $1 / T$ and the calculated activation energies of the anodic metal dissolution reactions for Type 316L SS in borate buffer solutions containing $400 \mathrm{ppb}$ dissolved oxygen.

Figure X.41 presents the changes in transfer coefficients for anodic metal dissolution reactions of Alloy 600 in borate buffer solutions at different temperatures. The transfer coefficient $\alpha_{2}$ for Reaction (2) shows a rising trend while all the other transfer coefficients $\alpha_{i}(i=3,5,6,7)$ decrease with increasing temperature. At the same temperature, the transfer coefficients $\alpha_{i}$ varies insignificantly with the change of solution $\mathrm{pH}$. 
Table X.12. Calculated values of activation energies for anodic metal dissolution of Alloy 600, Alloy 690, Type 304L SS, and Type 316L SS in oxygenated borate buffer solution.

\begin{tabular}{|c|c|c|c|c|}
\hline Activation Energy & & 2000 ppm B & 200 ppm B & 0 ppm B \\
\hline$(\mathrm{KJ} / \mathrm{mol})$ & & + 2 ppm Li & $+2 \mathrm{ppm} \mathrm{Li}$ & +2 ppm Li \\
\hline \multirow{4}{*}{$E_{a 2}$} & Alloy 600 & 146.1 & 167.4 & 182.2 \\
\hline & Alloy 690 & 167.3 & 153.6 & 186.7 \\
\hline & Type 304L & 103.6 & 118.5 & 115.6 \\
\hline & Type $316 \mathrm{~L}$ & 109.6 & 127.3 & 130.7 \\
\hline \multirow{4}{*}{$E_{a 3}$} & Alloy 600 & 54.2 & 60.0 & 64.3 \\
\hline & Alloy 690 & 66.3 & 56.8 & 71.2 \\
\hline & Type 304L & 62.1 & 67.0 & 106.3 \\
\hline & Type $316 \mathrm{~L}$ & 62.9 & 64.9 & 95.6 \\
\hline \multirow{4}{*}{$E_{a 5}$} & Alloy 600 & 19.3 & 38.1 & 38.2 \\
\hline & Alloy 690 & 25.3 & 27.7 & 56.7 \\
\hline & Type 304L & 39.8 & 70.2 & 46.8 \\
\hline & Type 316L & 73.3 & 50.0 & 49.0 \\
\hline \multirow{4}{*}{$E_{a 6}$} & Alloy 600 & 39.6 & 30.0 & 23.3 \\
\hline & Alloy 690 & 17.4 & 36.2 & 16.7 \\
\hline & Type 304L & 48.0 & 68.9 & 25.8 \\
\hline & Type $316 \mathrm{~L}$ & 59.5 & 51.9 & 58.2 \\
\hline \multirow{4}{*}{$E_{a 7}$} & Alloy 600 & 22.3 & 24.0 & 26.2 \\
\hline & Alloy 690 & 36.6 & 52.8 & 55.1 \\
\hline & Type 304L & 7.9 & 53.7 & 13.0 \\
\hline & Type $316 \mathrm{~L}$ & 18.1 & 47.9 & 9.2 \\
\hline
\end{tabular}




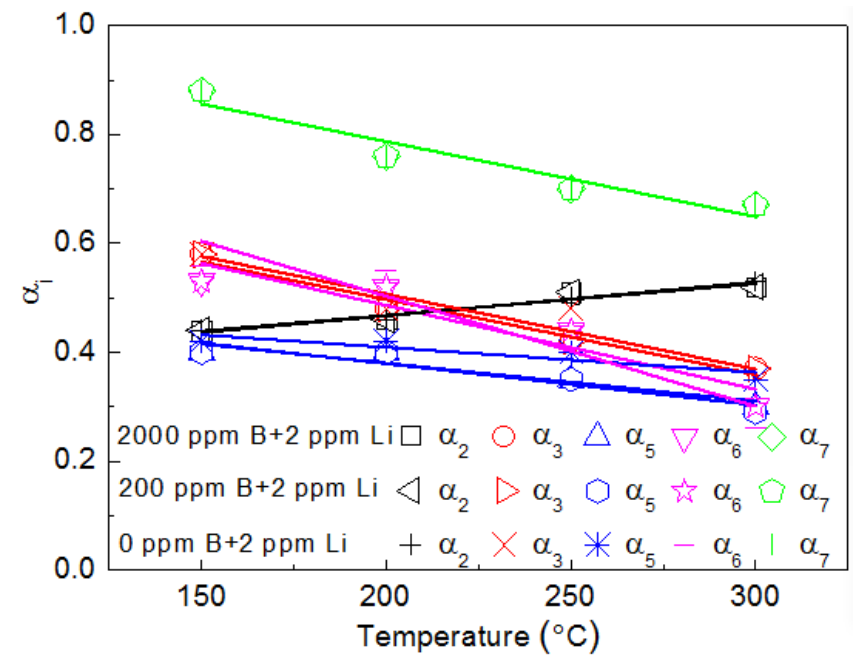

Figure X.41. Changes of transfer coefficient $\alpha_{i}(i=2,3,5,6,7)$ for anodic metal passive dissolution of Alloy 600 with increasing temperature in borate buffer solutions containing $400 \mathrm{ppb}$ dissolved oxygen.

The anodic $\left(I_{a}\right)$ and cathodic $\left(I_{c}\right)$ steady-state current density, the cathodic charge transfer impedance $Z_{R_{C}}$, the steady-state film thickness $L_{S S}$, the standard rate constants $k_{i}^{0}$ and rate constants $k_{i}(i=2,3,5,6,7)$ for anodic metal dissolution, the concentrations of cation interstitial $C_{i}^{0}\left(k_{2} / k_{5}\right)$ and oxygen vacancy $C_{V_{0}}^{0}\left(\chi k_{3} / 2 k_{6}\right)$ at the bl/ol interface, the ratio between $C_{i}^{0}$ and chromium sublattice concentration in stoichiometric $\mathrm{Cr}_{2} \mathrm{O}_{3}$ lattice $\left(\frac{2}{\Omega}\right)$, the ratio between $C_{V_{0}}^{0}$ and anion sublattice concentration in normal $\mathrm{Cr}_{2} \mathrm{O}_{3}\left(\frac{3}{\Omega}\right)$, the ratio of the growth rate $\left(R_{g}, \mathrm{~cm} / \mathrm{s}\right)$ of the barrier layer to the dissolution rate $\left(R_{d}, \mathrm{~cm} / \mathrm{s}\right)$ of the metal substrate, noted as $R_{g} / R_{d}$, and the diffusivity of cation interstitial $D_{i}$ were calculated based on the above-optimized coefficients for Alloy 600 (Tables X.9 to X.12), as indicated in Tables X.13 to X.16. At each temperature, the concentration ratio between metal interstitial and chromium atom in stoichiometric $\mathrm{Cr}_{2} \mathrm{O}_{3}$ is less than $4.00 \%$, and the concentration ratio between the oxygen vacancy and the oxygen atom in normal $\mathrm{Cr}_{2} \mathrm{O}_{3}$ lattice is less than $0.05 \%$. The barrier layer film grows into the metal substrate; the growth rate $R_{g}$ is calculated by:

$R_{g}=k_{3} \cdot \Omega$

and the dissolution rate of the metal substrate $R_{d}$ is expressed as 
$R_{d}=\left(k_{2}+k_{3}\right) \cdot \Omega^{\prime}$

where $\Omega^{\prime}$ is the mole volume of the chromium metal $\left(7.23 \mathrm{~cm}^{3} / \mathrm{mol}\right)$. The value of $R_{g} / R_{d}$ varies from 1.0 to 0.7 as the temperature increases from $150^{\circ} \mathrm{C}$ to $300{ }^{\circ} \mathrm{C}$, indicating that if the dissolution of the bl occurring at the bl/ol interface is negligible, the bl/ol interface will generally remain at the original metal surface as the bl grows into the metal, which is a common observation from marker experiments. The diffusivity of the dominant point defect metal interstitial $\left(D_{i}\right)$ was calculated according to Equation X.29 [75] as a function of temperature:

$D_{i}=2 \sigma^{2}(1-\alpha)^{2} I_{a}^{2} / \varepsilon^{2}$

The calculated diffusivity of metal interstitial increased with increasing temperature.

For passive films formed under OCP conditions, the net current density $\left(I_{a}+I_{c}\right)$ is nearby zero, which agrees with the charge conservation and Wagner-Traud theory. With increasing temperature, the anodic steady-state current density, film thickness, standard reaction rate constants and rate constants, total defect density $\left(C_{i}^{0}+C_{V_{\vec{o}}}^{0}\right)$ increased, and the charge transfer resistance of cathodic oxygen reduction decreased. At the same temperature, the magnitude of the interstitial metal concentration is much higher than the corresponding value for the oxygen vacancy, indicating the dominant point defect in the barrier layer formed on Alloy 600 under OCP in borate buffer solutions is the cation interstitial, which is also evident from $k_{2} / k_{3}>1$. The diffusivity of cation interstitials increased significantly with increasing temperature.

Figures X.42 to X.44 show the effects of temperature and $\mathrm{pH}$ on the anodic current density, steady-state thickness of the barrier layer, and defect density of barrier layer formed on Alloy 600 under OCP in oxygenated borate buffer solution more clearly. With increasing temperature, the logarithmic anodic current density (Figure X.42a) and barrier layer thickness increased linearly (Figure X.43a), while the defect density increased exponentially (Figure X.44a). The exponentially increasing defect density may dominant the corrosion resistance more than the linearly increasing barrier layer thickness, resulting in a significant increase in the anodic current density and in a decrease in the corrosion resistance at the elevated temperatures. With increasing $\mathrm{pH}$, the defect density decreased (Figure X.44b), and barrier layer thickness increased (Figure X.43b) slightly, leading to a slight linear increase of anodic current density (Figure X.42b) and impedance. 
Table X.13. Several properties that were calculated based on the extracted coefficients for passive films formed on Alloy 600 under OCP in a solution containing 2000 ppm B + 2 ppm Li, and 400 ppb dissolved oxygen at different temperatures.

\begin{tabular}{|c|c|c|c|c|}
\hline Parameters & $150^{\circ} \mathrm{C}$ & $200^{\circ} \mathrm{C}$ & $250^{\circ} \mathrm{C}$ & $300^{\circ} \mathrm{C}$ \\
\hline$I_{a}\left(\mathrm{~A} \cdot \mathrm{cm}^{-2}\right)$ & $5.46 \times 10^{-7}$ & $6.37 \times 10^{-7}$ & $1.82 \times 10^{-6}$ & $2.42 \times 10^{-6}$ \\
\hline$I_{c}\left(\mathrm{~A} \cdot \mathrm{cm}^{-2}\right)$ & $-5.42 \times 10^{-7}$ & $-6.29 \times 10^{-7}$ & $-1.84 \times 10^{-6}$ & $-2.40 \times 10^{-6}$ \\
\hline$Z_{R_{C}}\left(\Omega \cdot \mathrm{cm}^{2}\right)$ & $1.1 \times 10^{5}$ & $7.2 \times 10^{4}$ & $2.4 \times 10^{4}$ & $1.9 \times 10^{4}$ \\
\hline$L_{S S}(\mathrm{~nm})$ & 1.5 & 2.7 & 3.3 & 5.4 \\
\hline$k_{2}^{0}\left(\mathrm{~mol} \cdot \mathrm{cm}^{-2} \cdot \mathrm{s}^{-1}\right)$ & $1.7 \times 10^{-8}$ & $9.2 \times 10^{-7}$ & $1.7 \times 10^{-5}$ & $1.2 \times 10^{-3}$ \\
\hline$k_{3}^{0}\left(\mathrm{~mol} \cdot \mathrm{cm}^{-2} \cdot \mathrm{s}^{-1}\right)$ & $1.9 \times 10^{-7}$ & $8.2 \times 10^{-7}$ & $5.7 \times 10^{-7}$ & $4.5 \times 10^{-6}$ \\
\hline$k_{5}^{0}\left(\mathrm{~cm} \cdot \mathrm{s}^{-1}\right)$ & $5.9 \times 10^{-7}$ & $6.8 \times 10^{-7}$ & $2.1 \times 10^{-6}$ & $4.7 \times 10^{-6}$ \\
\hline$k_{6}^{0}\left(\mathrm{~cm} \cdot \mathrm{s}^{-1}\right)$ & $1.2 \times 10^{-6}$ & $1.6 \times 10^{-6}$ & $1.8 \times 10^{-5}$ & $3.9 \times 10^{-5}$ \\
\hline$k_{7}^{0}\left(\mathrm{~mol} \cdot \mathrm{cm}^{-2} \cdot \mathrm{s}^{-1}\right)$ & $4.5 \times 10^{-9}$ & $5.7 \times 10^{-9}$ & $6.5 \times 10^{-9}$ & $3.0 \times 10^{-8}$ \\
\hline$k_{2}\left(\mathrm{~mol} \cdot \mathrm{cm}^{-2} \cdot \mathrm{s}^{-1}\right)$ & $1.2 \times 10^{-12}$ & $1.5 \times 10^{-12}$ & $4.2 \times 10^{-12}$ & $4.2 \times 10^{-12}$ \\
\hline$k_{3}\left(\mathrm{~mol} \cdot \mathrm{cm}^{-2} \cdot \mathrm{s}^{-1}\right)$ & $6.5 \times 10^{-13}$ & $7.3 \times 10^{-13}$ & $2.1 \times 10^{-12}$ & $4.2 \times 10^{-12}$ \\
\hline$k_{5}\left(\mathrm{~cm} \cdot \mathrm{s}^{-1}\right)$ & $2.5 \times 10^{-9}$ & $2.0 \times 10^{-9}$ & $3.2 \times 10^{-9}$ & $8.3 \times 10^{-10}$ \\
\hline$k_{6}\left(\mathrm{~cm} \cdot \mathrm{s}^{-1}\right)$ & $8.7 \times 10^{-9}$ & $1.1 \times 10^{-8}$ & $8.1 \times 10^{-8}$ & $1.2 \times 10^{-7}$ \\
\hline$k_{7}\left(\mathrm{~mol} \cdot \mathrm{cm}^{-2} \cdot \mathrm{s}^{-1}\right)$ & $4.5 \times 10^{-9}$ & $5.7 \times 10^{-9}$ & $6.5 \times 10^{-9}$ & $3.0 \times 10^{-8}$ \\
\hline$C_{i}^{0}=k_{2} / k_{5}\left(\mathrm{~mol} \cdot \mathrm{cm}^{-3}\right)$ & $5.0 \times 10^{-4}$ & $7.2 \times 10^{-4}$ & $1.3 \times 10^{-3}$ & $5.0 \times 10^{-3}$ \\
\hline$C_{V_{\mathrm{o}}}^{0}=\chi k_{3} / 2 k_{6}\left(\mathrm{~mol} \cdot \mathrm{cm}^{-3}\right)$ & $1.1 \times 10^{-4}$ & $1.0 \times 10^{-4}$ & $3.9 \times 10^{-5}$ & $5.3 \times 10^{-5}$ \\
\hline$C_{i}^{0} /\left(\frac{2}{\Omega}\right)$ & $0.4 \%$ & $0.5 \%$ & $0.9 \%$ & $3.7 \%$ \\
\hline$C_{V_{\ddot{0}}}^{0} /\left(\frac{3}{\Omega}\right)$ & $0.06 \%$ & $0.05 \%$ & $0.02 \%$ & $0.03 \%$ \\
\hline$D_{i}\left(\mathrm{~cm}^{2} \cdot \mathrm{s}^{-1}\right)$ & $7.6 \times 10^{-18}$ & $2.0 \times 10^{-15}$ & $1.3 \times 10^{-13}$ & $2.9 \times 10^{-13}$ \\
\hline$R_{g} / R_{d}$ & 0.7 & 0.7 & 0.7 & 1.0 \\
\hline
\end{tabular}


Table X.14. Several properties that were calculated based on the extracted coefficients for passive films formed on Alloy 600 under OCP in a solution containing $200 \mathrm{ppm} \mathrm{B}+2 \mathrm{ppm} \mathrm{Li}$, and 400 ppb dissolved oxygen at different temperatures.

\begin{tabular}{lllll}
\hline Parameters & $150{ }^{\circ} \mathrm{C}$ & $200{ }^{\circ} \mathrm{C}$ & $250{ }^{\circ} \mathrm{C}$ & $300{ }^{\circ} \mathrm{C}$ \\
\hline$I_{a}\left(\mathrm{~A} \cdot \mathrm{cm}^{-2}\right)$ & $3.39 \times 10^{-7}$ & $6.29 \times 10^{-7}$ & $9.37 \times 10^{-7}$ & $2.00 \times 10^{-6}$ \\
\hline$I_{c}\left(\mathrm{~A} \cdot \mathrm{cm}^{-2}\right)$ & $-3.22 \times 10^{-7}$ & $-6.03 \times 10^{-7}$ & $-9.31 \times 10^{-7}$ & $-2.05 \times 10^{-6}$ \\
\hline$Z_{R_{C}}\left(\Omega \cdot \mathrm{cm}^{2}\right)$ & $2.1 \times 10^{5}$ & $7.9 \times 10^{4}$ & $5.1 \times 10^{4}$ & $2.2 \times 10^{4}$ \\
\hline$L_{s S}(\mathrm{~nm})$ & 1.4 & 2.5 & 3.6 & 5.6 \\
\hline$k_{2}^{0}\left(\mathrm{~mol} \cdot \mathrm{cm}^{-2} \cdot \mathrm{s}^{-1}\right)$ & $1.4 \times 10^{-8}$ & $1.3 \times 10^{-6}$ & $1.7 \times 10^{-4}$ & $2.2 \times 10^{-3}$ \\
\hline$k_{3}^{0}\left(\mathrm{~mol} \cdot \mathrm{cm}^{-2} \cdot \mathrm{s}^{-1}\right)$ & $3.7 \times 10^{-7}$ & $1.2 \times 10^{-6}$ & $3.7 \times 10^{-6}$ & $5.5 \times 10^{-6}$ \\
\hline$k_{5}^{0}\left(\mathrm{~cm} \cdot \mathrm{s}^{-1}\right)$ & $4.7 \times 10^{-7}$ & $6.3 \times 10^{-7}$ & $8.1 \times 10^{-6}$ & $1.5 \times 10^{-5}$ \\
\hline$k_{6}^{0}\left(\mathrm{~cm} \cdot \mathrm{s}^{-1}\right)$ & $3.1 \times 10^{-6}$ & $5.2 \times 10^{-6}$ & $3.6 \times 10^{-5}$ & $7.0 \times 10^{-5}$ \\
\hline$k_{7}^{0}\left(\mathrm{~mol} \cdot \mathrm{cm}^{-2} \cdot \mathrm{s}^{-1}\right)$ & $4.0 \times 10^{-9}$ & $5.4 \times 10^{-9}$ & $1.3 \times 10^{-8}$ & $2.2 \times 10^{-8}$ \\
\hline$k_{2}\left(\mathrm{~mol} \cdot \mathrm{cm}^{-2} \cdot \mathrm{s}^{-1}\right)$ & $5.7 \times 10^{-13}$ & $1.5 \times 10^{-12}$ & $2.1 \times 10^{-12}$ & $3.8 \times 10^{-12}$ \\
\hline$k_{3}\left(\mathrm{~mol} \cdot \mathrm{cm}^{-2} \cdot \mathrm{s}^{-1}\right)$ & $6.0 \times 10^{-13}$ & $7.3 \times 10^{-13}$ & $1.1 \times 10^{-12}$ & $3.1 \times 10^{-12}$ \\
\hline$k_{5}\left(\mathrm{~cm} \cdot \mathrm{s}^{-1}\right)$ & $1.2 \times 10^{-9}$ & $2.4 \times 10^{-9}$ & $1.7 \times 10^{-9}$ & $1.0 \times 10^{-9}$ \\
\hline$k_{6}\left(\mathrm{~cm} \cdot \mathrm{s}^{-1}\right)$ & $1.5 \times 10^{-8}$ & $4.4 \times 10^{-8}$ & $3.1 \times 10^{-8}$ & $9.4 \times 10^{-8}$ \\
\hline$k_{7}\left(\mathrm{~mol} \cdot \mathrm{cm}^{-2} \cdot \mathrm{s}^{-1}\right)$ & 4.0 & $5.4 \times 10^{-9}$ & $1.3 \times 10^{-8}$ & $2.2 \times 10^{-8}$ \\
\hline$C_{i}^{0}=k_{2} / k_{5}\left(\mathrm{~mol} \cdot \mathrm{cm}^{-3}\right)$ & $4.8 \times 10^{-4}$ & $6.2 \times 10^{-4}$ & $1.3 \times 10^{-3}$ & $3.7 \times 10^{-3}$ \\
\hline$C_{V_{0}^{0}}^{0}=\chi k_{3} / 2 k_{6}\left(\mathrm{~mol} \cdot \mathrm{cm}^{-3}\right)$ & $6.0 \times 10^{-5}$ & $2.5 \times 10^{-5}$ & $5.3 \times 10^{-5}$ & $4.9 \times 10^{-5}$ \\
\hline$C_{i}^{0} /\left(\frac{2}{\Omega}\right)$ & $0.4 \%$ & $0.4 \%$ & $0.9 \%$ & $2.7 \%$ \\
\hline$C_{V_{0}}^{0} /\left(\frac{3}{\Omega}\right)$ & $0.03 \%$ & $0.02 \%$ & $0.03 \%$ & $0.03 \%$ \\
\hline$R_{g} / R_{d}$ & $4.9 \times 10^{-17}$ & $8.7 \times 10^{-16}$ & $2.5 \times 10^{-15}$ & $9.7 \times 10^{-14}$ \\
\hline$D_{i}\left(\mathrm{~cm} \cdot \mathrm{s}^{-1}\right)$ & & & 0.7 & 0.9 \\
\hline & & & & \\
\hline
\end{tabular}


Table X.15. Several properties that were calculated based on the extracted coefficients for passive films formed on Alloy 600 at $+0.3 \mathrm{~V}_{\text {OCP }}$ in a solution containing $200 \mathrm{ppm} \mathrm{B}+2 \mathrm{ppm} \mathrm{Li}$, and 400 ppb dissolved oxygen at different temperatures.

\begin{tabular}{lllll}
\hline Parameters & $150{ }^{\circ} \mathrm{C}$ & $200{ }^{\circ} \mathrm{C}$ & $250{ }^{\circ} \mathrm{C}$ & $300{ }^{\circ} \mathrm{C}$ \\
\hline$I_{a}\left(\mathrm{~A} \cdot \mathrm{cm}^{-2}\right)$ & $2.49 \times 10^{-7}$ & $6.30 \times 10^{-7}$ & $9.35 \times 10^{-7}$ & $2.17 \times 10^{-6}$ \\
\hline$I_{C}\left(\mathrm{~A} \cdot \mathrm{cm}^{-2}\right)$ & $-4.08 \times 10^{-9}$ & $-1.28 \times 10^{-9}$ & $-1.83 \times 10^{-9}$ & $-3.70 \times 10^{-9}$ \\
\hline$Z_{R_{C}}\left(\Omega \cdot \mathrm{cm}^{2}\right)$ & $1.7 \times 10^{7}$ & $3.8 \times 10^{7}$ & $2.7 \times 10^{7}$ & $1.3 \times 10^{7}$ \\
\hline$L_{S S}(\mathrm{~nm})$ & 2.3 & 3.8 & 5.1 & 6.6 \\
\hline$k_{2}^{0}\left(\mathrm{~mol} \cdot \mathrm{cm}^{-2} \cdot \mathrm{s}^{-1}\right)$ & $1.4 \times 10^{-8}$ & $1.3 \times 10^{-6}$ & $1.7 \times 10^{-4}$ & $2.2 \times 10^{-3}$ \\
\hline$k_{3}^{0}\left(\mathrm{~mol} \cdot \mathrm{cm}^{-2} \cdot \mathrm{s}^{-1}\right)$ & $3.7 \times 10^{-7}$ & $1.2 \times 10^{-6}$ & $3.7 \times 10^{-6}$ & $5.5 \times 10^{-6}$ \\
\hline$k_{5}^{0}\left(\mathrm{~cm} \cdot \mathrm{s}^{-1}\right)$ & $4.7 \times 10^{-7}$ & $6.3 \times 10^{-7}$ & $8.1 \times 10^{-6}$ & $1.5 \times 10^{-5}$ \\
\hline$k_{6}^{0}\left(\mathrm{~cm} \cdot \mathrm{s}^{-1}\right)$ & $3.1 \times 10^{-6}$ & $5.2 \times 10^{-6}$ & $3.6 \times 10^{-5}$ & $7.0 \times 10^{-5}$ \\
\hline$k_{7}^{0}\left(\mathrm{~mol} \cdot \mathrm{cm}^{-2} \cdot \mathrm{s}^{-1}\right)$ & $4.0 \times 10^{-9}$ & $5.4 \times 10^{-9}$ & $1.3 \times 10^{-8}$ & $2.2 \times 10^{-8}$ \\
\hline$k_{2}\left(\mathrm{~mol} \cdot \mathrm{cm}^{-2} \cdot \mathrm{s}^{-1}\right)$ & $4.4 \times 10^{-13}$ & $1.5 \times 10^{-12}$ & $2.1 \times 10^{-12}$ & $4.2 \times 10^{-12}$ \\
\hline$k_{3}\left(\mathrm{~mol} \cdot \mathrm{cm}^{-2} \cdot \mathrm{s}^{-1}\right)$ & $4.2 \times 10^{-13}$ & $7.3 \times 10^{-13}$ & $1.1 \times 10^{-12}$ & $3.4 \times 10^{-12}$ \\
\hline$k_{5}\left(\mathrm{~cm} \cdot \mathrm{s}^{-1}\right)$ & $3.7 \times 10^{-9}$ & $4.8 \times 10^{-9}$ & $3.7 \times 10^{-9}$ & $2.7 \times 10^{-9}$ \\
\hline$k_{6}\left(\mathrm{~cm} \cdot \mathrm{s}^{-1}\right)$ & $4.0 \times 10^{-8}$ & $8.1 \times 10^{-8}$ & $5.9 \times 10^{-8}$ & $1.9 \times 10^{-7}$ \\
\hline$k_{7}\left(\mathrm{~mol} \cdot \mathrm{cm}^{-2} \cdot \mathrm{s}^{-1}\right)$ & 0.8 & $5.4 \times 10^{-9}$ & $1.3 \times 10^{-8}$ & $2.2 \times 10^{-8}$ \\
\hline$C_{i}^{0}=k_{2} / k_{5}\left(\mathrm{~mol} \cdot \mathrm{cm}{ }^{-3}\right)$ & $3.1 \times 10^{-4}$ & $3.8 \times 10^{-4}$ & $1.0 \times 10^{-3}$ & $3.7 \times 10^{-3}$ \\
\hline$C_{V_{0}}^{0}=\chi k_{3} / 2 k_{6}\left(\mathrm{~mol} \cdot \mathrm{cm}^{-3}\right)$ & $1.6 \times 10^{-5}$ & $1.4 \times 10^{-5}$ & $2.8 \times 10^{-5}$ & $2.7 \times 10^{-5}$ \\
\hline$C_{i}^{0} /\left(\frac{2}{\Omega}\right)$ & $0.2 \%$ & $0.2 \%$ & $0.7 \%$ & $2.7 \%$ \\
\hline$C_{V_{\mathrm{o}}}^{0} /\left(\frac{3}{s_{\iota}}\right)$ & $5.2 \times 10^{-18}$ & $8.1 \times 10^{-16}$ & $1.3 \times 10^{-15}$ & $4.2 \times 10^{-14}$ \\
\hline$R_{g} / R_{d}$ & $0.02 \%$ & $0.02 \%$ & $0.03 \%$ \\
\hline$D_{i}\left(\mathrm{~cm} \cdot \mathrm{s}^{-1}\right)$ & & 0.7 & 0.7 & 0.9 \\
\hline
\end{tabular}


Table X.16. Several properties that were calculated based on the extracted coefficients for passive films formed on Alloy 600 under OCP in a solution containing 0 ppm B +2 ppm Li, and $400 \mathrm{ppb}$ dissolved oxygen at different temperatures.

\begin{tabular}{|c|c|c|c|c|}
\hline Parameters & $150^{\circ} \mathrm{C}$ & $200^{\circ} \mathrm{C}$ & $250^{\circ} \mathrm{C}$ & $300^{\circ} \mathrm{C}$ \\
\hline$I_{a}\left(\mathrm{~A} \cdot \mathrm{cm}^{-2}\right)$ & $2.28 \times 10^{-7}$ & $4.95 \times 10^{-7}$ & $6.00 \times 10^{-7}$ & $1.92 \times 10^{-6}$ \\
\hline$I_{c}\left(\mathrm{~A} \cdot \mathrm{cm}^{-2}\right)$ & $-2.37 \times 10^{-7}$ & $-4.85 \times 10^{-7}$ & $-6.07 \times 10^{-7}$ & $-1.87 \times 10^{-6}$ \\
\hline$Z_{R_{C}}\left(\Omega \cdot \mathrm{cm}^{2}\right)$ & $2.8 \times 10^{5}$ & $9.7 \times 10^{4}$ & $7.5 \times 10^{4}$ & $2.3 \times 10^{4}$ \\
\hline$L_{S S}(\mathrm{~nm})$ & 1.6 & 2.6 & 3.6 & 6.4 \\
\hline$k_{2}^{0}\left(\mathrm{~mol} \cdot \mathrm{cm}^{-2} \cdot \mathrm{s}^{-1}\right)$ & $3.7 \times 10^{-8}$ & $1.8 \times 10^{-6}$ & $1.5 \times 10^{-5}$ & $6.1 \times 10^{-2}$ \\
\hline$k_{3}^{0}\left(\mathrm{~mol} \cdot \mathrm{cm}^{-2} \cdot \mathrm{s}^{-1}\right)$ & $9.4 \times 10^{-7}$ & $1.6 \times 10^{-6}$ & $5.6 \times 10^{-6}$ & $1.3 \times 10^{-5}$ \\
\hline$k_{5}^{0}\left(\mathrm{~cm} \cdot \mathrm{s}^{-1}\right)$ & $2.3 \times 10^{-6}$ & $3.0 \times 10^{-6}$ & $7.8 \times 10^{-6}$ & $1.7 \times 10^{-4}$ \\
\hline$k_{6}^{0}\left(\mathrm{~cm} \cdot \mathrm{s}^{-1}\right)$ & $1.6 \times 10^{-6}$ & $2.8 \times 10^{-6}$ & $1.5 \times 10^{-5}$ & $4.0 \times 10^{-5}$ \\
\hline$k_{7}^{0}\left(\mathrm{~mol} \cdot \mathrm{cm}^{-2} \cdot \mathrm{s}^{-1}\right)$ & $3.5 \times 10^{-9}$ & $4.6 \times 10^{-9}$ & $1.2 \times 10^{-8}$ & $2.3 \times 10^{-8}$ \\
\hline$k_{2}\left(\mathrm{~mol} \cdot \mathrm{cm}^{-2} \cdot \mathrm{s}^{-1}\right)$ & $4.6 \times 10^{-13}$ & $1.1 \times 10^{-12}$ & $1.2 \times 10^{-12}$ & $4.1 \times 10^{-12}$ \\
\hline$k_{3}\left(\mathrm{~mol} \cdot \mathrm{cm}^{-2} \cdot \mathrm{s}^{-1}\right)$ & $3.1 \times 10^{-13}$ & $5.7 \times 10^{-13}$ & $8.6 \times 10^{-13}$ & $2.6 \times 10^{-12}$ \\
\hline$k_{5}\left(\mathrm{~cm} \cdot \mathrm{s}^{-1}\right)$ & $2.4 \times 10^{-9}$ & $3.5 \times 10^{-9}$ & $1.4 \times 10^{-9}$ & $1.1 \times 10^{-9}$ \\
\hline$k_{6}\left(\mathrm{~cm} \cdot \mathrm{s}^{-1}\right)$ & $3.7 \times 10^{-9}$ & $8.2 \times 10^{-9}$ & $2.8 \times 10^{-8}$ & $1.1 \times 10^{-7}$ \\
\hline$k_{7}\left(\mathrm{~mol} \cdot \mathrm{cm}^{-2} \cdot \mathrm{s}^{-1}\right)$ & $3.5 \times 10^{-9}$ & $4.6 \times 10^{-9}$ & $1.2 \times 10^{-8}$ & $2.3 \times 10^{-8}$ \\
\hline$C_{i}^{0}=k_{2} / k_{5}\left(\mathrm{~mol} \cdot \mathrm{cm}^{-3}\right)$ & $2.0 \times 10^{-4}$ & $3.3 \times 10^{-4}$ & $8.6 \times 10^{-4}$ & $3.7 \times 10^{-3}$ \\
\hline$C_{V_{\ddot{o}}}^{0}=\chi k_{3} / 2 k_{6}\left(\mathrm{~mol} \cdot \mathrm{cm}^{-3}\right)$ & $1.3 \times 10^{-4}$ & $1.0 \times 10^{-4}$ & $4.6 \times 10^{-5}$ & $3.5 \times 10^{-5}$ \\
\hline$C_{i}^{0} /\left(\frac{2}{\Omega}\right)$ & $0.1 \%$ & $0.2 \%$ & $0.6 \%$ & $2.7 \%$ \\
\hline$C_{V_{\tilde{o}}}^{0} /\left(\frac{3}{\Omega}\right)$ & $0.06 \%$ & $0.05 \%$ & $0.02 \%$ & $0.02 \%$ \\
\hline$R_{g} / R_{d}$ & 0.8 & 0.7 & 0.8 & 0.8 \\
\hline$D_{i}\left(\mathrm{~cm}^{2} \cdot \mathrm{s}^{-1}\right)$ & $1.0 \times 10^{-18}$ & $3.9 \times 10^{-16}$ & $1.4 \times 10^{-14}$ & $9.3 \times 10^{-14}$ \\
\hline
\end{tabular}



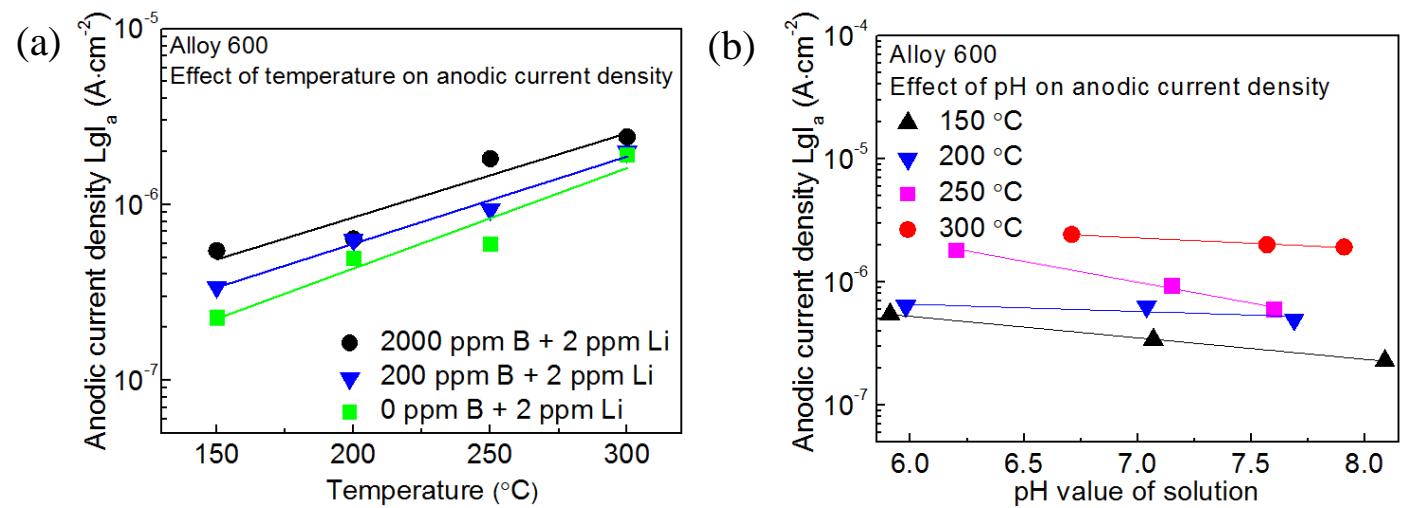

Figure X.42. Effects of (a) temperature and (b) $\mathrm{pH}$ on the anodic passive current density for passive films formed on Alloy 600 under OCP in borate buffer solutions with 400 ppb dissolved oxygen.

(a)

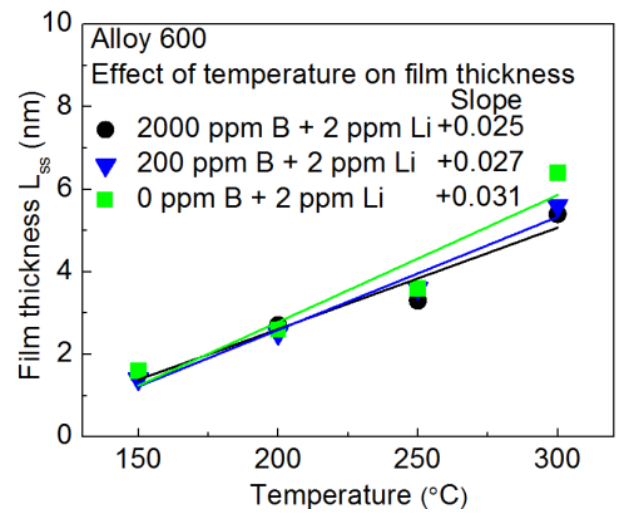

(b)

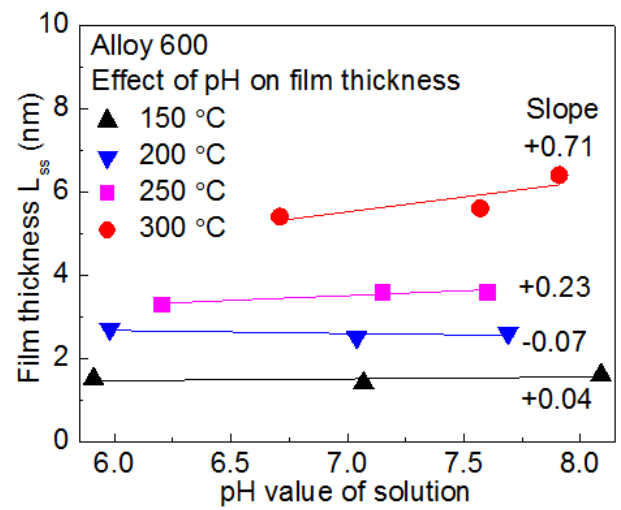

Figure X.43. Effects of (a) temperature and (b) $\mathrm{pH}$ on the thickness of passive films formed on Alloy 600 under OCP in borate buffer solutions with 400 ppb dissolved oxygen.

The properties of passive films formed on Alloy 600 at OCP and +0.3 VOCP in an oxygenated solution containing 200 ppm B +2 ppm Li are compared, as shown in Tables X.14 and X.15. At the same temperature, the anodic current density is independent of the film formation potential, corresponding to the n-Type film character and the above anodic potentiostatic polarization measurement results. The barrier layer of passive film formed at $+0.3 \mathrm{~V}$ OCP is much thicker than that formed at OCP, in concert with the prediction of the MPM. The increased barrier layer thickness and slightly decreased defect density of passive film formed at more positive potential contribute to the increased impedance and improved corrosion resistance. 
(a)

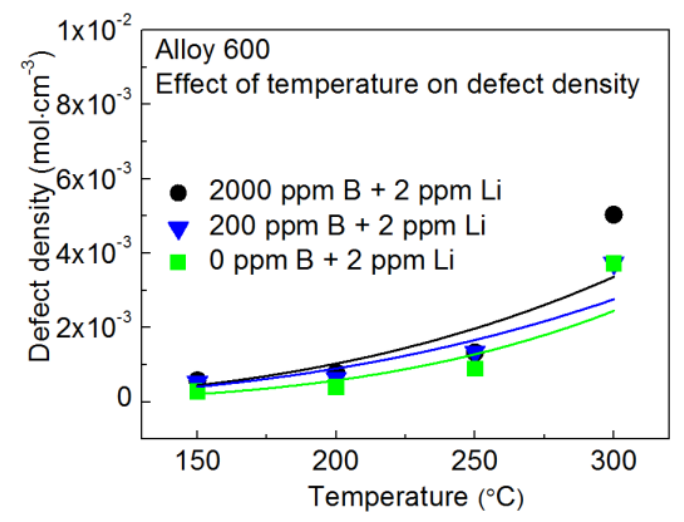

(b)

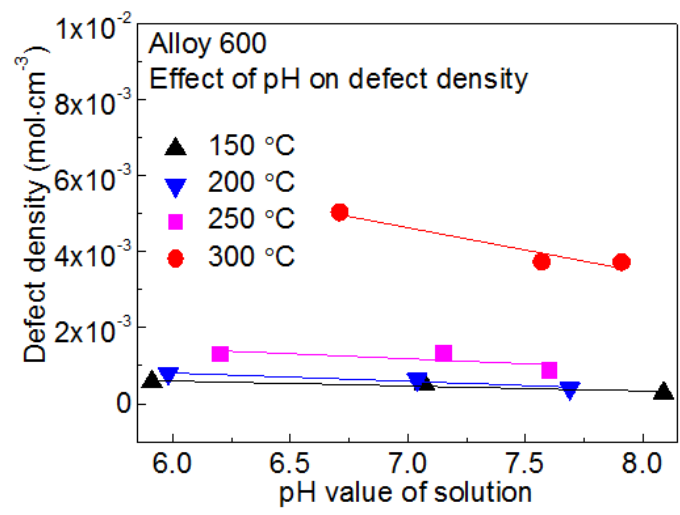

Figure X.44. Effects of (a) temperature and (b) $\mathrm{pH}$ on the defect density of passive films formed on Alloy 600 under OCP in borate buffer solutions with $400 \mathrm{ppb}$ dissolved oxygen.

\section{X.5. Discussion}

The measured OCP values for Alloy 600, Alloy 690, Type 304L SS, and Type 316L SS at different solution environments are compared in Figure X.45 and indicated in Table X.17. At the same temperature (in each solution), the OCP difference between these four alloys is insignificant. This may occur due to the similar composition of the barrier layer for each alloy, which comprises defective iron- and nickel-containing chromic oxide $(\mathrm{Fe}, \mathrm{Ni}, \mathrm{Cr})_{2+x} \mathrm{O}_{3-y}$.

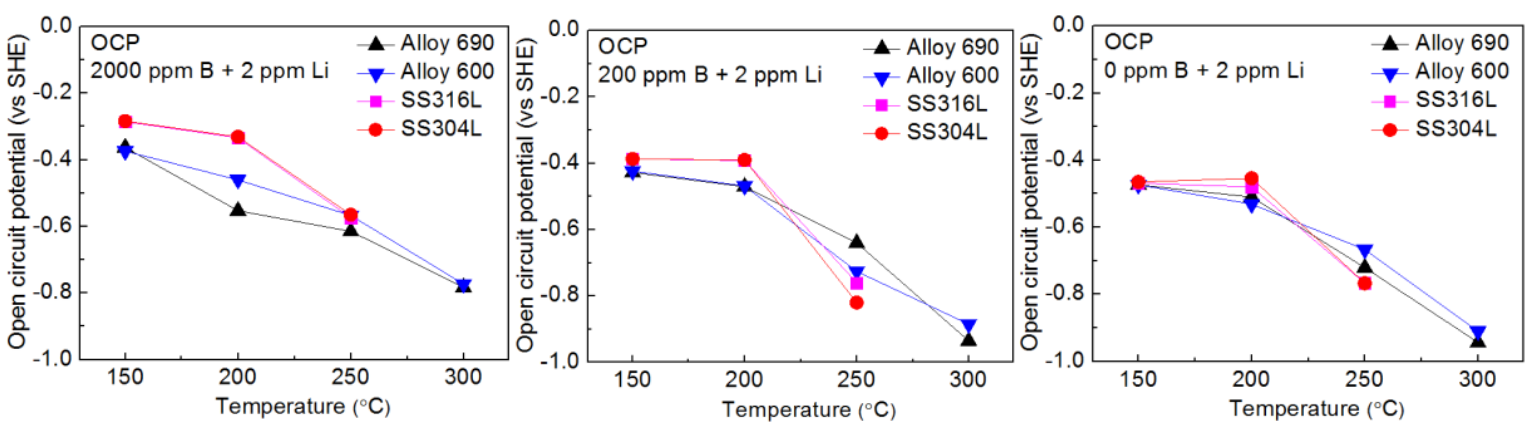

Figure X.45. Change of open circuit potential with temperature for Alloy 690, Alloy 600, Type 316L SS and Type 304L SS in a solution containing $\mathrm{H}_{3} \mathrm{BO}_{3}(2000$ ppm B, 200 ppm B, 0 ppm B) + $\mathrm{LiOH}(2$ ppm Li) with 400 ppb dissolved oxygen.

Figure X.46 presents the EIS plots recorded in the Bode plane for Alloy 690, Alloy 600, Type 316L SS, and Type 304L SS. 
Table X.17. OCP values for all alloys in borate buffer solutions with $400 \mathrm{ppb}$ dissolved oxygen.

\begin{tabular}{|c|c|c|c|c|c|c|c|c|c|c|c|c|}
\hline \multicolumn{13}{|c|}{ Open Circuit Potential $\left(\mathrm{V}_{\mathrm{SHE}}\right)$} \\
\hline \multirow{2}{*}{$\begin{array}{c}T \\
\left({ }^{\circ} \mathrm{C}\right)\end{array}$} & \multicolumn{4}{|c|}{2000 ppm B + 2 ppm Li } & \multicolumn{4}{|c|}{$200 \mathrm{ppm} \mathrm{B}+2 \mathrm{ppm} \mathrm{Li}$} & \multicolumn{4}{|c|}{$2 \mathrm{ppm} \mathrm{Li}$} \\
\hline & $\begin{array}{c}\text { Alloy } \\
690\end{array}$ & $\begin{array}{c}\text { Alloy } \\
600\end{array}$ & SS316L & SS304L & $\begin{array}{c}\text { Alloy } \\
690\end{array}$ & $\begin{array}{l}\text { Alloy } \\
600\end{array}$ & SS316L & SS304L & $\begin{array}{c}\text { Alloy } \\
690\end{array}$ & $\begin{array}{c}\text { Alloy } \\
600\end{array}$ & SS316L & SS304L \\
\hline 150 & -0.363 & -0.374 & -0.286 & -0.284 & -0.428 & -0.424 & -0.387 & -0.387 & -0.474 & -0.474 & -0.469 & -0.465 \\
\hline 200 & -0.553 & -0.460 & -0.334 & -0.331 & -0.471 & -0.469 & -0.392 & -0.390 & -0.510 & -0.531 & -0.480 & -0.454 \\
\hline 250 & -0.615 & -0.567 & -0.574 & -0.565 & -0.640 & -0.726 & -0.760 & -0.820 & -0.721 & -0.667 & -0.767 & -0.767 \\
\hline 300 & -0.783 & -0.773 & 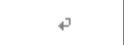 & 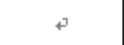 & -0.935 & -0.885 & 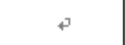 & 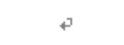 & -0.944 & -0.911 & 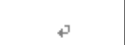 & 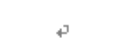 \\
\hline
\end{tabular}
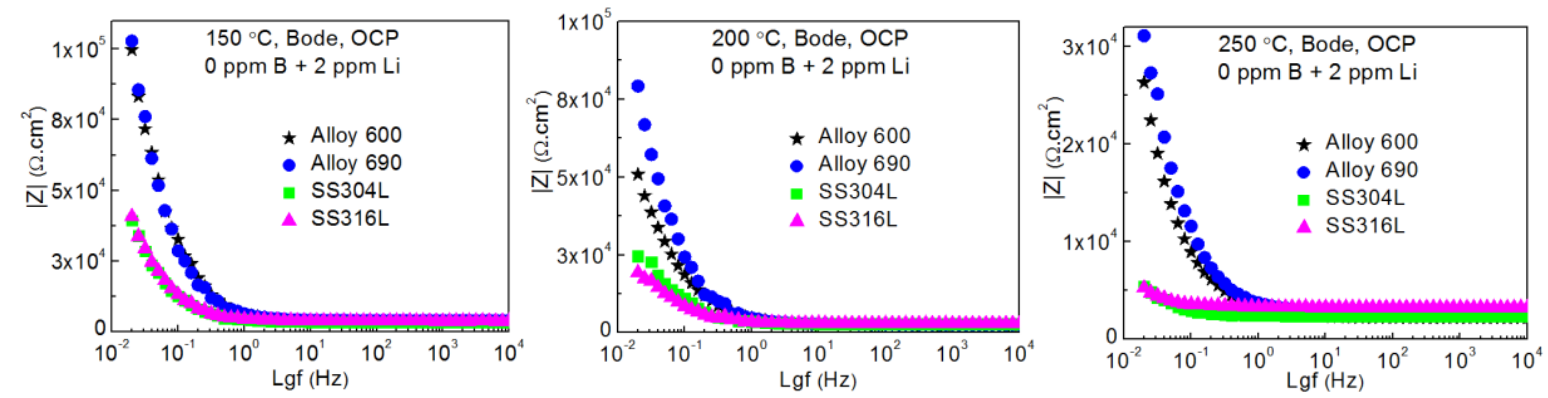

(a)
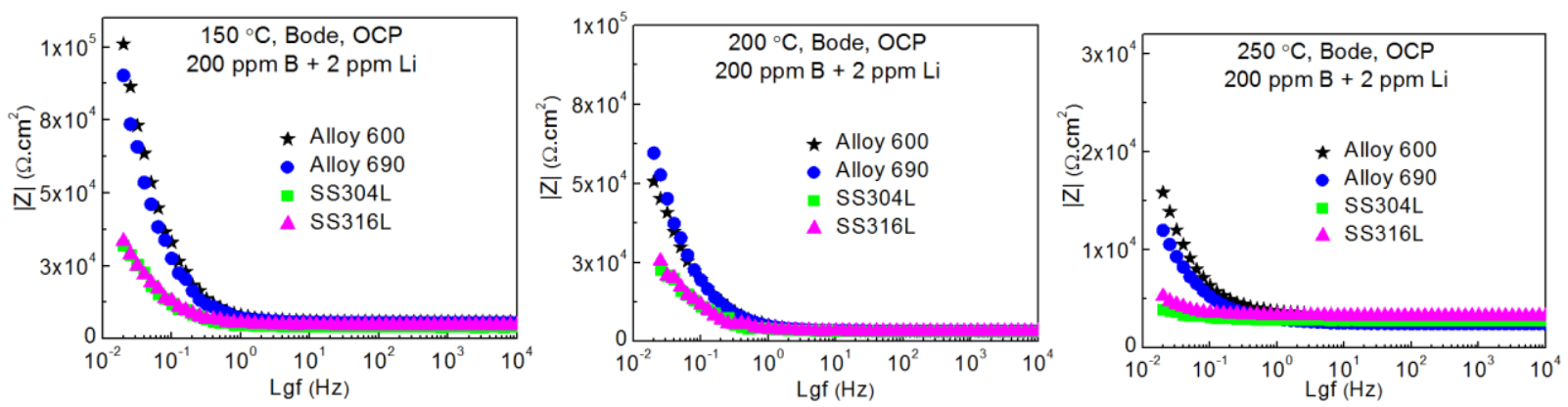

(b)
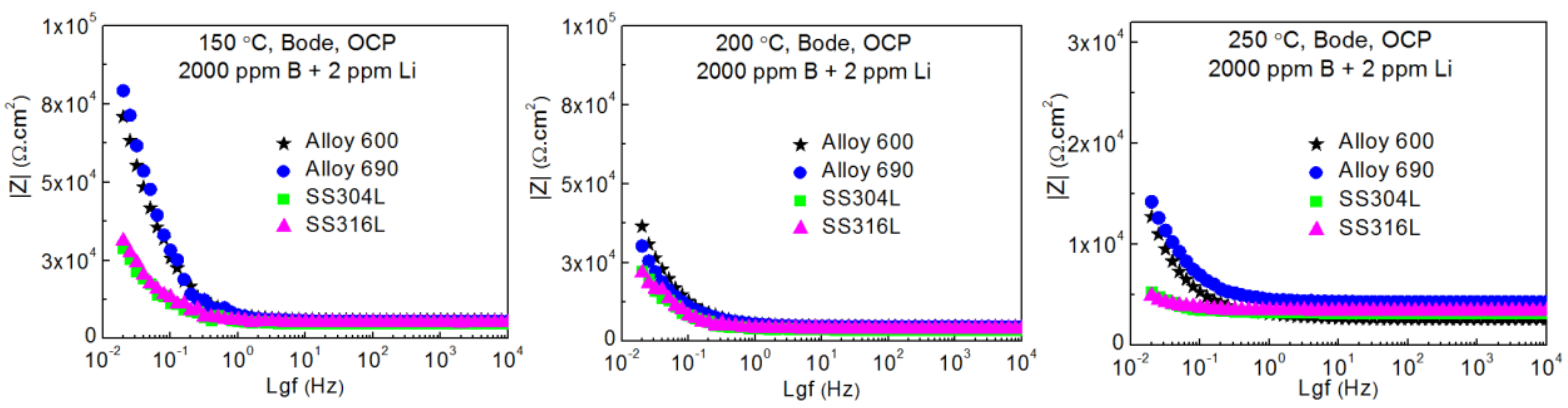

(c)

Figure X.46. Comparison of the absolute value of impedance for passive films formed on different alloys at OCP in borate buffer solutions containing $400 \mathrm{ppb}$ dissolved oxygen-containing (a) 2000 ppm B + 2 ppm Li, (b) 200 ppm B +2 ppm Li, and (c) 0 ppm B +2 ppm Li. 
At each electrochemical environment, obviously, the impedance value of nickel alloy is much higher than that of stainless-steel alloy at the same temperature, indicating the higher corrosion resistance. The corrosion resistance of Alloy 690 is slightly higher than that of Alloy 600, while the Type 304L SS and Type 316L SS show very similar resistance. In this research, the passive films formed on all alloys (Alloy 600, Alloy 690, Type 304L SS, and Type 316L SS) show n-Type semiconductor properties. For each alloy, the concentration of oxygen vacancy in the barrier layer of passive film is much less than that of metal interstitial in each solution (at the same temperature), as shown in Figure X.47.

(a)
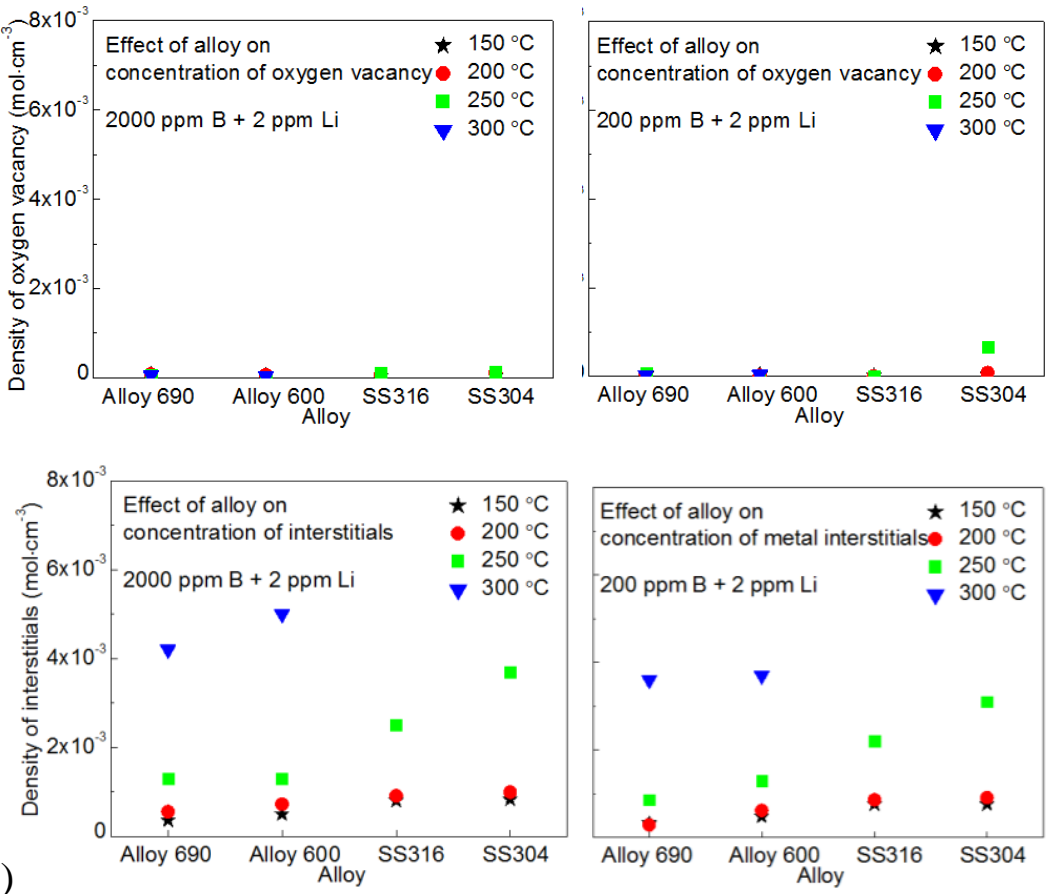

(b)

(c)

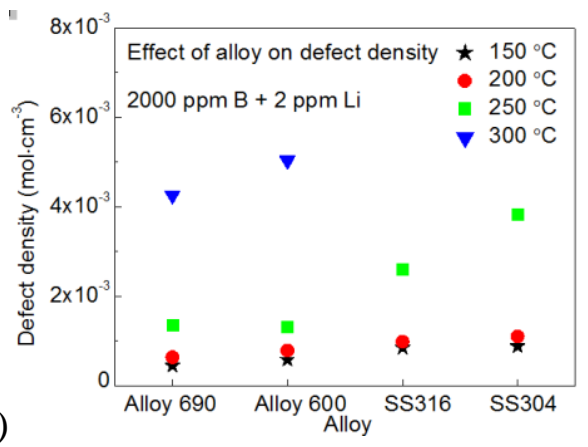

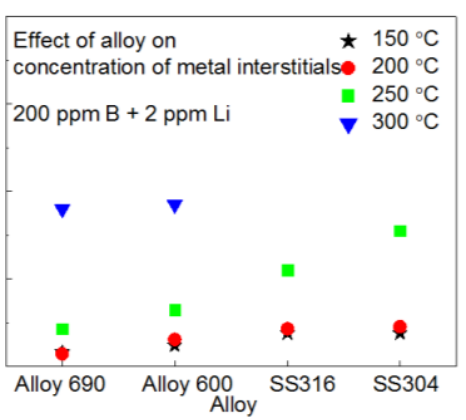

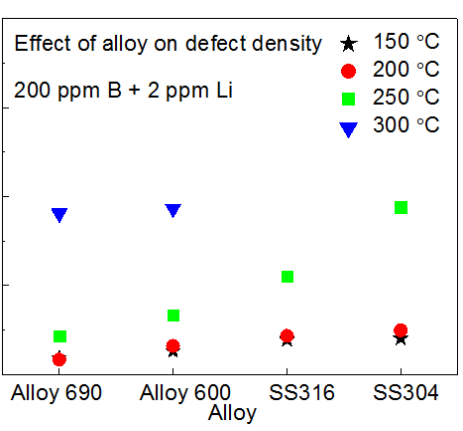

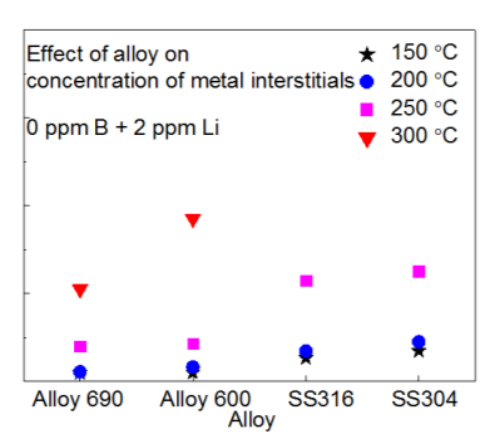
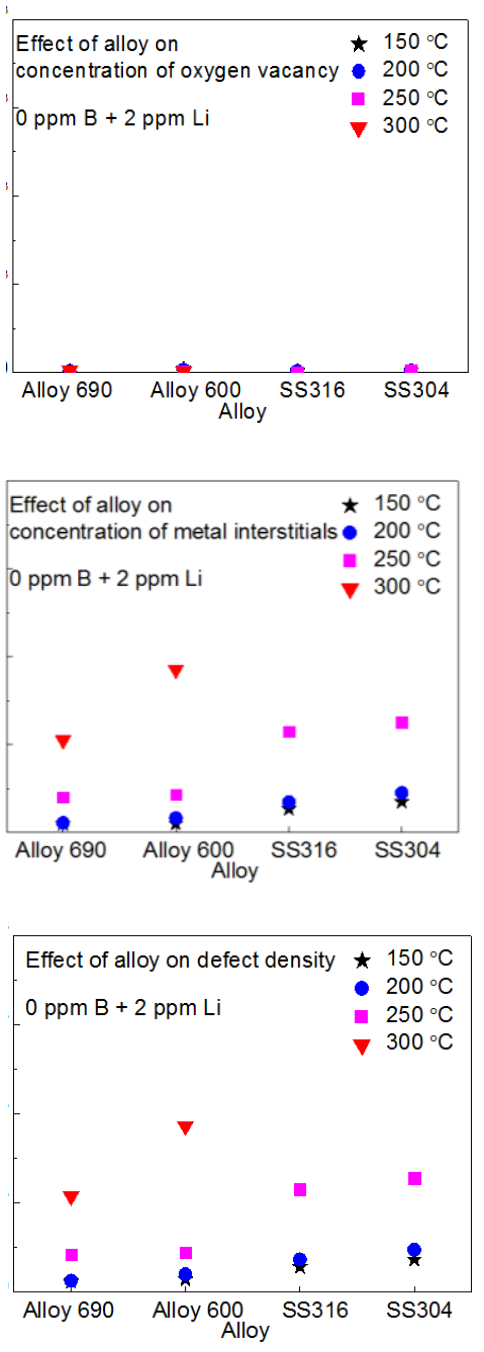

Figure X.47. Compare concentrations of (a) oxygen vacancy, (b) metal interstitial, and (c) total defect density in the barrier layers of passive films formed on Alloy 690, Alloy 600, Type 316L SS, and Type 304L SS. 
The concentration of metal interstitial is much higher in the passive films of stainless steel than nickel alloy, resulting in higher total defect density and lower corrosion resistance.

Figure X.48 indicates that the activation energy for the formation of metal interstitials (Reaction 2) is much higher for nickel alloy than stainless steel. Iron interstitials (as in SS) generates and move through the $\mathrm{Cr}_{2} \mathrm{O}_{3}$ lattice much more readily than do $\mathrm{Ni}$ interstitials, and since $99.9+\%$ of the passive current is carried by the interstitials, the passive current density is highest (and the corrosion resistance is lowest) in the case of the stainless steels than for Alloy 600 and Alloy 690. The concentration of Fe interstitials may much higher than $\mathrm{Ni}$ in the barrier layer; therefore, the passive film on stainless steel with much higher defect densities of Fe interstitials showed much lower corrosion resistance than nickel alloys (Figure X.47).

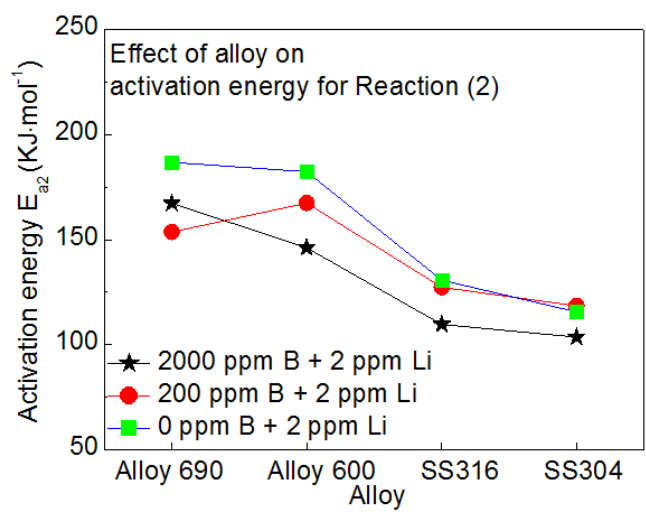

Figure X.48. Calculated values of activation energy for the formation of metal interstitials for Alloy 690, Alloy 600, Type 316L SS, and Type 304L SS in three borate buffer solutions with 400 ppb dissolved oxygen.

At the same temperature (in each solution), the thickness of the barrier layer for the passive film formed on each alloy is similar, as presented in Figure X.49. This confirmed that the effect of defect density on the corrosion properties of the alloy is much more significant than that of film thickness. The sharply decreased impedance value of stainless steel compared with nickel alloy (at the same temperature in the same solution) indicate rapidly increased defect density. 


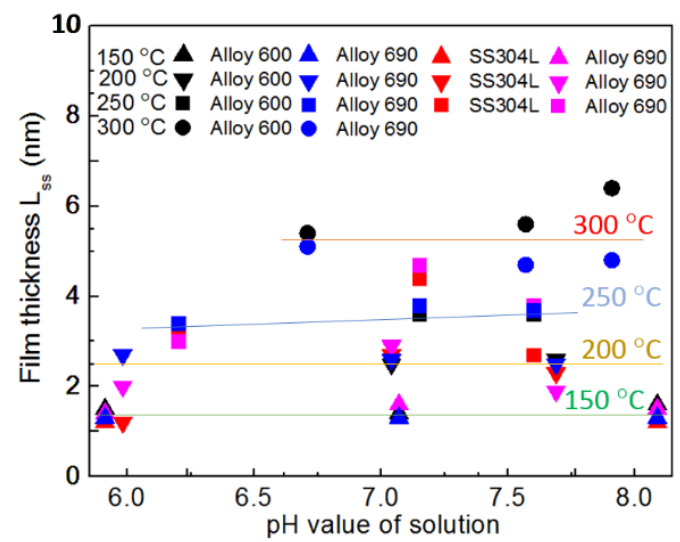

Figure X.49. Calculated thickness of barrier layers for passive films formed on Alloy 690, Alloy 600, Type 316L SS, and Type 304L SS in borate buffer solutions with 400 ppb dissolved oxygen at different temperatures.

\section{X.6. Conclusions}

The effects of temperature $\left(150{ }^{\circ} \mathrm{C}, 200{ }^{\circ} \mathrm{C}, 250{ }^{\circ} \mathrm{C}, 300{ }^{\circ} \mathrm{C}\right)$ and $\mathrm{pH}$ on the corrosion behavior of Alloy 600, Alloy 690, Type 304L SS, and Type 316L SS in oxygenated borate buffer solutions (2000 ppm B + 2 ppm Li, 200 ppm B + 2 Li, 0 ppm B +2 ppm Li ) were studied at the open circuit potential (OCP) and under anodic potentiostatic polarization using anodic potentiostatic polarization, Mott-Schottky analysis (MSA) and electrochemical impedance spectroscopy (EIS). The mixed potential model (MPM) was optimized on the experimental EIS data, and several parameters were extracted and calculated.

For each alloy:

1- The passive current density is independent of the formation potentials for passive films formed at the same temperature but at different anodic polarisation potential, which is consistent with the n-Type character of the barrier layer of the passive film as predicted by the Point Defect Model (PDM) provided that the oxidation state of the cation does not change upon ejection at the bl/ol interface. The n-Type characteristic was confirmed by the positive slopes of the MottSchottky (M-S) plots.

2- With increasing temperature (in the same solution), the anodic current density increases, the defect density calculated from the slope of M-S plots increases, and the impedance of the passive film decrease, resulting in a significant decrease in corrosion resistance. 
3- With increasing $\mathrm{pH}$ (at the same temperature), the anodic current density decreases, the defect density calculated from the slope of M-S plots shows no systematic change, and the impedance of the interface increases, leading to a slight increase in corrosion resistance. The deteriorative effect of the elevated temperature on the corrosion resistance of passive films is much more significant than the improvement indicated by the increased $\mathrm{pH}$.

4- The MPM, comprising the PDM for the anodic passive metal dissolution and the generalized Bulter-Volmer equation for cathodic oxygen reduction, along with a quantum-mechanical correction for the tunneling of charge carriers through the barrier oxide layer, was optimized on the experimental EIS data to acquire values for the kinetic and other parameters in the model. The thickness of the barrier layer and the defect densities increase with increasing temperature, resulting in a decrease in the corrosion resistance. The defect density of the barrier layer has a greater influence on the corrosion behavior of each alloy than does the film thickness. At each temperature, the concentration of metal interstitials is much higher than that of the anion vacancy, from which we conclude the metal interstitial is the dominant point defect in the barrier layer. With increasing $\mathrm{pH}$, the film defect density decreases, and the thickness of the passive film increases linearly, leading to a linear decrease in anodic current density and slightly improved corrosion resistance.

5- The activation energy for Reaction (2) is the highest.

For the four alloys in the same electrochemical corrosion environment:

1- The open-circuit potential is similar because of the similar composition of the barrier layer.

2- The thickness of the barrier layer is similar.

3- The concentration of metal interstitials in the barrier layer of Nickel alloy is much lower than that of stainless steel, leading to higher corrosion resistance.

4- The activation energy for the formation of metal interstitials (Reaction 2) is higher for Nickel alloy than stainless steel.

5- Generation and diffusion of iron interstitials are much easier that of nickel interstitials.

\section{X.7. References}

[1] D.D. Macdonald, 11-Electrochemical techniques for monitoring and controlling corrosion in water-cooled nuclear reactor systems, in D. Féron (Ed.) Nuclear Corrosion Science and Engineering, Woodhead Publishing, 331-407 (2012). 
[2] J. Panter, B. Viguier, J.M. Cloué, M. Foucault, P. Combrade, and E. Andrieu, J. Nucl. Mater., 348, 213-221 (2006).

[3] P.M. Scott, Corrosion, 56, 771-782 (2000).

[4] Q. Peng, J. Hou, K. Sakaguchi, Y. Takeda, and T. Shoji, Electrochim. Acta, 56, 8375-8386 (2011).

[5] Y. Qiu, T. Shoji, and Z. Lu, Corros. Sci., 53, 1983-1989 (2011).

[6] Z. Zhai, M.B. Toloczko, M.J. Olszta, and S.M. Bruemmer, Corros. Sci., 123, 76-87 (2017).

[7] F. Hamdani, Improvement of the corrosion and oxidation resistance of Ni-based alloys by optimizing the chromium content, Ph.D. Dissertation in Materials, INSA de Lyon, Tohoku Gakuin University, 2015.

[8] S.E. Ziemniak and M. Hanson, Corros. Sci., 48, 498-521 (2006).

[9] M. Sennour, L. Marchetti, F. Martin, S. Perrin, R. Molins, and M. Pijolat, J. Nucl. Mater., 402, 147-156 (2010).

[10] J. Shi, B. Fekete, J. Wang, and D.D. Macdonald, Corros. Sci., 139, 58-67 (2018).

[11] D.D. Macdonald and M. Urquidi-Macdonald, Corros. Sci., 32, 51-81 (1991).

[12] D.D. Macdonald and M. Urquidi-Macdonald, The Electrochemistry of Nuclear Reactor Coolant Circuits, Wiley Online Library, (2007).

[13] A. Machet, A. Galtayries, S. Zanna, L. Klein, V. Maurice, P. Jolivet, M. Foucault, P. Combrade, P. Scott, and P. Marcus, Electrochim. Acta, 49, 3957-3964 (2004).

[14] T. Nakagawa, N. Totsuka, T. Terachi, and N. Nakajima, J. Nucl. Sci. Technol., 40, 39-43 (2003).

[15] L. Marchetti, S. Perrin, Y. Wouters, F. Martin, and M. Pijolat, Electrochim. Acta, 55, 53845392 (2010).

[16] A. Machet, A. Galtayries, P. Marcus, P. Combrade, P. Jolivet, and P. Scott, Surf. Interface Anal., 34, 197-200 (2002).

[17] D.D. Macdonald, Electrochim. Acta, 56, 1761-1772 (2011).

[18] A. Loucif, J.P. Petit, and Y. Wouters, J. Nucl. Mater., 443, 222-229 (2013). 
[19] M.F. Montemor, M.G.S. Ferreira, N.E. Hakiki, and M. Da Cunha Belo, Corros. Sci., 42, 16351650 (2000).

[20] E.W.A. Young, P.C.M. Stiphout, and J.H.W. de Wit, J. Electrochem. Soc., 132, 884-886 (1985).

[21] E.W.A. Young, J.H. Gerretsen, and J.H.W. de Wit, J. Electrochem. Soc., 134, 2257-2260 (1987).

[22] T. Matsui and K. Naito, J. Nucl. Mater. 136, 78-82 (1985).

[23] J.S. Baek, J.G. Kim, D.H. Hur, and J.S. Kim, Corros. Sci., 45, 983-994 (2003).

[24] N. Totsuka and Z. Szklarska-Smialowska, Corrosion, 43, 734-738 (1987).

[25] G. Furutani, N. Nakajima, T. Konishi, and M. Kodama, J. Nucl. Mater., 288, 179-186 (2001).

[26] J. Yang, Y. Li, A. Xu, B. Fekete, and D.D. Macdonald, J. Nucl. Mater., 518, 305-315 (2019).

[27] R. Biswas, Investigation of electrochemical phenomena related to corrosion in hightemperature aqueous systems, Ph.D. Dissertation in Materials Science and Engineering, Pennsylvania State University, (1999).

[28] J.B. Ferguson and H.F. Lopez, Metall. Mater. Trans. A, 37, 2471-2479 (2006).

[29] J.R. Crum and R.C. Scarberry, J. Mater. Energy Syst., 4, 125-130 (1982).

[30] C.M. Brown and W.J. Mills, Corrosion, 55, 173-186 (1999).

[31] A.J. Smith and R.P. Stratton, Thermal treatment, grain boundary composition and intergranular attack resistance of Alloy 690, Proceedings of the fifth international symposium on environmental degradation of materials in nuclear power systems - water reactors, Monterey, CA, 25-29 August, American Nuclear Society Inc., La Grange Park, IL, 855-860 (1993).

[32] H. Lefaix-Jeuland, L. Marchetti, S. Perrin, M. Pijolat, M. Sennour, and R. Molins, Corros. Sci., 53, 3914-3922 (2011).

[33] J. Huang, X. Wu, and E.H. Han, Corros. Sci., 51, 2976-2982 (2009).

[34] W. Kuang, X. Wu, E.H. Han, and J. Rao, Corros. Sci., 53, 3853-3860 (2011). 
[35] H.S. Kim, A study for modeling electrochemistry in light water reactors, Ph.D. Dissertation in Mechanical and Nuclear Engineering, Pennsylvania State University, (2007).

[36] H. Sun, X.Q. Wu, and E.H. Han, Corros. Sci., 51, 2840-2847 (2009).

[37] J.J. Chen, Q. Xiao, Z.P. Lu, X.K. Ru, H. Peng, Q. Xiong, and H.J. Li, J. Nucl. Mater., 489, 137-149 (2017).

[38] Y. Gui, Z.J. Zheng, and Y. Gao, Thin Solid Films, 599, 64-71 (2016).

[39] G. Lorang, M.D.C. Belo, A.P. Simoes, and M.G.S. Ferreira, J. Electrochem. Soc., 12, 33473356 (1994).

[40] K. Shimizu, H. Habazaki, P. Skeldon, and G.E. Thompson, Surf. Interface Anal., 35, 564-574 (2003).

[41] B. Stellwag, Corros. Sci., 40, 337-370 (1998).

[42] S.E. Ziemniak and M. Hanson, Corros. Sci., 44, 2209-2230 (2002).

[43] J. Robertson, Corros. Sci., 32, 443-465 (1991).

[44] I. Nicic and D.D. Macdonald, J. Nucl. Mater., 379, 54-58 (2008).

[45] T. Terachi, K. Fujii, and K. Arioka, J. Nucl. Sci. Technol., 42, 225-232 (2005).

[46] W. Kuang, X. Wu, and E.H. Han, Corros. Sci., 52, 4081-4087 (2010).

[47] D.D. Macdonald, P.C. Lu, M. Urquidi-Macdonald, and T.K. Yeh, Corrosion, 52, 768-785 (1996).

[48] D.D. Macdonald, J. Electrochem. Soc., 139, 3434-3449 (1992).

[49] D.D. Macdonald, Pure Appl. Chem., 71, 951 (1999).

[50] D.D. Macdonald, A. Sun, N. Priyantha, and P. Jayaweera, J. Electroanal. Chem., 572, 421431 (2004).

[51] D.D. Macdonald and G. Engelhardt, ECS Transactions, 28, 123-144 (2010).

[52] D.D. Macdonald, Arabian J. Sci. Eng., 37, 1143-1185 (2012).

[53] S. Sharifi-Asl, M.L. Taylor, Z. Lu, G.R. Engelhardt, B. Kursten, and D.D. Macdonald, Electrochim. Acta, 102, 161-173 (2013). 
[54] J. Ai, Y. Chen, M. Urquidi-Macdonald, and D.D. Macdonald, J. Electrochem. Soc., 154, C43C51 (2007).

[55] Y. Zhang, D.D. Macdonald, M. Urquidi-Macdonald, G.R. Engelhardt, and R.B. Dooley, Corros. Sci., 48, 3812-3823 (2006).

[56] F. Sutanto and D.D. Macdonald, On the Role of Quantum Mechanics in Corrosion Processes, Corrosion \& Prevention 2016, Auckland, NZ (2016).

[57] P. Lu, B. Kursten, and D.D. Macdonald, Electrochim. Acta, 143, 312-323 (2014).

[58] Y. Chen, The oxidation and hydriding of zircaloy fuel cladding in high-temperature aqueous solutions, Ph.D. Dissertation in Mechanical and Nuclear Engineering, Pennsylvania State University, (2006).

[59] S. Sharifi-Asl, F.X. Mao, C.F. Dong, D.C. Kong, and D.D. Macdonald, Corrosion of copper in sodium chloride solution containing sulfide species, Unpublished, (2019).

[60] D.D. Macdonald, A.C. Scott, and P. Wentrcek, J. Electrochem. Soc., 128, 250-257 (1981).

[61] D.D. Macdonald, Corrosion, 48, 194-205 (1992).

[62] D.D. Macdonald, Corros. Sci., 38, 1003-1010 (1996).

[63] D.D. Macdonald and M. Urquidi-Macdonald, Corrosion, 46, 380-390 (1990).

[64] X. Li, J. Wang, E.H. Han, and W. Ke, Corros. Sci., 67, 169-178 (2013).

[65] H. Kim and D.D. Macdonald, Corros. Sci., 52, 1139-1145 (2010).

[66] M. Karaminezhaad-Ranjbar, J. Mankowski, and D.D. Macdonald, Corrosion, 41, 197-204 (1985).

[67] D.D. Macdonald, J. Mankowski, M. Karaminezhaad-Ranjbar, and Y.H. Hu, Corrosion, 44, 186-192 (1988).

[68] https://en.wikipedia.org/wiki/Henry\%27s_law.

[69] D.S. Kong, S.H. Chen, C. Wang, and W. Yang, Corros. Sci., 45, 747-758 (2003).

[70] B. Ter-Ovanessian, C. Alemany-Dumont, and B. Normand, Electrochim. Acta, 133, 373-381 (2014). 
[71] S.R. Morrison, Electrochemistry at Semiconductor and Oxidized Metal Electrodes, Plenum Press, NY (1980).

[72] M. Urquidi-Macdonald, S. Real, and D.D. Macdonald, J. Electrochem. Soc., 133, 2018-2024 (1986).

[73] M. Urquidi-Macdonald, S. Real, and D.D. Macdonald, Electrochim. Acta, 35, 1559-1566 (1990).

[74] D.D. Macdonald, Electrochim. Acta, 51, 1376-1388 (2006).

[75] C.Y. Chao, L.F. Lin, and D.D. Macdonald, J. Electrochem. Soc., 129, 1874-1879 (1982).

[76] C.Y. Chao, Z. Szklarska-Smialowska, and D.D. Macdonald, J. Electroanal. Chem. Interfacial Electrochem., 131, 279-287 (1982).

[77] J. Bao and D.D. Macdonald, J. Electroanal. Chem., 600, 205-216 (2007).

[78] J. Bao and D.D. Macdonald, ECS Transactions, 3, 1-13 (2007).

[79] M. Kamrunnahar, J. Bao, and D.D. Macdonald, Corros. Sci., 47, 3111-3139 (2005).

[80] T.P. Moffat, H. Yang, F.R.F. Fan, and A.J. Bard, J. Electrochem. Soc., 139, 3158-3167 (1992).

[81] B. Li, L. Wang, and G. Casati, Phys. Rev. Lett., 93, 184301 (2004).

\section{Lifetime estimation of a BWR core shroud in terms of IGSCC}

\section{XI.1. Background}

Continued operation of the aging fleet of BWRs worldwide requires gradually increasing expenditures for inspection, maintenance, and repair. An initiative to proactively address material aging issues in BWR reactor vessels and internals was initiated in the USA in 1994 to lead the industry toward a proactive, generic resolution of vessel and internals material condition issues and the development of cost-effective, remedial strategies [2]. Among the most critical reactor aging mechanisms, corrosion damage in the coolant circuits is known to be a reason for an estimated $80 \%$ of unscheduled shutdowns of commercial Light Water Reactors (LWRs) and results in significant financial losses to the plant owners [1]. IGSCC in austenitic stainless steel 
piping was accepted as a major issue for BWRs in the 1980s, at which time susceptibility of reactor internals to IGSCC was also recognized [2,3]. Shroud cracking, which was identified in 19931994, confirmed that IGSCC of internals is a significant issue for BWRs.

IGSCC is a time-dependent material degradation process that is known to be caused and accelerated by the presence of residual and operating stresses, material sensitization, irradiation, cold work, elevated temperature, and corrosive environments (Figure XI.1). Many internal components inside BWR vessels are made of materials that are susceptible to IGSCC, including austenitic stainless steels, Alloy 600, Alloy X750, and Alloy 182 weld metal.

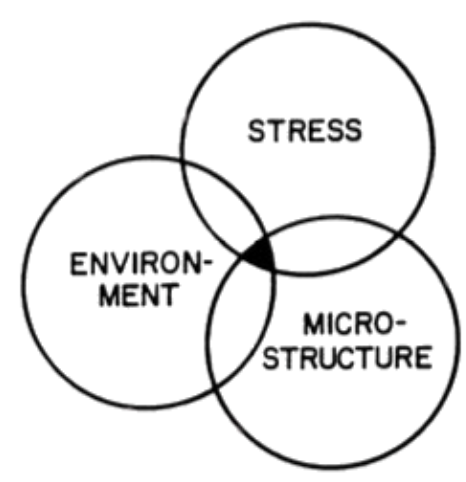

Figure XI.1. Synergy of driving forces for the stress corrosion cracking phenomenon.

IGSCC is a commonly observed phenomenon in BWR core shrouds [4]. It was first observed in 1990 at the Mtihleberg site in Switzerland [5]. As of 2013, the surface cracks being monitored in the core shroud of Mtihleberg ranged from few centimeters to over half a meter in length and had depths exceeding two centimeters. In response to this finding, the original equipment manufacturer (OEM) issued an information letter to all owners of BWRs designed by the OEM, recommending a visual examination of the shroud circumferential welds [6]. The NRC responded to the early observations of cracking at several plants by issuing Generic Letter 94-03 to request that all licensees inspect their BWR core shrouds no later than the next scheduled refueling outage [7]. By the mid-1990s, the cracking had progressed to the point that several plants had installed tie rods to bolster the integrity of the core shroud, in the presence of circumferential cracks $[5,6]$. By the late 1990s, a significant percentage of operating BWRs had installed tie rods $[8,9]$. 
The tie rods rely on the integrity of the vertical welds of the shroud to perform their key function, but by 1993, cracking had been observed in the vertical welds, as well [9]. Multiple visual and ultrasonic examinations were conducted after this observation, and these cracks have been observed to progress slowly. Inspection and evaluation guidelines for the BWR core shroud were developed and documented in BWRVIP-76-A [10], which was submitted to the NRC for review, and Revision 1 received a safety evaluation in 2014 [11]. This supported the establishment of a re-inspection interval that can be up to ten years for these welds. To date, plants have been able to continue operation with the observed flaws, despite increases in the number and size of the flaws.

As these inspections progressed, the number of flaws observed has increased, and flaw dimensions have changed. Figure XI. illustrates the various types of cracking that have been observed [8]. Cracks oriented in the heat-affected zones along the welds have been commonly observed and were the primary concern related to core shroud integrity when SCC was first identified in the early 1990s. More recently, "off-axis" cracking (i.e., cracks that propagate approximately perpendicular to the associated weld, see Figure XI.) has become more of a concern as inspections have revealed changes over time to the off-axis cracks along with new or deeper than expected cracks. These changes observed during inspections could be due to improvements in inspection techniques and equipment, rather than additional crack growth or initiation [8]. Analyses of BWR core shrouds indicate that the off-axis flaws are likely to grow through-wall, but are not likely to grow into long cracks due to the stress distribution predicted around the weld [12]. These analytical results are consistent with the majority of the reported off-axis flaws. 


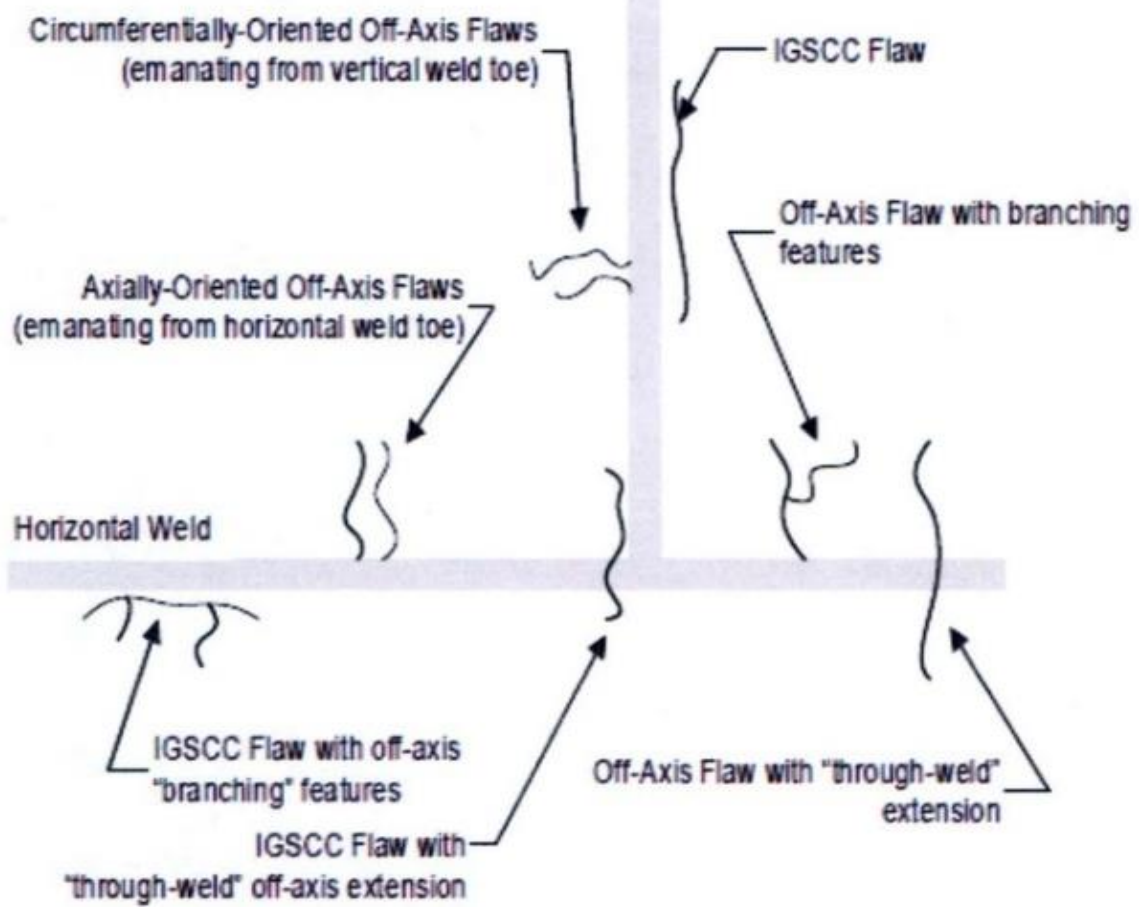

Figure XI.2. Locations and orientations of BWR shroud cracking [8].

Correlation studies have shown that off-axis cracking is most strongly related to plant design, neutron fluence, and shroud fabrication [8]. The location of most of the reported off-axis cracks has been the core shroud beltline region spanning beltline welds H3 and H6a (Figure XI.). Factors introduced during construction, such as cold work due to manipulation of the shroud during fit-up or local material changes due to the welding and subsequent removal of construction support and alignment lugs on the core shroud could have contributed to the cracking. The correlation with neutron fluence is driven by the location of the cracking in the beltline region welds (Figure XI.). Some of these correlated factors could be confounded with fabricator or design differences. The cause of this off-axis cracking remains under active investigation, but analytical results indicate that off-axis cracks are expected to remain short and do not have a significant impact on core shroud structural integrity [12]. To date, this has been confirmed by the results of field inspections. 
Many other BWR internal components have also shown evidence of environmental cracking; though the cracking in core shrouds is one of the most widespread [5]. The experience summarized above shows that stainless steel welds operating in BWR environments are highly susceptible to stress corrosion cracking. Neutron irradiation appears to increase this susceptibility, and this phenomenon has become known as "Irradiation Assisted Stress Corrosion Cracking (IASCC)". The experience of the BWR core shrouds provides evidence that much of the cracking initiated early in BWR plant life due to fabrication factors and the initial exposure to the oxidizing, Normal Water Chemistry (NWC) environment is important.

A reliable, predictive model is required for proactively resolving vessel and core internal material condition issues with generic, cost-effective strategies. A completely successful model must account for all of the phenomenological correlations that exist between IGSCC susceptibility and crack velocity and various environmental, electrochemical, and mechanical independent variables, as summarized above. To date, models have been developed that appear to account for the effects of electrochemical potential, loading waveform, and stress intensity on crack velocity, at least qualitatively, but only one, the Coupled Environment Fracture Model (described below), has succeeded in providing a comprehensive theoretical account of IGSCC in sensitized Type 304 SS. The empirical model developed by Ford and coworkers [13-18] perhaps came closest to this goal before the development of deterministic models. The Ford, et.al. model [13-18] does not account for the influence of the external environment on crack growth in an analytical (as opposed to empirical) manner. This latter aspect is particularly important, because the conductivity of the recirculating fluid in an operating BWR may vary significantly if upset chemistry conditions are experienced, resulting in enhanced crack growth rate. Extensive laboratory and in-plant studies indicate that impurity concentration (and hence conductivity) is one of the most important parameters in determining the rate of propagation of intergranular cracks [19-27]. In short, contemporary models for IGSCC, except the Coupled Environment Fracture Model (CEFM) [2835], fail to invoke charge conservation explicitly and hence fail to account for processes that occur in the environment external to the crack in an analytical manner. 


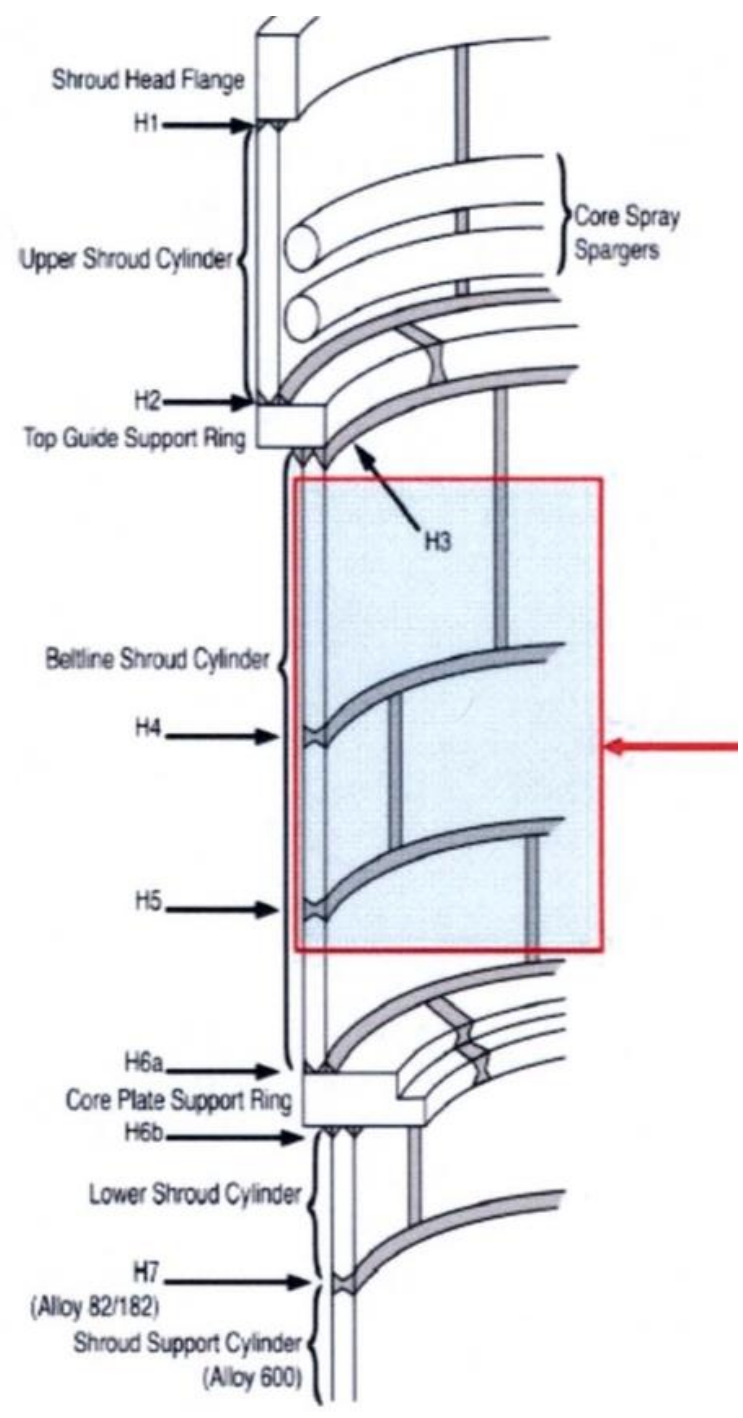

Figure XI.3. Location of BWR Shroud Welds and Off-axis Cracking. Nearly all off-axis cracking is between weld numbers $\mathrm{H} 3$ and H6a, as shown by the arrow [8].

The principal shortcoming in this regard is the failure to describe the cathodic reactions that must occur on the external surfaces to consume the current leaving the crack. Indeed, simple charge conservation considerations show that the internal and external environments must be strongly coupled, and this coupling is most likely responsible for the influence of solution conductivity, corrosion potential (ECP), and flow velocity on crack velocity. The CEFM was developed by Macdonald and co-workers to couple the internal and external environments in an analytical manner and to invoke charge conservation as a necessary condition for any deterministic 
treatment of crack growth [28-35]. Importantly, invocation of charge conservation leads to specification of the electrostatic potential in the solution at the crack mouth, which in turn allows calculation of the coupling current and hence the crack growth rate via Faraday's law. The main advantage of deterministic models for predicting corrosion damage in the coolant circuits of watercooled nuclear power reactors is that their output is constrained to physical reality by the natural laws and the impact of all independent variables can be captured if correctly identified and included in the model.

\section{XI.2. Deterministic modeling of environment, crack propagation, and component's lifetime.}

The typical deterministic modeling process for the evolution of SCC damage in the primary coolant circuit of a water-cooled nuclear power reactor involves three steps (Figure XI.):

1. Calculation of concentrations of reducing and oxidizing species in the reactor coolant based on known reactor operating parameters and the principles of water radiolysis and chemical kinetics;

2. Estimation of Electrochemical Corrosion Potential (ECP) from interfacial charge conservation based on calculated species concentrations, hydrodynamic conditions, and electrochemical parameters of the structural materials in reactor Heat Transport Circuit (HTC);

3. Estimation of Crack Growth Rates (CGR) and crack depth in HTC components based on known species concentrations, ECP, material degradation parameters, mechanical stress (stress intensity factor), and reactor operating history.
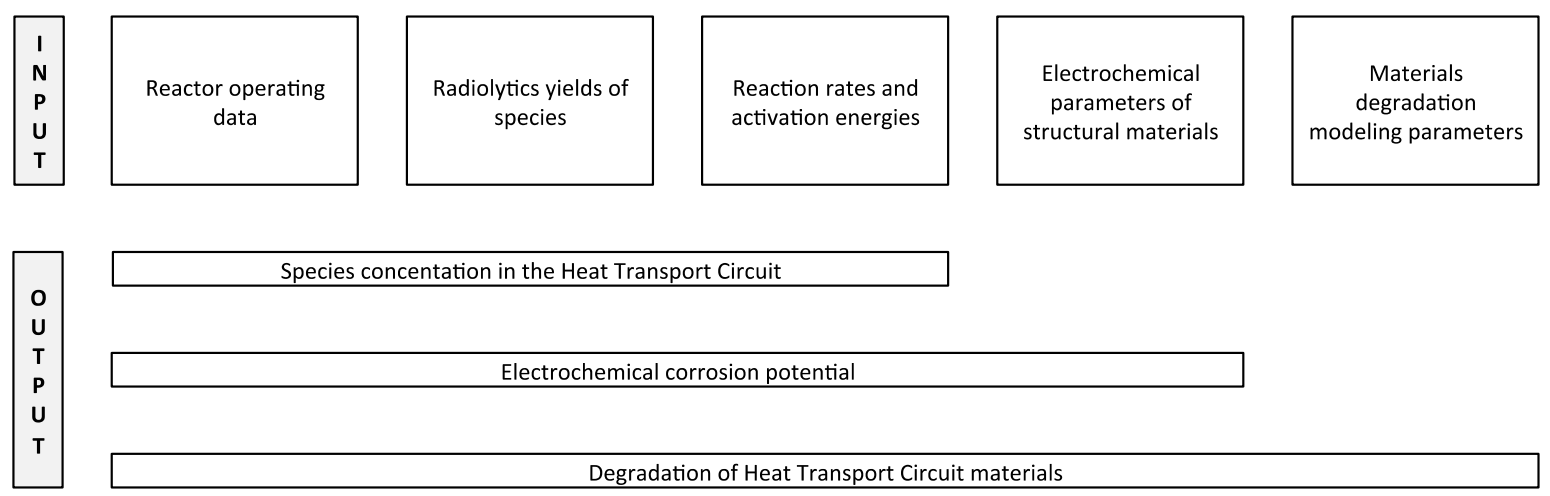

Species concentation in the Heat Transport Circuit

Electrochemical corrosion potential

Degradation of Heat Transport Circuit materials

Figure XI.4. Modeling of IGSCC in BWRs. 
As may be seen from Figure XI., such modeling requires the following five types of input data:

1. Reactor operating data that include neutron and gamma dose profiles, coolant temperatures and flow velocities, certain geometrical dimensions of the HTC "flow channels" and data on impurities and additions of chemicals, such as hydrogen over the reactor operating history. All these data usually are available from plant engineering personnel.

2. Radiolytic yields (or G-values) of species generated via neutron and gamma radiation of water at high temperatures. For gamma radiolysis, the G-values are well known at ambient temperature. Even for radiolysis with fast neutrons, the G-values at ambient temperature are known. The G-values for both gamma radiation and fast neutrons have been determined in recent years in experiments as a function of temperature by several groups, so that a moderately comprehensive database for these parameters is now available.

3. Reaction rate constants for water radiolysis reactions at reactor operating temperatures. Rate constants at ambient temperature are generally well known. In normal cases, rate constants at high temperature have been calculated from the rate constants at ambient temperature and the (often-assumed) activation energies. Exceptions to this rule are required for certain reactions. For diffusion-controlled reactions, activation energy of $12 \mathrm{~kJ} / \mathrm{mole}$ is assumed. For slower reactions, higher activation energies are commonly assumed, but are not always verified experimentally.

4. Electrochemical parameters of structural materials and major reducing and oxidizing species. Over the past thirty years, researchers and power plant operators alike have come to realize that corrosion damage in coolant circuits, including most, if not all, forms of general corrosion and localized corrosion, including pitting, stress corrosion cracking, corrosion fatigue, erosioncorrosion, hydrogen-induced cracking, and crevice corrosion, are primarily electrochemical phenomena that involve charge transfer at metal/coolant interfaces. As with any electrochemical charge transfer process, the rate at which charge transfer occurs (the current and hence the rate of accumulation of damage) depends upon the voltage that exists across the metal/environment interface. This potential difference, when measured under free corrosion conditions with respect to a suitable reference electrode, is referred to as the ECP. The corrosion potential, or the "ECP," as it is commonly known in the nuclear power technology arena, is accounted for by mixed potential theory and can be calculated using mixed potential 
models (MPM) [29,34], provided that the required thermodynamic, kinetic, and mass transport parameters are known.

5. Materials degradation modeling parameters, including mechanical, chemical, and electrochemical parameters required for the estimation of initiation and propagation of localized damage (cracks) in LWR components [35], including stress intensity factor $\left(K_{I}\right)$.

This paper compares deterministic and empirical predictions of IGSCC rates in sensitized austenitic stainless steel, in order to calculate the accumulated damage (crack depth versus time) in BWR in-vessel components and estimate component lifetimes for given operating conditions. Our extensive crack growth rate modeling work predicts that the crack growth rate should decrease with increasing crack depth; a relationship that is generally not gleaned from experimental data obtained from fracture mechanics specimens of fixed design. In this case, the electrochemical crack depth (shortest distance from the crack front to the external surface) is constant and independent of the mechanical crack depth. The impact that increasing electrochemical crack depth has on the CGR is a sensitive function of water conductivity, ECP, flow rate (especially for a high aspect ratio crack), and stress intensity factor. Accordingly, the predicted accumulated damage becomes a non-linear function of time and hence the operating history of the plant, which can be captured accurately only by deterministic models.

The accumulated damage is the expected crack depth, $L$, which is calculated on a component-by-component basis as a function of time, $T$, for an envisioned future operating protocol:

$$
L={ }_{0}^{T} \frac{L}{t} d t
$$

Crack growth rates at each state point $t$ of reactor operation, $\frac{L}{t}$, are calculated by the Coupled Environment Fracture Model [28-35] as:

$$
\frac{L}{t}=f[\operatorname{power}(t), \text { chemistry }(t), L(t)]
$$

The evolution of crack depth, $L$, over the anticipated service time of a component, $T$, is obtained by an accumulation of crack advances over $N$ periods of time (state point duration) $\Delta t_{1}, \ldots, \Delta t_{i}, \ldots \Delta t_{N}$. 


$$
\begin{aligned}
& L_{i}=L_{i 1}+\left(\frac{\partial L}{\partial t}\right)_{i} t_{i} \\
& T=\sum_{i=1}^{N} t_{i}
\end{aligned}
$$

The crack growth rate is assumed to be time-independent for each interval, $\Delta t_{i}$, in that it depends on the crack depth (due to dependence of $K_{I}$ on crack depth and because of changes in the current and potential distributions in the crack internal and external environments). The initial crack depth, $L_{0}$, corresponds to the depth of a pre-existing crack (as may have been detected during an inspection or assumed for a safety analysis scenario) or the length of the crevice at an initiation site.

A computer code REMAIN [36] has been developed for performing calculations for each step shown in Figure XI.. This code performs the calculations for each state point during BWR operation history of $40-60$ or more years within an hour on a desktop PC. Recently updated codes (PWR_MASTER and BWR_MASTER) with generic kinetics algorithm and advanced MPM and CEFM models for macOS and PC are described elsewhere [37-39]. The remaining service life of the core shroud was estimated using the BWR_MASTER code based on safety criteria (which require crack depth to be less than half the wall thickness) for the H6a weld.

\section{XI.3. Modeling of stress}

The BWR_MASTER code has no Finite Element Modeling (FEM) module for calculating stresses in in-vessel components. The code only calculates the stress intensity factor $\left(K_{I}\right.$ in $M P a \sqrt{m}$ ) of a pre-existing crack based on $K_{I}$ at the previous state point and an empirical

correction factor for $\mathrm{CT}$ specimens assuming constant stress but correcting for increased crack length:

$$
f_{C T}=\frac{(2+L)\left(0.886+4.64 L \quad 13.32 L^{2}+14.72 L^{3} \quad 5.6 L^{4}\right.}{(1 \quad L)^{3 / 2}}
$$

The values of $K_{I}$ at the beginning of the operating period are obtained from plant engineers. There are codes, such as ABAQUS [40], Grizzly [41,42], and several others capable of finite element method (FEM) analysis to obtain stresses and fracture mechanics parameters, such as $K_{I}$ 
in 3-D structures like the components of nuclear reactors. FEM is direct modeling based on first principles and no empirical correlations, such as in Eq. (XI.5), are required. The downside of the FEM modeling is the long input preparation and computation time that is frequently required in localized modeling of a component.

FEM modeling of the relationship between $K_{I}$ and $L$ for propagating crack in the H6a weld of a BWR core shroud (Figure XI.) was reported [43] as an attempt to estimate the remaining life of shroud. First, a 2-D model of the H6a weld (Figure XI.) for the weld's residual stresses was created.

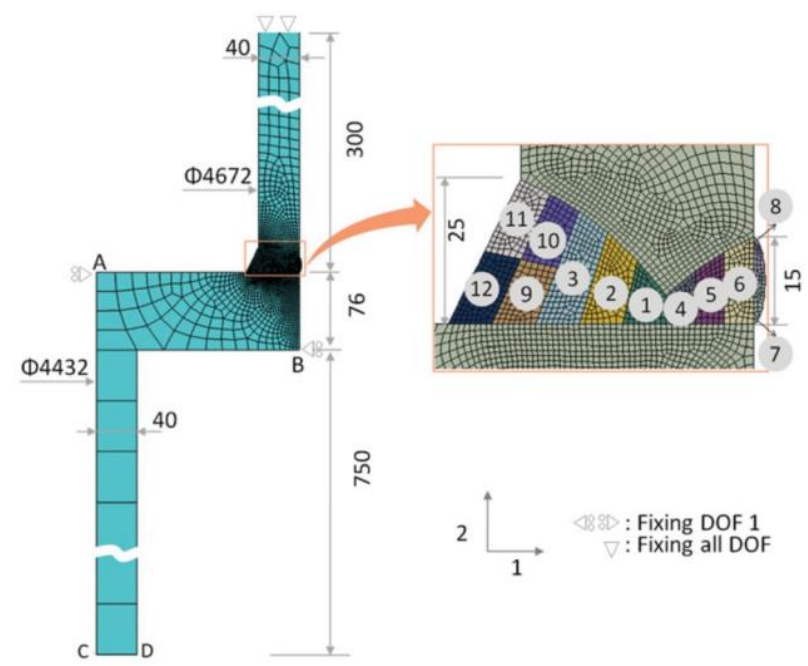

Figure XI.5. A Localized Model of the H6a Weld [43].

The redistribution of the stress field in the vicinity of the weld, which initially consists of welding residual stresses but is later affected by SCC propagation was simulated and, in combination with the J-integral method, the evolution of the stress intensity factor $K_{I}$ at the cracking tip was calculated.

\section{XI.4. Empirical modeling of crack propagation and component's lifetime}

The estimated SCC growth rate was calculated in [43] using empirical relations between $K_{I}$ and the SCC propagation rate $\frac{d L}{d t}$ that can be summarized as follows [44]: 


$$
\begin{aligned}
& \frac{d L}{d t}=2 \times 10{ }^{9} \mathrm{~mm} / \mathrm{s}, K_{I} \quad 6.7 M P a \sqrt{m} \\
& \frac{d L}{d t}=3.33 \times 10^{11} K_{I}^{2.161} \mathrm{~mm} / \mathrm{s}, 6.7 \mathrm{MPa} \sqrt{\mathrm{m}} \quad K_{I} \quad 59 M P a \sqrt{m} \\
& \frac{d L}{d t}=2 \times 01{ }^{7} \mathrm{~mm} / \mathrm{s}, K_{I} \quad 59 M P a \sqrt{m}
\end{aligned}
$$

The relations between the $K_{I}$ and SCC crack growth rate are obtained under normal water conditions at $288^{\circ} \mathrm{C}$ for $316 \mathrm{~L}$ stainless steel and are given by the Nuclear and Industrial Safety Agency in [44]. As suggested in [44], the SCC crack growth rate, while referring to "normal water" conditions, does not include the effect of various oxidizing conditions in the HTC regions under normal water chemistry on SCC and ignores SCC crack growth rate variations during temperature transients. There are several radiolytically produced reducing and oxidizing species, such as $\mathrm{H}_{2}, \mathrm{O}_{2}$, and $\mathrm{H}_{2} \mathrm{O}_{2}$, with concentrations varying significantly in the $\mathrm{HTC}$ regions and establishing various level of ECP and SCC crack growth rate. For example, concentrations of $\mathrm{H}_{2}$, $\mathrm{O}_{2}$, and $\mathrm{H}_{2} \mathrm{O}_{2}$, and resulting ECP and crack growth rate in core bypass and recirculation line vary significantly under normal water chemistry conditions. As a result, using the type correlations specified in [44] leads to large negative and positive biases, depending on the HTC region. The remaining service life of the core shroud was estimated based on safety criteria (requiring the crack depth to be less than half the wall thickness) for the H6a weld [43]. Of perhaps greater concern is that Equations (XI.6) to (XI.8) appear to have been established using crack growth rate data that are not purely "mechanical" in nature, but contain significant contributions from environmental effects. If so, the CGR data employed in $[43,44]$ inadvertently contain environmental effects.

\section{XI.5. Comparison of stress intensity factors}

According to the model for $K_{I}$ at the crack tip given in Equation (XI.5) and used by the BWR_MASTER code (Figure XI., solid bold line), there is a linear increase of $K_{I}$ from 28 $M P a \sqrt{m}$ to $62 M P a \sqrt{m}$ as the crack depth increases from $1 \mathrm{~mm}$ (assumed depth of a pre-existing crack) to $25 \mathrm{~mm}$. The variation of $K_{I}$ is within the allowed range for the CEFM, which is from 10 $\operatorname{MPa} \sqrt{m}$ to $80 \operatorname{MPa} \sqrt{m}$. 
In [43], an average J-integral from a FEM analysis was used to calculate $K_{I}$. The solid symbols in Figure XI. show the results of that analysis, which are fit to a polynomial shown by the black thin curve in that Figure. The $K_{I}$ increases with the cracking depth at the beginning stage and reaches a maximum value $(54.98 \mathrm{MPa} \sqrt{\mathrm{m}}$ ) at a cracking depth of $12.0 \mathrm{~mm}$. As the SCC continues to propagate through the wall, $K_{I}$ eventually decreases with crack depth, because of the stress relief that occurs due to the loss of constraint caused by the crack and the movement of the crack tip away from the region of maximum residual stress. When $K_{I}$ reaches zero, the SCC stops propagating, as $K_{I}$ halls below $K_{I S C C}$ [43].

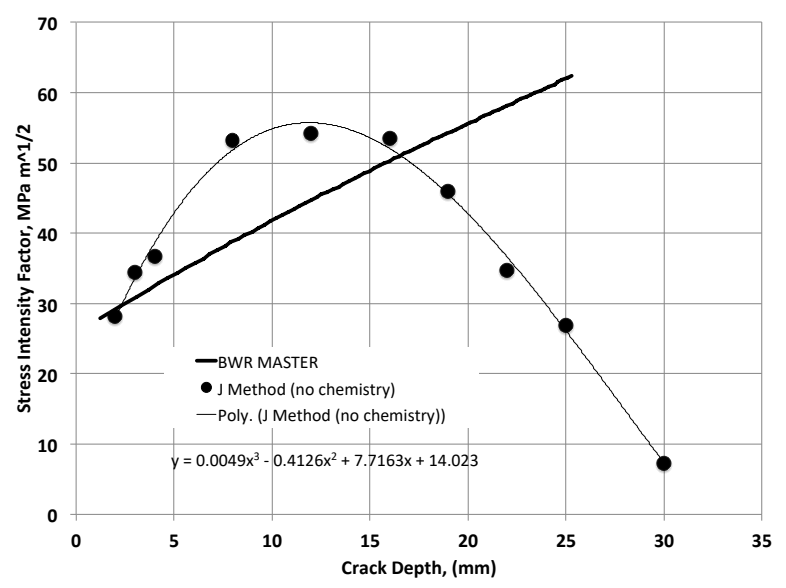

Figure XI.6. Stress intensity factor at the crack tip during crack propagation.

While the results of the $K_{I}$ calculations using the two approaches are drastically different, both of them are within the $10-60 M P a \sqrt{m}$ range. The effect of using the FEM based $K_{I}$ instead of the CT based $K_{I}$ on SCC crack growth rate and component lifetime in deterministic modeling using the BWR_MASTER code is explored in the next sections.

\section{XI.6. Comparison of crack growth rates}

Simulation of SCC crack propagation rate using the BWR MASTER code has been performed for two cases: (1) using the original relationship between $K_{I}$ and crack depth based on Equation (XI.5); and (2) using the FEM based relationship between $K_{I}$ and crack depth represented by a polynomial approximation of results from [43] shown as a solid line and a small dotted line respectively in Figure XI.. 


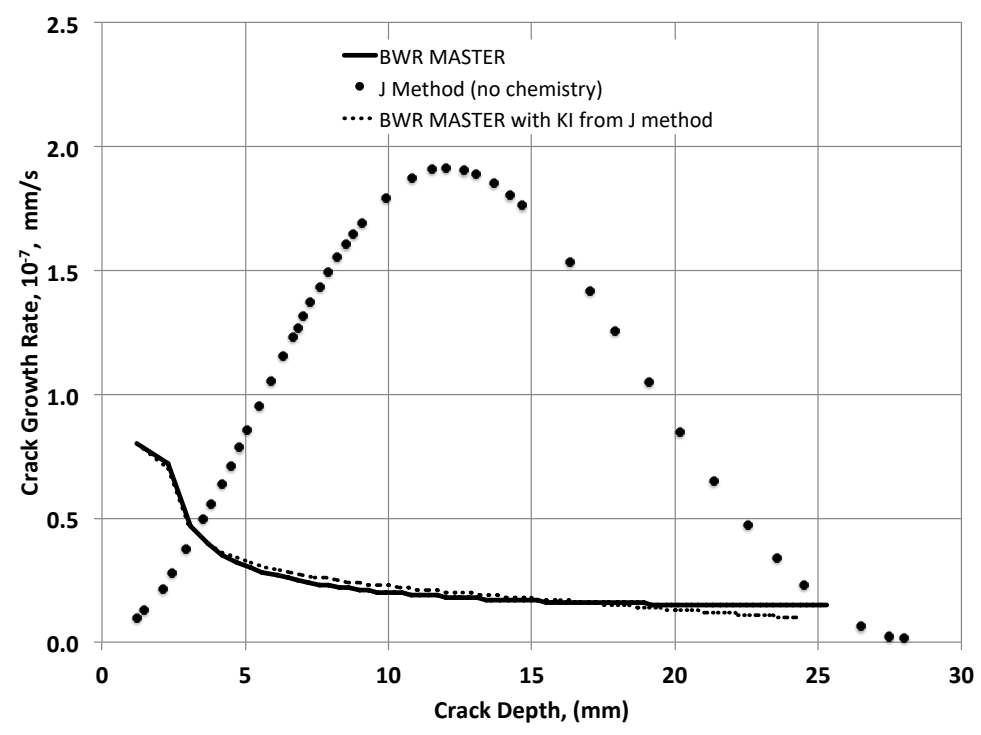

Figure XI.7. Crack growth rate during crack propagation through the H6a weld.

In both cases, a $1 \mathrm{~mm}$ deep preexisting crack was an input parameter, corresponding to field observed situation when multiple cracks from fabrication have been missed with existing detection techniques. While a crack initiation model has been developed, field data indicate that the majority of the observed cracks have been grown from pre-existing cracks created during components fabrication and installation process. The crack growth rate decreases from $8 \times 10^{-8}$ $\mathrm{mm} / \mathrm{s}$ to $1 \times 10^{-8} \mathrm{~mm} / \mathrm{s}$ with crack propagation, as predicted by CEFM. Substitution of the "simplified" relationship between $K_{I}$ and crack depth based on Equation (XI.5) (solid line in Figure XI.) with the "more accurate" FEM based relationship (small dots line in Figure XI.) did not result in significant changes in CEFM-predicted crack growth rates. This fact demonstrates the low sensitivity of crack growth rates to variations in $K_{I}$ in deterministic modeling based on the CEFM, because over the $K_{I}$ range of interest the crack growth rate is dominated by electrochemical factors (i.e., it is in the Stage II region of CGR vs $K_{I}$ ). These results agree well with the Artificial Neural Network based sensitivity analysis result for Type 304 SS [45], in which the $K_{I}$ comes out as the fourth most important parameter in determining the CGR after temperature, conductivity, and ECP, suggesting that the SCC in sensitized Type 304 SS in high temperature aqueous environments is primarily electrochemical in character. Such low sensitivity of crack growth rate to $K_{I}$ opens an opportunity to avoid the use of time-consuming FEM calculations and to use simplified approaches such as Equation (XI.5) instead. 
In the FEM analysis in [43], the crack was permitted to propagate by releasing nodes until the crack tip was no longer in tension, which occurred at a crack depth of $29.6 \mathrm{~mm}$ (indicated by the point where the crack growth rate shown by the bold dotted line in Figure XI. goes to zero). However, the SCC propagation rates are obtained using the relation between $K_{I}$ and the SCC propagation rate calculated using the empirically-derived Equations (XI.6)-(XI.8), which are continuously calculated during the simulation and the effect of environment (water chemistry) is ignored.

In BWR_MASTER, modeling crack propagation is driven, according to CEFM [36], by electrochemical dissolution of bare metal after rupture of surface oxide film caused by stress and by the injection of hydrogen into the matrix ahead of the crack tip. In order for such dissolution to occur, the internal environment of the crack and external (surface) environment must be coupled: i. e. an IR drop between the internal and external environments has to be low enough for current to flow from the crack tip to the external surface where it is annihilated by cathodic reactions, such as oxygen reduction and hydrogen evolution. In other words, crack growth rate should slow down as crack grows, because crack tip becomes more electrochemically isolated (IR drop increases) from the external surface, resulting in a lower voltage being available across the external interface to drive the cathodic reactions. Theory shows that the crack can grow no faster than the coupling current can be consumed on the external surfaces; that is, the crack is driven by a large cathode, which is an outcome of differential aeration hypothesis that is the basis of all localized corrosion processes, including SCC. The resulting dependence of SCC rate on crack depth predicted by BWR_MASTER is shown in Figure XI. as solid and small dots lines.

Parenthetically, it may be noticed that, when both BWR_MASTER and FM modeling are used to predict crack advance into the base alloy, BWR_MASTER predicts a higher CGR than does FEM initially (up to 5 years), because of the additional positive impact of oxidizing chemistry conditions in the former. Thus, such a comparison for higher crack depth is not appropriate: BWR_MASTER takes into account the decrease of the electrochemical activity at the crack tip (decrease of the CGR) as the crack grows in-depth, while the FEM model recognizes no dependence of CGR on crack depth and postulates a much stronger dependence on $K_{I}$ within the range $10-60 \mathrm{MPa} \sqrt{m}$ [i.e., Equation (XI.7)]. The practical implication of this difference is discussed below in the next section. 
The SCC propagation rate increase to a maximum value of $1.84 \times 10^{-7} \mathrm{~mm} / \mathrm{s}$ at a cracking depth of $9.0 \mathrm{~mm}$ and decreases to $2 \times 10^{-9} \mathrm{~mm} / \mathrm{s}$ at $26.5 \mathrm{~mm}$ and remains relatively constant to a depth of $27.4 \mathrm{~mm}$. The behavior of the FEM-based $K_{I}$ contradicts the CEFM-prediction that the CGR decreases as the crack depth increases, because the former is a purely mechanical model that does not consider electrochemistry, whereas the decrease of the crack growth rate with increasing crack length (for constant $K_{I}$ ) is an electrochemical phenomenon related to the IR potential drop down the crack brought on by the coupling current.

While the predictions of crack growth rate from these two methods are drastically different, the effect of using a FEM based $K_{I}$ instead of a CT based $K_{I}$ on a component's lifetime in deterministic modeling using the BWR_MASTER code and a comparison of these results with the results of empirical modeling [43] are explored in the next section.

\section{XI.7. Comparison of predicted component lifetime}

A component's lifetime is defined by a safety criterion, which limits crack penetration to half of the wall thickness. This criterion corresponds to a crack depth of $25 \mathrm{~mm}$ in the H6a BWR core shroud weld. There are two major differences between empirical approaches of estimating component's lifetime, as described in [43], and the deterministic approach implemented in the BWR_MASTER code:

1. Deterministic modeling using the BWR_MASTER code includes all three components that drive the SCC process, as shown in Figure XI.1: (1) environment (i.e., BWR heat transport coolant chemistry and electrochemistry), (2) stress (in a simplified way) and (3) material microstructure. Empirical modeling, as presented in [43], ignores the effect of the environment. This is problematic, as there are extensive plant, laboratory, and modeling evidence on the importance of water chemistry in SCC.

2. BWR_MASTER calculates an accumulated crack depth [i.e., the crack advancement process is modeled according to Equation (XI.1)] and BWR operating protocol, which includes periods of operating at full power and transients, such as startups and shutdowns. Operating protocol represents a stepwise function of time with reactor thermal power and the temperature is constant over time (a state point). Duration of a state point may be one hour or less during startup and shutdown to over a month-long during full-power operation. Simulating 60 years 
of operating history comprises 1,200 state points, in this case. The modeling presented in [43] assumes that the reactor operates at full power $100 \%$ of the time. This is another significant simplification as there are extensive plant and modeling evidence of the importance of operating transients on the progression of SCC.

A comparison of the component's lifetime predicted by [43] and by BWR_MASTER for a simplified case of operation at full power for $100 \%$ of the time is presented in Figure XI.. By ignoring the water chemistry (environmental) effect on SCC in [43] results in an estimated lifetime of about 9 years (bold dotted line in Figure XI.). BWR_MASTER predicts about 40 years of lifetime regardless of the type of model used for estimating $K_{I}$ used: a "simplified" one based on Equation (XI.5), which is shown as a solid line in Figure XI. or an "accurate" one based on FEM modeling and Equations (XI.6) - (XI.8), which is shown as a small dotted line in Figure XI.. Thus, in the case of the H6a weld, ignoring the effects of the chemistry of the BWR coolant and the electrochemistry at the metal/coolant interface on the SCC propagation rate leads to underestimating component's lifetime by a factor of four. The apparent paradox that a model that incorporates environmental effects (i.e., BWR_MASTER) should predict a longer life that one that explicitly does not (i.e., that described in [43]) needs to be resolved, but probably arises from the fact that the empirical model proposed in [43] may contain environmental effects inadvertently by being calibrated on SCC data rather than on data for purely mechanical cracking (creep) and because the model does not recognize a dependence of the CGR on crack length for constant $K_{I}$.

An assumption of full-power operation during $100 \%$ of the time is also unrealistic, and leads to significant underestimation of a component lifetime, thereby rendering the value of such predictions marginal for safety analysis. Modeling of SCC crack propagation, with power transients taken into account, indicates that a significant fraction of the SCC damage is accumulated during short transients. Additional modeling has been performed using BWR_MASTER to illustrate this claim. Reactor operation was modeled over 60+ years with 12 months of fuel cycles with refueling outages. Each outage includes 50 hours long shutdown, one month of standby at zero thermal power, and 50 hours long startup. Results of the accumulated crack depth are shown in Figure XI. as a solid gray line. The lifetime of the BWR core shroud under these conditions (i.e., time to produce a $25 \mathrm{~mm}$ crack) was estimated as 28 years. At the same time, ignoring transients in BWR_MASTER modeling results in ten years longer lifetime of about 40 years. These results indicate that including transients into [43] modeling would reduce 
the estimated component's lifetime also by a factor of $28 / 40=0.7$, i. e. from 9 years to 6.3 years. This estimation is four times lower than deterministic one of 28 years obtained from BWR_MASTER. Based on this analysis, it was concluded that typical SCC modeling, which ignores water chemistry and electrochemistry and does not take into account the actual operating history, would underestimate the component's lifetime by a factor of four. Again, this difference can be attributed to the much higher dependence of CGR on $K_{I}$ and the lack of dependence on crack length. Finally, modeling like that in Ref. [43] is controversial because, on the one hand, it overestimates SCC damage, even though it ignores water chemistry and electrochemistry, and on the other hand, it underestimates SCC damage by ignoring reactor operating transients during refueling outages.

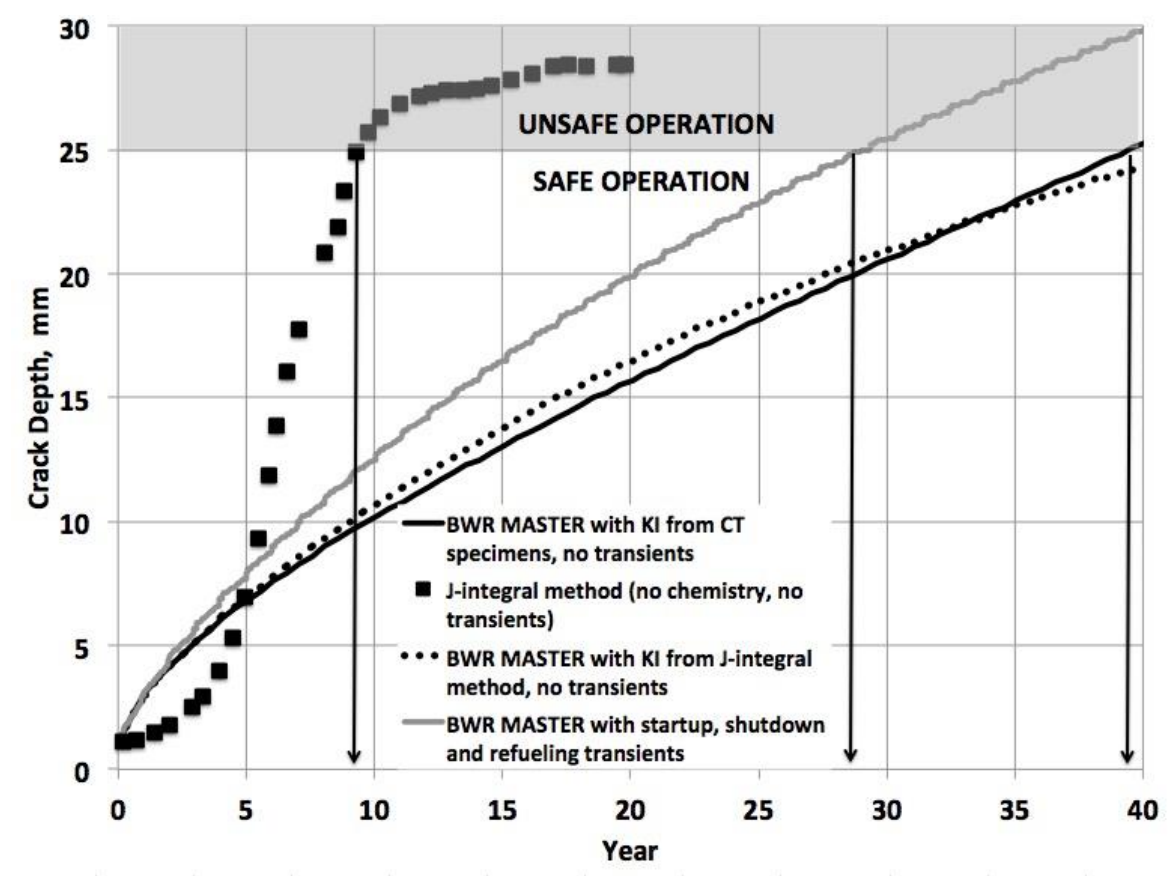

Figure XI.8. Predicted core shroud remaining lifetime based on crack propagation in H6a weld in the core shroud for BWR operating at full power throughout the calculation.

\section{XI.8. Summary and conclusions}

A comparison of deterministic and empirical predictions of IGSCC in sensitized austenitic stainless steel, in order to calculate the accumulated damage (crack depth versus time) in a BWR in-vessel component and estimate component's lifetime for given operating conditions is described. Counterintuitively, it was found that substitution of a "simplified" relationship between 
$K_{I}$ and crack depth based on Equation (XI.5) with a "more accurate" FEM based relationship, which gives significantly lower values of $K_{I}$ for higher crack depths, did not result in significant changes in the CEFM-predicted crack growth rates. This is due to the fact that the crack growth rate in the CEFM has a relatively low sensitivity to $K_{I}$. This low sensitivity of crack growth rate to $K_{I}$ opens an opportunity of avoiding the use of time-consuming FEM calculations and to use simplified models such as Equation (XI.5) instead. Based on the performed modeling, it was concluded that typical SCC modeling, which ignores water chemistry and electrochemistry and does not take into account the actual operating history, would underestimate the component's lifetime by a factor of 4. Finally, modeling like that in Ref. [43] is controversial because it overestimates SCC damage by using empirical fracture mechanical models that do not correspond to the actual mechanical condition of the core shroud. On the other hand, it underestimates SCC damage by ignoring water chemistry and reactor operating transients during refueling outages. Involving operating transients is particularly important, as, during start-up and shut-down, the change in the stress field around the crack increases the crack tip strain rate, which, as a consequence, increases the fracture frequency and the exposure of the bare metal to the environment, which results in a higher CGR.

\section{XI.9. References}

[1] Stress Corrosion Cracking in Light Water Reactors: Good Practices and Lessons Learned. Vienna: International Atomic Energy Agency, (2011).

[2] BWRVIP Vessel and Internals Project Update, Drew Odell, Exelon, BWRVIP Integration Committee Technical Chairman EPRI-NRC Technical Exchange Meetings, May 22-25, (2017).

[3] Status Report: Intergranular Stress Corrosion Cracking of BWR Core Shrouds and Other Internal Components, NUREG-1544, (1996).

[4] J.K. McKinley, W.H. Bamford, A.M. Ruminski, T.L. Meikle, and J.L. McFadden, Comparison of Boiling Water Reactor and Pressurized Water Reactor Experience with Cracking of Austenitic Stainless Steel, Enclosure 3 to MRP 2018-011, Westinghouse Electric Company, (2018). 
[5] A. Roth, Review of Intergranular Cracking in Austenitic Stainless Steel Components of BWR RPV-Internals, Fontevraud 8, Avignon, France, September 15-18, (2014).

[6] Nuclear Regulatory Commission Document, NUREG-1544, Status Report: Intergranular Stress Corrosion Cracking of BWR Core Shrouds and Other Internal Components, March (1996).

[7] Nuclear Regulatory Commission Generic Letter 94-03, Intergranular Stress Corrosion Cracking of Core Shrouds in Boiling Water Reactors, July 25, (1994).

[8] W. Lunceford, N. Palm, and D. Sommerville, BWR Core Shroud Off-Axis Cracking Inspection Experience, International Light Water Reactor Materials Reliability Conference and Exhibition, EPRI, Chicago, Illinois, August 1-4, (2016).

[9] BWR Vessel and Internals Project, BWR Core Shroud Repair Design Criteria, Rev. 2 (BWRVIP-02), EPRI, Palo Alto, CA: 1999, 112642.

[10] BWR Vessel and Internals Project: BWR Core Shroud Inspection and Flaw Evaluation Guidelines (BWRVIP-76, Revision 1-A), EPRI, Palo Alto, CA: (2015), 3002005566.

[11] Nuclear Regulatory Commission Final Safety Evaluation, Final Safety Evaluations of the Boiling Water Reactor Vessel and Internals Project 76, Rev. 1 Topical Report, 'Boiling Water Reactor Core Shroud Inspection and Flaw Evaluation Guidelines, November 12, (2014) (ML14266A227).

[12] BWRVIP-311: BWR Vessel and Internals Project: Core Shroud Weld Off-Axis Crack Growth Assessment. EPRI, Palo Alto, CA: (2017), 3002010682.

[13] F.P. Ford and M.J. Silverman, Corrosion, 36, 597-603 (1980).

[14] F.P. Ford, Mechanisms of Environmental Cracking in Systems Peculiar to the Power Generation Industry, EPRI NP-2589, Final Report, Electric Power Research Institute, Palo Alto, CA (1982).

[15] F.P. Ford and P.L. Andresen, Proc. 3rd Int. Symp. Environ. Degrad. Mat. Nucl. Power Syst.-Water Reactors, pp. 2789. Metallurgical Soc., Warrendale, PA (1988). 
[16] F.P. Ford and P.L. Andresen, The Theoretical Prediction of the Effect of System Variables on the Cracking of Stainless Steels and Its Use in Design, Paper No. 83, Corrosion/87, NACE, Houston, TX (1987).

[17] F.P. Ford, Mechanisms of Environmental Cracking of Systems Peculiar to the Power Generation Industry, NP-2589, Final Report, Electric Power Research Institute, Palo Alto, CA (1987).

[18] F.P. Ford, D.F. Taylor, P.L. Andresen, and R.G. Ballinger, Corrosion-Assisted Cracking of Stainless and Low-Alloy Steels in LWR Environments, NP-5064M, Final Report, Electric Power Research Institute, Palo Alto, CA (1987).

[19] P.L. Andresen and M.E. Indig, Corrosion, 38, 531 (1981).

[20] A, Molanderand, B. Rosberg, Proc. 3rd Int. Symp. Environ. Degrad. Mat. Nucl. Power Systems-Water Reactors, P. 333. The Metallurgical Soc., Warrendale, PA (1988).

[21] W.E. Ruther, W.K. Soppet, G. Ayrault, and T.F. Kassner, Corrosion, 40, 518-527 (1984).

[22] L.G. Ljungberg and D. Cubicciotti, Corrosion, 41, 290-295 (1985).

[23] L.G. Ljungberg, D. Cubicciotti, and M. Trolle, Corrosion, 44, 66-72 (1988).

[24] L.G. Ljungberg, C. Jannson, and D. Cubicciotti, Proc. 3rdlnt. Syrup. Environ. Degrad. Mat. Nucl. Power Syst.--Water Reactors, P. 315. The Metallurgical Soc., Warrendale, PA (1988).

[25] L. Ljungberg and E. Hallden, BWR Water Chemistry Impurity Studies: Literature Review Of Effects On Stress Corrosion Cracking, Epri Np-3663, Electric Power Research Institute, Palo Alto, CA (1984).

[26] J.Y. Park, W.E. Ruther, T.F. Kassner, and W.J. Shack, Trans. ASME 108, $20-24$ (1986).

[27] A.K. Eghan, Z. Szklarska-Smialowska, and D.D. Macdonald, Corrosion, 41, 474-484 (1985).

[28] D.D. Macdonald et al., Estimation of Corrosion Potentials in the Heat Transport Circuit of LWRs, Proc. Int. Conf. Chemistry in Water Reactors: Operating Experience \& New Developments, Nice, France, French Nuclear Energy Society, April 24-27 (1994).

[29] C.P. Ruiz, et al., Modeling Hydrogen Water Chemistry for BWR Applications, EPRI NP6386, Electric Power Research Institute, June (1989). 
[30] D.D. Macdonald and M. Urquidi-Macdonald, Corrosion, 46, 380-390 (1990).

[31] D.D. Macdonald, J. Mankowski, M. Karaminezhaad-Ranjbar, and Y.H. Hu, Corrosion, 44, 186-192 (1988).

[32] D.D. Macdonald, J. Electrochem. Soc, 139, 3434-3449 (1992).

[33] D.D. Macdonald, Corrosion, 48, 194-205 (1992).

[34] D.D. Macdonald, A.C. Scott, and P.R. Wentrcek, J. Electrochem. Soc., 126, 908-911 (1979).

[35] D.D. Macdonald and M. Urquidi-Macdonald, Corros. Sci., 32, 51-81 (1991).

[36] I. Balachov, D.D. Macdonald, B. Stellwag, N. Henzel, and R. Kilian, Prediction of Materials Damage History from Stress Corrosion Cracking in Boiling Water Reactors. Proc. ASMEIJSME Joint Press. Vess. Piping Conf., San Diego, CA, 376, 101-109 (July 26-30, 1998).

[37] B. Fekete, J. Ai, J. Yang, J. Han, W. Maeng, and D.D. Macdonald, Theor. Appl. Fract. Mec., 95, 233-241 (2018).

[38] B. Fekete, I. Balachov, and D.D. Macdonald, Deterministic Modeling of Stress Corrosion Cracking in Light Water Nuclear Reactors. Proc. $21^{\text {st }}$ Int. Conf. on Water Chem. in Nucl. React. Syst., San Francisco, CA (2018).

[39] I. Balachov, B. Fekete, and D.D. Macdonald, Parameter Sensitivity Analysis on Deterministic Models of Stress Corrosion Cracking in Light Water Nuclear Reactors. Proc. $21^{\text {st }}$ Int. Conf. on Water Chem. in Nucl. React. Syst., San Francisco, CA (2018).

[40] ABAQUS User Manual Version 6.9. Pennsylvania, PA: Karlsson \& Sorensen, Incorporated; (2009).

[41] B. Spencer, M. Backman, P. Chakraborty, and W. Hoffman, Reactor pressure vessel fracture analysis capabilities in Grizzly, INL/EXT-15-34736, Idaho National Laboratory, Idaho Falls, ID, Mar. (2015).

[42] B. Spencer, W. Hoffman, and M. Backman, Modular system for probabilistic fracture mechanics analysis of embrittled reactor pressure vessels in the Grizzly Code, Nucl. Eng. Des., 341, 25-37 (2019). 
[43] Y. Li, S. Lu, D. Li, and Y. Kaji, J. Nucl. Sci. Technol., 52, 96-103 (2015).

[44] Nuclear and Industrial Safety Agency. Report on Structural Integrity Evaluation for Core Shroud and PLR Piping. Tokyo (Japan): NISA; (2004).

[45] J. Shi, J. Wang, and D.D. Macdonald, Corros. Sci., 89, 69-80 (2014).

\section{Predicting transients in chemistry and crack growth rate in BWR start- ups.}

\section{XII.1. Introduction}

It has long been suspected that the accumulation of IGSCC in weld-sensitized, Type 304 SS in BWR primary coolant circuits is enhanced during reactor transients, such as shut-downs and start-ups and during other power excursions. For example, it has been known for several decades that the IGSCC crack growth rate for a constant stress intensity factor $\left(K_{I}\right)$ and invariant coolant chemistry (pure water), passes through a maximum as a function of temperature at about $150{ }^{\circ} \mathrm{C}$ [1]. This maximum arises because of the competing effects of increasing temperature in enhancing activation of the crack tip strain rate, and hence in enhancing the frequency of microfracture events at the crack tip, and in inducing a decrease of the electrochemical corrosion potential (ECP), noting that the CGR is exponentially-dependent on the ECP [2]. However, experiment and theory [3,4] demonstrate that IGSCC in sensitized Type 304 SS is primarily an electrochemical phenomenon, with the CGR being a function of the ECP, temperature $(T), K_{I}$, high-temperature conductivity $\left(\kappa^{T}\right)$, and degree of sensitization (DoS) of the steel. Artificial Neural Network (ANN) analysis [3] of a large body of laboratory and field data reveal that the dependencies lie in the order ECP >> $T$ $>\kappa^{T} \approx \operatorname{DoS}>K_{I}$, demonstrating that IGSCC is primarily an electrochemical phenomenon, as noted above. Thus, in actual reactors, transients, other than that in temperature, can significantly contribute to the accumulation of IGSCC damage (crack length), including transients in coolant conductivity $\left(\kappa^{T}\right)$, due to "hide-out" and "hide-out return" of electrolytes, such as $\mathrm{NaCl}, \mathrm{Na}_{2} \mathrm{SO}_{4}$, etc, and in the ECP.

In this Research Brief, we illustrate the ability of BWR_Master to predict transients in IGSCC upon start-up of a BWR in response to transients in temperature, $\mathrm{ECP}$, and chloride $(\mathrm{NaCl})$ concentration, and hence in conductivity. BWR_Master is the latest code developed by the authors 
for modeling stress corrosion cracking in BWRs under any set of operating and coolant conditions. The code, which was developed with funding from DOE-NEUP over the past three years [5], is the latest in a series of codes beginning with DAMAGE PREDICTOR [6-8] in the 1990s and transitioning through ALERT [9] (2000s) and FOCUS [10] (2010s). Each generation contained improved modules for describing the radiolysis of water and for calculating the ECP, crack growth rate, and accumulated damage (crack length vs. operating time). Major advances were made in reducing the execution time in each successive code.

\section{XII.2. Coolant chemistry and IGSCC damage transients during BWR start-up}

Typical transients in chloride concentration and conductivity measured in a BWR during start-up are shown in Figure XII.1 [11]. The close correspondence between the transients in these two parameters argues that the conductivity is dominated by $\mathrm{Cl}^{-}$and the assumed counter ion $\left(\mathrm{Na}^{+}\right)$, a conclusion that is largely accepted in the industry.

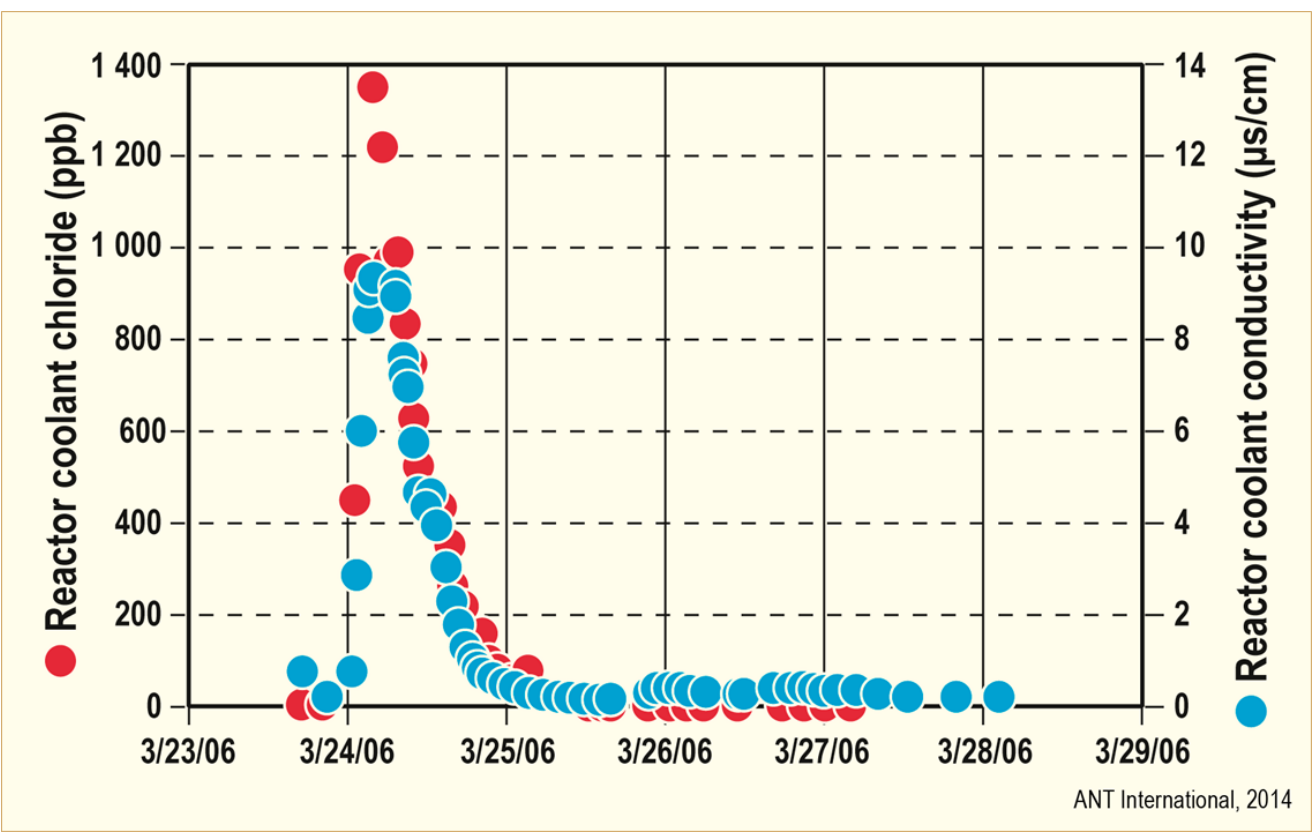

Figure XII.1. Typical transients in chloride concentration and conductivity in the start-up of a BWR [11]. 
However, transients in other ionic species also occur, as illustrated in Figure XII.2, which shows a transient in sulfate concentration. The reader will note that there is a large difference between $\left[\mathrm{Cl}^{-}\right]$and $\left[\mathrm{SO}_{4}{ }^{2-}\right]$ in Figures XII.1 and 2, with this difference accounting for the relatively higher importance of chloride transients over sulfate transients. In a private communication, Dr. Samson Hettiarachchi [11] states that he has seen sulfate transients as high as $60 \mathrm{ppb}$ and occasional transients in $\mathrm{Mg}^{2+}$ and $\mathrm{Ca}^{2+}$. It is also evident that the release of ionic species also depends on the Type of clean-up systems the plants employ. Accordingly, there is no one set of numbers for all plants, but plants that have better clean-up systems and higher clean-up capacity appear to have less chlorides and sulfates. Because each plant in the fleet is more-or-less unique, it is difficult to predict $a b$ initio the transients in the concentrations of various ionic species and hence predict the transient in conductivity. At present, it is recommended that these transients be constructed from plant conductivity vs. temperature data, if available. Although conductivity is an important water chemistry parameter, it is worth noting that it ranks third behind ECP and temperature in terms of impact on CGR in sensitized Type 304 SS in BWRs.

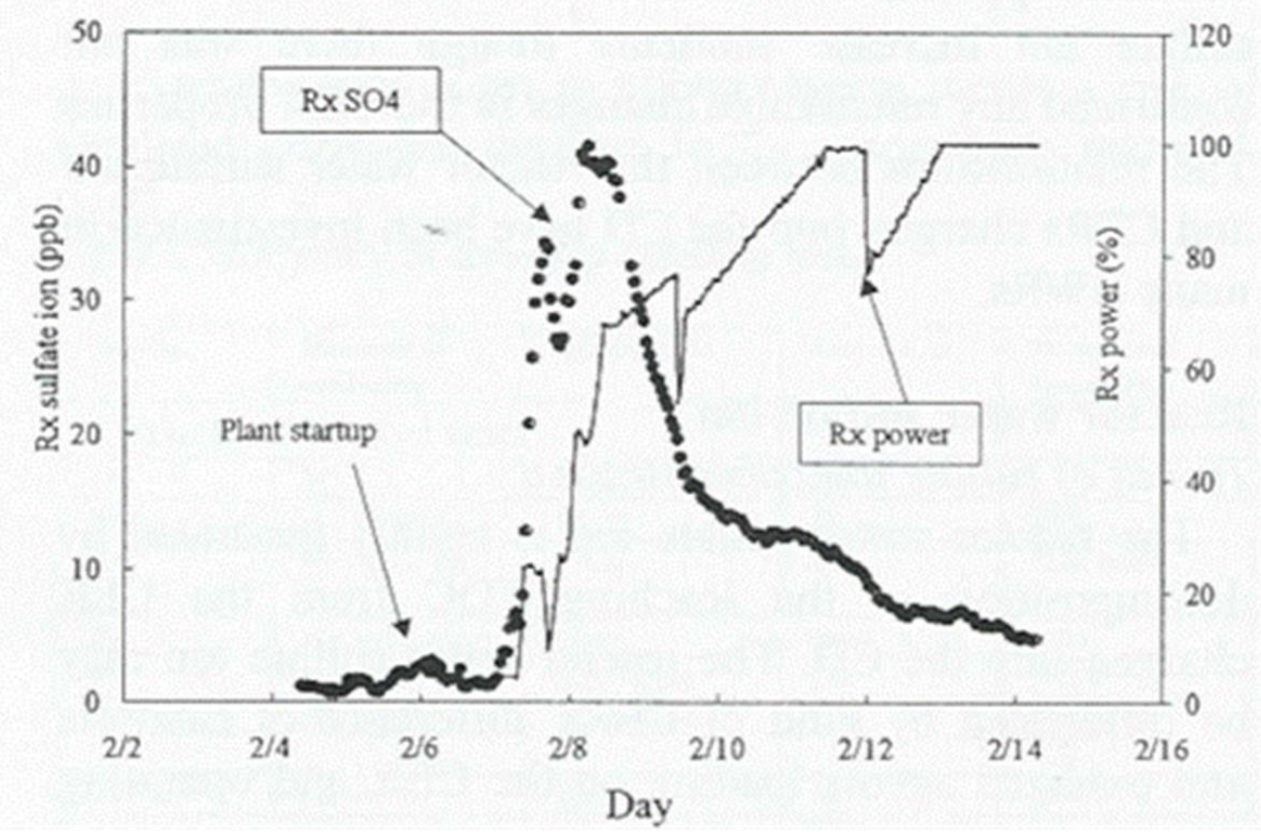

Figure XII.2. Comparison of transients in coolant sulfate concentration and reactor power for the start-up of a BWR [11]. 
The example given in Figure XII.2 indicates that the maximum in the chloride concentration due to "hide-out return" occurs at a power level of about $50 \%$. The cause of this phenomenon is often attributed to the segregation of ionic species into crevices during cool-down, with the species subsequently being released upon heat-up during the start-up of the reactor. Segregation of anionic species into active crevices is predicted and explained by the Differential Aeration Hypothesis (DAH) and is driven by the positive coupling current that exits the mouth of an active crevice and that is annihilated by redox reactions [e.g., the hydrogen electrode reaction (HER) and the oxygen electrode reaction (OER)] occurring on the surface external to the crack. However, the same theory predicts that cations (e.g., $\mathrm{Mg}^{2+}, \mathrm{Ca}^{2+}$ ) are inhibited from segregating into the crevices upon shutdown so that there are no cations to be released upon start-up in contradiction to the observed transients in $\mathrm{Mg}^{2+}$ and $\mathrm{Ca}^{2+}$ noted above. As an alternative explanation, it is sometimes assumed that hide-out occurs within crevices that are experiencing heat transfer. This issue has been examined at great length by Abela, et al. [12,13], with respect to crevices between Alloy 600 steam generator tubes and carbon steel tube sheets in PWRs. In that case, if heat transfer is from the tube toward the solution, the crevice exhibits polarity reversal, so that the cathode exists within the crevice and the anode exists on the external surfaces, with a positive coupling current flowing from the latter to the former. In this case, positive ions (e.g., $\mathrm{Cu}^{+}$) are transported into the magnetite-filled crevice leading to the accelerated corrosion of the carbon steel and, ultimately, to denting corrosion. It is not obvious how these phenomena lead to reversible hide-out/hide-out return in BWRs so that the fundamental mechanisms hide-out/hideout return remain controversial.

Under hydrogen water chemistry (HWC) operating conditions, several scenarios exist for adding hydrogen to the reactor feedwater, which can have a significant impact on ECP. Thus, the design, full-power, operating hydrogen concentration can be established soon after the initiation of start-up, when the steam jet air ejectors come into operation, or hydrogen may be added in stages at specified power levels (see Figure XII.3 below). 


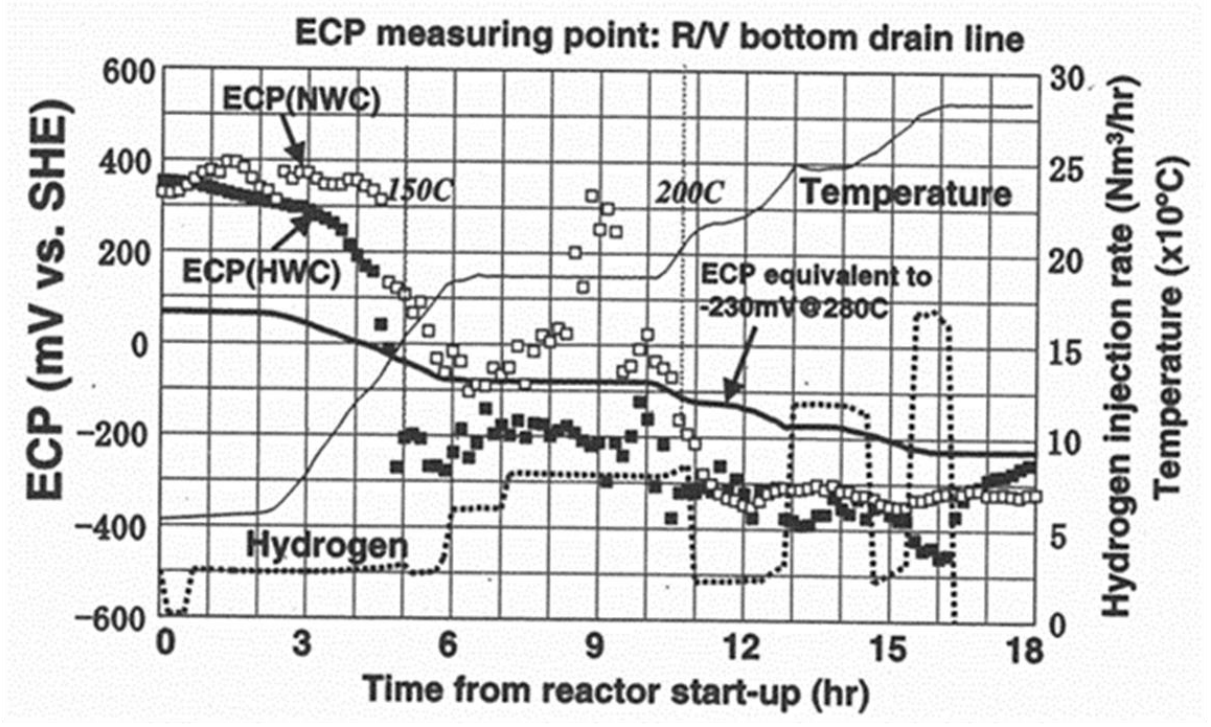

Figure XII.3. Transients in ECP, temperature, and $\left[\mathrm{H}_{2}\right]$ upon the start-up of a BWR on HWC. Also shown are ECP data for start-up under normal water chemistry (NWC). The ECP data were measured at the R/V bottom drain line [11].

Thus, Dr. Samson Hettiarachchi [11], who directed the application of HWC in the field for GE Nuclear, states: "Different plants add $\mathrm{H}_{2}$ at different levels of power. We have advised the plants to bring $\mathrm{H}_{2}$ on ASAP, but for a variety of reasons that are not always clear, some plants add $\mathrm{H}_{2}$ at $<10 \%$ power some add $\mathrm{H}_{2}$ at $25-50 \%$ power. This is their prerogative. In any event, plants cannot add $\mathrm{H}_{2}$ until the steam jet air ejectors come into operation to remove non-condensable gases, which occurs at a power level of > 5\%, typically. Plants have performed better in 2013 compared to 2010 because of injecting $\mathrm{H}_{2}$ at lower power". An example of the transient in ECP is shown in Figure XII.3.

Both Figures XII.2 and XII.3 contain data from Japanese plants. As noted by Dr. Hettiarachchi [11], there are two issues and challenges in measuring ECP during start-up. Firstly, as the temperature changes during start-up, the reference potential also changes, and it is not known how well any temperature corrections have been made. Secondly, it is not clear how quickly the reference potential stabilizes when the temperature changes from one value to another. He is aware of only one US plant that tried measuring ECP during power ascension. Apparently, that did not work well, so there are no data available on this issue, to the best of his knowledge. 
In illustrating the capabilities of the BWR_Master code, we have chosen to model cracking in the HAZ of the H6a weld in the core shroud of a GE Model 6 BWR. In this case, transients in several variables are envisioned to occur simultaneously, as is known to occur in operating reactors.

\section{XII.3. Simulation of BWR transients}

In the simulation described below, we combine simultaneous transients in $T$, [ $\left.\mathrm{H}_{2}\right],[\mathrm{NaOH}]$, $[\mathrm{HCl}]$ (i.e., $[\mathrm{NaCl}]$ ), and flow velocity $\left(V_{f}\right)$ to predict transients in the high temperature conductivity $\left(\kappa^{T}\right)$, ECP, CGR, and crack length $(L)$, in order to describe the evolution of damage due to IGSCC in the HAZ of the H6a weld in the core shroud. The assumed transients in $T$, $\left[\mathrm{H}_{2}\right]$, [NaOH], $\mathrm{HCl}]$, and $V_{f}$ is presented in Figure XII.4. While these transients were not taken from an actual reactor during start-up, we consider them to be reasonably realistic, if somewhat idealized. The flow velocity and $\left[\mathrm{H}_{2}\right]$ added to the feedwater are assumed to scale linearly with reactor power, which in turn was assumed to scale linearly with time (note that a log scale is employed on the vertical axis to accommodate the different scales). Also note that at full power, the temperature and flow velocity at the $\mathrm{H} 6 \mathrm{a}$ weld location is $288^{\circ} \mathrm{C}$ and $28.2 \mathrm{~cm} / \mathrm{s}$, respectively.

From Figure XII.5, it is evident that the high-temperature conductivity $\left(\kappa^{T}\right)$ passes through a maximum at $230{ }^{\circ} \mathrm{C}$, corresponding to $80 \%$ power or at about 14 hours into the start-up. This may be compared with the transients $[\mathrm{HCl}]$ and $[\mathrm{NaOH}]$ plotted in Figure XII.4, where the maxima occur at $50 \%$ power or 10 hours into the start-up. This shift in the maximum results from the positive effect of temperature on the ionic conductivities, which are superimposed upon the change in $\kappa^{T}$ due to the changes in concentrations alone. Note that the $\mathrm{NaOH}$ and $\mathrm{HCl}$ concentrations were chosen so that their molalities are equal, and hence, the $\mathrm{pH}$ is that of neutral water. This was done, so that the only electrolyte present is $\mathrm{NaCl}$ and that no transient in $\mathrm{pH}$ existed. However, BWR_MASTER is perfectly capable of handling transients in $\mathrm{pH}$, as the need arises. A transient in $\mathrm{pH}$ would affect not only the transient in $\kappa^{T}$ but also the transients in the ECP and CGR, because both the ECP and CGR depend upon the activity of hydrogen ion (i.e., $\mathrm{pH}$ ). These dependencies are contained in the rate expressions for the various reactions that determine the partial anodic and cathodic reactions on the steel surface $[1,6-8]$. 


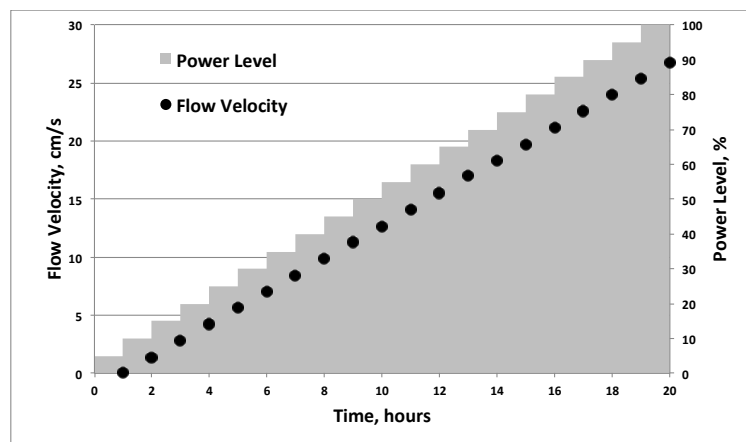

(a)

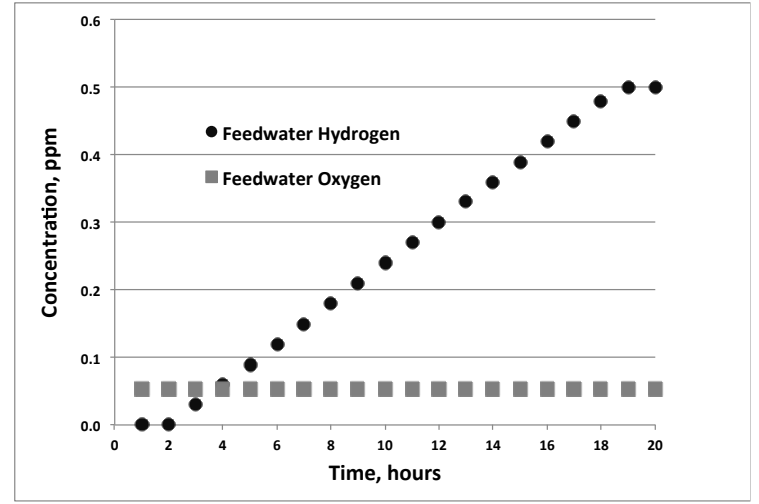

(b)

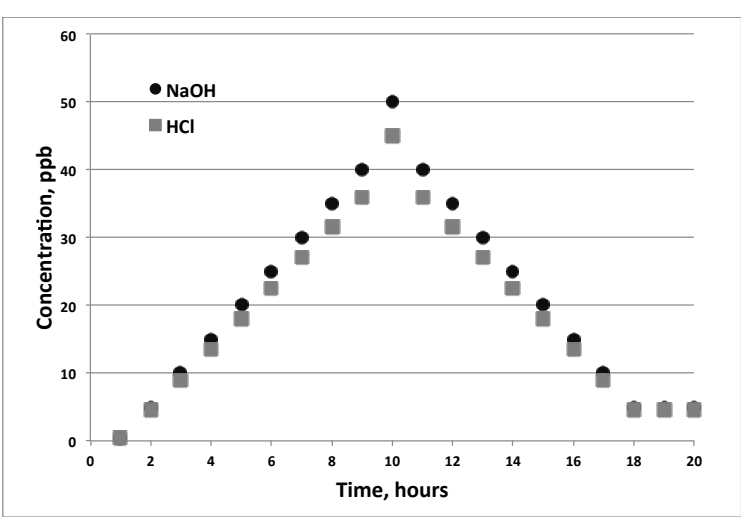

(c)

Figure XII.4. Transients in power and flow velocity (a), concentration of Hydrogen and Oxygen in Feedwater (b), and $[\mathrm{NaOH}],[\mathrm{HCl}]$ (c) during an idealized start of a BWR operating on HWC.

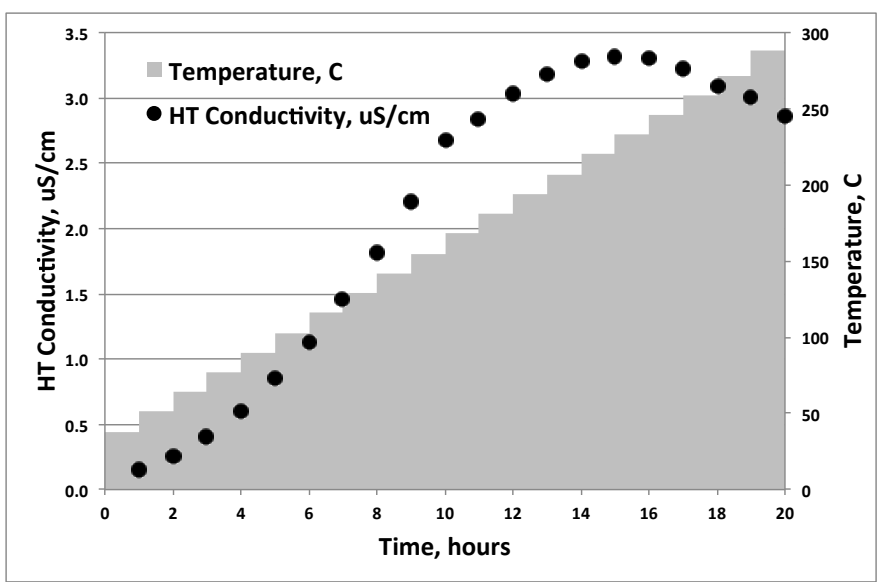

Figure XII.5. Time dependence of water temperature and water conductivity at H6a Core Shroud weld location during reactor startup under combined transient conditions. 
The predicted transients in ECP and CGR are plotted in Figure XII.6. Thus, the ECP is predicted to decrease monotonically with increasing temperature, which is the result of the dominating influence of temperature, as is well-known from fundamental studies. On the other hand, the CGR is predicted to pass through a clearly-defined maximum at about $150{ }^{\circ} \mathrm{C}$, as found experimentally [1], or at about $50 \%$ through the start-up. This primarily reflects the competing effects of increasing temperature in enhancing activation of the crack tip strain rate, and hence in enhancing the frequency of microfracture events at the crack tip, and in inducing a decrease of the electrochemical corrosion potential (ECP), noting that the CGR is exponentially-dependent on the ECP [2], as noted previously.

Note that the ECP calculated for the H6a weld location $\left(0.07 \mathrm{~V}_{\text {she }}\right)$ is considerably more positive than that reported in Figure XII. 3 at the R/V bottom drain line location ( -0.4 to $\left.-0.5 \mathrm{~V}_{\text {she }}\right)$. This demonstrates that for the measured ECP to be a useful parameter, it must be measured at the specific location of the crack, again noting that the CGR is a very strong (exponential) function of the ECP. Thus, if the measured ECP at the R/V bottom drain line was taken as being representative of that at the H6a weld location, no IGSCC could occur, because the ECP is more negative than the critical potential of $-0.23 \mathrm{~V}_{\text {she }}[1,6-8,15]$. Importantly, the ECP can only be measured at a few locations within the reactor vessel, e.g., at the bottom drain line or within instrumentation tubes, specifically in a local power range monitor (LPRM) housing, which is a stainless-steel tube that normally contains neutron detectors and is in the fuel channel bypass region in the BWR core [15]. Most of these locations are not particularly relevant to ascertaining the accumulation of IGSCC damage in critical components. Accordingly, an alternative to direct measurement must be employed to analyze the problem of cracking in BWR internals fully. We posit that BWR_MASTER provides that capability. 


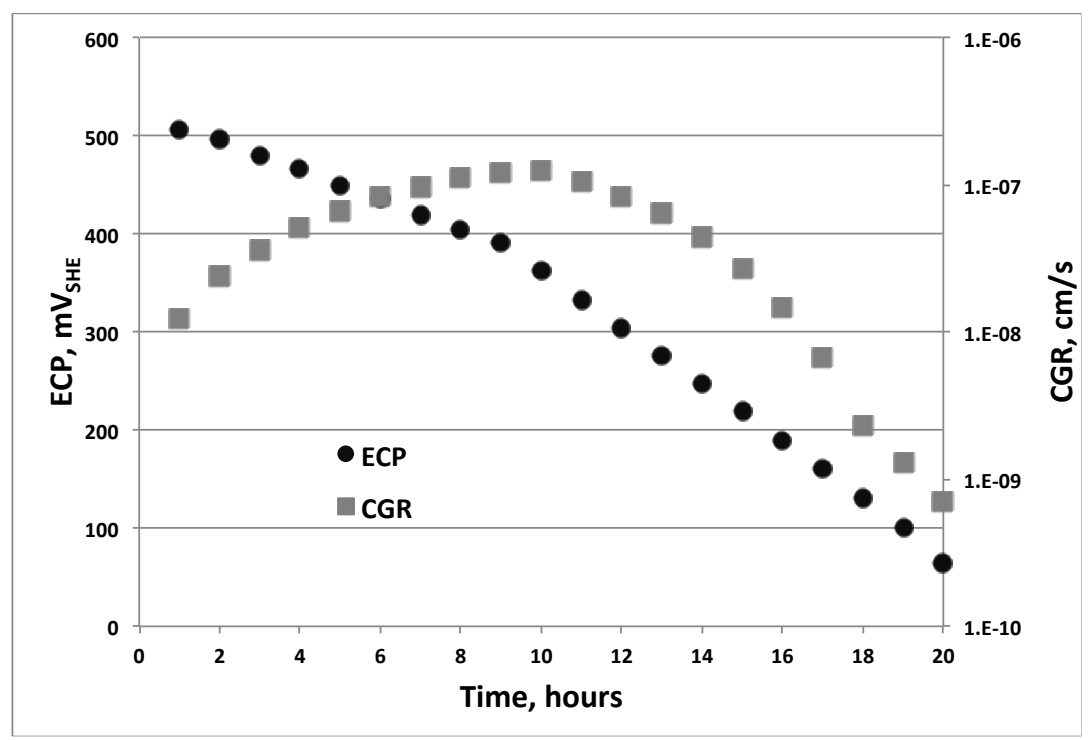

Figure XII.6. Time dependence of ECP and CGR at H6a Core Shroud weld location during reactor startup under combined transient conditions. While ECP monotonically diminished during the transient, CGR exhibits a maximum at about 10 hours and a temperature of about $160{ }^{\circ} \mathrm{C}$.

Finally, the accumulated damage (crack length) due to IGSCC at the H6a weld is plotted in Figure XII.7 as a function of time through start-up. The crack length is predicted to increase sigmoidally with time and is calculated to increase by about $38 \mu \mathrm{m}$ over the 20 -hour start-up time, corresponding to an average crack growth rate of about $0.5 \times 10^{-7} \mathrm{~cm} / \mathrm{s}$. Also plotted in Figure XII.7 is the contribution to "crack advance," which is defined as crack advance during any given hour of exposure divided by a crack advance over the entire 20-hr start-up period (x 100). The hourly contribution to crack advance exhibits a maximum at about 10 hours, corresponding to a temperature of about $150^{\circ} \mathrm{C}$. The advance within that hour is about $12 \%$ of the total. 


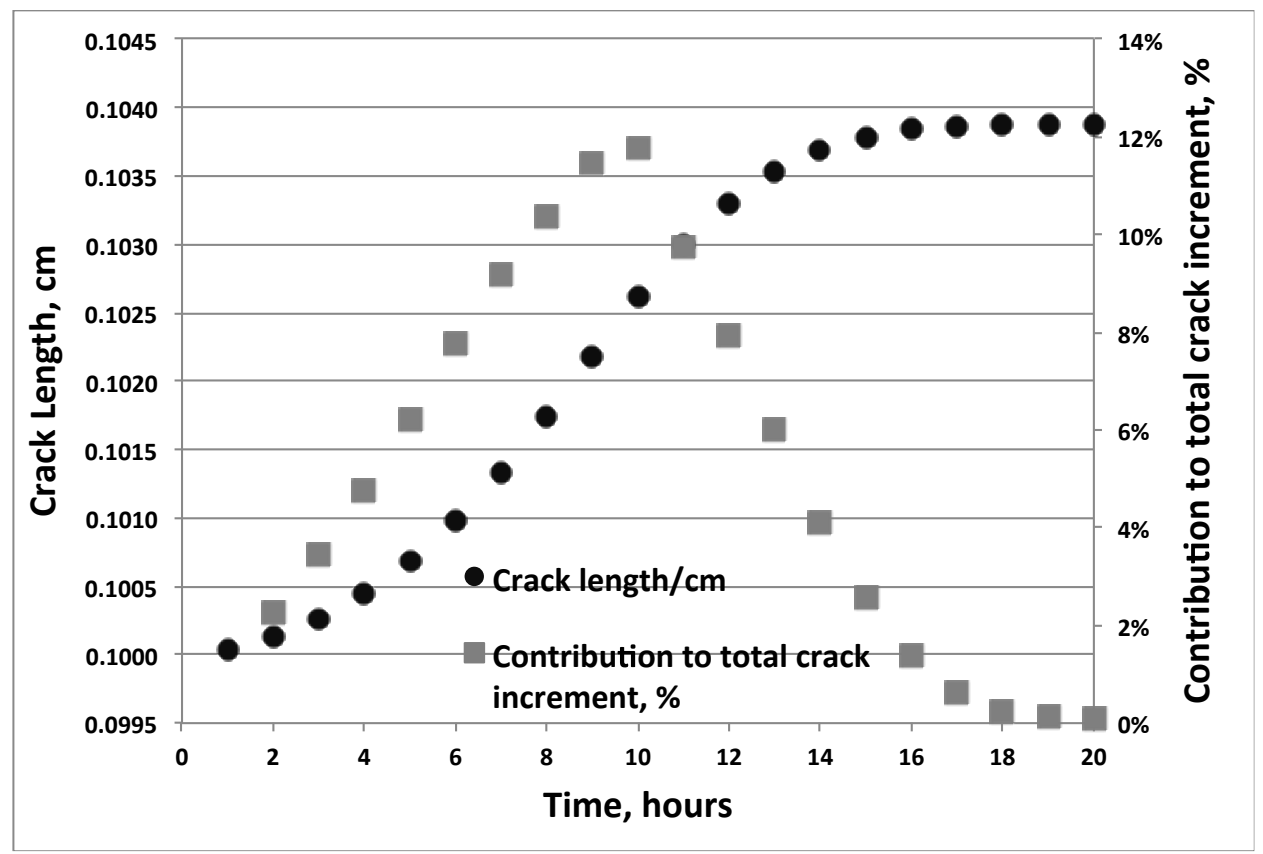

Figure XII.7. Time dependence of the crack length of a pre-existing, 0.1-cm deep crack at the H6a Core Shroud weld location and the incremental contribution of the crack advance at each time interval to the total crack advance over the 20-hr during reactor startup under combined transient conditions.

\section{XII.4. Advantages of deterministic modeling of IGSCC damage in BWRs.}

Several advantages accrue in using deterministic physico-electrochemical models for assessing and controlling corrosion phenomena in reactor primary coolant circuits. These advantages include:

- The transients presented in Figures XII.4 to XII.7 were derived for cracking on the outside of the core shroud at the location of the H6a weld. However, these calculations could have been made for any location in the primary (water phase) circuit of the BWR with a 1-cm resolution (distance between neighboring locations) for all components except for the recirculation piping system where the resolution is $100 \mathrm{~cm}$. There is no theoretical limit to the distance between neighboring locations that can be modeled, but of course, increasing the resolution (decreasing the distance between neighboring locations) increases the sizes of various matrices, correspondingly, in the algorithm resulting in increased execution time. 
- Despite almost heroic efforts by Indig and Nelson at GE in the US [15], Molander and coworkers at Studsvik in Sweden [16-18] and Abe, et al. [19] in Japan, in-reactor ECP measurements are few and far between and often are not made at locations of significant interest. Thus, Indig and Nelson [15] measured the ECP in an instrumentation tube that is not representative of the thermal-hydraulic environment that exists at, for example, the surface of the core shroud, noting that the ECP is a sensitive function of the coolant flow velocity as well as the local concentrations of the various redox species $\left(\mathrm{H}_{2}, \mathrm{O}_{2}, \mathrm{H}_{2} \mathrm{O}_{2}\right)$ [4].

- $\quad$ Because the ECP can only be measured at a few locations with the reactor vessel (e.g., at the bottom drain line or within instrumentation tubes [15]), most of which are not particularly relevant to ascertaining the accumulation of IGSCC damage in critical components, it is evident that an alternative to measurement must be employed to explore the problem of cracking in BWR internals fully. We posit that BWR_MASTER provides that capability.

- Although the MPM has been very successful in accounting for in-reactor ECP measurements [20], it is important to recognize that the measured ECP are, themselves, of limited accuracy. The principal problem lies with the viability of the reference electrode operating in a high temperature, high neutron/gamma flux environment in a reactor core. Over the past decade, there has been a move toward using platinum as a reference electrode, but this electrode is a classical redox sensor that responds to changes in $\left[\mathrm{H}_{2}\right],\left[\mathrm{O}_{2}\right]$, and $\left[\mathrm{H}_{2} \mathrm{O}_{2}\right]$ in much the same way as does the ECP of stainless steel. Accordingly, the measured potential difference between steel and Pt does not reflect the changes in the ECP of the steel alone. A viable reference electrode must yield a potential that is invariant to changes in the properties of the environment, and its potential must be determined by a single charge transfer reaction (i.e., the potential should not be a "mixed potential") [21]. In the case of platinum, this requirement is only satisfied if the concentration of $\mathrm{H}_{2} \gg \mathrm{O}_{2}, \mathrm{H}_{2} \mathrm{O}_{2}$, such that the potential is determined solely by the HER $\left(\mathrm{H}^{+}+\mathrm{e}^{-}=1 / 2 \mathrm{H}_{2}\right)$. However, in BWR in-core environments, where intense radiolysis continually produces $\mathrm{O}_{2}$ and $\mathrm{H}_{2} \mathrm{O}_{2}$ and recognizing that $\mathrm{H}_{2}$ is preferentially stripped from the coolant by boiling, it is difficult to see how this condition holds. Indeed, measurements by Arioka et al. [22] demonstrate the high sensitivity of the measured platinum potential to minor contamination of hydrogenated solutions by oxygen. In our opinion, after having compared calculated and measured ECP and CGR in laboratory and in-reactor 
environments for almost forty years, these important properties can be calculated at least as accurately as can be measured with no restriction on location in the reactor primary coolant circuit.

\section{XII.5. References}

[1] P.L. Andresen, Corrosion, 49, 714-725 (1993).

[2] D.D. Macdonald, Power Plant Chemistry, 4, 329-335 (2002).

[3] J Shi, J Wang, and D D Macdonald, Corros. Sci., 89, 69-80 (2014).

[4] P.C. Lu, D.D. Macdonald, M. Urquidi-Macdonald, and T.K. Yeh. Corrosion, 52, 768-785 (1996).

[5] D.D. Macdonald, J. Yang, B. Fekete, I. Balachov, and B. Spencer, Development and Integration of Light Water Reactor (LWR) Materials Corrosion Degradation Codes into Grizzly, Nuclear Energy University Program Final Technical Report, 2017-2019, Award DENE0008541, (2019).

[6] T.K. Yeh, D.D. Macdonald, and A.T. Motta, Nucl. Sci. Eng., 121, 468-482 (1995).

[7] T.K. Yeh, D.D. Macdonald, and A.T. Motta, Nucl. Sci. Eng., 123, 295-304 (1996).

[8] T.K. Yeh, D.D. Macdonald, and A.T. Motta, Nucl. Sci. Eng., 123, 305-316 (1996).

[9] I. Balachov and D.D. Macdonald, Enhancing the Operation of Boiling Water Reactors by Deterministic Simulation, Proc. Water Chemistry 98, 1998 JAIF Int. Conf. Water Chem. Nucl. Power Plants, Kashiwazaki, Japan, Oct. 13-16 (1998).

[10] H.S. Kim, A study for modeling electrochemistry in light water reactors, Ph.D. Thesis, Dept. Nucl. Eng., Pennsylvania State University, (2007).

[11] Dr. Samson Hettiarachchi, private communication.

[12] J. Abella, I. Balachov, G.R. Engelhardt, D.D. Macdonald, and P.J. Millett, Mater. Sci. Forum, 30, 895-914 (1998).

[13] J. Abellà, I. Balachov, D.D. Macdonald, and P.J. Millet, Corros. Sci., 44, 191 (2002).

[14] M.P. Manahan, D.D. Macdonald, and A.J. Peterson, Corros. Sci., 37, 189-208 (1995). 
[15] M.E. Indig and J.L. Nelson, Corrosion, 47, 202-209 (1991).

[16] A. Molander and M. Ullberg, The corrosion potential of stainless steel in BWR environment comparison of data and modeling results, TRN: JP0403460086466, Atomic Energy Society of Japan, Tokyo, 105-0004, Japan, (2004).

[17] A. Molander and G. Karlberg, Hydrogen water chemistry surveillance in a boiling water reactor, Proceedings: 1989 workshop on LWR radiation water chemistry and its influence on in-core structural materials, EPRI-NP-7033, Electric Power Research Inst., Palo Alto, CA (United States), 531, 13.1-13.12 (1991).

[18] A. Molander, Online electrochemical monitoring in light water reactor (LWR) systems, in Nuclear Corrosion Science and Engineering, Woodhead Publishing Series in Energy, 408437 (2012).

[19] H. Takiguchi, M. Sekiguchi, A. Abe, K. Akamine, M. Sakai, Y. Wada, and S. Uchida, J. Nucl. Sci. Tech., 36, 179-188 (1999).

[20] D.D. Macdonald, I. Balachov, and G. Engelhardt, PowerPlant Chemistry, 1, 9-16 (1999).

[21] D.D. Macdonald and C.C. Lin, Corrosion, 49, 90-93 (1993).

[22] K. Arioka, A. Bertuch, L. Kriksunov, D. D. Macdonald, and J. Pang. Modeling the Corrosion Behaviors of the Heat Transport Circuits of Light Water Nuclear Reactors. Proc. Sixth Intl. Symp. Environ. Degrad. Maters. Nucl. Power Systs. -Water Reactors. pp. 905-913. August 1-

5, 1993. San Diego, CA. Houston, TX, National Association of Corrosion Engineers, International (NACE International).

\section{Modeling the electrochemistry and SCC damage accumulation in BWRs.}

\section{XIII.1. The BWR_MASTER code}

The BWR_MASTER code [1] was designed for modeling water chemistry, electrochemistry CGR and crack length in the heat transport circuit (HTC) of Boiling Water Reactors (BWRs). Code development history is shown in Figure XIII.1.

The first version, called DAMAGE PREDICTOR [2], has been developed in the early 1990s at PennState University. DAMAGE PREDICTOR was capable of estimating the 
concentrations of electrochemically active species, Electrochemical Corrosion Potential (ECP), and CGR for several commercial BWRs. Models were developed for calculating ECP (Mixed Potential Model or MPM) [1] and CGR (Coupled Environment Fracture Model or CEFM) [3-5]. Water chemistry, ECP and CGR modeling were possible only for a single point in time of the reactor operating history. DAMAGE PREDICTOR had been programmed in FORTRAN, and a algorithm of low efficiency was devised for solving a system of stiff differential equations was used, resulting in more than 3 hours of calculation time for a single state point on a PC computer.

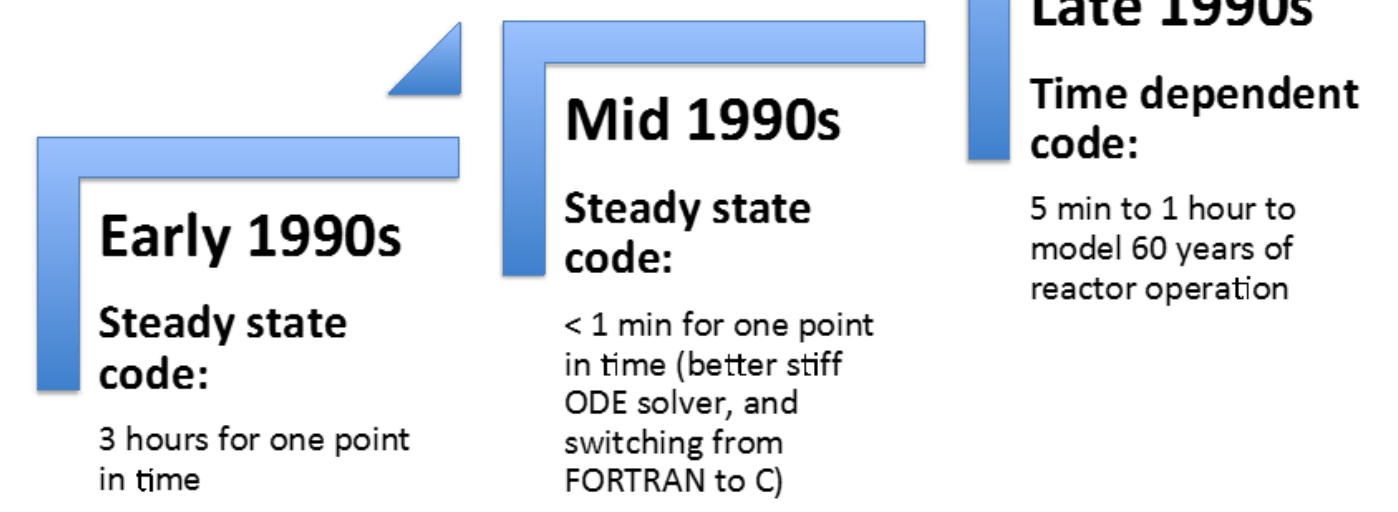

Figure XIII.1. Code development history.

A later version of DAMAGE PREDICTOR, called ALERT, was completed in the mid1990s at PennState University. The code was programmed in C, and a more efficient algorithm for solving system of stiff differential equations was implemented, resulting in less than one minute computational time for a single state point on a Windows PC computer. Several commercial BWRs in the US and Europe were modeled with considerable success. Predictions of water chemistry, ECP, and CGR agree well with in-plant measurements.

High speed of calculations allowed designing a time-dependent version in the late 1990s, which allows modeling of 60 and more years of reactor operation, including shutdowns and restarts on a Windows PC during 1-2 hours. Both the MPM and CEFM models had not been updated at that time, and are the same as the ones programmed in DAMAGE PREDICTOR. Meanwhile, significant progress was made in MPM and CEFM model development. The effects of catalysis 
and surface coatings were included in the last version of ALERT and are retained in the upgraded code, BWR_MASTER.

\section{Upgrading of ALERT into BWR_MASTER and embedding into Grizzly code}

The ultimate goal of this work is to augment the fracture mechanics capabilities in INL's Grizzly code [6], with models that account for environmental effects in the fracture of components in LWRs. Grizzly, which is a fracture-mechanics based code, will be used to compute the stress intensity factor as the crack propagates through a component of any geometry, particularly as the stresses (loading and residual) change (e.g., relax during shutdown) as the reactor progresses along with the corrosion evolutionary paths (CEPs).

\section{Workflow on embedding BWR_MASTER into the Grizzly code includes:}

1. Insertion of an advanced Mixed Potential Model (MPM) for calculating the corrosion potential (ECP); inclusion of advanced crack growth rate (ACGR) models; and insertion of models for crack initiation, in both the BWR and PWR codes (BWR_MASTER and PWR_MASTER, respectively), and inclusion of an algorithm for integrating the CGR in a PWR over the operating history of the reactor (such an algorithm already exists in the BWR codes). These upgrades will allow prediction of the accumulation of damage in stainless steels and nickel-based alloys, such as Type 304 SS and Alloy 600, respectively, in both types of reactors.

2. Incorporation of BWR_MATER into Grizzly, as appropriate, to yield a tool that will be capable of calculating the accumulated damage (crack length) in any component in the primary coolant circuit along any preconceived CEP or operating history of the reactor.

As the first step of this work, BWR_MASTER was upgraded into BWR_MASTER and was configured to supply time-dependent data on the concentrations of hydrogen, hydrogen peroxide and oxygen, ECP, CGR, crack length, and stress intensity factor to an external program, such as Grizzly as described.

\section{HTC representation in BWR_MASTER.}


The regions of a BWR heat transport circuit that are modeled are illustrated schematically in Figure XIII.2a. A total of ten regions in the circuit are considered. The concentration of each species is calculated, assuming transport in one dimension along the coolant path (Figure XIII.2. and Figure XIII.3. ).

In order to calculate the species concentrations, the combined effects of the radiolytic yield of each species due to radiation, and the changes in concentration due to chemical reactions and fluid convection have been taken into account. After the concentration of each radical species is calculated, the corrosion potential of the component can be calculated using the Mixed Potential Model. Once again, after the concentration of each electroactive species and the corrosion potential are calculated, the crack growth rate of any existing crack can then be calculated using the coupled environment fracture model (CEFM).

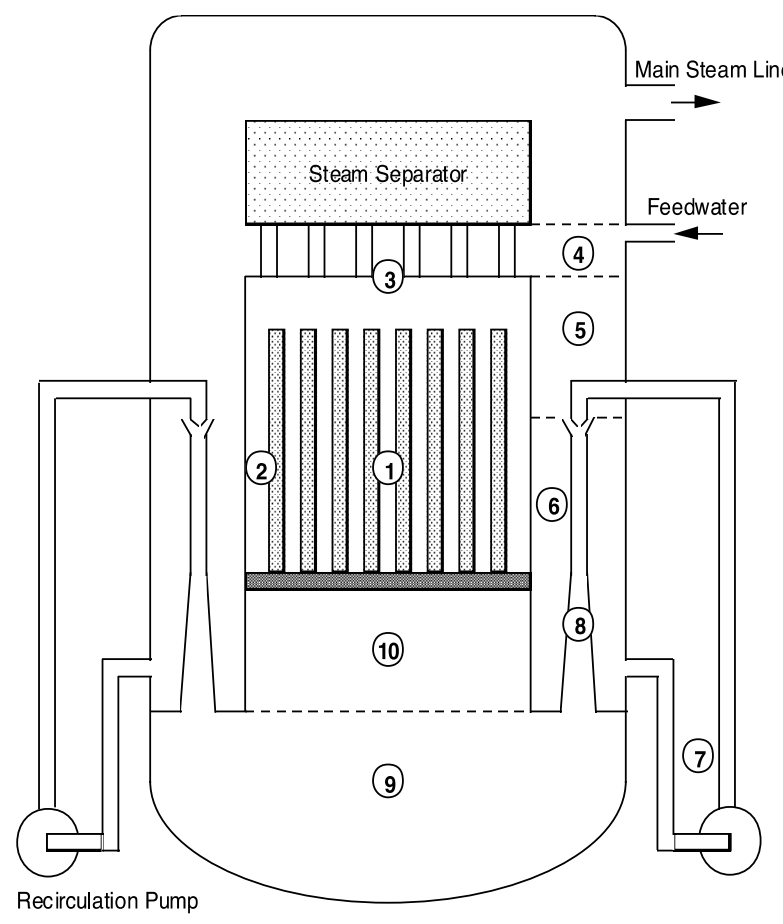

(a)

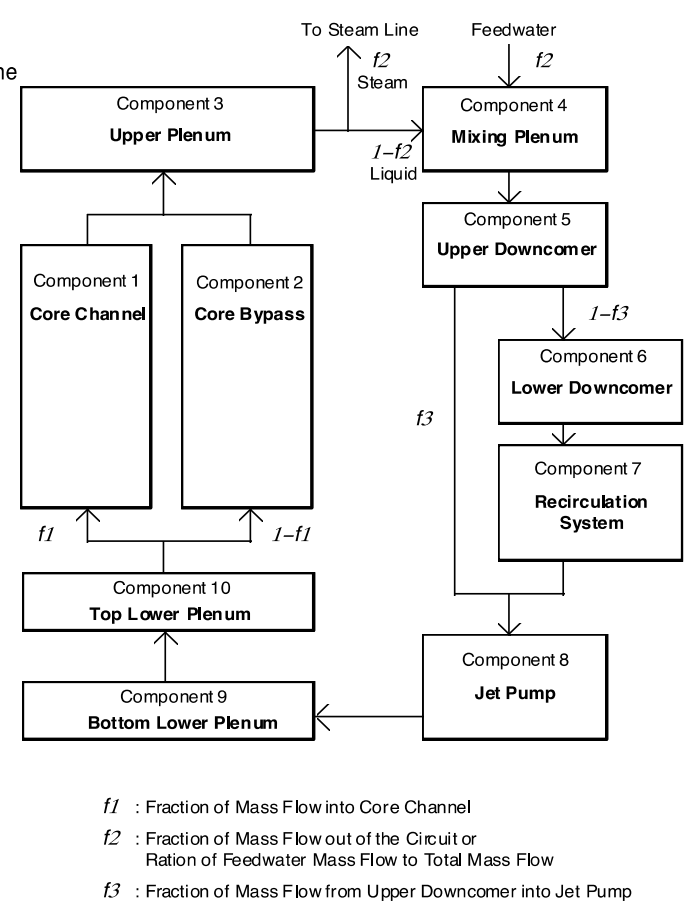

(b)

Figure XIII.2. BWR recirculating coolant model: simplified reactor geometry (a) and HTC components line-up in BWR_MASTER (b). 


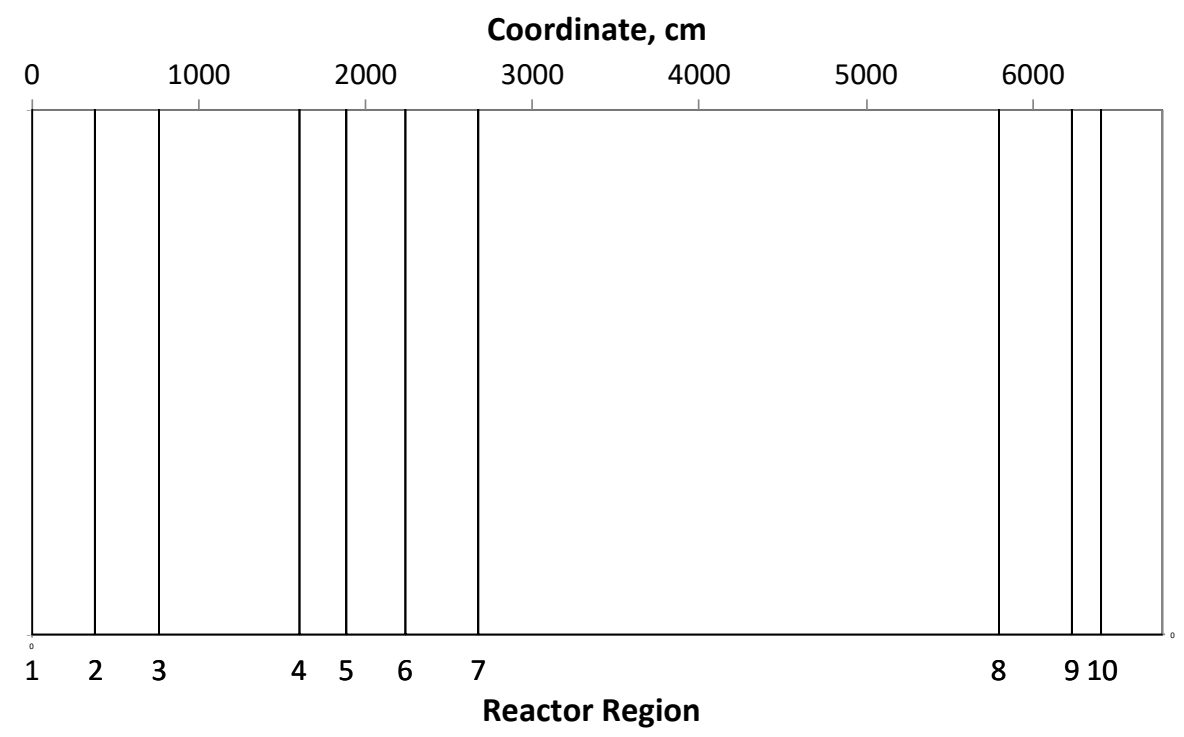

Figure XIII.3. A one-dimensional representation of BWR HTC in BWR_MASTER.

\section{BWR_MASTER input files}

The BWR_MASTER is a console application for MAC and Windows operating systems. Required files (Figure XIII.4. and Table XIII.1) include reactor geometry, reactor operating history, two BWR library data files, which are not to be changed by a user (reaction rates and radiolytic yields) and the BWR_MASTER executable file.

Input data files prepared by user (any name and extension)

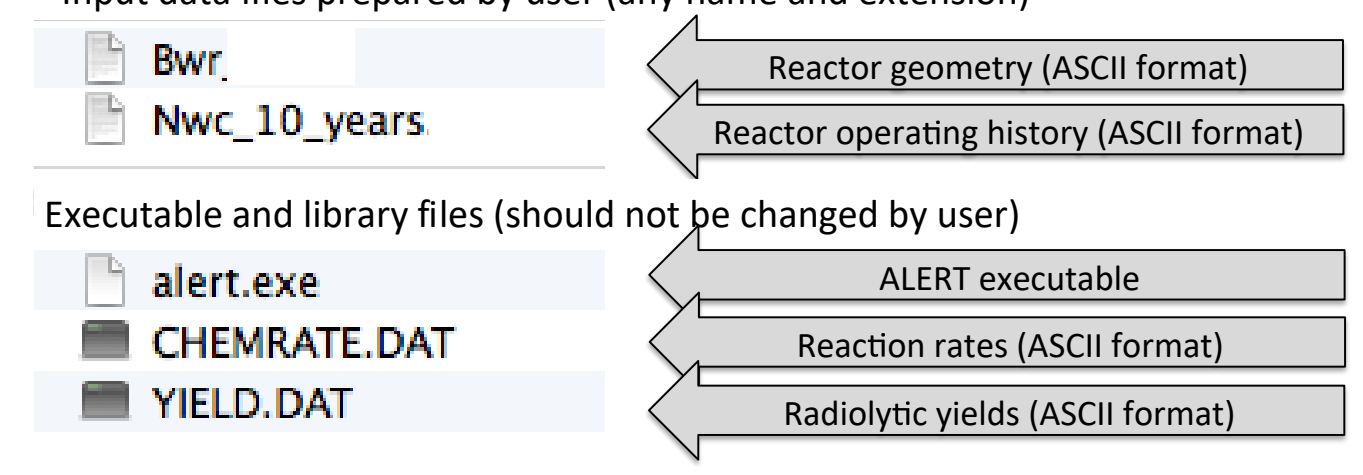

Figure XIII.4. The BWR_MASTER input files. 
Table XIII.1. Input files.

\begin{tabular}{|l|l|}
\hline File name & \multicolumn{1}{|c|}{ Description } \\
\hline Bwr & Reactor geometry (a descriptive file name recommended) \\
\hline Nwc_10_years & Reactor operating history (a descriptive file name recommended) \\
\hline alert.exe & ALERT executable file for Windows operating system \\
\hline CHEMRATE.DAT & Reaction rates for species in reactor HTC (file name can not be changed) \\
\hline YIELD.DAT & Radiolytic yields of species (file name can not be changed) \\
\hline
\end{tabular}

All input files are in ASCII format. Information contained in each file is included in the "printout" output file.

\section{Migrating the BWR_MASTER code to macOS operating system}

The Grizzly code [6,7] has been designed for macOS or Linux operating systems, and the Windows system is not supported. BWR_MASTER code [1] has been created under the Windows operating system, and the migration of BWR_MASTER to the macOS system is required in order to run a BWR_MASTER + Grizzly combined package. The BWR_MASTER source code was modified to be compatible with the GCC compiler [9] under macOS. The installation of the XCode development tool [10] is required in order to run the GCC compiler. Compiling of BWR_MASTER source files (Figure XIII.) requires execution of the following command to create executable file alert_G:

gcc cvdense.c cvode.c dense.c llnlmath.c vector.c cefm.c main_g.c -o alert_G 


\begin{tabular}{|c|c|c|c|c|c|c|}
\hline 哭 $\equiv$ & 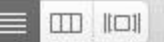 & 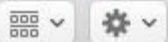 & & 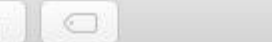 & 82 & Q. Search \\
\hline \multicolumn{2}{|l|}{ Name } & & $\wedge$ & Date Modified & Size & Kind \\
\hline h & CEFM.H & & 0 & Feb $6,1998,9: 49 \mathrm{PM}$ & $3 \mathrm{~KB}$ & C Header Source \\
\hline c & CVDENSE.C & & 0 & Oct $14,1997,5: 42 \mathrm{AM}$ & $11 \mathrm{~KB}$ & C Source \\
\hline h & CVDENSE.H & & 0 & Oct $14,1997,5: 42 \mathrm{AM}$ & $10 \mathrm{~KB}$ & C Header Source \\
\hline G & cvdense.o & & 0 & Mar 20, 2017, 6:59 PM & $5 \mathrm{~KB}$ & object code \\
\hline c & CVODE.C & & 0 & Oct $14,1997,5: 43$ AM & $73 \mathrm{~KB}$ & C Source \\
\hline h & CVODE.H & & 0 & Oct $14,1997,5: 43 \mathrm{AM}$ & $43 \mathrm{~KB}$ & C Header Source \\
\hline B & cvode.o & & 0 & Mar 20, 2017, 6:58 PM & $38 \mathrm{~KB}$ & object code \\
\hline c & DENSE.C & & 0 & Oct $14,1997,5: 43 \mathrm{AM}$ & $5 \mathrm{~KB}$ & C Source \\
\hline h & DENSE.H & & 0 & Oct $14,1997,5: 43 \mathrm{AM}$ & $26 \mathrm{~KB}$ & C Header Source \\
\hline 0 & dense.o & & 0 & Mar $20,2017,7: 00$ PM & $6 \mathrm{~KB}$ & object code \\
\hline c & LLNLMATH.C & & 0 & Oct $14,1997,5: 43 \mathrm{AM}$ & 859 bytes & C Source \\
\hline h & LLNLMATH.H & & 0 & Oct $14,1997,5: 43 \mathrm{AM}$ & $4 \mathrm{~KB}$ & C Header Source \\
\hline D & IInImath.o & & 0 & Mar $20,2017,7: 01 \mathrm{PM}$ & $2 \mathrm{~KB}$ & object code \\
\hline h & LLNLTYPS.H & & 0 & Oct $14,1997,5: 43 \mathrm{AM}$ & $6 \mathrm{~KB}$ & C Header Source \\
\hline c & main_g.c & & 0 & Today, 8:54 PM & $58 \mathrm{~KB}$ & C Source \\
\hline c & VECTOR.C & & $\theta$ & Oct $14,1997,5: 44$ AM & $9 \mathrm{~KB}$ & C Source \\
\hline h & VECTOR.H & & 0 & Oct $14,1997,5: 44 \mathrm{AM}$ & $22 \mathrm{~KB}$ & C Header Source \\
\hline B & vector.o & & 0 & Mar $20,2017,7: 01 \mathrm{PM}$ & $10 \mathrm{~KB}$ & object code \\
\hline
\end{tabular}

Figure XIII.5. The BWR_MASTER source files.

BWR_MASTER is a console application. The execution of simulations with BWR_MASTER code requires the creation of a folder with BWR_MASTER executable and input files (Figure XIII.1). The required files include reactor geometry, reactor operating history, two BWR library data files, which are not to be changed by a user (reaction rates and radiolytic yields), and the BWR_MASTER executable file alert_G.

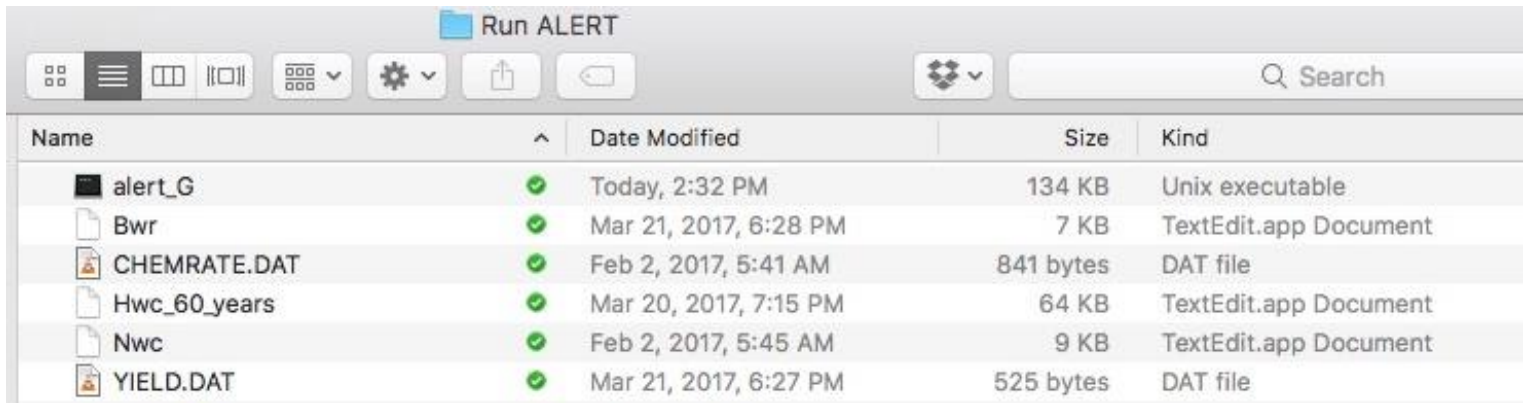

Figure XIII.6. The BWR_MASTER folder with input files in macOS Finder. 
Execution of BWR_MASTER must be done only from macOS Terminal Window (Figure XIII.) or XQuartz [11] window (Figure XIII.) with command ./alert_G

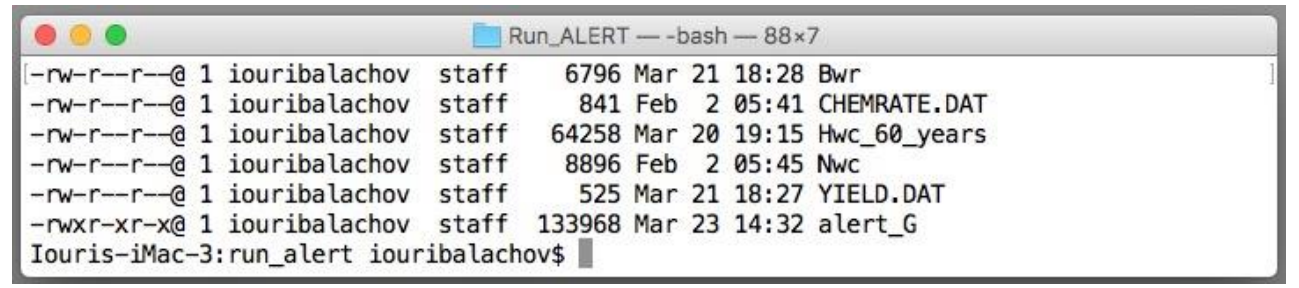

Figure XIII.7. BWR_MASTER folder with input files in Terminal Window.

\begin{tabular}{|c|c|c|c|}
\hline & & X xterm & \\
\hline 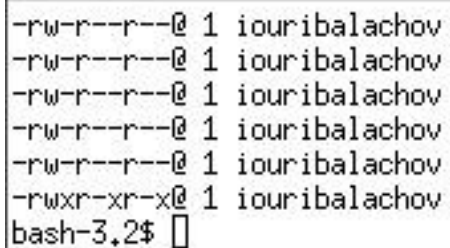 & $\begin{array}{l}\text { staff } \\
\text { staff } \\
\text { staff } \\
\text { staff } \\
\text { staff } \\
\text { staff }\end{array}$ & $\begin{array}{rlll}6796 & \text { Mar } 21 & 18 * 28 & \text { Bur } \\
841 & \text { Feb } 2 & 05 * 41 & \text { CHEHRATE+DAT } \\
64258 & \text { Mar } 20 & 19 \div 15 & \text { Hwc_60_years } \\
8896 & \text { Feb } 2 & 05 * 45 & \text { Nwc } \\
525 & \text { Mar } 21 & 18 * 27 & \text { YIELD,DAT } \\
133968 \text { Mar } 23 & 14+32 \text { alert_G }\end{array}$ & \\
\hline
\end{tabular}

Figure XIII.8. BWR_MASTER folder with input files in the XQuarts window.

An attempt to execute BWR_MASTER from macOS Finder by double-clicking on BWR_MASTER executable will set a default directory to /Users/YourUserName, which does not have required input files.

User should Type the name (and extension, if any) of reactor geometry file and press "Enter" and then Type the name (and extension, if any) of reactor operating history file and press "Enter” (Figure XIII.9). All input files should be accessible from alert_G location.

Progress will be displayed for each operating time interval, as shown in Figure XIII.3. Upon completion, the console window will be closed automatically. Files with output results will be stored in the same folder as alert_G. 


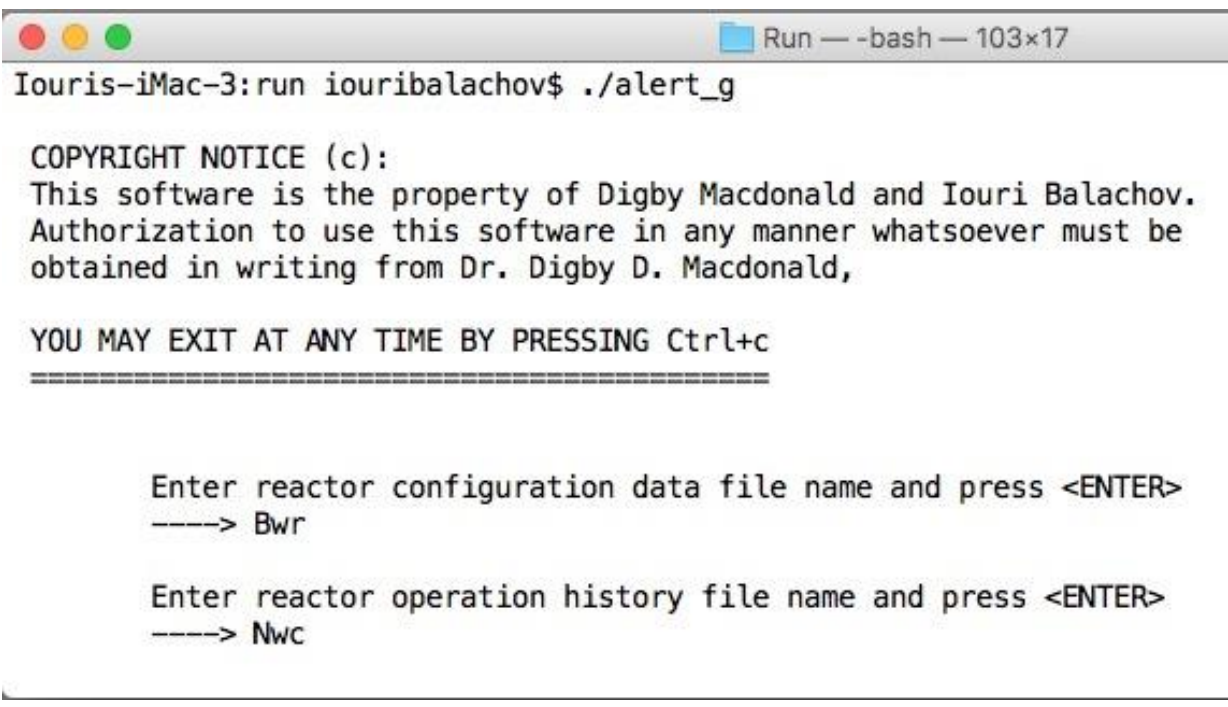

Figure XIII.9. BWR_MASTER execution.

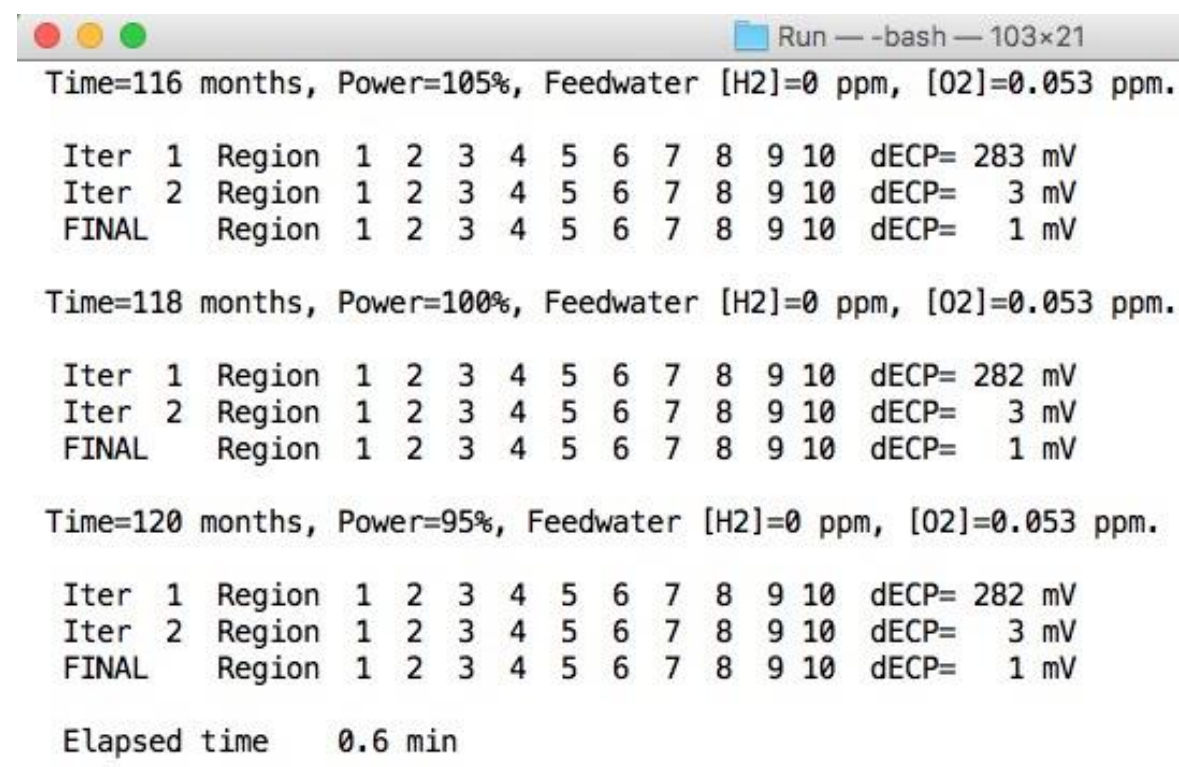

Figure XIII.3. The BWR_MASTER progress output.

Executing ALERT under XQuartz requires identical steps (

(b)

Figure XIII.4). 


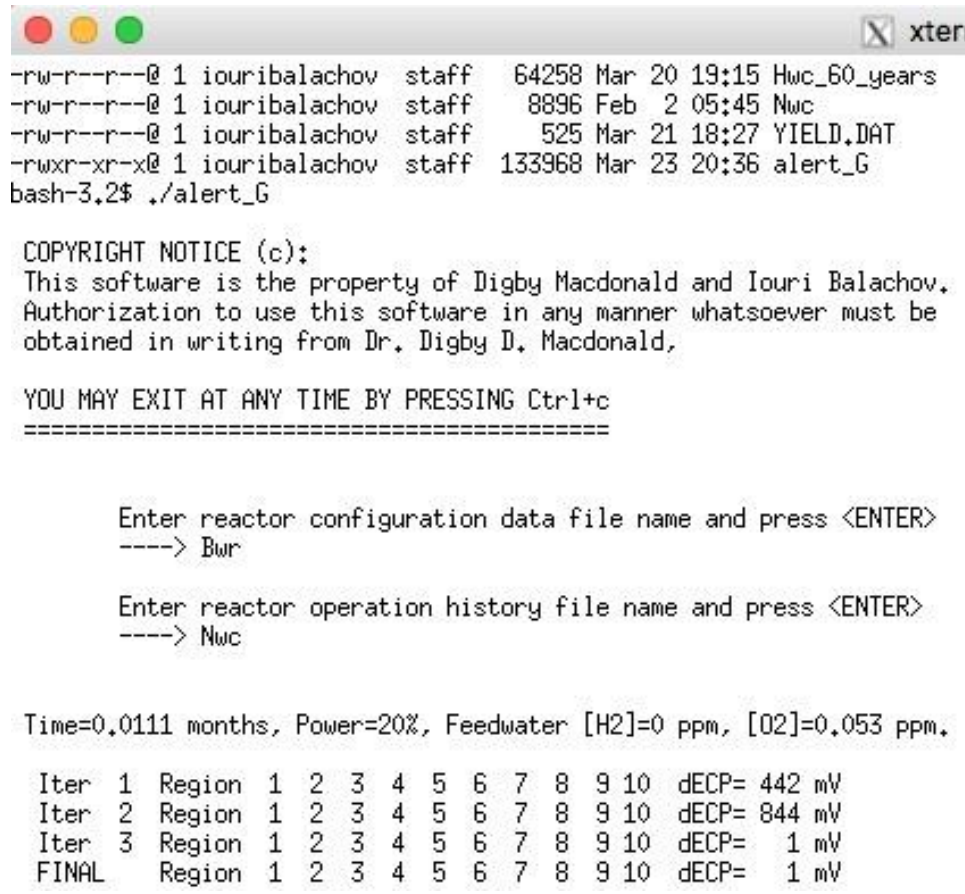

(a)

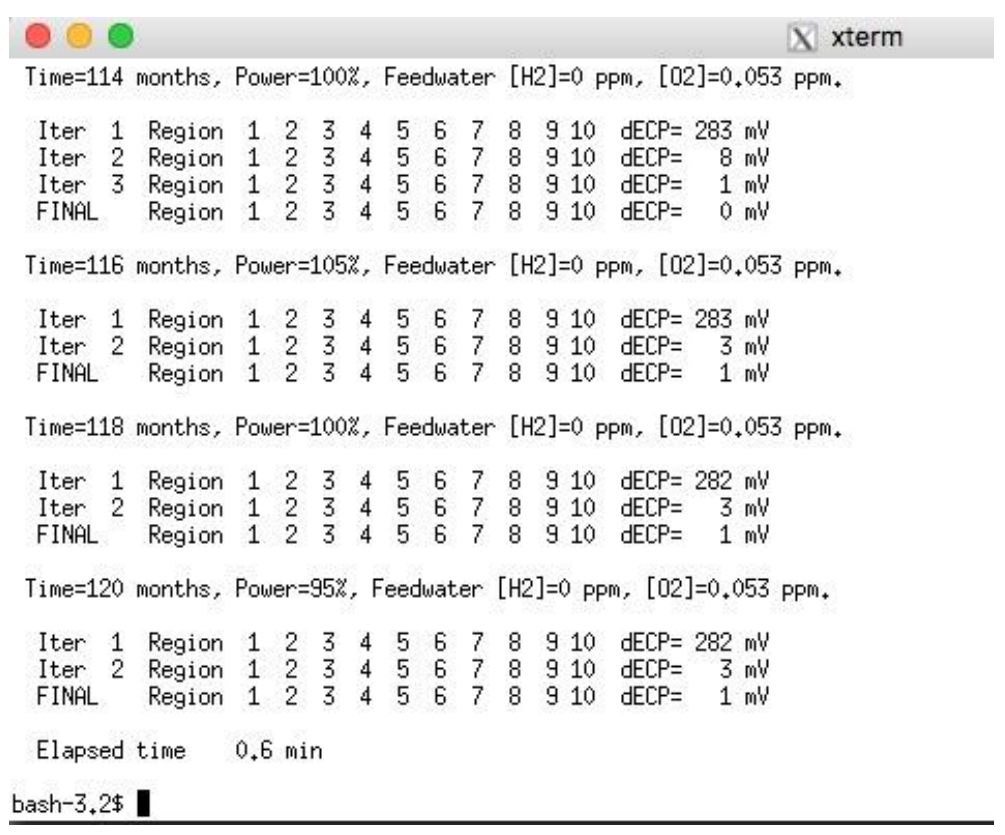

(b)

Figure XIII.4. The BWR_MASTER progress output in a XQuartz window: beginning (a) and end (b). 
BWR_MASTER generates two groups of output files (Figure XIII.5). Output file names are concatenated from $\{$ Reactor geometry $\}$ file name, $\{$ Reactor operating history $\}$ file name and \{File contents\}. First group includes one file, an analog to "printout" file with all results printed for each component and state point. \{File contents $\}$ is set to $\{\mathrm{WC}$ ECP_CGR_vs_time $\}$ for this file. The second group of files includes 7 files with \{File contents $\}$ set to \{Crack_Depth_vs_time\}, \{Crack_Growth_Rates_vs_time $\}, \quad\{$ ECP_vs_time $\}, \quad\left\{\mathrm{H} 2 \_v s \_t i m e\right\}, \quad\{\mathrm{H} 2 \mathrm{O} 2$ _vs_time $\}$, $\{\mathrm{O} 2$ _vs_time $\}$ and $\{$ Stress_Intencity_vs_time $\}$, accordingly. Files in the second group are "interface" files for post-processing and for visualization and analysis.

Computations have been performed on macOS to confirm identity with results of the ALERT and BWR_MASTER computations performed under the Windows operating system. Calculation time for 60 years of BWR operation is under 5 minutes on a iMac $2.7 \mathrm{GHz}$ Intel Core i5 processor, 8 GB 1333 MHz DDR3 memory, 2 TB hard drive, and a macOS Sierra operating system version 10.12 .3 .

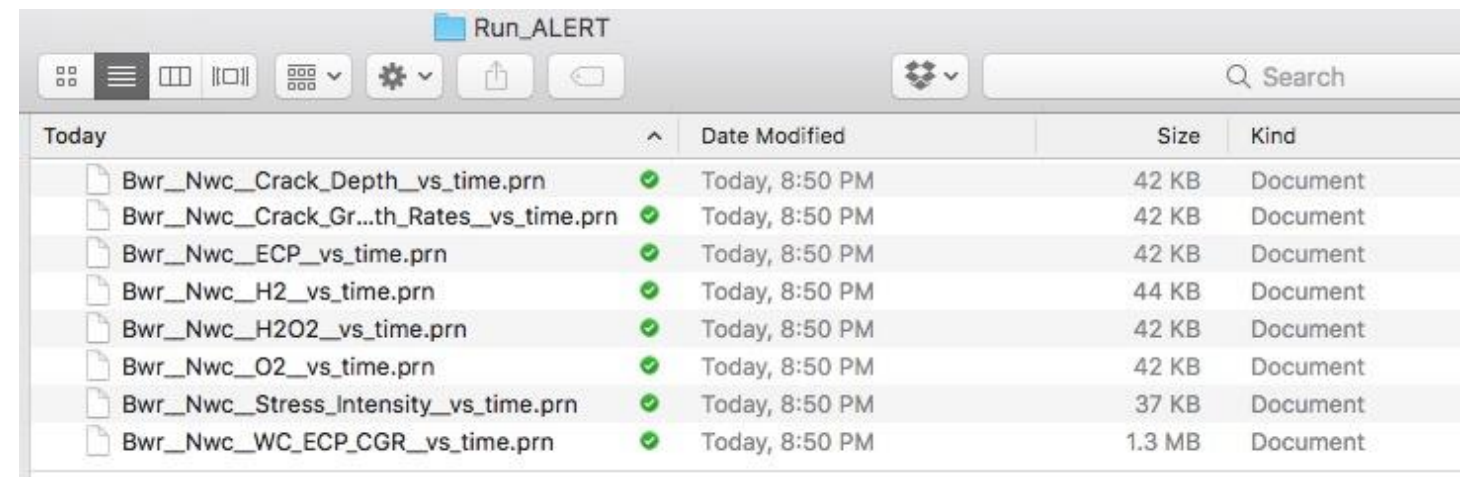

Figure XIII.5 BWR_MASTER output files.

\section{Running BWR_MASTER simulation under windows}

Once all input files are prepared, the user selects the executable file and presses "Enter" or double left-clicks on it. A Windows OS console window appears (Figure XIII.6) with prompts the user to enter the names of two input files: reactor geometry file and reactor operating history file. The user should type the name (and extension, if any) of the reactor geometry file and press "Enter" 
and then type the name (and extension, if any) of the reactor operating history file and press "Enter." All input files should be accessible from the alert.exe location.

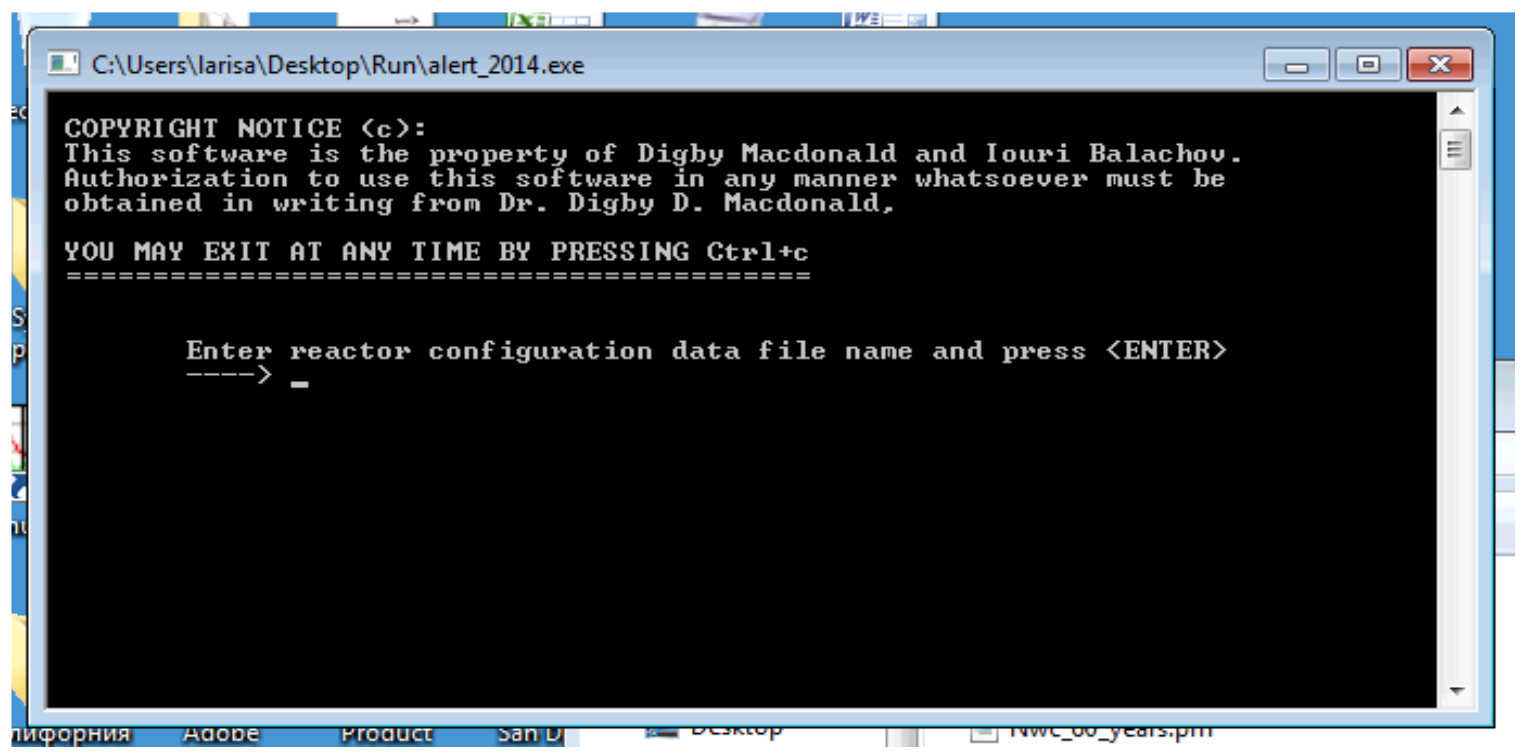

Figure XIII.6. BWR_MASTER input window.

Progress will be displayed for each operating time interval, as shown in Figure XIII.7:

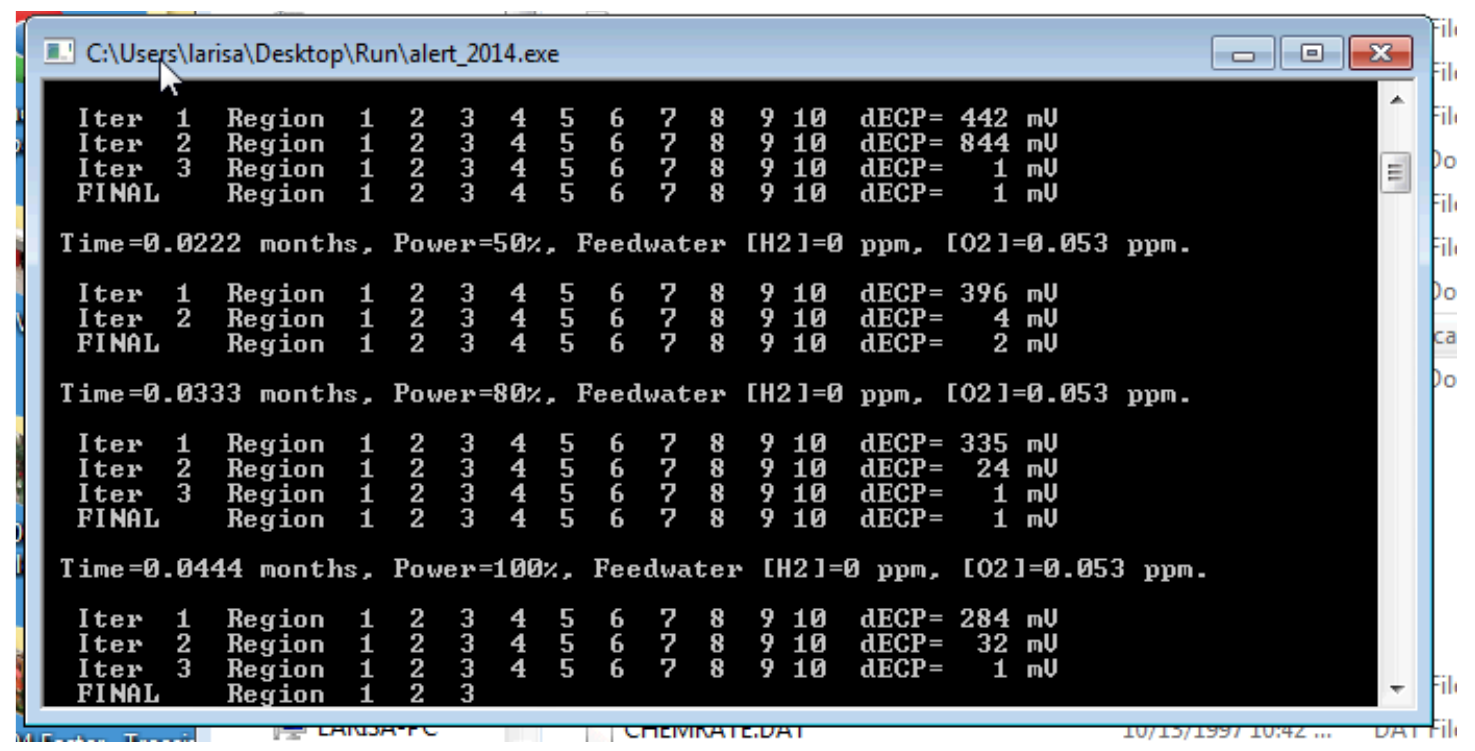

Figure XIII.7. BWR_MASTER progress window. 
Upon completion, the console window will be closed automatically. Files with output results will be stored in the same folder as alert.exe

\section{BWR_MASTER output files}

BWR_MASTER generates two groups of output files (Figure XIII.). The output file names are concatenated from the $\{$ Reactor geometry\} file name, $\{$ Reactor operating history $\}$ file name, and the $\{$ File contents $\}$ as described in Table XIII.. The first group includes one file, an analog to a "printout" file with all results printed for each component and state point. \{File contents $\}$ is set to $\{$ WC_ECP_CGR_vs_time\} for this file. The second group of files includes 7 files with \{File contents $\}$ set to $\{$ Crack_Depth_vs_time $\},\{$ Crack_Growth_Rates_vs_time $\},\{$ ECP_vs_time $\}$, $\left\{\mathrm{H}_{2} \_\mathrm{vs} \_t i m e\right\},\left\{\mathrm{H}_{2} \mathrm{O}_{2} \_\mathrm{vs} \_t i m e\right\},\left\{\mathrm{O}_{2} \_\mathrm{vs} \_t i m e\right\}$, and $\{$ Stress_Intencity_vs_time $\}$, accordingly. Files in the second group are "interface" files for interfacing with Grizzly or other codes and postprocessing and for visualization and analysis.
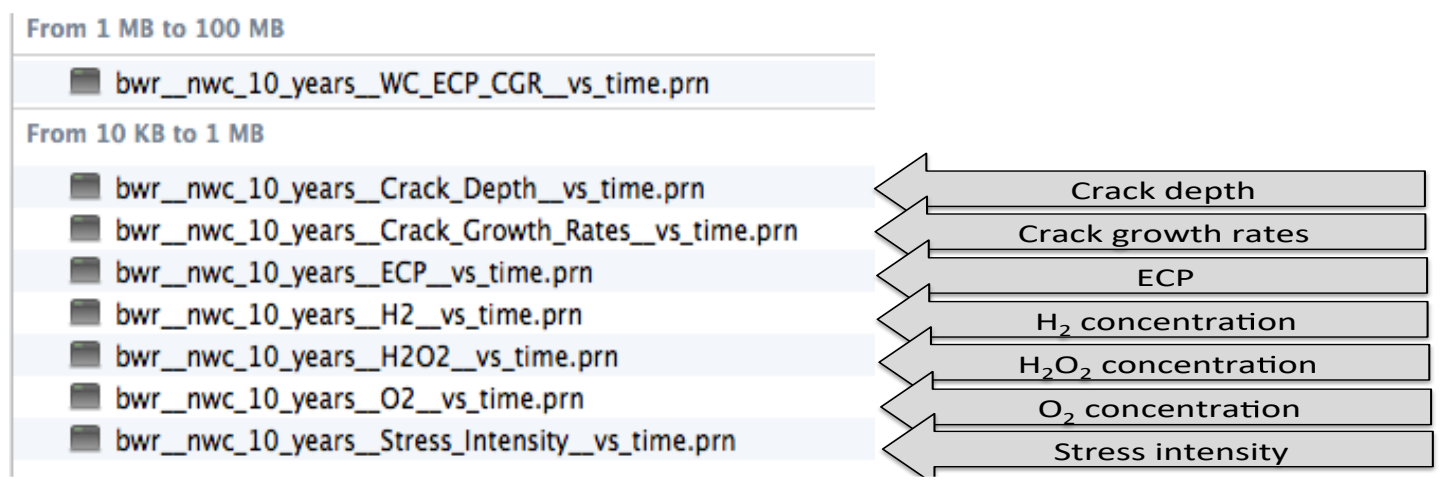

Figure XIII.15. BWR_MASTER output files.

\section{BWR_MASTER "Printout" file}

BWR_MASTER completes the output file in ASCII format with the concentrations of species, ECP, and CGR along the reactor HTC for each time interval for visual analysis. The file also includes a printout of all input files with comprehensive labeling. Up to the year 2016, this was a single output file for data analysis and visualization. 
Table XIII.2. Output file names and contents.

\begin{tabular}{|c|c|}
\hline File name & Description \\
\hline $\begin{array}{l}\text { Bwr_nwc_10_years_WC_E } \\
\text { CP_CGR_vs_time.prn }\end{array}$ & $\begin{array}{l}\text { BWR_MASTER output file in ASCII format with } \\
\text { concentrations of species, ECP and CGR along the reactor } \\
\text { HTC for each time interval for visual analysis }\end{array}$ \\
\hline $\begin{array}{l}\text { Bwr_nwc_10_years_Crack_ } \\
\text { Depth_vs_time.prn }\end{array}$ & $\begin{array}{l}\text { A new file with crack depth along the reactor HTC for each } \\
\text { time interval for post-processing or (and) interfacing }\end{array}$ \\
\hline $\begin{array}{l}\text { Bwr_nwc_10_years_Crack_ } \\
\text { Growth_Rates_vs_time.prn }\end{array}$ & $\begin{array}{l}\text { A new file with CGR along the reactor HTC for each time } \\
\text { interval for post-processing or (and) interfacing }\end{array}$ \\
\hline $\begin{array}{l}\text { Bwr_nwc_10_years_ECP_ } \\
\text { vs_time.prn }\end{array}$ & $\begin{array}{l}\text { A new file with ECP along the reactor HTC for each time } \\
\text { interval for post-processing or (and) interfacing }\end{array}$ \\
\hline $\begin{array}{l}\text { Bwr_nwc_10_years_H2_vs } \\
\text { _time.prn }\end{array}$ & $\begin{array}{l}\text { A new file with } \mathrm{H}_{2} \text { concentration along the reactor HTC for } \\
\text { each time interval for post-processing or (and) interfacing }\end{array}$ \\
\hline $\begin{array}{l}\text { Bwr_nwc_10_years_H2O2 } \\
\text { _vs_time.prn }\end{array}$ & $\begin{array}{l}\text { A new file with } \mathrm{H}_{2} \mathrm{O}_{2} \text { concentration along the reactor } \mathrm{HTC} \text { for } \\
\text { each time interval for post-processing or (and) interfacing }\end{array}$ \\
\hline $\begin{array}{l}\text { Bwr_nwc_10_years_O2_vs } \\
\text { time.prn }\end{array}$ & $\begin{array}{l}\text { A new file with } \mathrm{O}_{2} \text { concentration along the reactor HTC for } \\
\text { each time interval for post-processing or (and) interfacing }\end{array}$ \\
\hline $\begin{array}{l}\text { Bwr_nwc_10_years_Stress } \\
\text { _Intensity_vs_time.prn }\end{array}$ & $\begin{array}{l}\text { A new file with stress intensity factor along the reactor HTC } \\
\text { for each time interval for post-processing or (and) interfacing }\end{array}$ \\
\hline
\end{tabular}

\section{BWR_MASTER interface files}

The output of BWR_MASTER interface files was programmed in 2016 under Subaward 00009412 with the University of California Berkeley. Presently there are 7 interface files (Table XIII.), which may be used for data analysis and visualization and (or) interfacing with other computer codes, such as Grizzly. Each file is a two-dimensional array of $\{$ File contents $\}$ (x,time) with space and time meshes and comments. Upper left corner of the space-separated ASCII interface file for $\{$ File contents $\}=\{$ Crack_Depth_vs_time $\}$ is shown in Figure XIII.16 as an example. 


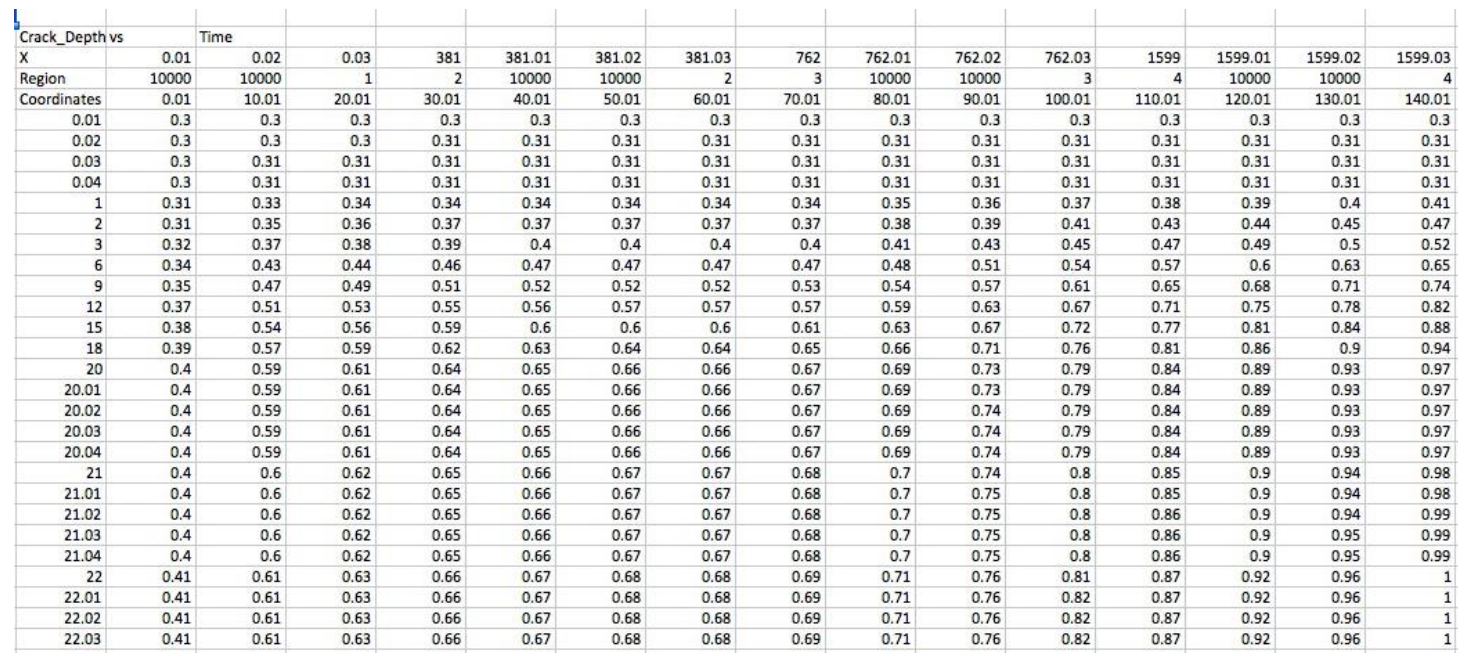

Figure XIII.16. BWR_MASTER interface file example: crack depth along HTC for each time point.

\section{Examples of BWR_MASTER post-processing data visualization}

Interface files may be imported into Excel for data visualization. Excel templates are available from 4D Power, LLC. An example of an importing interface file with $\{$ File contents $\}=$ $\{$ ECP_vs_time $\}$ for plotting the ECP along with the HTC coordinates for a selected period of time in a reactor operating history is shown in Figure XIII.18.

Visualization of particular \{File contents $\}$ in (HTC coordinate, the period of time) is also available. For example, the visualization of \{Crack_Depth_vs_time $\}$ using gnuplot [8] is illustrated in Figure XIII.18.

Interfacing with Grizzly using interface files is possible if BWR_MASTER and Grizzly calculations have been made sequentially or simultaneously by running BWR_MASTER on a Windows OS PC and running Grizzly on any suitable computer. The direct interfacing would require running both codes under the Windows operating system. 


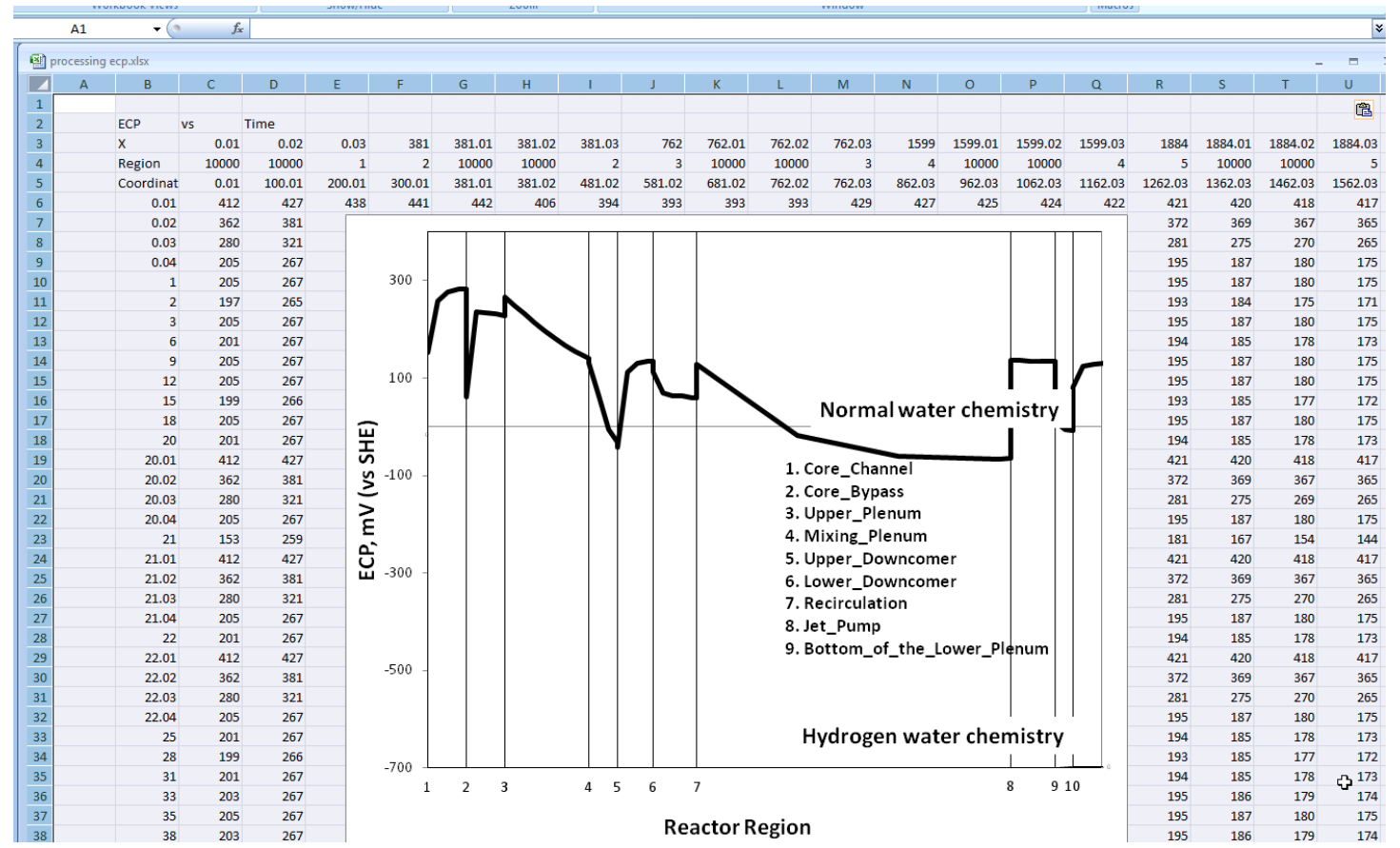

Figure XIII.17. Post-processing in Excel.

\section{XIII.2. Sensitivity analysis}

The goal of sensitivity analysis is to define a contribution of the input data variations to variation of the calculated species concentrations, ECP, and crack growth rate. Theoretical or (and) experimental update of the most sensitive input parameters is required to achieve a better agreement with plant measured data, such as the concentrations of $\mathrm{H}_{2}$ and $\mathrm{O}_{2}$, and the ECP.

The list of 79 input parameters in the sensitivity analysis (Table XIII.) includes radiolytic yields, reaction rate constants, parameters of the Mixed Potential Model, and reactor modeling parameters, such as dose rates, flow velocity, and the hydrodynamic diameters of the flow channels. 


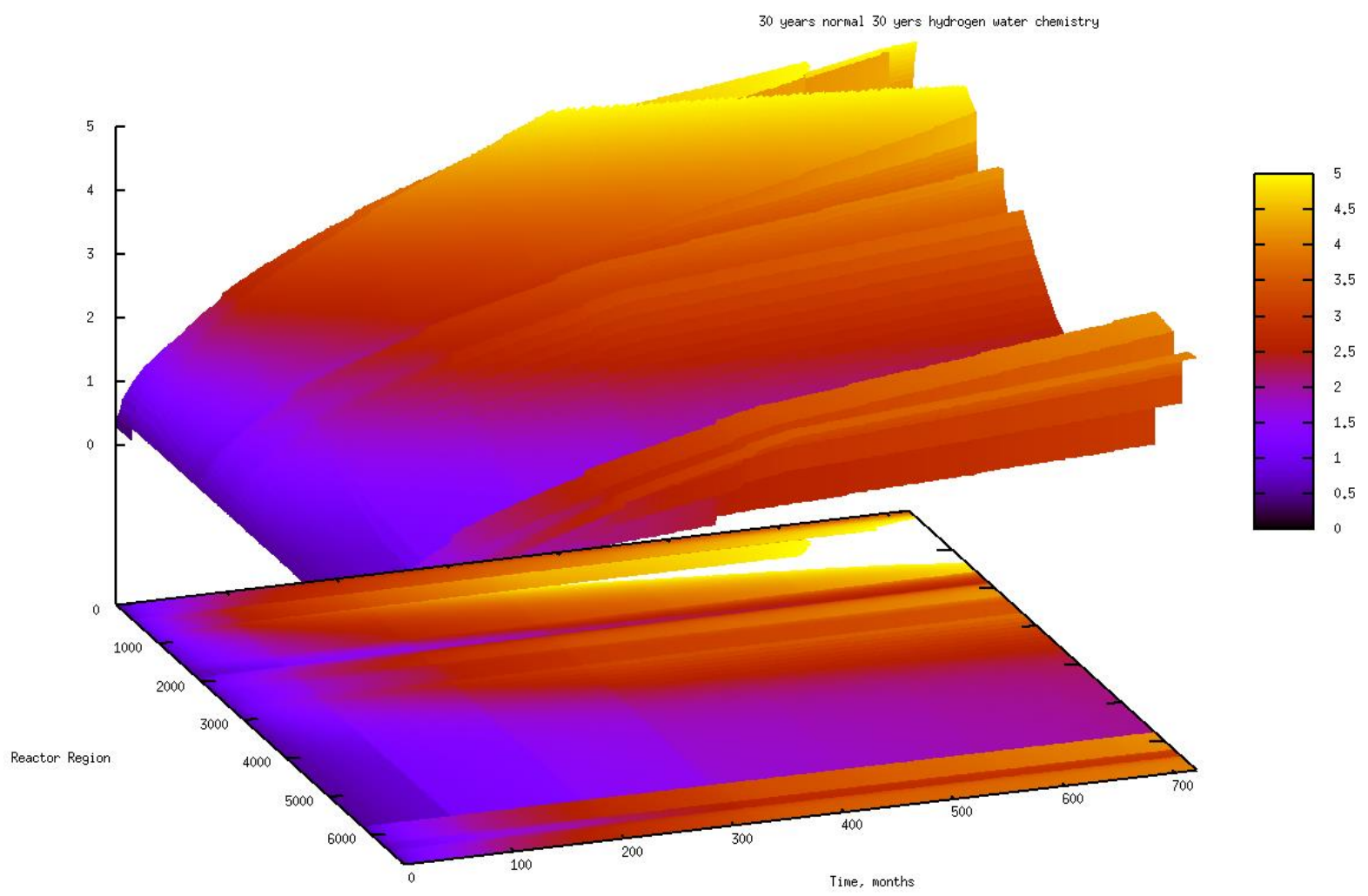

Figure XIII.18. Post-processing with Gnuplot.

The following list of integral parameters has been selected for sensitivity analysis: concentrations of $\mathrm{H}_{2}, \mathrm{O}_{2}$ and $\mathrm{H}_{2} \mathrm{O}_{2}, E C P$, and crack growth rate. Sensitivities of integral parameters to variations in input parameters have been calculated using the "Direct Substitution Method" under 1\% variations in the input parameter. Absolute sensitivities of hydrogen and oxygen concentrations (change in ppb resulting from $1 \%$ change in input parameter) and ECP (change in $\mathrm{mV}$ resulting from $1 \%$ change in input parameter) and relative sensitivity of crack growth rate (change in $\%$ resulting from $1 \%$ change in input parameter) have been calculated for each coordinate point in the reactor heat transport circuit. All calculations have been performed for normal and hydrogen $\left(0.5 \mathrm{ppm}\right.$ of $\mathrm{H}_{2}$ in feedwater) water chemistry conditions. 
Table XIII.3. List of input parameters for sensitivity analysis.

\begin{tabular}{|c|c|c|}
\hline Parameter's group & Parameter & Description \\
\hline Radiolytic yields & Yield of species & $\begin{array}{l}\text { Under gamma radiation }\left(G_{i}\right) \text { and neutron radiation }\left(N_{i}\right) \text { (Error! } \\
\text { Reference source not found. }\end{array}$ \\
\hline Reaction rates & Rate constants & According to Error! Reference source not found. \\
\hline MPM parameters & 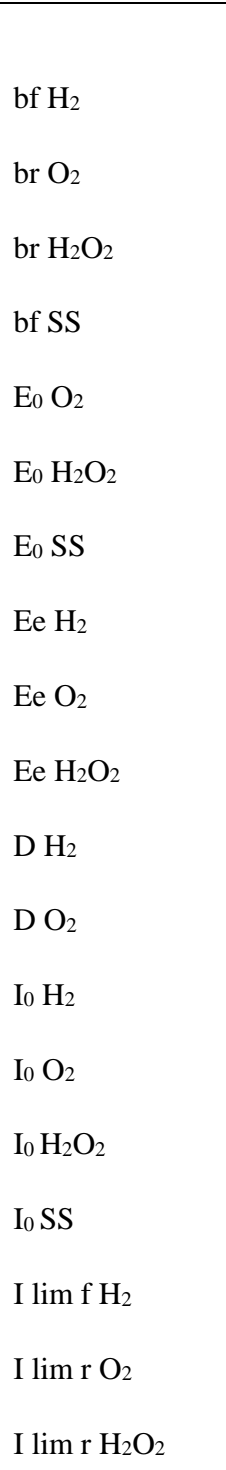 & $\begin{array}{l}\text { According to the section "Error! Reference source not found.": } \\
\text { Tafel constants } \\
\text { Standard potentials } \\
\text { Equilibrium potentials } \\
\text { Limiting currents } \\
\text { Exchange current densities } \\
\text { Diffusivity of species }\end{array}$ \\
\hline $\begin{array}{l}\text { Reactor modeling } \\
\text { parameters }\end{array}$ & $\begin{array}{l}\text { D n } \\
\text { D g } \\
\text { Flow vel } \\
\text { Hydr diam }\end{array}$ & $\begin{array}{l}\text { Neutron dose rates } \\
\text { Gamma dose rates } \\
\text { Flow velocity } \\
\text { Hydrodynamic diameters }\end{array}$ \\
\hline
\end{tabular}




\section{Highest sensitivity to input parameters}

The results of the sensitivity analyses demonstrate that the integral parameters are much more sensitive to radiolytic yields of species in comparison to other input parameters (Table XIII.4 to Table XIII.8.). An update of the presently used yields with more recent data is required in an attempt to improve agreement with measured plant data.

Table XIII.4. The highest sensitivity of $\mathrm{H}_{2}$ concentration to input parameters under normal and hydrogen water chemistry conditions.

\begin{tabular}{l|l|l|l|l|l}
\hline ID & Parameter & $\Delta \mathrm{C}_{\mathrm{H} 2}, \mathrm{ppb}, \mathrm{NWC}$ & $\mathrm{ID}$ & Parameter & $\Delta \mathrm{C}_{\mathrm{H} 2}, \mathrm{ppb}, \mathrm{HWC} 0.5 \mathrm{ppm} \mathrm{H}_{2}$ \\
\hline G 03 & $\mathrm{H}+$ & 18.5 & $\mathrm{G} \mathrm{04}$ & $\mathrm{OH}$ & 27.1 \\
\hline G 01 & $\mathrm{e}$ & 12.23 & $\mathrm{G} \mathrm{01}$ & $\mathrm{e}$ & 25.1 \\
\hline N 03 & $\mathrm{H}+$ & 10.84 & G 03 & $\mathrm{H}+$ & 19.3 \\
\hline N 01 & $\mathrm{e}$ & 5.05 & G 06 & $\mathrm{H} 2 \mathrm{O} 2$ & 17.2 \\
\hline G 04 & OH & 4.07 & N 03 & $\mathrm{H}+$ & 14.2 \\
\hline
\end{tabular}

ECP and crack growth rates are also very sensitive to the standard potential of the hydrogen peroxide reaction. Estimation of actual variations on integral parameters to input data sets will require replacement of the radiolytic data set and the reaction rate (and list) set with updated sets and the MPM parameters with re-evaluated ones.

\section{Sensitivity to radiolytic yields}

The sensitivity of the integral parameters to the radiolytic yields reveals (Table XIII.9 and Figure XIII.19 to Figure XIII.11) that the yields of electrons and protons are of primary importance, followed by the yields of $\mathrm{OH}, \mathrm{H}_{2} \mathrm{O}_{2}$, and $\mathrm{H}_{2}$. The sensitivity seems to be slightly higher under hydrogen water chemistry conditions. 
Table XIII.5. The highest sensitivity of $\mathrm{O}_{2}$ concentration to input parameters under normal and hydrogen water chemistry conditions.

\begin{tabular}{|c|c|c|c|c|c|}
\hline ID & Parameter & $\begin{array}{r}\Delta \mathrm{C}_{\mathrm{O} 2}, \mathrm{ppb} \\
\mathrm{NWC}\end{array}$ & ID & Parameter & $\begin{array}{l}\Delta \mathrm{C}_{\mathrm{O} 2}, \mathrm{ppb} \\
\mathrm{HWC} 0.5 \mathrm{ppm} \\
\mathrm{H}_{2}\end{array}$ \\
\hline G 03 & $\mathrm{H}+$ & 209 & G 03 & $\mathrm{H}+$ & 191 \\
\hline G 04 & $\mathrm{OH}$ & 180 & $\mathrm{~N} 03$ & $\mathrm{H}+$ & 142 \\
\hline $\mathrm{N} 03$ & $\mathrm{H}+$ & 151 & G 01 & $\mathrm{e}$ & 48 \\
\hline G 01 & $\mathrm{e}$ & 150 & N 01 & $\mathrm{e}$ & 43 \\
\hline G06 & $\mathrm{H}_{2} \mathrm{O}_{2}$ & 114 & G 04 & $\mathrm{OH}$ & 8.5 \\
\hline
\end{tabular}

Table XIII.6. The highest sensitivity of $\mathrm{H}_{2} \mathrm{O}_{2}$ concentration to input parameters under normal and hydrogen water chemistry conditions

\begin{tabular}{|c|c|c|c|c|c|}
\hline ID & Parameter & $\begin{array}{l}\Delta \mathrm{CH}_{2} \mathrm{O}_{2}, \mathrm{ppb} \\
\mathrm{NWC}\end{array}$ & ID & Parameter & $\begin{array}{l}\Delta \mathrm{CH}_{2} \mathrm{O}_{2}, \mathrm{ppb} \\
\mathrm{HWC} 0.5 \mathrm{ppm} \\
\mathrm{H}_{2}\end{array}$ \\
\hline G 03 & $\overline{\mathrm{H}^{+}}$ & 656 & G 03 & $\overline{\mathrm{H}^{+}}$ & 560 \\
\hline N 03 & $\mathrm{H}^{+}$ & 451 & $\mathrm{~N} 03$ & $\mathrm{H}^{+}$ & 412 \\
\hline G 01 & $\mathrm{e}$ & 283 & G 01 & $\mathrm{e}$ & 240 \\
\hline G 04 & $\mathrm{OH}$ & 134 & G 04 & $\mathrm{OH}$ & 18.5 \\
\hline G 06 & $\mathrm{H}_{2} \mathrm{O}_{2}$ & 91.4 & N 06 & $\mathrm{H}_{2} \mathrm{O}_{2}$ & 16.3 \\
\hline
\end{tabular}

The sensitivity of hydrogen concentration to a $1 \%$ increase in the radiolytic yield of electrons is low, with the highest values of about $20 \mathrm{ppb}$ in the Core Bypass region (Figure XIII.12). The sensitivity of the oxygen concentration to a $1 \%$ increase in the radiolytic yield of electrons is considerable (Figure XIII.13), especially at the exit from the Upper Plenum (+200 ppb) and in the Recirculation system (+170 ppb under normal and +120 ppb under hydrogen water 
chemistry conditions). The sensitivity of hydrogen peroxide concentration to $\% 1$ increase in radiolytic yield of electrons is high $(+500-600 \mathrm{ppb})$ only in the Core Channel and Core Bypass regions under normal water chemistry conditions and in the lower part of the Upper Plenum (>+100 ppb) under normal and hydrogen water chemistry conditions (Figure XIII.26).

Table XIII.7. The highest sensitivity of $\mathrm{ECP}_{\mathrm{SHE}}$ to input parameters under normal and hydrogen water chemistry conditions.

\begin{tabular}{|c|c|c|c|c|c|}
\hline ID & Parameter & $\begin{array}{c}\Delta \mathrm{ECP}_{\mathrm{SHE}}, \mathrm{mV} \\
\mathrm{NWC}\end{array}$ & ID & Parameter & 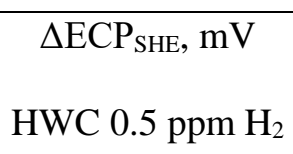 \\
\hline G 01 & $\mathrm{e}$ & 510 & G 03 & $\mathrm{H}^{+}$ & 943 \\
\hline G 03 & $\mathrm{H}^{+}$ & 224 & N 03 & $\mathrm{H}^{+}$ & 908 \\
\hline $\mathrm{N} 01$ & $\mathrm{e}$ & 178 & G 01 & $\mathrm{e}$ & 807 \\
\hline $\mathrm{N} 03$ & $\mathrm{H}^{+}$ & 154 & $\mathrm{~N} 01$ & $\mathrm{e}$ & 650 \\
\hline MPM & $\mathrm{E}_{0} \mathrm{H}_{2} \mathrm{O}_{2}$ & 13 & $\overline{G 04}$ & $\mathrm{OH}$ & 590 \\
\hline
\end{tabular}

Table XIII.8. The highest sensitivity of CGR to input parameters under normal and hydrogen water chemistry conditions.

\begin{tabular}{c|c|c|c|c|c}
\hline ID & Parameter & $\begin{array}{c}\Delta \text { CGR/CGR, } \% \\
\text { NWC }\end{array}$ & ID & Parameter & $\begin{array}{c}\Delta \text { CGR/CGR, } \% \\
\text { HWC 0.5 ppm H }\end{array}$ \\
\hline G 03 & H+ & $1380 . \%$ & G 03 & H+ & $1500 \%$ \\
\hline N 03 & H+ & $490 . \%$ & N 03 & H+ & $590 \%$ \\
\hline G 01 & e & $99.6 \%$ & G 01 & e & $99 \%$ \\
\hline N 01 & e & $87.0 \%$ & N 01 & e & $99 \%$ \\
\hline MPM & E0 H2O2 & $18.0 \%$ & G 04 & OH & $50 \%$ \\
\hline
\end{tabular}

There is also a considerable sensitivity of the ECP (from $+100 \mathrm{mV}$ to above $+300 \mathrm{mV}$ ) in all reactor regions under both normal and hydrogen water chemistry conditions (Figure XIII.27). 
The sensitivity of crack growth rate to a $+1 \%$ variation in the radiolytic yield of the electron is extremely high (Figure XIII.28) and may reach a two or more times increase in the Recirculation region and even more in the Core Channel and Core Bypass under both normal and hydrogen water chemistry conditions. The sensitivity of the crack growth rate is higher under hydrogen water chemistry conditions. At reactor temperatures, the G-values become more uncertain. The variation between different results is quite large, but when the highest and lowest values are disregarded, the variation is acceptable, with the exception of the values of $\mathrm{G}\left(\mathrm{H}_{2} \mathrm{O}_{2}\right)$. The G-values for fast neutrons at reactor temperatures are author estimates as redox-balanced mean values, which have been calculated by neglecting the highest and lowest values. These values are probably correct within about $25 \%$.

There is a general agreement that the radical yields and the yield of $\mathrm{H}_{2}$ increase with increasing temperature. Most results show that the yield of $\mathrm{H}_{2} \mathrm{O}_{2}$ decreases with increasing temperature. Generally, the yields of radicals decrease with increasing LET of the radiation, whereas the opposite is the case for molecular yields.

Table XIII.9. Sensitivity to radiolytic yields.

\begin{tabular}{|c|c|c|c|c|c|c|c|c|c|c|c|}
\hline \multirow[b]{2}{*}{ ID } & \multirow{2}{*}{$\begin{array}{c}\text { Paramete } \\
r\end{array}$} & \multicolumn{2}{|c|}{$\Delta \mathrm{C}_{\mathrm{H} 2}, \mathrm{ppb}$} & \multicolumn{2}{|c|}{$\Delta \mathrm{C}_{02, \mathrm{ppb}}$} & \multicolumn{2}{|c|}{$\Delta \mathrm{C}_{\mathrm{H} 2 \mathrm{O} 2}, \mathrm{ppb}$} & \multicolumn{2}{|c|}{$\triangle \mathrm{ECP}_{\text {SHE }}, \mathrm{mV}$} & \multicolumn{2}{|c|}{$\Delta \mathrm{CGR} / \mathrm{CGR}, \%$} \\
\hline & & NWC & $\begin{array}{c}\mathrm{HWC} 0.5 \\
\mathrm{ppm} \mathrm{H}_{2}\end{array}$ & NWC & $\begin{array}{l}\text { HWC } 0.5 \\
\text { ppm H2 }\end{array}$ & NWC & $\begin{array}{c}\text { HWC } 0.5 \\
\text { ppm } \mathrm{H}_{2}\end{array}$ & NWC & $\begin{array}{c}\mathrm{HWC} 0.5 \\
\mathrm{ppm} \mathrm{H} \mathrm{H}_{2}\end{array}$ & NWC & $\begin{array}{c}\mathrm{HWC} 0.5 \\
\mathrm{ppm} \mathrm{H} \mathrm{H}_{2}\end{array}$ \\
\hline N 01 & e- & -5.05 & 5.81 & -60.5 & -43 & 6.17 & 6.17 & -178 & -650 & $-87.0 \%$ & $-99.0 \%$ \\
\hline N 02 & $\mathrm{H}$ & -0.79 & 3.22 & -22.5 & -1.2 & -19 & -4.28 & -2.28 & -2 & $-3.0 \%$ & $-2.0 \%$ \\
\hline N 03 & $\mathrm{H}+$ & 10.84 & -14.26 & 151 & 142 & 451 & 412 & 154 & 908 & $490.0 \%$ & $590.0 \%$ \\
\hline N 04 & $\mathrm{OH}$ & 1.41 & -7.44 & 49.1 & 2.72 & 39.9 & 7.64 & 4.88 & 7 & $5.2 \%$ & $10.0 \%$ \\
\hline N 05 & $\mathrm{OH}-$ & 0 & 0 & 0 & 0 & 0 & 0 & 0 & 0 & $0.0 \%$ & $0.0 \%$ \\
\hline N 06 & $\mathrm{H} 2 \mathrm{O} 2$ & 2.44 & -13.6 & 90 & 6.99 & 73.3 & 16.3 & 7.2 & 9 & $10.1 \%$ & $10.0 \%$ \\
\hline N 07 & $\mathrm{HO} 2$ & 0.19 & -0.94 & 5.55 & 0.35 & 4.8 & 1.38 & 1.21 & 2 & $2.1 \%$ & $3.0 \%$ \\
\hline N 08 & $\mathrm{HO} 2-$ & 0 & 0 & 0 & 0 & 0 & 0 & 0 & 0 & $0.0 \%$ & $0.0 \%$ \\
\hline N 09 & $\mathrm{O} 2$ & 0 & 0 & 0 & 0 & 0 & 0 & 0 & 0 & $0.0 \%$ & $0.0 \%$ \\
\hline N 10 & O2- & 0 & 0 & 0 & 0 & 0 & 0 & 0 & 0 & $0.0 \%$ & $0.0 \%$ \\
\hline N 11 & $\mathrm{H} 2$ & -3.85 & 11.77 & -85 & -3.85 & -76 & -11.1 & -5.4 & -9 & $-7.6 \%$ & $-12.0 \%$ \\
\hline G 01 & e- & -12.23 & 25.1 & -150 & -48.1 & -283 & -240 & -510 & -807 & $-99.6 \%$ & $-99.2 \%$ \\
\hline G 02 & $\mathrm{H}$ & -2.02 & 7.23 & -51.6 & -2.16 & -45 & -7.73 & -3.89 & -6 & $-5.1 \%$ & $-10.0 \%$ \\
\hline G 03 & $\mathrm{H}+$ & 18.5 & -19.3 & 209 & 191 & 656 & 560 & 224 & 943 & $1380.0 \%$ & $1500.0 \%$ \\
\hline G 04 & $\mathrm{OH}$ & 4.07 & -27.12 & 180 & 8.56 & 134 & 18.5 & 12.1 & 590 & $16.3 \%$ & $50.0 \%$ \\
\hline G 05 & $\mathrm{OH}-$ & 0 & 0 & 0 & 0 & 0 & 0 & 0 & 0 & $0.0 \%$ & $0.0 \%$ \\
\hline G 06 & $\mathrm{H} 2 \mathrm{O} 2$ & 2.96 & -17.2 & 114 & 5.77 & 91.4 & 13.6 & 8.8 & 20 & $10.6 \%$ & $30.0 \%$ \\
\hline G 07 & $\mathrm{HO} 2$ & 0 & 0 & 0 & 0 & 0 & 0 & 0 & 0 & $0.0 \%$ & $0.0 \%$ \\
\hline G 08 & $\mathrm{HO} 2-$ & 0 & 0 & 0 & 0 & 0 & 0 & 0 & 0 & $0.0 \%$ & $0.0 \%$ \\
\hline G 09 & $\mathrm{O} 2$ & 0 & 0 & 0 & 0 & 0 & 0 & 0 & 0 & $0.0 \%$ & $0.0 \%$ \\
\hline G 10 & O2- & 0 & 0 & 0 & 0 & 0 & 0 & 0 & 0 & $0.0 \%$ & $0.0 \%$ \\
\hline G 11 & $\mathrm{H} 2$ & -2.33 & 8.34 & -59.3 & -2.32 & -51.9 & -7.59 & -4.68 & -7 & $-5.2 \%$ & $-9.0 \%$ \\
\hline
\end{tabular}




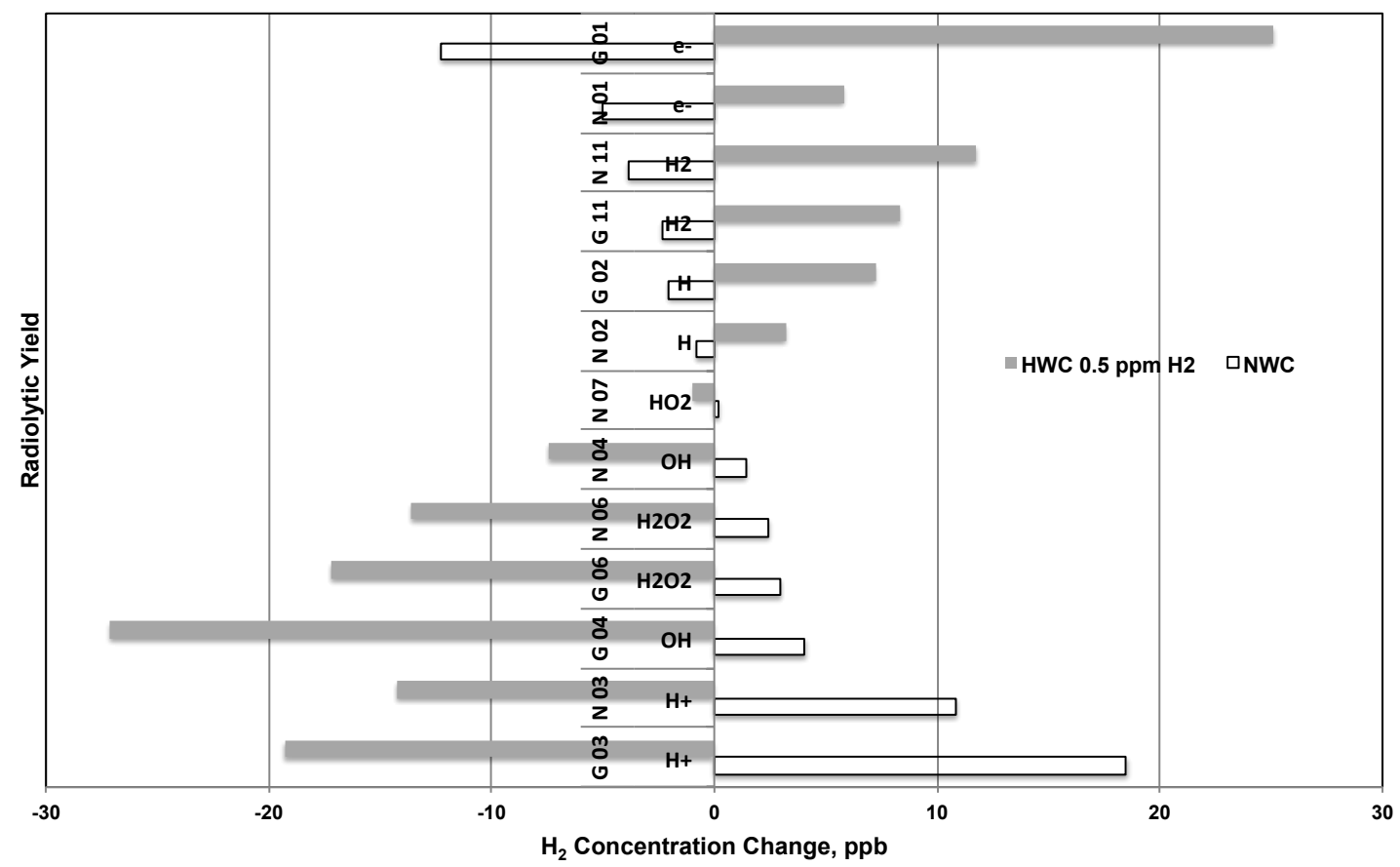

Figure XIII.19. The sensitivity of $\mathrm{H}_{2}$ concentration to radiolytic yields.

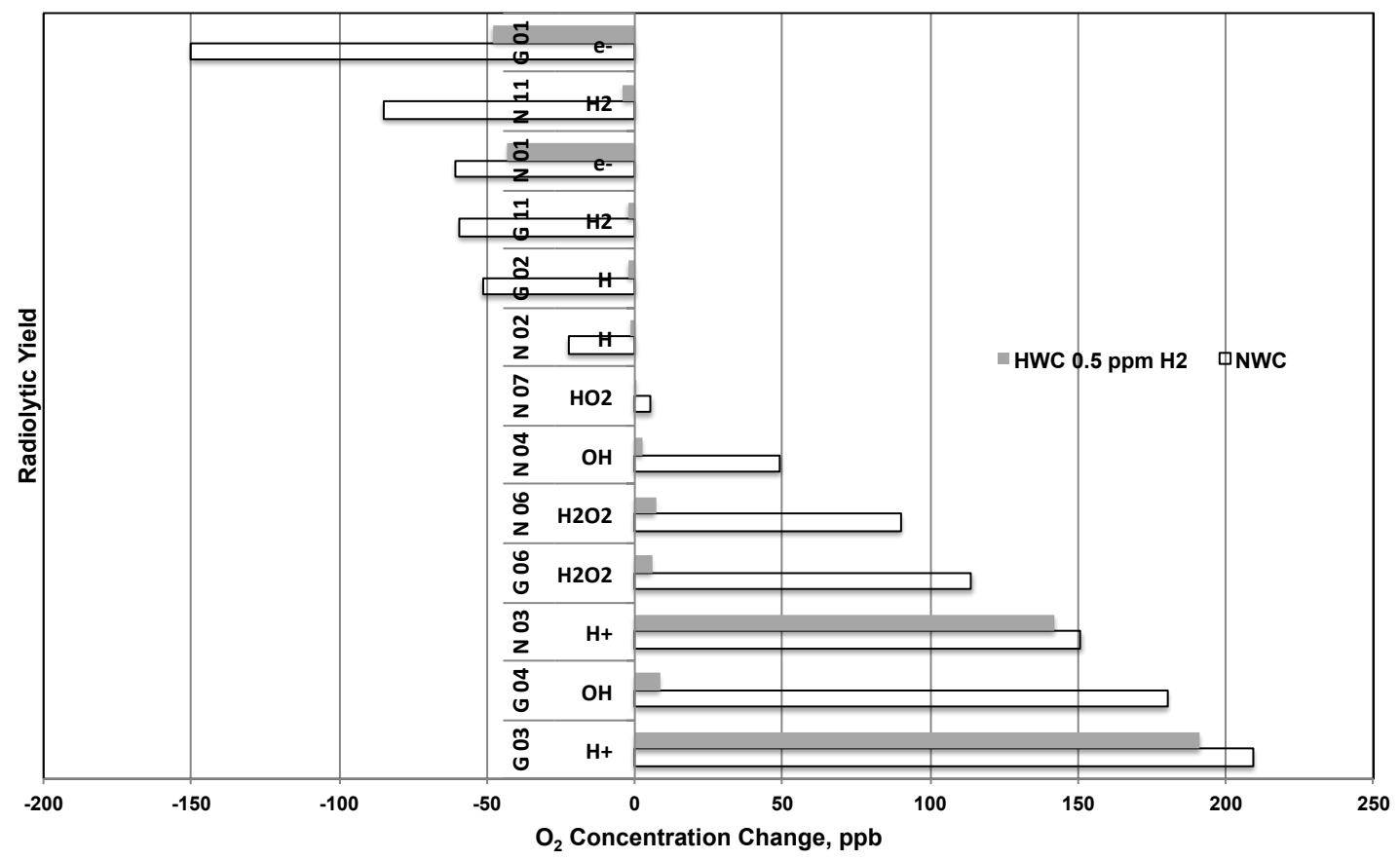

Figure XIII.8. The sensitivity of $\mathrm{O}_{2}$ concentration to radiolytic yields. 


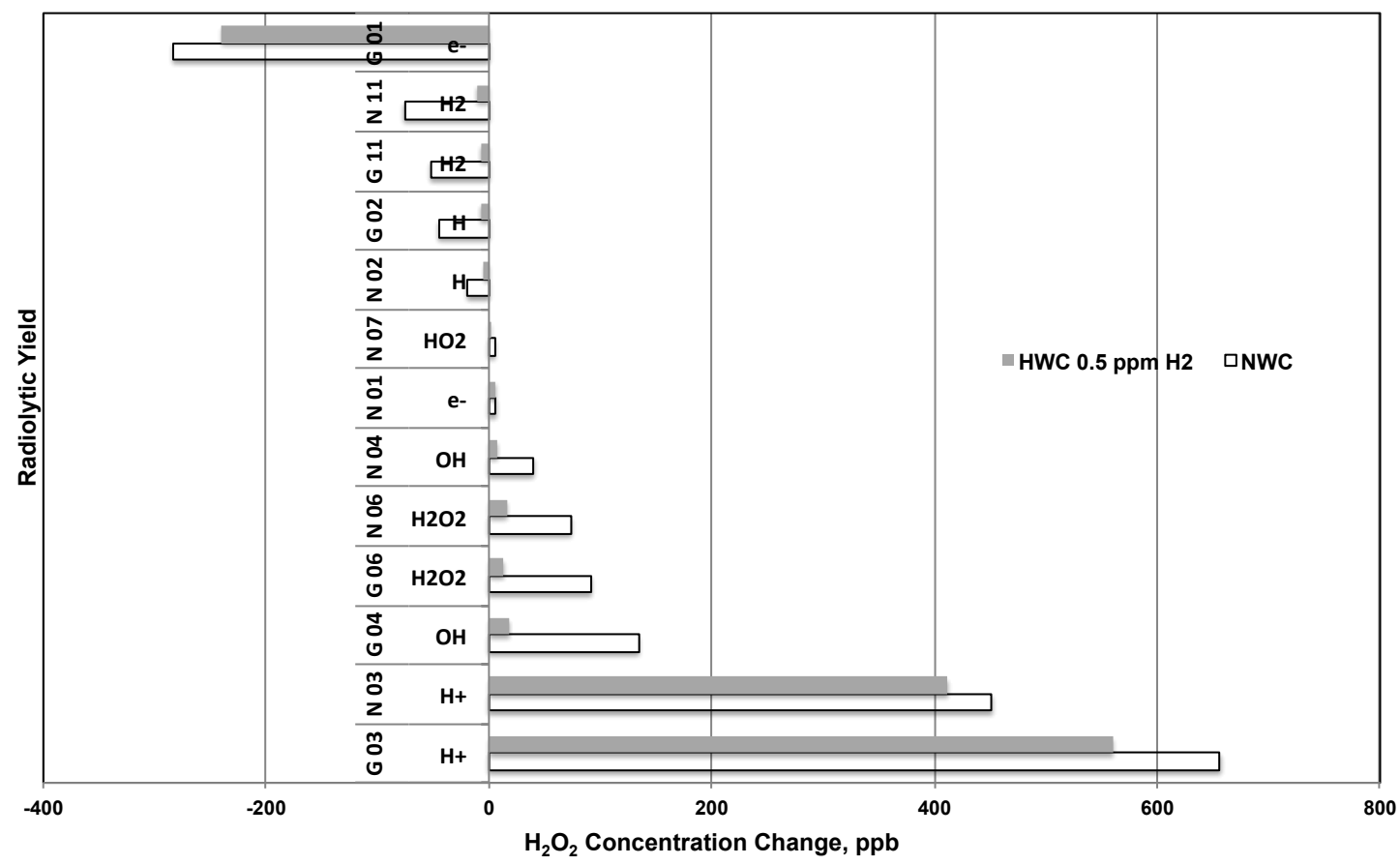

Figure XIII.9. The sensitivity of $\mathrm{H}_{2} \mathrm{O}_{2}$ concentration to radiolytic yields.

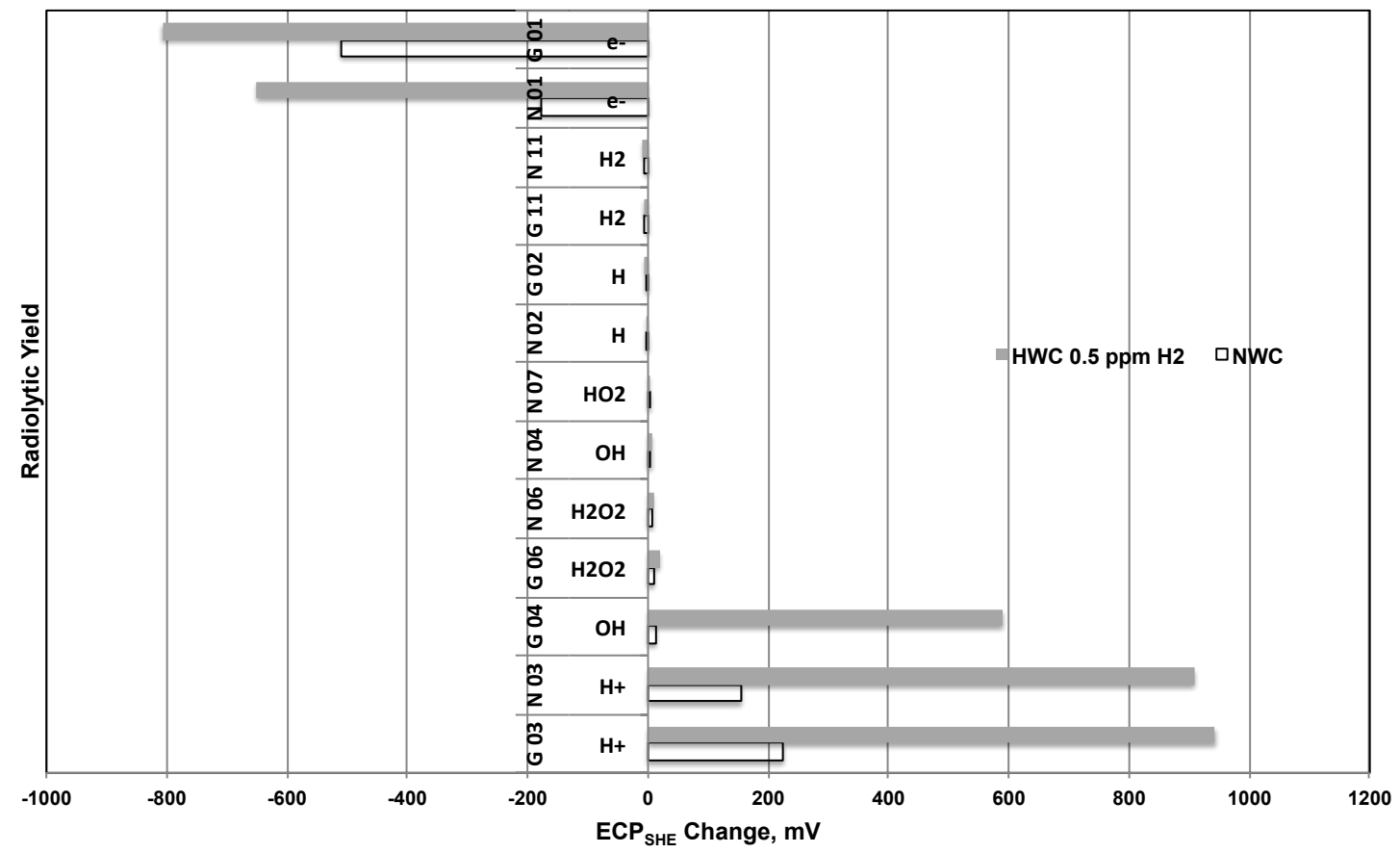

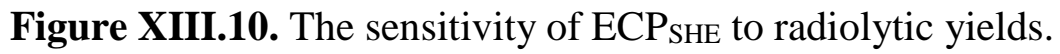




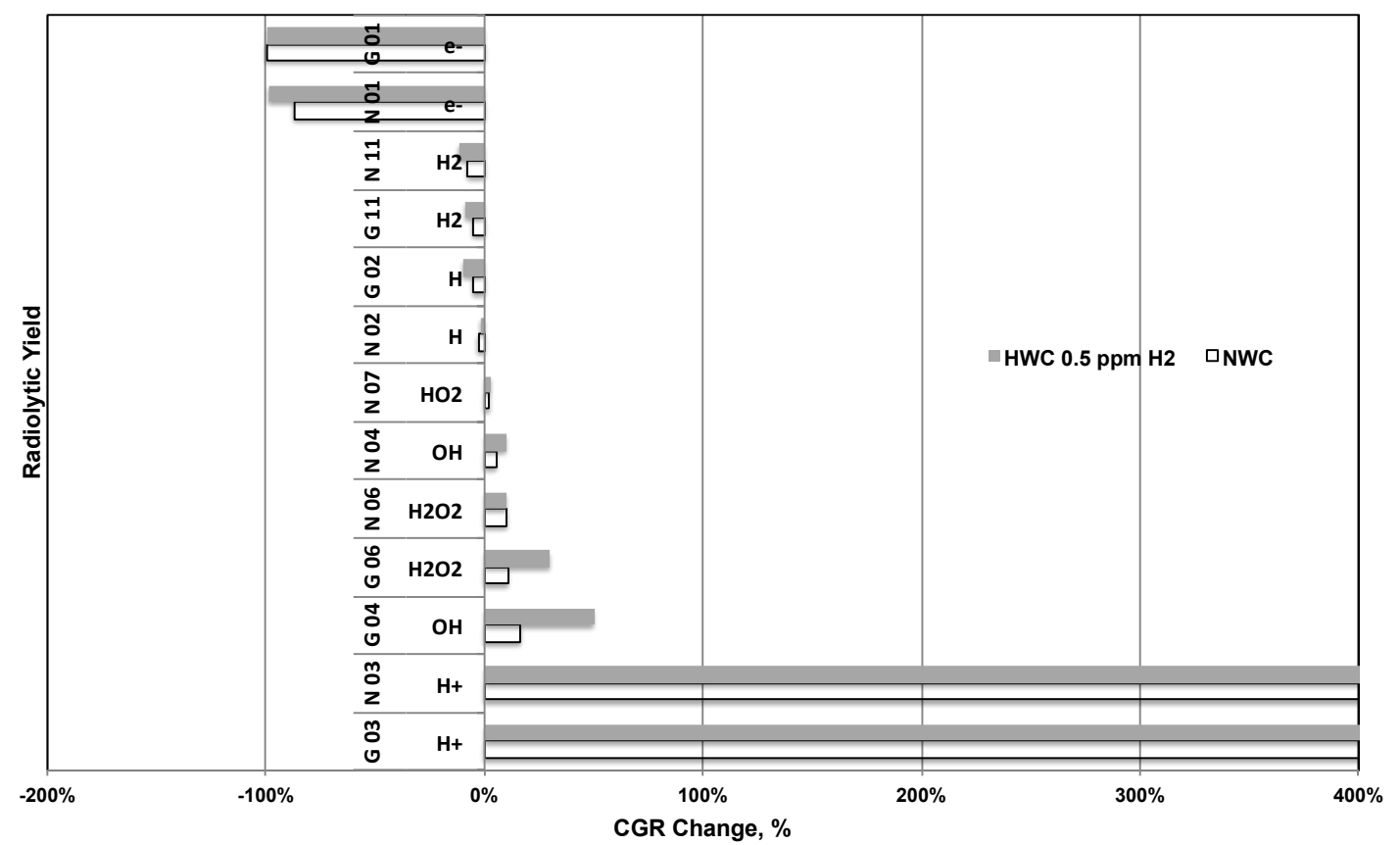

Figure XIII.11. The sensitivity of crack growth rate to radiolytic yields.

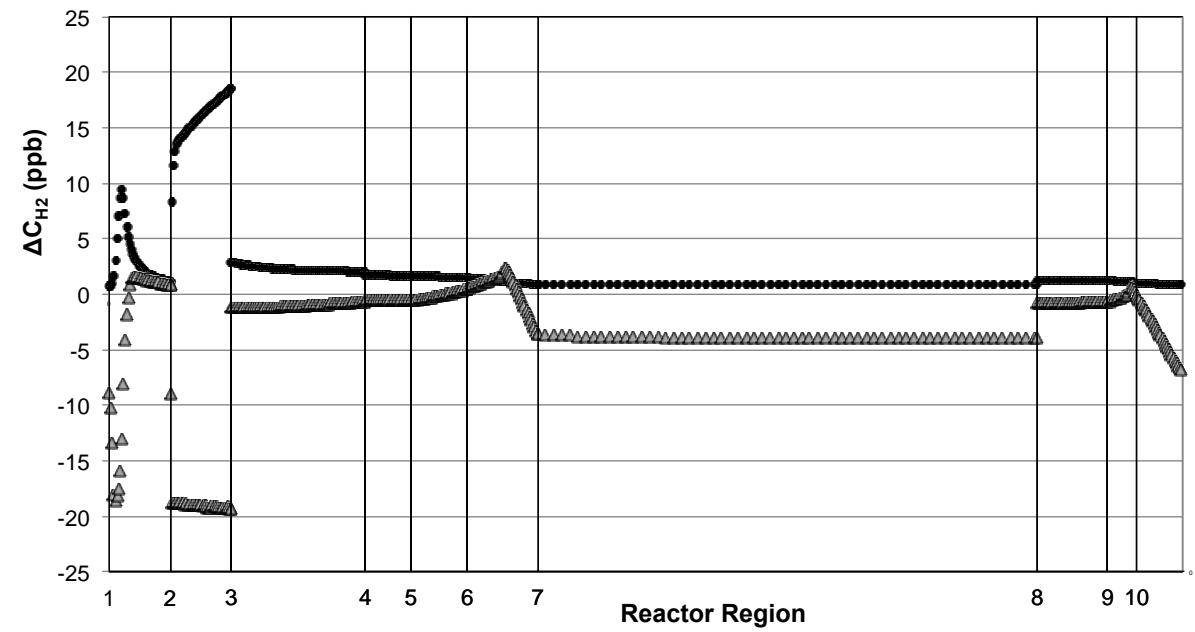

- NWC

$\triangle$ HWC 0.5 ppm H2

1. Core_Channel

2. Core_Bypass

3. Upper_Plenum

5. Upper_Downcomer

6. Lower_Downcomer

7. Recirculation

8. Jet_Pump

9. Bottom of the Lower_Plenum

10. Top_of_the_Lower_Plenum

Figure XIII.12. The sensitivity of $\mathrm{H}_{2}$ concentration to the radiolytic yield of $\mathrm{e}^{-}$under gamma radiation.

Given the high sensitivity to the radiolytic yields, a most recent data set has been used in BWR_MASTER in an attempt to reduce discrepancies with measurements of hydrogen and oxygen concentrations and the ECP in the recirculation sampling line performed during a hydrogen 
water chemistry mini test at the Leibstadt BWR reactor. The impact of using the updated data set is described in the the section "Comparison With Plant Measured Data."

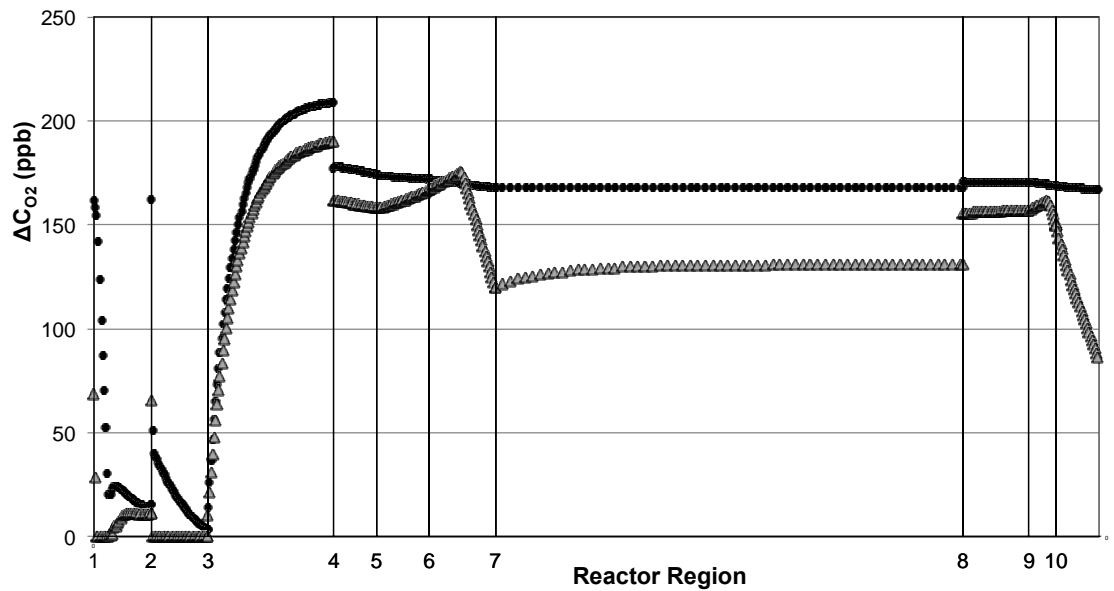

- NWC

$\triangle$ HWC 0.5 ppm H2

1. Core_Channel

2. Core_Bypass

4. Upper_Plenum

5. Upper_Downcomer

6. Lower_Downcomer

7. Recirculation

9. Bottom_of_the_Lower_Plenum

10. Top_of_the_Lower_Plenum

Figure XIII.13. The sensitivity of $\mathrm{O}_{2}$ concentration to the radiolytic yield of $\mathrm{e}^{-}$under gamma radiation.

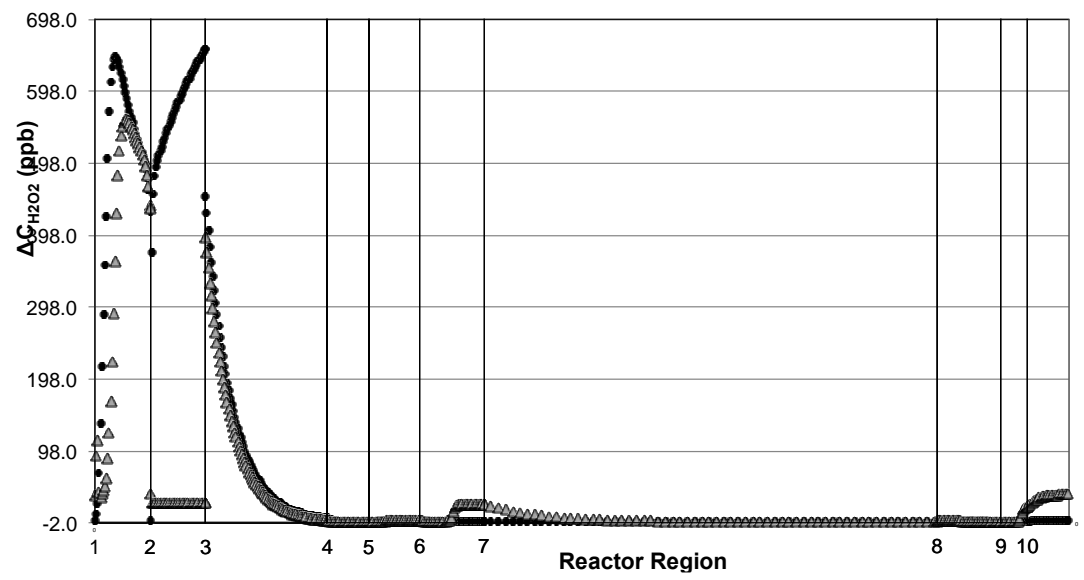

- NWC

$\triangle$ HWC 0.5 ppm H2

Core_Channel

3. Upper_Plenum

4. Mixing_Plenum

5. Upper_Downcomer

6. Lower_Downcomer

7. Recirculation

8. Jet_Pump

9. Bottom_of_the_Lower_Plenum

10. Top_of_the_Lower_Plenum

Figure XIII.26. The sensitivity of $\mathrm{H}_{2} \mathrm{O}_{2}$ concentration to the radiolytic yield of $\mathrm{e}^{-}$under gamma radiation. 


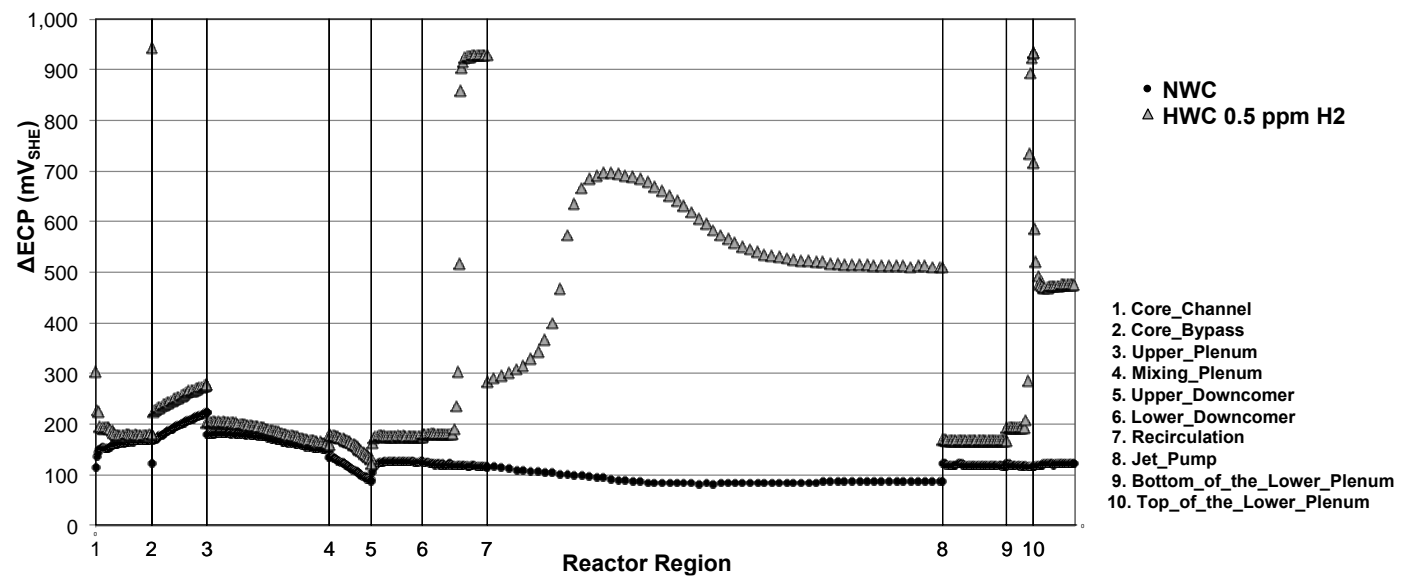

Figure XIII.27. The sensitivity of $\mathrm{ECP}_{\mathrm{SHE}}$ to the radiolytic yield of $\mathrm{e}^{-}$under gamma radiation.

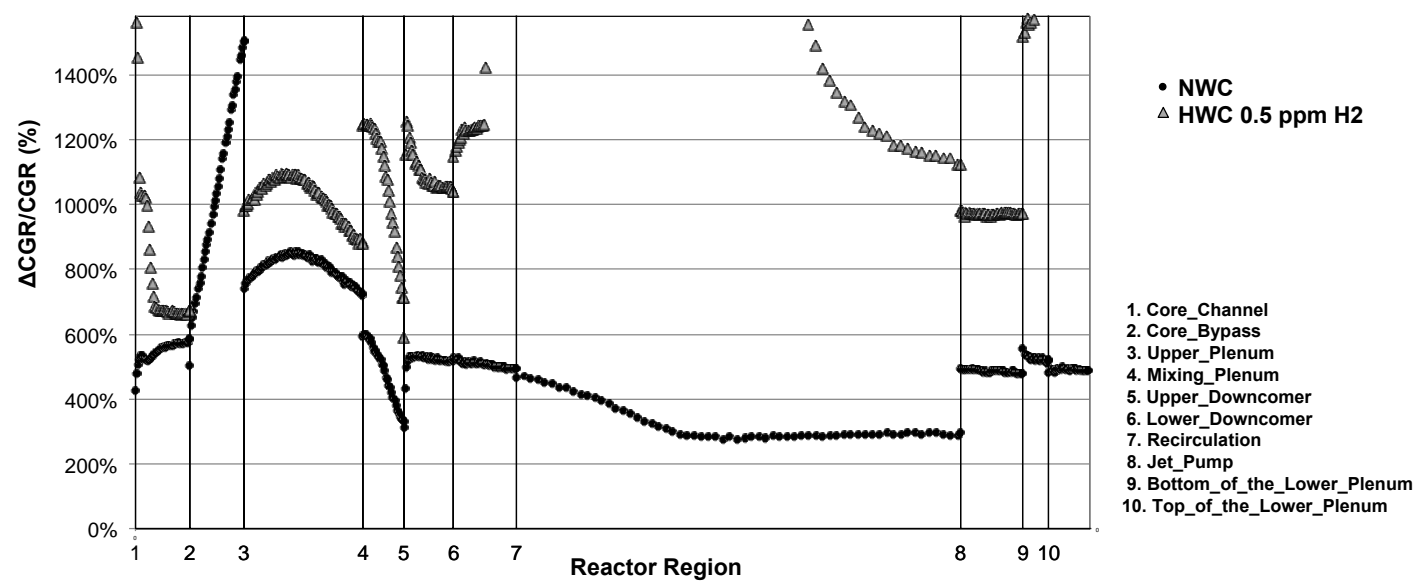

Figure XIII.28. The sensitivity of the crack growth rate to the radiolytic yield of $\mathrm{e}^{-}$under gamma radiation.

\section{Sensitivity to reaction rates}

The sensitivity of integral parameters to $+1 \%$ variation in reaction rates under normal and hydrogen water chemistry conditions (Table XIII.10) does not exceed a few ppb for hydrogen, oxygen, and hydrogen peroxide concentrations, few $\mathrm{mV}$ for ECP and few \% for crack growth rate. Integral parameters are most sensitive to rates of reactions $22\left(\mathrm{HO}_{2}=\mathrm{O}_{2}{ }^{-}+\mathrm{H}^{+}\right), 18\left(\mathrm{OH}+\mathrm{H}_{2}=\mathrm{H}\right.$ $\left.+\mathrm{H}_{2} \mathrm{O}\right)$ and $11\left(\mathrm{OH}+\mathrm{O}_{2}^{-}=\mathrm{OH}^{-}+\mathrm{O}_{2}\right)$. 
Table XIII.10. Sensitivity to reaction rates.

\begin{tabular}{|c|c|c|c|c|c|c|c|c|c|c|c|}
\hline \multirow[b]{2}{*}{ ID } & \multirow[b]{2}{*}{ Parameter } & \multicolumn{2}{|c|}{$\Delta \mathrm{C}_{\mathrm{H} 2}, \mathrm{ppb}$} & \multicolumn{2}{|c|}{$\Delta \mathrm{C}_{02, \mathrm{ppb}}$} & \multicolumn{2}{|c|}{$\Delta \mathrm{C}_{\mathrm{H} 2 \mathrm{O} 2, \mathrm{ppb}}$} & \multicolumn{2}{|c|}{$\triangle \mathrm{ECP}_{\mathrm{SHE}}, \mathrm{mV}$} & \multicolumn{2}{|c|}{$\begin{array}{c}\Delta \text { CGR/CGR, } \\
\%\end{array}$} \\
\hline & & NWC & $\begin{array}{c}\mathrm{HWC} \\
0.5 \\
\mathrm{ppm} \\
\mathrm{H}_{2}\end{array}$ & NWC & $\begin{array}{c}\mathrm{HWC} \\
0.5 \\
\mathrm{ppm} \\
\mathrm{H}_{2}\end{array}$ & NWC & $\begin{array}{c}\mathrm{HWC} \\
0.5 \\
\mathrm{ppm} \\
\mathrm{H}_{2}\end{array}$ & NWC & $\begin{array}{c}\mathrm{HWC} \\
0.5 \\
\mathrm{ppm} \\
\mathrm{H}_{2}\end{array}$ & NWC & $\begin{array}{c}\mathrm{HWC} \\
0.5 \\
\mathrm{ppm} \\
\mathrm{H}_{2}\end{array}$ \\
\hline R Rate 01 & $\mathrm{e}-\mathrm{H}_{2} \mathrm{O}=\mathrm{H}+\mathrm{OH}-$ & 0.03 & -0.20 & 0.33 & -0.06 & 0.63 & -0.12 & -1.35 & 2.65 & $-2 \%$ & $5 \%$ \\
\hline R Rate 02 & $\mathrm{e}-+\mathrm{H}+=\mathrm{H}$ & 0.06 & -0.25 & 0.74 & 0.67 & 2.12 & 2.73 & 1.19 & 2.00 & $2 \%$ & $5 \%$ \\
\hline R Rate 03 & $\mathrm{e}-+\mathrm{OH}=\mathrm{OH}-$ & 0.04 & 0.20 & 0.74 & 0.51 & 0.99 & 0.48 & -2.20 & -1.70 & $-2 \%$ & $-3 \%$ \\
\hline R Rate 04 & $\mathrm{e}-+\mathrm{H}_{2} \mathrm{O} 2=\mathrm{OH}+\mathrm{OH}-$ & -0.07 & 0.34 & -1.13 & -0.89 & -3.94 & -3.57 & -1.88 & -2.00 & $-2 \%$ & $-7 \%$ \\
\hline R Rate 05 & $\mathrm{H}+\mathrm{H}=\mathrm{H} 2$ & 0.03 & -0.16 & 0.28 & 0.08 & 0.56 & 0.45 & 0.60 & 12.20 & $1 \%$ & $6 \%$ \\
\hline R Rate 06 & $\mathrm{e}-+\mathrm{HO} 2=\mathrm{HO}_{2}-$ & 0.03 & -0.13 & 0.33 & 0.04 & 0.64 & 0.09 & 0.93 & 1.72 & $1 \%$ & $3 \%$ \\
\hline R Rate 07 & $\mathrm{e}-+\mathrm{O} 2=\mathrm{O} 2$ & 0.07 & 0.22 & 0.39 & 0.22 & 1.95 & 0.99 & 1.12 & 1.50 & $2 \%$ & $2 \%$ \\
\hline R Rate 08 & $2 \mathrm{e}-+2 \mathrm{H} 2 \mathrm{O}=2 \mathrm{OH}-+\mathrm{H}_{2}$ & 0.03 & 0.09 & -0.24 & -0.05 & -0.48 & -0.12 & -1.12 & -1.21 & $-1 \%$ & $-2 \%$ \\
\hline R Rate 09 & $\mathrm{OH}+\mathrm{OH}=\mathrm{H} 2 \mathrm{O} 2$ & 0.03 & -0.17 & 0.36 & 0.36 & 0.79 & 1.43 & 1.03 & 2.00 & $2 \%$ & $3 \%$ \\
\hline R Rate 10 & $\mathrm{OH}+\mathrm{HO} 2=\mathrm{H} 2 \mathrm{O}+\mathrm{O} 2$ & 0.05 & 0.19 & 0.41 & 0.03 & 0.68 & 0.12 & 0.95 & 5.09 & $1 \%$ & $-5 \%$ \\
\hline R Rate 11 & $\mathrm{OH}+\mathrm{O} 2-=\mathrm{OH}-+\mathrm{O} 2$ & 0.13 & 0.18 & 0.79 & 0.24 & 0.79 & 1.06 & 0.47 & 1.00 & $1 \%$ & $3 \%$ \\
\hline R Rate 12 & $\mathrm{OH}-+\mathrm{H}=\mathrm{e}_{-}+\mathrm{H} 2 \mathrm{O}$ & 0.03 & -0.20 & 0.25 & -0.07 & 0.48 & -0.13 & -1.08 & -2.03 & $1 \%$ & $3 \%$ \\
\hline R Rate 13 & $\mathrm{e}-+\mathrm{H}+\mathrm{H}_{2} \mathrm{O}=\mathrm{OH}-+\mathrm{H}_{2}$ & 0.02 & 0.07 & -0.17 & -0.05 & -0.33 & -0.10 & -1.00 & -0.90 & $-1 \%$ & $-2 \%$ \\
\hline R Rate 14 & $\mathrm{e}-+\mathrm{HO} 2-+\mathrm{H} 2 \mathrm{O}=\mathrm{OH}+2 \mathrm{OH}-$ & -0.02 & 0.21 & -0.21 & -0.06 & -0.40 & 0.12 & 1.08 & -2.78 & $1 \%$ & $-5 \%$ \\
\hline R Rate 15 & $\mathrm{H}++\mathrm{OH}-=\mathrm{H}_{2} \mathrm{O}$ & 0.04 & 0.21 & -0.21 & -0.12 & 0.73 & -0.31 & -1.49 & -3.00 & $-2 \%$ & $-6 \%$ \\
\hline R Rate 16 & $\mathrm{H}_{2} \mathrm{O}=\mathrm{H}_{+}+\mathrm{OH}_{-}$ & 0.06 & -0.24 & 0.51 & 0.41 & 1.37 & 1.77 & 1.59 & 2.00 & $2 \%$ & $3 \%$ \\
\hline R Rate 17 & $\mathrm{H}+\mathrm{OH}=\mathrm{H} 2 \mathrm{O}$ & -0.02 & 0.20 & -0.21 & 0.08 & -0.43 & 0.26 & -1.00 & 3.04 & $-1 \%$ & $4 \%$ \\
\hline R Rate 18 & $\mathrm{OH}+\mathrm{H} 2=\mathrm{H}+\mathrm{H} 2 \mathrm{O}$ & -0.21 & -0.17 & -1.39 & -0.79 & -1.30 & -2.83 & -1.29 & -1.50 & $-2 \%$ & $1 \%$ \\
\hline R Rate 19 & $\mathrm{OH}+\mathrm{H}_{2} \mathrm{O} 2=\mathrm{H} 2 \mathrm{O}+\mathrm{HO} 2$ & 0.09 & 0.19 & 0.44 & 0.29 & -1.03 & -1.11 & -1.07 & -1.50 & $-2 \%$ & $-2 \%$ \\
\hline R Rate 20 & $\mathrm{H}+\mathrm{H}_{2} \mathrm{O} 2=\mathrm{OH}+\mathrm{H} 2 \mathrm{O}$ & 0.03 & 0.12 & -0.27 & -0.20 & -0.55 & -1.16 & -1.01 & -1.20 & $-2 \%$ & $-4 \%$ \\
\hline R Rate 21 & $\mathrm{H}+\mathrm{O} 2=\mathrm{HO} 2$ & 0.07 & 0.15 & 0.58 & 0.08 & 1.07 & 0.36 & -1.35 & -2.04 & $-2 \%$ & $8 \%$ \\
\hline R Rate 22 & $\mathrm{HO} 2=\mathrm{O} 2-+\mathrm{H}^{+}$ & -0.63 & -0.56 & -4.47 & -2.38 & -2.80 & -8.36 & 1.98 & -3.00 & $2 \%$ & $-3 \%$ \\
\hline R Rate 23 & $\mathrm{O} 2-+\mathrm{H}+=\mathrm{HO} 2$ & 0.02 & 0.20 & 0.18 & 0.04 & 0.30 & 0.12 & -1.01 & -1.58 & $1 \%$ & $4 \%$ \\
\hline R Rate 24 & $2 \mathrm{HO} 2=\mathrm{H} 2 \mathrm{O} 2+\mathrm{O} 2$ & 0.03 & 0.20 & 0.32 & -0.06 & 0.62 & 0.12 & -1.57 & -1.99 & $-2 \%$ & $-4 \%$ \\
\hline R Rate 25 & $2 \mathrm{O} 2-+2 \mathrm{H} 2 \mathrm{O}=\mathrm{H} 2 \mathrm{O} 2+\mathrm{O} 2+2 \mathrm{OH}-$ & -0.05 & 0.17 & -0.32 & -0.16 & -0.33 & -0.67 & -1.03 & -3.00 & $2 \%$ & $-3 \%$ \\
\hline R Rate 26 & $\mathrm{H}+\mathrm{HO} 2=\mathrm{H} 2 \mathrm{O} 2$ & 0.03 & -0.15 & 0.35 & -0.60 & 0.69 & 0.06 & 1.04 & 2.03 & $1 \%$ & $4 \%$ \\
\hline R Rate 27 & $\mathrm{H}+\mathrm{O} 2-=\mathrm{HO} 2-$ & -0.04 & -0.13 & 0.34 & -0.16 & 0.73 & 0.31 & 1.20 & 1.50 & $2 \%$ & $4 \%$ \\
\hline R Rate 28 & $\mathrm{e}-+\mathrm{O} 2-+\mathrm{H} 2 \mathrm{O}=\mathrm{HO}_{2}-+\mathrm{OH}-$ & 0.04 & -0.20 & 0.39 & 0.04 & 0.75 & -0.12 & -1.13 & 2.62 & $-2 \%$ & $3 \%$ \\
\hline R Rate 29 & $\mathrm{OH}-+\mathrm{H}_{2} \mathrm{O} 2=\mathrm{HO}_{2}-+\mathrm{H}_{2} \mathrm{O}$ & 0.04 & 0.20 & 0.39 & 0.23 & 0.82 & 0.91 & 1.33 & 1.00 & $2 \%$ & $1 \%$ \\
\hline R Rate 30 & $2 \mathrm{H} 2 \mathrm{O} 2=2 \mathrm{H} 2 \mathrm{O}+\mathrm{O} 2$ & 0.03 & -0.16 & 0.40 & 0.38 & -1.31 & 1.34 & -3.00 & -2.00 & $-4 \%$ & $-3 \%$ \\
\hline R Rate 31 & $\mathrm{H}+\mathrm{H} 2 \mathrm{O}=\mathrm{H} 2+\mathrm{OH}$ & 0.03 & -0.18 & 0.32 & -0.06 & 0.62 & -0.11 & -1.00 & 2.44 & $-1 \%$ & $5 \%$ \\
\hline R Rate 32 & $\mathrm{H} 2 \mathrm{O}+\mathrm{HO}_{2}-=\mathrm{H}_{2} \mathrm{O} 2+\mathrm{OH}-$ & 0.03 & -0.17 & 0.29 & -0.25 & 0.49 & -0.88 & -1.94 & -1.00 & $-2 \%$ & $-3 \%$ \\
\hline R Rate 33 & $\mathrm{HO} 2+\mathrm{O} 2-=\mathrm{O} 2+\mathrm{HO} 2-$ & 0.04 & 0.16 & 0.41 & -0.05 & 0.83 & -0.09 & -1.43 & -3.05 & $-2 \%$ & $-3 \%$ \\
\hline R Rate 34 & $\mathrm{H} 2 \mathrm{O} 2=2 \mathrm{OH}$ & 0.04 & -0.15 & 0.39 & -0.36 & 0.67 & -1.56 & -1.20 & -2.00 & $-2 \%$ & $-2 \%$ \\
\hline
\end{tabular}

Rate constants at ambient temperature are generally well-known and were given together with assumed activation energies. For diffusion-controlled reactions, an activation energy of 3 $\mathrm{kcal} / \mathrm{mole}(12.6 \mathrm{~kJ} / \mathrm{mole})$ was assumed. For slower reactions, higher activation energies were assumed. However, in order to be able to make more reliable calculations of radiolysis in the coolant, it is necessary to determine rate constants experimentally at reactor temperature.

It is preferable to use experimentally determined rate constants in radiolysis calculations at high temperatures only in the temperature range in which the experiments were carried out. However, in some cases, it is necessary to use the experimental activation energy in order to 
extrapolate rate constants outside this range. This is the case for experiments with added $\mathrm{H}_{2} \mathrm{O}_{2}$, because of the low thermal stability of $\mathrm{H}_{2} \mathrm{O}_{2}$ in the small experimental cell.

There are considerable differences in the rate constants used at reactor temperatures. There is a general agreement about the mechanism and rate constants to be used at ambient temperature. However, there is a lack of experimental data for the high-temperature rate constants of a number

of reactions. Normally, an activation energy for diffusion-controlled reactions of 12.614 .2 $\mathrm{kJ} / \mathrm{mole}$ is assumed when no experimental data exist. However, this is an uncertain assumption. A more reasonable approach would be to use values based on experimentally-determined activation energies when they can be found and assumed activation energies when no experimental values are available. Additional uncertainties arise when rate constants are used outside of the temperature range in which they were determined.

\section{Sensitivity to MPM Input Parameters}

Determining sensitivity to the MPM input parameters will aid in determining a priority of experimental and theoretical re-evaluation of certain parameters in order to achieve better agreement with the measured plant data. The sensitivity of the ECP (in $\mathrm{mV}$ ) and crack growth rate (in \%) have been calculated for a $+1 \%$ increase in input parameters under normal and hydrogen (0.5 ppm of hydrogen in feedwater) water chemistry conditions (

\section{Table XIII.1).}

The ECP is most sensitive to the exchange current density for stainless steel (under hydrogen water chemistry conditions), standard potentials for oxygen and hydrogen peroxide (both under normal and hydrogen water chemistry conditions), and to the Tafel constants for hydrogen, oxygen and hydrogen peroxide (both under normal and hydrogen water chemistry conditions). 
Experimental re-evaluation of the exchange current density for stainless steel and the Tafel constants has been performed in the current project. The additional effort on re-estimation of standard potentials for oxygen and hydrogen peroxide is recommended.

Table XIII.1. Sensitivity to MPM Parameters.

\begin{tabular}{|c|c|c|c|c|c|}
\hline \multirow[b]{2}{*}{ ID } & \multirow[b]{2}{*}{ Parameter } & \multicolumn{2}{|c|}{$\Delta \mathrm{ECP}_{\mathrm{SHE}}, \mathrm{mV}$} & \multicolumn{2}{|c|}{$\Delta \mathrm{CGR} / \mathrm{CGR}, \%$} \\
\hline & & NWC & $\begin{array}{l}\text { HWC } 0.5 \mathrm{ppm} \\
\mathrm{H}_{2}\end{array}$ & NWC & $\begin{array}{l}\text { HWC } 0.5 \text { ppm } \\
\mathrm{H}_{2}\end{array}$ \\
\hline MPM & bf $\mathrm{H}_{2}$ & 4 & 5 & $4.0 \%$ & $8.0 \%$ \\
\hline MPM & br $\mathrm{O}_{2}$ & -4 & -7 & $-6.0 \%$ & $-4.0 \%$ \\
\hline MPM & br $\mathrm{H}_{2} \mathrm{O}_{2}$ & -4 & -4 & $-6.0 \%$ & $-5.0 \%$ \\
\hline MPM & bf SS & 0 & 1 & $0.0 \%$ & $0.0 \%$ \\
\hline MPM & $\mathrm{E}_{0} \mathrm{O}_{2}$ & 10 & 9 & $17.0 \%$ & $8.0 \%$ \\
\hline MPM & $\mathrm{E}_{0} \mathrm{H}_{2} \mathrm{O}_{2}$ & 13 & 10 & $18.0 \%$ & $13.0 \%$ \\
\hline MPM & $\mathrm{E}_{0} \mathrm{SS}$ & -1 & -8 & $-1.9 \%$ & $-2.6 \%$ \\
\hline MPM & $\mathrm{E}_{\mathrm{e}} \mathrm{H}_{2}$ & -3 & -4 & $3.0 \%$ & $6.0 \%$ \\
\hline MPM & $\mathrm{E}_{\mathrm{e}} \mathrm{O} 2$ & 4 & 3 & $6.0 \%$ & $3.0 \%$ \\
\hline MPM & $\mathrm{E}_{\mathrm{e}} \mathrm{H}_{2} \mathrm{O}_{2}$ & 5 & 4 & $7.0 \%$ & $6.0 \%$ \\
\hline MPM & $\mathrm{D} \mathrm{H}_{2}$ & -1 & 0 & $-1.0 \%$ & $0.0 \%$ \\
\hline MPM & $\mathrm{D} \mathrm{O}_{2}$ & 1 & 12 & $2.0 \%$ & $8.0 \%$ \\
\hline MPM & $\mathrm{I}_{0} \mathrm{H}_{2}$ & -1 & -1 & $-1.5 \%$ & $-1.6 \%$ \\
\hline MPM & $\mathrm{I}_{0} \mathrm{O}_{2}$ & 1 & 1 & $1.9 \%$ & $1.5 \%$ \\
\hline MPM & $\mathrm{I}_{0} \mathrm{H}_{2} \mathrm{O}_{2}$ & 1 & 1 & $1.9 \%$ & $1.6 \%$ \\
\hline MPM & $\mathrm{I}_{0} \mathrm{SS}$ & -2 & -18 & $-3.0 \%$ & $-8.0 \%$ \\
\hline
\end{tabular}




\begin{tabular}{c|l|cccc} 
MPM & $\mathrm{I} \lim \mathrm{f} \mathrm{H}_{2}$ & 0 & 0 & $0.0 \%$ & $0.0 \%$ \\
$\mathrm{MPM}$ & $\mathrm{I} \lim \mathrm{r} \mathrm{O}_{2}$ & 0 & 1 & $0.0 \%$ & $0.0 \%$ \\
$\mathrm{MPM}$ & $\mathrm{I} \operatorname{lim~} \mathrm{r} \mathrm{H}_{2} \mathrm{O}_{2}$ & 1 & 18 & $2.0 \%$ & $8.0 \%$ \\
\hline
\end{tabular}

\section{The sensitivity to reactor modeling parameters}

Reactor modeling parameters include neutron and gamma dose rate profiles, coolant velocity, and hydrodynamic diameter of the flow channels. These data are obtained from plant engineers, and an estimation of their accuracy is necessary.

The sensitivity of BWR_MASTER to changes in the $\mathrm{H}_{2}, \mathrm{O}_{2}$ and $\mathrm{H}_{2} \mathrm{O}_{2}$ concentrations (in $\mathrm{ppb}$ ), the ECP (in $\mathrm{mV}$ ), and the crack growth rate (in \%) have been calculated for $+1 \%$ increase in reactor modeling parameters under normal and hydrogen $(0.5 \mathrm{ppm}$ of hydrogen in feedwater $)$ water chemistry conditions (Table XIII.2).

The sensitivity of the $\mathrm{H}_{2}, \mathrm{O}_{2}$, and $\mathrm{H}_{2} \mathrm{O}_{2}$ concentrations does not exceed a few ppb, and it is the highest to neutron dose rate for $\mathrm{H}_{2} \mathrm{O}_{2}$ concentrations under normal and hydrogen water chemistry conditions and to gamma dose rate under hydrogen water chemistry conditions. The ECP is most sensitive to the gamma dose rate under hydrogen water chemistry conditions and to flow velocity under both normal and hydrogen water chemistry conditions. The crack growth rate is most sensitive to flow velocity under normal and hydrogen water chemistry conditions and to the gamma dose rate under hydrogen water chemistry conditions.

While, in general, plant data are reliable, continuous attention is required during input set preparation to make sure than dose rate profiles and flow velocities are properly estimated by plant engineers. 
Table XIII.2. Sensitivity to Reactor Modeling Parameters.

\begin{tabular}{|c|c|c|c|c|c|c|c|c|c|c|c|}
\hline \multirow[b]{2}{*}{ ID } & \multirow[b]{2}{*}{ Parameter } & \multicolumn{2}{|c|}{$\Delta \mathrm{C}_{\mathrm{H} 2}, \mathrm{ppb}$} & \multicolumn{2}{|c|}{$\Delta \mathrm{C}_{02}, \mathrm{ppb}$} & \multicolumn{2}{|c|}{$\Delta \mathrm{C}_{\mathrm{H} 2 \mathrm{O}}, \mathrm{ppb}$} & \multicolumn{2}{|c|}{$\triangle \mathrm{ECP}_{\mathrm{SHE}}, \mathrm{mV}$} & \multicolumn{2}{|c|}{$\Delta \mathrm{CGR} / \mathrm{CGR}, \%$} \\
\hline & & NWC & $\begin{array}{c}\text { HWC } \\
0.5 \\
\text { ppm H }\end{array}$ & NWC & $\begin{array}{c}\text { HWC } \\
0.5 \\
\text { ppm } \mathrm{H}_{2}\end{array}$ & NWC & $\begin{array}{c}\text { HWC } \\
0.5 \\
\text { ppm H }\end{array}$ & NWC & $\begin{array}{c}\text { HWC } \\
0.5 \\
\text { ppm } \mathrm{H}_{2}\end{array}$ & NWC & $\begin{array}{c}\text { HWC } \\
0.5 \\
\text { ppm } \mathrm{H}_{2}\end{array}$ \\
\hline Reactor & $\mathrm{Dn}$ & 0.24 & -0.3 & 1.78 & 1.45 & 5.46 & 3.6 & 1.25 & 1 & $2.0 \%$ & $1.0 \%$ \\
\hline Reactor & $\mathrm{Dg}$ & -0.09 & -0.4 & -0.57 & -1.77 & -1.76 & -6.56 & 1.69 & 5.47 & $2.0 \%$ & $4.0 \%$ \\
\hline Reactor & Flow vel & -0.12 & -0.09 & -0.54 & 1.33 & 1.24 & 1 & 3.08 & 2.5 & $5.0 \%$ & $7.0 \%$ \\
\hline Reactor & Hydr diam & 0.02 & -0.21 & 0.33 & 0.17 & 0.53 & 0.66 & -1.4 & -2.33 & $-1.0 \%$ & $-3.0 \%$ \\
\hline
\end{tabular}

\section{New data need}

Based on the results of performed sensitivity analysis, new data sets are required for radiolytic yields, reaction rates, and exchange current densities for stainless steel, Tafel constants, and for the standard potentials for the oxygen and hydrogen peroxide electrode reactions.

\section{XIII.3. Available data sets \\ Radiolytic yields}

G-values at high temperatures have been determined both for gamma and fast neutron radiation and even for radiation with higher LET at ambient temperature. For gamma radiolysis, the G-values are well-known at ambient temperature. Even for radiolysis with fast neutrons, the G-values at ambient temperature are known. G-values for both gamma and fast neutrons have been determined in recent years in experiments as a function of temperature by several groups.

In a number of journal articles and reports from Chalk River Laboratories, McCracken and Elliot and co-workers have reported determination of G-values for gamma radiation, fast neutrons, and a number of particles with various energies as a function of temperature [12-24]. They have compared yields for neutron radiolysis from their own work with literature data, all at temperatures around $285^{\circ} \mathrm{C}$, and found a considerable variation between the values from different sources.

The results from various research groups for fast neutrons at high temperatures are and for gamma radiation at high temperature are shown in the summary Table XIII.3 and

Table XIII.4 [24]. 


\section{XIII.4. Comparison with plant measured data}

The current set of radiolytic yields under neutron and gamma radiation has been replaced with the most recent data [29] listed in Table XIII.3, and

Table XIII.4 in an attempt to improve agreement with plant measured data. Experimental data on the concentrations of hydrogen and oxygen and the ECP in the sampling/recirculation line of the Leibstadt BWR under normal and hydrogen water chemistry conditions have been selected for comparison with BWR_MASTER calculations.

Table XIII.3. Primary G-values for fast neutrons at high temperature.

\begin{tabular}{|c|c|c|c|c|c|c|c|c|c|}
\hline \multirow{2}{*}{ ID } & Source & $\begin{array}{c}{[25]} \\
(1988)\end{array}$ & $\begin{array}{c}{[19]} \\
(1994)\end{array}$ & $\begin{array}{c}{[22]} \\
(1996)\end{array}$ & $\begin{array}{c}{[23]} \\
(1998)\end{array}$ & $\begin{array}{c}{[26]} \\
(1995)\end{array}$ & $\begin{array}{c}{[31]} \\
(1987)\end{array}$ & $\begin{array}{c}{[27]} \\
(1991)\end{array}$ & $\begin{array}{c}{[29]} \\
(2002)\end{array}$ \\
\cline { 2 - 9 } & $\begin{array}{c}\text { Cemp., } \\
\text { Species }\end{array}$ & 280 & 300 & 300 & 285 & 250 & 290 & 250 & 289 \\
\hline N 01 & $\mathrm{e}^{-}$ & 0.9 & 0.61 & 0.61 & 1.0 & 0.77 & 0.93 & 0.19 & 0.65 \\
N 02 & $\mathrm{H}$ & 0.3 & 0.34 & 0.34 & 0.49 & 0.64 & 0.50 & 0.70 & 0.45 \\
N 03 & $\mathrm{H}^{+}$ & 0.9 & 0.61 & 0.61 & 1 & 0.77 & 0.93 & 0.19 & 0.65 \\
N 04 & $\mathrm{OH}^{2}$ & 2 & 2.02 & 2.76 & 2.7 & 1.74 & 1.09 & 2.85 & 1.79 \\
N 06 & $\mathrm{H}_{2} \mathrm{O}_{2}$ & 0.7 & 0.65 & 0.19 & 1.2 & 1.33 & 0.99 & 0.03 & 0.84 \\
N 07 & $\mathrm{HO}_{2}$ & & 0.05 & 0.05 & 0.05 & & 0.04 & & 0.05 \\
N 11 & $\mathrm{H}_{2}$ & 1.2 & 1.26 & 1.21 & 1.08 & 1.49 & 0.88 & 1.04 & 1.26 \\
\hline
\end{tabular}

Table XIII.4. Primary G-values for gamma radiation at high temperature.

\begin{tabular}{|c|c|c|c|c|c|c|c|}
\hline \multirow{2}{*}{ ID } & Source & $\begin{array}{c}{[28]} \\
(1992)\end{array}$ & $\begin{array}{c}{[16]} \\
(1991)\end{array}$ & $\begin{array}{c}{[14]} \\
(1993)\end{array}$ & $\begin{array}{c}{[26]} \\
(1995)\end{array}$ & $\begin{array}{c}{[30]} \\
(1993)\end{array}$ & $\begin{array}{c}{[29]} \\
(2002)\end{array}$ \\
\cline { 2 - 8 } & $\begin{array}{c}\text { Temp., } \\
\text { C/ } \\
\text { Species }\end{array}$ & 270 & 300 & 300 & 250 & 300 & 289 \\
\hline G 01 & $\mathrm{e}^{-}$ & 3.6 & 4.15 & 2.6 & 3.54 & 3.5 & 3.51 \\
G 02 & $\mathrm{H}$ & 0.68 & 1.08 & 0.87 & 0.94 & 0.8 & 0.91 \\
G 03 & $\mathrm{H}^{+}$ & 3.6 & 4.15 & 2.6 & 3.54 & 3.5 & 3.51 \\
G 04 & $\mathrm{OH}$ & 5.7 & 3.9 & 4.6 & 3.66 & 4.2 & 4.52 \\
G 06 & $\mathrm{H}_{2} \mathrm{O}_{2}$ & 0.28 & 1.32 & 0.27 & 1.16 & 0.3 & 0.55 \\
G 11 & $\mathrm{H}_{2}$ & 0.7 & 0.62 & 0.66 & 0.56 & 0.2 & 0.60 \\
\hline
\end{tabular}

The use of new data on radiolytic yields did not improve the agreement of measured and calculated hydrogen concentration significantly. While deviations under normal water chemistry 
(4 ppb) and $0.5 \mathrm{ppm}$ hydrogen injection $(30 \mathrm{ppb})$ are seemed to be within the margin of measurements and calculations, deviation increases from $88 \mathrm{ppb}$ to $343 \mathrm{ppb}$ when hydrogen injection increases from 0.8 to 2 ppm (Figure XIII.29.).

The agreement of measured and calculated oxygen concentration is better with a new set of yields for normal water chemistry conditions and remains unchanged for hydrogen water chemistry conditions (Figure XIII.14).

Deviation of calculated from measured data has been reduced from $132 \mathrm{ppb}$ to $41 \mathrm{ppb}$ under normal water chemistry conditions. Oxygen concentration under hydrogen water chemistry conditions drops dramatically, and deviation of calculated and measured concentrations does not exceed $2.4 \mathrm{ppb}$.

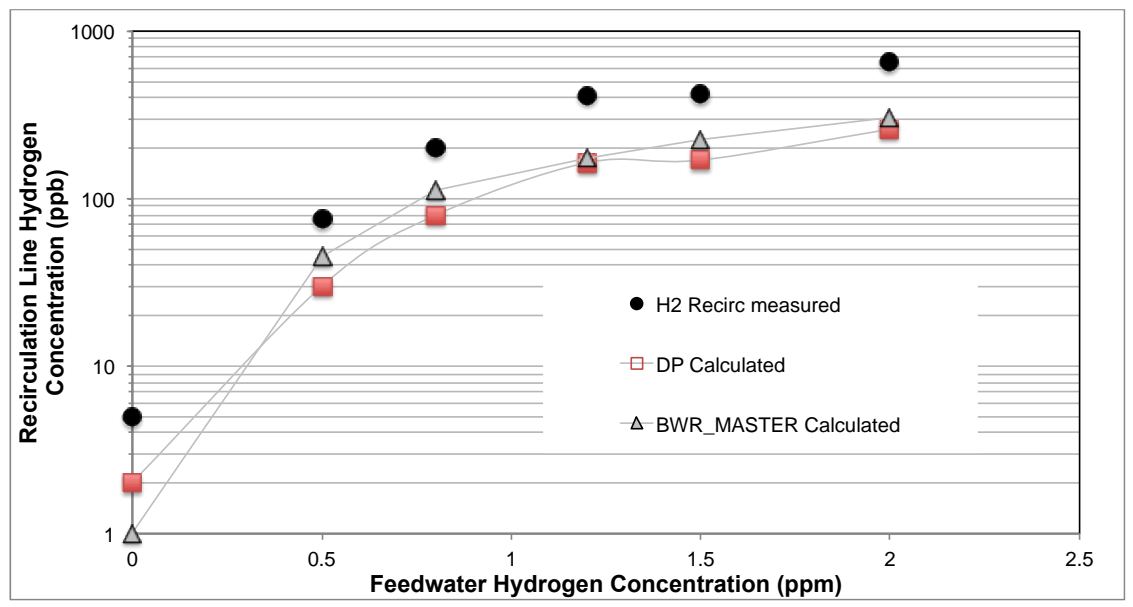

Figure XIII.29. Comparison of measured and calculated $\mathrm{H}_{2}$ concentration. 


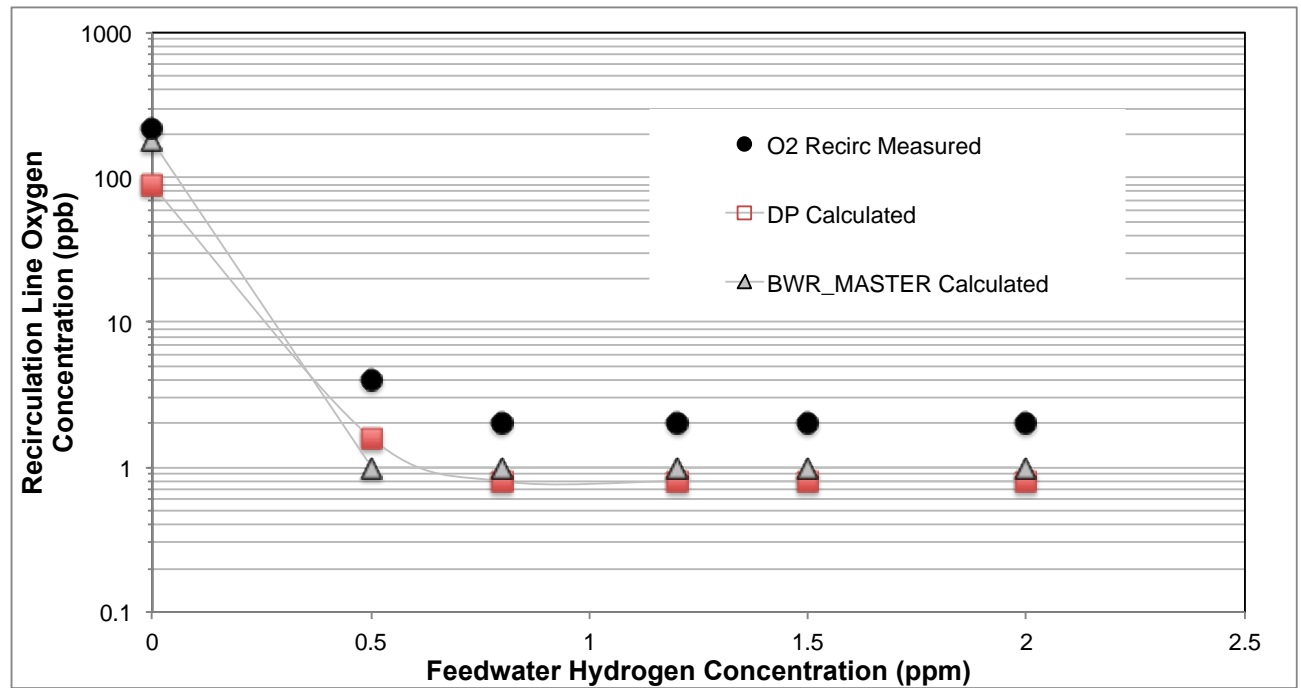

Figure XIII.14. Comparison of measured and calculated $\mathrm{O}_{2}$ concentration.

Most important, the agreement with the experiment has been significantly improved for ECP (Figure XIII.15). The deviation was reduced from $96 \mathrm{mV}$ to $1 \mathrm{mV}$ under normal water chemistry conditions and from $110 \mathrm{mV}$ to $41 \mathrm{mV}$ under 0.5 ppm hydrogen injection.

While increasing deviations under high hydrogen injection ( 0.8 to $2 \mathrm{ppm})$ have limited practical value, the formal approach requires understanding and reducing existing $100-200 \mathrm{mV}$ discrepancies. Recommended steps are to use another set of radiolytic yields and reaction rates.

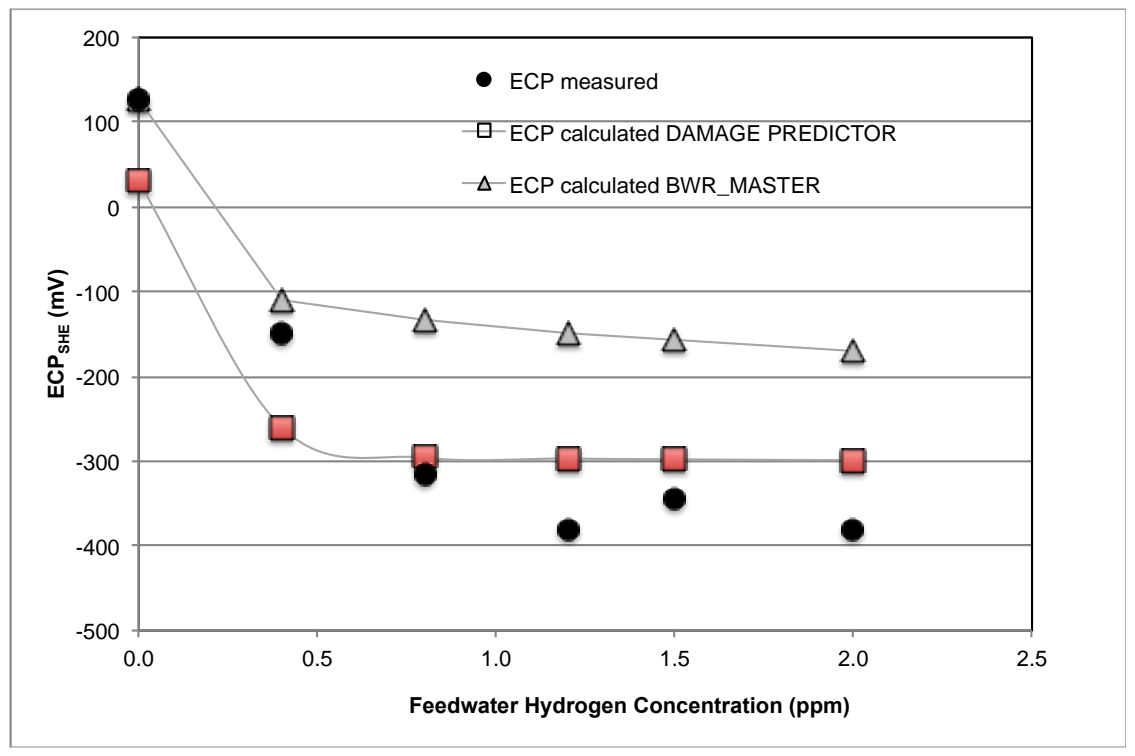


Figure XIII.15 Comparison of measured and calculated ECP ( $\left.\mathrm{V}_{\mathrm{SHE}}\right)$.

\section{XIII.5. Implementation of generic chemical kinetics algorithm in BWR_MASTER radiolysis code}

The present version of the BWR_MASTER code includes a "fixed" chemical kinetics algorithm, which allows the use of only one fixed set of species, chemical reactions, and radiolytic yields of species. This set was developed at CAM PSU and originally was used in DAMAGE PREDICTOR code [32] to simulate water chemistry, corrosion potential (ECP) and crack growth rates (CGR) in heat transport circuit HTC) of operating Boiling Water Reactors (BWRs). Comparison with plant measure data $(\mathrm{C} / \mathrm{E})$ on species concentrations, ECP, and CGR demonstrated good agreement. Nevertheless, the $\mathrm{C} / \mathrm{E}$ deviations of some parameters require upgrading of BWR_MASTER simulation capabilities using new available sets of radiolytic yields and chemical reactions. The use of these data would require a re-programming of BWR_MASTER chemical kinetics and radiolysis model. Two options are available: (1) creating a "fixed" BWR_MASTER model for each new data set or (2) implementing a generic algorithm for chemical kinetics and radiolysis model. The second option has been selected.

Generic chemical kinetics algorithm

$$
\left(\frac{G_{i}^{\gamma} \Gamma^{\gamma}}{100 N_{V}}+\frac{G_{i}^{n} \Gamma^{n}}{100 N_{V}}\right) \tilde{F} \rho d V+\left[\sum_{s=1}^{N} \sum_{m=1}^{N} k_{s m} C_{s} C_{m}-C_{i} \sum_{s=1}^{N} k_{s i} C_{s}\right] d V+\left[\frac{d\left(u C_{i}\right)}{d x} d V\right] \pm\left(\mu_{i}^{*} C_{i}^{g}-\mu_{i} C_{i}^{f}\right) d V_{g}=0
$$

was implemented by replacing "fixed" model radiolysis function Chemical_Kinetics as shown in Figure XIII.16 to calculate gains and losses of species as:

$$
{ }_{s=1}^{N=1}{ }_{s m}^{N} C_{s} C_{m} C_{i}^{N}{ }_{s=1}^{N} k_{s i} C_{s}
$$

and modifying radiolysis function for calculating radiolytic yields from neutron and gamma radiation

$$
\left(\frac{G_{i}^{\gamma} \Gamma^{\gamma}}{100 N_{V}}+\frac{G_{i}^{n} \Gamma^{n}}{100 N_{V}}\right) \tilde{F} \rho
$$

and convection and two-phase flow terms 


$$
\left[\frac{d\left(u C_{i}\right)}{d x} d V\right] \pm\left({ }_{i} C_{i}^{g} \quad{ }_{i} C_{i}^{f}\right) d V_{g}
$$

A fragment of the previous ("fixed") version of Chemical_Kinetics is shown in Figure XIII.17. BWR_MASTER function radiolysis was modified to migrate from "fixed" lists of species and chemical reactions to generic ones.

A list of chemical species is defined in the input file YIELDS.DAT as string constants, which allows expansion or reduction of the number of species. Arbitrary lists of chemical reactions, reaction rates, and activation energies are taken from input file CHEMRAT.DAT, where chemical reactions are also defined as string variables. More information on BWR_MASTER input and output files is contained in Sections 4 and 5, respectively. 


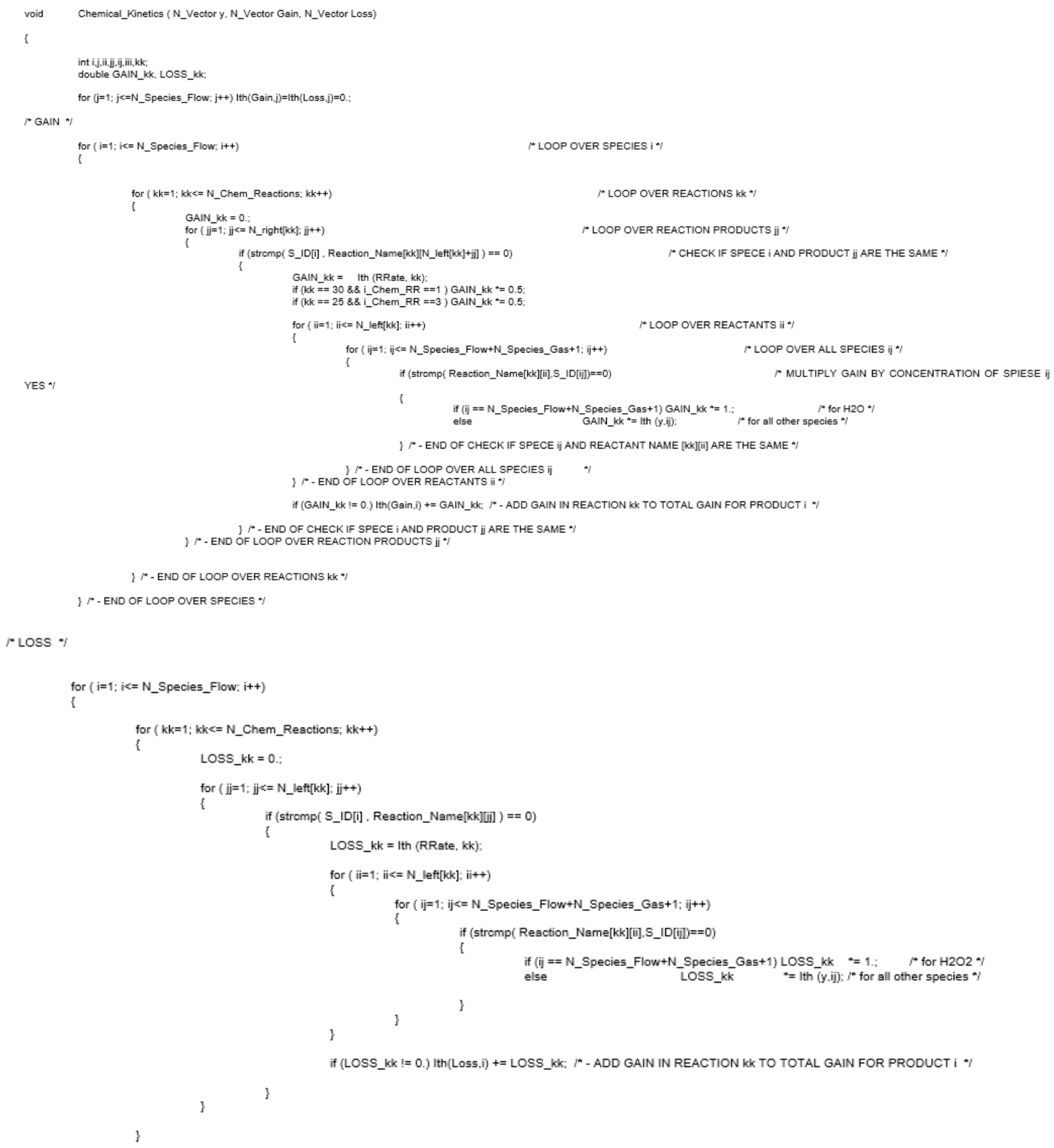

Figure XIII.16 Source code of generic chemical kinetics algorithm. 


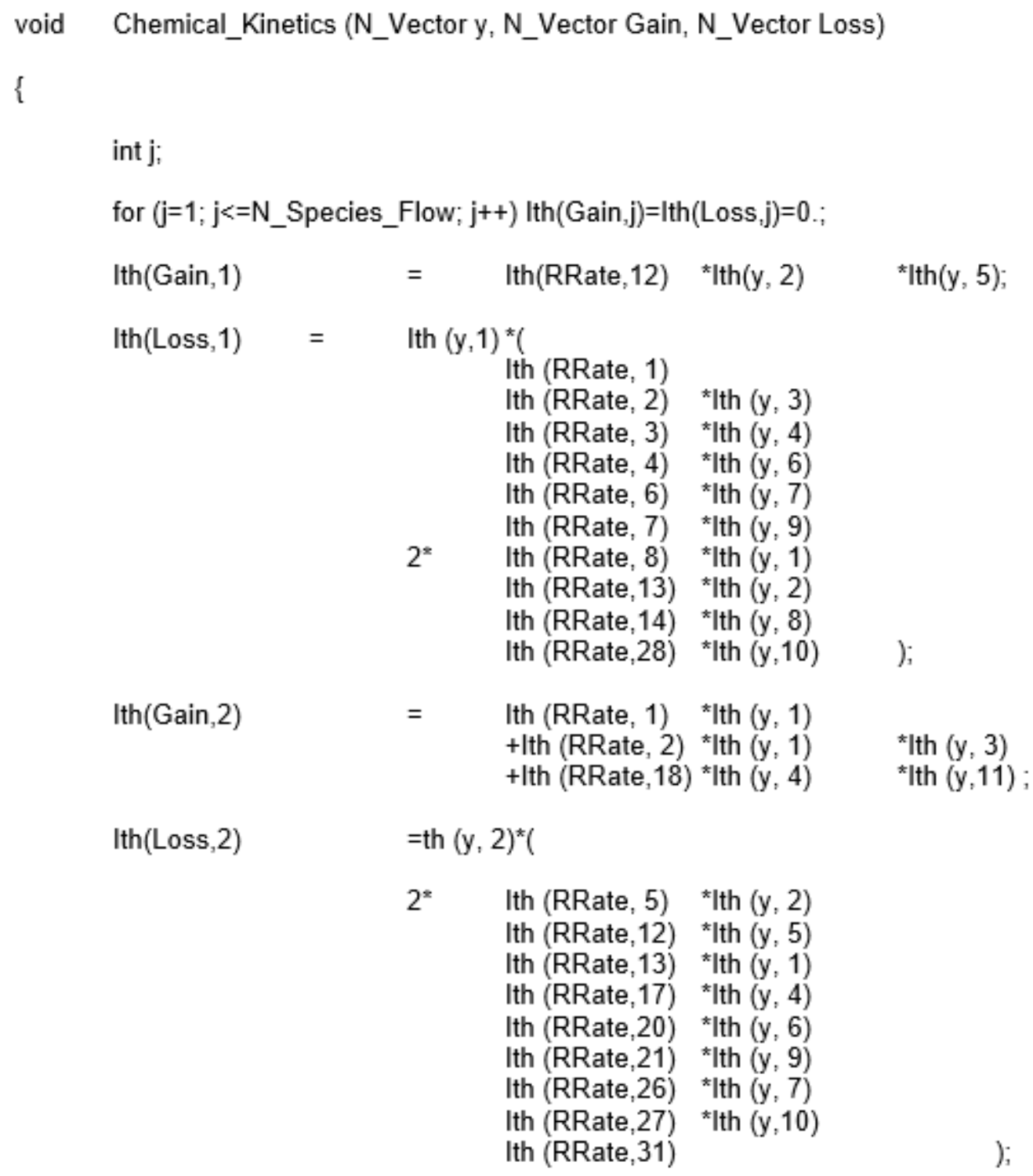

And so on for reactions from 3 to 34

Figure XIII.17 A fragment of the source code of the previously used "fixed" chemical kinetics algorithm.

\section{Verification of generic chemical kinetics algorithm}

Four sets of calculations have been performed to verify that the use of the "fixed" and generic algorithms yield identical results: 
1. The simulation of water chemistry, ECP and CGR in a BWR using the previous version of BWR_MASTER with "fixed" algorithm and "fixed" versions of input data files with radiolytic yields and CAM PSU set of chemical reactions

2. The same simulation using a modified version of BWR_MASTER with a generic algorithm and generic versions of input data files with radiolytic yields and chemical reactions has been performed. The species sequence in YIELD.DAT has been changed intentionally to perform additional software check

3. The simulation of water chemistry, ECP, and CGR in a BWR using the previous version of BWR_MASTER with "fixed" algorithm and "fixed" versions of input data files with radiolytic yields and chemical reactions. Rates of 5 chemical reactions in CHEMRATE.DAT have been set to zero

4. The same simulation using a modified version of BWR_MASTER with a generic algorithm and with generic versions of the input data files of radiolytic yields and chemical reactions. The same five reactions have been removed from input data file CHEMRATE.DAT

Verification of the modified BWR_MASTER was performed by comparison of the results from Simulations \#1 and \#2 and, as additional verification, by comparison of the results for Cases \#3 and \#4.

Comparison of distribution of calculated ECP, CGR and concentrations of $\mathrm{O}_{2}, \mathrm{H}_{2} \mathrm{O}_{2}$ and $\mathrm{H}_{2}$ along the BWR HTC under Normal Water Chemistry (NWC) and Hydrogen Water Chemistry ${ }^{1}$ ) (HWC conditions (Figure XIII.1 to Figure XIII.22) confirms that previous "fixed" version of BWR_MASTER and a modified BWR_MASTER with generic chemical kinetics delivered identical results. Additional visual examination of the printout files confirmed the identity of Case $\# 1$ and Case\#2 results.

An additional verification has been performed to confirm that the modified BWR_MASTER can handle chemical reaction sets with an increased or decreased number of chemical reactions. Comparison of Case \#3 and Case \#4 results for the distribution of ECP along BWR HTC is shown in Figure XIII.23 (for NWC) and in Figure XIII.424 (for HWC). Both results are identical. Also, the identity of distribution of CGR and species concentrations along BWR

\footnotetext{
${ }^{1}$ Hydrogen injection of 0.4 ppm
} 
HTC have been observed, but not reported here. Additional visual examination of the printout files confirmed the identity of Case \#3 and Case\#4 results.

Based on the presented verification results, it was concluded that the modified BWR_MASTER code could handle generic lists of chemical species and chemical reactions.

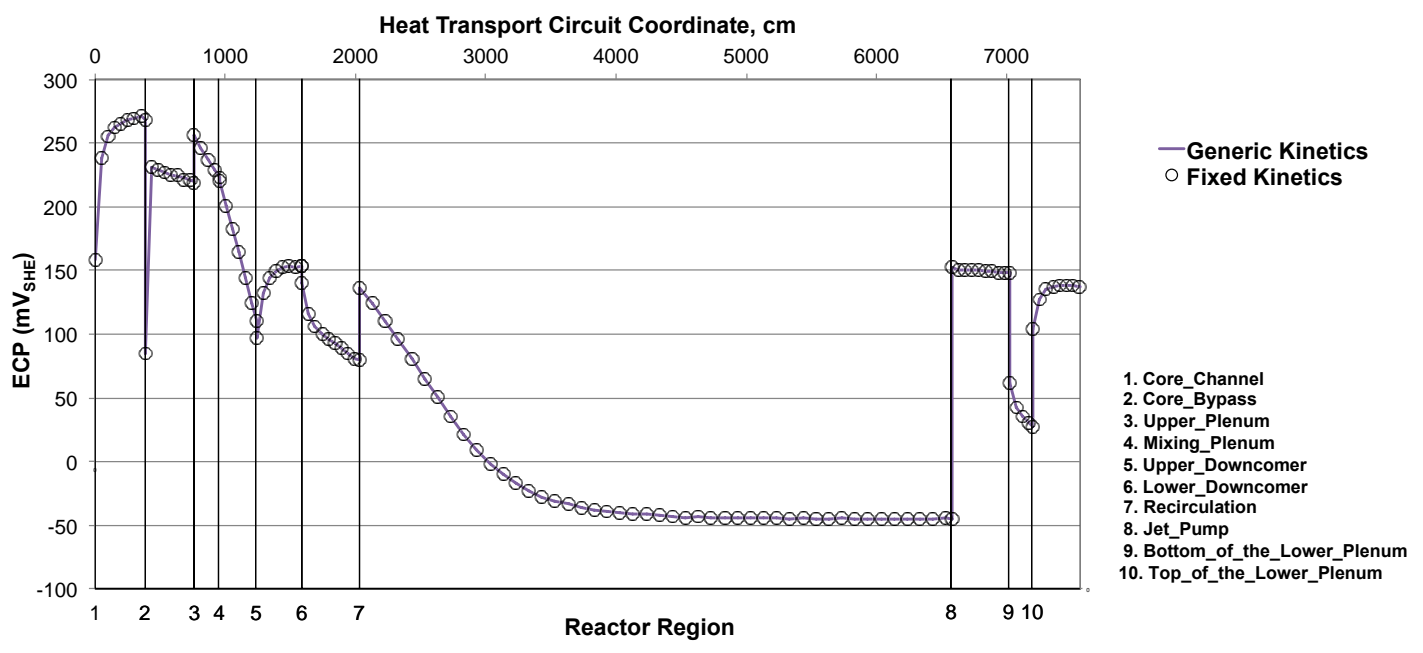

Figure XIII.18 Comparison of ECP under Normal Water Chemistry conditions calculated with "Fixed" (old version of BWR_MASTER) and "Generic" kinetics algorithms (new version of BWR_MASTER).

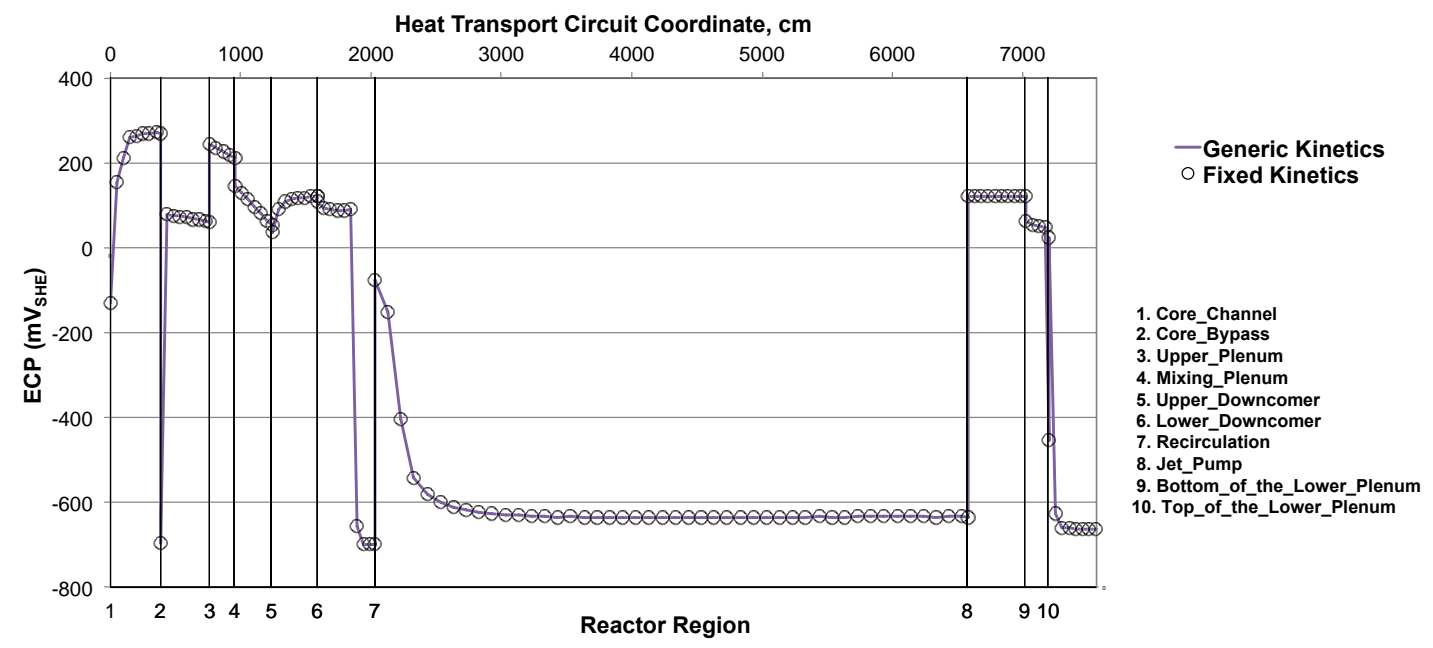

Figure XIII.35. Comparison of ECP under Hydrogen Water Chemistry conditions calculated with "Fixed" (old version of BWR_MASTER) and "Generic" kinetics algorithms (new version of BWR_MASTER). 


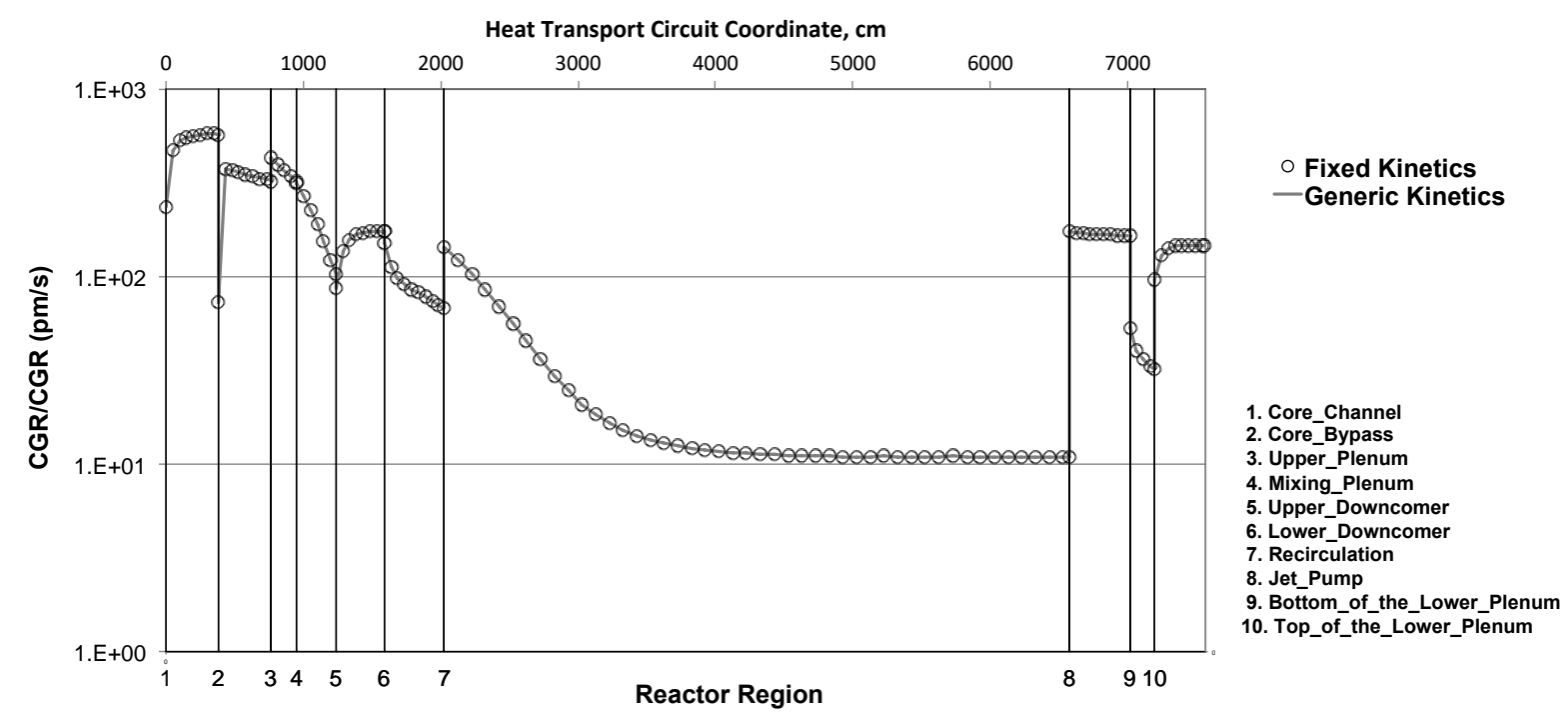

Figure XIII.36. Comparison of Crack Growth Rates under Normal Water Chemistry conditions calculated with "Fixed" (old version of BWR_MASTER) and "Generic" kinetics algorithms (new version of BWR_MASTER).

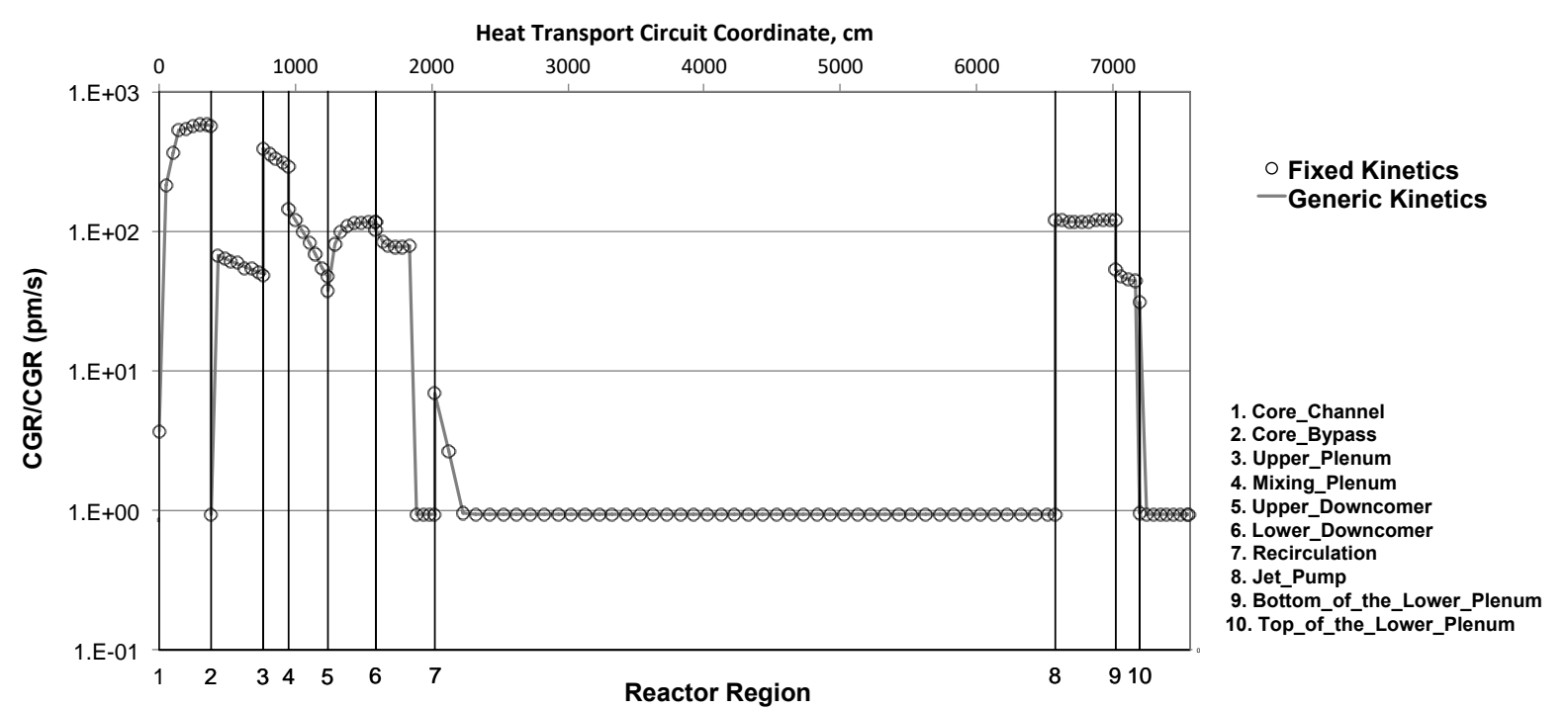

Figure XIII.37. Comparison of Crack Growth Rates under Hydrogen Water Chemistry conditions calculated with "Fixed" (old version of BWR_MASTER) and "Generic" kinetics algorithms (new version of BWR_MASTER). 


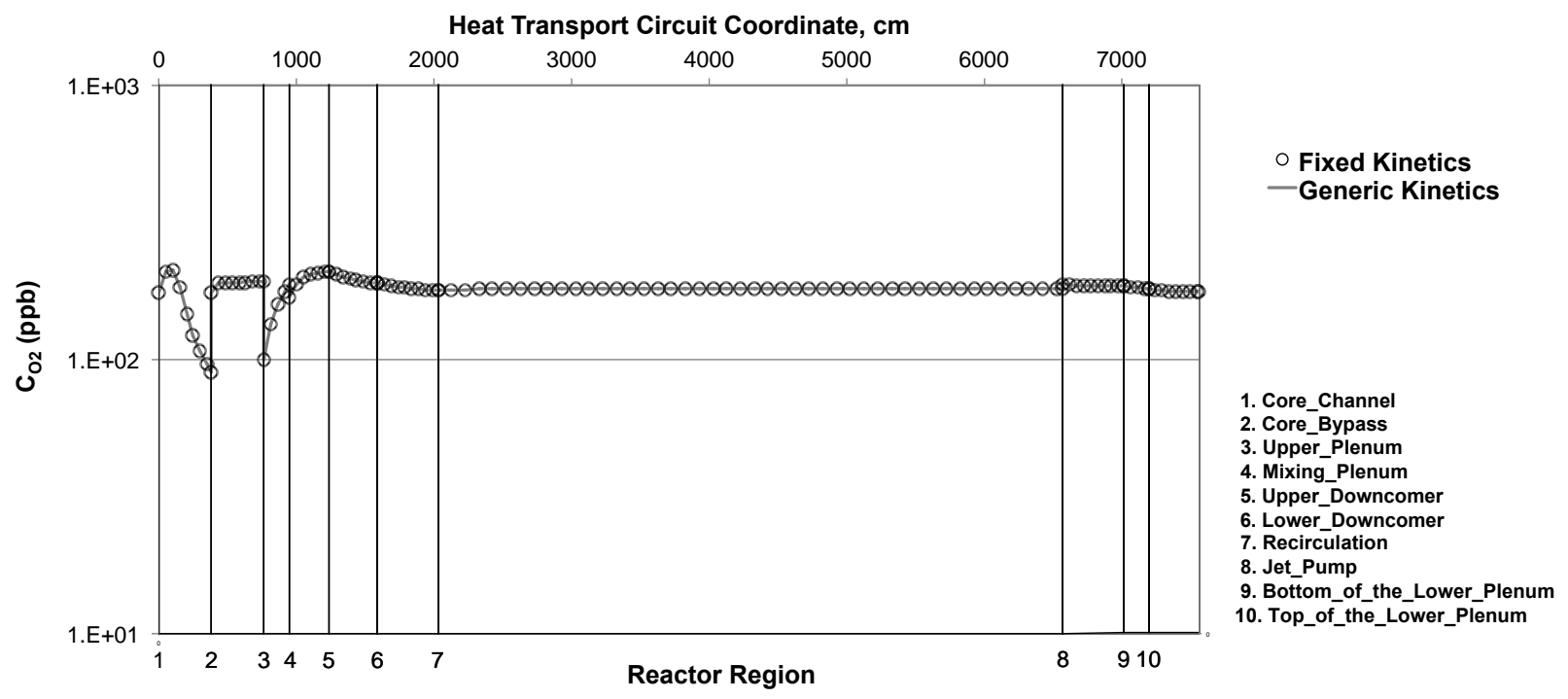

Figure XIII.38. Comparison of oxygen concentration under Normal Water Chemistry conditions calculated with "Fixed" (old version of BWR_MASTER) and "Generic" kinetics algorithms (new version of BWR_MASTER).

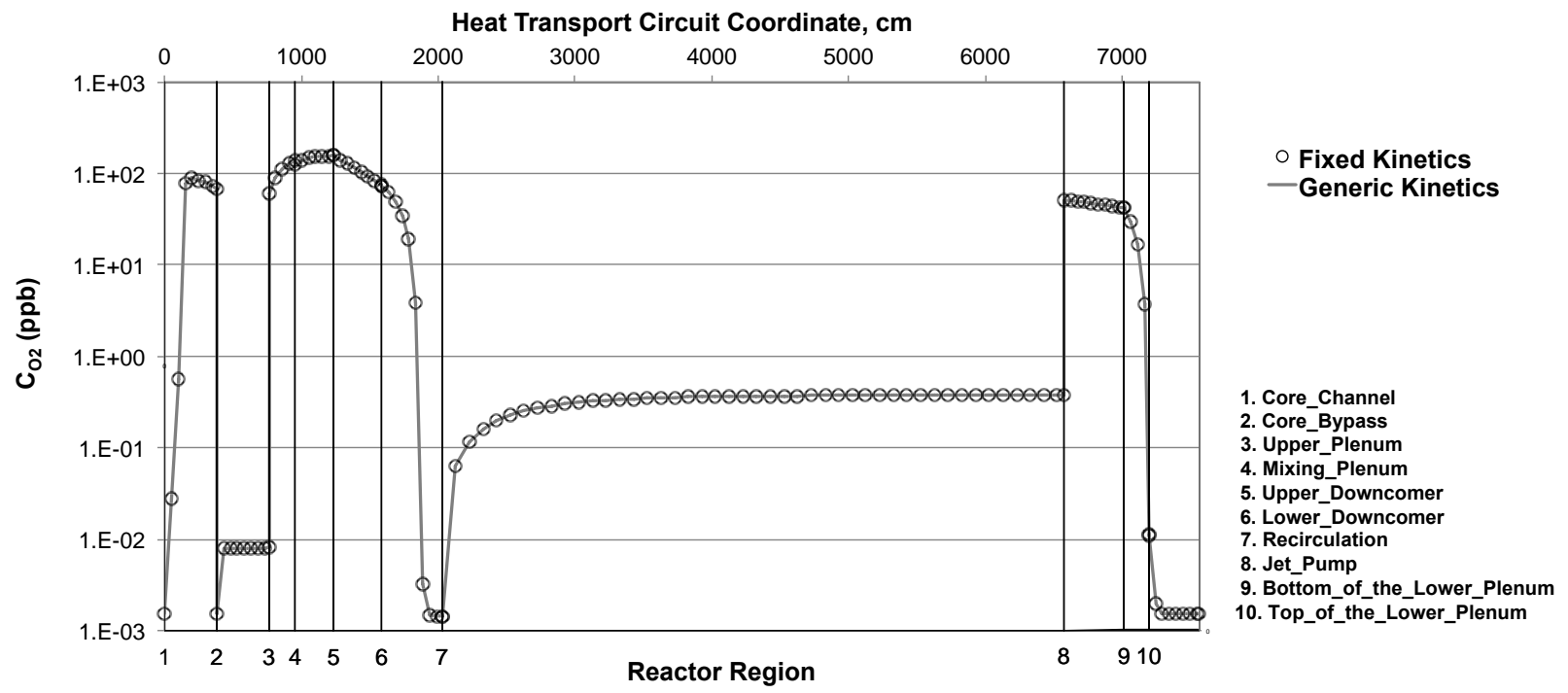

Figure XIII.39. Comparison of oxygen concentration under Hydrogen Water Chemistry conditions calculated with "Fixed" (old version of BWR_MASTER) and "Generic" kinetics algorithms (new version of BWR_MASTER). 


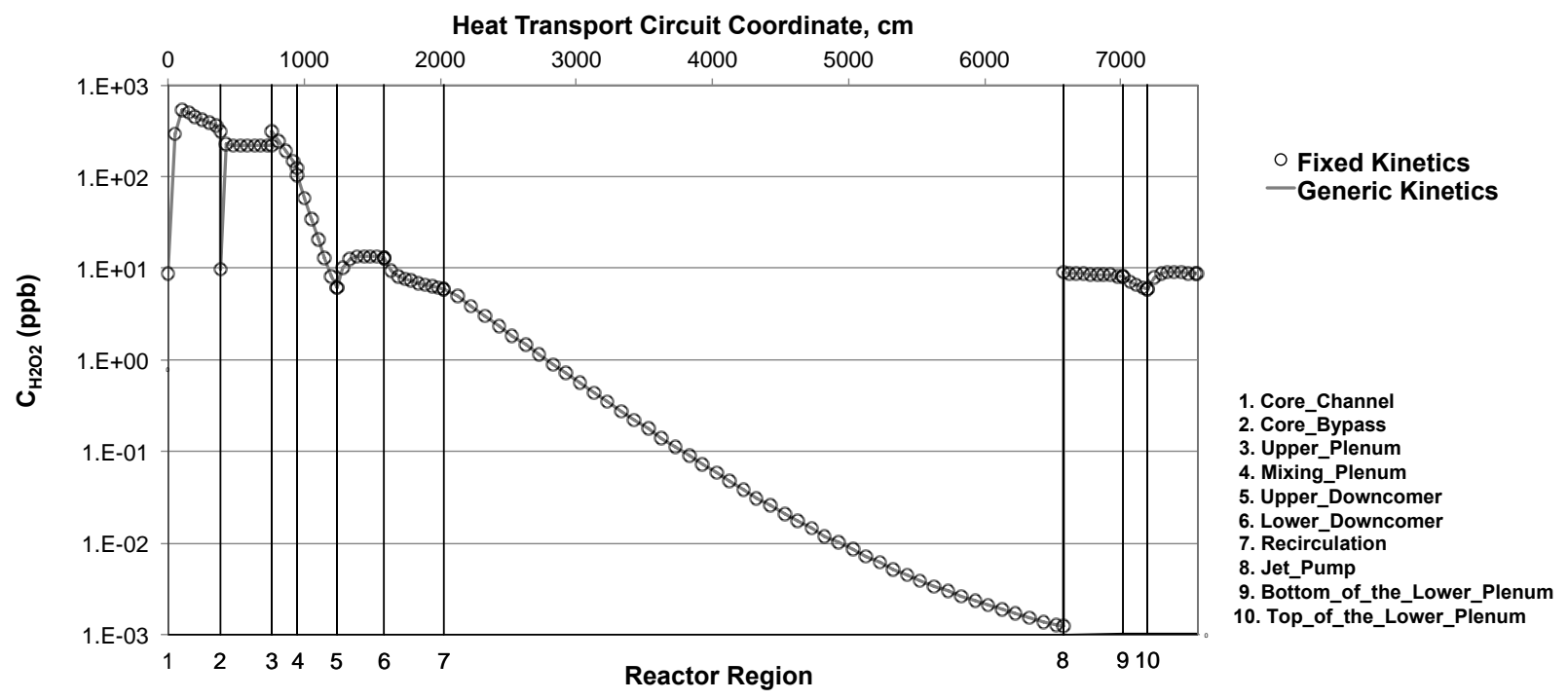

Figure XIII.19 Comparison of hydrogen peroxide concentration under Normal Water Chemistry conditions calculated with "Fixed" (old version of BWR_MASTER) and "Generic" kinetics algorithms (new version of BWR_MASTER).

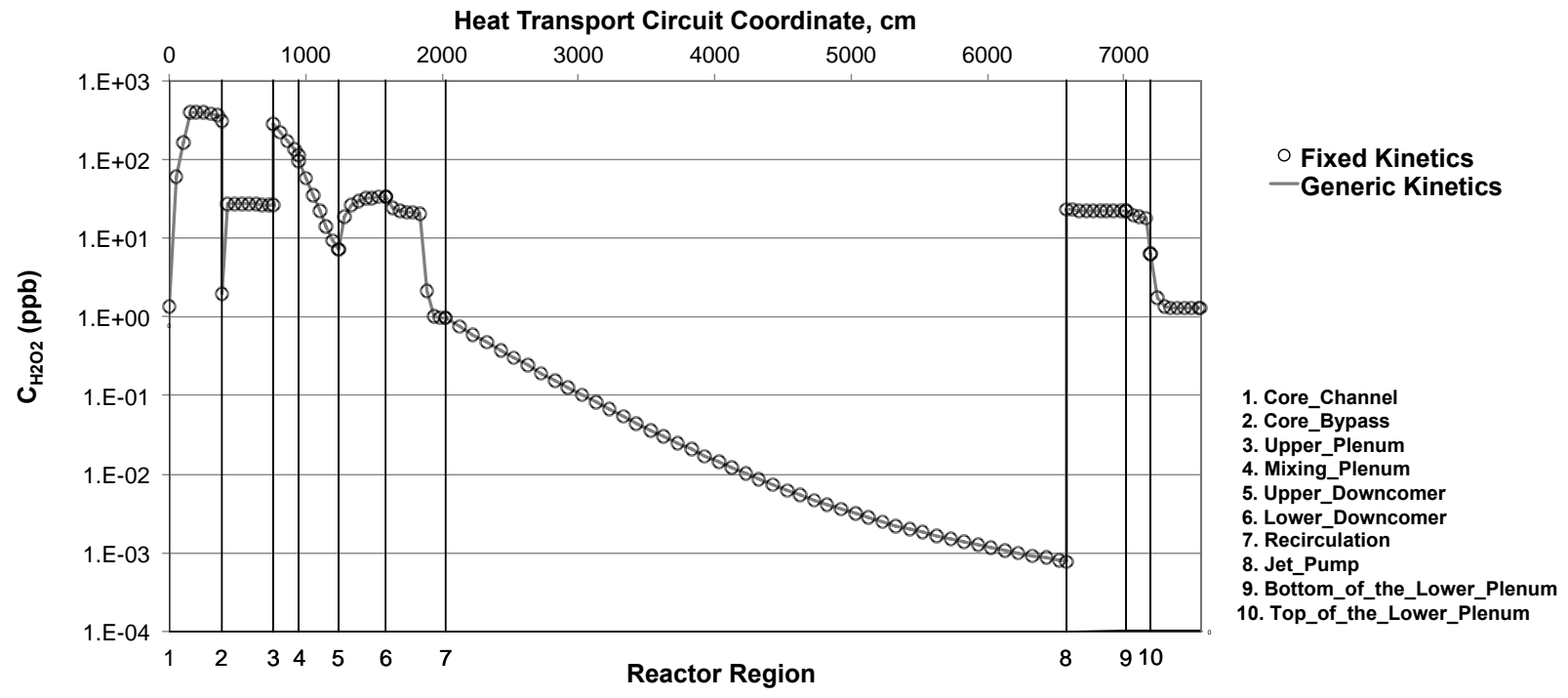

Figure XIII.20 Comparison of hydrogen peroxide concentration under Hydrogen Water Chemistry conditions calculated with "Fixed" (old version of BWR_MASTER) and "Generic" kinetics algorithms (new version of BWR_MASTER). 


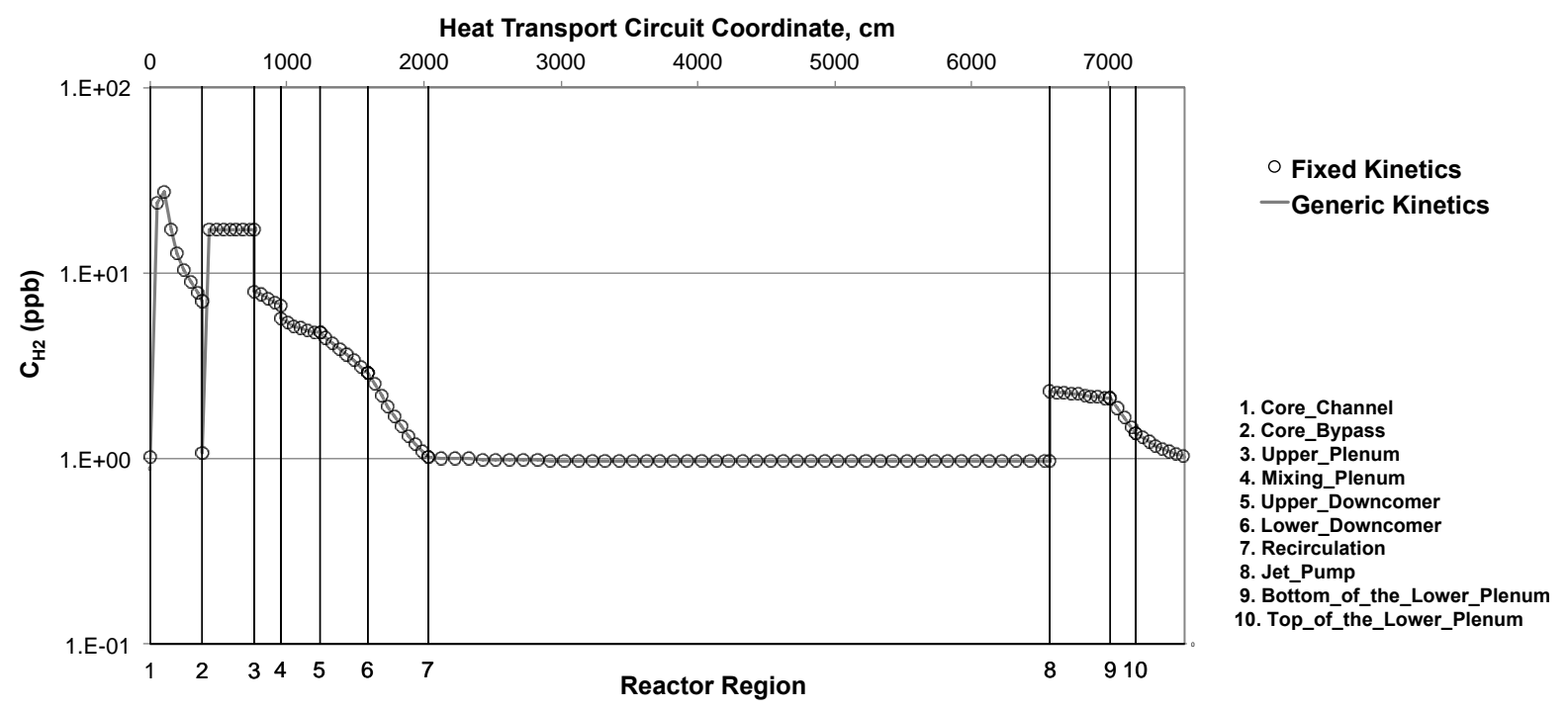

Figure XIII.21 Comparison of hydrogen concentration under Normal Water Chemistry conditions calculated with "Fixed" (old version of BWR_MASTER) and "Generic" kinetics algorithms (new version of BWR_MASTER).

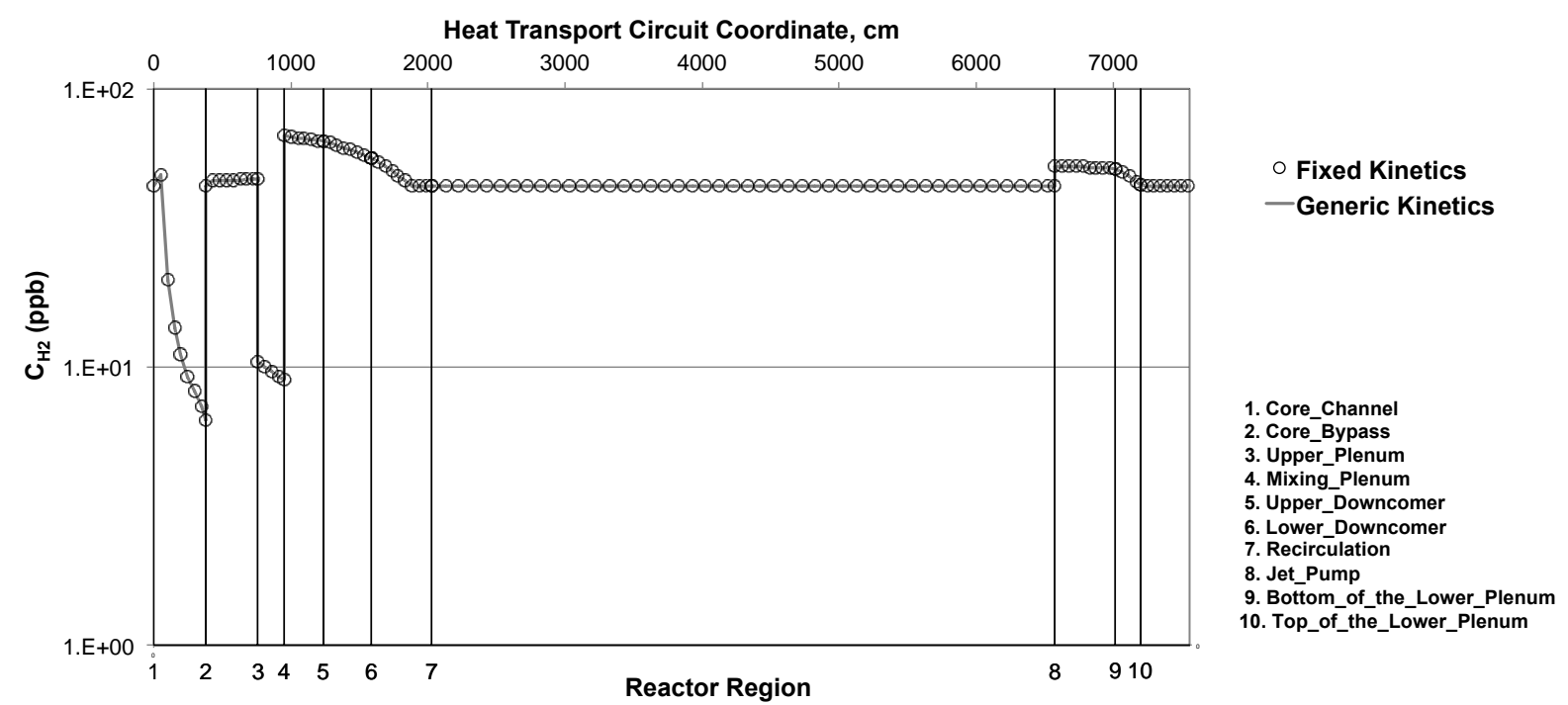

Figure XIII.22 Comparison of hydrogen concentration under Hydrogen Water Chemistry conditions calculated with "Fixed" (old version of BWR_MASTER) and "Generic" kinetics algorithms (new version of BWR_MASTER). 


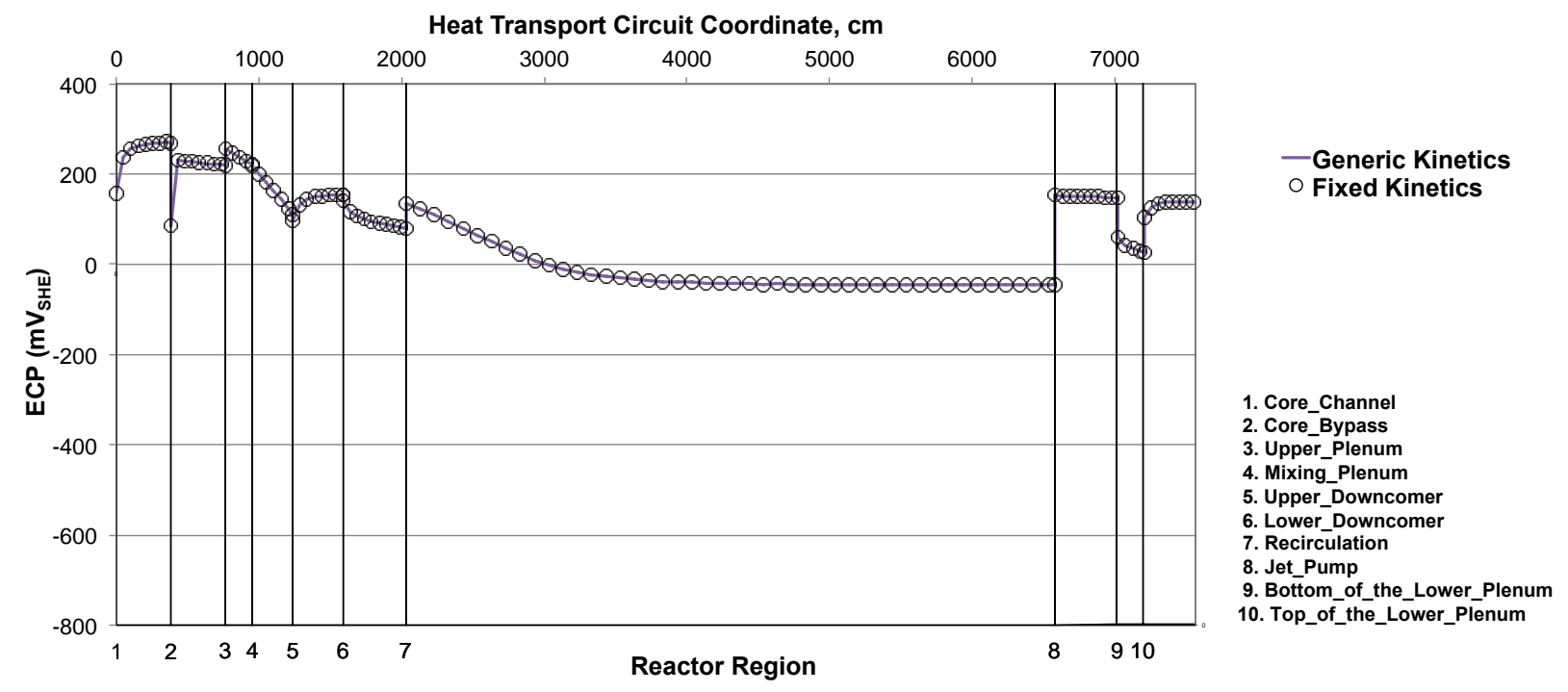

Figure XIII.23 Comparison of ECP under Normal Water Chemistry (NWC) Conditions calculated using a generic kinetics algorithm with five reaction rates in CAM PSU set are set to zero (open circles) and the same reactions removed from CAM PSU set.

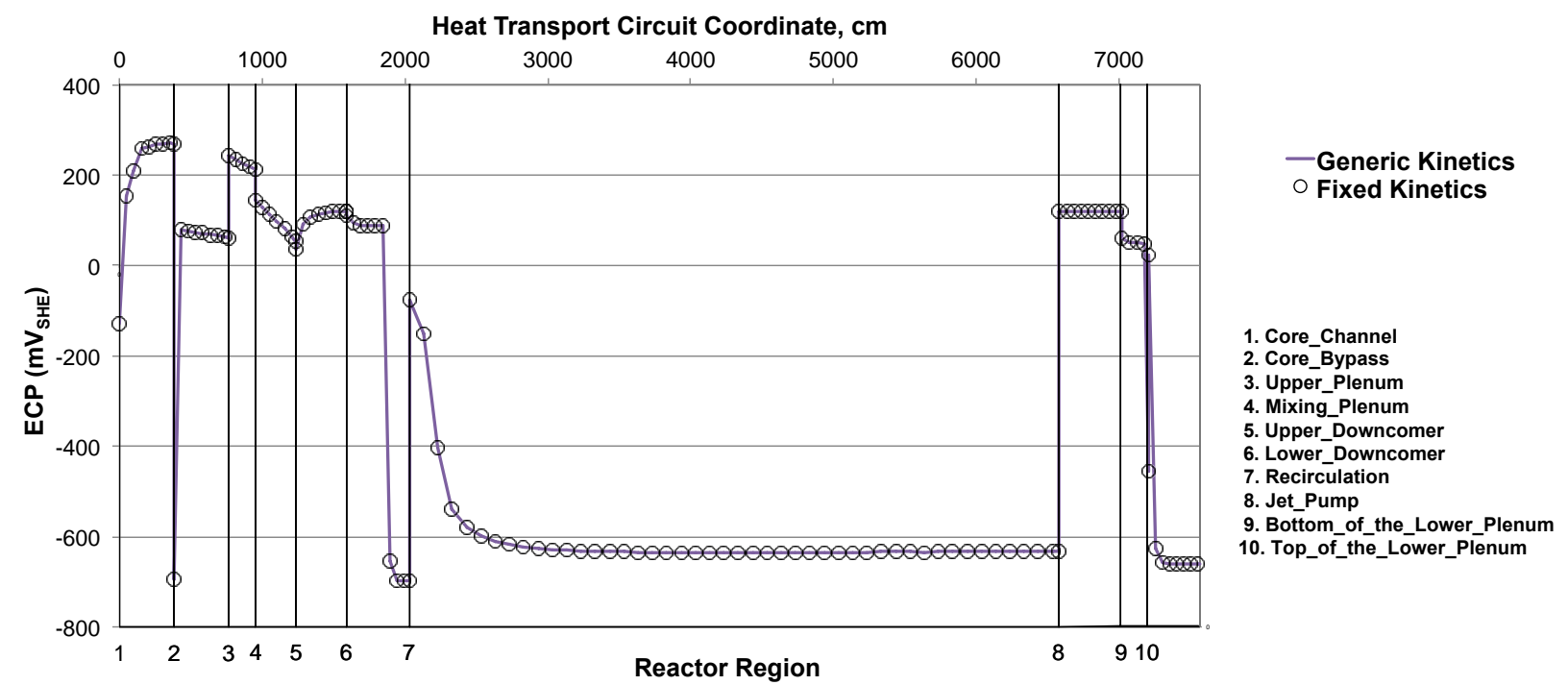

Figure XIII.424 Comparison of ECP under Hydrogen Water Chemistry (HWC) Conditions calculated using a generic kinetics algorithm with five reaction rates in CAM PSU set are set to zero (open circles) and the same reactions removed from CAM PSU set. 


\section{Changes in BWR_MASTER code input data files}

The format of two input data files YIELD.DAT and CHEMRATE.DAT has been slightly modified, as described below.

The format of the modified YIELD.DAT file is shown in Figure XIII.46.. The first two parameters specify the number of species in the liquid flow and in the gas flow, respectively. An additional column (\#2) with the molecular weight of the species has been added. Also, all YIELD.DAT files have to have $\mathrm{H}_{2} \mathrm{O}$ listed at the end. Users may change the sequence and number of the liquid flow species and place blank line(s) between data lines. The user-selected sequence of species will determine the sequence of species concentrations in printout files. At this time, the number of gas flow species is set to two.

$\begin{array}{llllll}112 & & & & & \\ \mathrm{e}^{-} & 1 & 0.65 & 3.51 & 0 . & 0 . \\ \mathrm{H} & 1 & 0.45 & 0.91 & 0 . & 0 . \\ \mathrm{OH} & 17 & 1.79 & 4.52 & 0 . & 0 . \\ \mathrm{H} 2 & 33 & 0.05 & 0.00 & 0 . & 0 . \\ \mathrm{HO2-} & 33 & 0 . & 0.00 & 0 . & 0 . \\ \mathrm{O} 2- & 32 & 0 . & 0.00 & 0 . & 0 . \\ \mathrm{H}+ & 1 & 0.65 & 3.51 & 0 . & 0 . \\ \mathrm{OH}- & 17 & 0 . & 0.00 & 0 . & 0 . \\ \mathrm{H} 2 \mathrm{O} 2 & 34 & 0.84 & 0.55 & 0 . & 0 . \\ \mathrm{H} 2 & 2 & 1.26 & 0.60 & 255 . & 15 . \\ \mathrm{O} 2 & 32 & 0 . & 0.00 & 228 . & 21.5 \\ & & & & & \\ \mathrm{H} 2 \mathrm{~g} & 2 & 0 . & 0.00 & 0 . & 0 . \\ \mathrm{O} 2 \mathrm{~g} & 32 & 0 . & 0.00 & 0 . & 0 . \\ & & & & & \end{array}$

Figure XIII.46. YIELD.DAT file format.

The format of the modified CHEMRATE.DAT file is shown in Figure XIII.47.. First two parameters specify the total number of chemical reactions and Type of reaction rate set: 1 for CAM PSU set with room temperature reaction rates and a need for special treatment of Reactions \#16 and \#30 and 2 for the generic set with high-temperature reaction rates and no need for special treatments of some chemical reactions. Each chemical reaction specified as a set of string-Type species names and "+" and " =" symbols. 
Lists of species in YIELD.DAT and CHEMRATE.DAT files must be consistent: all species present in the YIELD.DAT file must be present in the CHEMRATE.DAT file and vise versa. Otherwise, BWR_MASTER will stop with a warning message. According to this requirement, even species with zero yields from neutron or gamma radiation must be included in YIELD.DAT

\begin{tabular}{|c|c|c|c|c|c|c|c|c|c|c|c|c|c|c|c|c|c|c|c|}
\hline 34 & 1 & & & & & & & & & & & & & & & & & & \\
\hline 1 & $1.60 \mathrm{E}+01$ & 3 & 3 & 4 & e- & + & $\mathrm{H} 2 \mathrm{O}$ & $=$ & $\mathrm{H}$ & + & $\mathrm{OH}-$ & & & & & & & & \\
\hline 2 & $2.40 \mathrm{E}+10$ & 3 & 3 & 2 & e- & + & $\mathrm{H}+$ & $=$ & $\mathrm{H}$ & & & & & & & & & & \\
\hline 3 & $2.40 \mathrm{E}+10$ & 3 & 3 & 2 & e- & + & $\mathrm{OH}$ & $=$ & $\mathrm{OH}-$ & & & & & & & & & & \\
\hline 4 & $1.30 \mathrm{E}+10$ & 3 & 3 & 4 & e- & + & $\mathrm{H} 2 \mathrm{O} 2$ & $=$ & $\mathrm{OH}$ & + & $\mathrm{OH}-$ & & & & & & & & \\
\hline 5 & $1.00 \mathrm{E}+10$ & 3 & 3 & 2 & $\mathrm{H}$ & + & $\mathrm{H}$ & $=$ & $\mathrm{H} 2$ & & & & & & & & & & \\
\hline 6 & $2.00 \mathrm{E}+10$ & 3 & 3 & 2 & e- & + & $\mathrm{HO} 2$ & $=$ & HO2- & & & & & & & & & & \\
\hline 7 & $1.90 \mathrm{E}+10$ & 3 & 3 & 2 & e- & + & 02 & $=$ & O2- & & & & & & & & & & \\
\hline 8 & $5.00 \mathrm{E}+09$ & 3 & 7 & 6 & e- & + & e- & + & $\mathrm{H} 2 \mathrm{O}$ & + & $\mathrm{H} 2 \mathrm{O}$ & $=$ & $\mathrm{OH}-$ & + & $\mathrm{OH}-$ & + & $\mathrm{H} 2$ & & \\
\hline 9 & $4.50 \mathrm{E}+09$ & 3 & 3 & 2 & $\mathrm{OH}$ & + & $\mathrm{OH}$ & $=$ & $\mathrm{H} 2 \mathrm{O} 2$ & & & & & & & & & & \\
\hline 10 & $1.20 \mathrm{E}+10$ & 3 & 3 & 4 & $\mathrm{OH}$ & + & $\mathrm{HO} 2$ & $=$ & $\mathrm{H} 2 \mathrm{O}$ & + & 02 & & & & & & & & \\
\hline 11 & $1.20 \mathrm{E}+10$ & 3 & 3 & 4 & $\mathrm{OH}$ & + & O2- & $=$ & $\mathrm{OH}-$ & + & 02 & & & & & & & & \\
\hline 12 & $2.00 \mathrm{E}+07$ & 3 & 3 & 4 & $\mathrm{OH}-$ & + & $\mathrm{H}$ & $=$ & $e^{-}$ & + & $\mathrm{H} 2 \mathrm{O}$ & & & & & & & & \\
\hline 13 & $4.50 \mathrm{E}+08$ & 3 & 5 & 4 & $\mathrm{e}^{-}$ & + & $\mathrm{H}$ & + & $\mathrm{H} 2 \mathrm{O}$ & $=$ & $\mathrm{OH}-$ & + & $\mathrm{H} 2$ & & & & & & \\
\hline 14 & $6.30 \mathrm{E}+07$ & 3 & 5 & 6 & e- & + & HO2- & + & $\mathrm{H} 2 \mathrm{O}$ & $=$ & $\mathrm{OH}$ & + & $\mathrm{OH}-$ & + & $\mathrm{OH}^{-}$ & & & & \\
\hline 15 & $1.44 \mathrm{E}+11$ & 3 & 3 & 2 & $\mathrm{H}+$ & + & $\mathrm{OH}-$ & $=$ & $\mathrm{H} 2 \mathrm{O}$ & & & & & & & & & & \\
\hline 16 & $2.60 \mathrm{E}-05$ & 3 & 1 & 4 & $\mathrm{H} 2 \mathrm{O}$ & $=$ & $\mathrm{H}+$ & + & $\mathrm{OH}-$ & & & & & & & & & & \\
\hline 17 & $2.00 \mathrm{E}+10$ & 3 & 3 & 2 & $\mathrm{H}$ & + & $\mathrm{OH}$ & $=$ & $\mathrm{H} 2 \mathrm{O}$ & & & & & & & & & & \\
\hline 18 & $3.40 \mathrm{E}+07$ & 4.6 & 3 & 4 & $\mathrm{OH}$ & + & $\mathrm{H} 2$ & $=$ & $\mathrm{H}$ & + & $\mathrm{H} 2 \mathrm{O}$ & & & & & & & & \\
\hline 19 & $2.70 \mathrm{E}+07$ & 3.45 & 3 & 4 & $\mathrm{OH}$ & + & $\mathrm{H} 2 \mathrm{O} 2$ & $=$ & $\mathrm{H} 2 \mathrm{O}$ & + & $\mathrm{HO} 2$ & & & & & & & & \\
\hline 20 & $4.40 E+07$ & 4.5 & 3 & 4 & $\mathrm{H}$ & + & $\mathrm{H} 2 \mathrm{O} 2$ & $=$ & $\mathrm{OH}$ & + & $\mathrm{H} 2 \mathrm{O}$ & & & & & & & & \\
\hline 21 & $1.90 \mathrm{E}+10$ & 3 & 3 & 2 & $\mathrm{H}$ & + & 02 & $=$ & $\mathrm{HO2}$ & & & & & & & & & & \\
\hline 22 & $8.00 \mathrm{E}+05$ & 3 & 1 & 4 & $\mathrm{HO2}$ & $=$ & O2- & + & $\mathrm{H}+$ & & & & & & & & & & \\
\hline 23 & $5.00 \mathrm{E}+10$ & 3 & 3 & 2 & O2- & + & $\mathrm{H}+$ & $=$ & $\mathrm{HO2}$ & & & & & & & & & & \\
\hline 24 & $2.70 \mathrm{E}+06$ & 4.5 & 3 & 4 & $\mathrm{HO2}$ & + & $\mathrm{HO2}$ & $=$ & $\mathrm{H} 2 \mathrm{O} 2$ & + & 02 & & & & & & & & \\
\hline 25 & $1.70 \mathrm{E}+07$ & 4.5 & 7 & 8 & O2- & + & O2- & + & $\mathrm{H} 2 \mathrm{O}$ & + & $\mathrm{H} 2 \mathrm{O}$ & $=$ & $\mathrm{H} 2 \mathrm{O} 2$ & + & $\mathrm{O} 2$ & + & $\mathrm{OH}-$ & + & $\mathrm{OH}-$ \\
\hline 26 & $2.00 \mathrm{E}+10$ & 3 & 3 & 2 & $\mathrm{H}$ & + & $\mathrm{HO2}$ & $=$ & $\mathrm{H} 2 \mathrm{O} 2$ & & & & & & & & & & \\
\hline 27 & $2.00 \mathrm{E}+10$ & 3 & 3 & 2 & $\mathrm{H}$ & + & O2- & $=$ & HO2- & & & & & & & & & & \\
\hline 28 & $1.30 \mathrm{E}+08$ & 4.5 & 5 & 4 & $\mathrm{e}-$ & + & O2- & + & $\mathrm{H} 2 \mathrm{O}$ & $=$ & HO2- & + & $\mathrm{OH}-$ & & & & & & \\
\hline 29 & $1.80 \mathrm{E}+08$ & 4.5 & 3 & 4 & $\mathrm{OH}-$ & + & $\mathrm{H} 2 \mathrm{O} 2$ & $=$ & $\mathrm{HO2-}$ & + & $\mathrm{H} 2 \mathrm{O}$ & & & & & & & & \\
\hline 30 & 0 & 0 & 1 & 5 & $\mathrm{H} 2 \mathrm{O} 2$ & $=$ & $\mathrm{H} 2 \mathrm{O}$ & + & 0.5 & 02 & & & & & & & & & \\
\hline 31 & $1.04 \mathrm{E}-04$ & 3 & 3 & 4 & $\mathrm{H}$ & + & $\mathrm{H} 2 \mathrm{O}$ & $=$ & $\mathrm{H} 2$ & + & $\mathrm{OH}$ & & & & & & & & \\
\hline 32 & $1.02 E+04$ & 3 & 3 & 4 & $\mathrm{H} 2 \mathrm{O}$ & + & HO2- & $=$ & $\mathrm{H} 2 \mathrm{O} 2$ & + & $\mathrm{OH}-$ & & & & & & & & \\
\hline 33 & $1.50 \mathrm{E}+07$ & 4.5 & 3 & 4 & $\mathrm{HO2}$ & + & O2- & $=$ & 02 & + & HO2- & & & & & & & & \\
\hline 34 & $7.70 \mathrm{E}-04$ & 7.3 & 1 & 4 & $\mathrm{H} 2 \mathrm{O} 2$ & $=$ & $\mathrm{OH}$ & + & $\mathrm{OH}$ & & & & & & & & & & \\
\hline
\end{tabular}

Figure XIII.47. CHEMRATE.DAT file format.

\section{Changes in BWR_MASTER code output data files}

The format of the BWR_MASTER printout file has been slightly modified as shown in Figure XIII.48.. The calculated concentration of gas and liquid flow species have been moved to the last columns. Concentrations of gas flow species are printed first, followed by concentrations of the liquid flow species. The sequence of printing concentrations of the liquid flow species is defined by the sequence of listing species in YIELD.DAT. 


\begin{tabular}{|c|c|c|c|c|c|c|c|c|c|c|c|c|c|c|c|c|c|c|c|c|c|}
\hline $\mathrm{x}$ & $\mathrm{T}$ & $\mathrm{pH}$ & Cond25 & CondHT & $\mathrm{ECP}$ & REDOX & CGR & Crack d & Steam, & ppm & & qui & , p & & & & & & & & \\
\hline$(\mathrm{cm})$ & (C) & & $\mathrm{us} / \mathrm{cm}$ & $\mathrm{us} / \mathrm{cm}$ & $\mathrm{mV}$ SHE & $\mathrm{mV}$ & $\mathrm{pm} / \mathrm{s}$ & $\mathrm{cm}$ & 02 & $\mathrm{H} 2$ & $e^{-}$ & $\mathrm{H}$ & $\mathrm{OH}$ & HO2 & HO2- & $02-$ & $\mathrm{H}+$ & $\mathrm{OH}-$ & $\mathrm{H} 2 \mathrm{O} 2$ & H2 & 02 \\
\hline 0.0 & 276.7 & 5.6 & 0.11 & 3.91 & 158 & 95 & 234 & 0.30 & --- & --- & 0 & 0 & 0 & 0 & 1 & 1 & 3 & 50 & 9 & 1 & 176 \\
\hline 50.0 & 280.9 & 5.6 & 0.11 & 3.88 & 238 & 123 & 472 & 0.30 & 3.7 & 0.5 & 0 & 0 & 0 & 1 & 30 & 8 & 4 & 42 & 291 & 24 & 209 \\
\hline 100.0 & 285.0 & 5.5 & 0.10 & 3.85 & 256 & 145 & 532 & 0.30 & 25.9 & 4.8 & 0 & 0 & 0 & 2 & 50 & 9 & 4 & 38 & 542 & 27 & 212 \\
\hline 150.0 & 288.3 & 5.5 & 0.10 & 3.83 & 262 & 156 & 546 & 0.30 & 29.4 & 4.2 & 0 & 0 & 1 & 1 & 49 & 7 & 4 & 39 & 508 & 17 & 184 \\
\hline 200.0 & 289.5 & 5.5 & 0.10 & 3.82 & 265 & 160 & 554 & 0.30 & 25.7 & 3.4 & 0 & 0 & 1 & 1 & 45 & 6 & 4 & 41 & 450 & 13 & 147 \\
\hline 250.0 & 289.9 & 5.5 & 0.10 & 3.81 & 268 & 164 & 567 & 0.30 & 22.2 & 2.9 & 0 & 0 & 1 & 1 & 43 & 5 & 4 & 42 & 415 & 10 & 122 \\
\hline 300.0 & 290.0 & 5.5 & 0.10 & 3.81 & 269 & 167 & 575 & 0.30 & 19.9 & 2.5 & 0 & 0 & 1 & 1 & 41 & 5 & 4 & 43 & 394 & 9 & 108 \\
\hline 350.0 & 290.0 & 5.5 & 0.10 & 3.81 & 271 & 170 & 582 & 0.30 & 18.3 & 2.3 & 0 & 0 & 1 & 1 & 40 & 4 & 4 & 44 & 370 & 8 & 97 \\
\hline 381.0 & 289.6 & 5.6 & 0.10 & 3.81 & 268 & 167 & 570 & 0.30 & 18.1 & 2.3 & 0 & 0 & 0 & 1 & 35 & 3 & 4 & 45 & 318 & 7 & 90 \\
\hline
\end{tabular}

Figure XIII.48. BWR_MASTER code printout file format.

\section{XIII.6. Simulation of BWR water chemistry, ECP and CGR with alternative chemical reaction sets}

Trial simulation of species concentrations in BWR HTC has been performed with two "as is" alternative sets of chemical reactions: one by Christensen and another by Ishigure. Both sets have reaction rates for $288{ }^{\circ} \mathrm{C}$, which have been used as an input. No special treatment of reactions has been performed within the BWR_MASTER source code.

The reaction set from Christensen includes 37 chemical reactions (Table XIII.5), the one from Ishigure - 33 reactions (Table XIII.6), while presently used CAM PSU set - 34 reactions. Two new species: $\mathrm{O}$ and $\mathrm{O}^{-}$are present in the Christensen set in comparison with CAM PSU and Ishigure sets.

Calculated concentrations of $\mathrm{H}_{2}, \mathrm{O}_{2}$, and $\mathrm{H}_{2} \mathrm{O}_{2}$ in a BWR HTC under NWC and HWC with Christensen and CAM PSU data are shown in Figure XIII.49. to Figure XIII.29.

Calculated concentrations of $\mathrm{H}_{2}$ are in good agreement, except for the Core Bypass region under NWC (Figure XIII.49. and Figure XIII.25).

Calculated concentrations of $\mathrm{H}_{2} \mathrm{O}_{2}$ deviate significantly from each other, both for NWC and HWC (Figure XIII.26 and Figure XIII.27). There obvious under prediction of $\mathrm{H}_{2} \mathrm{O}_{2}$ decomposition when Christensen data are used. The possibility of adjusting reactions in Christensen's set is discussed briefly in the "Adjustment of Rate Constants" section. 
Table XIII.5. Christensen's (2002) reaction set.

\begin{tabular}{|c|c|c|c|c|}
\hline No. & Reactions & $\mathrm{k}_{22}$ & $\begin{array}{l}\mathrm{E}_{\mathrm{A}} \\
\mathrm{J} \times \mathrm{mol}^{-1}\end{array}$ & $\mathrm{k}_{288}$ \\
\hline 1 & $\mathrm{OH}+\mathrm{OH} \rightarrow \mathrm{H}_{2} \mathrm{O}_{2}$ & $5.5000 \mathrm{E}+9$ & $7.90 \mathrm{E}+3$ & $2.540 \mathrm{E}+10$ \\
\hline 2 & $\mathrm{OH}+\mathrm{E}^{-} \rightarrow \mathrm{OH}$ & $3.100 \mathrm{E}+10$ & $1.47 \mathrm{E}+4$ & $5.345 \mathrm{E}+11$ \\
\hline 3 & $\mathrm{OH}+\mathrm{H} \rightarrow \mathrm{H}_{2} \mathrm{O}$ & $9.300 \mathrm{E}+9$ & $8.20 \mathrm{E}+3$ & $4.553 \mathrm{E}+10$ \\
\hline 4 & $\mathrm{OH}+\mathrm{HO}_{2} \rightarrow \mathrm{H}_{2} \mathrm{O}+\mathrm{O}_{2}$ & $7.100 \mathrm{E}+9$ & $1.42 \mathrm{E}+4$ & $1.111 \mathrm{E}+11$ \\
\hline s & $\mathrm{OH}+\mathrm{O}_{2}^{-} \rightarrow \mathrm{O}_{2}+\mathrm{OH}$ & $1.000 \mathrm{E}+10$ & $1.76 \mathrm{E}+4$ & $3.024 \mathrm{E}+11$ \\
\hline j & $\mathrm{OH}+\mathrm{H}_{2} \mathrm{O}_{2} \rightarrow \mathrm{HO}_{2}+\mathrm{H}_{2} \mathrm{C}$ & $2.700 \mathrm{E}+7$ & $1.42 \mathrm{E}+4$ & $4.225 \mathrm{E}+8$ \\
\hline 1 & $\mathrm{OH}+\mathrm{H}_{2} \rightarrow \mathrm{H}+\mathrm{H}_{2} \mathrm{C}$ & $3.4000 \mathrm{E}+7$ & $1.92 \mathrm{E}+4$ & $1.401 \mathrm{E}+9$ \\
\hline 8 & $\mathrm{OH}+\mathrm{OH}^{-} \rightarrow \mathrm{H}_{2} \mathrm{O}+\mathrm{O}^{-}$ & $1.200 \mathrm{E}+10$ & $1.42 \mathrm{E}+4$ & $1.878 \mathrm{E}+11$ \\
\hline 9 & $\mathrm{OH}+\mathrm{HO}_{2}^{-} \rightarrow \mathrm{HO}_{2}+\mathrm{OH}^{-}$ & $7.500 \mathrm{E}+9$ & $1.42 \mathrm{E}+4$ & $1.174 \mathrm{E}+11$ \\
\hline 10 & $\mathrm{OH}+\mathrm{O}^{-} \rightarrow \mathrm{HO}_{2}^{-}$ & $1.800 \mathrm{E}+10$ & $1.42 \mathrm{E}+4$ & $2.817 \mathrm{E}+11$ \\
\hline 11 & $\mathrm{O}^{-}+\mathrm{H}_{2} \mathrm{O} \rightarrow \mathrm{OH}+\mathrm{OH}^{-}$ & $1.700 \mathrm{E}+6$ & $\mathrm{Na}$ & $1.500 \mathrm{E}+8$ \\
\hline 12 & $\mathrm{E}^{-}+\mathrm{E}^{-} \rightarrow \mathrm{H}_{2}+\mathrm{OH}^{-}+\mathrm{OH}^{-}$ & $5.500 \mathrm{E}+9$ & $\mathrm{Na}$ & $5.500 \mathrm{E}+9$ \\
\hline 13 & $\mathrm{E}^{-}+\mathrm{H} \rightarrow \mathrm{H}_{2}+\mathrm{OH}^{-}-\mathrm{H}_{2} \mathrm{C}$ & $2.400 \mathrm{E}+10$ & $1.40 \mathrm{E}+4$ & $3.613 \mathrm{E}+11$ \\
\hline 14 & $\mathrm{E}^{-}+\mathrm{O}_{2}^{-} \rightarrow \mathrm{HO}_{2}^{-}+\mathrm{OH}^{-}-\mathrm{H}_{2} \mathrm{C}$ & $1.300 \mathrm{E}+10$ & $1.42 \mathrm{E}+4$ & $2.034 \mathrm{E}+11$ \\
\hline 15 & $\mathrm{E}^{-}+\mathrm{HO}_{2} \rightarrow \mathrm{HO}_{2}^{-}$ & $2.000 \mathrm{E}+10$ & $1.42 \mathrm{E}+4$ & $3.130 \mathrm{E}+11$ \\
\hline 16 & $\mathrm{E}^{-}+\mathrm{H}_{2} \mathrm{O}_{2} \rightarrow \mathrm{OH}+\mathrm{OH}$ & $1.200 \mathrm{E}+10$ & $1.56 \mathrm{E}+4$ & $2.463 \mathrm{E}+11$ \\
\hline 17 & $\mathrm{E}^{-}+\mathrm{O}_{2} \rightarrow \mathrm{O}_{2}^{-}$ & $1.900 \mathrm{E}+10$ & $1.36 \mathrm{E}+4$ & $2.647 \mathrm{E}+11$ \\
\hline 18 & $\mathrm{E}^{-}+\mathrm{H}^{+} \rightarrow \mathrm{H}$ & $2.300 \mathrm{E}+10$ & $1.26 \mathrm{E}+4$ & $2.640 \mathrm{E}+11$ \\
\hline 19 & $\mathrm{E}^{-}+\mathrm{H}_{2} \mathrm{O} \rightarrow \mathrm{H}+\mathrm{OH}^{-}$ & $1.900 \mathrm{E}+1$ & $1.42 \mathrm{E}+4$ & $2.973 \mathrm{E}+2$ \\
\hline 20 & $\mathrm{E}^{-}+\mathrm{HO}_{2}^{-} \rightarrow \mathrm{O}^{-}+\mathrm{OH}^{-}$ & $3.500 \mathrm{E}+9$ & $1.42 \mathrm{E}+4$ & $5.477 \mathrm{E}+10$ \\
\hline 21 & $\mathrm{H}+\mathrm{H} \rightarrow \mathrm{H}_{2}$ & $5.500 \mathrm{E}+9$ & $1.46 \mathrm{E}+4$ & $9.301 \mathrm{E}+10$ \\
\hline 22 & $\mathrm{H}+\mathrm{O}_{2}^{-} \rightarrow \mathrm{HO}_{2}^{-}$ & $2.000 \mathrm{E}+10$ & $1.42 \mathrm{E}+4$ & $3.130 \mathrm{E}+11$ \\
\hline 23 & $\mathrm{H}+\mathrm{HO}_{2} \rightarrow \mathrm{H}_{2} \mathrm{O}_{2}$ & $2.000 \mathrm{E}+10$ & $1.42 \mathrm{E}+4$ & $3.130 \mathrm{E}+11$ \\
\hline 24 & $\mathrm{H}+\mathrm{H}_{2} \mathrm{O}_{2} \rightarrow \mathrm{H}_{2} \mathrm{O}+\mathrm{OH}$ & $5.100 \mathrm{E}+7$ & $1.07 \mathrm{E}+4$ & $4.052 \mathrm{E}+8$ \\
\hline 25 & $\mathrm{H}+\mathrm{O}_{2} \rightarrow \mathrm{HO}_{2}$ & $2.100 \mathrm{E}+10$ & $1.03 \mathrm{E}+4$ & $1.544 \mathrm{E}+11$ \\
\hline 26 & $\mathrm{H}+\mathrm{OH}^{-} \rightarrow \mathrm{E}^{-}+\mathrm{H}_{2} \mathrm{O}$ & $2.200 \mathrm{E}+7$ & $3.76 \mathrm{E}+4$ & $3.201 \mathrm{E}+10$ \\
\hline 21 & $\mathrm{HO}_{2}+\mathrm{HO}_{2} \rightarrow \mathrm{H}_{2} \mathrm{O}_{2}+\mathrm{O}_{2}$ & $8.400 \mathrm{E}+5$ & $2.06 \mathrm{E}+4$ & $4.541 \mathrm{E}+7$ \\
\hline $2 \gamma$ & $\mathrm{HO}_{2}+\mathrm{O}_{2}^{-} \rightarrow \mathrm{O}_{2}+\mathrm{HO}_{2}^{-}$ & $9.600 \mathrm{E}+7$ & $7.60 \mathrm{E}+3$ & $4.184 \mathrm{E}+8$ \\
\hline 29 & $\mathrm{HO}_{2} \rightarrow \mathrm{H}^{+}+\mathrm{O}_{2}^{-}$ & $8.000 \mathrm{E}+5$ & $\mathrm{Na}$ & $3.900 \mathrm{E}+5$ \\
\hline 30 & $\mathrm{H}^{+}+\mathrm{O}_{2}^{-} \rightarrow \mathrm{HO}_{2}$ & $5.000 \mathrm{E}+10$ & $1.42 \mathrm{E}+4$ & $7.825 \mathrm{E}+11$ \\
\hline 31 & $\mathrm{H}_{2} \mathrm{O}_{2}+\mathrm{OH}^{-} \rightarrow \mathrm{HO}_{2}^{-}+\mathrm{H}_{2} \mathrm{O}$ & $5.000 \mathrm{E}+8$ & $1.88 \mathrm{E}+4$ & $1.907 \mathrm{E}+10$ \\
\hline 32 & $\mathrm{HO}_{2}^{-}+\mathrm{H}_{2} \mathrm{O} \rightarrow \mathrm{H}_{2} \mathrm{O}_{2}+\mathrm{OH}$ & $5.700 \mathrm{E}+4$ & $\mathrm{Na}$ & $1.200 \mathrm{E}+7$ \\
\hline 33 & $\mathrm{H}_{2} \mathrm{O}_{2} \rightarrow \mathrm{H}_{2} \mathrm{O}+\mathrm{O}$ & $4.000 \mathrm{E}-8$ & $6.30 \mathrm{E}+4$ & $3.000 \mathrm{E}-2$ \\
\hline 34 & $\mathrm{O}+\mathrm{O} \rightarrow \mathrm{O}_{2}$ & $5.000 \mathrm{E}+9$ & $1.42 \mathrm{E}+4$ & $7.825 \mathrm{E}+10$ \\
\hline 35 & $\mathrm{H}_{2} \mathrm{O} \rightarrow \mathrm{H}^{+}+\mathrm{OH}^{-}$ & $2.599 \mathrm{E}-5$ & $\mathrm{Na}$ & $1400 \mathrm{E}-1$ \\
\hline 36 & $\mathrm{H}^{+}+\mathrm{OH}^{-} \rightarrow \mathrm{H}_{2} \mathrm{C}$ & $1.430 \mathrm{E}+11$ & $1.42 \mathrm{E}+4$ & $2.238 \mathrm{E}+12$ \\
\hline 37 & $\mathrm{O}_{2}^{-}+\mathrm{O}_{2}^{-} \rightarrow \mathrm{HO}_{2}^{-}+\mathrm{O}_{2}-\mathrm{H}^{+}$ & $1.770 \mathrm{E}+9$ & $8.00 \mathrm{E}+4$ & $9.497 \mathrm{E}+15$ \\
\hline
\end{tabular}


Table XIII.6. Ishigure's (1987) reaction set.

\begin{tabular}{|c|c|c|}
\hline No. & Reactions & $\mathrm{k}_{280}$ \\
\hline 1 & $\mathrm{e}^{-}{ }_{\mathrm{aa}}+\mathrm{H}_{2} \mathrm{O} \rightarrow \mathrm{H}+\mathrm{OH}$ & $1.65 \mathrm{E}+2$ \\
\hline 2 & $\mathrm{e}_{\mathrm{aa}}^{-}+\mathrm{H}^{+} \rightarrow \mathrm{H}$ & $2.48 \mathrm{E}+11$ \\
\hline 3 & $\mathrm{e}_{\mathrm{aa}}^{-}+\mathrm{OH} \rightarrow \mathrm{OH}$ & $3.10 \mathrm{E}+11$ \\
\hline 4 & $\mathrm{e}_{\mathrm{aa}}^{-}+\mathrm{H}_{2} \mathrm{O}_{2} \rightarrow \mathrm{OH}+\mathrm{OH}$ & $1.34 \mathrm{E}+11$ \\
\hline p & $\mathrm{H}+\mathrm{H} \rightarrow \mathrm{H}_{2}$ & $1.03 \mathrm{E}+11$ \\
\hline 5 & $\mathrm{e}_{\mathrm{aq}}^{-}+\mathrm{HO}_{2} \rightarrow \mathrm{HO}_{2}^{-}$ & $2.06 \mathrm{E}+11$ \\
\hline I & $\mathrm{e}_{\mathrm{aa}}^{-}+\mathrm{O}_{2} \rightarrow \mathrm{O}_{2}^{-}$ & $1.96 \mathrm{E}+11$ \\
\hline 3 & $2 \mathrm{H}_{2} \mathrm{O}+2 \mathrm{e}_{\mathrm{aa}}^{-} \rightarrow \mathrm{H}_{2}+2 \mathrm{OH}$ & $3.00 \mathrm{E}+7$ \\
\hline$y$ & $\mathrm{OH}+\mathrm{OH} \rightarrow \mathrm{H}_{2} \mathrm{O}_{2}$ & $4.65 \mathrm{E}+10$ \\
\hline 10 & $\mathrm{OH}^{-}+\mathrm{H} \rightarrow \mathrm{e}^{-}{ }_{\mathrm{aa}}+\mathrm{H}_{2} \mathrm{C}$ & $6.63 \mathrm{E}+8$ \\
\hline 11 & $\mathrm{H}_{2} \mathrm{O}+\mathrm{e}^{-}{ }_{\mathrm{aa}}+\mathrm{H} \rightarrow \mathrm{H}_{2}+\mathrm{OH}$ & $6.22 \mathrm{E}+9$ \\
\hline 12 & $\mathrm{H}_{2} \mathrm{O}+\mathrm{e}_{\mathrm{aa}}^{-}+\mathrm{HO}_{2}^{-} \rightarrow \mathrm{OH}+2 \mathrm{OH}$ & $8.70 \mathrm{E}+8$ \\
\hline 13 & $\mathrm{H}+\mathrm{OH} \rightarrow \mathrm{H}_{2} \mathrm{C}$ & $2.06 \mathrm{E}+11$ \\
\hline 14 & $\mathrm{OH}+\mathrm{H}_{2} \rightarrow \mathrm{H}+\mathrm{H}_{2} \mathrm{C}$ & 4. $8 \angle \mathrm{E}+8$ \\
\hline 12 & $\mathrm{H}+\mathrm{H}_{2} \mathrm{O} \rightarrow \mathrm{H}_{2}+\mathrm{OH}$ & $8.14 \mathrm{E}+2$ \\
\hline 16 & $\mathrm{H}+\mathrm{OH} \rightarrow \mathrm{HO}_{2}$ & $1.96 \mathrm{E}+11$ \\
\hline 17 & $\mathrm{H}+\mathrm{HO}_{2} \rightarrow \mathrm{H}_{2} \mathrm{O}_{2}$ & $2.06 \mathrm{E}+11$ \\
\hline 18 & $\mathrm{H}+\mathrm{O}_{2}^{-} \rightarrow \mathrm{HO}_{2}^{-}$ & $2.06 \mathrm{E}+11$ \\
\hline 19 & $\mathrm{H}_{2} \mathrm{O}+\mathrm{e}^{-}{ }_{\mathrm{aq}}+\mathrm{O}^{-}{ }_{2} \rightarrow \mathrm{HO}_{2}^{-}+\mathrm{OH}^{-}$ & $1.04 \mathrm{E}+10$ \\
\hline 20 & $\mathrm{H}+\mathrm{H}_{2} \mathrm{O}_{2} \rightarrow \mathrm{OH}+\mathrm{H}_{2} \mathrm{O}$ & $1.13 \mathrm{E}+9$ \\
\hline 21 & $\mathrm{OH}+\mathrm{H}_{2} \mathrm{O}_{2} \rightarrow \mathrm{HO}_{2}+\mathrm{H}_{2} \mathrm{C}$ & $1.03 \mathrm{E}+8$ \\
\hline 22 & $\mathrm{OH}+\mathrm{HO}_{2} \rightarrow \mathrm{O}_{2}+\mathrm{H}_{2} \mathrm{O}$ & $1.24 \mathrm{E}+11$ \\
\hline 23 & $\mathrm{OH}^{-}+\mathrm{H}_{2} \mathrm{O}_{2} \rightarrow \mathrm{HO}_{2}^{-}+\mathrm{H}_{2} \mathrm{C}$ & $5.97 \mathrm{E}+0$ \\
\hline 24 & $\mathrm{HO}_{2}^{-}+\mathrm{H}_{2} \mathrm{O} \rightarrow \mathrm{OH}^{-}+\mathrm{H}_{2} \mathrm{O}_{2}$ & $1.89 \mathrm{E}+7$ \\
\hline 25 & $\mathrm{H}^{+}+\mathrm{O}_{2}^{-} \rightarrow \mathrm{HO}_{2}$ & $5.16 \mathrm{E}+11$ \\
\hline 26 & $\mathrm{HO}_{2} \rightarrow \mathrm{H}^{+}+\mathrm{O}_{2}^{-}$ & $3.40 \mathrm{E}+7$ \\
\hline 27 & $\mathrm{HO}^{2}+\mathrm{O}_{2}^{-} \rightarrow \mathrm{O}_{2}+\mathrm{HO}_{2}^{-}$ & $4.97 \mathrm{E}+8$ \\
\hline 28 & $2 \mathrm{H}_{2} \mathrm{O}+2 \mathrm{O}_{2}^{-} \rightarrow \mathrm{H}_{2} \mathrm{O}_{2}+\mathrm{O}_{2}+2 \mathrm{OH}$ & $3.27 \mathrm{E}+5$ \\
\hline 29 & $\mathrm{HO}_{2}+\mathrm{HO}_{2} \rightarrow \mathrm{H}_{2} \mathrm{O}_{2}+\mathrm{O}_{2}$ & $8.95 \mathrm{E}+7$ \\
\hline 30 & $\mathrm{H}^{+}+\mathrm{OH}^{-} \rightarrow \mathrm{H}_{2} \mathrm{O}$ & $1.49 \mathrm{E}+12$ \\
\hline 31 & $\mathrm{H}_{2} \mathrm{O} \rightarrow \mathrm{H}^{+}+\mathrm{OH}^{-}$ & $1.33 \mathrm{E}-1$ \\
\hline 32 & $\mathrm{OH}+\mathrm{O}_{2}^{-} \rightarrow \mathrm{O}_{2}+\mathrm{OH}^{-}$ & $1.24 \mathrm{E}+11$ \\
\hline 33 & $\mathrm{H}_{2} \mathrm{O}_{2} \rightarrow 2 \mathrm{OH}$ & $1.26 \mathrm{E}-1 *$ \\
\hline
\end{tabular}




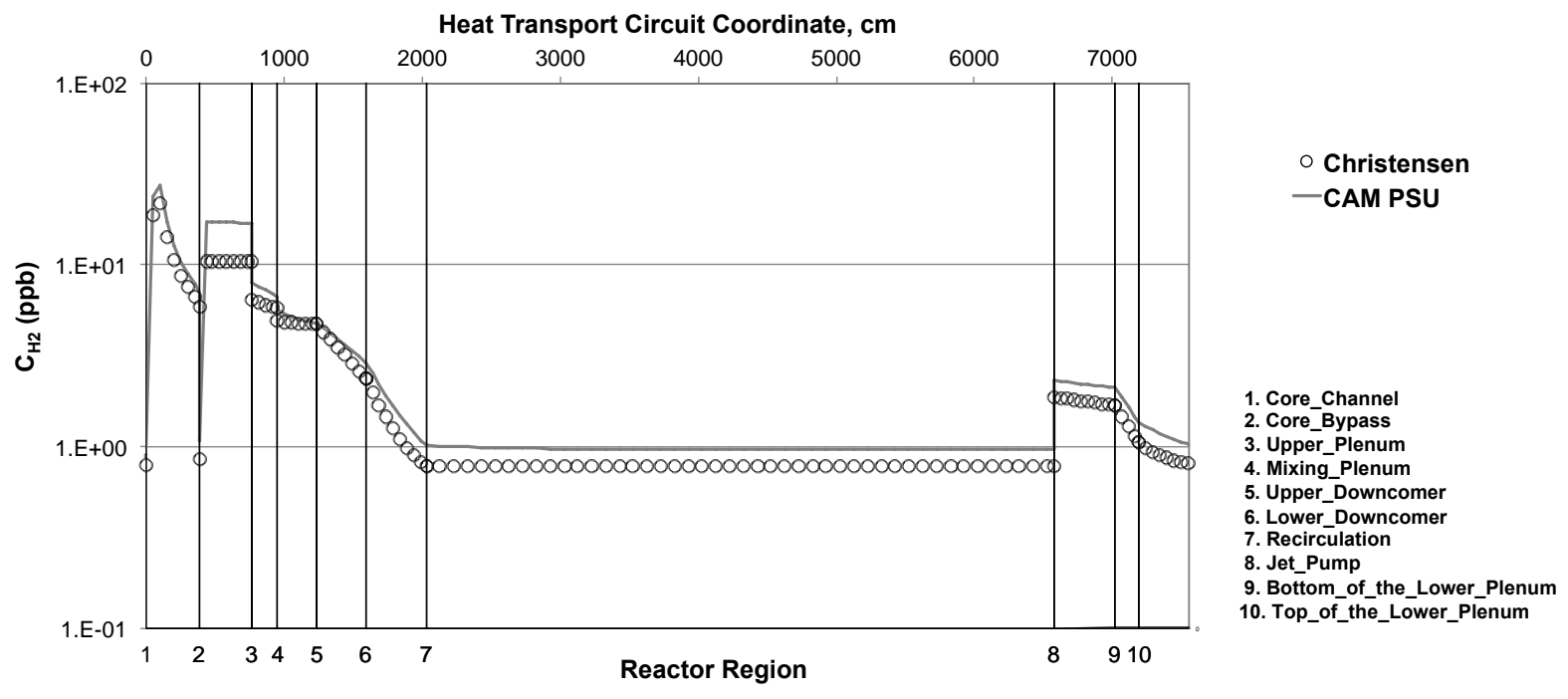

Figure XIII.49. Comparison of hydrogen concentration under Normal Water Chemistry conditions calculated with Christensen set (open circles) and CAM PSU set (solid line).

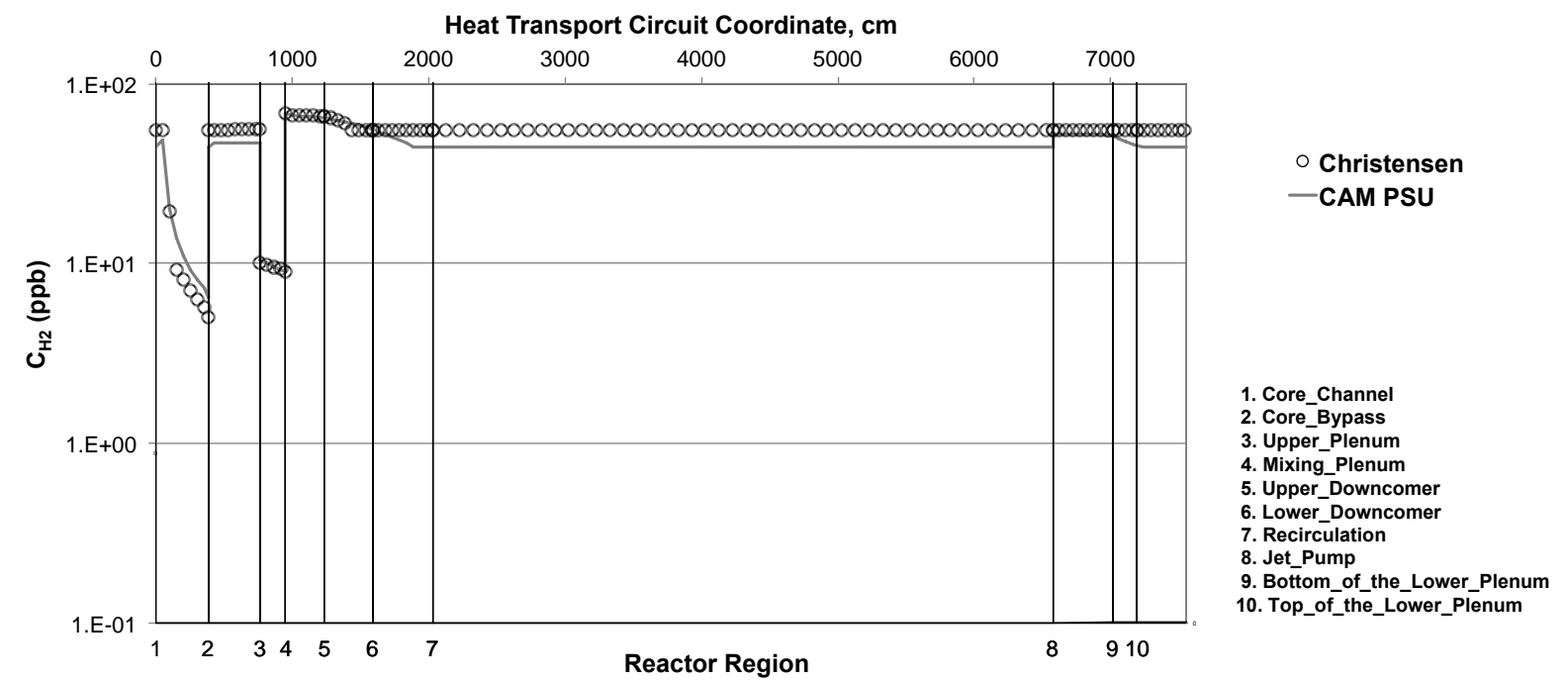

Figure XIII.25 Comparison of hydrogen concentration under Hydrogen Water Chemistry conditions calculated with Christensen set (open circles) and CAM PSU set (solid line). 


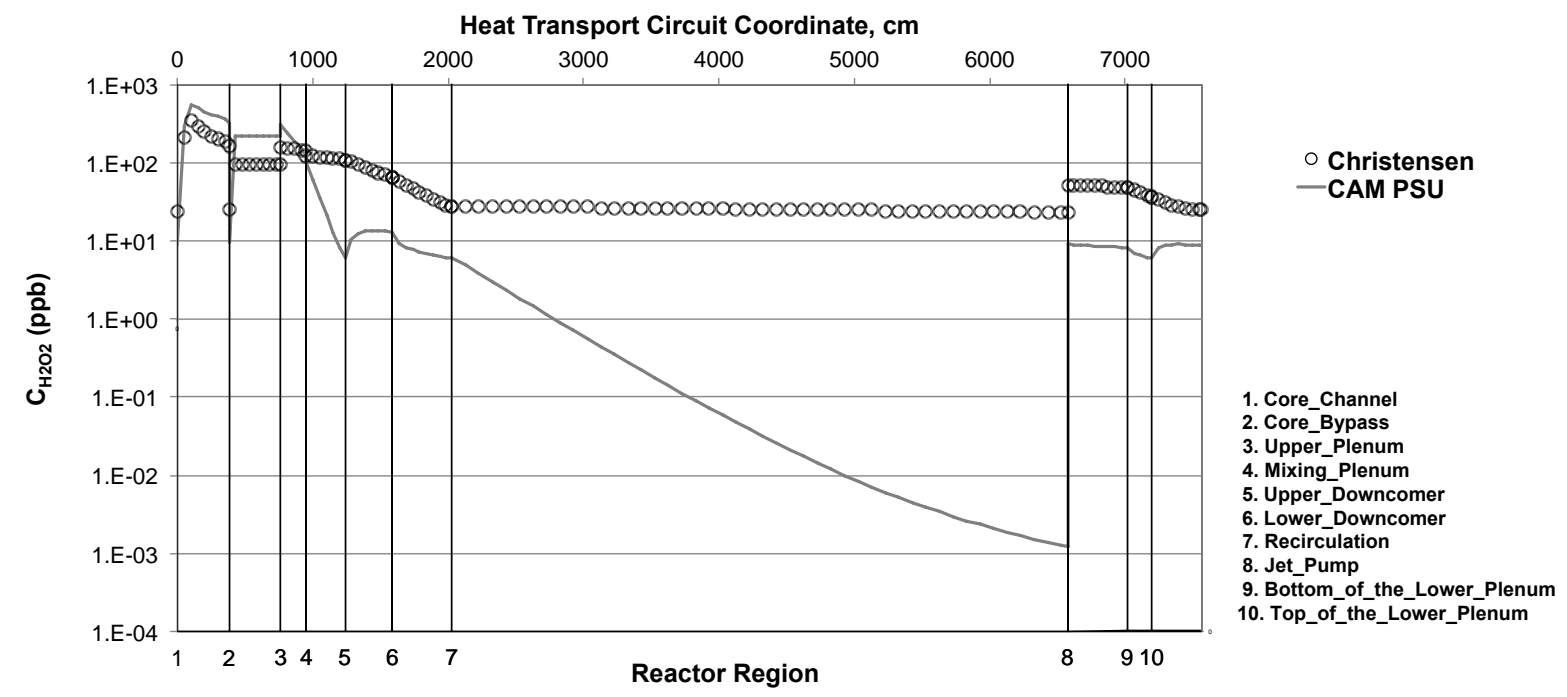

Figure XIII.26 Comparison of hydrogen peroxide concentration under Normal Water Chemistry conditions calculated with Christensen set (open circles) and CAM PSU set (solid line).

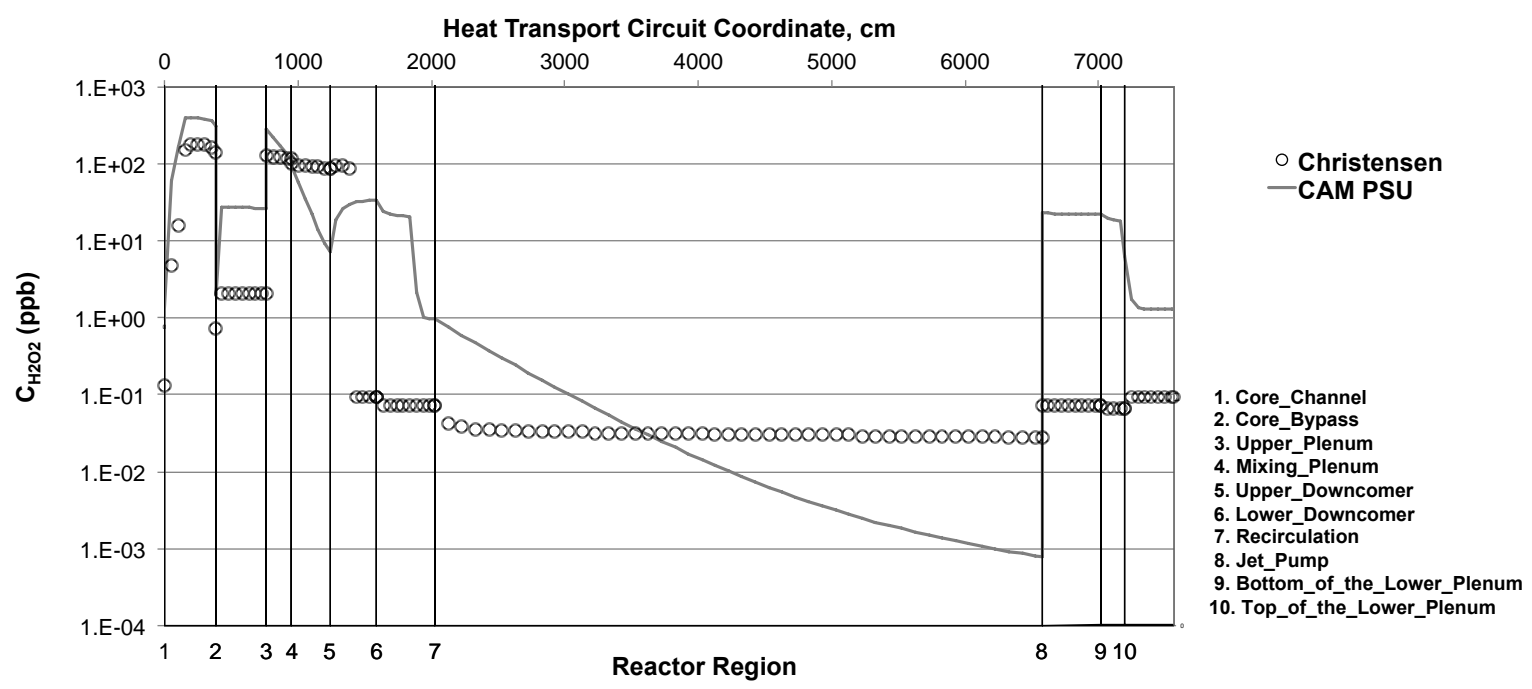

Figure XIII.27 Comparison of hydrogen peroxide concentration under Hydrogen Water Chemistry conditions calculated with Christensen set (open circles) and CAM PSU set (solid line).

Calculated concentrations of $\mathrm{O}_{2}$ are in satisfactory agreement for NWC and deviate significantly under HWC (Figure XIII.28 and Figure XIII.29). 


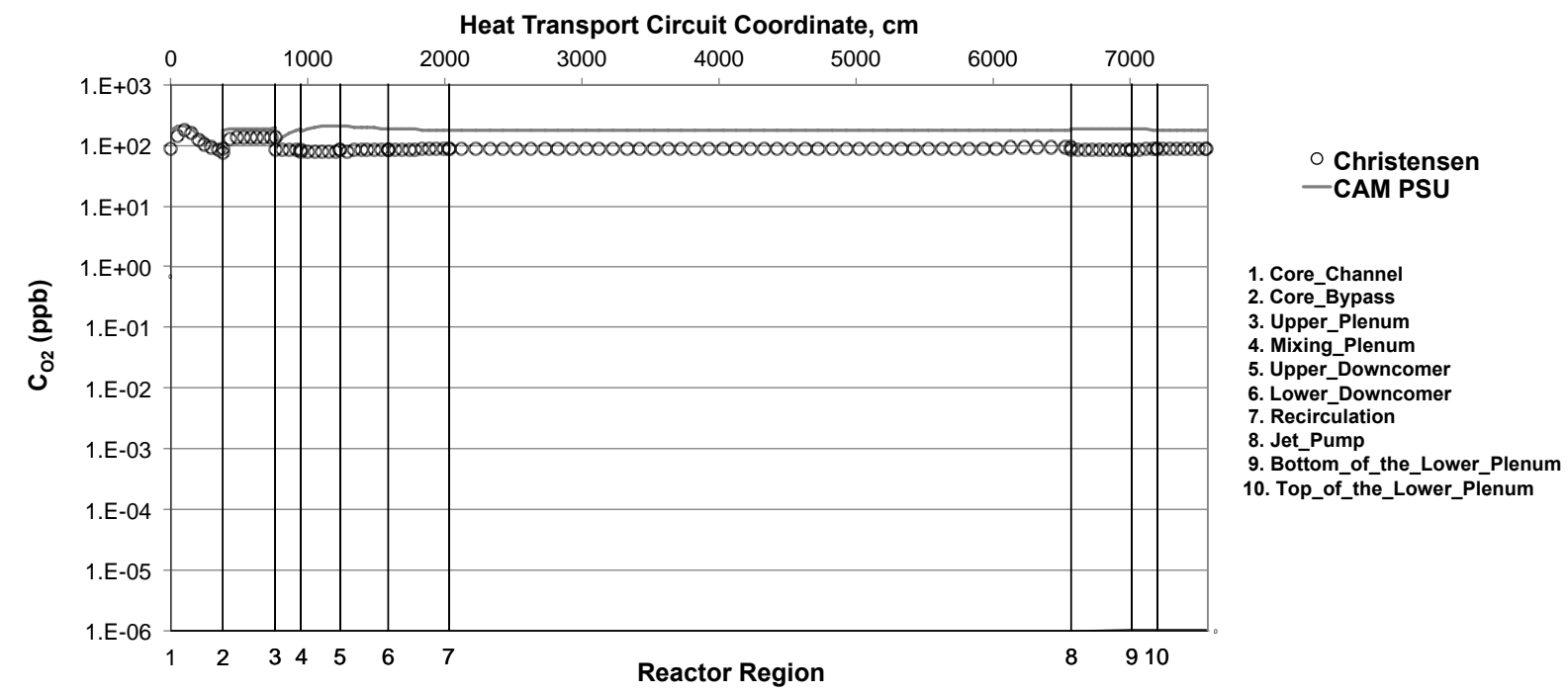

Figure XIII.28 Comparison of oxygen concentration under Normal Water Chemistry conditions calculated with Christensen set (open circles) and CAM PSU set (solid line).

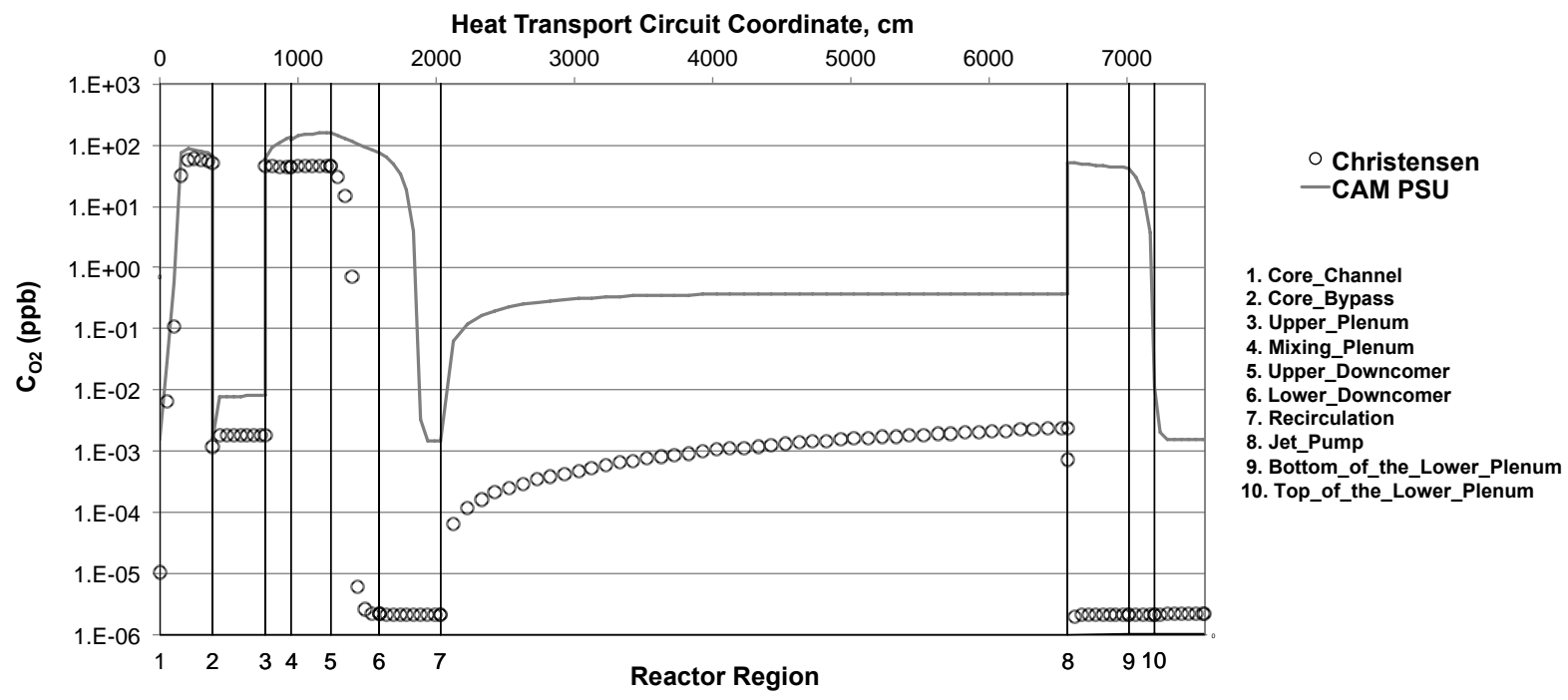

Figure XIII.29 Comparison of oxygen concentration under Hydrogen Water Chemistry conditions calculated with Christensen set (open circles) and CAM PSU set (solid line).

The discrepancies in calculated species concentration are higher if Isigure data are being used (not included in this report). 
The previously used CAM PSU data on chemical reactions are much more adequate and produce a better agreement with plant measure data (see "XIII.7. Comparison of calculated species concentrations and ECP with plant measured data" section).

The Christensen data are more recent than Ishigure data and may be considered to be an alternative data set after proper adjustment of high-temperature reaction rates.

\section{XIII.7. Comparison of calculated species concentrations and ECP with plant measured data}

There are BWR plants without detected cracks using the mitigation methods. In other plants, where cracks were detected, it was not possible to show significant crack growth reduction up to now. New developments such as the on-line NMCA technique will provide new results and additional experience. However, in conjunction with NMCA applications, several secondary negative impacts were also reported in some cases: increase of fuel cladding corrosion rates, increased deposition of corrosion products, mobilization of activities, and increasing radiation fields.

At the Leibstadt BWR core internals, no cracks were detected so far. Nevertheless, based on worldwide experience, it was decided to implement HWC. On the other hand, due to possible problems and uncertainties associated with the efficiency of the NMCA technique as reported above and taking into account the near-irreversibility of the noble metal addition, the application of NMCA is not intended.

The concentrations of $\mathrm{H}_{2}$ and $\mathrm{O}_{2}$ and the ECP were determined during a "Hydrogen Water Chemistry Minitest" at Leibstadt BWR/6 (German: Kernkraftwerk Leibstadt, KKL) located near Leibstadt, canton of Aargau, Switzerland, on the Rhine River and close to the border to Germany. A boiling water reactor built there by General Electric with 1,220 MW of electrical power. This power station is owned by Leibstadt AG (KKL).

Hydrogen and oxygen sensors and an ECP probe have been installed in the sampling line. Reported measured concentrations and ECP are shown in Figure XIII.55 as black circles. Measured plant data demonstrate that Leibstadt BWR responds quickly to hydrogen addition. Essentially no further decrease in ECP and oxygen concentration has been observed when hydrogen injection increased above $0.8 \mathrm{ppm}$. 
(a)

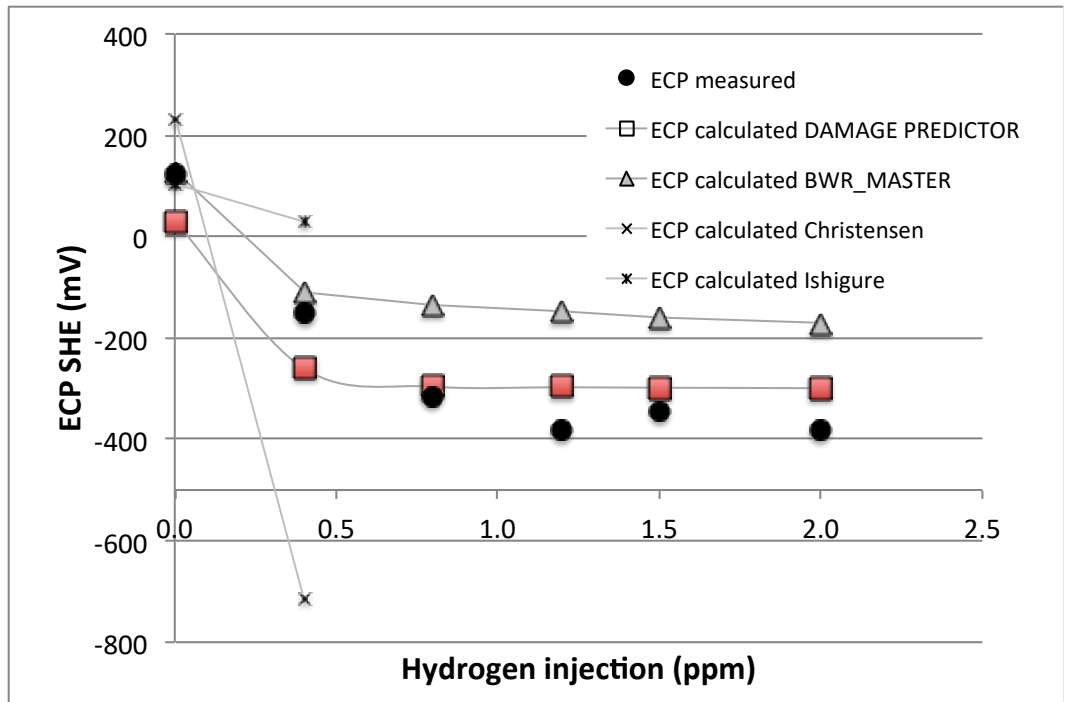

(b)
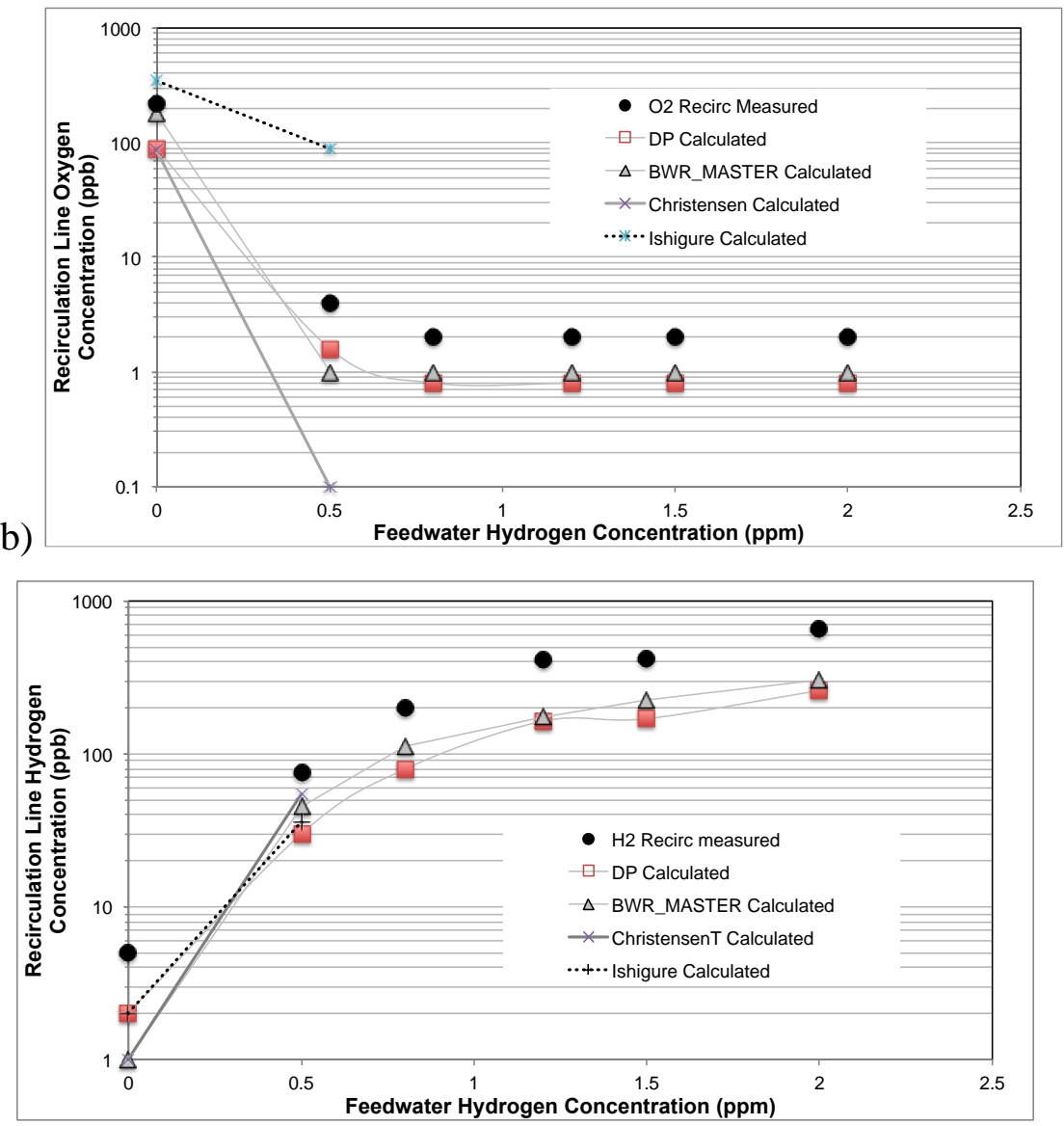

(c)

Figure XIII.55. Comparison of measured during Hydrogen Water Chemistry minitest and calculated ECP and species concentrations. 
The ECP calculated by BWR_MASTER with CAM PSU reaction set and Christensen (2002) data on radiolytic yields are in perfect agreement with measurements for 0 and $0.4 \mathrm{ppm}$ of hydrogen injection (Figure XIII.55a). The agreement is poor for hydrogen injection above 0.8 ppm: calculated ECP is still above the protection level.

The ECP calculated by DAMAGE PREDICTOR is in poor agreement with measurements for 0 and $0.4 \mathrm{ppm}$ of hydrogen injection (Figure XIII.55a). The agreement is perfect for hydrogen injection above $0.8 \mathrm{ppm}$.

The ECP and species concentrations calculated by BWR_MASTER with Christensen and Ishigure data on chemical reactions are in poor agreement in all cases with one exception: measured and calculated ECP agree under NWC if Christensen data on chemical reactions are used.

While using CAM PSU data on chemical reactions provides the best agreement with measurements, concentrations of oxygen and hydrogen are systematically under-predicted (Figure XIII.55 a and b). Under-prediction of hydrogen concentrations at HWC conditions by a factor of 2 definitely contributes to over-predictions of ECP at the same conditions. Under-prediction of oxygen concentrations under HWC is not critical since oxygen is rapidly depressed by HWC to single digits ppb in the case of the Leibstadt reactor.

Given a good agreement of previous DAMAGE PREDICTOR with measured data, it is not clear if improper modeling or input data are the major reason for disagreements between the calculated $\mathrm{H}_{2}$ concentrations and the ECP with the Leibstadt measured data.

\section{XIII.8. Reduced reaction rate sets}

An attempt to estimate feasibility and cost/benefit ratio of reducing the number of chemical reactions was undertaken. The only benefit from reducing the number of reactions is reduced simulation time, which may be desirable if the simulation of 60 or so years of reactor operation is reduced significantly (by a factor of 2 and more).

The candidate reaction for removal from the input data set has been identified by performing a sensitivity analysis of variations in species concentrations and ECP along BWR HTC resulting from a 10 times increase in a particular reaction rate. CAM PSU reaction rate set was 
selected for analysis because it appears more reliable, as judged by good agreement of calculated and measured data for several operating BWRs.

An example of sensitivity of ECP in the Core Bypass and in recirculation line to variation of reaction rates (Figure XIII.56) shows, that a list of most and least sensitive reactions depends on position along BWR HTC, Type of integral parameter (concentrations or ECP) and water chemistry conditions (Figure XIII.57 and Figure XIII.58.).

The candidate reactions for deletion (Table XIII.7) have been selected according to three, progressively more softening criteria:

(1) "strong" - deviations of ECP and concentrations of $\mathrm{H}_{2}, \mathrm{O}_{2}$, and $\mathrm{H}_{2} \mathrm{O}_{2}$ are less than $0.5 \%$

(2) "medium" - only deviations of ECP are less than $0.5 \%$

(3) "weak" - only deviations of ECP are less than $2 \%$

No chemical reactions may be removed from CAM PSU set if "strong" criteria are used. While 4 reactions may be removed to keep adequate predictions in the Core Bypass under NWC, keeping reliable predictions in the Recirculation Line does not allow that any reaction is removed. The sensitivity to reaction removal under HWC conditions is lower than under NWC, and 6 to 10 reactions may be removed without reducing the reliability of the predictions of species concentrations and ECP in the Core Bypass and in Recirculation Line, respectively. However, it does not change the final conclusion.

In case, if "medium" or "weak" selection criteria are used, 5 or 10 reactions may be removed, as it is illustrated by Table XIII.7.

The impact of using reduced reactions set on the calculated ECP under NWC is illustrated in Figure XIII.59.. A maximal deviation of about $100 \mathrm{mV}$ is observed in the Upper Plenum and $25 \mathrm{mV}$, the Recirculation Line, and in the Bottom of the Lower Plenum.

The predicted crack depth in the Core Bypass and Recirculation Line after 60 years of operation under NWC deviates deviate significantly if the original CAM PSU set with 34 reactions is reduced to 24 reactions (Figure XIII.30). 


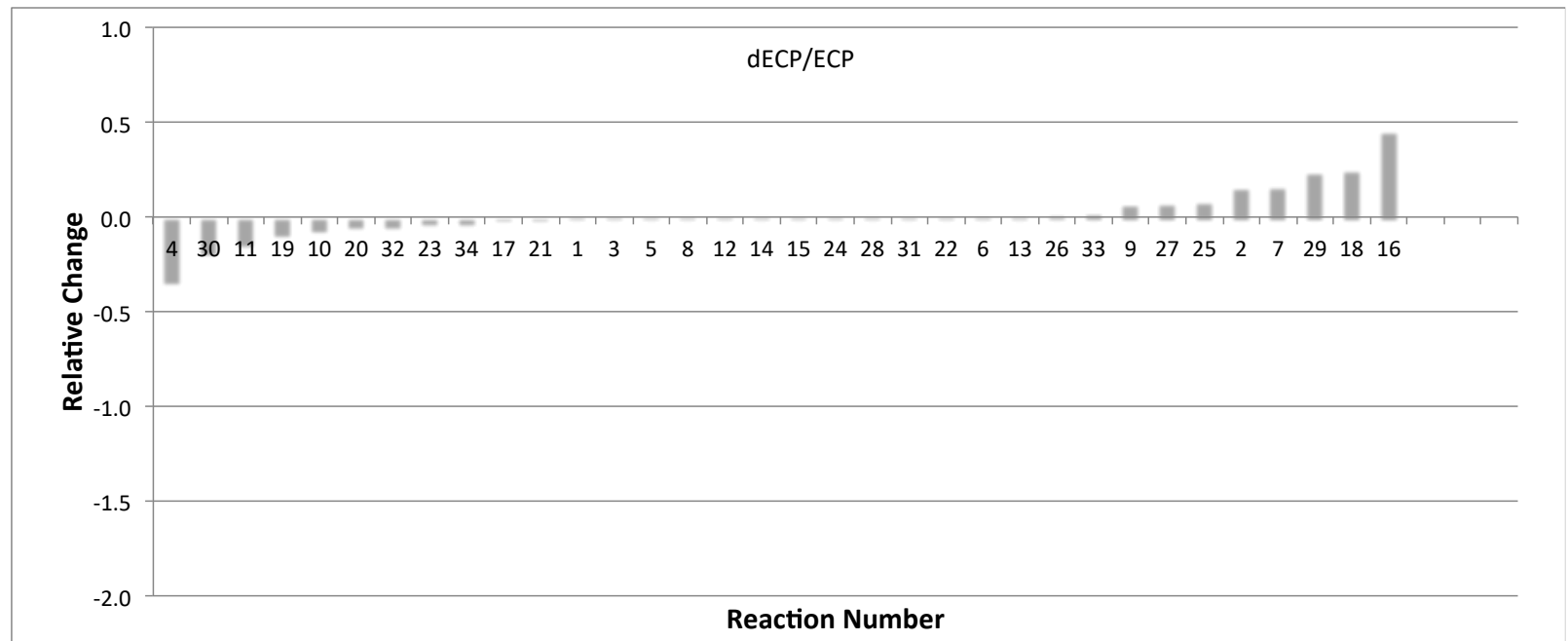

(a)

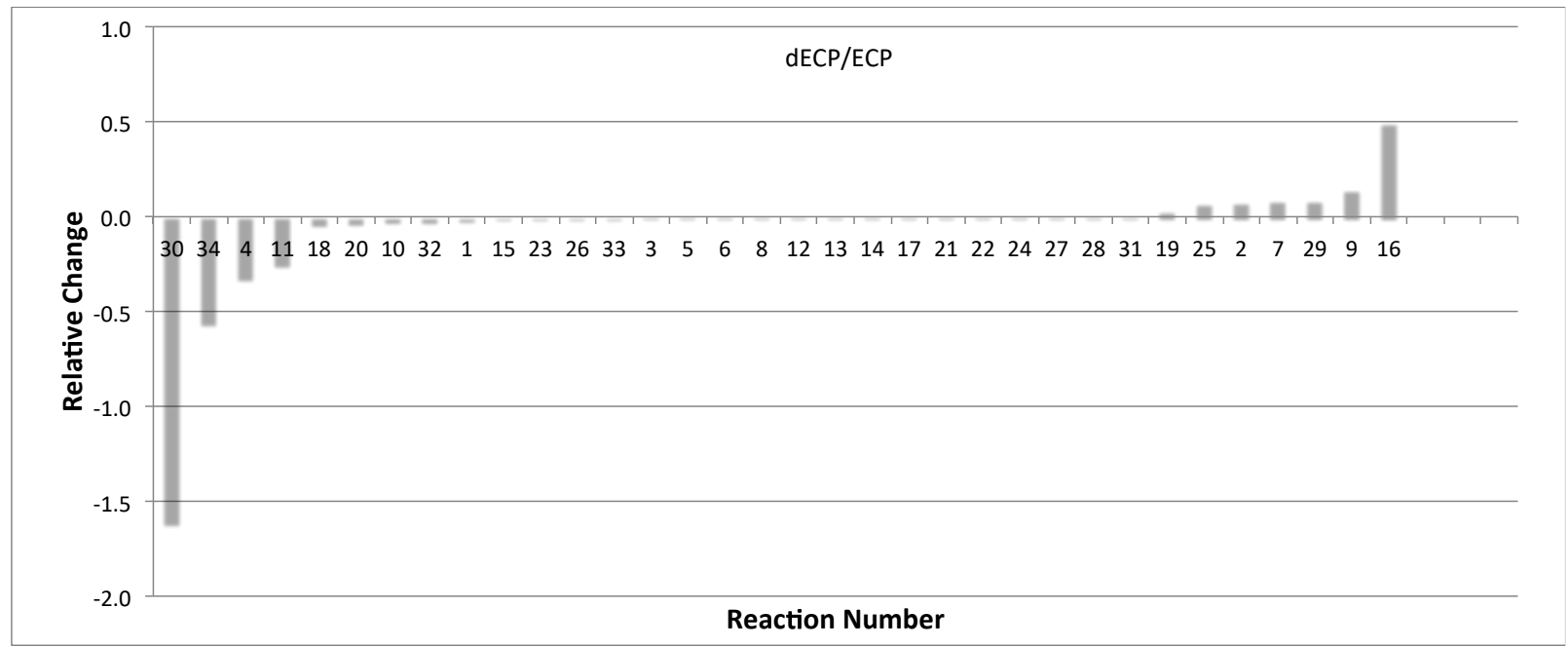

(b)

Figure XIII.56. The sensitivity of ECP in Core Bypass (a) and Recirculation Line (b) to 10x increase in reaction rates in CAM PSU reaction set under NWC. 

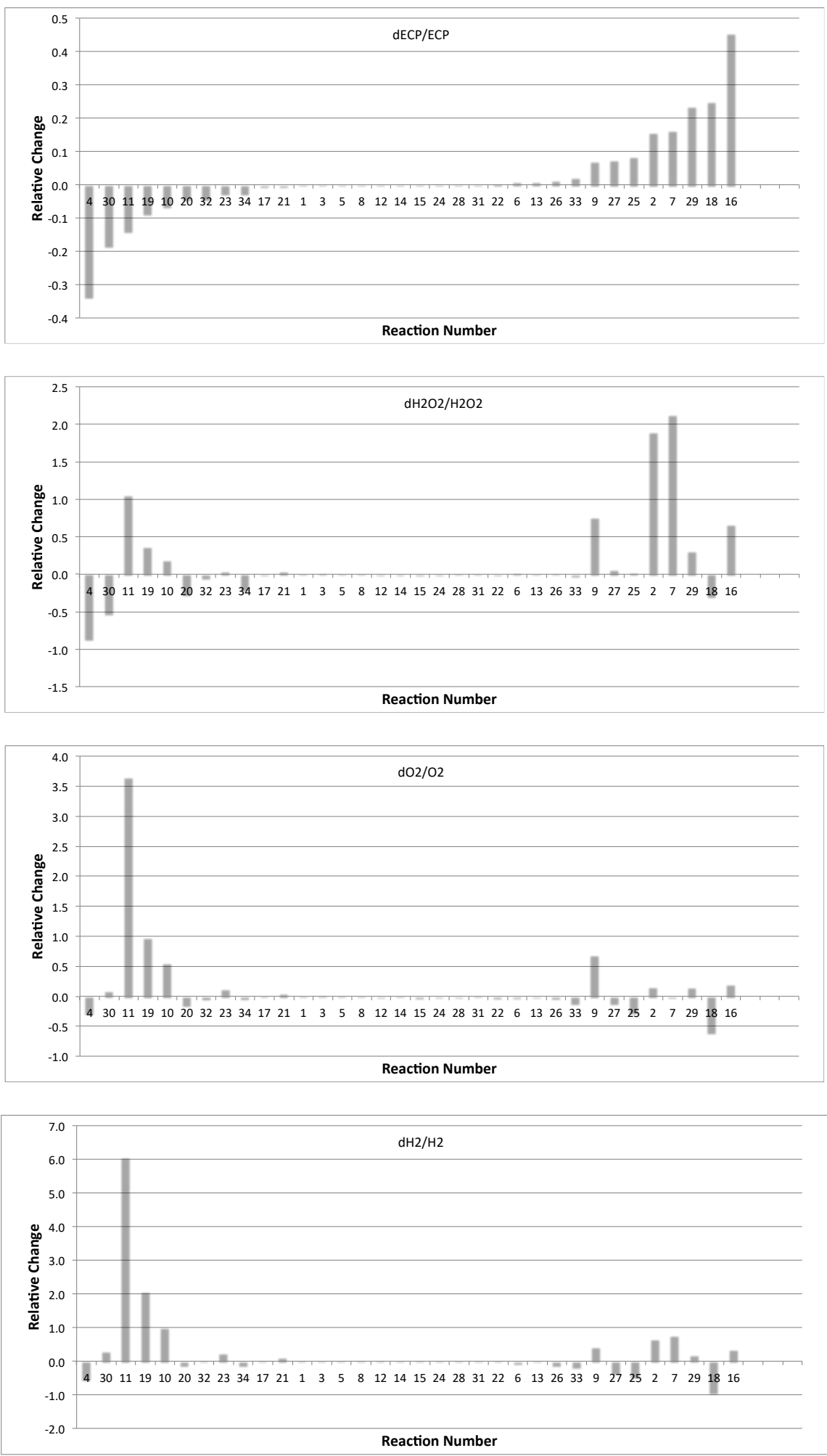

Figure XIII.57. The sensitivity of species concentrations and ECP in core bypass to 10x increase in reaction rates in CAM PSU reaction set under NWC. 

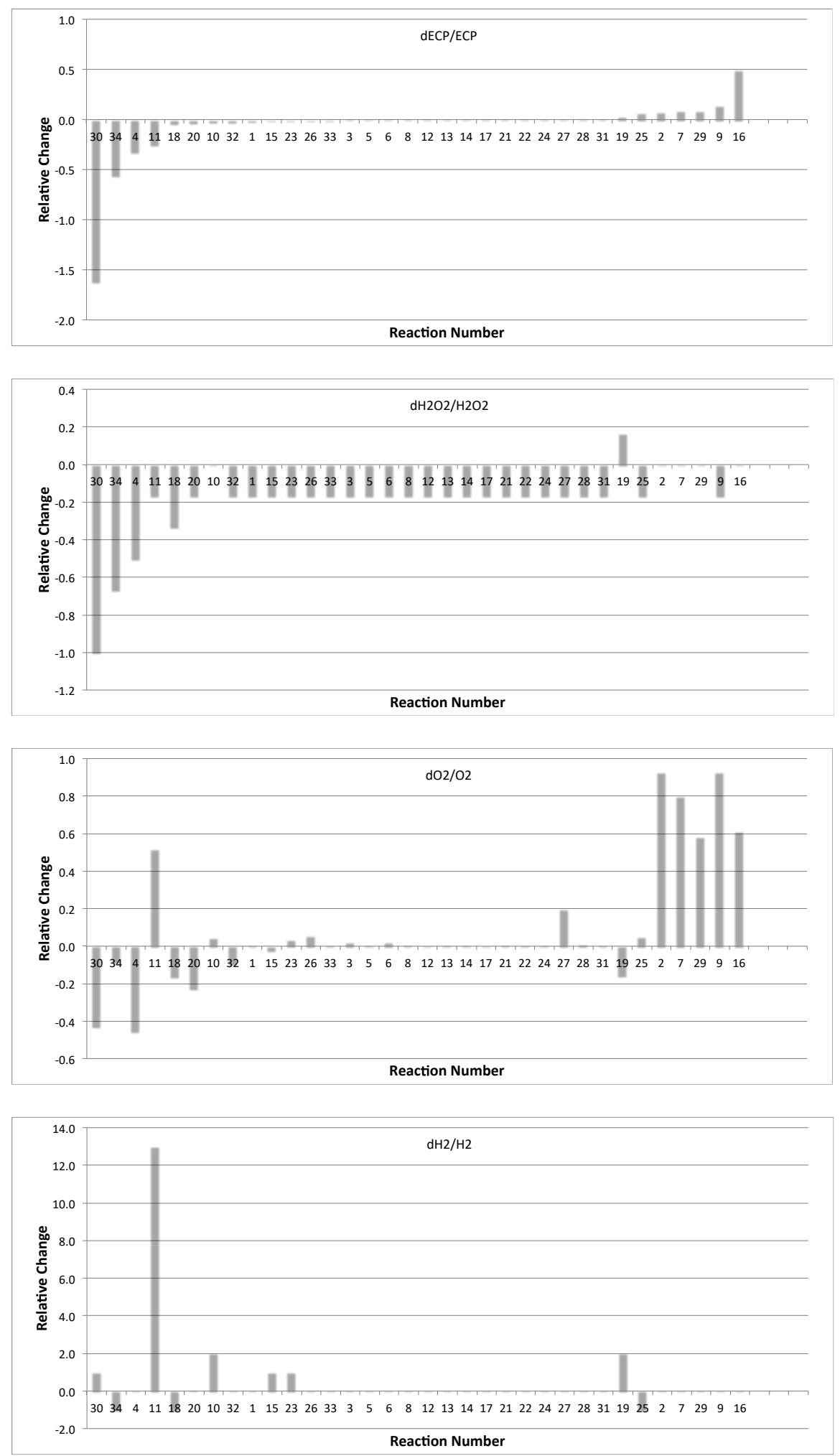

Figure XIII.58. The sensitivity of species concentrations and ECP in recirculation line to $10 \mathrm{x}$ increase in reaction rates in CAM PSU reaction set under NWC. 
Table XIII.7. Reduced reaction rate sets.
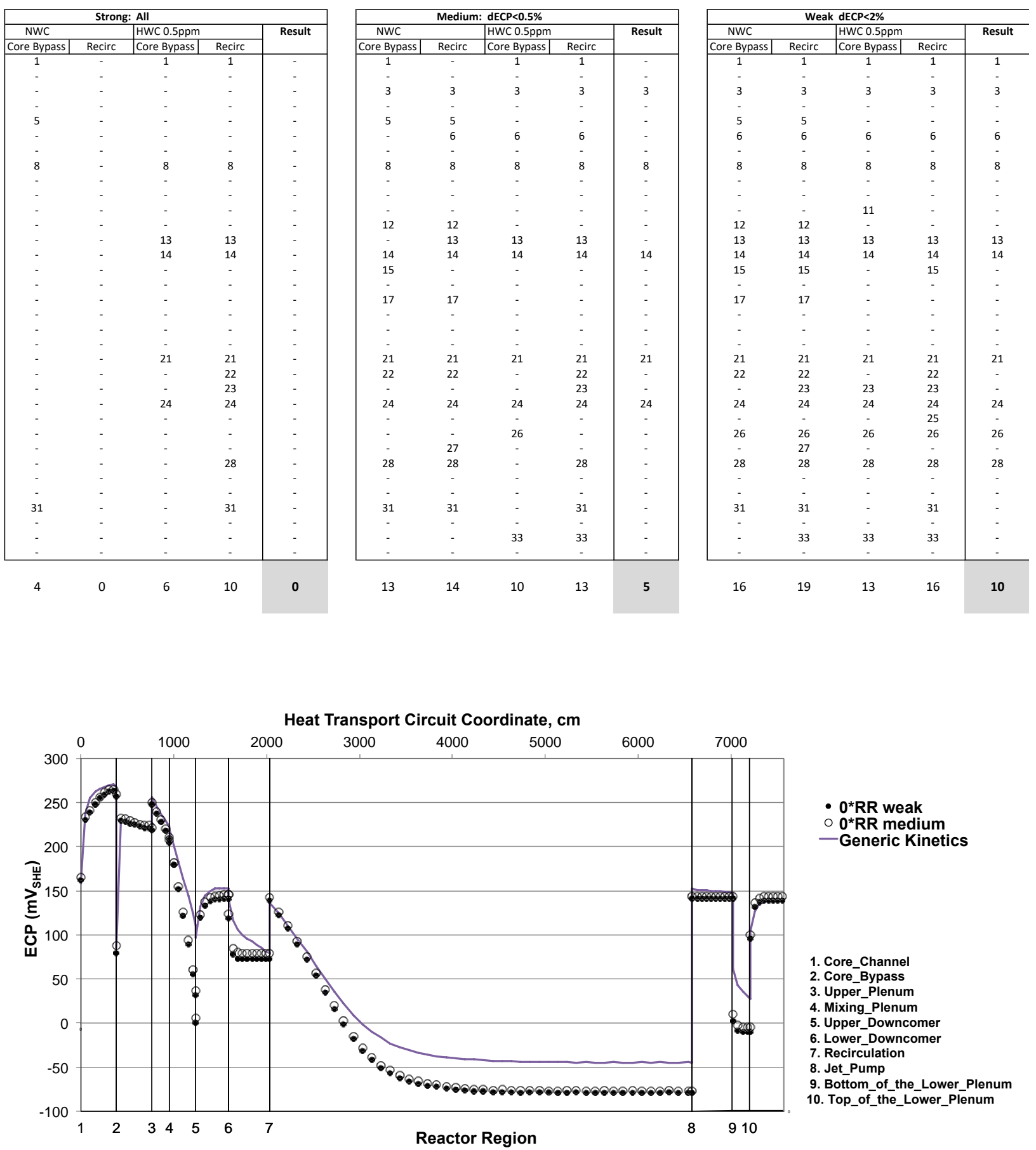

Figure XIII.59. Calculated ECP under NWC with original CAM PSU data (solid line), with 5 reactions removed (open circles) and 10 reactions removed (closed circles). 

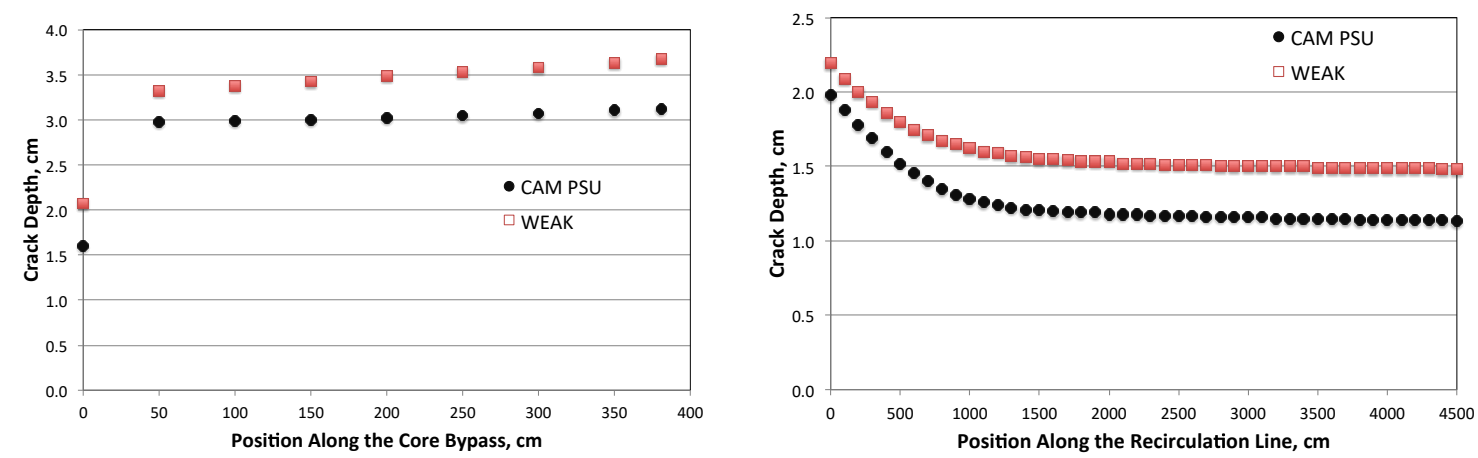

(a)

(b)

Figure XIII.30. Calculated crack depth in the Bypass Region (a) and in the Recirculation Line (b) after 60 years of operation under NWC with original CAM PSU data (closed circles) and with 10 reactions removed (closed squares).

The impact of using reduced reactions sets on the simulation time of 60 years of reactor operation is illustrated in Figure XIII.31. The duration of the simulation on an iMAC computer with a $2.7 \mathrm{GHz}$ Intel Core i5 $8 \mathrm{~GB} 1333 \mathrm{MHz}$ DDR3 processor is reduced from 38.8 minutes to 24.2 minutes.

While, in general, a reduction of the reaction rates does not result in a more attractive cost/benefit ratio, in some "envelope estimation" cases, it may be justified, for example, in parametric scientific or engineering studies.

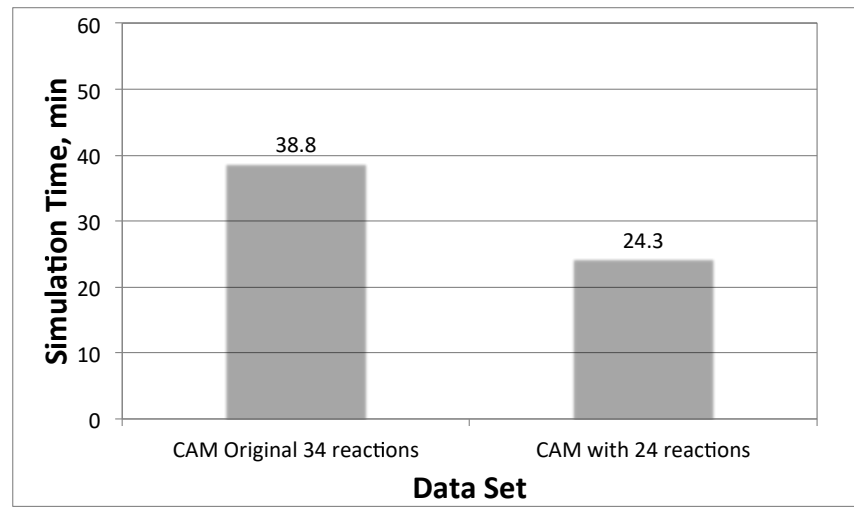

Figure XIII.31 Effect of the number of chemical reactions in the reaction rate set on simulation time. 


\section{XIII.9. Adjustment of rate constants}

Trial runs of BWR_MASTER with Ishigure and Christensen reaction rate sets (see section "XIII.6. Simulation of BWR water chemistry, ECP and CGR with alternative chemical reaction sets") reveled significant discrepancies with CAM PSU set based simulations and Leibstadt measured data, which points to a necessity to adjust published reaction rates.

An attempt to adjust the reaction rates in Christensen's set to match the calculated concentration of $\mathrm{H}_{2} \mathrm{O}_{2}$ has been made, as an example. A proper adjustment procedure should be a two-step process:

1. Adjusting reaction rates to achieve the best possible agreement with CAM PSU based concentrations of $\mathrm{H}_{2}, \mathrm{O}_{2}$, and $\mathrm{H}_{2} \mathrm{O}_{2}$

2. Adjusting reaction rates to achieve the best possible agreement with one arbitrary selected set of experimental data, for example, such as the one reported by Leibstadt

3. Verification of the adjusted data by comparison with other available sets of experimental data from operating BWRs.

As was mentioned in Section "XIII.6. Simulation of BWR water chemistry, ECP and CGR with alternative chemical reaction sets") there is an obvious problem with the predicted decomposition of $\mathrm{H}_{2} \mathrm{O}_{2}$ when using Christensen's data set (Figure XIII.32). A list of relevant reactions is shown in Table XIII.8. A decision was made to adjust the rate of Reaction \#33 only. The agreement has been improved significantly after increasing the rate of Reaction \#33 by two orders of magnitude (Figure XIII.33).

Table XIII.8. Hydrogen peroxide decomposition reactions in Christensen data set.

\begin{tabular}{r|c|l}
\hline \multicolumn{1}{c|}{ R } & Rate at $288{ }^{\circ} \mathbf{C}$ & Reaction \\
\hline 16 & $2.46 \mathrm{e}+11$ & $\mathrm{e}-+\mathrm{H}_{2} \mathrm{O}_{2}=\mathrm{OH}+\mathrm{OH}^{-}$ \\
\hline 24 & $4.05 \mathrm{e}+08$ & $\mathrm{H}+\mathrm{H}_{2} \mathrm{O}_{2}=\mathrm{H}_{2} \mathrm{O}+\mathrm{OH}$ \\
\hline 31 & $1.91 \mathrm{e}+10$ & $\mathrm{H}_{2} \mathrm{O}_{2}+\mathrm{OH}^{-}=\mathrm{HO}^{2-}+\mathrm{H}_{2} \mathrm{O}$ \\
\hline 33 & $3.00 \mathrm{e}-02$ & $\mathrm{H}_{2} \mathrm{O}_{2}=\mathrm{H}_{2} \mathrm{O}+\mathrm{O}$ \\
\hline
\end{tabular}




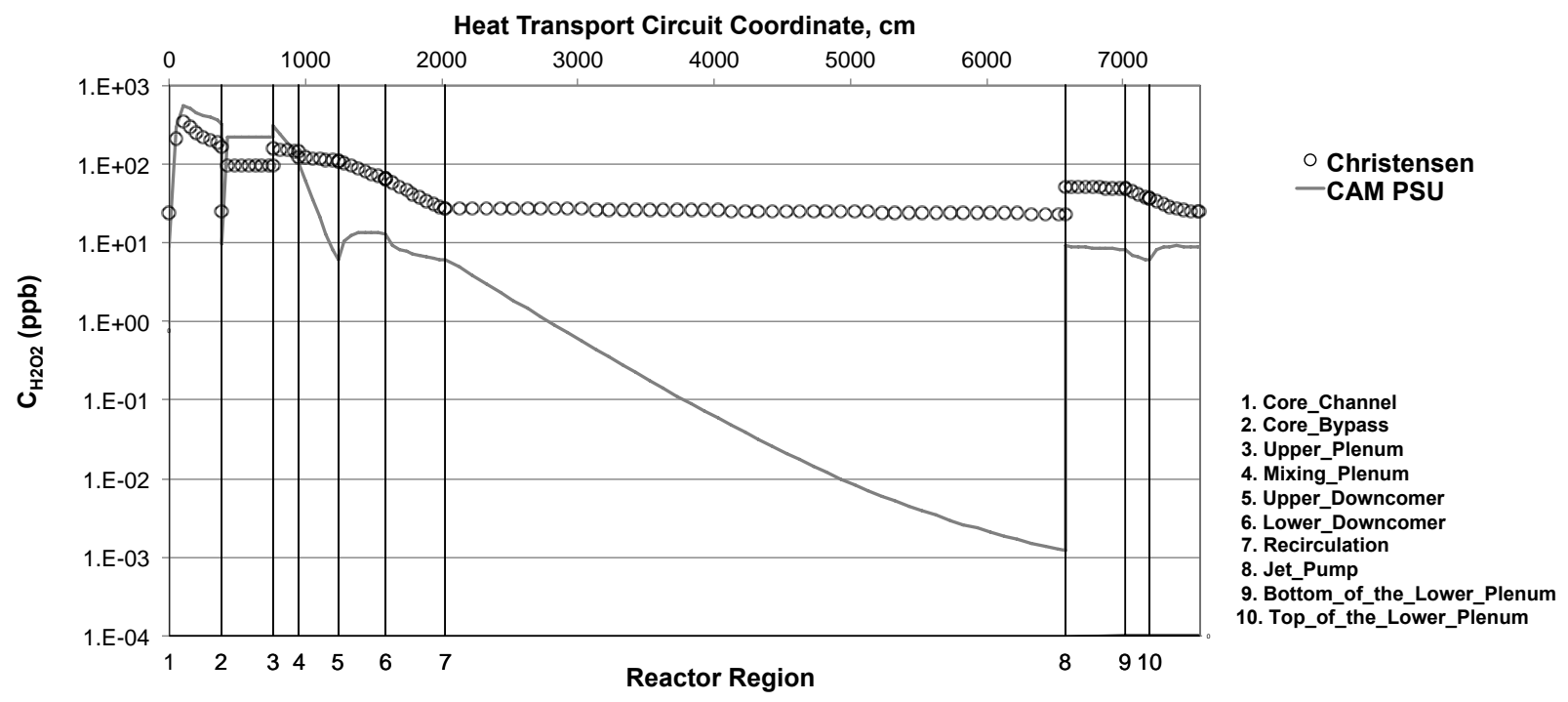

Figure XIII.32. Comparison of hydrogen peroxide concentration under Normal Water Chemistry conditions calculated with Christensen's original set (open circles) and CAM PSU set (solid line).

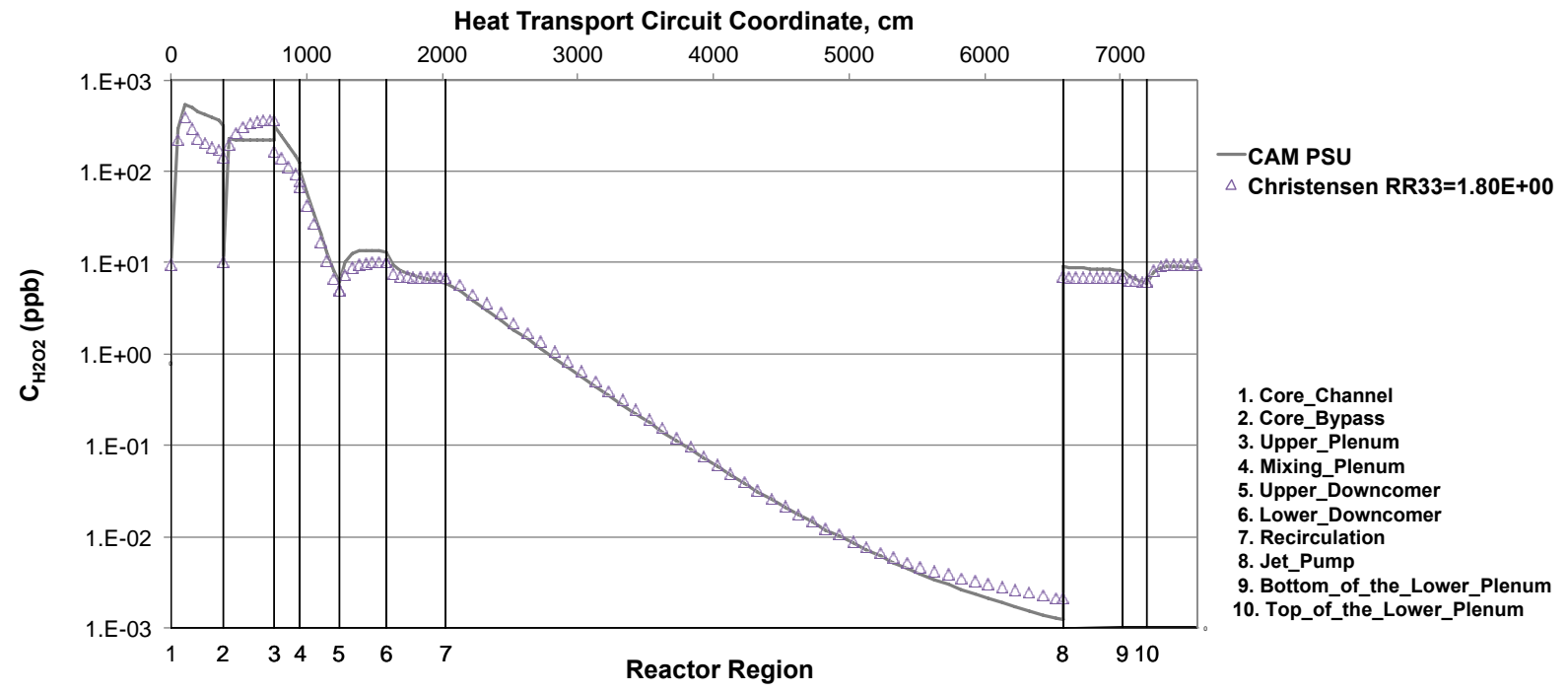

Figure XIII.33. Comparison of hydrogen peroxide concentration under Normal Water Chemistry conditions calculated with adjusted Christensen original set (open triangles) and CAM PSU set (solid line). 
A conclusion was made that reaction rates in Christensen's data set must be adjusted in order to produce adequate simulation results for species concentrations, if desired.

The use and adjustment of the Ishigure data set are not recommended for further consideration.

\section{XIII.10. Implementation of the generic chemical kinetics algorithm in PWR_MASTER code}

The modern version of the PWR_MASTER code includes a "fixed" chemical kinetics algorithm, which allows the use of only one fixed set of species, chemical reactions, and radiolytic yields of species. This set was developed at CAM PSU and originally was used in DAMAGE PREDICTOR code to simulate water chemistry, corrosion potential (ECP), and crack growth rates (CGR) in heat transport circuit HTC) of operating Boiling Water Reactors (BWRs). Ten chemical reactions (Table XIII.19) have been added to the original 34 reactions in CAM PSU reaction set based on an assumption of their relevance to PWR water chemistry. These additional reactions involve three other species: $\mathrm{O}, \mathrm{O}^{-}$and $\mathrm{O}_{2}{ }^{2-}$, making a total of 14 chemical species involved in PWR water chemistry simulations. Radiolytic yields of additional species from neutron and gamma radiation have been assumed to be equal to zero in the original PWR_MASTER code.

Table XIII.9. List of additional chemical reactions.

\begin{tabular}{|c|l|c|c|}
\hline 35 & $\mathrm{OH}+\mathrm{HO}_{2}^{-}=\mathrm{O}_{2}^{-}+\mathrm{H}_{2} \mathrm{O}$ & $7.88 \mathrm{E}+09$ & 3.0 \\
\hline 36 & $\mathrm{OH}+\mathrm{OH}^{-}=\mathrm{O}^{-}+\mathrm{H}_{2} \mathrm{O}$ & $1.28 \mathrm{E}+10$ & 3.0 \\
\hline 37 & $\mathrm{e}^{-}+\mathrm{HO}_{2}^{-}=\mathrm{O}^{-}+\mathrm{OH}^{-}$ & $3.97 \mathrm{E}+09$ & 3.0 \\
\hline 38 & $\mathrm{O}_{2}^{-}+\mathrm{O}_{2}^{-}+\mathrm{H}^{+}=\mathrm{HO}_{2}^{-}+\mathrm{O}_{2}$ & $6.42 \mathrm{E}+14$ & 3.0 \\
\hline 39 & $\mathrm{H}_{2} \mathrm{O}_{2}=\mathrm{H}_{2} \mathrm{O}+\mathrm{O}$ & $2.72 \mathrm{E}-03$ & 15 \\
\hline 40 & $\mathrm{e}^{-}+\mathrm{O}_{2}^{-}=\mathrm{O}_{2}{ }^{2-}$ & $1.3 \mathrm{E}+10$ & 3.0 \\
\hline 41 & $\mathrm{H}_{2} \mathrm{O}_{2}+\mathrm{HO}_{2}=\mathrm{H}_{2} \mathrm{O}+\mathrm{O}_{2}+\mathrm{OH}$ & $0.5 \mathrm{E}+00$ & 3.0 \\
\hline 42 & $\mathrm{O}_{2}^{-}+\mathrm{H}_{2} \mathrm{O}_{2}=\mathrm{OH}^{-} \mathrm{OH}^{-}+\mathrm{O}_{2}$ & $0.13 \mathrm{E}+00$ & 3.0 \\
\hline 43 & $\mathrm{H}_{2} \mathrm{O}_{2}=\mathrm{H}^{+}+\mathrm{HO}_{2}^{-}$ & $2.56 \mathrm{E}-08$ & 3.0 \\
\hline 44 & $\mathrm{e}^{-}+\mathrm{HO}_{2}+\mathrm{H}_{2} \mathrm{O}=\mathrm{H}_{2} \mathrm{O}_{2}+\mathrm{OH}^{-}$ & $1.39 \mathrm{E}+10$ & 3.2 \\
\hline 45 & $\mathrm{e}^{-}+\mathrm{O}_{2}^{-}+\mathrm{H}_{2} \mathrm{O}=\mathrm{HO}_{2}^{-}+\mathrm{OH}^{-}$ & $1.39 \mathrm{E}+10$ & 3.2 \\
\hline
\end{tabular}

Generic chemical kinetics algorithm 


$$
\left(\frac{G_{i}^{\gamma} \Gamma^{\prime}}{100 N_{V}}+\frac{G_{i}^{n} \Gamma^{n}}{100 N_{V}}\right) \tilde{F} \rho d V+\left[\sum_{s=1}^{N} \sum_{m=1}^{N} k_{s m} C_{s} C_{m}-C_{i} \sum_{s=1}^{N} k_{s i} C_{s}\right] d V+\left[\frac{d\left(u C_{i}\right)}{d x} d V\right] \pm\left(\mu_{i}^{*} C_{i}^{g}-\mu_{i} C_{i}^{f}\right) d V_{g}=0 .
$$

was implemented by replacing "fixed" model radiolysis function Chemical_Kinetics as shown in Figure XIII.16 to calculate gains and losses of species as:

$$
{ }_{s=1}^{N=1}{ }_{s=1}^{N} C_{s} C_{m} C_{i}^{N}{ }_{s=1}^{N} k_{s i} C_{s}
$$

and modifying radiolysis function for calculating radiolytic yields from neutron and gamma radiation

$$
\left(\frac{G_{i}^{\gamma} \Gamma^{\gamma}}{100 N_{V}}+\frac{G_{i}^{n} \Gamma^{n}}{100 N_{V}}\right) \tilde{F} \rho
$$

and convection and two-phase flow terms

$$
\left[\frac{d\left(u C_{i}\right)}{d x} d V\right] \pm\left({ }_{i} C_{i}^{g} \quad{ }_{i} C_{i}^{f}\right) d V_{g}
$$

A fragment of the previous ("fixed") version of Chemical_Kinetics is shown in Figure XIII.17. PWR_MASTER function radiolysis was modified to migrate from "fixed" lists of species and chemical reactions to generic ones.

A list of chemical species is defined in the input file YIELDS.DAT as string constants, which allows expansion or reduction of the number of species. Arbitrary lists of chemical reactions, reaction rates, and activation energies are taken from input file CHEMRAT.DAT, where chemical reactions are also defined as string variables. More information on PWR_MASTER input and output files are contained in Sections "Changes in PWR_MASTER code input data files" and "Changes in PWR_MASTER code output data files", respectively. 


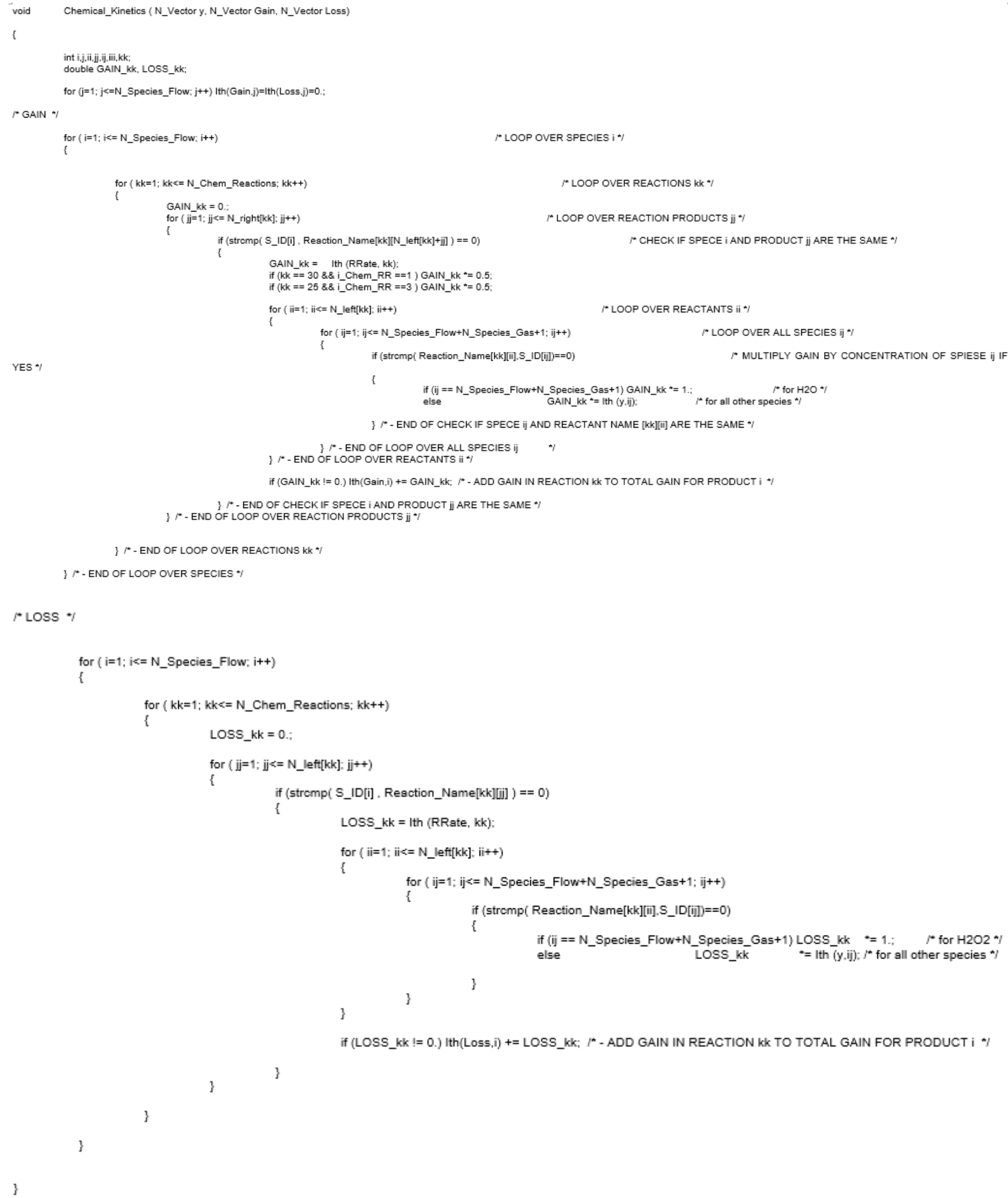

Figure XIII.34. Source code of generic chemical kinetics algorithm. 


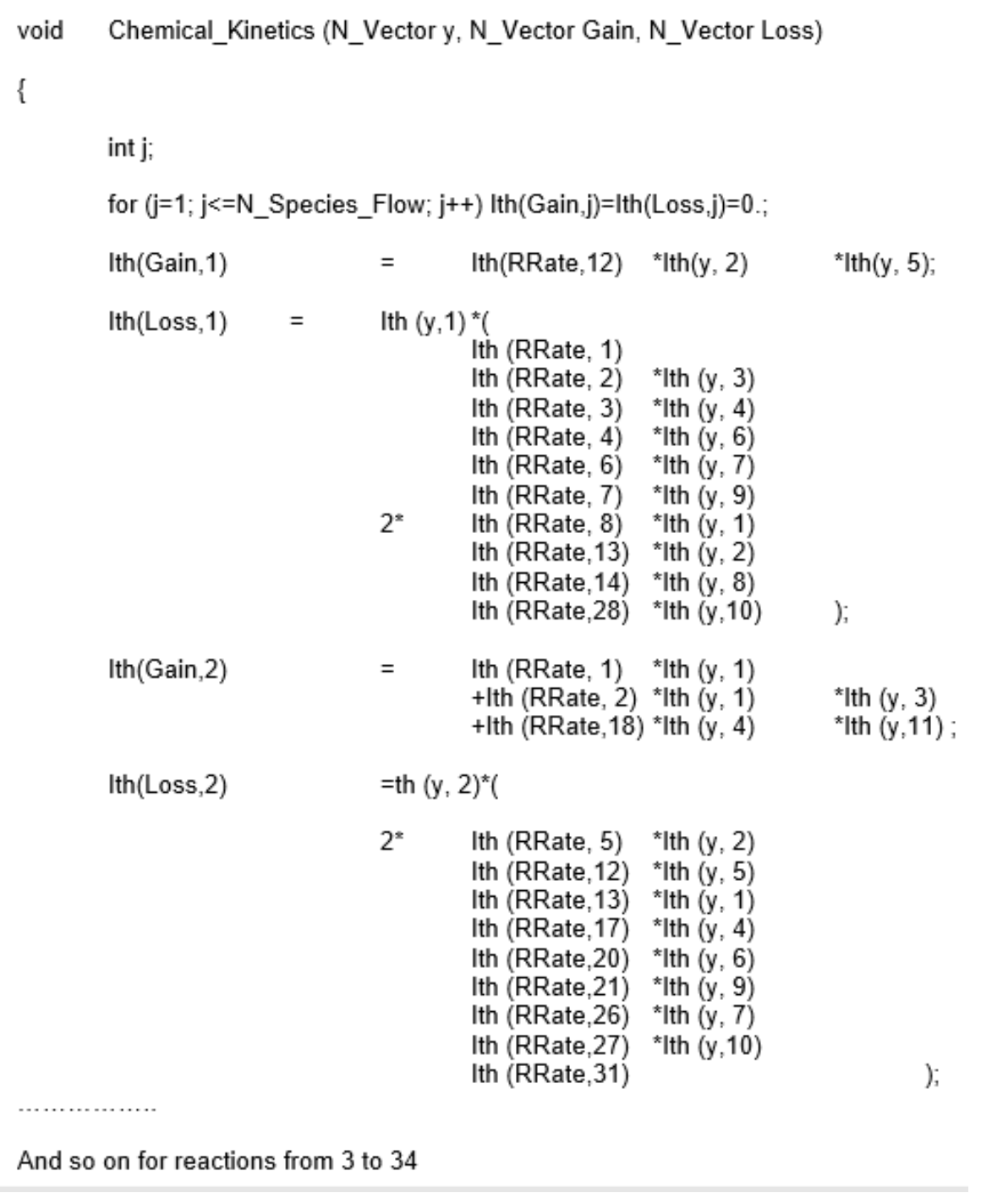

Figure XIII.65. A fragment of the source code of the previously used "fixed" chemical kinetics algorithm.

\section{Verification of generic chemical kinetics algorithm}

Three sets of calculations have been performed to verify that the use of "fixed" and generic algorithms yield identical results:

(1) Simulation of water chemistry, ECP, and CGR in a PWR using the previous version of PWR_MASTER with a "fixed" algorithm.

(2) The same simulation using a modified version of PWR_MASTER with generic algorithm and generic versions of input data files with radiolytic yields and chemical reactions. The sequence of 
species in YIELD.DAT and reactions CHEMRATE.DAT has been changed ${ }^{2}$ intentionally to perform additional software check

Comparison of the distribution of calculated ECP, CGR, and concentrations of $\mathrm{O}_{2}, \mathrm{H}_{2} \mathrm{O}_{2}$, and $\mathrm{H}_{2}$ along the PWR HTC confirms that the previous "fixed" version of BWR Master and modified BWR Master with generic chemical kinetics delivered essentially ${ }^{3}$ identical results. Additional visual examination of the printout files confirmed the identity of Case \#1 and Case\#2 results.

Based on the presented verification results, it was concluded that the modified PWR_MASTER code could handle generic lists of chemical species and chemical reactions.

\section{Platform dependence of the original PWR_MASTER code}

The PWR_MASTER code was developed from an earlier code, PWR_ECP. PWR_ECP was written in FORTRAN and hence required a complete rewrite into $\mathrm{C}++$ for execution in macOS. Additionally, the radiolysis, pH, ECP (MPM) sub-models were upgraded, and a model was added for calculating the CGR in MA Alloy 600. In the following changes were also made to the original PWR_Master code received from UC Berkeley in order to render it compatible with the macOS:

1. C-language function abs have been used instead of fabs for calculation of floating absolute value of floating arguments, which results in high calculated conductivity of water and affected calculated crack growth rates.

2. Chemical gains and losses for additional species $O, \mathrm{O}^{-}$and $\mathrm{O}_{2}{ }^{2-}$ have not been included in Chemical_Kinetics function, which resulted in zero calculated concentrations of these species.

Replacement of abs with function fabs with function resulted in more realistic calculated conductivities of water (Table XIII.20.). Inclusion of gains and losses of species in chemical reactions have been done automatically after expanding the list of species and inclusion of ten additional chemical reactions in modified PWR_MASTER code with generic kinetics algorithm (Table XIII.10). Proper inclusion of additional species and gains and losses in chemical reactions

\footnotetext{
${ }^{2}$ Except reactions \#\# 15, 16 and 30 as their reaction rates require special treatment

${ }^{3}$ See Section for more information
} 
had no effect on the calculated concentration of $\mathrm{H}_{2}, \mathrm{ECP}$, and crack growth rate, as may be seen from a comparison of the simulated results presented in Table XIII.20. and Table XIII.10.

A comparison of simulated results obtained from the original PWR_MASTER code obtained at UC Berkeley and by PWR_MASTER with generic chemical kinetics algorithm are shown in Table XIII.11.

Table XIII.20. PWR simulation results before and after replacement "abs" with "fabs."

Original PWR_MASTER

\begin{tabular}{|c|c|c|c|c|c|c|c|c|c|c|c|c|c|c|c|c|}
\hline$\underset{(\mathrm{cm})}{\mathrm{X}}$ & $\begin{array}{c}\mathrm{T} \\
\text { (C) }\end{array}$ & $\mathrm{pH}$ & $\begin{array}{l}\mathrm{OH}- \\
\mathrm{ppb}\end{array}$ & $\begin{array}{r}\mathrm{H} 202 \\
\mathrm{ppb}\end{array}$ & $\begin{array}{l}\mathrm{H} 02 \\
\mathrm{ppb}\end{array}$ & $\begin{array}{l}\mathrm{H} 02- \\
\mathrm{ppb}\end{array}$ & $\begin{array}{r}02 \\
\text { ppb }\end{array}$ & $\begin{array}{l}02- \\
\text { ppb }\end{array}$ & $\begin{array}{r}\mathrm{H} 2 \\
\mathrm{ppb}\end{array}$ & $\begin{array}{l}02 \mathrm{~g} \\
\mathrm{ppm}\end{array}$ & $\begin{array}{l}\mathrm{H} 2 \mathrm{~g} \\
\mathrm{ppm}\end{array}$ & $\begin{array}{c}\text { ECP } \\
\mathrm{mV} \text { SHE }\end{array}$ & $\begin{array}{l}\text { REDOX } \\
\mathrm{mV}\end{array}$ & $\begin{array}{l}\text { CGR } \\
\mathrm{pm} / \mathrm{s}\end{array}$ & $\begin{array}{r}\text { Cond } 25 \\
\mathrm{uS} / \mathrm{cm}\end{array}$ & $\begin{array}{r}\text { COND } \\
\mathrm{uS} / \mathrm{cm}\end{array}$ \\
\hline $\begin{array}{r}0.0 \\
366.0\end{array}$ & $\begin{array}{l}292.0 \\
326.0\end{array}$ & $\begin{array}{l}5.9 \\
6.3\end{array}$ & $\begin{array}{r}89 \\
181\end{array}$ & $\begin{array}{l}0 \\
0\end{array}$ & $\begin{array}{l}0 \\
0\end{array}$ & $\begin{array}{l}0 \\
0\end{array}$ & $\begin{array}{l}0 \\
0\end{array}$ & $\begin{array}{l}0 \\
0\end{array}$ & $\begin{array}{l}3143 \\
3925\end{array}$ & --- & -- & $\begin{array}{l}-664 \\
-715\end{array}$ & $\begin{array}{l}-664 \\
-716\end{array}$ & $\begin{array}{l}437 \\
284\end{array}$ & $\begin{array}{l}536.61 \\
332.07\end{array}$ & $\begin{array}{l}4679.69 \\
3176.33\end{array}$ \\
\hline
\end{tabular}

\section{Revised PWR_MASTER}

\begin{tabular}{|c|c|c|c|c|c|c|c|c|c|c|c|c|c|c|c|c|}
\hline$\underset{(\mathrm{cm})}{\mathrm{X}}$ & $\begin{array}{c}T \\
\text { (C) }\end{array}$ & $\mathrm{pH}$ & $\begin{array}{l}\mathrm{OH}- \\
\mathrm{ppb}\end{array}$ & $\begin{array}{r}\mathrm{H} 202 \\
\mathrm{ppb}\end{array}$ & $\begin{array}{l}\mathrm{H} 02 \\
\mathrm{ppb}\end{array}$ & $\begin{array}{l}\mathrm{H} 02- \\
\mathrm{ppb}\end{array}$ & $\begin{array}{r}02 \\
\mathrm{ppb}\end{array}$ & $\begin{array}{l}02- \\
\text { ppb }\end{array}$ & $\begin{array}{r}\mathrm{H} 2 \\
\mathrm{ppb}\end{array}$ & $\begin{array}{l}02 \mathrm{~g} \\
\mathrm{ppm}\end{array}$ & $\begin{array}{l}\mathrm{H} 2 \mathrm{~g} \\
\mathrm{ppm}\end{array}$ & $\begin{array}{c}\text { ECP } \\
\mathrm{mV} \text { SHE }\end{array}$ & $\begin{array}{l}\text { REDOX } \\
\mathrm{mV}\end{array}$ & $\begin{array}{l}\text { CGR } \\
\mathrm{pm} / \mathrm{s}\end{array}$ & $\begin{array}{r}\text { Cond } 25 \\
\text { uS } / \mathrm{cm}\end{array}$ & $\begin{array}{l}\text { COND } \\
\mathrm{uS} / \mathrm{cm}\end{array}$ \\
\hline $\begin{array}{r}0.0 \\
366.0\end{array}$ & $\begin{array}{l}292.0 \\
326.0\end{array}$ & $\begin{array}{l}5.9 \\
6.2\end{array}$ & $\begin{array}{r}89 \\
181\end{array}$ & $\begin{array}{l}0 \\
0\end{array}$ & $\begin{array}{l}0 \\
0\end{array}$ & $\begin{array}{l}0 \\
0\end{array}$ & $\begin{array}{l}0 \\
0\end{array}$ & $\begin{array}{l}0 \\
0\end{array}$ & $\begin{array}{l}3143 \\
3925\end{array}$ & -- & --- & $\begin{array}{l}-664 \\
-716\end{array}$ & $\begin{array}{l}-664 \\
-716\end{array}$ & $\begin{array}{l}160 \\
210\end{array}$ & $\begin{array}{l}53.74 \\
47.28\end{array}$ & $\begin{array}{l}409.94 \\
394.29\end{array}$ \\
\hline
\end{tabular}

Table XIII.10 PWR simulation results after implementing a generic kinetics algorithm.

\begin{tabular}{|c|c|c|c|c|c|c|c|c|c|c|c|c|c|c|c|c|c|c|c|c|c|c|}
\hline $\begin{array}{c}x \\
(\mathrm{~cm})\end{array}$ & $\begin{array}{c}\top \\
\text { (C) }\end{array}$ & $\mathrm{pH}$ & $\begin{array}{l}\text { Cond25 } \\
\mathrm{uS} / \mathrm{cm}\end{array}$ & $\begin{array}{l}\text { CondHT. } \\
\mathrm{uS} / \mathrm{cm} \text {. }\end{array}$ & $\begin{array}{c}\text { ECP } \\
\mathrm{mV} \text { SHE }\end{array}$ & $\begin{array}{l}\text { REDOX } \\
\text { mV }\end{array}$ & $\begin{array}{l}\mathrm{CGR} \\
\mathrm{pm} / \mathrm{s}\end{array}$ & $\begin{array}{c}\text { Crack d } \\
\mathrm{cm}\end{array}$ & e- & $\begin{array}{c}\text { quid } \\
\text { H }\end{array}$ & $\underset{\mathrm{H}+}{\mathrm{ppb}}$ & $\mathrm{OH}$ & OH- & $\mathrm{H} 202$ & HO2 & HO2- & 02 & 02- & $\mathrm{H} 2$ & 0 & & -02_2- \\
\hline $\begin{array}{r}0.0 \\
366.0\end{array}$ & $\begin{array}{l}292.0 \\
326.2\end{array}$ & $\begin{array}{l}5.9 \\
6.2\end{array}$ & $\begin{array}{l}53.7 \\
47.2\end{array}$ & $\begin{array}{r}409.9 \\
394.1\end{array}$ & $\begin{array}{l}-664 \\
-716\end{array}$ & $\begin{array}{l}-664 \\
-716\end{array}$ & $\begin{array}{l}160 \\
211\end{array}$ & $\begin{array}{l}0.50 \\
0.50\end{array}$ & $\begin{array}{l}\theta \\
0\end{array}$ & $\begin{array}{l}0 \\
0\end{array}$ & $\begin{array}{l}2 \\
1\end{array}$ & $\begin{array}{l}0 \\
0\end{array}$ & $\begin{array}{r}89 \\
180\end{array}$ & $\ddot{\theta}$ & $\begin{array}{l}0 \\
0\end{array}$ & $\ddot{0}$ & $\begin{array}{l}0 \\
0\end{array}$ & $\ddot{\theta}$ & $\begin{array}{l}3173 \\
3964\end{array}$ & $\begin{array}{l}7382 \\
9149\end{array}$ & $\begin{array}{l}11825 \\
14764\end{array}$ & $\begin{array}{l}0 \\
1\end{array}$ \\
\hline
\end{tabular}

These hardware-dependent statements are somewhere in PWR_MASTER source files generated before the inclusion of the Artificial Neural Network (ANN) files into the code package: the same ANN files are present in BWR Master code, which is hardware-independent. 
Table XIII.11 PWR simulation results from UC Berkeley and from author.

\begin{tabular}{|c|c|c|c|c|c|c|c|c|c|c|c|c|c|c|c|c|}
\hline \multicolumn{17}{|c|}{ 1.Core_Channel } \\
\hline$\underset{(\mathrm{cm})}{\mathrm{X}}$ & $\begin{array}{l}T \\
\text { (C) }\end{array}$ & $\mathrm{pH}$ & $\begin{array}{l}\mathrm{OH}-\mathrm{H} \\
\mathrm{ppb}\end{array}$ & $\begin{array}{r}\mathrm{H} 202 \\
\mathrm{ppb}\end{array}$ & $\begin{array}{l}\mathrm{H} 02 \\
\mathrm{ppb}\end{array}$ & $\begin{array}{l}\mathrm{HO2-} \\
\mathrm{ppb}\end{array}$ & $\begin{array}{r}02 \\
\mathrm{ppb}\end{array}$ & $\begin{array}{l}02- \\
\text { ppb }\end{array}$ & $\begin{array}{r}\mathrm{H} 2 \\
\mathrm{ppb}\end{array}$ & $\begin{array}{l}02 \mathrm{~g} \\
\mathrm{ppm}\end{array}$ & $\begin{array}{l}\mathrm{H} 2 \mathrm{~g} \\
\mathrm{ppm}\end{array}$ & $\begin{array}{c}\text { ECP } \\
\mathrm{mV} \text { SHE }\end{array}$ & $\begin{array}{l}\text { REDOX } \\
\mathrm{mV}\end{array}$ & $\begin{array}{l}\text { CGR } \\
\mathrm{pm} / \mathrm{s}\end{array}$ & $\begin{array}{l}\text { Cond25 } \\
\text { uS } / \mathrm{cm}\end{array}$ & $\begin{array}{r}\text { COND } \\
\mathrm{uS} / \mathrm{cm}\end{array}$ \\
\hline $\begin{array}{r}0.0 \\
366.0\end{array}$ & $\begin{array}{l}292.0 \\
326.2\end{array}$ & $\begin{array}{l}5.9 \\
6.3\end{array}$ & $\begin{array}{r}89 \\
181\end{array}$ & $\begin{array}{l}0 \\
0\end{array}$ & $\begin{array}{l}0 \\
0\end{array}$ & $\begin{array}{l}0 \\
0\end{array}$ & $\begin{array}{l}0 \\
0\end{array}$ & $\begin{array}{l}0 \\
0\end{array}$ & $\begin{array}{l}3143 \\
3928\end{array}$ & -- & --- & $\begin{array}{l}-664 \\
-717\end{array}$ & $\begin{array}{l}-664 \\
-717\end{array}$ & $\begin{array}{l}437 \\
577\end{array}$ & $\begin{array}{l}536.61 \\
331.25\end{array}$ & $\begin{array}{l}4679.69 \\
3169.99\end{array}$ \\
\hline \multicolumn{17}{|c|}{ Revised PWR_MASTER } \\
\hline \multicolumn{17}{|c|}{ 1.Core_Channel } \\
\hline$\underset{(\mathrm{cm})}{\mathrm{X}}$ & $\begin{array}{c}\top \\
\text { (C) }\end{array}$ & $\mathrm{pH}$ & $\begin{array}{l}\mathrm{OH}- \\
\mathrm{ppb}\end{array}$ & $\begin{array}{r}\mathrm{H} 202 \\
\mathrm{ppb}\end{array}$ & $\begin{array}{l}\mathrm{H} 02 \\
\mathrm{ppb}\end{array}$ & $\begin{array}{l}\mathrm{H} 02- \\
\mathrm{ppb}\end{array}$ & $\begin{array}{r}02 \\
\mathrm{ppb}\end{array}$ & $\begin{array}{l}02- \\
\mathrm{ppb}\end{array}$ & $\begin{array}{r}\mathrm{H} 2 \\
\mathrm{ppb}\end{array}$ & $\begin{array}{l}02 \mathrm{~g} \\
\mathrm{ppm}\end{array}$ & $\begin{array}{l}\mathrm{H} 2 \mathrm{~g} \\
\mathrm{ppm}\end{array}$ & $\begin{array}{c}\text { ECP } \\
\mathrm{mV} \text { SHE }\end{array}$ & $\begin{array}{l}\text { REDOX } \\
\mathrm{mV}\end{array}$ & $\begin{array}{l}\text { CGR } \\
\mathrm{pm} / \mathrm{s}\end{array}$ & $\begin{array}{r}\text { Cond25 } \\
\text { uS } / \mathrm{cm}\end{array}$ & $\begin{array}{r}\text { COND } \\
\mathrm{uS} / \mathrm{cm}\end{array}$ \\
\hline $\begin{array}{r}0.0 \\
366.0\end{array}$ & $\begin{array}{l}292.0 \\
326.0\end{array}$ & $\begin{array}{l}5.9 \\
6.3\end{array}$ & $\begin{array}{r}89 \\
181\end{array}$ & $\begin{array}{l}0 \\
0\end{array}$ & $\begin{array}{l}0 \\
0\end{array}$ & $\begin{array}{l}0 \\
0\end{array}$ & $\begin{array}{l}0 \\
0\end{array}$ & & $\begin{array}{l}3143 \\
3925\end{array}$ & --- & --- & $\begin{array}{l}-664 \\
-715\end{array}$ & $\begin{array}{l}-664 \\
-716\end{array}$ & $\begin{array}{l}437 \\
284\end{array}$ & $\begin{array}{l}536.61 \\
332.07\end{array}$ & $\begin{array}{l}4679.69 \\
3176.33\end{array}$ \\
\hline
\end{tabular}

\section{Changes in PWR_MASTER code input data files}

The format of two input data files YIELD.DAT and CHEMRATE.DAT has been slightly modified, as described below.

The format of the modified YIELD.DAT file is shown in Figure XIII.46.. The first two parameters specify the number of species in the liquid flow and in the gas flow, respectively. An additional column (\#2) with the molecular weight of the species has been added. Also, all YIELD.DAT files have to have $\mathrm{H}_{2} \mathrm{O}$ listed at the end. Users may change the sequence and number of the liquid flow species and place blank line(s) between data lines. A user-selected sequence of species will determine the sequence of species concentrations in printout files. At this time, the number of gas flow species is set to two.

The format of the modified CHEMRATE.DAT file is shown in Figure XIII.47.. The first two parameters specify the total number of chemical reactions and Type of reaction rate set: 1 for the CAM PSU set with room temperature reaction rates and a need for special treatment of reactions \#16 and \#30 and 2 for the generic set with high-temperature reaction rates and no need for special treatments of some chemical reactions. Each chemical reaction specified as a set of string-Type species names and "+" and "=" symbols. 
Lists of species in YIELD.DAT and CHEMRATE.DAT files must be consistent: all species present in the YIELD.DAT file must be present in the CHEMRATE.DAT file and vise versa. Otherwise, PWR_MASTER will stop with a warning message. According to this requirement, even species with zero yields from neutron or gamma radiation must be included in YIELD.DAT.

$\begin{array}{llll}14 & & & \\ \mathrm{e}- & 0 & 0.662 & 3.565 \\ \mathrm{H} & 1 & 0.453 & 0.927 \\ \mathrm{H}+ & 1 & 1.278 & 0.612 \\ \mathrm{OH} & 17 & 1.849 & 4.632 \\ \mathrm{OH}- & 17 & 0.00 & 0.00 \\ \mathrm{H} 202 & 34.016 & 0.836 & 0.542 \\ \mathrm{H} 2 & 33 & 0.05 & 0.00 \\ \mathrm{H} 02- & 33 & 0.00 & 0.00 \\ 02 & 32 & 0.00 & 0.00 \\ 02- & 32 & 0.00 & 0.00 \\ \mathrm{H} 2 & 2.016 & 0.662 & 3.565 \\ 0 & 16 & 0 & 0 \\ 0- & 16 & 0 & 0 \\ 02-2- & 32 & 0 & 0 \\ \mathrm{H} 20 & & & \end{array}$

Figure XIII.66. YIELD.DAT file format.

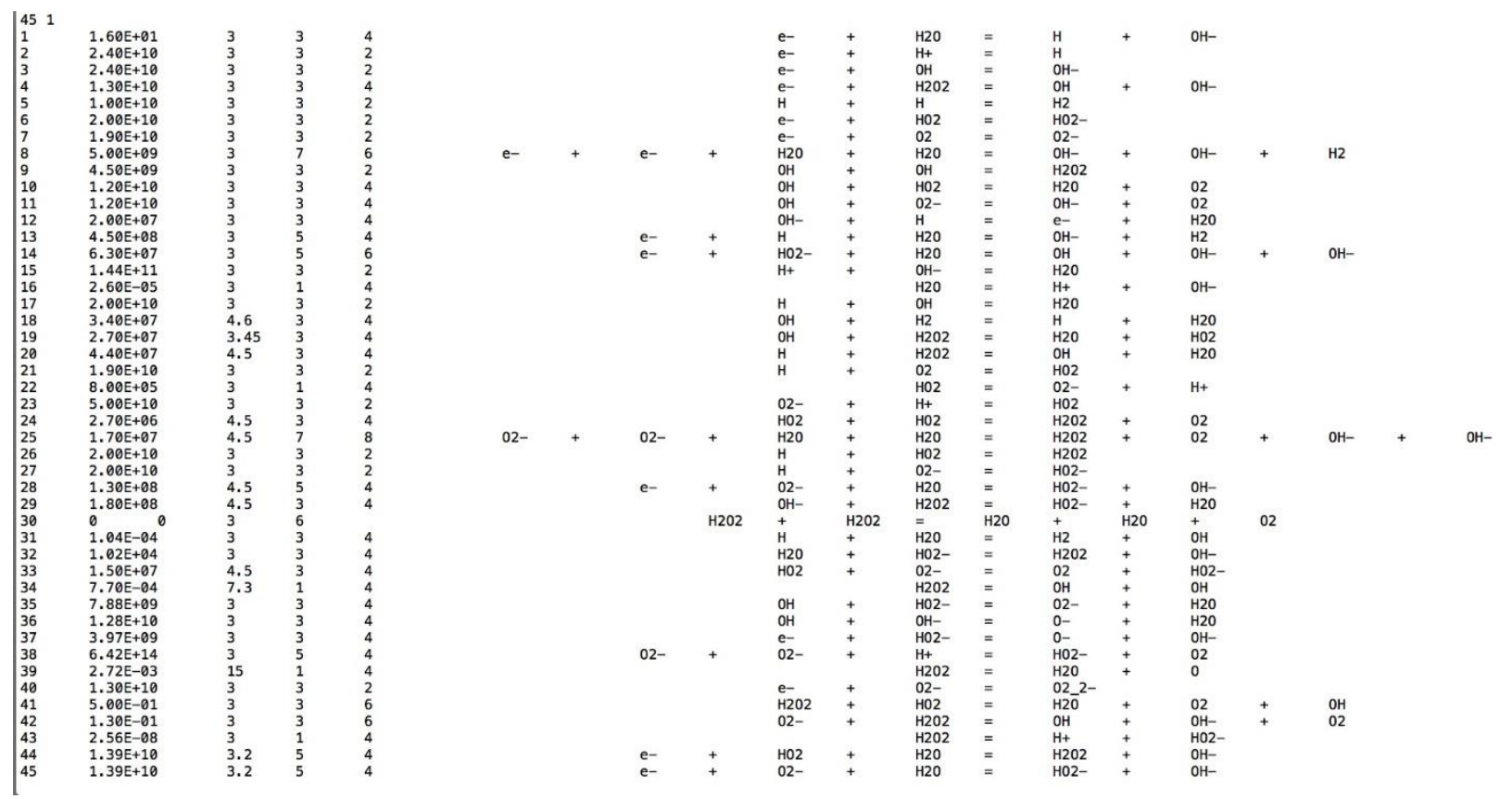

Figure XIII.635 CHEMRATE.DAT file format.

\section{Changes in PWR_MASTER code output data files}

The format of the PWR_MASTER printout file has been modified, as shown in Figure XIII.48.. The calculated concentration of gas and liquid flow species have been moved to the last 
columns. The sequence of printing concentrations of the liquid flow species is defined by the sequence of listing species in YIELD.DAT.

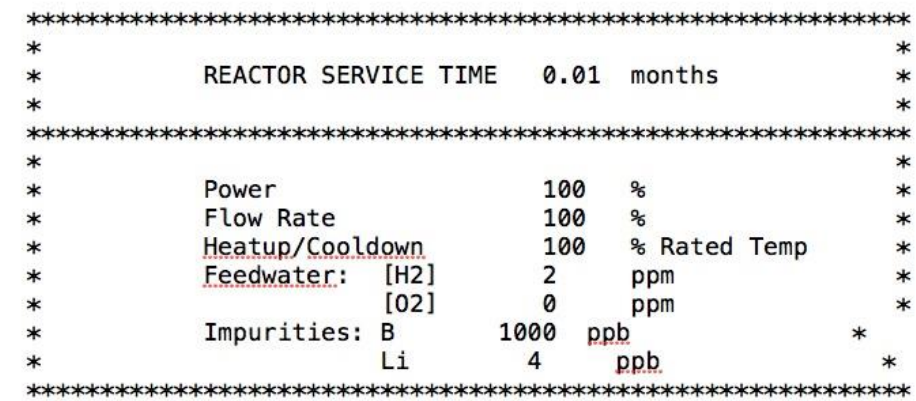

1.Core_Channel

\begin{tabular}{|c|c|c|c|c|c|c|c|c|c|c|c|c|c|c|c|c|}
\hline$\underset{(\mathrm{cm})}{X}$ & $\begin{array}{c}T \\
(\mathrm{C})\end{array}$ & $\mathrm{pH}$ & $\begin{array}{l}\mathrm{OH}- \\
\mathrm{ppb}\end{array}$ & $\begin{array}{r}\mathrm{H} 202 \\
\mathrm{ppb}\end{array}$ & $\begin{array}{l}\mathrm{H} 02 \\
\mathrm{ppb}\end{array}$ & $\begin{array}{l}\mathrm{H} 02- \\
\mathrm{ppb}\end{array}$ & $\begin{array}{r}02 \\
p p b\end{array}$ & $\begin{array}{l}02- \\
\text { ppb }\end{array}$ & $\begin{array}{r}\mathrm{H} 2 \\
\mathrm{ppb}\end{array}$ & $\begin{array}{l}02 \mathrm{~g} \\
\mathrm{ppm}\end{array}$ & $\begin{array}{l}\mathrm{H} 2 \mathrm{~g} \\
\mathrm{ppm}\end{array}$ & $\begin{array}{c}\text { ECP } \\
\mathrm{mV} \text { SHE }\end{array}$ & $\begin{array}{c}\text { REDOX } \\
\mathrm{mV}\end{array}$ & $\begin{array}{l}\text { CGR } \\
\mathrm{pm} / \mathrm{s}\end{array}$ & $\begin{array}{c}\text { Cond25 } \\
\text { uS/cm }\end{array}$ & $\begin{array}{r}\text { COND } \\
\mathrm{uS} / \mathrm{cm}\end{array}$ \\
\hline $\begin{array}{r}0.0 \\
366.0\end{array}$ & $\begin{array}{l}292.0 \\
326.0\end{array}$ & $\begin{array}{l}5.9 \\
6.2\end{array}$ & $\begin{array}{r}89 \\
181\end{array}$ & $\begin{array}{l}0 \\
0\end{array}$ & $\begin{array}{l}0 \\
0\end{array}$ & $\begin{array}{l}0 \\
0\end{array}$ & $\begin{array}{l}0 \\
0\end{array}$ & $\begin{array}{l}0 \\
0\end{array}$ & $\begin{array}{l}3143 \\
3925\end{array}$ & --- & $\begin{array}{l}--- \\
---\end{array}$ & $\begin{array}{l}-664 \\
-716\end{array}$ & $\begin{array}{l}-664 \\
-716\end{array}$ & $\begin{array}{l}160 \\
210\end{array}$ & $\begin{array}{l}53.74 \\
47.28\end{array}$ & $\begin{array}{l}409.94 \\
394.29\end{array}$ \\
\hline
\end{tabular}

2.Upper_Plenum

\begin{tabular}{|c|c|c|c|c|c|c|c|c|c|c|c|c|c|c|c|c|}
\hline $\begin{array}{c}X \\
(\mathrm{~cm})\end{array}$ & $\begin{array}{c}\mathrm{T} \\
(\mathrm{C})\end{array}$ & $\mathrm{pH}$ & $\begin{array}{l}\mathrm{OH}- \\
\mathrm{ppb}\end{array}$ & $\begin{array}{r}\mathrm{H} 202 \\
\mathrm{ppb}\end{array}$ & $\begin{array}{l}\mathrm{HO2} \\
\mathrm{ppb}\end{array}$ & $\begin{array}{l}\mathrm{HO2-} \\
\mathrm{ppb}\end{array}$ & $\begin{array}{r}02 \\
p p b\end{array}$ & $\begin{array}{l}02- \\
\text { ppb. }\end{array}$ & $\begin{array}{r}\mathrm{H} 2 \\
\mathrm{ppb} .\end{array}$ & $\begin{array}{l}02 \mathrm{~g} \\
\mathrm{ppm}\end{array}$ & $\begin{array}{l}\mathrm{H} 2 \mathrm{~g} \\
\mathrm{ppm}\end{array}$ & $\begin{array}{c}\text { ECP } \\
\mathrm{mV} \text { SHE }\end{array}$ & $\begin{array}{c}\text { REDOX } \\
\text { mV }\end{array}$ & $\begin{array}{l}\text { CGR } \\
\mathrm{pm} / \mathrm{s}\end{array}$ & $\begin{array}{r}\text { Cond } 25 \\
\text { uS } / \mathrm{cm}\end{array}$ & $\begin{array}{l}\text { COND } \\
\mathrm{uS} / \mathrm{cm}\end{array}$ \\
\hline $\begin{array}{r}0.0 \\
400.0\end{array}$ & $\begin{array}{l}330.0 \\
330.0\end{array}$ & $\begin{array}{l}6.3 \\
6.3\end{array}$ & $\begin{array}{l}165 \\
165\end{array}$ & $\begin{array}{l}0 \\
0\end{array}$ & $\begin{array}{l}0 \\
0\end{array}$ & $\begin{array}{l}0 \\
0\end{array}$ & $\begin{array}{l}0 \\
0\end{array}$ & $\begin{array}{l}0 \\
0\end{array}$ & $\begin{array}{l}3594 \\
3594\end{array}$ & $\begin{array}{l}--- \\
---\end{array}$ & $\begin{array}{l}--- \\
---\end{array}$ & $\begin{array}{l}-722 \\
-721\end{array}$ & $\begin{array}{l}-722 \\
-722\end{array}$ & $\begin{array}{l}432 \\
216\end{array}$ & $\begin{array}{l}46.30 \\
46.30\end{array}$ & $\begin{array}{l}390.21 \\
390.22\end{array}$ \\
\hline
\end{tabular}

\begin{tabular}{|c|c|c|c|c|c|c|c|c|c|c|c|c|c|c|c|c|}
\hline$\underset{(\mathrm{cm})}{X}$ & $\begin{array}{c}T \\
(\mathrm{C})\end{array}$ & $\mathrm{pH}$ & $\begin{array}{l}\mathrm{OH}- \\
\mathrm{ppb}\end{array}$ & $\begin{array}{r}\mathrm{H} 202 \\
\mathrm{ppb} .\end{array}$ & $\begin{array}{l}\mathrm{H} 02 \\
\mathrm{ppb}\end{array}$ & $\begin{array}{l}\mathrm{HO2-} \\
\mathrm{ppb}\end{array}$ & $\begin{array}{r}02 \\
p p b\end{array}$ & $\begin{array}{l}02- \\
\text { ppb. }\end{array}$ & $\begin{array}{r}\mathrm{H} 2 \\
\mathrm{ppb} .\end{array}$ & $\begin{array}{l}02 \mathrm{~g} \\
\mathrm{ppm}\end{array}$ & $\begin{array}{l}\mathrm{H} 2 \mathrm{~g} \\
\mathrm{ppm}\end{array}$ & $\begin{array}{c}\text { ECP } \\
\mathrm{mV} \text { SHE }\end{array}$ & $\begin{array}{l}\text { REDOX } \\
\mathrm{mV}\end{array}$ & $\begin{array}{l}\text { CGR } \\
\mathrm{pm} / \mathrm{s}\end{array}$ & $\begin{array}{r}\text { Cond25 } \\
\text { uS } / \mathrm{cm}\end{array}$ & $\begin{array}{r}\text { COND } \\
\mathrm{uS} / \mathrm{cm}\end{array}$ \\
\hline $\begin{array}{r}0.0 \\
900.0\end{array}$ & $\begin{array}{l}326.0 \\
326.0\end{array}$ & $\begin{array}{l}6.3 \\
6.3\end{array}$ & $\begin{array}{l}180 \\
180\end{array}$ & $\begin{array}{l}0 \\
0\end{array}$ & $\begin{array}{l}0 \\
0\end{array}$ & $\begin{array}{l}0 \\
0\end{array}$ & $\begin{array}{l}0 \\
0\end{array}$ & $\begin{array}{l}0 \\
0\end{array}$ & $\begin{array}{l}3920 \\
3920\end{array}$ & --- & --- & $\begin{array}{l}-716 \\
-716\end{array}$ & $\begin{array}{l}-716 \\
-716\end{array}$ & $\begin{array}{l}211 \\
211\end{array}$ & $\begin{array}{l}47.27 \\
47.27\end{array}$ & $\begin{array}{l}394.28 \\
394.28\end{array}$ \\
\hline
\end{tabular}

4.SG_Tube_HotLeg

\begin{tabular}{|c|c|c|c|c|c|c|c|c|c|c|c|c|c|c|c|c|}
\hline$\underset{(\mathrm{cm})}{X}$ & $\begin{array}{c}\mathrm{T} \\
(\mathrm{C})\end{array}$ & $\mathrm{pH}$ & $\begin{array}{l}\mathrm{OH}- \\
\mathrm{ppb}\end{array}$ & $\begin{array}{r}\mathrm{H} 202 \\
\mathrm{ppb}\end{array}$ & $\begin{array}{l}\mathrm{H} 02 \\
\text { ppb. }\end{array}$ & $\begin{array}{l}\mathrm{H} 02- \\
\mathrm{ppb}\end{array}$ & $\begin{array}{r}02 \\
\mathrm{ppb}\end{array}$ & $\begin{array}{l}02- \\
\text { ppb. }\end{array}$ & $\begin{array}{r}\mathrm{H} 2 \\
\mathrm{ppb}\end{array}$ & $\begin{array}{l}02 \mathrm{~g} \\
\mathrm{ppm}\end{array}$ & $\begin{array}{l}\mathrm{H} 2 \mathrm{~g} \\
\mathrm{ppm}\end{array}$ & $\begin{array}{c}\text { ECP } \\
\mathrm{mV} \text { SHE }\end{array}$ & $\begin{array}{l}\text { REDOX } \\
\mathrm{mV}\end{array}$ & $\begin{array}{l}\text { CGR } \\
\mathrm{pm} / \mathrm{s}\end{array}$ & $\begin{array}{l}\text { Cond25 } \\
\text { uS/cm }\end{array}$ & $\begin{array}{r}\text { COND } \\
\mathrm{uS} / \mathrm{cm}\end{array}$ \\
\hline $\begin{array}{r}0.0 \\
600.0\end{array}$ & $\begin{array}{l}325.0 \\
325.0\end{array}$ & $\begin{array}{l}6.3 \\
6.3\end{array}$ & $\begin{array}{l}179 \\
179\end{array}$ & $\begin{array}{l}0 \\
0\end{array}$ & $\begin{array}{l}0 \\
0\end{array}$ & $\begin{array}{l}0 \\
0\end{array}$ & $\begin{array}{l}0 \\
0\end{array}$ & $\begin{array}{l}0 \\
0\end{array}$ & $\begin{array}{l}3904 \\
3904\end{array}$ & --- & $\begin{array}{l}--- \\
---\end{array}$ & $\begin{array}{l}-729 \\
-729\end{array}$ & $\begin{array}{l}-727 \\
-727\end{array}$ & $\begin{array}{l}2595 \\
1551\end{array}$ & $\begin{array}{l}47.49 \\
47.49\end{array}$ & $\begin{array}{l}395.16 \\
395.16\end{array}$ \\
\hline
\end{tabular}

5.SG_Tube_ColdLeg

\begin{tabular}{|c|c|c|c|c|c|c|c|c|c|c|c|c|c|c|c|c|}
\hline $\begin{array}{c}X \\
(\mathrm{~cm})\end{array}$ & $\begin{array}{c}T \\
(\mathrm{C})\end{array}$ & $\mathrm{pH}$ & $\begin{array}{l}\mathrm{OH}- \\
\mathrm{ppb}\end{array}$ & $\begin{array}{r}\mathrm{H} 202 \\
\mathrm{ppb}\end{array}$ & $\begin{array}{l}\mathrm{H} 02 \\
\mathrm{ppb}\end{array}$ & $\begin{array}{l}\mathrm{H} 02- \\
\mathrm{ppb}\end{array}$ & $\begin{array}{r}02 \\
\mathrm{ppb}\end{array}$ & $\begin{array}{l}02- \\
\text { ppb. }\end{array}$ & $\begin{array}{r}\mathrm{H} 2 \\
\mathrm{ppb}\end{array}$ & $\begin{array}{l}02 \mathrm{~g} \\
\mathrm{ppm}\end{array}$ & $\begin{array}{l}\mathrm{H} 2 \mathrm{~g} \\
\mathrm{ppm}\end{array}$ & $\begin{array}{c}\text { ECP } \\
\mathrm{mV} \text { SHE }\end{array}$ & $\begin{array}{c}\text { REDOX } \\
\text { mV }\end{array}$ & $\begin{array}{l}\text { CGR } \\
\mathrm{pm} / \mathrm{s}\end{array}$ & $\begin{array}{r}\text { Cond } 25 \\
\text { uS } / \mathrm{cm}\end{array}$ & $\begin{array}{r}\text { COND } \\
\mathrm{uS} / \mathrm{cm}\end{array}$ \\
\hline $\begin{array}{r}0.0 \\
600.0\end{array}$ & $\begin{array}{l}320.0 \\
320.0\end{array}$ & $\begin{array}{l}6.2 \\
6.2\end{array}$ & $\begin{array}{l}176 \\
176\end{array}$ & $\begin{array}{l}0 \\
0\end{array}$ & $\begin{array}{l}0 \\
0\end{array}$ & $\begin{array}{l}0 \\
0\end{array}$ & $\begin{array}{l}0 \\
0\end{array}$ & $\begin{array}{l}0 \\
0\end{array}$ & $\begin{array}{l}3824 \\
3824\end{array}$ & --- & $\begin{array}{l}--- \\
---\end{array}$ & $\begin{array}{l}-722 \\
-722\end{array}$ & $\begin{array}{l}-720 \\
-720\end{array}$ & $\begin{array}{l}1357 \\
1357\end{array}$ & $\begin{array}{l}48.57 \\
48.57\end{array}$ & $\begin{array}{l}399.18 \\
399.18\end{array}$ \\
\hline
\end{tabular}

Figure XIII.68. PWR_MASTER code printout file format. 


\section{XIII.11. Integration of BWR_MASTER with Grizzly}

BWR MASTER code has been integrated with Grizzly to use Grizzly-generated stress intensity factors for predicting crack propagation. The integrated version of BWR MASTER has been transferred to INL (Dr. Benjamin Spencer).

\section{Changes in BWR_MASTER code input and output files}

The second from the last line in the reactor description input file changed: one additional parameter is added as shown in Figure XIII.69: if the last parameter is equal 1, externally generated stress intensity factors will be expected in an external file (Figure XIII.36 to XIII.72); otherwise, internal algorithm in BWR_MASTER code will be used to calculate stress intensity factors at a given crack depth based on CT specimens approximation (Figure XIII.73).

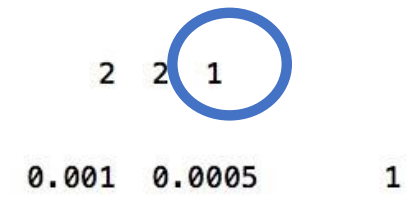

Figure XIII.69. A modified portion of the reactor description input file.

CRACK INTERNAL \& EXTERNAL ENVIRONMENT
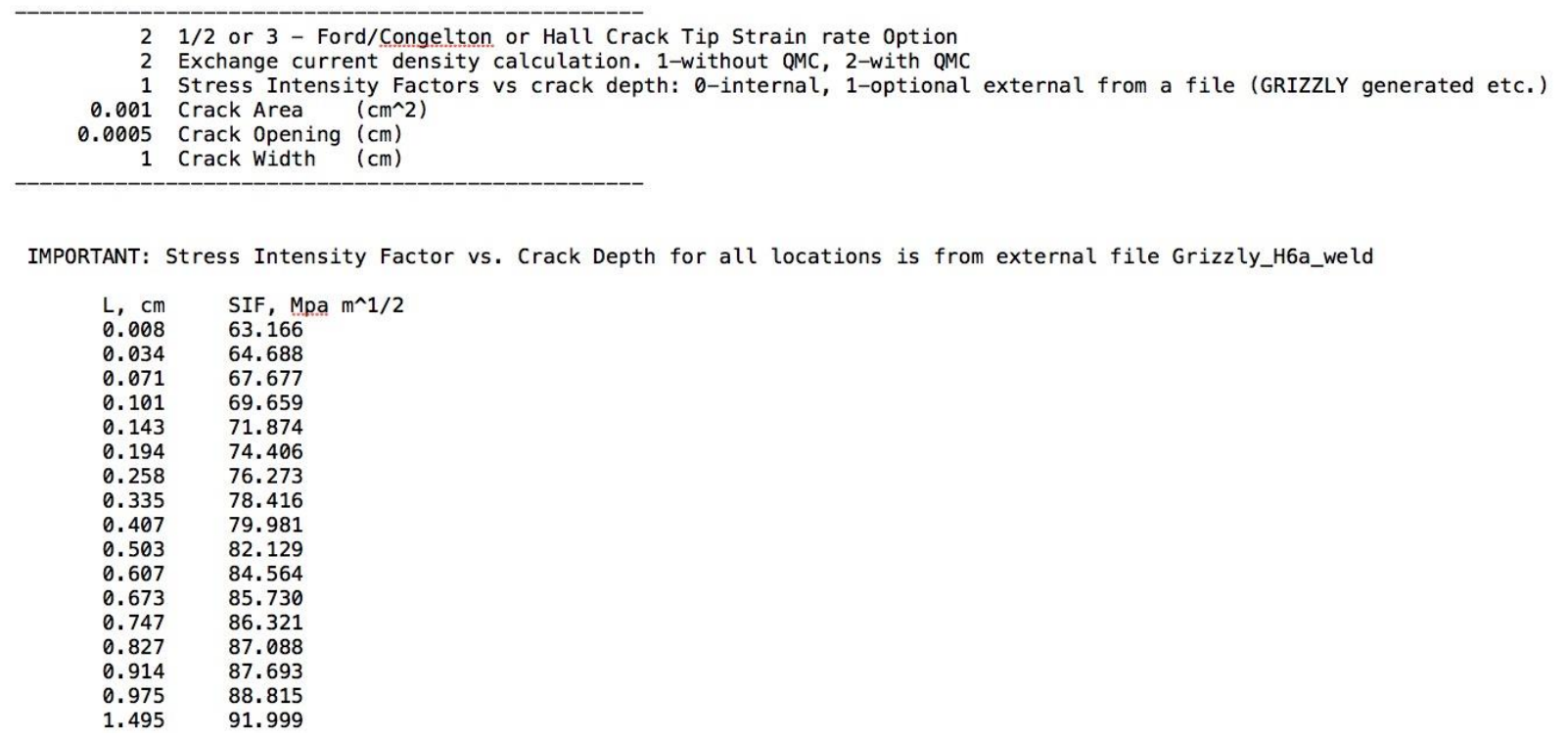

Figure XIII.36 The modified portion of printout file format. 
Additional prompt at the console to input the name of stress intensity factors file will appear as shown if the third parameter is equal to 1 . No additional prompt at the console will take place if the third parameter is not equal to 1 .

$\begin{array}{ll}0.0 & \\ 0.0077 & 63.166 \\ 0.0344 & 64.688 \\ 0.071 & 67.677 \\ 0.1011 & 69.659 \\ 0.1429 & 71.874 \\ 0.194 & 74.406 \\ 0.2576 & 76.273 \\ 0.335 & 78.416 \\ 0.4066 & 79.981 \\ 0.503 & 82.129 \\ 0.6065 & 84.564 \\ 0.6734 & 85.73 \\ 0.7474 & 86.321 \\ 0.8271 & 87.088 \\ 0.9145 & 87.693 \\ 0.9749 & 88.815 \\ 1.4946 & 91.999 \\ 1.8351 & 93.363 \\ 2.1662 & 94.037 \\ 2.4287 & 94.176 \\ 2.6424 & 94.98 \\ 2.7976 & 95.421 \\ 2.9294 & 95.845 \\ 2.979 & 96.088\end{array}$

Figure XIII.37 Stress Intensity Factors input file format: number of input lines, crack depth in cm, and stress intensity factor in $\mathrm{MPa} \mathrm{m}^{\wedge} 1 / 2$.

\section{Transmitted package}

The integrated version of BWR_MASTER has been transferred to INL (Dr. Benjamin Spencer) as a compressed zip file. The file contains the Contents of the Root Directory (Figure XIII.38) and includes the source file in c-language (file extension is *.c) and the header files (file extension is *.h). GNU c-compiler GCC has been used for assembling source files into executable under macOS.

Subdirectory Run in the root directory contains a BWR_MASTER executable file in a set of input files to run simulations with internal and external stress intensity factors (Figure XIII.39).

Sub-directory outputs in the Run sub-directory contains BWR_MASTER outputs for three various sources of stress intensity factors: factors from the original BWR_MASTER code, Grizzly (using a reduced order model for fracture mechanics), and J-integral method (Figure XIII.76). 


\begin{tabular}{|c|c|c|c|c|c|}
\hline Today & & ^ & Date Modified & Size & Kind \\
\hline c & MASTER_BWR_main_3.c & 0 & Today, $10: 58$ AM & $67 \mathrm{~KB}$ & C Source \\
\hline$\square$ & Run & 0 & Today, 11:28 AM & -- & Folder \\
\hline \multicolumn{6}{|l|}{2017} \\
\hline & CEFM.C & 0 & Aug $14,2017,2: 09 \mathrm{PM}$ & $59 \mathrm{~KB}$ & C Source \\
\hline h & cefm.h & 0 & Aug $14,2017,2: 51 \mathrm{PM}$ & $8 \mathrm{~KB}$ & C Hea...Source \\
\hline c & CVDENSE.C & 0 & Aug $14,2017,2: 09 \mathrm{PM}$ & $11 \mathrm{~KB}$ & C Source \\
\hline c & CVODE.C & 0 & Aug $14,2017,2: 09 \mathrm{PM}$ & $71 \mathrm{~KB}$ & C Source \\
\hline c & DENSE.C & 0 & Aug $14,2017,2: 09 \mathrm{PM}$ & $5 \mathrm{~KB}$ & C Source \\
\hline c & LLNLMATH.C & 0 & Aug $14,2017,2: 09 \mathrm{PM}$ & 802 bytes & C Source \\
\hline 回 & master & 0 & Oct $29,2017,5: 10$ PM & $148 \mathrm{~KB}$ & Unix e...cutable \\
\hline c & myNeuralNetworkFunction_initialize.c & 0 & Aug $14,2017,2: 18$ PM & 512 bytes & C Source \\
\hline h & myNeuralNetworkFunction_initialize.h & 0 & May $22,2017,10: 20 \mathrm{AM}$ & 600 bytes & C Hea...Source \\
\hline c & myNeuralNetworkFunction_terminate.c & 0 & Aug 14, 2017, 2:09 PM & 518 bytes & C Source \\
\hline h & myNeuralNetworkFunction_terminate.h & 0 & May $22,2017,10: 20 \mathrm{AM}$ & 595 bytes & C Hea...Source \\
\hline h & myNeuralNetworkFunction_types.h & 0 & May $22,2017,10: 20 \mathrm{AM}$ & 373 bytes & C Hea...Source \\
\hline c & myNeuralNetworkFunction.c & 0 & Aug 14, 2017, 2:09 PM & $7 \mathrm{~KB}$ & C Source \\
\hline h & myNeuralNetworkFunction. $h$ & 0 & May $22,2017,10: 20 \mathrm{AM}$ & 565 bytes & C Hea...Source \\
\hline c & rt_nonfinite.c & 0 & Aug 14, 2017, 2:09 PM & $2 \mathrm{~KB}$ & C Source \\
\hline h & rt_nonfinite.h & 0 & May $22,2017,12: 26$ PM & $1 \mathrm{~KB}$ & C Hea...Source \\
\hline c & rtGetinf.c & 0 & Aug $14,2017,2: 09 \mathrm{PM}$ & $3 \mathrm{~KB}$ & C Source \\
\hline h & rtGetinf.h & 0 & May $22,2017,10: 20 \mathrm{AM}$ & 465 bytes & C Hea...Source \\
\hline c & rtGetNaN.C & 0 & Aug $14,2017,2: 09 \mathrm{PM}$ & $2 \mathrm{~KB}$ & C Source \\
\hline h & rtGetNaN.h & 0 & May $22,2017,10: 20 \mathrm{AM}$ & 390 bytes & C Hea...Source \\
\hline h & rtwtypes.h & 0 & May $22,2017,10: 20 \mathrm{AM}$ & $5 \mathrm{~KB}$ & C Hea...Source \\
\hline c & VECTOR.C & 0 & Aug $14,2017,2: 22 \mathrm{PM}$ & $8 \mathrm{~KB}$ & C Source \\
\hline \multicolumn{6}{|l|}{2007} \\
\hline h & cvdense.h & 0 & Nov $29,2007,11: 39$ AM & $10 \mathrm{~KB}$ & C Hea...Source \\
\hline h & cvode.h & 0 & Nov $29,2007,11: 39$ AM & $43 \mathrm{~KB}$ & C Hea...Source \\
\hline h & dense.h & 0 & Nov $29,2007,11: 39 \mathrm{AM}$ & $26 \mathrm{~KB}$ & C Hea...Source \\
\hline h & IInlmath.h & 0 & Nov $29,2007,11: 39$ AM & $4 \mathrm{~KB}$ & C Hea...Source \\
\hline h & IInltyps.h & 0 & Nov $29,2007,11: 39$ AM & $6 \mathrm{~KB}$ & C Hea...Source \\
\hline h & vector.h & 0 & Nov $29,2007,11: 39 \mathrm{AM}$ & $22 \mathrm{~KB}$ & C Hea...Source \\
\hline
\end{tabular}

Figure XIII.38 Content of the root directory.

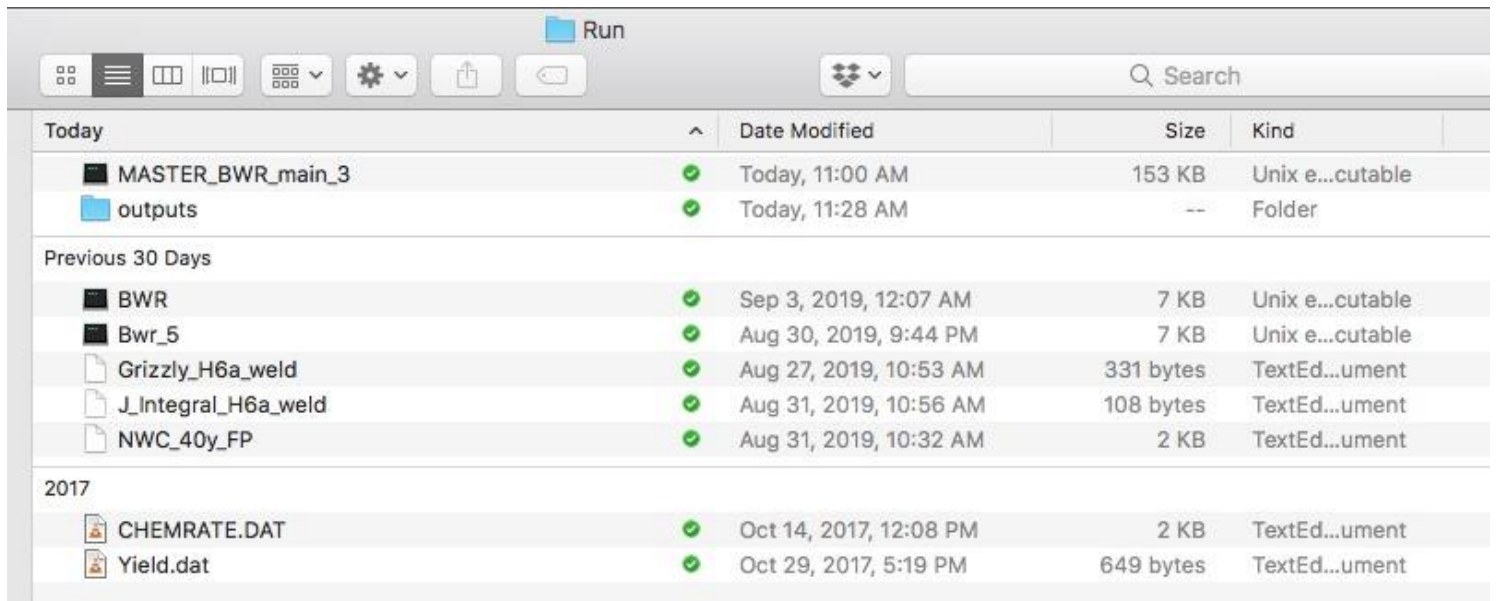

Figure XIII.39 Content of the "Run" sub-directory. 


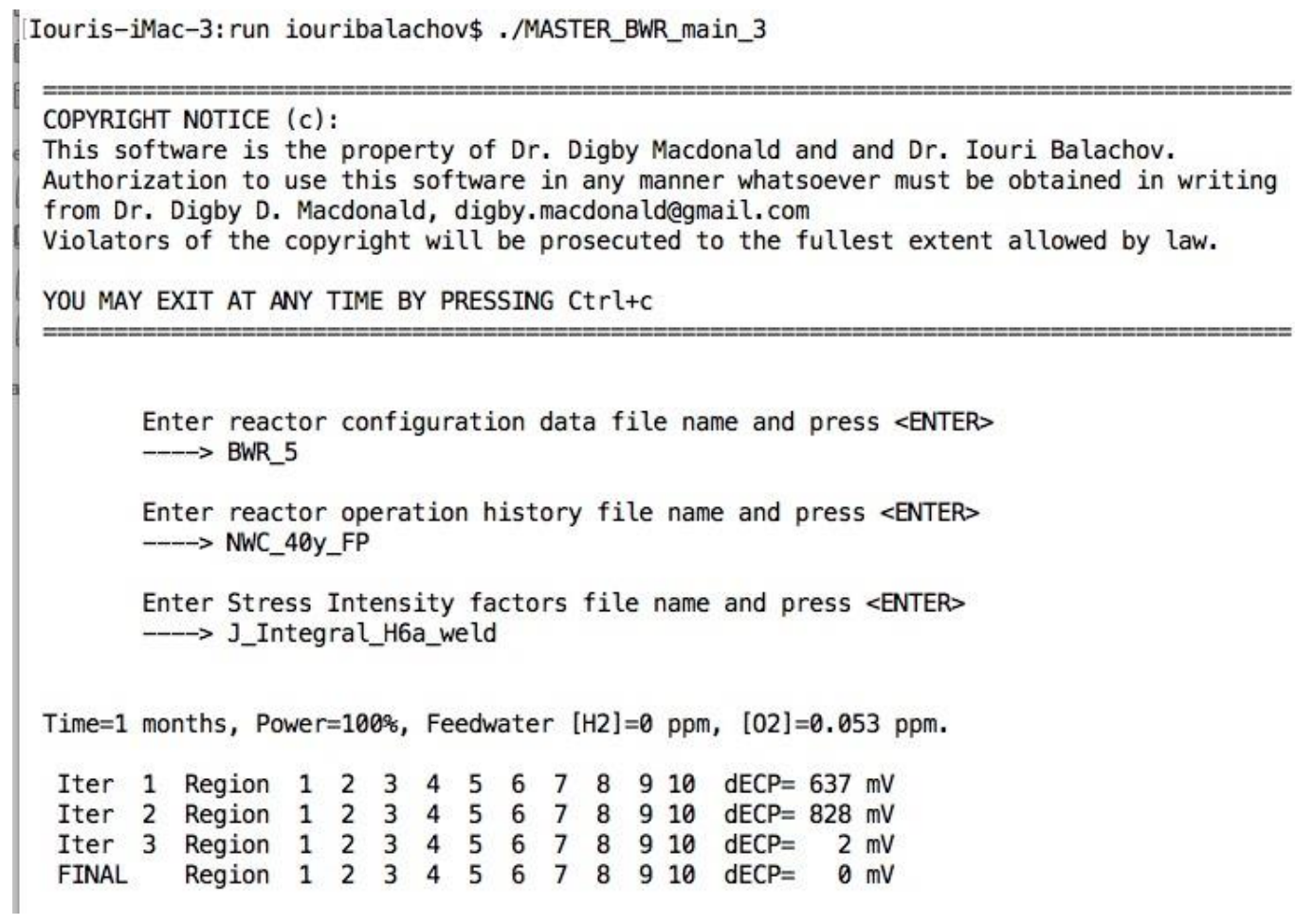

Figure XIII.40 Running BWR_MASTER simulation with external stress intensity factor data file.

Iouris-iMac-3: run iouribalachov\$ ./MASTER_BWR_main_3

COPYRIGHT NOTICE (c):

This software is the property of Dr. Digby Macdonald and and Dr. Iouri Balachov.

Authorization to use this software in any manner whatsoever must be obtained in writing

from Dr. Digby D. Macdonald, digby.macdonald@gmail.com

Violators of the copyright will be prosecuted to the fullest extent allowed by law.

YOU MAY EXIT AT ANY TIME BY PRESSING Ctrl+C

\author{
Enter reactor configuration data file name and press <ENTER> \\ $\rightarrow$ BWR \\ Enter reactor operation history file name and press <ENTER> \\ $\rightarrow$ NWC_40y_FP
}

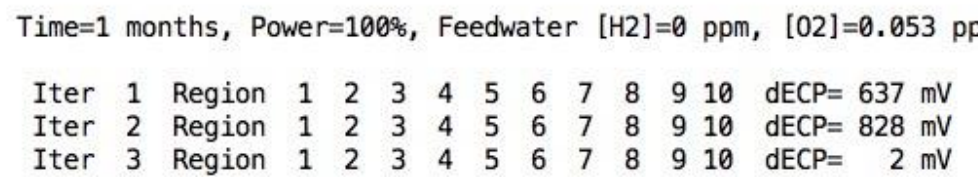

Figure XIII.75. Running BWR_MASTER simulation with internally generated stress intensity factors. 


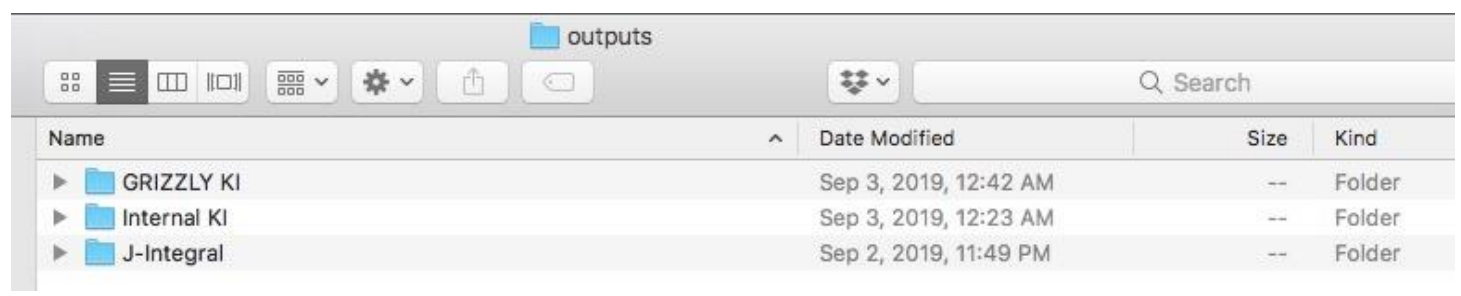

Figure XIII.76. Content of the "outputs" sub-directory.

\section{Comparison of crack propagation}

A comparison of crack propagation in H6a weld of BWR Core Shroud during 40 years of full power operation under normal water chemistry condition is shown in Figure XIII.77.. As explained in Section XIV, the values of KI predicted by Grizzly are known to be overly conservative because of current limitations of the code in modeling weld residual stresses that can be addressed with additional development. This is intended only as a proof of concept analysis.

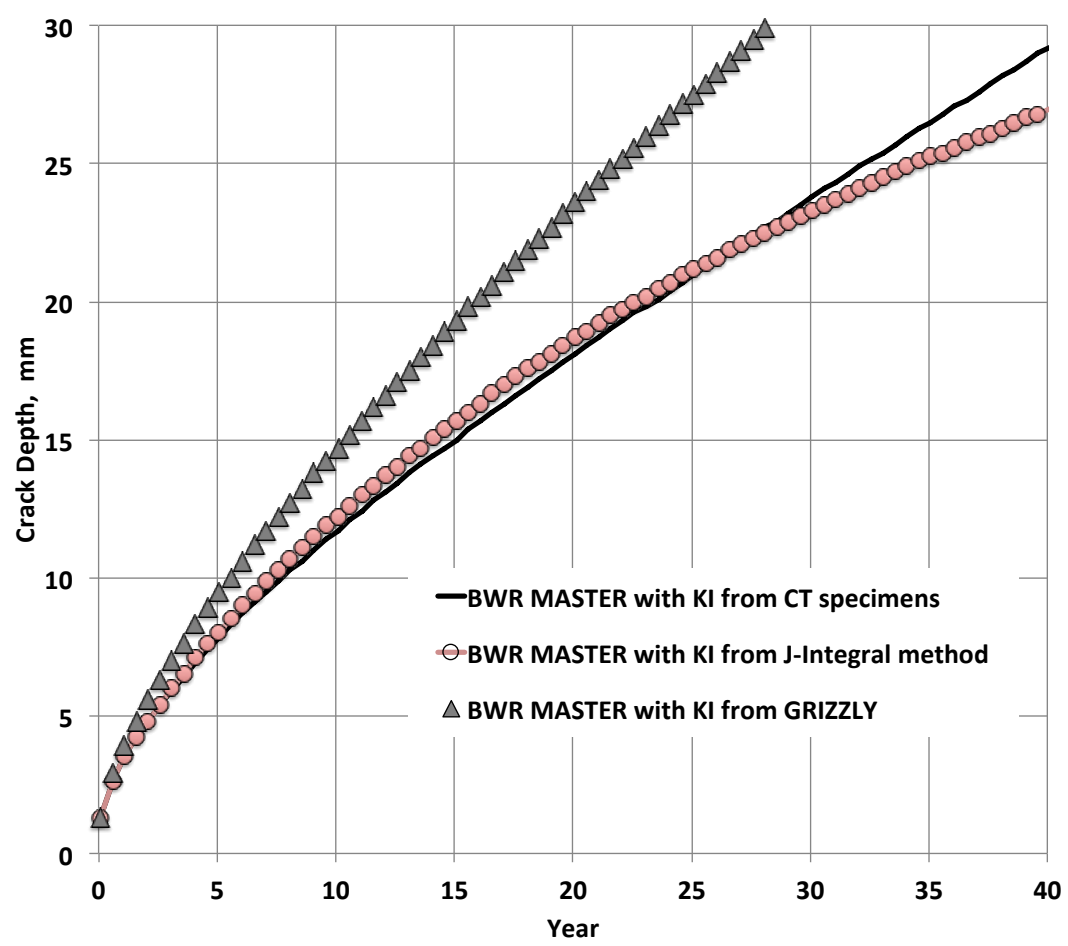

Figure XIII.77. Comparison of crack propagation in H6a weld of BWR Core Shroud during 40 years of full power operation under normal water chemistry condition. 


\section{XIII.12. Lifetime estimation of a BWR core shroud in terms of IGSCC}

The continued operation of aging Boiling Water Reactors (BWRs) worldwide requires gradually increasing the cost of the inspection, maintenance, and repair. Intergranular Stress Corrosion Cracking (IGSCC) in sensitized austenitic stainless steel piping first became a major issue for BWRs in the 1980s, resulting in recognition of the susceptibility of reactor internals to IGSCC. Shroud cracking identified in 1993-1994 confirmed that IGSCC of internals is a significant issue for BWRs. IGSCC is a time-dependent, material degradation process, which is caused and accelerated by the presence of residual stresses, material sensitization, irradiation, cold work, elevated temperature, and corrosive environments. This section paper emphasizes the importance of accounting for corrosive environments, or more exactly, electrochemical phenomena in modeling IGSCC and predicting the service life of BWR in-vessel components and stresses the necessity of performing such modeling not just for a single state point under full power conditions, but for the whole operating history of the reactor, including startups and shutdowns. This section demonstrates that ignoring electrochemical considerations may result in underestimating component lifetime and lead to unnecessary expenses for inspection and repair (Figure XIII.78). The manuscript has been submitted for publication to "Nuclear Engineering and Design."

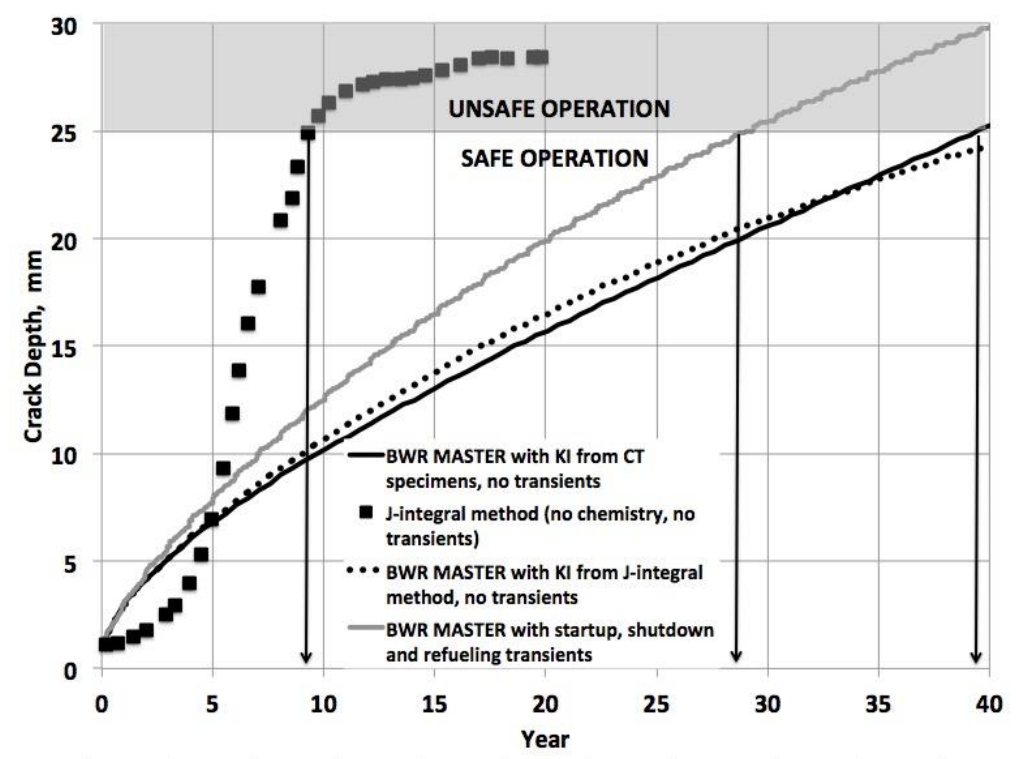

Figure XIII.78. Predicted core shroud remaining lifetime based on crack propagation in H6a weld in the core shroud for BWR. 
A comparison of deterministic and empirical predictions of IGSCC in sensitized austenitic stainless steel, in order to calculate the accumulated damage (crack depth versus time) in a BWR in-vessel component and estimate component's lifetime for given operating conditions is described. Counterintuitively, it was found that substitution of a "simplified" relationship between $K_{I}$ and crack depth based on CT specimens with a "more accurate" FEM based relationship, which gives significantly lower values of $K_{I}$ for higher crack depths, did not result in significant changes in the CEFM-predicted crack growth rates. This is due to the fact that the crack growth rate in the CEFM has a relatively low sensitivity to $K_{I}$. This low sensitivity of crack growth rate to $K_{I}$ opens an opportunity for avoiding the use of time-consuming FEM calculations by using simplified models instead. Based on the performed modeling, it was concluded that typical SCC modeling, which ignores water chemistry and electrochemistry and does not take into account the actual operating history, would underestimate the component's lifetime by a factor of 5 . Finally, such modeling is controversial because it overestimates SCC damage by using empirical fracture mechanical models that do not correspond to the actual mechanical condition of the core shroud. On the other hand, it underestimates SCC damage by ignoring water chemistry and reactor operating transients during refueling outages. Involving operating transients is particularly important, as, during start-up and shut-down, the change in the stress field around the crack increases the crack tip strain rate, which, as a consequence, increases the fracture frequency and the exposure of the bare metal to the environment, which results in a higher CGR.

\section{XIII.13. References}

[1] D.D. Macdonald and I. Balachov, Modeling the Accumulation and Mitigation of SCC Damage in BWRs, Proc. 9-th. Int. Symp. Mats. Degrad. Nucl. Power Systs.-Water Reactors, (Ed. F. P. Ford, S. M. Bruemmer, and G. S. Was), TMS, Warrendale, PA., 399-407 (1999).

[2] D.D. Macdonald and M. Urquidi-Macdonald, The Electrochemistry of Nuclear Reactor Coolant Circuits, Encyclopedia of Electrochemistry, A.J. Bard and M. Stratmann eds. Vol 5 Electrochemical Engineering, Edited by D.D. Macdonald and Patrik Schmuki, Wiley-VCH Verlag GmbH \& Co. KGaA, Weinheim, 665-720 (2007).

[3] X. Zhou, I. Balachov, and D.D. Macdonald, Corros. Sci., 40, 1349-1362 (1998). 
[4] C. Liu and D.D. Macdonald, ASME J. Pressure Vessel Technol., 119, 393-400 (1997).

[5] N. Totsuka and Z. Szklarska-Smialowska, Corrosion, 43, 734 (1987).

[6] B. Spencer, M. Backman, P. Chakraborty, and W. Hoffman, Reactor Pressure Vessel Fracture Analysis Capabilities in Grizzly, INL/EXT-15-34736, Idaho National Laboratory, Idaho Falls, ID, Mar. (2015).

[7] B. Spencer, W. Hoffman, S. Sen, C. Rabiti, T. Dickson, and R. Bass, Initial Probabilistic Evaluation of Reactor Pressure Vessel Fracture with Grizzly and RAVEN, INL/EXT-1537121, Idaho National Laboratory, Idaho Falls, ID, Oct. (2015).

[8] T. Williams and C. Kelley, Gnuplot 4.6. An Interactive Plotting Program, http://gnuplot.sourceforge.net/docs_4.6/gnuplot.pdf.

[9] GCC, the GNU Compiler Collection, https://gcc.gnu.org

[10] Xcode 8, https://developer.apple.com/xcode/

[11] XQuartz, https://www.xquartz.org

[12] A.J. Elliot, Rate constants and g-values for the simulation of the radiolysis of light water over the range $0300^{\circ} \mathrm{C}$, AECL-11073, Atomic Energy of Canada Ltd. (1994).

[13] A.J. Elliot and G. Buxton, J. Chem. Soc. Faraday Trans., 88, 2465-2470 (1992).

[14] A.J. Elliot, M.P. Chenier, and D.C. Ouellete, J. Chem. Soc, Faraday Trans, 89, 1193-1197 (1993).

[15] A.J. Elliot, M.P. Chenier, D.C. Ouellette, and V.T Koslowsky, The Temperature dependence of $\mathrm{g}$-values for aqueous solutions irradiated with a $23 \mathrm{MeV} 2 \mathrm{H}^{+}$beam, AECL-10659, Atomic Energy of Canada Ltd., (1992).

[16] G.V. Buxton and A.J. Elliot, High-temperature water radiolysis and its relevance to reactor coolant, Proc. 1991 JAIF Intl. Conf. Water Chem. Nucl. Power Plants, 283-288, Fukui City, (1991).

[17] A.J. Elliot, M.P. Chenier, D.C. Ouellette, and V.T. Koslowski, J. Phys. Chem., 100, 9014 (1996). 
[18] A.J. Elliot, D.R. McCracken, G.V. Buxton, and N.D. Wood, J. Chem. Soc., Faraday Trans. 86, 1539-1547 (1990).

[19] A.J. Elliot and D.C. Ouellette, J. Chem. Soc. Faraday Trans., 90, 837-841 (1994).

[20] A.J. Elliot, D.C. Ouellette, and D.R. McCracken, AECL-10 667, Atomic Energy of Canada, (1992).

[21] A.J. Elliot, D.C. Ouellette, D. Reid, and D.R. McCracken, Radiat. Phys. Chem., 34, 747-751 (1989).

[22] A.J. Elliot, D.C. Ouellette, and C.R. Stuart, The temperature dependence of the rate constants and yields for the simulation of the radiolysis of heavy water, AECL- 11658, Atomic Energy of Canada Ltd., (1996).

[23] D.R. McCracken, K.T. Tsang, and P.J. Laughton, Aspects of the Physics and chemistry of water radiolysis by fast neutrons and fast electrons in nuclear reactors, AECL- 11895, Atomic Energy of Canada Ltd., (1998).

[24] H. Christensen, Fundamental Aspects of Water Coolant Radiolysis, SKI Report 2006:16, (2006).

[25] H. Sims, Radiation chemical yields, Proc. Workshop on LWR coolant water radiolysis, Tokyo (1998).

[26] G.R. Sunaryo, Y. Katsumura, D. Hiroishi, and K. Ishigure, Radiat. Phys. Chem., 45, 131-139, (1995).

[27] Y. Katsumura, D. Yamamoto, D. Hiroishi and K. Ishigure, Radiolysis of water with high LET radiations at elevated temperatures, Proc. JAIF Intl. Conf. Water Chem. Nucl. Power Plants, Fukui City (1991).

[28] M.C. Kent and H.E. Sims, The yield of radiolysis products from water at temperatures up to $300^{\circ} \mathrm{C}$, Conf. Water Chem. Nucl. Reactor systems 6, BNES 1992 and AEA-RS-2302, Harwell (1992).

[29] H.C. Christensen, Studsvik Nuclear AB, STUDSVIK/N(K)-0214, (2002).

[30] J.A. LaVerne and S. Pimblott, J. Phys. Chem. 97, 3291-3297, (1993). 
[31] C.P. Ruiz, et al., Modeling Hydrogen Water Chemistry for BWR Applications, EPRI NP6386, Electric Power Research Institute, (1989).

[32] T.K. Yeh, D.D. Macdonald, and A.T. Motta, Nucl. Sci. and Eng., 121, 468 (1995).

\section{Integration of damage prediction codes with Grizzly.}

XIV.1. Grizzly analysis of stress intensity factors near the weld region of the core shroud.

In addition to the electrochemical potential, the mode-I stress intensity factor, $K_{I}$, is an important factor determining the rate of stress corrosion crack (SCC) growth in structures exposed to the coolant. One of the goals of the present work is to use the Grizzly code to compute $K_{I}$ for propagating cracks so it can be tied with the crack propagation models in the damage prediction codes.

Much of the Grizzly development to-date has focused on LWR reactor pressure vessel (RPV) integrity.

The issues related to RPV integrity are largely centered around the evaluation of the probability of a fracture initiating at the location of a pre-existing flaw introduced during the manufacturing process during a transient event. Long-term exposure to radiation embrittles the RPV steel, making it more susceptible to fracture over time. Grizzly has extensive fracture mechanics capabilities that were developed for probabilistic assessment of the susceptibility of embrittled RPVs to fracture [1]. These can be readily repurposed for the evaluation of SCC in other nuclear power plant structures.

Grizzly has two main ways of evaluating stress intensity factors and other fracture mechanics parameters for an existing crack. The first is to use fracture domain integrals to evaluate a number of quantities, including J-Integrals and interaction integrals using the techniques documented in [2], which can both be used to provide the stress intensity factor for a crack in a 2D or 3D model. Evaluating these fracture integrals requires including the crack in the finite element model, which can be done either by constructing a mesh that conforms to the crack topology, or by using the extended finite element method (XFEM), which allows for cracks to be defined in an arbitrary, mesh-independent manner. 
The second technique provided by Grizzly for evaluating stress intensity factors is the weight function (WF) technique [4], which allows for very rapid evaluation of flaws of specific geometries. In this technique, which is limited to linear elastic fracture mechanics problems, the through-wall stress distribution is described by a fitted polynomial, which is typical of third or fourth-order. A set of weight functions, also known as stress intensity factor influence coefficients (SIFICs), is pre-computed for a given crack geometry by evaluating the stress intensity factor for that crack geometry with loading corresponding to each of the terms in the polynomial describing through-wall stress variation. The stress intensity factor is evaluated by taking the sum of the products of each of the SIFICs and the corresponding term in the polynomial describing the through-wall stress variation. Techniques for computing SIFICs for axis-aligned flaws with standard geometries are readily available in the FAVOR code [5] and the ASME Boiler and Pressure Vessel code [6]. This technique is limited to crack geometries for which SIFICs are available, but it allows for extremely rapid evaluation of stress intensity factors for such flaws.

To demonstrate the use of Grizzly to compute the variation in the stress intensity factor as a function of crack depth in an SCC problem, the boiling water reactor (BWR) core shroud problem demonstrated in [7] has been evaluated in Grizzly. The primary stresses driving SCC crack growth are the residual stresses originating from the welding process. In this demonstration, the propagation of a 360-degree circumferential crack starting on the inner surface of a BWR core shroud $1 \mathrm{~mm}$ above the top of the weld shown in Figure XIV.1 was evaluated. Figure XIV.1 shows the residual stress field obtained from the axisymmetric model of the core shroud performed in $[7]$.

In that study, a series of direct fracture analyses were performed on the shroud with this residual stress field with cracks of varying depths. This type of analysis would be straightforward to perform using Grizzly’s XFEM capability. However, Grizzly does not yet have the capability to simulate the welding process to compute the residual stress field, so this analysis could not yet be performed in Grizzly. Instead, a polynomial fit of the through-wall distribution of the stress field shown in Figure XIV.1 computed in [7] was performed in Grizzly, and the weight function technique was used to compute the stress intensity factor for a 360-degree circumferential crack of varying depth in Grizzly. For short cracks, this approach would be expected to give stress intensity factors like those reported in [7], but because the weight function approach does not 
account for stress relaxation due to the presence of the crack, it will tend to over-predict the stress intensity factor for deeper cracks.

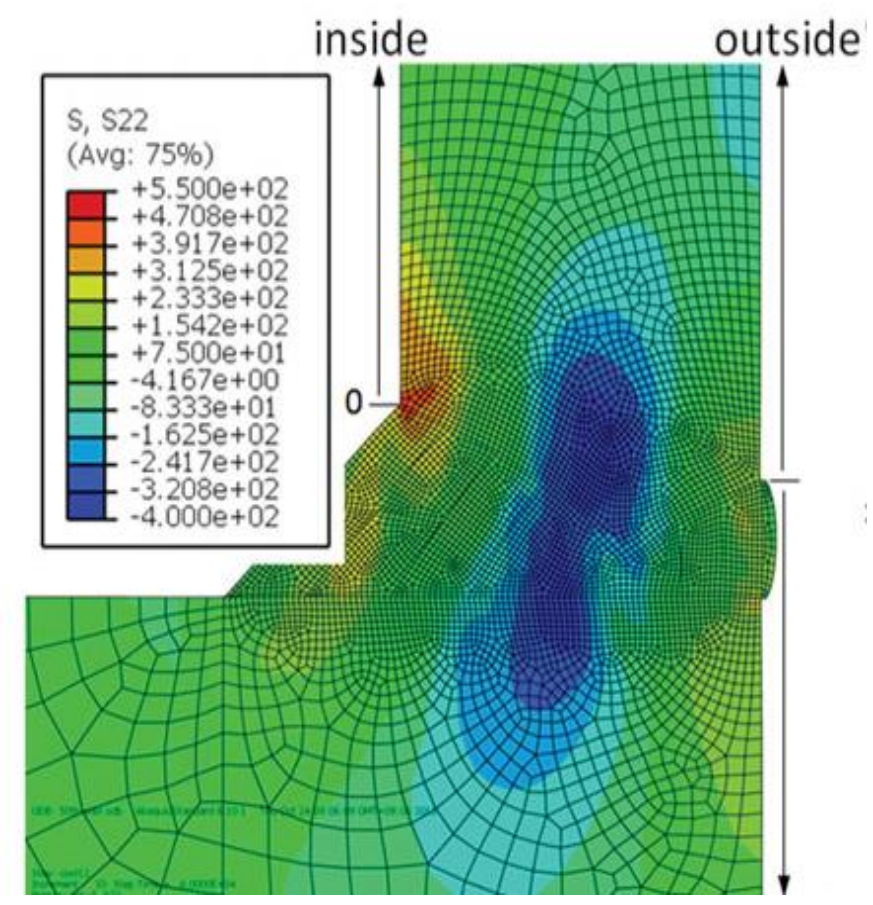

Figure XIV.1. Residual stress profile in the vicinity of a weld in a BWR core shroud from [7].

Figure XIV.2 shows the third and fourth-order polynomial fits of the through-wall residual stress variation obtained by extracting results from Figure XIV.1.

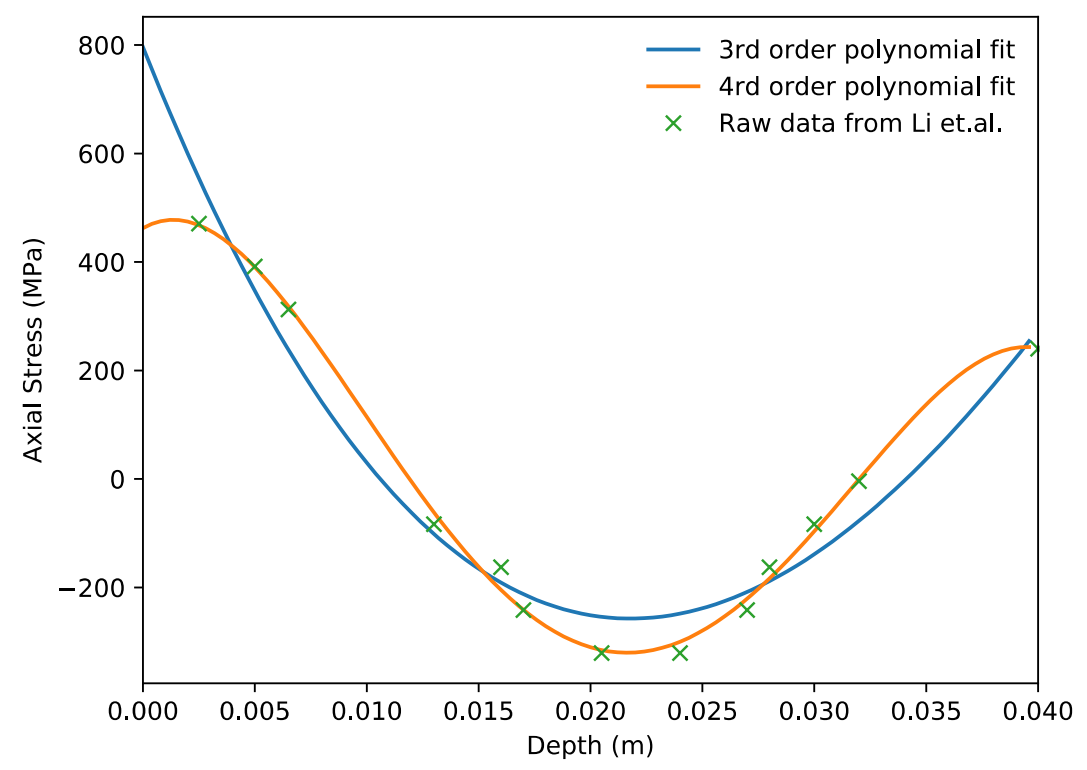


Figure XIV.2. Through-wall residual axial stress showing the raw data extracted from the image in Figure XIV.1, as reported in [7], together with third and fourth-order polynomial fits of that data used in a weight function analysis in Grizzly.

The third-order polynomial somewhat over-represents the stress near the inner surface of the shroud. Figure XIV.3 shows the stress intensity factor computed using the weight function technique in Grizzly using these third and fourth-order polynomial fits. As would be expected, the fourth-order polynomial results in stress intensity factors that are lower for shorter cracks (because the fourth-order polynomial fit gives lower stresses near the inner surface). Although these values are lower than those for the third order polynomial, they are still somewhat high relative to the values reported in [7], and the reason for that discrepancy is unclear. It is also important to note that as expected, for deeper cracks, the weight function approach used in the Grizzly analyses give higher values than those computed using the direct approach because they do not capture stress relaxation due to the presence of the crack.

Although these stress intensity factors are conservative, they can still be used for a proofof-concept demonstration of the coupling of Grizzly with the damage prediction code. Development of an ability in Grizzly to simulate welding processes will be an important area of future work in Grizzly to allow for direct fracture simulations that account for stress relaxation to permit this code to be used for simulation of SCC.

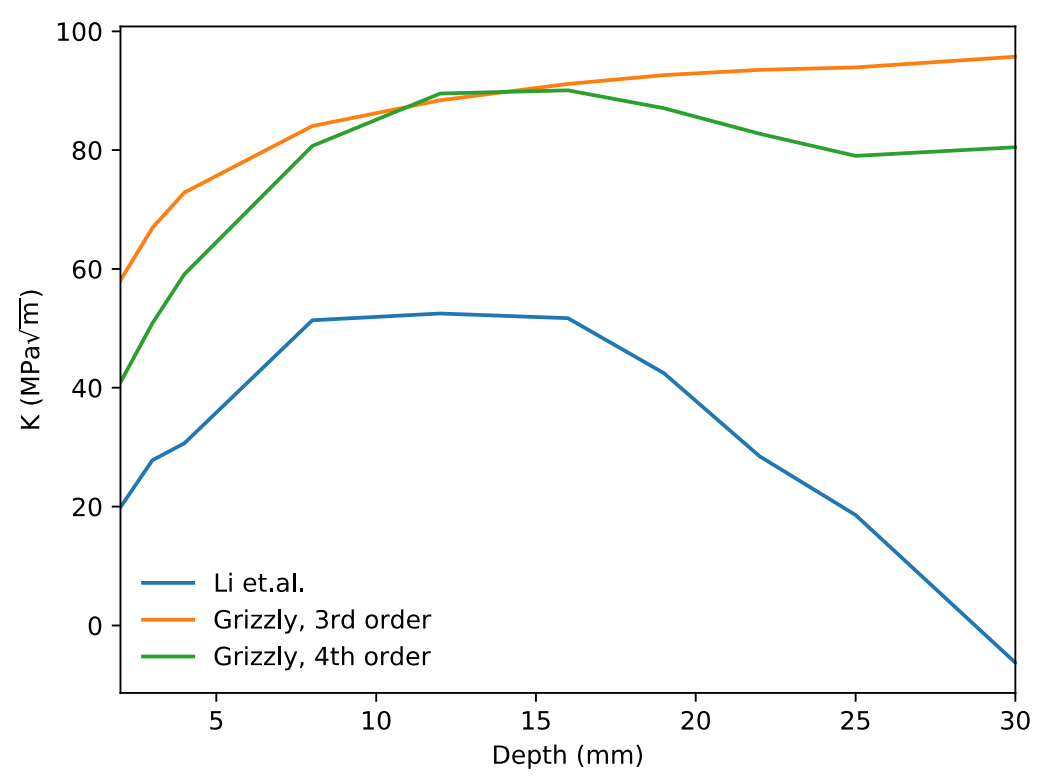


Figure XIV.3. Stress intensity factor for a 360-degree circumferential crack computed using the weight function technique in Grizzly using the third and fourth-order polynomial expansions of the residual stress field shown in Figure XIV.2, along with the results computed in [7].

\section{XIV.2. References}

[1] B. Spencer, W. Hoffman, and M. Backman, Nucl. Eng. Des., 341, 25-37 (2019).

[2] B. Healy, A. Gullerud, K. Koppenhoefer, A. Roy, S. Roy-Chowdhury, M. Walters, B. Bichon, K. Cochran, A. Carlyle, J. Sobotka, M. Messner, and R. Dodds, Warp3D-Release 17.3.1. Technical Report UILU-ENG-95-2012, the University of Illinois at Urbana-Champaign, (2012).

[3] Z. Zhang, W. Jiang, J.E. Dolbow, and B.W. Spencer, Comput. Mech., 62, 233-252 (1970).

[4] H.F. Bückner, Z.angew Math. Mech., 50, 529-546 (1970).

[5] P. Williams, T. Dickson, B.R. Bass, and H.B. Klasky, Fracture Analysis of Vessels - Oak Ridge, FAVOR, v16.1, computer code: Theory and implementation of algorithms, methods, and correlations. Technical Report ORNL/LTR-2016/309, Oak Ridge National Laboratory, Oak Ridge, TN, September 2016.

[6] ASME Boiler and Pressure Vessel Code, Section XI, Rules for Inservice Inspection of Nuclear Power Plant Components, ASME BPVC.XI-2017, ASME, 2017.

[7] Y. Li, S. Lu, D. Li, and Y. Kaji, J. Nucl. Sci. Technol., 52, 96-103 (2015).

\section{Comprehensive summary and conclusions}

The project sought to further develop theories and models for prediction the evolution of stress corrosion cracking damage in the primary coolant circuits (PCCs) of Boiling Water Reactors (BWRs) and Pressurized Water Reactors (PWRs) and to provide the codes to the Idaho National Laboratory (INL) for embedment into their Grizzly protocol for predicting damage due to stress corrosion cracking. Grizzly is a fracture mechanics code that does not address the electrochemical aspects of localized corrosion, including stress corrosion cracking. The inclusion of the codes developed in this work will address that issue. 
The previously-developed ALERT code was modified into BWR_MASTER with considerable upgrading of the component models to be compatible with the macOS c compiler. The macOS compatible version of the BWR_MASTER executable file has been created. Computations have been performed on a macOS to confirm identity with the results of BWR_MASTER computations performed under the Windows operating system. The calculation time for simulating 60 years of BWR operation is under 5 minutes with the iMac $2.7 \mathrm{GHz}$ Intel Core i5 processor, 8 GB 1333 MHz DDR3 memory, 2 TB hard drive and macOS Sierra operating system version 10.12.3. The Interface files are created for post-processing for data analysis and visualization with Excel and Gnuplot. The sensitivity analysis of integral output parameters to variation of input parameters has been performed. The following integral parameters that have been selected include: the concentrations of hydrogen, oxygen, and hydrogen peroxide, EPC, and crack growth rate. A total of 79 input model parameters have been selected, including reactor modeling parameters, reaction rates, radiolytic yields of species, and electrochemical input parameters for the Mixed Potential Model (MPM). Sensitivities have been estimated for each point along the coolant flow under normal and hydrogen (0.5 $\mathrm{ppm} \mathrm{H}_{2}$ in feedwater) water chemistry (HWC) conditions. Integral parameters, including the integrated damage (crack length), have been found to be most sensitive to variations in radiolytic yields, Tafel constants, and exchange current density for the hydrogen electrode reaction (HER), oxygen electrode reaction (OER), hydrogen peroxide electrode reaction (HPER), passive dissolution stainless steel, and the standard potentials for these reactions. Incorporation of the most recent data on radiolytic yields into BWR_MASTER resulted in much better agreement with measured oxygen concentration and ECP in Leibstadt mini-test under normal and $0.5 \mathrm{ppm}$ hydrogen water chemistry conditions. However, further re-evaluation of the standard potentials for the OER and HPER reactions is recommended. A generic chemical kinetics algorithm was implemented by replacing the "fixed" model radiolysis function Chemical_Kinetics that was contained in ALERT to calculate gains and losses of species and modifying the radiolysis function for calculating radiolytic yields from neutron and gamma radiation, considering coolant convection and two-phase flow terms. A list of chemical species is defined in the input file YIELDS.DAT as string constants, which allows expansion or reduction of the number of species as necessary. Arbitrary lists of chemical reactions, reaction rates constants, and activation energies are taken from input file CHEMRAT.DAT, where chemical reactions are also defined as string variables. 
Comparison of distribution in calculated ECP, CGR, and in the concentrations of $\mathrm{O}_{2}, \mathrm{H}_{2} \mathrm{O}_{2}$, and $\mathrm{H}_{2}$ along the BWR HTC under NWC and HWC conditions confirms that the previous "fixed" version of BWR_MASTER and modified BWR_MASTER with generic chemical kinetics delivered identical results. Trial simulation of species concentrations in BWR PCC has been performed with two "as is" alternative sets of chemical reactions: one by Christensen and another by Ishigure. Both sets have reaction rates for $288^{\circ} \mathrm{C}$, which have been used as an input. No special treatment of reactions has been performed within the BWR_MASTER source code. For Christensen's reaction set, calculated concentrations of $\mathrm{H}_{2}$ are in good agreement, except for the Core Bypass region under NWC, calculated concentrations of $\mathrm{H}_{2} \mathrm{O}_{2}$ deviate significantly from each other, both for NWC and HWC. The apparent, underprediction of $\mathrm{H}_{2} \mathrm{O}_{2}$ decomposition and calculated concentrations of $\mathrm{O}_{2}$, are in satisfactory agreement for NWC but deviate significantly from plant data under HWC. Discrepancies in the calculated species concentrations are higher if Ishigure's data are being used (not included in this report). Previously used CAM PSU data on chemical reactions are much more adequate and produce a better agreement with plant measure data. An attempt to estimate feasibility and cost/benefit ratio of reducing the number of chemical reactions was undertaken. The candidate reaction for removing from the input data set has been identified by performing a sensitivity analysis. The impact of using reduced reactions set on calculated ECP under NWC was estimated. Maximal deviation of about $100 \mathrm{mV}$ was observed in the Upper Plenum and $25 \mathrm{mV}$ the recirculation line and in the bottom of the Lower Plenum. While, in general, reduction of the reaction rates does not result in a more attractive cost/benefit ratio, in some practical cases, it may be justified, for example, in parametric scientific or engineering studies. An attempt to adjust reaction rates in Christensen set to match the calculated concentration of $\mathrm{H}_{2} \mathrm{O}_{2}$ has been made as an example. Agreement with CAM PSU based data has been improved significantly after increasing the rate of reaction \#33 (decomposition of hydrogen peroxide) by two orders of magnitude. Christensen's data set must be adjusted in order to produce adequate simulation results for species concentrations, if desired. The use and adjustment of the Ishigure data set are not recommended for further consideration. The generic kinetics algorithm was implemented into advanced by UCB BWR_MASTER code for macOS/UNIX operating systems. A paper on crack propagation in H6a has been submitted to "Nuclear Engineering and Design." As noted above, BWR_MASTER may be used on macOS or Windows platforms, as selected by the user. BWR_MASTER code has been integrated with Grizzly to use Grizzly generated stress 
intensity factors for predicting crack propagation. An integrated version of BWR_MASTER has been transferred to INL (Benjamin Spencer). Simulation of crack propagation in BWR Core Shroud weld H6a has been performed for stress intensity factors from the reduced order model approach in Grizzly and J-integral methods. Importance of using electrochemical consideration and transients during start-ups and shutdowns on crack propagation and predicted component lifetime has been demonstrated through simulation.

We determined that it was necessary to introduce a quantum mechanical tunneling correction to describe electronic charge transfer across the barrier oxide layer on the alloy surface. This was necessary, because the redox reactions (HER, OER, and HPER) occur on the outer surface of the barrier layer, and the electronic charge carriers (electrons and/or electron holes) must, therefore, be transported across the barrier layer from the metal to the redox reaction center at the barrier layer/solution interface. Thus, it is known from theory and experiment that the thickness of the barrier layer $\left(L_{b l}\right)$ increases linearly with voltage, and hence, the tunneling probability is a decreasing exponential function of $L_{b l}$. Thus, the exchange current densities for the HER, OER, and HPER are voltage-dependent, but no such dependencies are incorporated in the classical Generalized Butler-Volmer equation. This fundamental addition to electrochemical kinetic theory corrects that omission. Perhaps most importantly, it is now necessary to specify the kinetic parameters for the HER, OER, and HPER on the hypothetical bare metal surface, rather on the passive surface at each of an infinite number of potentials, each of which depends upon the conditions of the system.

In parallel with developing BWR_MASTER, a refined PWR_MASTER code has been developed to model electrochemical phenomena, including stress corrosion cracking. The codes contain a refined Coupled Environment Fracture Model (CEFM) and a refined Mixed Potential Model (MPM) for calculating crack growth rate in and the ECP, respectively, in sensitized Type 304 SS and in MA Alloys 600 and 690 have been developed. Thus, in developing PWR_MASTER in this project, we have modified and calibrated the CEFM, which has been used extensively to predict IGSCC in sensitized austenitic stainless-steel components in BWR environment, to predict CGRs in Alloys 600 and 690 in PWR primary coolant environments (see below). Calibration involved optimization of the CEFM on measured CGR data and, after extracting values for essential parameters, calculating CGR data as a function of the independent variables of interest. The customized CEFM for nickel-based, Alloy 600 provides quantitative predictions of the effects 
of temperature, yield strength, hydrogen concentration, and stress intensity factor on CGR in simulated PWR environment. Good agreement has been obtained between experimental data obtained from the artificial neural network analysis of CGR data in the literature, from which were distilled relationships between CGR and the various independent variables (temperature, ECP, $\left[\mathrm{H}_{2}\right]$, solution conductivity, yield strength, stress intensity factor) and the CEFM-predicted results. It should be noted that all of the error bars associated with the CGR are those indicated by ANN analysis, which indicates that the uncertainty in the CGR is about $\pm 0.3 \log$ (CGR). The CEFM also captured the well-known dependence of CGR on $\mathrm{K}_{\mathrm{I}}$ that is observed experimentally. Estimation of the value of the microfracture dimension confirms the likely presence of $\mathrm{HIC}$ because $\mathrm{H}$ is the only element that could possibly diffuse microns within the lattice at the crack tip over the period of seconds between microfracture events. Although microstructural effects were not incorporated in our recent Artificial Neural Network (ANN) analysis of SCC in Alloy 600 in PWR primary coolant, the ANN model successfully reproduced the CGR vs independent variables $\left[K_{I}\right.$, Yield Strength, temperature, solution conductivity, Electrochemical Corrosion Potential, $\mathrm{pH}$ (B and Li concentrations)] when tested against an evaluation data set suggesting that either the variation in the microstructure of the specimens involved was not significant, or the microstructural features do not strongly influence the CGR. This issue has yet to be resolved and was considered to be well beyond the scope of the current project.

Comparison of experimental and computed values showed that the Mixed Potential Model, including the quantum mechanical correction for electronic charge carrier tunneling through the barrier layer for the calculating ECP, is viable and that the exchange current density calculated for the bare ("film-free") surface satisfactorily accounts for the ECP. As noted above, this represents a major advance in the theory of electrochemical kinetics, because it is now only necessary to specify a single set of exchange current density and Tafel constants for each redox reaction, rather than a set for each value of the ECP (i.e., for each temperature, $\left[\mathrm{H}_{2}\right],\left[\mathrm{O}_{2}\right],\left[\mathrm{H}_{2} \mathrm{O}_{2}\right]$, flow velocity) in order to calculate the ECP and CGR under any given set of environmental conditions. This innovation greatly reduces the dimensionality of the problem of predicting the evolution of damage in nuclear power reactor primary coolant circuits. This accomplishment is in addition to the work proposed.

During this project, we have developed a theoretical model for the hydrogen-induced cracking to estimate the inter-granular crack growth rate in Alloy 600 in aqueous environments. 
In this model, a crack growth is assumed to occur by the combined effect of the anodic dissolution and the nucleation and growth of voids ahead of the crack tip along a grain boundary, and then linkage with the main crack due to stress-induced fracture of the remaining ligaments to yield the micro-fracture events that have been previously reported for IGSCC in Alloy 600 in high temperature water. Under proper conditions, hydrogen evolution at the crack tip injects atomic hydrogen into the crack tip matrix. The hydrogen atoms diffuse ahead of the crack tip and recombine to form $\mathrm{H}_{2}$ in the voids. The hydrogen generates pressure within a void that increases with time. The hydrogen pressure adds to the hydrostatic stress on the void due to mechanical loading, and once the effective stress exceeds the fracture stress of the ligament, the void links with the crack marking a micro-fracture event. The theoretical model for the hydrogen-induced cracking based on grain boundary void pressurization was incorporated into the CEFM. The CGR was calculated for Alloy 600 in the PWR heat transport circuit for different electrochemical and mechanical conditions. The calculated results indicate that the hydrogen evolution at the crack tip under PWR conditions has a significant effect on the CGR.

A viable, mechanism-based model for crack initiation in BWRs has never been previously developed. Experimental investigations have shown that crack is often observed to nucleate within an emergent grain face (probably from a corrosion pit), grow transgranularly until it intersects a grain boundary, and then grows intergranularly thereafter. Our goal has been to develop a model for calculating the crack initiation time in BWRs that considers the properties of the external environment. We have developed a model that predicts the time that it takes to nucleate and grow a critical pit; i.e., one whose stress intensity factor exceeds $\mathrm{K}_{\mathrm{ISCC}}$ for the prevailing stress includes residual and operational. The CEFM has been modified to calculate the growth rate of a hemispherical pit in terms of the properties of the pit external and internal environment.

A literature survey revealed that the internal oxidation of Alloy 600 had received the most experimental support as a viable mechanism of stress corrosion crack initiation mechanism in PWRs. Based on the information collected from the literature, we have developed a model for calculating crack initiation time in PWRs. The model postulates that the water penetrates the emergent grain boundaries and reacts with the $\mathrm{Cr}$ of Alloy 600, forming a $\mathrm{Cr}_{2} \mathrm{O}_{3}$ oxide layer that wedges open the grain boundary. The model can reproduce the effects of the following parameters: applied stress, temperature, cold work, grain boundary segregations, water chemistry, $\mathrm{pH}$, electrochemical potential. The model calculates the film thickness with the PDM and applies the 
Griffith criterion for modeling the delamination of grain boundaries. Mechanical creep rate is calculated by the Wilkinson and Vitek model. The crack initiation time is a sensitive function of the temperature and ECP under PWR primary coolant conditions.

In addition to the proposed work, we have initiated the development of the theory to predict the large distribution in observed crack initiation times. The model proposes that the local, potential breakdown sites are normally distributed with respect to the residual surface stress. To our knowledge, this is the first attempt to develop a theoretical edifice for accounting for and predicting the wide distributions in crack initiation times under nominally invariant conditions.

Work was initiated on predicting the experimentally-observed, wide distribution in crack initiation time by assuming that the local crack initiation sites are normally distributed with respect to local, surface stress (PWR) and/or with respect to pit depth (BWR). We identify the next steps that should be taken to resolve the "greatest theoretical problem in corrosion science" and hence, in the prediction of localized corrosion damage in nuclear power reactor coolant circuits. This accomplishment was in addition to the proposed work.

We demonstrated theoretically, using both the CEFM and ANN, that if the independent variables in the IGSCC in sensitized BWR primary coolant conditions are individually distributed normally, the resulting crack growth rate (CGR) is distributed log-normally, in agreement with experiment. These calculations define the ultimate accuracy with which the CGR can be determined under practical conditions. This accomplishment was in addition to the proposed work.

The electrochemical properties of Alloy 600, Alloy 690, Type 304L SS, and Type 316L SS were investigated at four temperatures $\left(150{ }^{\circ} \mathrm{C}, 200{ }^{\circ} \mathrm{C}, 250{ }^{\circ} \mathrm{C}, 300{ }^{\circ} \mathrm{C}\right)$ in three oxygenated borate buffer solutions (2000 ppm B + 2 ppm Li, 200 ppm B + 2 ppm Li, 0 ppm B + 2 ppm Li) by the measurements of open circuit potential (OCP), anodic potentiostatic polarization, MottSchottky analysis (MSA), and electrochemical impedance spectroscopy (EIS). For each alloy at each temperature and $\mathrm{pH}$ value, the stabilized anodic passive current density was independent of the formation potential, and the slope of the M-S plot for the passive film formed at $+0.3 \mathrm{~V}_{\mathrm{OCP}}$ $(\mathrm{OCP}+0.3 \mathrm{~V})$ is positive, indicating that the passive films formed on all alloys have an n-type semiconductor character. With an increase in temperature (in each solution), the OCP and the impedance modulus decreased, while both the passive current density and the defect density calculated from MSA increased, which indicated a significant reduction in corrosion resistance. 
With the increase in $\mathrm{pH}$ value (at the same temperature), the OCP and the passive current density decreased; however, the absolute value of impedance increased slightly, revealing a relatively slight increase in corrosion resistance. According to the optimization of Mixed Potential Model (MPM) on the experimentally measured EIS data for all alloys at OCP and $+0.3 \mathrm{~V}_{\text {OCP }}$, a series of fundamental parameters related to the micro-characteristic of the formed passive film, such as the concentrations of specific point defect, film thickness, and the kinetics of atomic-scale reactions occurring at the substrate/film interface, were extracted. In the barrier layer of the passive film, the concentration of cation interstitials is much higher than that of anion vacancies, indicating that the former is the dominant defect. With increasing temperature, the linear increases in the defect density and film thickness resulted in a linear increase in the anodic current density of the passive film. However, with increasing $\mathrm{pH}$, the decrease of defect density and the slight increase of film thickness caused a linear reduction in anodic current density. The elevated temperature has a more significant deteriorative effect on the corrosion resistance of each alloy than an increase in the $\mathrm{pH}$ value. Additionally, all the kinetics parameters describing the generation and annihilation of cation interstitials and oxygen vacancies and the cathodic reactions for alloys exposed to simulated solutions in pressurized water reactor primary circuit over wide ranges in temperatures and $\mathrm{pH}$ values were obtained. These parameters were employed to predict and understand the correlations between the corrosion resistance of alloys and the properties of the passive film, such as the film thickness and the electronic and defect structures; meanwhile, they are necessary fundamental data for calculating the ECP value of alloys in serviced in pressurized water reactor primary circuit from atomic and molecular scale information and for predicting the corrosion processes, such as the generation of cracking at the atomic level.

At the same corrosion environment: the OCP difference between these four alloys (Alloy 600, Alloy 690, Type 304L SS, and Type 316L SS) is found to be insignificant. This may be due to the similar composition of the barrier layer for each alloy, which comprises defective iron- and nickel-containing, chromic oxide $(\mathrm{Fe}, \mathrm{Ni}, \mathrm{Cr})_{2+x} \mathrm{O}_{3-y}$. The impedance values of nickel alloys are much higher than those of stainless-steel alloys, indicating higher corrosion resistance. The defect density of the barrier layer of the passive films formed on nickel alloy is much lower than that of stainless steel, while the thickness of the barrier layers is very similar. This confirmed that the effect of defect density on the corrosion properties of an alloy is much more significant than that of film thickness. The activation energy for the formation of metal interstitials is much higher for 
nickel alloy compared with stainless steel. Iron interstitials (as in SS) are generated at the metal/barrier layer interface and move through the $\mathrm{Cr}_{2} \mathrm{O}_{3}$ lattice much more readily than do $\mathrm{Ni}$ interstitials, and since $99.9+\%$ of the passive current is carrier by the interstitials, the passive current density is higher (and the corrosion resistance is lower) in the case of the stainless steels than for Alloy 600 and Alloy 690. The concentration of Fe interstitials is much higher than Ni in the barrier layers on stainless steels; therefore, the passive films on stainless steels exhibit much higher defect densities of Fe interstitials and show much lower corrosion resistance than do the nickel alloys. The corrosion resistance of Alloy 690 is slightly higher than that of Alloy 600, while the Type 304L SS and Type 316L SS show very similar corrosion resistance.

Finally, also outside of the Statement of Work, we have developed a new, in situ method to monitor the hydriding in zirconium alloy, nuclear fuel cladding. The method involves optimizing the Point Defect Model for the growth and breakdown of passive films on metal surfaces that were reformulated to describe the formation of a $\mathrm{ZrH}_{2-\mathrm{x}}$ barrier layer and a porous, $\mathrm{ZrO}_{2}$ outer layer, on experimental electrochemical impedance (EIS) data. The optimized model parameters are then used to calculate the thickness of the $\mathrm{ZrH}_{2-x}$ barrier layer, which provides a measure of the inventory of hydrogen in the alloy. The method has been evaluated on pure zirconium in PWR primary coolant $\left[0.1 \mathrm{~m} \mathrm{~B}(\mathrm{OH})_{3}+0.001 \mathrm{~m} \mathrm{LiOH}+25 \mathrm{~cm}^{3}(\mathrm{STP}) / \mathrm{kg} \mathrm{H}_{2} \mathrm{O}\right.$ of $\left.\mathrm{H}_{2}\right]$ at $250{ }^{\circ} \mathrm{C}$ on EIS data obtained in a previous NEUP-supported study.

\section{Appendix: Optimization of the mixed potential model on the experimental EIS data for passive films formed on Alloy 690, Type 304L SS, and Type 316L SS in oxygenated borate buffer solution.}

Various parameters in the mixed potential model (MPM) were acquired for passive films formed on Alloy 690, Type 304L SS, and Type 316L SS in solutions with $400 \mathrm{ppb}$ dissolved oxygen containing 2000 ppm $B+2$ ppm $L i, 200$ ppm $B+2$ ppm $L i$, and 0 ppm $B+2$ ppm $L i$ at different temperatures, as shown from Tables A.1 to A.18. Tables A.1 to A.9 present the values of model parameters, which were directly extracted from the optimization of MPM. Based on these extracted coefficients, several parameters were calculated and indicated from Tables A.10 to A.18. 
The rate constants $k_{i}$ and standard rate constant $k_{i}^{0}$ for Reaction $i$ are expressed as:

$$
\begin{array}{ll}
k_{i}=k_{i}^{0} \cdot e^{\left.a_{i \cdot\left(V-I_{\text {total }} \cdot R_{o l}\right)}\right)} e^{b_{i} \cdot L} \cdot e^{c_{i} \cdot p H} ; k_{i}^{0}=k_{i}^{00} \cdot e^{-\chi \alpha_{i} \gamma \phi_{f s}^{0}} & i=1,2,3 \\
k_{i}=k_{i}^{0} \cdot e^{\left.a_{i \cdot\left(V-I_{\text {total }} \cdot R_{o l}\right)}\right)} \cdot e^{c_{i} \cdot p H} ; k_{i}^{0}=k_{i}^{00} \cdot e^{-\delta \alpha_{i} \gamma \phi_{f s}^{0}} & i=4,5,6 \\
k_{7}=k_{7}^{0} \cdot e^{\left.a_{7 \cdot\left(V-I_{\text {total }} \cdot R_{o l}\right)}\right)} \cdot e^{c_{7} \cdot p H} ; k_{7}^{0}=k_{7}^{00} \cdot e^{(\delta-\chi) \alpha_{7} \gamma \phi_{f s}^{0}} &
\end{array}
$$

where $k_{i}^{00}$ is the base rate constant for Reaction $i, a_{i}, b_{i}, c_{i}$ are exponential coefficients, $V$ is the applied external potential, $I_{\text {total }}$ is the total current density, $R_{o l}$ is the resistance of the passive film outer layer, $L$ is the barrier layer thickness, $\phi_{f s}^{0}$ is the standard potential of film/solution interface, $\alpha_{i}$ is the transfer coefficients, $\chi$ and $\delta$ are oxidation states of chromium in barrier layer and in solution, respectively, and all assumed to be $3, \gamma=F / R T, F$ is Faraday's constant, $R$ represents molar gas constant, and $T$ is the temperature in Kelvin. 
Table A.1. Values of the parameters extracted from the optimization of the mixed potential model on the experimental EIS data for passive films formed on Alloy 690 at OCP in solution containing $2000 \mathrm{ppm} \mathrm{B}+2 \mathrm{ppm} \mathrm{Li}$ and $400 \mathrm{ppb}$ dissolved oxygen at different temperatures.

\begin{tabular}{|c|c|c|c|c|c|}
\hline Parameters & $150^{\circ} \mathrm{C}$ & $200^{\circ} \mathrm{C}$ & $250^{\circ} \mathrm{C}$ & $300^{\circ} \mathrm{C}$ & Method \\
\hline$O C P\left(V_{S H E}\right)$ & -0.363 & -0.553 & -0.615 & -0.783 & Measured \\
\hline$p H$ & 5.91 & 5.98 & 6.20 & 6.71 & $\begin{array}{l}\text { Calculated by } \\
\text { pH_Calc }\end{array}$ \\
\hline$\varepsilon\left(\mathrm{V} \cdot \mathrm{cm}^{-1}\right)$ & $1.1 \times 10^{6}$ & $9.0 \times 10^{5}$ & $8.7 \times 10^{5}$ & $8.4 \times 10^{5}$ & $3^{\text {rd }}$ stage \\
\hline$\sigma$ & $1.4 \times 10^{6}$ & $7.4 \times 10^{4}$ & $2.2 \times 10^{5}$ & $3.9 \times 10^{5}$ & $3^{\text {rd }}$ stage \\
\hline$R_{s}(\Omega)$ & 5757 & 4676 & 4292 & 4100 & $3^{\text {rd }}$ stage \\
\hline$R_{e, h}\left(\Omega \cdot \mathrm{cm}^{2}\right)$ & $7.5 \times 10^{6}$ & $2.0 \times 10^{5}$ & $1.8 \times 10^{5}$ & $1.5 \times 10^{5}$ & $3^{\text {rd }}$ stage \\
\hline$C_{g}\left(\mathrm{~F} \cdot \mathrm{cm}^{-2}\right)$ & $6.3 \times 10^{-5}$ & $1.4 \times 10^{-4}$ & $4.0 \times 10^{-4}$ & $1.2 \times 10^{-3}$ & $3^{\text {rd }}$ stage \\
\hline$C_{d l}\left(\mathrm{~F} \cdot \mathrm{cm}^{-2}\right)$ & $2.2 \times 10^{-3}$ & $2.0 \times 10^{-4}$ & $5.2 \times 10^{-4}$ & $1.9 \times 10^{-3}$ & $3^{\text {rd }}$ stage \\
\hline$R_{o l}\left(\Omega \cdot \mathrm{cm}^{2}\right)$ & 19988 & 4427 & 3430 & 3271 & $3^{\text {rd }}$ stage \\
\hline$C_{o l}\left(\mathrm{~F} \cdot \mathrm{cm}^{-2}\right)$ & $1.1 \times 10^{-4}$ & $5.7 \times 10^{-2}$ & $1.6 \times 10^{-3}$ & $1.8 \times 10^{-3}$ & $3^{\text {rd }}$ stage \\
\hline$\alpha_{c}$ & 0.29 & 0.40 & 0.43 & 0.62 & $3^{\text {rd }}$ stage \\
\hline$\widehat{\tau_{0}}\left(\mathrm{~A} \cdot \mathrm{cm}^{-2}\right)$ & $4.5 \times 10^{-9}$ & $6.6 \times 10^{-9}$ & $6.4 \times 10^{-8}$ & $6.2 \times 10^{-7}$ & $3^{\text {rd }}$ stage \\
\hline$i_{l, c}\left(\mathrm{~A} \cdot \mathrm{cm}^{-2}\right)$ & $1.0 \times 10^{-5}$ & $2.5 \times 10^{-5}$ & $2.5 \times 10^{-5}$ & $7.2 \times 10^{-5}$ & $3^{\text {rd }}$ stage \\
\hline$k_{2}^{00}\left(\mathrm{~mol} \cdot \mathrm{cm}^{-2} \cdot \mathrm{s}^{-1}\right)$ & $4.5 \times 10^{-10}$ & $1.6 \times 10^{-7}$ & $1.2 \times 10^{-5}$ & $8.9 \times 10^{-5}$ & $2^{\text {nd }}$ stage \\
\hline$k_{3}^{00}\left(\mathrm{~mol} \cdot \mathrm{cm}^{-2} \cdot \mathrm{s}^{-1}\right)$ & $3.2 \times 10^{-9}$ & $1.0 \times 10^{-7}$ & $2.3 \times 10^{-7}$ & $5.0 \times 10^{-7}$ & $2^{\text {nd }}$ stage \\
\hline$k_{5}^{00}\left(\mathrm{~cm} \cdot \mathrm{s}^{-1}\right)$ & $2.9 \times 10^{-6}$ & $6.3 \times 10^{-6}$ & $6.8 \times 10^{-6}$ & $2.4 \times 10^{-5}$ & $2^{\text {nd }}$ stage \\
\hline$k_{6}^{00}\left(\mathrm{~cm} \cdot \mathrm{s}^{-1}\right)$ & $1.4 \times 10^{-5}$ & $1.9 \times 10^{-5}$ & $2.0 \times 10^{-5}$ & $6.0 \times 10^{-5}$ & $2^{\text {nd }}$ stage \\
\hline$k_{7}^{00}\left(\mathrm{~mol} \cdot \mathrm{cm}^{-2} \cdot \mathrm{s}^{-1}\right)$ & $4.0 \times 10^{-9}$ & $4.5 \times 10^{-9}$ & $4.7 \times 10^{-9}$ & $1.0 \times 10^{-7}$ & $2^{\text {nd }}$ stage \\
\hline$n$ & 0.61 & 0.65 & 0.56 & 0.65 & $1^{\text {st }}$ stage \\
\hline $\begin{array}{l}\alpha ; \alpha_{2} ; \\
\alpha_{3} ; \alpha_{5} ; \\
\alpha_{6} ; \alpha_{7}\end{array}$ & $\begin{array}{l}0.63 ; 0.44 ; \\
0.58 ; 0.35 \\
0.53 ; 0.88\end{array}$ & $\begin{array}{l}0.60 ; 0.46 \\
0.47 ; 0.33 \\
0.48 ; 0.76\end{array}$ & $\begin{array}{l}0.55 ; 0.51 \\
0.40 ; 0.35 \\
0.44 ; 0.70\end{array}$ & $\begin{array}{l}0.70 ; 0.52 \\
0.36 ; 0.30 \\
0.30 ; 0.67\end{array}$ & $1^{\text {st }}$ stage \\
\hline
\end{tabular}


Table A.2. Values of the parameters extracted from the optimization of the mixed potential model on the experimental EIS data for passive films formed on Alloy 690 at OCP in solution containing $200 \mathrm{ppm} \mathrm{B}+2$ ppm Li and $400 \mathrm{ppb}$ dissolved oxygen at different temperatures.

\begin{tabular}{|c|c|c|c|c|c|}
\hline Parameters & $150^{\circ} \mathrm{C}$ & $200^{\circ} \mathrm{C}$ & $250^{\circ} \mathrm{C}$ & $300^{\circ} \mathrm{C}$ & Method \\
\hline$O C P\left(V_{S H E}\right)$ & -0.428 & -0.471 & -0.640 & -0.935 & Measured \\
\hline$p H$ & 7.07 & 7.04 & 7.15 & 7.57 & $\begin{array}{l}\text { Calculated by } \\
\text { pH_Calc }\end{array}$ \\
\hline$\varepsilon\left(\mathrm{V} \cdot \mathrm{cm}^{-1}\right)$ & $1.3 \times 10^{6}$ & $1.1 \times 10^{6}$ & $8.7 \times 10^{5}$ & $8.1 \times 10^{5}$ & $3^{\text {rd }}$ stage \\
\hline$\sigma$ & $1.9 \times 10^{6}$ & $9.8 \times 10^{4}$ & $2.3 \times 10^{5}$ & $4.9 \times 10^{5}$ & $3^{\text {rd }}$ stage \\
\hline$R_{s}(\Omega)$ & 5726 & 2773 & 2469 & 2322 & $3^{\text {rd }}$ stage \\
\hline$R_{e, h}\left(\Omega \cdot \mathrm{cm}^{2}\right)$ & $3.1 \times 10^{6}$ & $1.4 \times 10^{5}$ & $1.6 \times 10^{5}$ & $1.0 \times 10^{5}$ & $3^{\text {rd }}$ stage \\
\hline$C_{g}\left(\mathrm{~F} \cdot \mathrm{cm}^{-2}\right)$ & $5.5 \times 10^{-3}$ & $6.2 \times 10^{-4}$ & $4.5 \times 10^{-4}$ & $7.0 \times 10^{-4}$ & $3^{\text {rd }}$ stage \\
\hline$C_{d l}\left(\mathrm{~F} \cdot \mathrm{cm}^{-2}\right)$ & $8.3 \times 10^{-5}$ & $1.2 \times 10^{-4}$ & $4.2 \times 10^{-3}$ & $7.9 \times 10^{-3}$ & $3^{\text {rd }}$ stage \\
\hline$R_{o l}\left(\Omega \cdot \mathrm{cm}^{2}\right)$ & 8797 & 4897 & 1911 & 962 & $3^{\text {rd }}$ stage \\
\hline$C_{o l}\left(\mathrm{~F} \cdot \mathrm{cm}^{-2}\right)$ & $1.2 \times 10^{-4}$ & $1.9 \times 10^{-4}$ & $3.8 \times 10^{-3}$ & $2.8 \times 10^{-3}$ & $3^{\text {rd }}$ stage \\
\hline$\alpha_{c}$ & 0.29 & 0.47 & 0.55 & 0.55 & $3^{\text {rd }}$ stage \\
\hline$\widehat{t_{0}}\left(\mathrm{~A} \cdot \mathrm{cm}^{-2}\right)$ & $4.4 \times 10^{-9}$ & $6.2 \times 10^{-9}$ & $6.2 \times 10^{-8}$ & $6.1 \times 10^{-7}$ & $3^{\text {rd }}$ stage \\
\hline$i_{l, c}\left(\mathrm{~A} \cdot \mathrm{cm}^{-2}\right)$ & $9.7 \times 10^{-6}$ & $2.4 \times 10^{-5}$ & $2.5 \times 10^{-5}$ & $7.1 \times 10^{-5}$ & $3^{\text {rd }}$ stage \\
\hline$k_{2}^{00}\left(\mathrm{~mol} \cdot \mathrm{cm}^{-2} \cdot \mathrm{s}^{-1}\right)$ & $2.4 \times 10^{-9}$ & $7.8 \times 10^{-8}$ & $6.8 \times 10^{-6}$ & $1.9 \times 10^{-4}$ & $2^{\text {nd }}$ stage \\
\hline$k_{3}^{00}\left(\mathrm{~mol} \cdot \mathrm{cm}^{-2} \cdot \mathrm{s}^{-1}\right)$ & $1.6 \times 10^{-8}$ & $6.4 \times 10^{-8}$ & $4.4 \times 10^{-7}$ & $8.8 \times 10^{-7}$ & $2^{\text {nd }}$ stage \\
\hline$k_{5}^{00}\left(\mathrm{~cm} \cdot \mathrm{s}^{-1}\right)$ & $6.4 \times 10^{-6}$ & $9.6 \times 10^{-6}$ & $2.3 \times 10^{-5}$ & $4.9 \times 10^{-5}$ & $2^{\text {nd }}$ stage \\
\hline$k_{6}^{00}\left(\mathrm{~cm} \cdot \mathrm{s}^{-1}\right)$ & $1.3 \times 10^{-5}$ & $1.2 \times 10^{-5}$ & $2.8 \times 10^{-5}$ & $2.2 \times 10^{-4}$ & $2^{\text {nd }}$ stage \\
\hline$k_{7}^{00}\left(\mathrm{~mol} \cdot \mathrm{cm}^{-2} \cdot \mathrm{s}^{-1}\right)$ & $1.1 \times 10^{-9}$ & $3.6 \times 10^{-9}$ & $9.5 \times 10^{-9}$ & $6.9 \times 10^{-8}$ & $2^{\text {nd }}$ stage \\
\hline$n$ & 0.48 & 0.55 & 0.55 & 0.58 & $1^{\text {st }}$ stage \\
\hline $\begin{array}{l}\alpha ; \alpha_{2} ; \\
\alpha_{3} ; \alpha_{5} ; \\
\alpha_{6} ; \alpha_{7}\end{array}$ & $\begin{array}{l}0.55 ; 0.44 ; \\
0.58 ; 0.39 \\
0.53 ; 0.88\end{array}$ & $\begin{array}{l}0.57 ; 0.46 \\
0.48 ; 0.40 \\
0.52 ; 0.76\end{array}$ & $\begin{array}{l}0.61 ; 0.51 \\
0.44 ; 0.35 \\
0.44 ; 0.70\end{array}$ & $\begin{array}{l}0.65 ; 0.52 \\
0.37 ; 0.29 \\
0.30 ; 0.67\end{array}$ & $1^{\text {st }}$ stage \\
\hline
\end{tabular}


Table A.3. Values of the parameters extracted from the optimization of the mixed potential model on the experimental EIS data for passive films formed on Alloy 690 at OCP in solution containing 0 ppm B +2 ppm Li and 400 ppb dissolved oxygen at different temperatures.

\begin{tabular}{|c|c|c|c|c|c|}
\hline Parameters & $150^{\circ} \mathrm{C}$ & $200^{\circ} \mathrm{C}$ & $250^{\circ} \mathrm{C}$ & $300^{\circ} \mathrm{C}$ & Method \\
\hline$O C P\left(V_{S H E}\right)$ & -0.474 & -0.510 & -0.721 & -0.944 & Measured \\
\hline$p H$ & 8.09 & 7.69 & 7.60 & 7.91 & $\begin{array}{l}\text { Calculated by } \\
\text { pH_Calc }\end{array}$ \\
\hline$\varepsilon\left(\mathrm{V} \cdot \mathrm{cm}^{-1}\right)$ & $1.3 \times 10^{6}$ & $1.0 \times 10^{6}$ & $8.5 \times 10^{5}$ & $8.0 \times 10^{5}$ & $3^{\text {rd }}$ stage \\
\hline$\sigma$ & $3.9 \times 10^{4}$ & $8.7 \times 10^{4}$ & $3.1 \times 10^{4}$ & $3.0 \times 10^{5}$ & $3^{\text {rd }}$ stage \\
\hline$R_{s}(\Omega)$ & 4058 & 2637 & 2826 & 2648 & $3^{\text {rd }}$ stage \\
\hline$R_{e, h}\left(\Omega \cdot \mathrm{cm}^{2}\right)$ & $4.58 \times 10^{6}$ & $1.67 \times 10^{5}$ & $1.27 \times 10^{5}$ & $7.49 \times 10^{4}$ & $3^{\text {rd }}$ stage \\
\hline$C_{g}\left(\mathrm{~F} \cdot \mathrm{cm}^{-2}\right)$ & $8.8 \times 10^{-4}$ & $3.3 \times 10^{-4}$ & $1.5 \times 10^{-4}$ & $9.1 \times 10^{-4}$ & $3^{\text {rd }}$ stage \\
\hline$C_{d l}\left(\mathrm{~F} \cdot \mathrm{cm}^{-2}\right)$ & $7.0 \times 10^{-5}$ & $1.0 \times 10^{-4}$ & $4.7 \times 10^{-3}$ & $1.0 \times 10^{-2}$ & $3^{\text {rd }}$ stage \\
\hline$R_{o l}\left(\Omega \cdot \mathrm{cm}^{2}\right)$ & 7178 & 6840 & 2410 & 941 & $3^{\text {rd }}$ stage \\
\hline$C_{o l}\left(\mathrm{~F} \cdot \mathrm{cm}^{-2}\right)$ & $1.8 \times 10^{-4}$ & $2.9 \times 10^{-4}$ & $2.5 \times 10^{-3}$ & $5.8 \times 10^{-3}$ & $3^{\text {rd }}$ stage \\
\hline$\alpha_{c}$ & 0.30 & 0.47 & 0.51 & 0.58 & $3^{\text {rd }}$ stage \\
\hline$\widehat{\tau_{0}}\left(\mathrm{~A} \cdot \mathrm{cm}^{-2}\right)$ & $4.2 \times 10^{-9}$ & $6.2 \times 10^{-9}$ & $6.2 \times 10^{-8}$ & $6.0 \times 10^{-7}$ & $3^{\text {rd }}$ stage \\
\hline$i_{l, c}\left(\mathrm{~A} \cdot \mathrm{cm}^{-2}\right)$ & $1.0 \times 10^{-5}$ & $2.4 \times 10^{-5}$ & $2.5 \times 10^{-5}$ & $7.1 \times 10^{-5}$ & $3^{\text {rd }}$ stage \\
\hline$k_{2}^{00}\left(\mathrm{~mol} \cdot \mathrm{cm}^{-2} \cdot \mathrm{s}^{-1}\right)$ & $4.2 \times 10^{-10}$ & $6.2 \times 10^{-8}$ & $1.9 \times 10^{-5}$ & $3.0 \times 10^{-4}$ & $2^{\text {nd }}$ stage \\
\hline$k_{3}^{00}\left(\mathrm{~mol} \cdot \mathrm{cm}^{-2} \cdot \mathrm{s}^{-1}\right)$ & $3.2 \times 10^{-9}$ & $5.0 \times 10^{-8}$ & $4.0 \times 10^{-7}$ & $5.0 \times 10^{-7}$ & $2^{\text {nd }}$ stage \\
\hline$k_{5}^{00}\left(\mathrm{~cm} \cdot \mathrm{s}^{-1}\right)$ & $2.0 \times 10^{-5}$ & $3.3 \times 10^{-5}$ & $7.9 \times 10^{-5}$ & $2.0 \times 10^{-3}$ & $2^{\text {nd }}$ stage \\
\hline$k_{6}^{00}\left(\mathrm{~cm} \cdot \mathrm{s}^{-1}\right)$ & $2.1 \times 10^{-5}$ & $3.3 \times 10^{-5}$ & $5.8 \times 10^{-5}$ & $6.9 \times 10^{-5}$ & $2^{\text {nd }}$ stage \\
\hline$k_{7}^{00}\left(\mathrm{~mol} \cdot \mathrm{cm}^{-2} \cdot \mathrm{s}^{-1}\right)$ & $2.1 \times 10^{-9}$ & $3.6 \times 10^{-9}$ & $3.3 \times 10^{-8}$ & $1.0 \times 10^{-7}$ & $2^{\text {nd }}$ stage \\
\hline$n$ & 0.49 & 0.50 & 0.60 & 0.55 & $1^{\text {st }}$ stage \\
\hline $\begin{array}{l}\alpha ; \alpha_{2} ; \\
\alpha_{3} ; \alpha_{5} ; \\
\alpha_{6} ; \alpha_{7}\end{array}$ & $\begin{array}{l}0.58 ; 0.44 ; \\
0.58 ; 0.42 ; \\
0.56 ; 0.88\end{array}$ & $\begin{array}{l}0.55 ; 0.46 ; \\
0.48 ; 0.42 \\
0.55 ; 0.76\end{array}$ & $\begin{array}{l}0.55 ; 0.50 \\
0.40 ; 0.40 \\
0.44 ; 0.70\end{array}$ & $\begin{array}{l}0.67 ; 0.53 ; \\
0.35 ; 0.35 \\
0.26 ; 0.67\end{array}$ & $1^{\text {st }}$ stage \\
\hline
\end{tabular}


Table A.4. Values of the parameters extracted from the optimization of the mixed potential model on the experimental EIS data for passive films formed on Type 304L SS at OCP in solution containing 2000 ppm B +2 ppm Li and 400 ppb dissolved oxygen at different temperatures.

\begin{tabular}{|c|c|c|c|c|}
\hline Parameters & $150^{\circ} \mathrm{C}$ & $200^{\circ} \mathrm{C}$ & $250^{\circ} \mathrm{C}$ & Method \\
\hline$O C P\left(V_{S H E}\right)$ & -0.284 & -0.331 & -0.565 & Measured \\
\hline$p H$ & 5.91 & 5.98 & 6.20 & $\begin{array}{l}\text { Calculated by } \\
\text { pH_Calc }\end{array}$ \\
\hline$\varepsilon\left(\mathrm{V} \cdot \mathrm{cm}^{-1}\right)$ & $1.2 \times 10^{6}$ & $1.0 \times 10^{6}$ & $8.7 \times 10^{5}$ & $3^{\text {rd }}$ stage \\
\hline$\sigma$ & $1.2 \times 10^{5}$ & $1.3 \times 10^{4}$ & $1.4 \times 10^{5}$ & $3^{\text {rd }}$ stage \\
\hline$R_{s}(\Omega)$ & 4810 & 3760 & 3270 & $3^{\text {rd }}$ stage \\
\hline$R_{e, h}\left(\Omega \cdot \mathrm{cm}^{2}\right)$ & $6.6 \times 10^{4}$ & $1.1 \times 10^{5}$ & $1.6 \times 10^{5}$ & $3^{\text {rd }}$ stage \\
\hline$C_{g}\left(\mathrm{~F} \cdot \mathrm{cm}^{-2}\right)$ & $1.7 \times 10^{-4}$ & $2.2 \times 10^{-4}$ & $3.5 \times 10^{-3}$ & $3^{\text {rd }}$ stage \\
\hline$C_{d l}\left(\mathrm{~F} \cdot \mathrm{cm}^{-2}\right)$ & $1.4 \times 10^{-3}$ & $6.3 \times 10^{-3}$ & $4.9 \times 10^{-3}$ & $3^{\text {rd }}$ stage \\
\hline$R_{o l}\left(\Omega \cdot \mathrm{cm}^{2}\right)$ & 8561 & 3360 & 3303 & $3^{\text {rd }}$ stage \\
\hline$C_{o l}\left(\mathrm{~F} \cdot \mathrm{cm}^{-2}\right)$ & $4.5 \times 10^{-2}$ & $8.1 \times 10^{-2}$ & $8.7 \times 10^{-3}$ & $3^{\text {rd }}$ stage \\
\hline$\alpha_{c}$ & 0.33 & 0.44 & 0.47 & $3^{\text {rd }}$ stage \\
\hline$\widehat{t_{0}}\left(\mathrm{~A} \cdot \mathrm{cm}^{-2}\right)$ & $4.5 \times 10^{-9}$ & $6.6 \times 10^{-9}$ & $6.4 \times 10^{-8}$ & $3^{\text {rd }}$ stage \\
\hline$i_{l, c}\left(\mathrm{~A} \cdot \mathrm{cm}^{-2}\right)$ & $1.0 \times 10^{-5}$ & $2.5 \times 10^{-5}$ & $2.5 \times 10^{-5}$ & $3^{\text {rd }}$ stage \\
\hline$k_{2}^{00}\left(\mathrm{~mol} \cdot \mathrm{cm}^{-2} \cdot \mathrm{s}^{-1}\right)$ & $1.2 \times 10^{-9}$ & $2.1 \times 10^{-9}$ & $4.1 \times 10^{-7}$ & $2^{\text {nd }}$ stage \\
\hline$k_{3}^{00}\left(\mathrm{~mol} \cdot \mathrm{cm}^{-2} \cdot \mathrm{s}^{-1}\right)$ & $2.8 \times 10^{-9}$ & $1.0 \times 10^{-8}$ & $8.7 \times 10^{-8}$ & $2^{\text {nd }}$ stage \\
\hline$k_{5}^{00}\left(\mathrm{~cm} \cdot \mathrm{s}^{-1}\right)$ & $3.7 \times 10^{-7}$ & $4.3 \times 10^{-7}$ & $3.5 \times 10^{-6}$ & $2^{\text {nd }}$ stage \\
\hline$k_{6}^{00}\left(\mathrm{~cm} \cdot \mathrm{s}^{-1}\right)$ & $1.6 \times 10^{-6}$ & $1.7 \times 10^{-6}$ & $2.4 \times 10^{-5}$ & $2^{\text {nd }}$ stage \\
\hline$k_{7}^{00}\left(\mathrm{~mol} \cdot \mathrm{cm}^{-2} \cdot \mathrm{s}^{-1}\right)$ & $7.2 \times 10^{-9}$ & $1.0 \times 10^{-8}$ & $1.1 \times 10^{-8}$ & $2^{\text {nd }}$ stage \\
\hline$n$ & 0.65 & 0.64 & 0.57 & $1^{\text {st }}$ stage \\
\hline $\begin{array}{l}\alpha ; \alpha_{2} ; \\
\alpha_{3} ; \alpha_{5} ; \\
\alpha_{6} ; \alpha_{7}\end{array}$ & $\begin{array}{l}0.60 ; 0.47 \\
0.58 ; 0.35 \\
0.44 ; 0.88\end{array}$ & $\begin{array}{l}0.59 ; 0.46 \\
0.56 ; 0.39 \\
0.50 ; 0.76\end{array}$ & $\begin{array}{l}0.63 ; 0.51 \\
0.44 ; 0.35 \\
0.44 ; 0.70\end{array}$ & $1^{\text {st }}$ stage \\
\hline
\end{tabular}


Table A.5. Values of the parameters extracted from the optimization of the mixed potential model on the experimental EIS data for passive films formed on Type 304L SS at OCP in solution containing $200 \mathrm{ppm} \mathrm{B}+2 \mathrm{ppm} \mathrm{Li}$ and $400 \mathrm{ppb}$ dissolved oxygen at different temperatures.

\begin{tabular}{lllll}
\hline Parameters & $150^{\circ} \mathrm{C}$ & $200{ }^{\circ} \mathrm{C}$ & $250^{\circ} \mathrm{C}$ & Method \\
\hline$O C P\left(V_{S H E}\right)$ & -0.387 & -0.390 & -0.820 & Measured \\
\hline$p H$ & 7.07 & 7.04 & 7.15 & $\begin{array}{l}\text { Calculated by } \\
\text { pHadcalc }\end{array}$ \\
\hline$\varepsilon\left(\mathrm{V} \cdot \mathrm{cm}^{-1}\right)$ & $1.2 \times 10^{6}$ & $1.1 \times 10^{6}$ & $7.9 \times 10^{5}$ & $3^{\text {rd }}$ stage \\
\hline$\sigma$ & $2.4 \times 10^{6}$ & $1.2 \times 10^{4}$ & $1.6 \times 10^{5}$ & $3^{\text {rd }}$ stage \\
\hline$R_{s}(\Omega)$ & 4240 & 3400 & 2877 & $3^{\text {rd }}$ stage \\
\hline$R_{e, h}\left(\Omega \cdot \mathrm{cm}^{2}\right)$ & $6.5 \times 10^{4}$ & $1.0 \times 10^{5}$ & $1.1 \times 10^{5}$ & $3^{\text {rd }}$ stage \\
\hline$C_{g}\left(\mathrm{~F} \cdot \mathrm{cm}^{-2}\right)$ & $1.4 \times 10^{-4}$ & $1.4 \times 10^{-4}$ & $1.6 \times 10^{-2}$ & $3^{\text {rd }}$ stage \\
\hline$C_{d l}\left(\mathrm{~F} \cdot \mathrm{cm}^{-2}\right)$ & $5.3 \times 10^{-3}$ & $1.0 \times 10^{-2}$ & $1.0 \times 10^{-2}$ & $3^{\text {rd }}$ stage \\
\hline$R_{o l}\left(\Omega \cdot \mathrm{cm}^{2}\right)$ & 14526 & 3007 & 1000 & $3^{\text {rd }}$ stage \\
\hline$C_{o l}\left(\mathrm{~F} \cdot \mathrm{cm}^{-2}\right)$ & $3.1 \times 10^{-2}$ & $1.0 \times 10^{-1}$ & $5.3 \times 10^{-3}$ & $3^{\text {rd }}$ stage \\
\hline$\alpha_{c}$ & 0.33 & 0.58 & 0.55 & $3^{\text {rd }}$ stage \\
\hline$\hat{o}_{0}\left(\mathrm{~A} \cdot \mathrm{cm}^{-2}\right)$ & $4.4 \times 10^{-9}$ & $6.2 \times 10^{-9}$ & $6.2 \times 10^{-8}$ & $3^{\text {rd }}$ stage \\
\hline$i_{l, c}\left(\mathrm{~A} \cdot \mathrm{cm}^{-2}\right)$ & $9.7 \times 10^{-6}$ & $2.4 \times 10^{-5}$ & $2.2 \times 10^{-5}$ & $3^{\text {rd }}$ stage \\
\hline$k_{2}^{00}\left(\mathrm{~mol} \cdot \mathrm{cm}^{-2} \cdot \mathrm{s}^{-1}\right)$ & $4.4 \times 10^{-9}$ & $4.1 \times 10^{-8}$ & $3.1 \times 10^{-6}$ & $2^{\text {nd }}$ stage \\
\hline$k_{3}^{00}\left(\mathrm{~mol} \cdot \mathrm{cm}^{-2} \cdot \mathrm{s}^{-1}\right)$ & $2.8 \times 10^{-9}$ & $6.4 \times 10^{-8}$ & $1.0 \times 10^{-7}$ & $2^{\text {nd }}$ stage \\
\hline$k_{5}^{00}\left(\mathrm{~cm} \cdot \mathrm{s}^{-1}\right)$ & $5.4 \times 10^{-6}$ & $7.9 \times 10^{-6}$ & $2.8 \times 10^{-4}$ & $2^{\text {nd }}$ stage \\
\hline$k_{6}^{00}\left(\mathrm{~cm} \cdot \mathrm{s}^{-1}\right)$ & $1.7 \times 10^{-5}$ & $1.8 \times 10^{-5}$ & $8.5 \times 10^{-4}$ & $2^{\text {nd }}$ stage \\
\hline$k_{7}^{00}\left(\mathrm{~mol} \cdot \mathrm{cm}^{-2} \cdot \mathrm{s}^{-1}\right)$ & $5.5 \times 10^{-9}$ & $1.0 \times 10^{-8}$ & $1.1 \times 10^{-7}$ & $2^{\text {nd }}$ stage \\
\hline$n$ & 0.58 & 0.55 & 0.67 & $1^{\text {st }}$ stage \\
\hline$\alpha ; \alpha_{2} ;$ & $0.65 ; 0.56 ;$ & $0.66 ; 0.46 ;$ & $0.65 ; 0.40 ;$ & $1^{\text {st }}$ stage \\
$\alpha_{3} ; \alpha_{5} ;$ & $0.58 ; 0.39 ;$ & $0.48 ; 0.45 ;$ & $0.32 ; 0.35 ;$ & \\
\hline$\alpha_{6} ; \alpha_{7}$ & $0.53 ; 0.88$ & $0.55 ; 0.76$ & $0.44 ; 0.70 ;$ & \\
\hline
\end{tabular}


Table A.6. Values of the parameters extracted from the optimization of the mixed potential model on the experimental EIS data for passive films formed on Type 304L SS at OCP in solution containing 0 ppm B +2 ppm Li and 400 ppb dissolved oxygen at different temperatures.

\begin{tabular}{|c|c|c|c|c|}
\hline Parameters & $150^{\circ} \mathrm{C}$ & $200^{\circ} \mathrm{C}$ & $250^{\circ} \mathrm{C}$ & Method \\
\hline$O C P\left(V_{S H E}\right)$ & -0.465 & -0.454 & -0.767 & Measured \\
\hline$p H$ & 8.09 & 7.69 & 7.60 & $\begin{array}{l}\text { Calculated by } \\
\text { pH.Calc }\end{array}$ \\
\hline$\varepsilon\left(\mathrm{V} \cdot \mathrm{cm}^{-1}\right)$ & $1.1 \times 10^{6}$ & $1.0 \times 10^{6}$ & $8.5 \times 10^{5}$ & $3^{\text {rd }}$ stage \\
\hline$\sigma$ & $1.0 \times 10^{4}$ & $8.7 \times 10^{4}$ & $2.5 \times 10^{5}$ & $3^{\text {rd }}$ stage \\
\hline$R_{s}(\Omega)$ & 3260 & 2750 & 2365 & $3^{\text {rd }}$ stage \\
\hline$R_{e, h}\left(\Omega \cdot \mathrm{cm}^{2}\right)$ & $7.04 \times 10^{6}$ & $1.67 \times 10^{5}$ & $1.38 \times 10^{5}$ & $3^{\text {rd }}$ stage \\
\hline$C_{g}\left(\mathrm{~F} \cdot \mathrm{cm}^{-2}\right)$ & $4.4 \times 10^{-4}$ & $2.3 \times 10^{-4}$ & $2.0 \times 10^{-3}$ & $3^{\text {rd }}$ stage \\
\hline$C_{d l}\left(\mathrm{~F} \cdot \mathrm{cm}^{-2}\right)$ & $4.4 \times 10^{-4}$ & $1.0 \times 10^{-2}$ & $8.2 \times 10^{-3}$ & $3^{\text {rd }}$ stage \\
\hline$R_{o l}\left(\Omega \cdot \mathrm{cm}^{2}\right)$ & 19998 & 6840 & 2398 & $3^{\text {rd }}$ stage \\
\hline$C_{o l}\left(\mathrm{~F} \cdot \mathrm{cm}^{-2}\right)$ & $2.7 \times 10^{-4}$ & $2.9 \times 10^{-4}$ & $5.9 \times 10^{-3}$ & $3^{\text {rd }}$ stage \\
\hline$\alpha_{c}$ & 0.32 & 0.47 & 0.51 & $3^{\text {rd }}$ stage \\
\hline$\widehat{\tau_{0}}\left(\mathrm{~A} \cdot \mathrm{cm}^{-2}\right)$ & $4.5 \times 10^{-9}$ & $6.2 \times 10^{-9}$ & $6.2 \times 10^{-8}$ & $3^{\text {rd }}$ stage \\
\hline$i_{l, c}\left(\mathrm{~A} \cdot \mathrm{cm}^{-2}\right)$ & $1.0 \times 10^{-5}$ & $2.4 \times 10^{-5}$ & $2.5 \times 10^{-5}$ & $3^{\text {rd }}$ stage \\
\hline$k_{2}^{00}\left(\mathrm{~mol} \cdot \mathrm{cm}^{-2} \cdot \mathrm{s}^{-1}\right)$ & $1.2 \times 10^{-8}$ & $1.7 \times 10^{-8}$ & $7.8 \times 10^{-6}$ & $2^{\text {nd }}$ stage \\
\hline$k_{3}^{00}\left(\mathrm{~mol} \cdot \mathrm{cm}^{-2} \cdot \mathrm{s}^{-1}\right)$ & $1.0 \times 10^{-8}$ & $1.3 \times 10^{-8}$ & $4.0 \times 10^{-6}$ & $2^{\text {nd }}$ stage \\
\hline$k_{5}^{00}\left(\mathrm{~cm} \cdot \mathrm{s}^{-1}\right)$ & $1.9 \times 10^{-6}$ & $3.3 \times 10^{-6}$ & $2.5 \times 10^{-5}$ & $2^{\text {nd }}$ stage \\
\hline$k_{6}^{00}\left(\mathrm{~cm} \cdot \mathrm{s}^{-1}\right)$ & $9.1 \times 10^{-6}$ & $1.2 \times 10^{-5}$ & $3.9 \times 10^{-5}$ & $2^{\text {nd }}$ stage \\
\hline$k_{7}^{00}\left(\mathrm{~mol} \cdot \mathrm{cm}^{-2} \cdot \mathrm{s}^{-1}\right)$ & $4.6 \times 10^{-9}$ & $7.8 \times 10^{-9}$ & $9.3 \times 10^{-9}$ & $2^{\text {nd }}$ stage \\
\hline$n$ & 0.51 & 0.50 & 0.50 & $1^{\text {st }}$ stage \\
\hline $\begin{array}{l}\alpha ; \alpha_{2} ; \\
\alpha_{3} ; \alpha_{5} ; \\
\alpha_{6} ; \alpha_{7}\end{array}$ & $\begin{array}{l}0.54 ; 0.54 \\
0.57 ; 0.35 \\
0.48 ; 0.88\end{array}$ & $\begin{array}{l}0.50 ; 0.46 \\
0.48 ; 0.42 \\
0.55 ; 0.76\end{array}$ & $\begin{array}{l}0.49 ; 0.40 \\
0.40 ; 0.40 \\
0.44 ; 0.70\end{array}$ & $1^{\text {st }}$ stage \\
\hline
\end{tabular}


Table A.7. Values of the parameters extracted from the optimization of the mixed potential model on the experimental EIS data for passive films formed on Type 316L SS at OCP in solution containing 2000 ppm B +2 ppm Li and 400 ppb dissolved oxygen at different temperatures.

\begin{tabular}{lllll}
\hline Parameters & $150^{\circ} \mathrm{C}$ & $200{ }^{\circ} \mathrm{C}$ & $250^{\circ} \mathrm{C}$ & Method \\
\hline$O C P\left(V_{S H E}\right)$ & -0.286 & -0.334 & -0.574 & Measured \\
\hline$p H$ & 8.09 & 7.69 & 7.60 & $\begin{array}{l}\text { Calculated by } \\
\text { pHaecalc }\end{array}$ \\
\hline$\varepsilon\left(\mathrm{V} \cdot \mathrm{cm}^{-1}\right)$ & $1.2 \times 10^{6}$ & $1.0 \times 10^{6}$ & $8.7 \times 10^{5}$ & $3^{\text {rd }}$ stage \\
\hline$\sigma$ & $1.0 \times 10^{4}$ & $8.7 \times 10^{4}$ & $2.5 \times 10^{5}$ & $3^{\text {rd }}$ stage \\
\hline$R_{s}(\Omega)$ & 5070 & 3960 & 3420 & $3^{\text {rd }}$ stage \\
\hline$R_{e, h}\left(\Omega \cdot \mathrm{cm}^{2}\right)$ & $7.39 \times 10^{4}$ & $1.02 \times 10^{5}$ & $1.73 \times 10^{5}$ & $3^{\text {rd }}$ stage \\
\hline$C_{g}\left(\mathrm{~F} \cdot \mathrm{cm}^{-2}\right)$ & $6.1 \times 10^{-4}$ & $9.7 \times 10^{-4}$ & $3.9 \times 10^{-3}$ & $3^{\text {rd }}$ stage \\
\hline$C_{d l}\left(\mathrm{~F} \cdot \mathrm{cm}^{-2}\right)$ & $4.5 \times 10^{-4}$ & $5.3 \times 10^{-4}$ & $6.2 \times 10^{-3}$ & $3^{\text {rd }}$ stage \\
\hline$R_{o l}\left(\Omega \cdot \mathrm{cm}^{2}\right)$ & 10444 & 4900 & 3707 & $3^{\text {rd }}$ stage \\
\hline$C_{o l}\left(\mathrm{~F} \cdot \mathrm{cm}^{-2}\right)$ & $2.8 \times 10^{-4}$ & $4.7 \times 10^{-4}$ & $1.0 \times 10^{-2}$ & $3^{\text {rd }}$ stage \\
\hline$\alpha_{c}$ & 0.32 & 0.38 & 0.48 & $3^{\text {rd }}$ stage \\
\hline$\hat{l}_{0}\left(\mathrm{~A} \cdot \mathrm{cm}^{-2}\right)$ & $4.4 \times 10^{-9}$ & $6.6 \times 10^{-9}$ & $6.4 \times 10^{-8}$ & $3^{\text {rd }}$ stage \\
\hline$i_{l, c}\left(\mathrm{~A} \cdot \mathrm{cm}^{-2}\right)$ & $1.0 \times 10^{-5}$ & $2.5 \times 10^{-5}$ & $2.5 \times 10^{-5}$ & $3^{\text {rd }}$ stage \\
\hline$k_{2}^{00}\left(\mathrm{~mol} \cdot \mathrm{cm}^{-2} \cdot \mathrm{s}^{-1}\right)$ & $5.2 \times 10^{-10}$ & $5.6 \times 10^{-10}$ & $2.6 \times 10^{-7}$ & $2^{\text {nd }}$ stage \\
\hline$k_{3}^{00}\left(\mathrm{~mol} \cdot \mathrm{cm}^{-2} \cdot \mathrm{s}^{-1}\right)$ & $2.8 \times 10^{-9}$ & $2.8 \times 10^{-9}$ & $1.0 \times 10^{-7}$ & $2^{\text {nd }}$ stage \\
\hline$k_{5}^{00}\left(\mathrm{~cm} \cdot \mathrm{s}^{-1}\right)$ & $1.1 \times 10^{-7}$ & $1.8 \times 10^{-7}$ & $6.7 \times 10^{-6}$ & $2^{\text {nd }}$ stage \\
\hline$k_{6}^{00}\left(\mathrm{~cm} \cdot \mathrm{s}^{-1}\right)$ & $1.0 \times 10^{-6}$ & $1.6 \times 10^{-6}$ & $2.9 \times 10^{-5}$ & $2^{\text {nd }}$ stage \\
\hline$k_{7}^{00}\left(\mathrm{~mol} \cdot \mathrm{cm}^{-2} \cdot \mathrm{s}^{-1}\right)$ & $7.2 \times 10^{-9}$ & $1.5 \times 10^{-8}$ & $1.9 \times 10^{-8}$ & $2^{\text {nd }}$ stage \\
\hline$n$ & 0.64 & 0.66 & 0.62 & $1^{\text {st }}$ stage \\
\hline$\alpha_{;} \alpha_{2} ;$ & $0.50 ; 0.44 ;$ & $0.50 ; 0.46 ;$ & $0.63 ; 0.47 ;$ & $1^{\text {st }}$ stage \\
$\alpha_{3} ; \alpha_{5} ;$ & $0.56 ; 0.35 ;$ & $0.59 ; 0.36 ;$ & $0.44 ; 0.35 ;$ & \\
$\alpha_{6} ; \alpha_{7}$ & $0.50 ; 0.76$ & $0.44 ; 0.70$ & \\
\hline
\end{tabular}


Table A.8. Values of the parameters extracted from the optimization of the mixed potential model on the experimental EIS data for passive films formed on Type 316L SS at OCP in solution containing $200 \mathrm{ppm} \mathrm{B}+2$ ppm Li and 400 ppb dissolved oxygen at different temperatures.

\begin{tabular}{|c|c|c|c|c|}
\hline Parameters & $150^{\circ} \mathrm{C}$ & $200^{\circ} \mathrm{C}$ & $250^{\circ} \mathrm{C}$ & Method \\
\hline$O C P\left(V_{S H E}\right)$ & -0.387 & -0.392 & -0.760 & Measured \\
\hline$p H$ & 8.09 & 7.69 & 7.60 & $\begin{array}{l}\text { Calculated by } \\
\text { pH.Calc }\end{array}$ \\
\hline$\varepsilon\left(\mathrm{V} \cdot \mathrm{cm}^{-1}\right)$ & $1.2 \times 10^{6}$ & $1.0 \times 10^{6}$ & $7.9 \times 10^{5}$ & $3^{\text {rd }}$ stage \\
\hline$\sigma$ & $1.5 \times 10^{6}$ & $1.2 \times 10^{5}$ & $2.5 \times 10^{5}$ & $3^{\text {rd }}$ stage \\
\hline$R_{s}(\Omega)$ & 4700 & 3304 & 2770 & $3^{\text {rd }}$ stage \\
\hline$R_{e, h}\left(\Omega \cdot \mathrm{cm}^{2}\right)$ & $6.66 \times 10^{4}$ & $1.00 \times 10^{5}$ & $2.51 \times 10^{5}$ & $3^{\text {rd }}$ stage \\
\hline$C_{g}\left(\mathrm{~F} \cdot \mathrm{cm}^{-2}\right)$ & $1.4 \times 10^{-4}$ & $1.7 \times 10^{-4}$ & $3.2 \times 10^{-3}$ & $3^{\text {rd }}$ stage \\
\hline$C_{d l}\left(\mathrm{~F} \cdot \mathrm{cm}^{-2}\right)$ & $9.9 \times 10^{-3}$ & $2.5 \times 10^{-3}$ & $9.4 \times 10^{-3}$ & $3^{\text {rd }}$ stage \\
\hline$R_{o l}\left(\Omega \cdot \mathrm{cm}^{2}\right)$ & 12013 & 4897 & 3200 & $3^{\text {rd }}$ stage \\
\hline$C_{o l}\left(\mathrm{~F} \cdot \mathrm{cm}^{-2}\right)$ & $9.3 \times 10^{-2}$ & $9.9 \times 10^{-4}$ & $1.0 \times 10^{-2}$ & $3^{\text {rd }}$ stage \\
\hline$\alpha_{c}$ & 0.33 & 0.56 & 0.55 & $3^{\text {rd }}$ stage \\
\hline$\widehat{\tau_{0}}\left(\mathrm{~A} \cdot \mathrm{cm}^{-2}\right)$ & $4.4 \times 10^{-9}$ & $6.2 \times 10^{-9}$ & $6.2 \times 10^{-8}$ & $3^{\text {rd }}$ stage \\
\hline$i_{l, c}\left(\mathrm{~A} \cdot \mathrm{cm}^{-2}\right)$ & $9.7 \times 10^{-6}$ & $2.4 \times 10^{-5}$ & $2.2 \times 10^{-5}$ & $3^{\text {rd }}$ stage \\
\hline$k_{2}^{00}\left(\mathrm{~mol} \cdot \mathrm{cm}^{-2} \cdot \mathrm{s}^{-1}\right)$ & $1.0 \times 10^{-9}$ & $1.6 \times 10^{-8}$ & $1.1 \times 10^{-6}$ & $2^{\text {nd }}$ stage \\
\hline$k_{3}^{00}\left(\mathrm{~mol} \cdot \mathrm{cm}^{-2} \cdot \mathrm{s}^{-1}\right)$ & $2.8 \times 10^{-9}$ & $1.3 \times 10^{-8}$ & $1.0 \times 10^{-7}$ & $2^{\text {nd }}$ stage \\
\hline$k_{5}^{00}\left(\mathrm{~cm} \cdot \mathrm{s}^{-1}\right)$ & $3.7 \times 10^{-6}$ & $1.4 \times 10^{-5}$ & $5.5 \times 10^{-5}$ & $2^{\text {nd }}$ stage \\
\hline$k_{6}^{00}\left(\mathrm{~cm} \cdot \mathrm{s}^{-1}\right)$ & $7.2 \times 10^{-5}$ & $2.1 \times 10^{-4}$ & $1.3 \times 10^{-3}$ & $2^{\text {nd }}$ stage \\
\hline$k_{7}^{00}\left(\mathrm{~mol} \cdot \mathrm{cm}^{-2} \cdot \mathrm{s}^{-1}\right)$ & $7.2 \times 10^{-9}$ & $6.0 \times 10^{-9}$ & $1.1 \times 10^{-7}$ & $2^{\text {nd }}$ stage \\
\hline$n$ & 0.58 & 0.53 & 0.67 & $1^{\text {st }}$ stage \\
\hline $\begin{array}{l}\alpha ; \alpha_{2} ; \\
\alpha_{3} ; \alpha_{5} ; \\
\alpha_{6} ; \alpha_{7}\end{array}$ & $\begin{array}{l}0.65 ; 0.50 ; \\
0.58 ; 0.39 \\
0.53 ; 0.88\end{array}$ & $\begin{array}{l}0.66 ; 0.46 \\
0.48 ; 0.45 \\
0.55 ; 0.76\end{array}$ & $\begin{array}{l}0.59 ; 0.37 \\
0.32 ; 0.35 \\
0.44 ; 0.70\end{array}$ & $1^{\text {st }}$ stage \\
\hline
\end{tabular}


Table A.9. Values of the parameters extracted from the optimization of the mixed potential model on the experimental EIS data for passive films formed on Type 316L SS at OCP in solution containing 0 ppm B +2 ppm Li and 400 ppb dissolved oxygen at different temperatures.

\begin{tabular}{|c|c|c|c|c|}
\hline Parameters & $150^{\circ} \mathrm{C}$ & $200^{\circ} \mathrm{C}$ & $250^{\circ} \mathrm{C}$ & Method \\
\hline$O C P\left(V_{S H E}\right)$ & -0.469 & -0.480 & -0.767 & Measured \\
\hline$p H$ & 8.09 & 7.69 & 7.60 & $\begin{array}{l}\text { Calculated by } \\
\text { pH Calc }\end{array}$ \\
\hline$\varepsilon\left(\mathrm{V} \cdot \mathrm{cm}^{-1}\right)$ & $1.1 \times 10^{6}$ & $1.0 \times 10^{6}$ & $8.5 \times 10^{5}$ & $3^{\text {rd }}$ stage \\
\hline$\sigma$ & $4.4 \times 10^{4}$ & $1.2 \times 10^{4}$ & $1.6 \times 10^{5}$ & $3^{\text {rd }}$ stage \\
\hline$R_{s}(\Omega)$ & 3780 & 2862 & 2530 & $3^{\text {rd }}$ stage \\
\hline$R_{e, h}\left(\Omega \cdot \mathrm{cm}^{2}\right)$ & $7.07 \times 10^{4}$ & $1.00 \times 10^{5}$ & $1.80 \times 10^{5}$ & $3^{\text {rd }}$ stage \\
\hline$C_{g}\left(\mathrm{~F} \cdot \mathrm{cm}^{-2}\right)$ & $2.9 \times 10^{-4}$ & $3.2 \times 10^{-4}$ & $1.8 \times 10^{-3}$ & $3^{\text {rd }}$ stage \\
\hline$C_{d l}\left(\mathrm{~F} \cdot \mathrm{cm}^{-2}\right)$ & $5.7 \times 10^{-4}$ & $1.0 \times 10^{-2}$ & $9.9 \times 10^{-3}$ & $3^{\text {rd }}$ stage \\
\hline$R_{o l}\left(\Omega \cdot \mathrm{cm}^{2}\right)$ & 19947 & 6937 & 1959 & $3^{\text {rd }}$ stage \\
\hline$C_{o l}\left(\mathrm{~F} \cdot \mathrm{cm}^{-2}\right)$ & $3.3 \times 10^{-4}$ & $4.8 \times 10^{-4}$ & $1.0 \times 10^{-2}$ & $3^{\text {rd }}$ stage \\
\hline$\alpha_{c}$ & 0.28 & 0.50 & 0.51 & $3^{\text {rd }}$ stage \\
\hline$\widehat{\iota_{0}}\left(\mathrm{~A} \cdot \mathrm{cm}^{-2}\right)$ & $4.5 \times 10^{-9}$ & $6.2 \times 10^{-9}$ & $6.2 \times 10^{-8}$ & $3^{\text {rd }}$ stage \\
\hline$i_{l, c}\left(\mathrm{~A} \cdot \mathrm{cm}^{-2}\right)$ & $1.0 \times 10^{-5}$ & $2.4 \times 10^{-5}$ & $2.5 \times 10^{-5}$ & $3^{\text {rd }}$ stage \\
\hline$k_{2}^{00}\left(\mathrm{~mol} \cdot \mathrm{cm}^{-2} \cdot \mathrm{s}^{-1}\right)$ & $1.0 \times 10^{-9}$ & $5.1 \times 10^{-8}$ & $1.2 \times 10^{-6}$ & $2^{\text {nd }}$ stage \\
\hline$k_{3}^{00}\left(\mathrm{~mol} \cdot \mathrm{cm}^{-2} \cdot \mathrm{s}^{-1}\right)$ & $2.9 \times 10^{-9}$ & $8.0 \times 10^{-8}$ & $5.0 \times 10^{-7}$ & $2^{\text {nd }}$ stage \\
\hline$k_{5}^{00}\left(\mathrm{~cm} \cdot \mathrm{s}^{-1}\right)$ & $2.5 \times 10^{-6}$ & $7.5 \times 10^{-6}$ & $4.1 \times 10^{-5}$ & $2^{\text {nd }}$ stage \\
\hline$k_{6}^{00}\left(\mathrm{~cm} \cdot \mathrm{s}^{-1}\right)$ & $2.1 \times 10^{-5}$ & $4.2 \times 10^{-5}$ & $4.9 \times 10^{-4}$ & $2^{\text {nd }}$ stage \\
\hline$k_{7}^{00}\left(\mathrm{~mol} \cdot \mathrm{cm}^{-2} \cdot \mathrm{s}^{-1}\right)$ & $5.3 \times 10^{-9}$ & $5.2 \times 10^{-9}$ & $8.8 \times 10^{-9}$ & $2^{\text {nd }}$ stage \\
\hline$n$ & 0.49 & 0.46 & 0.47 & $1^{\text {st }}$ stage \\
\hline $\begin{array}{l}\alpha ; \alpha_{2} ; \\
\alpha_{3} ; \alpha_{5} ; \\
\alpha_{6} ; \alpha_{7}\end{array}$ & $\begin{array}{l}0.55 ; 0.50 \\
0.57 ; 0.35 \\
0.48 ; 0.88\end{array}$ & $\begin{array}{l}0.53 ; 0.46 \\
0.48 ; 0.42 \\
0.55 ; 0.76\end{array}$ & $\begin{array}{l}0.50 ; 0.42 ; \\
0.40 ; 0.40 ; \\
0.44 ; 0.70\end{array}$ & $1^{\text {st }}$ stage \\
\hline
\end{tabular}


Table A.10. Several properties that were calculated based on the extracted coefficients for passive films formed on Alloy 690 under OCP in solution containing 2000 ppm B + 2 ppm Li, and 400 ppb dissolved oxygen at different temperatures.

\begin{tabular}{|c|c|c|c|c|}
\hline Parameters & $150{ }^{0} \mathrm{C}$ & $200{ }^{\circ} \mathrm{C}$ & $250{ }^{\circ} \mathrm{C}$ & $300{ }^{\circ} \mathrm{C}$ \\
\hline$I_{a}\left(\mathrm{~A} \cdot \mathrm{cm}^{-2}\right)$ & $5.73 \times 10^{-7}$ & $5.07 \times 10^{-7}$ & $1.38 \times 10^{-6}$ & $2.52 \times 10^{-6}$ \\
\hline$I_{c}\left(\mathrm{~A} \cdot \mathrm{cm}^{-2}\right)$ & $-5.53 \times 10^{-7}$ & $-5.26 \times 10^{-7}$ & $-1.31 \times 10^{-6}$ & $-2.21 \times 10^{-6}$ \\
\hline$Z_{R_{C}}\left(\Omega \cdot \mathrm{cm}^{2}\right)$ & $1.1 \times 10^{5}$ & $9.7 \times 10^{4}$ & $3.4 \times 10^{4}$ & $2.2 \times 10^{4}$ \\
\hline$L_{S S}(\mathrm{~nm})$ & 1.3 & 2.7 & 3.4 & 5.1 \\
\hline$k_{2}^{0}\left(\mathrm{~mol} \cdot \mathrm{cm}^{-2} \cdot \mathrm{s}^{-1}\right)$ & $3.7 \times 10^{-9}$ & $1.2 \times 10^{-6}$ & $9.6 \times 10^{-5}$ & $7.4 \times 10^{-4}$ \\
\hline$k_{3}^{0}\left(\mathrm{~mol} \cdot \mathrm{cm}^{-2} \cdot \mathrm{s}^{-1}\right)$ & $5.3 \times 10^{-8}$ & $7.9 \times 10^{-7}$ & $1.2 \times 10^{-6}$ & $2.2 \times 10^{-6}$ \\
\hline$k_{5}^{0}\left(\mathrm{~cm} \cdot \mathrm{s}^{-1}\right)$ & $5.3 \times 10^{-7}$ & $1.5 \times 10^{-6}$ & $1.5 \times 10^{-6}$ & $7.0 \times 10^{-6}$ \\
\hline$k_{6}^{0}\left(\mathrm{~cm} \cdot \mathrm{s}^{-1}\right)$ & $2.5 \times 10^{-6}$ & $4.6 \times 10^{-6}$ & $5.8 \times 10^{-6}$ & $2.7 \times 10^{-5}$ \\
\hline$k_{7}^{0}\left(\mathrm{~mol} \cdot \mathrm{cm}^{-2} \cdot \mathrm{s}^{-1}\right)$ & $4.0 \times 10^{-9}$ & $4.5 \times 10^{-9}$ & $4.7 \times 10^{-9}$ & $1.0 \times 10^{-7}$ \\
\hline$k_{2}\left(\mathrm{~mol} \cdot \mathrm{cm}^{-2} \cdot \mathrm{s}^{-1}\right)$ & $9.9 \times 10^{-13}$ & $1.2 \times 10^{-12}$ & $3.2 \times 10^{-12}$ & $4.4 \times 10^{-12}$ \\
\hline$k_{3}\left(\mathrm{~mol} \cdot \mathrm{cm}^{-2} \cdot \mathrm{s}^{-1}\right)$ & $9.9 \times 10^{-13}$ & $5.8 \times 10^{-13}$ & $1.6 \times 10^{-12}$ & $4.3 \times 10^{-12}$ \\
\hline$k_{5}\left(\mathrm{~cm} \cdot \mathrm{s}^{-1}\right)$ & $2.8 \times 10^{-9}$ & $2.1 \times 10^{-9}$ & $2.5 \times 10^{-9}$ & $1.1 \times 10^{-9}$ \\
\hline$k_{6}\left(\mathrm{~cm} \cdot \mathrm{s}^{-1}\right)$ & $1.2 \times 10^{-8}$ & $7.1 \times 10^{-9}$ & $2.7 \times 10^{-8}$ & $7.5 \times 10^{-8}$ \\
\hline$k_{7}\left(\mathrm{~mol} \cdot \mathrm{cm}^{-2} \cdot \mathrm{s}^{-1}\right)$ & $4.0 \times 10^{-9}$ & $4.5 \times 10^{-9}$ & $4.7 \times 10^{-9}$ & $1.0 \times 10^{-7}$ \\
\hline$C_{i}^{0}=k_{2} / k_{5}\left(\mathrm{~mol} \cdot \mathrm{cm}^{-3}\right)$ & $3.6 \times 10^{-4}$ & $5.5 \times 10^{-4}$ & $1.3 \times 10^{-3}$ & $4.2 \times 10^{-3}$ \\
\hline$C_{V_{\ddot{o}}}^{0}=\chi k_{3} / 2 k_{6}\left(\mathrm{~mol} \cdot \mathrm{cm}^{-3}\right)$ & $1.2 \times 10^{-4}$ & $1.2 \times 10^{-4}$ & $8.9 \times 10^{-5}$ & $8.6 \times 10^{-5}$ \\
\hline$C_{i}^{0} /\left(\frac{2}{\Omega}\right)$ & $0.3 \%$ & $0.4 \%$ & $0.9 \%$ & $3.0 \%$ \\
\hline$C_{V_{\ddot{o}}}^{0} /\left(\frac{3}{\Omega}\right)$ & $0.06 \%$ & $0.06 \%$ & $0.05 \%$ & $0.05 \%$ \\
\hline$D_{i}\left(\mathrm{~cm}^{2} \cdot \mathrm{s}^{-1}\right)$ & $1.4 \times 10^{-13}$ & $5.7 \times 10^{-16}$ & $4.9 \times 10^{-13}$ & $2.5 \times 10^{-13}$ \\
\hline$R_{g} / R_{d}$ & 1.0 & 6.7 & 6.7 & 1.0 \\
\hline
\end{tabular}


Table A.11. Several properties that were calculated based on the extracted coefficients for passive films formed on Alloy 690 under OCP in solution containing $200 \mathrm{ppm} \mathrm{B}+2 \mathrm{ppm} \mathrm{Li}$, and $400 \mathrm{ppb}$ dissolved oxygen at different temperatures.

\begin{tabular}{lllll}
\hline Parameters & $150{ }^{0} \mathrm{C}$ & $200{ }^{0} \mathrm{C}$ & $250{ }^{0} \mathrm{C}$ & $300{ }^{0} \mathrm{C}$ \\
\hline$I_{a}\left(\mathrm{~A} \cdot \mathrm{cm}^{-2}\right)$ & $3.76 \times 10^{-7}$ & $4.16 \times 10^{-7}$ & $9.61 \times 10^{-7}$ & $2.02 \times 10^{-6}$ \\
\hline$I_{c}\left(\mathrm{~A} \cdot \mathrm{cm}^{-2}\right)$ & $-3.77 \times 10^{-7}$ & $-4.94 \times 10^{-7}$ & $-9.25 \times 10^{-7}$ & $-2.20 \times 10^{-6}$ \\
\hline$Z_{R_{C}}\left(\Omega \cdot \mathrm{cm}^{2}\right)$ & $1.7 \times 10^{5}$ & $9.9 \times 10^{4}$ & $4.8 \times 10^{4}$ & $2.2 \times 10^{4}$ \\
\hline$L_{s s}(\mathrm{~nm})$ & 1.3 & 2.6 & 3.8 & 4.7 \\
\hline$k_{2}^{0}\left(\mathrm{~mol} \cdot \mathrm{cm}^{-2} \cdot \mathrm{s}^{-1}\right)$ & $3.0 \times 10^{-8}$ & $8.4 \times 10^{-7}$ & $7.7 \times 10^{-4}$ & $2.0 \times 10^{-3}$ \\
\hline$k_{3}^{0}\left(\mathrm{~mol} \cdot \mathrm{cm}^{-2} \cdot \mathrm{s}^{-1}\right)$ & $4.5 \times 10^{-7}$ & $7.6 \times 10^{-7}$ & $3.5 \times 10^{-6}$ & $4.8 \times 10^{-6}$ \\
\hline$k_{5}^{0}\left(\mathrm{~cm} \cdot \mathrm{s}^{-1}\right)$ & $6.4 \times 10^{-7}$ & $1.2 \times 10^{-6}$ & $4.2 \times 10^{-6}$ & $1.3 \times 10^{-5}$ \\
\hline$k_{6}^{0}\left(\mathrm{~cm} \cdot \mathrm{s}^{-1}\right)$ & $1.6 \times 10^{-6}$ & $1.9 \times 10^{-6}$ & $7.0 \times 10^{-6}$ & $8.9 \times 10^{-5}$ \\
\hline$k_{7}^{0}\left(\mathrm{~mol} \cdot \mathrm{cm}^{-2} \cdot \mathrm{s}^{-1}\right)$ & $1.1 \times 10^{-9}$ & $3.6 \times 10^{-9}$ & $9.5 \times 10^{-9}$ & $6.9 \times 10^{-8}$ \\
\hline$k_{2}\left(\mathrm{~mol} \cdot \mathrm{cm}^{-2} \cdot \mathrm{s}^{-1}\right)$ & $8.5 \times 10^{-13}$ & $9.6 \times 10^{-13}$ & $2.2 \times 10^{-12}$ & $3.9 \times 10^{-12}$ \\
\hline$k_{3}\left(\mathrm{~mol} \cdot \mathrm{cm}^{-2} \cdot \mathrm{s}^{-1}\right)$ & $4.5 \times 10^{-13}$ & $4.8 \times 10^{-13}$ & $1.1 \times 10^{-12}$ & $3.0 \times 10^{-12}$ \\
\hline$k_{5}\left(\mathrm{~cm} \cdot \mathrm{s}^{-1}\right)$ & $2.6 \times 10^{-9}$ & $3.3 \times 10^{-9}$ & $2.6 \times 10^{-9}$ & $1.1 \times 10^{-9}$ \\
\hline$k_{6}\left(\mathrm{~cm} \cdot \mathrm{s}^{-1}\right)$ & $1.2 \times 10^{-8}$ & $1.2 \times 10^{-8}$ & $1.4 \times 10^{-8}$ & $1.4 \times 10^{-7}$ \\
\hline$k_{7}\left(\mathrm{~mol} \cdot \mathrm{cm}^{-2} \cdot \mathrm{s}^{-1}\right)$ & $1.1 \times 10^{-9}$ & $3.6 \times 10^{-9}$ & $9.5 \times 10^{-9}$ & $6.9 \times 10^{-8}$ \\
\hline$C_{i}^{0}=k_{2} / k_{5}(\mathrm{~mol} \cdot \mathrm{cm}-3)$ & $3.3 \times 10^{-4}$ & $2.9 \times 10^{-4}$ & $8.6 \times 10^{-4}$ & $3.6 \times 10^{-3}$ \\
\hline$C_{V_{\tilde{o}}^{0}}^{0}=\chi k_{3} / 2 k_{6}\left(\mathrm{~mol} \cdot \mathrm{cm}^{-3}\right)$ & $5.6 \times 10^{-5}$ & $6.0 \times 10^{-5}$ & $1.2 \times 10^{-4}$ & $3.2 \times 10^{-5}$ \\
\hline$C_{i}^{0} /\left(\frac{2}{\Omega}\right)^{\mathrm{a}}$ & $0.2 \%$ & $0.2 \%$ & $0.6 \%$ & $2.7 \%$ \\
\hline$C_{V_{\mathrm{o}}^{0}}^{0} /\left(\frac{3}{\Omega}\right)^{\mathrm{b}}$ & $0.03 \%$ & $0.03 \%$ & $0.06 \%$ & $0.02 \%$ \\
\hline$R_{g} / R_{d}^{\mathrm{c}}$ & 6.9 & 6.7 & 6.7 & 8.8 \\
\hline$D_{i}\left(\mathrm{~cm} \cdot \mathrm{s}^{-1}\right)$ & $1.1 \times 10^{-13}$ & $5.5 \times 10^{-16}$ & $2.0 \times 10^{-14}$ & $3.7 \times 10^{-13}$ \\
\hline
\end{tabular}


Table A.12. Several properties that were calculated based on the extracted coefficients for passive films formed on Alloy 690 under OCP in solution containing 0 ppm B +2 ppm Li, and 400 ppb dissolved oxygen at different temperatures.

\begin{tabular}{lllll}
\hline Parameters & $150{ }^{0} \mathrm{C}$ & $200{ }^{0} \mathrm{C}$ & $250{ }^{0} \mathrm{C}$ & $300{ }^{0} \mathrm{C}$ \\
\hline$I_{a}\left(\mathrm{~A} \cdot \mathrm{cm}^{-2}\right)$ & $1.43 \times 10^{-7}$ & $4.42 \times 10^{-7}$ & $7.36 \times 10^{-7}$ & $3.26 \times 10^{-6}$ \\
\hline$I_{c}\left(\mathrm{~A} \cdot \mathrm{cm}^{-2}\right)$ & $-3.98 \times 10^{-7}$ & $-4.77 \times 10^{-7}$ & $-7.76 \times 10^{-7}$ & $-2.52 \times 10^{-6}$ \\
\hline$Z_{R_{C}}\left(\Omega \cdot \mathrm{cm}^{2}\right)$ & $1.6 \times 10^{6}$ & $9.8 \times 10^{4}$ & $5.5 \times 10^{4}$ & $1.9 \times 10^{4}$ \\
\hline$L_{s s}(\mathrm{~nm})$ & 1.3 & 2.5 & 3.7 & 4.8 \\
\hline$k_{2}^{0}\left(\mathrm{~mol} \cdot \mathrm{cm}^{-2} \cdot \mathrm{s}^{-1}\right)$ & $7.7 \times 10^{-9}$ & $8.4 \times 10^{-7}$ & $2.3 \times 10^{-4}$ & $3.8 \times 10^{-3}$ \\
\hline$k_{3}^{0}\left(\mathrm{~mol} \cdot \mathrm{cm}^{-2} \cdot \mathrm{s}^{-1}\right)$ & $1.5 \times 10^{-7}$ & $7.6 \times 10^{-7}$ & $3.0 \times 10^{-6}$ & $2.7 \times 10^{-6}$ \\
\hline$k_{5}^{0}\left(\mathrm{~cm} \cdot \mathrm{s}^{-1}\right)$ & $1.2 \times 10^{-6}$ & $3.0 \times 10^{-6}$ & $1.0 \times 10^{-5}$ & $3.8 \times 10^{-4}$ \\
\hline$k_{6}^{0}\left(\mathrm{~cm} \cdot \mathrm{s}^{-1}\right)$ & $1.8 \times 10^{-6}$ & $4.2 \times 10^{-6}$ & $1.3 \times 10^{-5}$ & $3.0 \times 10^{-5}$ \\
\hline$k_{7}^{0}\left(\mathrm{~mol} \cdot \mathrm{cm}^{-2} \cdot \mathrm{s}^{-1}\right)$ & $2.1 \times 10^{-9}$ & $3.6 \times 10^{-9}$ & $3.3 \times 10^{-8}$ & $1.0 \times 10^{-7}$ \\
\hline$k_{2}\left(\mathrm{~mol} \cdot \mathrm{cm}^{-2} \cdot \mathrm{s}^{-1}\right)$ & $2.8 \times 10^{-13}$ & $1.0 \times 10^{-12}$ & $1.6 \times 10^{-12}$ & $6.8 \times 10^{-12}$ \\
\hline$k_{3}\left(\mathrm{~mol} \cdot \mathrm{cm}^{-2} \cdot \mathrm{s}^{-1}\right)$ & $2.1 \times 10^{-13}$ & $5.1 \times 10^{-13}$ & $9.1 \times 10^{-13}$ & $4.5 \times 10^{-12}$ \\
\hline$k_{5}\left(\mathrm{~cm} \cdot \mathrm{s}^{-1}\right)$ & $1.5 \times 10^{-9}$ & $4.7 \times 10^{-9}$ & $2.0 \times 10^{-9}$ & $3.2 \times 10^{-9}$ \\
\hline$k_{6}\left(\mathrm{~cm} \cdot \mathrm{s}^{-1}\right)$ & $4.9 \times 10^{-9}$ & $1.6 \times 10^{-8}$ & $2.6 \times 10^{-8}$ & $9.3 \times 10^{-8}$ \\
\hline$k_{7}\left(\mathrm{~mol} \cdot \mathrm{cm}^{-2} \cdot \mathrm{s}^{-1}\right)$ & $2.1 \times 10^{-9}$ & $3.6 \times 10^{-9}$ & $3.3 \times 10^{-8}$ & $1.0 \times 10^{-7}$ \\
\hline$C_{i}^{0}=k_{2} / k_{5}(\mathrm{~mol} \cdot \mathrm{cm}-3)$ & $1.9 \times 10^{-4}$ & $2.2 \times 10^{-4}$ & $8.0 \times 10^{-4}$ & $2.1 \times 10^{-3}$ \\
\hline$C_{V_{\tilde{o}}^{0}}^{0}=\chi k_{3} / 2 k_{6}\left(\mathrm{~mol} \cdot \mathrm{cm}^{-3}\right)$ & $6.4 \times 10^{-5}$ & $4.8 \times 10^{-5}$ & $5.3 \times 10^{-5}$ & $7.3 \times 10^{-5}$ \\
\hline$C_{i}^{0} /\left(\frac{2}{\Omega}\right)^{\mathrm{a}}$ & $0.1 \%$ & $0.2 \%$ & $0.6 \%$ & $1.6 \%$ \\
\hline$C_{V_{\mathrm{o}}^{0}}^{0} /\left(\frac{3}{\Omega}\right)^{\mathrm{b}}$ & $0.03 \%$ & $0.03 \%$ & $0.03 \%$ & $0.03 \%$ \\
\hline$R_{g} / R_{d}^{\mathrm{c}}$ & 0.9 & 0.7 & 0.7 & 0.8 \\
\hline$D_{i}\left(\mathrm{~cm} \cdot \mathrm{s}^{-1}\right)$ & $7.2 \times 10^{-18}$ & $5.9 \times 10^{-16}$ & $2.9 \times 10^{-16}$ & $3.3 \times 10^{-13}$ \\
\hline
\end{tabular}


Table A.13. Several properties that were calculated based on the extracted coefficients for passive films formed on Type 304L SS under OCP in solution containing $2000 \mathrm{ppm} \mathrm{B}+2 \mathrm{ppm} \mathrm{Li}$, and $400 \mathrm{ppb}$ dissolved oxygen at different temperatures.

\begin{tabular}{llll}
\hline Parameters & $150{ }^{0} \mathrm{C}$ & $200{ }^{0} \mathrm{C}$ & $250{ }^{0} \mathrm{C}$ \\
\hline$I_{a}\left(\mathrm{~A} \cdot \mathrm{cm}^{-2}\right)$ & $8.69 \times 10^{-7}$ & $8.65 \times 10^{-7}$ & $1.86 \times 10^{-6}$ \\
\hline$I_{c}\left(\mathrm{~A} \cdot \mathrm{cm}^{-2}\right)$ & $-6.81 \times 10^{-7}$ & $-6.90 \times 10^{-7}$ & $-1.71 \times 10^{-6}$ \\
\hline$Z_{R_{C}}\left(\Omega \cdot \mathrm{cm}^{2}\right)$ & $8.5 \times 10^{4}$ & $7.3 \times 10^{4}$ & $2.8 \times 10^{4}$ \\
\hline$L_{s S}(\mathrm{~nm})$ & 1.4 & 2.0 & 3.0 \\
\hline$k_{2}^{0}\left(\mathrm{~mol} \cdot \mathrm{cm}^{-2} \cdot \mathrm{s}^{-1}\right)$ & $1.2 \times 10^{-8}$ & $1.6 \times 10^{-8}$ & $3.3 \times 10^{-6}$ \\
\hline$k_{3}^{0}\left(\mathrm{~mol} \cdot \mathrm{cm}^{-2} \cdot \mathrm{s}^{-1}\right)$ & $4.8 \times 10^{-8}$ & $1.2 \times 10^{-7}$ & $5.4 \times 10^{-7}$ \\
\hline$k_{5}^{0}\left(\mathrm{~cm} \cdot \mathrm{s}^{-1}\right)$ & $6.8 \times 10^{-8}$ & $7.8 \times 10^{-8}$ & $8.3 \times 10^{-7}$ \\
\hline$k_{6}^{0}\left(\mathrm{~cm} \cdot \mathrm{s}^{-1}\right)$ & $3.8 \times 10^{-7}$ & $4.0 \times 10^{-7}$ & $7.2 \times 10^{-6}$ \\
\hline$k_{7}^{0}\left(\mathrm{~mol} \cdot \mathrm{cm}^{-2} \cdot \mathrm{s}^{-1}\right)$ & $7.2 \times 10^{-9}$ & $1.0 \times 10^{-8}$ & $1.1 \times 10^{-8}$ \\
\hline$k_{2}\left(\mathrm{~mol} \cdot \mathrm{cm}^{-2} \cdot \mathrm{s}^{-1}\right)$ & $2.0 \times 10^{-12}$ & $1.5 \times 10^{-12}$ & $3.2 \times 10^{-12}$ \\
\hline$k_{3}\left(\mathrm{~mol} \cdot \mathrm{cm}^{-2} \cdot \mathrm{s}^{-1}\right)$ & $1.0 \times 10^{-12}$ & $1.5 \times 10^{-12}$ & $3.2 \times 10^{-12}$ \\
\hline$k_{5}\left(\mathrm{~cm} \cdot \mathrm{s}^{-1}\right)$ & $2.4 \times 10^{-9}$ & $1.5 \times 10^{-9}$ & $8.8 \times 10^{-10}$ \\
\hline$k_{6}\left(\mathrm{~cm} \cdot \mathrm{s}^{-1}\right)$ & $2.3 \times 10^{-8}$ & $1.4 \times 10^{-8}$ & $2.4 \times 10^{-8}$ \\
\hline$k_{7}\left(\mathrm{~mol} \cdot \mathrm{cm}^{-2} \cdot \mathrm{s}^{-1}\right)$ & $7.2 \times 10^{-9}$ & $1.0 \times 10^{-8}$ & $1.1 \times 10^{-8}$ \\
\hline$C_{i}^{0}=k_{2} / k_{5}\left(\mathrm{~mol} \cdot \mathrm{cm}^{-3}\right)$ & $8.3 \times 10^{-4}$ & $9.9 \times 10^{-4}$ & $3.7 \times 10^{-3}$ \\
\hline$C_{V_{0}}^{0}=\chi k_{3} / 2 k_{6}\left(\mathrm{~mol} \cdot \mathrm{cm}^{-3}\right)$ & $6.5 \times 10^{-5}$ & $1.6 \times 10^{-4}$ & $2.0 \times 10^{-4}$ \\
\hline$C_{i}^{0} /\left(\frac{2}{\Omega}\right)$ & $0.6 \%$ & $0.7 \%$ & $2.7 \%$ \\
\hline$C_{V_{0}^{0}}^{0} /\left(\frac{3}{\Omega}\right)$ & $0.03 \%$ & $0.08 \%$ & $0.09 \%$ \\
\hline$D_{i}\left(\mathrm{~cm} \cdot \mathrm{s}^{-1}\right)$ & $2.5 \times 10^{-15}$ & $4.0 \times 10^{-17}$ & $2.5 \times 10^{-14}$ \\
\hline$R_{g} / R_{d}$ & 1.0 & 1.0 \\
\hline & & & \\
\hline
\end{tabular}


Table A.14. Several properties that were calculated based on the extracted coefficients for passive films formed on Type 304L SS under OCP in solution containing $200 \mathrm{ppm} \mathrm{B}+2 \mathrm{ppm} \mathrm{Li}$, and 400 ppb dissolved oxygen at different temperatures.

\begin{tabular}{llll}
\hline Parameters & $150{ }^{0} \mathrm{C}$ & $200{ }^{0} \mathrm{C}$ & $250{ }^{0} \mathrm{C}$ \\
\hline$I_{a}\left(\mathrm{~A} \cdot \mathrm{cm}^{-2}\right)$ & $3.78 \times 10^{-7}$ & $7.76 \times 10^{-7}$ & $1.53 \times 10^{-6}$ \\
\hline$I_{c}\left(\mathrm{~A} \cdot \mathrm{cm}^{-2}\right)$ & $-4.27 \times 10^{-7}$ & $-7.58 \times 10^{-7}$ & $-1.70 \times 10^{-6}$ \\
\hline$Z_{R_{C}}\left(\Omega \cdot \mathrm{cm}^{2}\right)$ & $1.5 \times 10^{5}$ & $6.1 \times 10^{4}$ & $2.5 \times 10^{4}$ \\
\hline$L_{s S}(\mathrm{~nm})$ & 1.6 & 2.9 & 4.7 \\
\hline$k_{2}^{0}\left(\mathrm{~mol} \cdot \mathrm{cm}^{-2} \cdot \mathrm{s}^{-1}\right)$ & $1.1 \times 10^{-7}$ & $1.1 \times 10^{-7}$ & $2.0 \times 10^{-5}$ \\
\hline$k_{3}^{0}\left(\mathrm{~mol} \cdot \mathrm{cm}^{-2} \cdot \mathrm{s}^{-1}\right)$ & $8.2 \times 10^{-8}$ & $8.2 \times 10^{-8}$ & $4.6 \times 10^{-7}$ \\
\hline$k_{5}^{0}\left(\mathrm{~cm} \cdot \mathrm{s}^{-1}\right)$ & $5.5 \times 10^{-7}$ & $5.5 \times 10^{-7}$ & $5.3 \times 10^{-5}$ \\
\hline$k_{6}^{0}\left(\mathrm{~cm} \cdot \mathrm{s}^{-1}\right)$ & $2.2 \times 10^{-6}$ & $2.2 \times 10^{-6}$ & $2.1 \times 10^{-4}$ \\
\hline$k_{7}^{0}\left(\mathrm{~mol} \cdot \mathrm{cm}^{-2} \cdot \mathrm{s}^{-1}\right)$ & $5.5 \times 10^{-9}$ & $5.5 \times 10^{-9}$ & $1.1 \times 10^{-7}$ \\
\hline$k_{2}\left(\mathrm{~mol} \cdot \mathrm{cm}^{-2} \cdot \mathrm{s}^{-1}\right)$ & $8.7 \times 10^{-13}$ & $1.3 \times 10^{-12}$ & $3.5 \times 10^{-12}$ \\
\hline$k_{3}\left(\mathrm{~mol} \cdot \mathrm{cm}^{-2} \cdot \mathrm{s}^{-1}\right)$ & $4.4 \times 10^{-13}$ & $1.3 \times 10^{-12}$ & $1.8 \times 10^{-12}$ \\
\hline$k_{5}\left(\mathrm{~cm} \cdot \mathrm{s}^{-1}\right)$ & $1.1 \times 10^{-9}$ & $1.5 \times 10^{-9}$ & $1.1 \times 10^{-9}$ \\
\hline$k_{6}\left(\mathrm{~cm} \cdot \mathrm{s}^{-1}\right)$ & $8.7 \times 10^{-9}$ & $1.6 \times 10^{-8}$ & $2.7 \times 10^{-8}$ \\
\hline$k_{7}\left(\mathrm{~mol} \cdot \mathrm{cm}^{-2} \cdot \mathrm{s}^{-1}\right)$ & $5.5 \times 10^{-9}$ & $1.0 \times 10^{-8}$ & $1.1 \times 10^{-7}$ \\
\hline$C_{i}^{0}=k_{2} / k_{5}\left(\mathrm{~mol} \cdot \mathrm{cm}^{-3}\right)$ & $7.6 \times 10^{-4}$ & $9.1 \times 10^{-4}$ & $3.1 \times 10^{-3}$ \\
\hline$C_{V_{0}}^{0}=\chi k_{3} / 2 k_{6}\left(\mathrm{~mol} \cdot \mathrm{cm}^{-3}\right)$ & $7.6 \times 10^{-5}$ & $1.2 \times 10^{-4}$ & $1.0 \times 10^{-3}$ \\
\hline$C_{i}^{0} /\left(\frac{2}{\Omega}\right)$ & $0.6 \%$ & $0.7 \%$ & $2.3 \%$ \\
\hline$C_{V_{0}^{0}}^{0} /\left(\frac{3}{\Omega}\right)$ & $0.03 \%$ & $0.06 \%$ & $0.05 \%$ \\
\hline$D_{i}\left(\mathrm{~cm} \cdot \mathrm{s}^{-1}\right)$ & 0.7 & $1.5 \times 10^{-13}$ & $2.4 \times 10^{-14}$ \\
\hline$R_{g} / R_{d}$ & 1.0 & 0.7 \\
\hline
\end{tabular}


Table A.15. Several properties that were calculated based on the extracted coefficients for passive films formed on Type 304L SS under OCP in solution containing $0 \mathrm{ppm} \mathrm{B}+2 \mathrm{ppm} \mathrm{Li}$, and 400 ppb dissolved oxygen at different temperatures.

\begin{tabular}{llll}
\hline Parameters & $150{ }^{0} \mathrm{C}$ & $200{ }^{0} \mathrm{C}$ & $250{ }^{0} \mathrm{C}$ \\
\hline$I_{a}\left(\mathrm{~A} \cdot \mathrm{cm}^{-2}\right)$ & $2.98 \times 10^{-7}$ & $9.64 \times 10^{-7}$ & $1.25 \times 10^{-6}$ \\
\hline$I_{c}\left(\mathrm{~A} \cdot \mathrm{cm}^{-2}\right)$ & $-4.06 \times 10^{-7}$ & $-8.78 \times 10^{-7}$ & $-1.28 \times 10^{-6}$ \\
\hline$Z_{R_{C}}\left(\Omega \cdot \mathrm{cm}^{2}\right)$ & $1.3 \times 10^{5}$ & $5.0 \times 10^{4}$ & $3.1 \times 10^{4}$ \\
\hline$L_{s S}(\mathrm{~nm})$ & 1.5 & 1.9 & 3.8 \\
\hline$k_{2}^{0}\left(\mathrm{~mol} \cdot \mathrm{cm}^{-2} \cdot \mathrm{s}^{-1}\right)$ & $4.2 \times 10^{-7}$ & $8.4 \times 10^{-7}$ & $5.9 \times 10^{-5}$ \\
\hline$k_{3}^{0}\left(\mathrm{~mol} \cdot \mathrm{cm}^{-2} \cdot \mathrm{s}^{-1}\right)$ & $4.4 \times 10^{-7}$ & $1.9 \times 10^{-7}$ & $3.0 \times 10^{-5}$ \\
\hline$k_{5}^{0}\left(\mathrm{~cm} \cdot \mathrm{s}^{-1}\right)$ & $1.8 \times 10^{-7}$ & $3.0 \times 10^{-7}$ & $3.4 \times 10^{-6}$ \\
\hline$k_{6}^{0}\left(\mathrm{~cm} \cdot \mathrm{s}^{-1}\right)$ & $1.1 \times 10^{-6}$ & $1.4 \times 10^{-6}$ & $8.8 \times 10^{-6}$ \\
\hline$k_{7}^{0}\left(\mathrm{~mol} \cdot \mathrm{cm}^{-2} \cdot \mathrm{s}^{-1}\right)$ & $4.6 \times 10^{-9}$ & $7.8 \times 10^{-9}$ & $9.3 \times 10^{-9}$ \\
\hline$k_{2}\left(\mathrm{~mol} \cdot \mathrm{cm}^{-2} \cdot \mathrm{s}^{-1}\right)$ & $6.9 \times 10^{-13}$ & $2.2 \times 10^{-12}$ & $2.9 \times 10^{-12}$ \\
\hline$k_{3}\left(\mathrm{~mol} \cdot \mathrm{cm}^{-2} \cdot \mathrm{s}^{-1}\right)$ & $3.5 \times 10^{-13}$ & $1.1 \times 10^{-12}$ & $1.5 \times 10^{-12}$ \\
\hline$k_{5}\left(\mathrm{~cm} \cdot \mathrm{s}^{-1}\right)$ & $9.9 \times 10^{-10}$ & $2.5 \times 10^{-9}$ & $1.2 \times 10^{-9}$ \\
\hline$k_{6}\left(\mathrm{~cm} \cdot \mathrm{s}^{-1}\right)$ & $9.2 \times 10^{-9}$ & $2.3 \times 10^{-8}$ & $2.6 \times 10^{-8}$ \\
\hline$k_{7}\left(\mathrm{~mol} \cdot \mathrm{cm}^{-2} \cdot \mathrm{s}^{-1}\right)$ & $4.6 \times 10^{-9}$ & $7.8 \times 10^{-9}$ & $9.3 \times 10^{-9}$ \\
\hline$C_{i}^{0}=k_{2} / k_{5}\left(\mathrm{~mol} \cdot \mathrm{cm}^{-3}\right)$ & $6.9 \times 10^{-4}$ & $9.0 \times 10^{-4}$ & $2.5 \times 10^{-3}$ \\
\hline$C_{V_{0}}^{0}=\chi k_{3} / 2 k_{6}\left(\mathrm{~mol} \cdot \mathrm{cm}^{-3}\right)$ & $5.7 \times 10^{-5}$ & $7.2 \times 10^{-5}$ & $8.7 \times 10^{-5}$ \\
\hline$C_{i}^{0} /\left(\frac{2}{\Omega}\right)$ & $0.5 \%$ & $0.7 \%$ & $1.8 \%$ \\
\hline$C_{V_{0}^{0}}^{0} /\left(\frac{3}{\Omega}\right)$ & $0.03 \%$ & $0.03 \%$ & $0.05 \%$ \\
\hline$D_{i}\left(\mathrm{~cm} \cdot \mathrm{s}^{-1}\right)$ & $3.4 \times 10^{-18}$ & $3.4 \times 10^{-15}$ & $6.9 \times 10^{-14}$ \\
\hline$R_{g} / R_{d}$ & 0.7 & 0.7 & 0.7 \\
\hline
\end{tabular}


Table A.16. Several properties that were calculated based on the extracted coefficients for passive films formed on Type 316L SS under OCP in solution containing $2000 \mathrm{ppm} \mathrm{B}+2 \mathrm{ppm} \mathrm{Li}$, and $400 \mathrm{ppb}$ dissolved oxygen at different temperatures.

\begin{tabular}{llll}
\hline Parameters & $150{ }^{0} \mathrm{C}$ & $200^{0} \mathrm{C}$ & $250{ }^{0} \mathrm{C}$ \\
\hline$I_{a}\left(\mathrm{~A} \cdot \mathrm{cm}^{-2}\right)$ & $6.86 \times 10^{-7}$ & $1.05 \times 10^{-6}$ & $1.85 \times 10^{-6}$ \\
\hline$I_{c}\left(\mathrm{~A} \cdot \mathrm{cm}^{-2}\right)$ & $-8.93 \times 10^{-7}$ & $-1.10 \times 10^{-6}$ & $-1.68 \times 10^{-6}$ \\
\hline$Z_{R_{C}}\left(\Omega \cdot \mathrm{cm}^{2}\right)$ & $5.8 \times 10^{4}$ & $4.4 \times 10^{4}$ & $2.9 \times 10^{4}$ \\
\hline$L_{S s}(\mathrm{~nm})$ & 1.2 & 1.2 & 3.1 \\
\hline$k_{2}^{0}\left(\mathrm{~mol} \cdot \mathrm{cm}^{-2} \cdot \mathrm{s}^{-1}\right)$ & $4.4 \times 10^{-9}$ & $4.3 \times 10^{-9}$ & $1.8 \times 10^{-6}$ \\
\hline$k_{3}^{0}\left(\mathrm{~mol} \cdot \mathrm{cm}^{-2} \cdot \mathrm{s}^{-1}\right)$ & $4.3 \times 10^{-8}$ & $3.8 \times 10^{-8}$ & $6.2 \times 10^{-7}$ \\
\hline$k_{5}^{0}\left(\mathrm{~cm} \cdot \mathrm{s}^{-1}\right)$ & $1.9 \times 10^{-8}$ & $3.7 \times 10^{-8}$ & $1.6 \times 10^{-6}$ \\
\hline$k_{6}^{0}\left(\mathrm{~cm} \cdot \mathrm{s}^{-1}\right)$ & $2.5 \times 10^{-7}$ & $3.7 \times 10^{-7}$ & $8.6 \times 10^{-6}$ \\
\hline$k_{7}^{0}\left(\mathrm{~mol} \cdot \mathrm{cm}^{-2} \cdot \mathrm{s}^{-1}\right)$ & $7.2 \times 10^{-9}$ & $1.5 \times 10^{-8}$ & $1.9 \times 10^{-8}$ \\
\hline$k_{2}\left(\mathrm{~mol} \cdot \mathrm{cm}^{-2} \cdot \mathrm{s}^{-1}\right)$ & $1.2 \times 10^{-12}$ & $1.8 \times 10^{-12}$ & $3.7 \times 10^{-12}$ \\
\hline$k_{3}\left(\mathrm{~mol} \cdot \mathrm{cm}^{-2} \cdot \mathrm{s}^{-1}\right)$ & $1.2 \times 10^{-12}$ & $1.8 \times 10^{-12}$ & $2.7 \times 10^{-12}$ \\
\hline$k_{5}\left(\mathrm{~cm} \cdot \mathrm{s}^{-1}\right)$ & $1.5 \times 10^{-9}$ & $2.0 \times 10^{-9}$ & $1.5 \times 10^{-9}$ \\
\hline$k_{6}\left(\mathrm{~cm} \cdot \mathrm{s}^{-1}\right)$ & $2.9 \times 10^{-8}$ & $2.5 \times 10^{-8}$ & $2.5 \times 10^{-8}$ \\
\hline$k_{7}\left(\mathrm{~mol} \cdot \mathrm{cm}^{-2} \cdot \mathrm{s}^{-1}\right)$ & $7.2 \times 10^{-9}$ & $1.5 \times 10^{-8}$ & $1.9 \times 10^{-8}$ \\
\hline$C_{i}^{0}=k_{2} / k_{5}\left(\mathrm{~mol} \cdot \mathrm{cm}^{-3}\right)$ & $8.0 \times 10^{-4}$ & $9.1 \times 10^{-4}$ & $2.5 \times 10^{-3}$ \\
\hline$C_{V_{0}^{0}}^{0}=\chi k_{3} / 2 k_{6}\left(\mathrm{~mol} \cdot \mathrm{cm}^{-3}\right)$ & $6.2 \times 10^{-5}$ & $1.1 \times 10^{-4}$ & $1.6 \times 10^{-4}$ \\
\hline$C_{i}^{0} /\left(\frac{2}{\Omega}\right)$ & $0.6 \%$ & $0.7 \%$ & $1.8 \%$ \\
\hline$C_{V_{\mathrm{o}}^{0}}^{0} /\left(\frac{3}{\Omega}\right)$ & $0.03 \%$ & $0.05 \%$ & $0.08 \%$ \\
\hline$D_{i}\left(\mathrm{~cm} \cdot \mathrm{s}^{2}\right)$ & $1.7 \times 10^{-13}$ & $6.8 \times 10^{-15}$ & $8.7 \times 10^{-14}$ \\
\hline$R_{g} / R_{d}$ & 1.0 & 1.0 & 0.9 \\
\hline & & & \\
\hline
\end{tabular}


Table A.17. Several properties that were calculated based on the extracted coefficients for passive films formed on Type 316L SS under OCP in solution containing $200 \mathrm{ppm} \mathrm{B}+2 \mathrm{ppm} \mathrm{Li}$, and 400 ppb dissolved oxygen at different temperatures.

\begin{tabular}{llll}
\hline Parameters & $150{ }^{0} \mathrm{C}$ & $200{ }^{0} \mathrm{C}$ & $250{ }^{0} \mathrm{C}$ \\
\hline$I_{a}\left(\mathrm{~A} \cdot \mathrm{cm}^{-2}\right)$ & $3.47 \times 10^{-7}$ & $9.02 \times 10^{-7}$ & $1.54 \times 10^{-6}$ \\
\hline$I_{c}\left(\mathrm{~A} \cdot \mathrm{cm}^{-2}\right)$ & $-4.67 \times 10^{-7}$ & $-8.83 \times 10^{-7}$ & $-1.56 \times 10^{-6}$ \\
\hline$Z_{R_{C}}\left(\Omega \cdot \mathrm{cm}^{2}\right)$ & $1.3 \times 10^{5}$ & $5.2 \times 10^{4}$ & $2.6 \times 10^{4}$ \\
\hline$L_{s S}(\mathrm{~nm})$ & 1.6 & 2.7 & 4.4 \\
\hline$k_{2}^{0}\left(\mathrm{~mol} \cdot \mathrm{cm}^{-2} \cdot \mathrm{s}^{-1}\right)$ & $1.8 \times 10^{-8}$ & $2.5 \times 10^{-7}$ & $6.4 \times 10^{-6}$ \\
\hline$k_{3}^{0}\left(\mathrm{~mol} \cdot \mathrm{cm}^{-2} \cdot \mathrm{s}^{-1}\right)$ & $8.2 \times 10^{-8}$ & $1.5 \times 10^{-7}$ & $4.6 \times 10^{-7}$ \\
\hline$k_{5}^{0}\left(\mathrm{~cm} \cdot \mathrm{s}^{-1}\right)$ & $3.8 \times 10^{-7}$ & $1.3 \times 10^{-6}$ & $1.0 \times 10^{-5}$ \\
\hline$k_{6}^{0}\left(\mathrm{~cm} \cdot \mathrm{s}^{-1}\right)$ & $9.2 \times 10^{-6}$ & $3.1 \times 10^{-5}$ & $3.1 \times 10^{-4}$ \\
\hline$k_{7}^{0}\left(\mathrm{~mol} \cdot \mathrm{cm}^{-2} \cdot \mathrm{s}^{-1}\right)$ & $7.2 \times 10^{-9}$ & $6.0 \times 10^{-9}$ & $1.1 \times 10^{-7}$ \\
\hline$k_{2}\left(\mathrm{~mol} \cdot \mathrm{cm}^{-2} \cdot \mathrm{s}^{-1}\right)$ & $6.3 \times 10^{-13}$ & $2.0 \times 10^{-12}$ & $3.6 \times 10^{-12}$ \\
\hline$k_{3}\left(\mathrm{~mol} \cdot \mathrm{cm}^{-2} \cdot \mathrm{s}^{-1}\right)$ & $5.7 \times 10^{-13}$ & $1.1 \times 10^{-12}$ & $1.8 \times 10^{-12}$ \\
\hline$k_{5}\left(\mathrm{~cm} \cdot \mathrm{s}^{-1}\right)$ & $8.3 \times 10^{-10}$ & $2.3 \times 10^{-9}$ & $1.6 \times 10^{-9}$ \\
\hline$k_{6}\left(\mathrm{~cm} \cdot \mathrm{s}^{-1}\right)$ & $3.8 \times 10^{-8}$ & $1.8 \times 10^{-7}$ & $2.0 \times 10^{-7}$ \\
\hline$k_{7}\left(\mathrm{~mol} \cdot \mathrm{cm}^{-2} \cdot \mathrm{s}^{-1}\right)$ & $7.2 \times 10^{-9}$ & $6.0 \times 10^{-9}$ & $1.1 \times 10^{-7}$ \\
\hline$C_{i}^{0}=k_{2} / k_{5}\left(\mathrm{~mol} \cdot \mathrm{cm}^{-3}\right)$ & $7.6 \times 10^{-4}$ & $8.6 \times 10^{-4}$ & $2.2 \times 10^{-3}$ \\
\hline$C_{V_{0}}^{0}=\chi k_{3} / 2 k_{6}\left(\mathrm{~mol} \cdot \mathrm{cm}^{-3}\right)$ & $2.3 \times 10^{-5}$ & $9.2 \times 10^{-6}$ & $1.4 \times 10^{-5}$ \\
\hline$C_{i}^{0} /\left(\frac{2}{\Omega}\right)$ & $0.6 \%$ & $0.6 \%$ & $1.6 \%$ \\
\hline$C_{V_{0}}^{0} /\left(\frac{3}{\Omega}\right)$ & $0.02 \%$ & $0.005 \%$ & $0.006 \%$ \\
\hline$D_{i}\left(\mathrm{~cm} \cdot \mathrm{s}^{-1}\right)$ & $4.9 \times 10^{-14}$ & $2.7 \times 10^{-15}$ & $8.1 \times 10^{-14}$ \\
\hline$R_{g} / R_{d}$ & 0.7 & 0.7 \\
\hline
\end{tabular}


Table A.18. Several properties that were calculated based on the extracted coefficients for passive films formed on Type 316L SS under OCP in solution containing $0 \mathrm{ppm} \mathrm{B}+2 \mathrm{ppm} \mathrm{Li}$, and 400 ppb dissolved oxygen at different temperatures.

\begin{tabular}{llll}
\hline Parameters & $150{ }^{0} \mathrm{C}$ & $200{ }^{0} \mathrm{C}$ & $250{ }^{0} \mathrm{C}$ \\
\hline$I_{a}\left(\mathrm{~A} \cdot \mathrm{cm}^{-2}\right)$ & $3.30 \times 10^{-7}$ & $9.54 \times 10^{-7}$ & $1.64 \times 10^{-6}$ \\
\hline$I_{c}\left(\mathrm{~A} \cdot \mathrm{cm}^{-2}\right)$ & $-3.53 \times 10^{-7}$ & $-8.86 \times 10^{-7}$ & $-1.65 \times 10^{-6}$ \\
\hline$Z_{R_{C}}\left(\Omega \cdot \mathrm{cm}^{2}\right)$ & $1.7 \times 10^{5}$ & $4.9 \times 10^{4}$ & $2.6 \times 10^{4}$ \\
\hline$L_{s s}(\mathrm{~nm})$ & 1.2 & 2.3 & 2.7 \\
\hline$k_{2}^{0}\left(\mathrm{~mol} \cdot \mathrm{cm}^{-2} \cdot \mathrm{s}^{-1}\right)$ & $2.8 \times 10^{-8}$ & $6.9 \times 10^{-7}$ & $9.7 \times 10^{-6}$ \\
\hline$k_{3}^{0}\left(\mathrm{~mol} \cdot \mathrm{cm}^{-2} \cdot \mathrm{s}^{-1}\right)$ & $1.3 \times 10^{-7}$ & $1.2 \times 10^{-6}$ & $3.8 \times 10^{-6}$ \\
\hline$k_{5}^{0}\left(\mathrm{~cm} \cdot \mathrm{s}^{-1}\right)$ & $2.4 \times 10^{-7}$ & $6.8 \times 10^{-7}$ & $5.4 \times 10^{-6}$ \\
\hline$k_{6}^{0}\left(\mathrm{~cm} \cdot \mathrm{s}^{-1}\right)$ & $2.5 \times 10^{-6}$ & $5.3 \times 10^{-6}$ & $1.1 \times 10^{-4}$ \\
\hline$k_{7}^{0}\left(\mathrm{~mol} \cdot \mathrm{cm}^{-2} \cdot \mathrm{s}^{-1}\right)$ & $5.3 \times 10^{-9}$ & $5.2 \times 10^{-9}$ & $8.8 \times 10^{-9}$ \\
\hline$k_{2}\left(\mathrm{~mol} \cdot \mathrm{cm}^{-2} \cdot \mathrm{s}^{-1}\right)$ & $5.7 \times 10^{-13}$ & $1.7 \times 10^{-12}$ & $3.4 \times 10^{-12}$ \\
\hline$k_{3}\left(\mathrm{~mol} \cdot \mathrm{cm}^{-2} \cdot \mathrm{s}^{-1}\right)$ & $5.7 \times 10^{-13}$ & $1.7 \times 10^{-12}$ & $2.3 \times 10^{-12}$ \\
\hline$k_{5}\left(\mathrm{~cm} \cdot \mathrm{s}^{-1}\right)$ & $1.1 \times 10^{-9}$ & $2.4 \times 10^{-9}$ & $1.5 \times 10^{-9}$ \\
\hline$k_{6}\left(\mathrm{~cm} \cdot \mathrm{s}^{-1}\right)$ & $1.8 \times 10^{-8}$ & $4.0 \times 10^{-8}$ & $2.9 \times 10^{-7}$ \\
\hline$k_{7}\left(\mathrm{~mol} \cdot \mathrm{cm}^{-2} \cdot \mathrm{s}^{-1}\right)$ & $7.5 \times 10^{-17}$ & $5.6 \times 10^{-17}$ & $4.8 \times 10^{-14}$ \\
\hline$C_{i}^{0}=k_{2} / k_{5}\left(\mathrm{~mol} \cdot \mathrm{cm}^{-3}\right)$ & $5.3 \times 10^{-9}$ & $5.2 \times 10^{-9}$ & $8.8 \times 10^{-9}$ \\
\hline$C_{V_{\mathrm{o}}^{0}}^{0}=\chi k_{3} / 2 k_{6}\left(\mathrm{~mol} \cdot \mathrm{cm}^{-3}\right)$ & $4.8 \times 10^{-5}$ & $6.4 \times 10^{-5}$ & $1.2 \times 10^{-5}$ \\
\hline$C_{i}^{0} /\left(\frac{2}{\Omega}\right)$ & $0.4 \%$ & $0.5 \%$ & $1.6 \%$ \\
\hline$C_{V_{\mathrm{o}}^{0}}^{0} /\left(\frac{3}{\Omega}\right)$ & $0.03 \%$ & 1.0 & 0.8 \\
\hline$D_{i}\left(\mathrm{~cm} \cdot \mathrm{s}^{-1}\right)$ & & $0.03 \% 10^{-4}$ & $2.3 \times 10^{-3}$ \\
\hline$R_{g} / R_{d}$ & & & $0.006 \%$ \\
\hline
\end{tabular}

